\title{
The Heart Valve Society
}

\author{
2nd Annual Meeting, March 17-19, 2016, New York City, N.Y., USA
}

\section{Oral Award Presentations}

\section{Tissue Engineered Heart Valves}

\section{In-situ Heart Valve Tissue Engineering - Is On-the- Fly Preseeding with Bone Marrow-Derived Cells Required?}

\section{Anthal I.P.M. Smits, Virginia Ballotta, Anita Driessen-Mol, Frank P.T. Baaijens, Carlijn V.C. Bouten}

Eindhoven University of Technology, Eindhoven, Netherlands

Objective: In-situ heart valve tissue engineering is a prospering new concept based on bioresorbable, self-regenerating synthetic grafts, which accommodate host cell colonization and subsequent tissue formation upon implantation. On-the-fly preseeding of bone marrow-derived cells (BMCs) into such grafts has been employed as a one-step intervention to stimulate the in-situ regeneration, although the underlying mechanisms remain largely unknown. Using a combination of in-vitro and in-vivo studies, our aim here was to systematically investigate the role of preseeded BMCs in a synthetic heart valve scaffold on the regenerative process in-situ. Methods: 3-dimensional fibrous scaffolds were produced from custom-developed elastomers by electrospinning. To study the cross-talk between preseeded BMCs and circulating leukocytes, we employed in-vitro co-culture models, using human cells, in which preseeded scaffolds were subjected to hemodynamic loads (i.e. cyclic strain, shear stress). The cellular response was analyzed in terms of immunomodulatory cytokine secretion and extracellular matrix production. To study the in-vivo effect of preseeding, heart valves were manufactured from the electrospun material, which were implanted in the pulmonary position in sheep up to 6 months. Results: In-vitro, preseeded human BMCs abun- dantly secreted trophic factors, such as VEGF, MCP-1, and bFGF. This effect was synergistically enhanced in the presence of leukocytes, which secreted a cocktail of pro- and anti-inflammatory factors in response to the elastomeric scaffold (e.g. TNFa, IL-10). Surprisingly, leukocytes in the scaffold were found to express a-SMA and contribute directly to ECM production (mainly sGAG and collagen III), depending on the level of strain. In-vivo, we observed active remodeling of the valve scaffold in-situ. The long-term invivo effect of $\mathrm{BMC}$ preseeding on this regenerative process is currently investigated with our clinical partner. Conclusions: Our results illustrate that preseeded BMCs secrete paracrine factors, important to the in-situ tissue engineering process, in synergy with the inflammatory milieu as set by the circulating leukocytes. These findings contribute to the development of completely cell-free synthetic heart valve grafts, which would be advantageous for clinical translation.

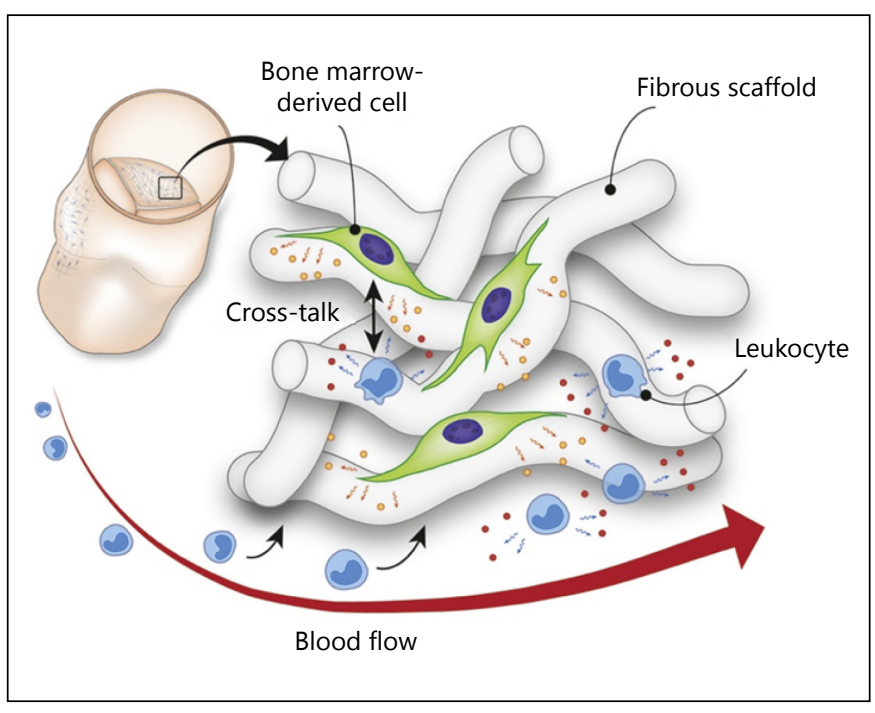

Fig. 1. (for Abstract of Anthal I.P.M. Smits).

\section{KARGER}

E-Mail karger@karger.com www.karger.com/crd
C) 2016 S. Karger AG, Basel

$0008-6312 / 16 / 1342-0136 \$ 39.50 / 0$ 


\section{Performance of Size-Reduced Bicuspidalized Decellularized Pulmonary Valve Conduits In Growing Sheep Model}

Karolina Theodoridis, Igor Tudorache, Andres Hilfiker, Tanja Mayer, Samir Sarikouch, Christoph Bara, Alexandru Calistru, Thomas Breymann, Alexander Horke, Axel Haverich, Serghei Cebotari

Hannover Medical School, Hannover, Germany

Background: Decellularization of pulmonary valve allografts leads to reduction of tissue immunogenicity, in-vivo autologous repopulation and ability to grow. On the other hand, the shortage of small-size homograft valves, represents an essential constrain for pediatric RVOT reconstructive surgery. In this study we evaluated the performance of decellularized and surgically size-reduced (bicuspidalized) allografts in juvenile growing sheep model. Methods: Ovine pulmonary valve conduits (PVC) were harvested from adult animals (75 to $80 \mathrm{~kg}$ ). PVC were decellularized using a detergent mixture of sodium desoxycholate and sodium dodecylsulphate. Orthotopic implantations were conducted in juvenile sheep $(27.1 \pm 2.2 \mathrm{~kg})$. Before implantation, one of the pulmonary leaflets was resected and the opened PVC was reclosed by getting both free commissures in the suture line. Functional performance and size development of decellularized bicuspidalized PVC were investigated by transesophageal echocardiography and cardiac magnetic resonance. PVC development was compared to physiological RVOT development of non-operated animals (control group). Bicuspidalized PVC were evaluated histologically after explantation. Results: The size of PVC harvested from adult animals varied from 21 to $24 \mathrm{~mm}$ (mean $22.8 \pm 2.4 \mathrm{~mm}$ ). Detergent decellularization converted PVC in cell-free scaffolds. Surgical size reduction of decellularized PVC resulted in competent bicuspid valves, except one, which exhibited a moderate insufficiency. Implantations were successful in all cases. There were 22 months follow-up in 5 animals. No valve dysfunction was observed, mean gradient was $3.0 \pm$ $2.2 \mathrm{~mm} \mathrm{Hg}$ and PVC moderate regurgitation rate was revealed in two animals. During the follow-up, animal body weight increased from $27.1 \pm 2.2$ to $71.9 \pm 12.1 \mathrm{~kg}$, and accordingly annulus size increased from $14.6 \pm 3.0 \mathrm{~mm}$ to $20.6 \pm 3.6 \mathrm{~mm}$, which was comparable to non-operated group (weight from $25.0 \pm 0.7$ to $71.0 \pm 4.4$ $\mathrm{kg}$ and annulus size from $18.3 \pm 2.0$ to $22.4 \pm 2.0 \mathrm{~mm}$ ). Histological analyses of the explants revealed repopulation of decellularized PVC scaffolds with autologous cells, and no signs of valve degeneration were documented. Conclusions: Decellularized bicuspid PVC exhibited good functional in-vivo performance, autologous cell repopulation and annulus enlargement together with physiologic growth of the animal. Surgical size reduction of decellularized PVC may expand the availability of small sized-allografts for RVOT reconstruction in children.

\section{How Close Are We to Fully Engineering a Living Heart Valve? A Tale of Scaffolds, Stem Cells, Bioreactors and Sheep}

Marius M. Harpa ${ }^{1}$, lonela Movileanu' ${ }^{1}$, Allison Kennamer' Nicholas Rierson ${ }^{2}$, Ovidiu Cotoi ${ }^{1}$, Horatiu Suciu ${ }^{1}$, Victor Raicea ${ }^{1}$, Terezia Preda ${ }^{1}$, Dan Nistor ${ }^{1}$, Klara Branzaniuc ${ }^{1}$, Radu Deac ${ }^{1}$, Simona Gurzu ${ }^{1}$, Lucian Harceaga ${ }^{1}$, Peter Olah ${ }^{1}$, Carmen Sircuta ${ }^{1}$, Agneta Simionescu${ }^{2}$, Michael Dandel ${ }^{1}$, Dan Simionescu ${ }^{2}$

${ }^{1}$ University of Medicine and Pharmacy, Targu Mures, Romania; ${ }^{2}$ Clemson University, Clemson, SC, USA

Objective: Our long term goal is to create implantable living heart valves. This research is in its infancy and progress has shown that the potential and challenges are significant. Our current goal was to investigate the in vitro and in vivo regenerative potential of xenogeneic acellular aortic and pulmonary roots seeded with autologous stem cells. Methods: First we prepared fully acellular porcine aortic and pulmonary roots using detergents and enzymes. We then injected adipose-derived stem cells (ASCs) in the interstitium of the valve cusps and tested them in our sterile pulsatile bioreactor systems at physiologic pressures and flow conditions for 2 weeks. Controls consisted of seeded valves incubated in a rotator device which allowed for fluid exchange and slight movement of the cusps. For in vivo studies we isolated autologous ASCs from juvenile sheep, injected them within the acellular valve cusps and implanted them as valved conduits ( $n=6$ per group) in the RVOT for 20 weeks. Unseeded valves served as controls. Results: Acellular aortic and pulmonary roots showed excellent preservation of the matrix fibers and suitable hemodynamics in vitro. Seeded control valves which were only rotated slowly retained most ASCs and their phenotype resembled that of valvular interstitial cells. Unexpectedly, ASC-seeded valves subjected immediately to bioreactor testing were found completely devoid of seeded cells. In vivo shortterm echographic follow-up showed that all implanted valves were fully functional in the first 4 weeks. However, after 5 weeks, ASCseeded valves failed prematurely by progressive covering with intra-luminal host fibrous tissue overgrowth resulting in right ventricular failure, while non-seeded valves were completely free of fibrous overgrowth at 20-22 weeks post-operatively. Cusps explanted from all implant groups showed complete absence of any interstitial cells (seeded or infiltrated) and lack of inflammation, calcification or thrombogenesis. Conclusions: Acellular roots are excellent valve scaffolds with great potential for tissue engineering. ASCs appear to be very vulnerable to valve-specific mechanical forces and the early demise of stem cell seeded implanted valves could be related to pannus formation in sheep. Ongoing studies focus on progressive mechanical conditioning of ASC-seeded valves in bioreactors for optimal ASC adaptation before implantation. 


\section{Echocardiographic Leaflet Performance of Stentless Extracellular Matrix Tricuspid Tube Graft in a Porcine Model}

Diana M. Ropcke, Christine Ilkjaer, Soren N. Skov, Marcell J. Tjornild, Anders V. Sorensen, Henrik Jensen, Sten L. Nielsen

Aarhus University Hospital, Aarhus C, Denmark

Background: Stentless porcine extracellular matrix tricuspid tube grafts have been developed for tricuspid valve reconstruction (see figure 1). The study purpose was to compare echocardiographic performance and coaptation of native and tube graft tricuspid anterior and septal leaflets in an acute porcine model. Methods: Fourteen $65 \mathrm{~kg}$ pigs were randomized to tube graft $(\mathrm{n}=$ 7 ) or control with native valve preservation $(n=7)$. Epicardial echocardiography was performed at two time points: Firstly, at baseline after sternotomy, and secondly following valve surgery according to randomization. Data collection was performed after weaning off cardiopulmonary bypass and hemodynamic stabilization. Results: No baseline differences were found ( $p>0.05)$. All valves were competent with only a mild central jet in two tube grafts and two native valves. The posterior leaflet was not assessed because of vast irregularity and size differences between pigs. Anterior-septal leaflet coaptation length $(18 \pm 6 \mathrm{~mm}$ vs. $6 \pm 1 \mathrm{~mm}, \mathrm{p}<$ $0.0005)$, coaptation height $(20 \pm 9 \mathrm{~mm}$ vs. $9 \pm 4 \mathrm{~mm}, \mathrm{p}<0.005)$ and anterior leaflet length $(31 \pm 1 \mathrm{~mm}$ vs. $22 \pm 2 \mathrm{~mm}, \mathrm{p}<0.00005)$ were all significantly larger in the tube graft valves. The billowing index $(0.8 \pm 0.1$ vs. $0.9 \pm 0.1, \mathrm{p}>0.05)$ and tenting height $(5 \pm 1 \mathrm{~mm}$ vs. $4 \pm 2 \mathrm{~mm}, \mathrm{p}>0.1$ ) were not significantly different. Leaflet excursion angles for both leaflets were not significantly different between native and tube graft valves $(p>0.1)$. The systolic angle for both anterior and septal leaflets was significantly smaller in the tube graft valve with a negative value, indicative of prolapse into the atrium $(\mathrm{p}=0.01)$. Conclusions: In a porcine experimental model, a competent tricuspid valve was constructed using extracellular matrix tube graft material. Coaptation geometry between the anterior and septal leaflet was significantly different from na-
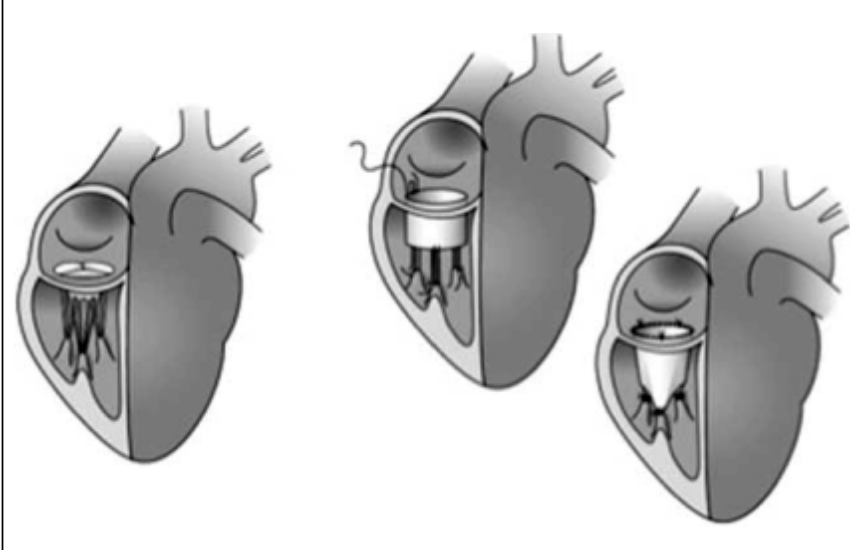

Fig. 1. (for Abstract of Diana M. Ropcke). tive valves with an increased coaptation zone due to excessive leaflet tissue in the tube graft valves. The tube graft tends to prolapse into the atrium due to excessive tissue, but this does not compromise the motion and competence of the leaflets.

\section{Bioresorbable Reinforcement of Neopulmonary Trunk Avoids Sovrapulmonary Stenosis in Arterial Switch Operation: Biomechanics, Growth and Remodelling}

\author{
Mario Fittipaldi ${ }^{1}$, Cristiano Spadaccio ${ }^{2}$, Antonio Nenna ${ }^{3}$, \\ Massimiliano Fraldi ${ }^{4}$, Francesco Nappi ${ }^{5}$ \\ ${ }^{1}$ Great Ormond Street Hospital for Children NHS Foundation \\ Trust, London, United Kingdom; ${ }^{2}$ Golden Jubilee National \\ Hospital, Glasgow, United Kingdom; ${ }^{3}$ University Campus \\ Bio-Medico, Rome, Italy; ${ }^{4}$ University Federico II of Naplesples, \\ Naples, Italy; ${ }^{5}$ Centre Cardiologique du Nord, Paris, France
}

Background: Supravalvular pulmonary stenosis (SPS) remains a worrisome complication in the long term of simple transposition of the great arteries. Issues of scar formation and inability to growth are considered at the base of this phenomenon. We pioneered the use of a tissue engineering approach to guide the maturation and the growth of pulmonary autograft in Ross procedure with encouraging results. We therefore sought to investigate the use of a similar approach in the reconstruction of neopulmonary trunk (NPT) with the aim to recreate a vascular conduit that retains the structural architecture and the same biological potential of native pulmonary artery and prevents long-term SPS. Methods: A model of NPT reconstruction in growing lambs was used. NPT was constructed with autologous pericardium and reinforced with a four-layered knitted polydioxanone mesh (PDS $n=15$ ) or left unreinforced (control $\mathrm{n}=15$ ). Animals were left growing for 6 months and angiographic and transesophageal echocardiographic measurements were performed at day 1,3 months and at the end of the study together with histological analysis. A physicalmathematical model was developed to integrate and validate the experimental animal model. Results: Control group developed SPS while PDS reinforcement allowed a progressive increase in diameter with an optimal size match to the pulmonary artery of healthy growing controls. Histological analysis showed in the control group disruption of endothelial lining with fibrosis, while demonstrated in the PDS group a trilaminar vessel-like structure. Conclusions: The bioresorbable reinforcement induced the formation over time of a neopulmonary conduit able to both face the hemodynamic load of the pulmonary system and guarantee a harmonious increase in size during the somatic growth. 


\section{Bioabsorbable Vascular Graft - The First Step Towards Development of the Next Generation Heart Valves}

\author{
Leo Bockeria ${ }^{1}$, Alex Kim ${ }^{1}$, Konstantin Shatalov', \\ Vladimir Makarenko ${ }^{1}$, Oleg Svanidze ${ }^{2}$ \\ ${ }^{1}$ Bakoulev Center for Cardiovascular Surgery, Moscow, \\ Russian Federation; ${ }^{2}$ Xeltis AG, Zurich, Switzerland
}

Objective: The bioabsorbable graft material is designed to attract patient's own cells that trigger a cascade of physiological events leading to natural tissue growth. After implantation the graft is gradually replaced by the components of native tissue, developing and organizing themselves into a fully functioning blood vessel. The vascular graft is an essential part of the valved conduit being developed for RVOT reconstruction. This feasibility study evaluated safety and performance of a novel bioabsorbable vascular graft in pediatric patients with univentricular congenital malformation, undergoing hemodynamic correction with an extracardiac cavopulmonary conduit. Methods: From October 2013 to February 2014 five patients (aged 4 to 12 years) with single ventricle congenital malformation have been implanted with the newly developed biodegradable vascular grafts (18 and $20 \mathrm{~mm}$ in diameter) connecting inferior vena cava with the right pulmonary artery. Patients were followed for 1, 3, 6 and 12 months after surgery with the graft assessment performed by echocardiography, CT-scan and MRI, including 4-D flow. Results: All 5 patients have been successfully recovered from the procedure and completed follow-up according to the study protocol. No device related adverse events were reported. Two patients with persistent pleural effusions required catheter based interventions to occlude collaterals between aorta and pulmonary artery. At six months postoperatively significant improvement in patients' general condition were noticed. Imaging studies demonstrated anatomical (conduit diam-

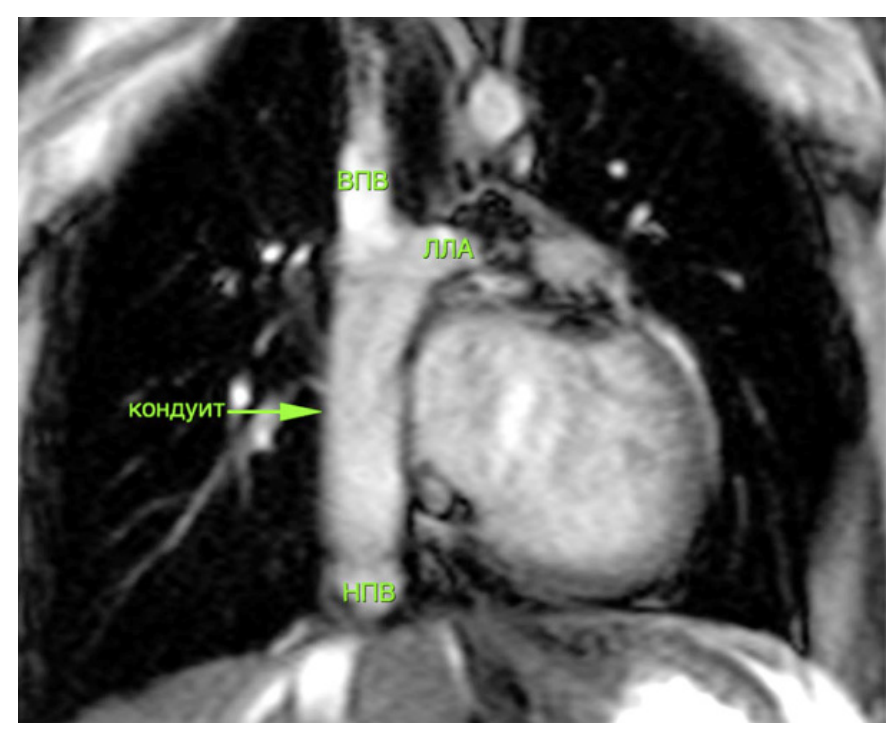

Fig. 1. (for Abstract of Leo Bockeria). eter, length and wall thickness) and functional (blood flow pattern) stability of the grafts in all patients with no significant changes at twelve months compared to early post-operative data. Conclusions: Initial clinical experience and study outcomes thus far suggest that this novel technology has the potential to improve cardiac and vascular surgical procedures by reducing permanent implant related complications. Longer follow-up, however, is needed to fully assess the long-term effectiveness of biodegradable vascular grafts including ability to grow.

\section{Experimental and Computational Valve Tissue Studies}

A Nitinol Mesh Scaffold for Heart Valve Engineering

Seyedhamed Alavi, Marc S. Baliarda, Lorenzo Valdevit, Arash Kheradvar

University of California, Irvine, Irvine, CA, USA

Objective: Valvular heart disease is the third-most common cause of heart problems in the United States. Replacement of dysfunctional valves markedly reduces the morbidity and mortality associated with valve disease. We are in process of developing a novel hybrid heart valve whose leaflets are composed of an extrathin superelastic Nitinol mesh, to be tightly enclosed by multiple layers of smooth muscle, fibroblast/myofibroblast, and endothelial cells of the recipient. The current work explores dynamic characteristics of the tri-leaflet valve scaffold with Nitinol mesh leaflets. Methods: The superelastic nitinol mesh leaflets were developed through acid etching of flat 25 microns thick nitinol sheets. Each three mesh leaflets were sewn to a tri-leaflet valve frame made of titanium that came with three separate stands made of flexible mylar all covered and connected with biocompatible polyester surgical fabric (figure 1a). The developed valves were tested in a heart flow simulator system under physiological conditions. A finite element model was also defined to computationally simulate the function and to optimize the design of the valve components. Results: The valve dynamics, computationally and experimentally, were similar with calculated effective orifice area (EOA) of $76.28 \%$ and $75 \%$, respectively. Based on our computational results, we noticed EOA reduces as the leaflet thickness increased, where thicknesses between 20 and 35 microns allowed for an EOA larger than $70 \%$. The material stiffness of the stands seems not to significantly alter the EOA; however, it strongly affects the stress distribution over the leaflets. The optimal condition was achieved with stands' Young's modulus of $3000 \mathrm{MPa}$ and leaflets' thickness of $25 \mathrm{mi}$ crons (figure $1 \mathrm{~b}$ and $1 \mathrm{c}$ ). We also observed that the dynamic movement of the stands and the presence of a fabric connecting the nitinol leaflets to the frame are the key features for proper function of these valves. Conclusions: A comprehensive computational model that properly predicts valve dynamics has been developed. The experimental and computational studies both suggest that nitinol valves display superior hemodynamic behavior compared to many bioprosthetic valves. 
Fig. 1.a The tri-leaflet valve scaffold with Nitinol mesh leaflets; b Von Mises stresses on the Nitinol leaflets at the instant of maximum negative pressure (i.e. closed configuration); c The instant of maximum positive pressure (open configuration) for the situation where leaflets are connected to the frame with an intermediary surgical fabric (for Abstract of Arash Kheradvar).
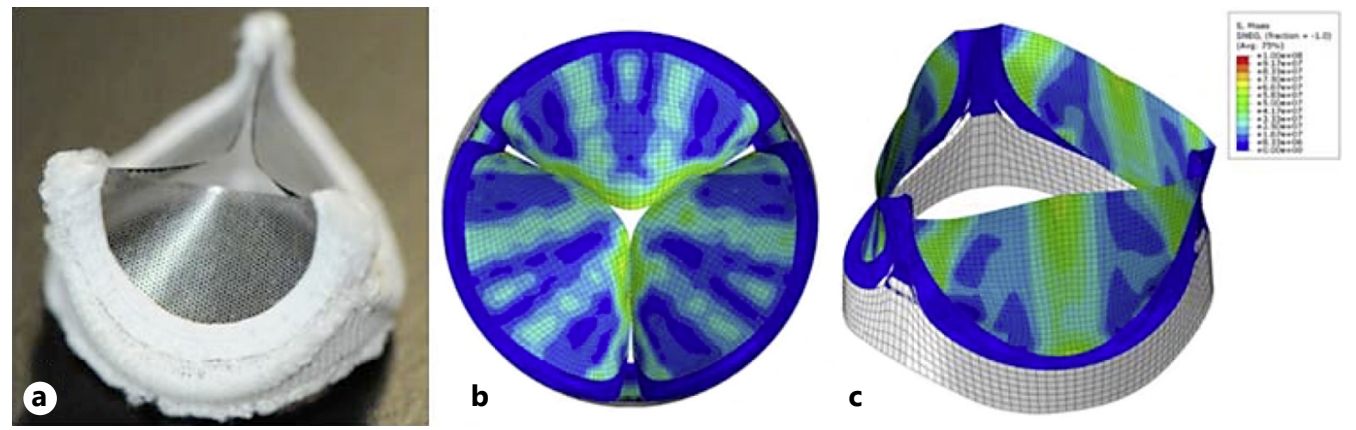

\section{Heart Valve Outcomes}

\section{Fresh Decellularized Pulmonary Valve Allografts for RVOT Reconstruction during the Ross Operation - Clinical Outcomes at 10 Years}

\section{Francisco D. Costa ${ }^{1}$, Eduardo Balbi Filho², Rafael Torres², Daniele Colatusso ${ }^{1}$, Claudinei Colatusso ${ }^{1}$, Allysson Calixto ${ }^{2}$, Marise Costa \\ ${ }^{1}$ Santa Casa de Curitiba PUCPR, Curitiba, Brazil; ${ }^{2}$ Instituto de Neurologia e Cardiologia de Curitiba, Curitiba, Brazil}

Background: Decellularized allografts are a promising alternative for RVOT reconstruction, however, limited long term data is available in the literature. The aim of this study is to evaluate the long-term clinical, echocardiographic and CT scan results of fresh decellularized pulmonary allografts for RVOT reconstruction during the Ross operation. Methods: Between October 2005 and September 2015, 155 Ross operations were performed using a fresh decellularized pulmonary valve allograft. 121 were male, and mean age was $31 \pm 15$ years. Fortyeight patients were less than 21 years of age. Conduit dysfunction was defined as any peak gradient greater than $40 \mathrm{~mm} \mathrm{Hg}$ or insufficiency grade III or IV. Graft diameter and conduit or leaflet calcification was analyzed by CT scan studies. Mean followup was 4.8 years ( $0.1-10$ years) and was complete in $95 \%$ of the cases. Results: Early mean peak gradient was $10 \pm 3 \mathrm{~mm} \mathrm{Hg}$ and at the latest follow-up $15 \pm 4 \mathrm{~mm} \mathrm{Hg}$. Only two patients exhibited a late peak gradient $>40 \mathrm{~mm} \mathrm{Hg}$. Moderate or severe regurgitation was present in two cases. Freedom from conduit dysfunction at 10 years was $92 \%$ (CL 95\%, 85-99\%). Two patients were reoperated due to bacterial endocarditis on the allograft but there was no reoperation due to primary conduit dysfunction. Late CT scan studies revealed preserved allograft diameters with no retraction and minimal or absent calcification in the cusps and/or conduit wall. Conclusions: Fresh decellularized allografts for RVOT reconstruction during the Ross operation are associated with excellent hemodynamic performance and low incidence of conduit dysfunction up to ten years of follow-up. The most striking feature was absence of calcification, even in patients younger than 20 years of age. Our results suggest that de- cellularized allografts may be superior to conventional cryopreserved allografts, especially in children, adolescents and young adults.

\section{Human Factors during Mitral Valve Replacement Surgery Are Associated with Adverse Events}

Jock N. McCullough ${ }^{1}$, Alexander Iribarne ${ }^{1}$, David C. Charlesworth ${ }^{2}$, Robert A. Cough ${ }^{3}$, Joseph P. DeSimone ${ }^{1}$, Carmine Frumiento ${ }^{4}$, Robert S. Kramer ${ }^{5}$, Bruce J. Leavitt 6 , Charles A. Marrin ${ }^{1}$, Jeremy R. Morton ${ }^{5}$, Joseph D. Schmoker 6 , Benjamin M. Westbrook ${ }^{2}$, Anthony W. DiScipio

'Dartmouth-Hitchcock Medical Center, Lebanon, $\mathrm{NH},{ }^{2}$ Catholic Medical Center, Manchester, $\mathrm{NH},{ }^{3}$ Eastern Maine Medical Center, Bangor, ME, ${ }^{4}$ Central Maine Medical Center, Lewiston, $\mathrm{ME},{ }^{5}$ Maine Medical Center, Portland, ME, ${ }^{6}$ University of Vermont Medical Center, Burlington, VT, USA

Objective: This study was designed to identify variables associated with excess mortality and adverse events following mitral valve replacement (MVR) surgery. Methods: We identified 153 deaths in the Northern New England Cardiovascular Disease Registry among patients undergoing MVR between 1/1/1996 through $6 / 1 / 2013(n=1987)$. Emergent, endocarditis and multi valve procedures were excluded. The operative reports of the patients who died were reviewed for patient specific and intraoperative complications. Patients who died were then matched by propensity score to a similar group of survivors based on preoperative characteristics. There was no significant difference between death and survivor groups in preoperative characteristics after propensity matching. The operative reports of the matched groups were then retrieved and data abstracted. All operative reports were then blinded and submitted to a committee of cardiac surgeons for scoring of intraoperative surgical human factors using predefined coding rules. The primary factors analyzed included patient-sick, technical-surgeon, technical-patient, smooth case or cannot assess. The blinded panel was also asked whether they agreed with intraoperative decision making. Results: Patients who died following MVR were more likely to be left with a paravalvular leak $(11.7 \%$ versus $3.1 \%, \mathrm{p}=0.028$ ) and/or have had a leaflet sparing MVR $(61.7 \%$ versus $38.2 \%, \mathrm{p}<0.001)$. Blinded analysis of operative reports demonstrated that patients who died following surgery were 
equally likely to be classified as sick preoperatively (11.8\% versus $6.3 \%, \mathrm{p}=0.12$ ), had a higher incidence of technical issues attributed to the surgeon ( $43.3 \%$ versus $18.0 \%, \mathrm{p}<0.001)$, and were less likely to be classified as a smooth case $(25.2 \%$ versus $62.5 \%$, p < 0.001 ). The blinded reviewing surgeons were more likely to agree with intraoperative decision making in patients who survived ( $90.3 \%$ versus $78.7 \%, p=0.012$ ). Conclusions: Technical decisions and unanticipated complications during MVR are significantly associated with procedural mortality. Even after controlling for baseline characteristics mortality following MVR is associated with human factors.

\section{Familial Clustering of Mitral Regurgitation in the Community}

\author{
Francesca N. Delling ${ }^{1}$, Shuo $L^{2}{ }^{2}$, Qiong Yang ${ }^{3}$, \\ Vanessa Xanthakis' ${ }^{2}$ Birgitta Lehman ${ }^{4}$,Ewa Osypiuk ${ }^{4}$, \\ Plamen Stantchev ${ }^{4}$, Emelia J. Benjamin ${ }^{2}$, \\ Ramachandran S. Vasan ${ }^{2}$
}

${ }^{1}$ Beth Israel Deaconess Medical Center, Boston, MA, ${ }^{2}$ Boston University School of Medicine, Boston, MA, ${ }^{3}$ Boston University School of Public Health, Boston, MA, ${ }^{4}$ Framingham Heart Study, Framingham, MA, USA

Objective: As in mitral valve prolapse, leaflet growth in response to tethering in secondary mitral regurgitation (MR) is linked to a reactivation of developmental pathways. These findings suggest similar regulatory mechanisms and common genetic susceptibility for primary and secondary MR. Our objective is to evaluate the potential familial clustering of MR regardless of etiology. Methods: Study participants were Framingham Heart Study Generation 3 (Gen 3; Exam 1) and Offspring (Gen 2; Exam 6 or 8) cohort participants undergoing routine echocardiography. MR was defined as $\geq$ mild regurgitation on Doppler color flow imaging. For each participant, presence of parental or sibling MR was defined as having at least one Gen 2 parent (for Gen 3) or Gen $2 /$ Gen 3 sibling, respectively, with $\geq$ mild MR. We estimated the association of parental MR and sibling MR with the prevalence of MR in Gen 3 offspring and in Gen 2/Gen 3 siblings using multivariable logistic regression with generalized estimating equations. We also estimated heritability of MR in full pedigrees (539 pedigrees, 7580 individuals) using the SOLAR liability threshold model. We adjusted for age, sex, systolic/diastolic blood pressure, and body mass index. Results: Among $2682 \mathrm{Gen}$ 3 participants (53.2\% women, mean age 40 years) with parental information, 352 had MR (24 [0.9\%] had mitral valve prolapse). Presence of both parental and sibling MR was associated with a greater odds of prevalent MR in offspring and sibs, respectively: $14 \%(\mathrm{n}=262)$ offspring with parental MR had MR compared with $10 \%(\mathrm{n}=90)$ without parental MR (multivariable-adjusted odds ratio [OR], 1.34; 95\% confidence interval [CI] 0.98-1.73; $\mathrm{p}=$ $0.045) ; 28 \%(\mathrm{n}=630)$ of siblings with sibling MR had MR compared with $18 \%(\mathrm{n}=1630)$ without sibling MR; multivariableadjusted OR, 1.25; 95\% CI, 1.05-1.47; $\mathrm{p}=0.008$ ]. Heritability of MR estimated by SOLAR was 0.15 (standard error $=0.04, \mathrm{p}=$ 1e-4). Conclusions: Familial clustering of MR exists in the community, supporting a genetic susceptibility common to primary and secondary etiologies of MR. Further studies are needed to elucidate the common regulatory pathways leading to MR irrespective of etiology.

\section{Outcome after Mechanical Aortic Valve Replacement in Adult Patients Under 55 Years: A Systematic Review, Meta-Analysis and Microsimulation}

\section{Nelleke M. Korteland, Jonathan R.G. Etnel, Bardia Arabkhani, Arezo Mohamad, M. Mostafa Mokhles, Ad J.J.C. Bogers, Johanna J.M. Takkenberg}

Erasmus MC, Rotterdam, Netherlands

Background: Our study aims to provide a detailed overview of reported outcome after mechanical aortic valve replacement (AVR) in young adults and to estimate life expectancy and lifetime risk of morbidity. Methods: A systematic literature search was conducted for papers published between January 1995 and April 2014 reporting clinical outcome after isolated AVR with a mechanical prosthesis in cohorts with a mean age between 18 and 55 years. A microsimulation model based on the pooled outcome estimates of studies on bileaflet prostheses was used to calculate age-specific life expectancy and lifetime risk of valve-related morbidity. Results: Forty-seven publications, encompassing a total of 13044 patients with 84,171 patientyears of follow-up (pooled mean follow-up: 6.45 years), were included. Mean age at surgery was $49.8 \pm 12.1$ years. Early and late mortality were $4.09 \%$ (95\% CI: 3.28-5.10\%) and 1.90\%/yr (95\% CI: $1.61-2.24 \% / \mathrm{yr}$ ), respectively, and $41.1 \%$ of late deaths were valverelated. Thromboembolism (1.00\%/yr; 95\% CI: 0.79-1.26\%/yr), major bleeding (0.87\%/yr; 95\% CI: $0.70-1.09 \% / y r)$ and endocarditis (0.44\%/yr; 95\% CI: $0.36-0.55 \% / y r)$ were the most frequent valverelated events, followed by nonstructural valve dysfunction $(0.50 \% /$ yr; 95\% CI: $0.31-0.80 \% / y r)$ and valve thrombosis (0.16\%/yr; 95\% CI: $0.10-0.25 \% / \mathrm{yr})$. Although structural valve deterioration was rare (0.07\%/yr; 95\% CI: 0.04-0.12\% yr), there was a considerable risk of reintervention $(0.63 \% / \mathrm{yr}$; $95 \% \mathrm{CI}: 0.50-0.80 \% / \mathrm{yr})$, mostly due to nonstructural valve dysfunction and endocarditis. Microsimulation-based estimates of age-specific life expectancy and lifetime risk of valve-related morbidity are presented in figure 1 . Conclusions: This review shows excellent long-term durability of mechanical prostheses in young adult patients, although a substantial proportion of patients experience valve-related morbidity and life expectancy remains impaired compared to the general population. 


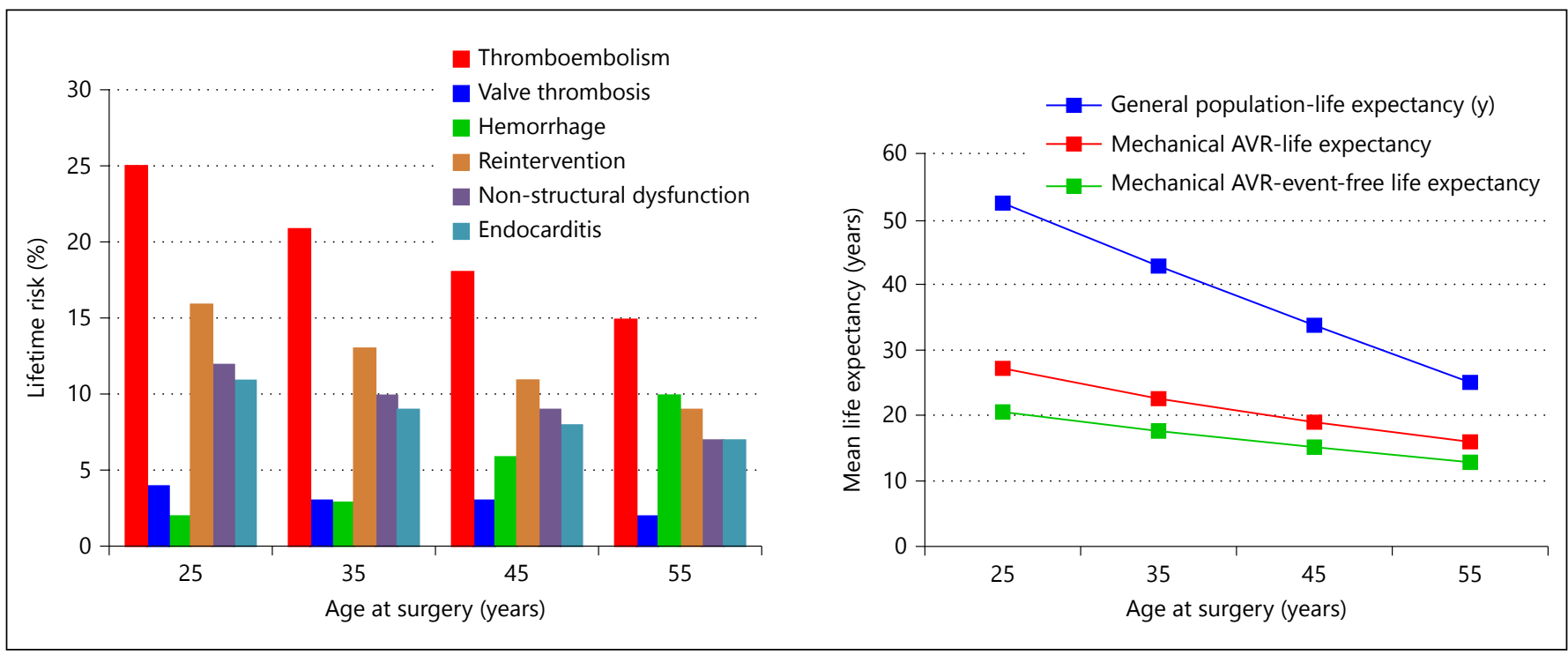

Fig. 1. (for Abstract of Nelleke M. Korteland).

Table 1. (for Abstract of Zhenhua Li)

\begin{tabular}{|c|c|c|c|c|c|c|}
\hline & Men MBP group & Men FED group & $\begin{array}{l}\text { Men } \\
\text { p value }\end{array}$ & Women MBP group & Women FED group & $\begin{array}{l}\text { Women } \\
\mathrm{p} \text { value }\end{array}$ \\
\hline Age, years & $55 \pm 11(\mathrm{n}=88)$ & $57 \pm 10(\mathrm{n}=252)$ & 0.07 & $52 \pm 13(\mathrm{n}=54)$ & $57 \pm 13(n=62)$ & 0.06 \\
\hline $\mathrm{LVEDV} / \mathrm{BSA}, \mathrm{ml} / \mathrm{m}^{2}$ & $125.41 \pm 24.30$ & $115.76 \pm 25.10$ & $0.003^{*}$ & $107.15 \pm 17.57$ & $108.78 \pm 20.61$ & 0.67 \\
\hline LVESV/BSA, $\mathrm{ml} / \mathrm{m}^{2}$ & $41.31 \pm 18.88$ & $38.67 \pm 16.58$ & 0.3 & $40.44 \pm 16.69$ & $33.89 \pm 17.28$ & 0.06 \\
\hline LVEDD/BSA, $\mathrm{mm} / \mathrm{m}^{2}$ & $29.39 \pm 4.14$ & $27.72 \pm 5.32$ & $0.008^{*}$ & $32.06 \pm 3.15$ & $30.81 \pm 5.16$ & 0.13 \\
\hline LVESD/BSA, $\mathrm{mm} / \mathrm{m}^{2}$ & $17.89 \pm 2.72$ & $16.78 \pm 3.98$ & $0.01^{*}$ & $19.91 \pm 2.48$ & $18.64 \pm 3.43$ & $0.03^{*}$ \\
\hline LVEF, \% & $65.68 \pm 4.9$ & $65.64 \pm 4.60$ & 0.57 & $64.41 \pm 5.4$ & $66.12 \pm 4.95$ & 0.1 \\
\hline Rvol, ml/beat & $74.91 \pm 28.37$ & $81.16 \pm 28.90$ & 0.08 & $55.66 \pm 19.09$ & $76.42 \pm 25.38$ & $<0.0001^{*}$ \\
\hline LV mass index & $125.57 \pm 24.72$ & $119.89 \pm 22.05$ & $0.047^{*}$ & $112.98 \pm 21.33$ & $110.54 \pm 23.70$ & 0.57 \\
\hline
\end{tabular}

* Statistically significant.

\section{Computed Tomography and Echo Evaluation of Left Ventricular Remodeling in Patients with Severe Degenerative Mitral Regurgitation}

Zhenhua Li ${ }^{1}$, Rakesh M. Suri ${ }^{2}$, Thomas A. Foley ${ }^{3}$, Eric E. Williamson ${ }^{3}$, Maurice Enriquez-Sarano', Hector I. Michelena ${ }^{1}$

${ }^{1}$ Division of Cardiovascular Diseases and Internal Medicine, Mayo Clinic, Rochester, MN, ${ }^{2}$ Department of Thoracic and Cardiovascular Surgery, Cleveland Clinic, Cleveland, $\mathrm{OH}$, ${ }^{3}$ Department of Radiology, Mayo Clinic, Rochester, MN, USA

Objective: Valvular phenotypes in degenerative mitral regurgitation (MR) range from myxomatous bileaflet prolapse (MBP) to fibroelastic deficiency (FED). We sought to determine patterns and predictors of left ventricular (LV) remodeling within these degenerative phenotypes. Methods: Between 2008 and 2014, 459 consecutive patients with severe degenerative MR underwent ECG-gated cardiac CT prior to robotically-assisted mitral valve repair. LV ejection fraction (LVEF), LV end-diastolic volume (LVEDV) and LV end-systolic volume (LVESV) were quantitated by CT and corrected by body surface area (BSA). LV mass index, LV end-diastolic diameter (LVEDD), LV end-systolic diameter (LVESD), and regurgitant volume (Rvol) were quantitated by echo. Mitral valve phenotype was determined by direct surgeon inspection. We compared cardiac remodeling parameters between MBP and FED stratified by gender. Results: A total of $456(75 \%$ men) patients had complete CT and echo data (table 1). Despite a higher Rvol in FED men ( $p=0.08$ for a trend), MBP men had larger LVEDV/BSA ( $\mathrm{p}=0.003)$, as well as larger LV mass index $(\mathrm{p}=0.047)$. Despite a significantly higher Rvol in FED women $(\mathrm{p}<$ 
0.0001), their LVEDV/BSA and LV mass index were not larger than MBP women ( $p>0.5$ ), but LVESV/BSA and LVESD/BSA were larger in $\mathrm{BD}$ women ( $\mathrm{p}=0.06$ and $\mathrm{p}=0.03$, respectively). Multivariate analysis of the entire group revealed that mitral valve MBP phenotype $(\mathrm{p}=0.001)$, higher Rvol $(\mathrm{p}<0.001)$, and male gender $(\mathrm{p}=0.008)$ were independently associated with LVEDV/ BSA, while only MBP phenotype $(\mathrm{p}=0.03)$ was independently associated with LVESV/BSA. Conclusions: In severe degenerative $\mathrm{MR}$, cardiac remodeling is a function of MR severity but also dependent on valvular phenotype and gender. The MBP phenotype is associated with a more prominent adverse diastolic LV remodeling response in men, and worse systolic remodeling in women. These findings have potential outcomes and surgical-timing thresholds implications.

\section{Restrictive Indications for the Ross Procedure Improve Long Term Outcomes}

\section{Zohair Y. Alhalees, Valeria Pergola, Giovanni Di Salvo, Maie Alshahid, Bahaa Fadel, Mohammed Alamri, Ziad Issa, Mamdouh Alahmadi, Saud Aloufi}

KFSHRC, Riyadh, Saudi Arabia

Background: Aortic valve replacement using the patient's own pulmonary valve (Ross Procedure) has proven to be a procedure with limited risks and good long term results in experienced hands. It is particularly suited for younger patients including neonates and infants. There is concern however in reference to progressive aortic root dilatation with time that may lead to aortic regurgitation (AR) and need for reoperation. Methods: January 1990 to December 2012, 537 patients underwent the Ross Procedure at our institutions. Among these, 357 (69\% males, mean age $29.4 \pm 3.9$ years) had complete clinical and echocardiographic follow up (mean $13.4 \pm 3.9$ years). The autograft was implanted as a fullstanding root with coronary transfer. Selective aortic root reinforcement was utilized in patients with dilated aortic roots. Seventy four patients with small aortic annulus \pm left ventricular outflow tract (LVOT) hypoplasia. Patients were divided into 2 groups according to pathology (group I with rheumatic etiology and group II with nonrheumatic etiology - mostly congenital aortic valve disease). Results: Hospital mortality was low (1.2\%). Reoperation was required in $36 \%$ of patients with a mean time for redo surgery of $6.7 \pm 4.2$ years. A second redo surgery was required in $3 \%$. Homograft survival was $13 \pm 4$ years. Reoperation on the neoaortic valve was required mostly for patients in group I (rheumatic patients with pure aortic valve regurgitation being the main hemodynamic manifestation and with dilated aortic roots $>27 \mathrm{~mm}$ ). These were mostly from our early experience. Patients in group II with aortic stenosis being the main hemodynamic manifestation had almost no autograft related reoperations. Children who required the Mini-Ross-Konno procedure showed no progressive AR, no recurrent AS or LVOTO in addition to normal growth. Conclusion: Autograft failure is higher in patients with rheumatic etiology, pure AR and dilated aortic root. Avoiding the Ross procedure in this group of patients and restricting it to patients with aortic roots $<27 \mathrm{~mm}$ and with stenosis as the main hemodynamic manifestation will result in improved long term results.

\section{Aortic Valve Replacement - Paradox in Patients with Paradoxical Low Flow}

\section{Anthony Poulin, Maxime Berthelot-Richer, Lionel Tastet, Louis Simard, Dimitri Kalavrouziotis, Philippe Pibarot, Marie-Annick Clavel}

Quebec Heart and Lung Institute, Québec, QC, Canada

Background: Patients with classical low flow (LEF) and paradoxical low flow (PLF) aortic stenosis (AS) have a higher risk of perioperative mortality following surgical aortic valve replacement (AVR). Stroke volume indexed to body surface area (SVi) before surgery (SViP) has been demonstrated to be an independent predictor of mortality in patients with PLF AS. The objective of this study was to assess the impact of postoperative SVi at discharge (SViD) on outcomes in patients with PLF and normal flow (NF) AS. Methods: Among 903 patients, we examined 820 patients with AS and preserved left ventricular ejection fraction who underwent surgical aortic valve replacement \pm coronary artery bypass grafting (CABG). 63 patients were excluded due to absent postoperative echography or technically limited echography. Results: Among these patients, 449 (55\%) had isolated AVR and 371 (45\%) had AVR + CABG. 312 (38\%) were in PLF as defined by SViP of $\leq 35$ $\mathrm{ml} / \mathrm{m}^{2}$; and $508(62 \%)$ were in NF as defined by SViP of $>35 \mathrm{ml} /$ $\mathrm{m}^{2}$. Groups were subdivided according to SViD: 248 (30\%) had PLF and low SViD (Persisting low flow (LF)); 64 (8\%) had PLF and normal SViD (Normalized flow group); 305 (37\%) had NF and low SViD (New onset LF); and 203 (25\%) had NF and normal SViD

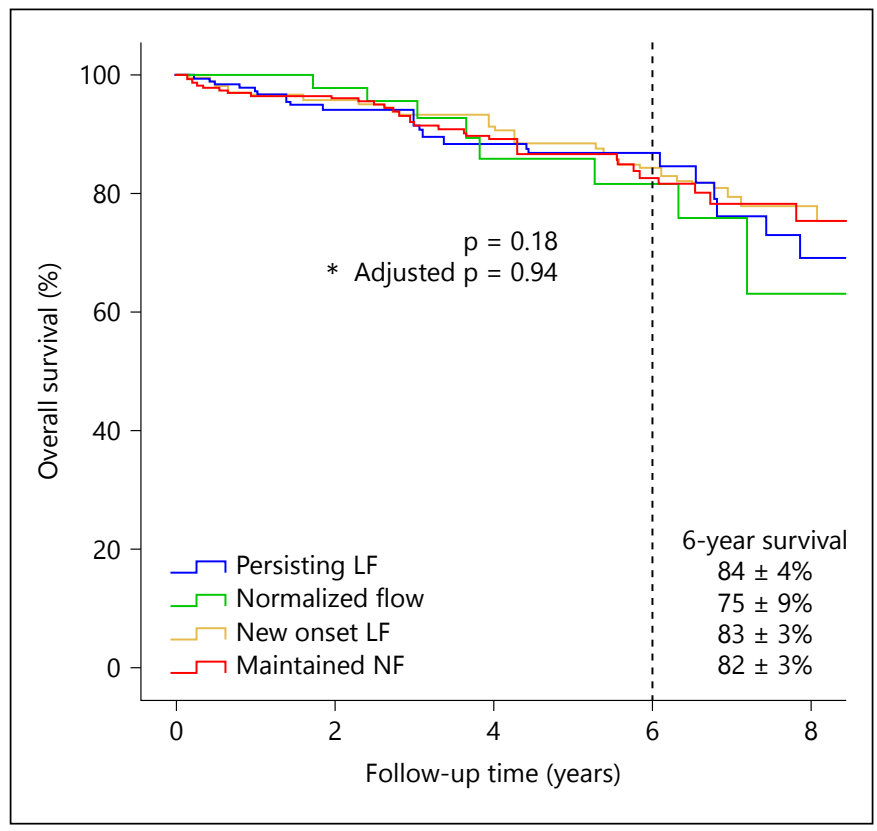

Fig. 1. Overall survival after SAVR. * Adjusted for age, sex, New York Heart Association functional class III or IV, atrial fibrillation, chronic kidney failure, diabetes, coronary artery disease, chronic obstructive pulmonary disease, left ventricular mass index, and mean gradient (for Abstract of Anthony Poulin). 
(Maintained NF). 101 deaths (12\%) occurred at a mean follow-up of $4.0 \pm 2.6$ years. With univariate analysis and comprehensive adjustment, SViD was not associated with late postoperative mortality. There was also no significant difference between persisting LF, new onset LF, normalized flow and maintained NF groups (figure $1)$. Significant independent predictors of mortality were age $(\mathrm{p}<$ $0.01)$, presence of chronic kidney disease $(p<0.01)$ and atrial fibrillation ( $\mathrm{p}=0.03)$. Conclusion: In patients with PLF and NF AS, postoperative SVi has no impact on late survival. Excluding the higher rates of perioperative mortality, patients with PLF AS have similar long-term outcomes compared to those with NF AS. These findings suggest that patients with PLF should be considered for surgical AVR.

\section{Experimental and Computational Valve Tissue Studies}

\section{A Study of Tunable Biaxial Strain Fields on Aortic Heart Valve Tissues}

\section{Ying Lei, Zannatul Ferdous}

The University of Tennessee, Knoxville, TN, USA

Objective: Tissue-engineered heart valves (TEHVs) are a promising option for valve replacement, since they can function as native valves and grow with the patients; however, they mostly lack ability to withstand native mechanical forces. Since cyclic stretch is known to modulate cells differentiation, extracellular matrix (ECM) synthesis and organization, understanding the interactions between stretch, cells and ECM will not only facilitate the design of functional TEHVs, but also will provide insight on valve biology and pathology. In this study, using a novel biaxial stretch bioreactor, we tested various physiological strain fields on heart valve tissues. Methods: A custom bioreactor has been designed using three stepper motors to achieve a maximum displacement of $25-35 \%$ in both radial and circumferential directions. The culture chambers were sterilized by
UV light for 30 minutes on each side. The bioreactor was kept in a humidified culture incubator for 7 days, with the media changed every 2 days. A rectangular silicone rubber membrane, mitral, pulmonary, and aortic valve tissues (figure 1) were tested under three strain regimens controlled using Labview software. Real-time images of the specimens subjected to cyclic stretch were captured by a camera, and the strain fields were obtained through the Digital Image Correlation technique. The theoretical strain fields of the silicone rubber membrane were obtained in simulations though finite element method. Results: During the 7 day culture, no contamination was observed. For the silicone rubber membrane, the strain fields were similar to the results generated in the simulations. They distributed relatively evenly on majority of the substrate in all three cases, except for the clamping area, where the gradient was higher. For the aortic valve tissue, strain was larger on area with thinner thickness due to the anisotropic distribution of the ECM in aortic valve. Strain fields along the circumferential direction were larger than the radial direction due to the alignment of collagen fibers. Conclusions: Our custom device can apply tunable biaxial stretch under aseptic culture condition to freshly isolated aortic valve tissues. In future, the variation of ECM and cell composition under physiological biaxial stretch on aortic valve tissues will be studied.

\section{Infectious Agents Related with Collagen Degradation and Inflammation in Myxomatous Mitral Valve Degeneration}

Marcos G. Tiveron, Sr., Pablo M.A. Pomerantzeff, Sr., Maria L. Higuchi, Marcia Reis, Jaqueline Pereira, Joyce Kawakami, Renata Ikegami, Carlos M. Brandao, Sr., Fabio B. Jatene, Sr.

Heart Institute, Sao Paulo, Brazil

Objective: The etiology of myxomatous mitral valve degeneration (MVD) is not fully understood and may depend on time or environmental factors for which the interaction of infectious agents has not been documented. The aim of this study is to analyze if the presence and effect of Mycoplasma pneumoniae (Mp), Chlamydophila pneumoniae $(\mathrm{Cp})$ and Borrelia burgdorferi $(\mathrm{Bb})$ on

Table 1. Inflammation, bacterial and MMP9 expression of antigens in mixomatous degeneration and control group (for Abstract of Marcos G. Tiveron, Sr.)

\begin{tabular}{lccc}
\hline & MD & CO & $\mathrm{p}$ \\
\hline $\mathrm{CD} 20$, cell $/ \mathrm{mm}^{2}$ & $17.8(6.7-27.9)$ & $4.6(3.6-9.8)$ & 0.007 \\
$\mathrm{CD} 45$, cell $/ \mathrm{mm}^{2}$ & $17.3(3.4-92.5)$ & $2.8(1.4-10.1)$ & 0.008 \\
$\mathrm{CD} 68, \mathrm{cell} / \mathrm{mm}^{2}$ & $38.7(26.6-81.8)$ & $70(5.4-182)$ & 0.098 \\
$\mathrm{Mp}, \mu \mathrm{m}^{2} \mathrm{Ag}-1 \mathrm{~mm}$ & $180,993(24,856-387,477)$ & $7,970(2,736-15,992)$ & $<0.001$ \\
$\mathrm{Cp}, \mu \mathrm{m}^{2} \mathrm{Ag}-1 \mathrm{~mm}$ & $9,905(4,716-16,912)$ & $5,864(2,382-8,692)$ & 0.2 \\
$\mathrm{Bp}, \mu \mathrm{m}^{2} \mathrm{Ag}-1 \mathrm{~mm}$ & $7,596(3,203-13,519)$ & $10,584(7,223-15,974)$ & 0.14 \\
MMP9, $\mu \mathrm{m}^{2} \mathrm{Ag}-1 \mathrm{~mm}$ & $389,844(214,459-679,711)$ & $144,397(29,894-247,453)$ & $<0.001$ \\
\hline
\end{tabular}

Date are presented as median value (25th, 75th percentile). 

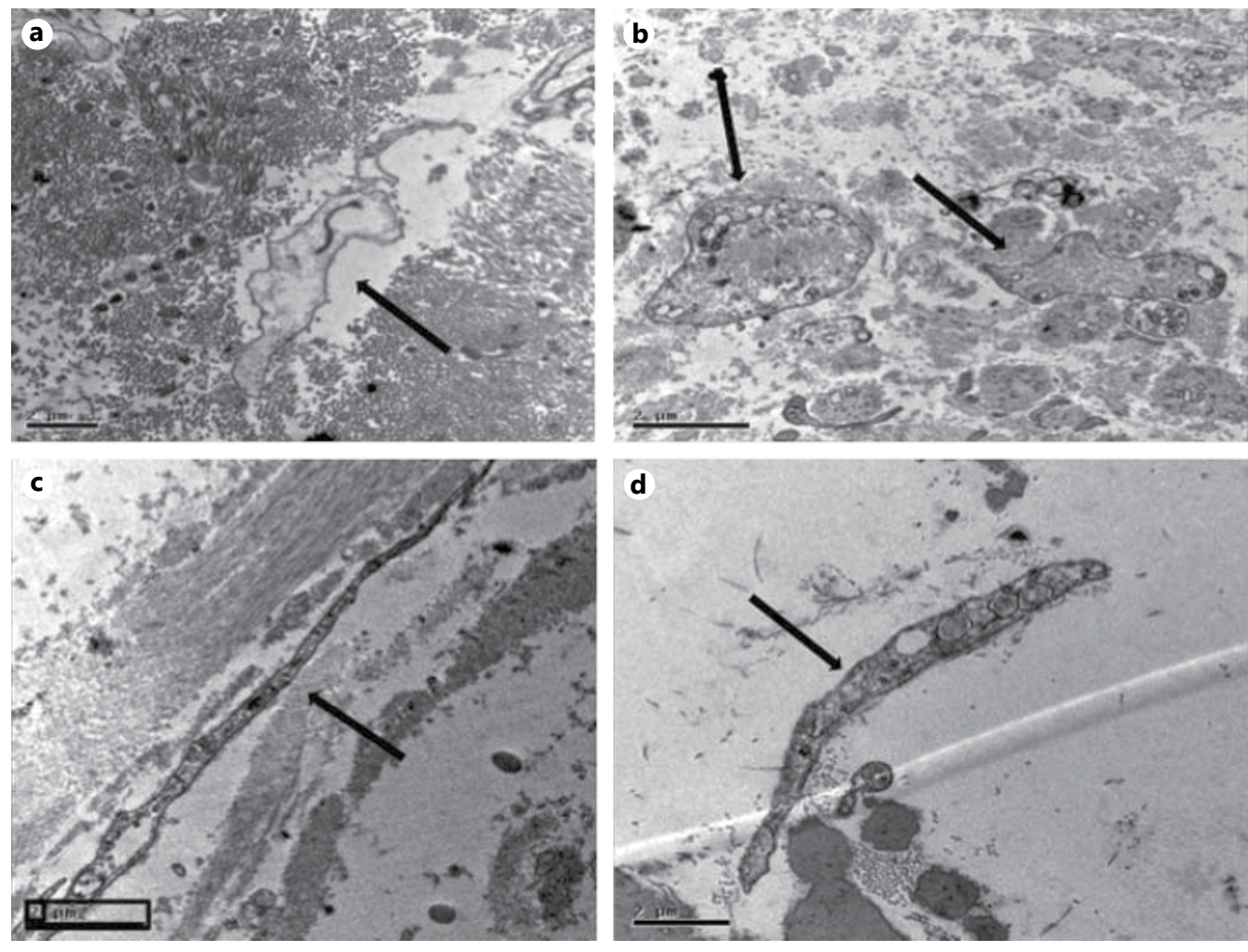

Fig. 1. (for Abstract of Marcos G. Tiveron, Sr.).

\section{Patient-Specific Computational Modeling of Mitral Valve Repair Techniques}

Thuy Pham ${ }^{1}$, Fanwei Kong ${ }^{1}$, Caitlin Martin ${ }^{1}$, Qian Wang ${ }^{1}$, Charles Primiano ${ }^{2}$, Raymond McKay ${ }^{2}$, John Elefteriades ${ }^{3}$, Wei Sun ${ }^{1}$

${ }^{1}$ Georgia Institute of Technology, Atlanta, GA, ${ }^{2}$ Hartford Hospital, Hartford, CT, ${ }^{3}$ Yale University, New Haven, CT, USA

Objective: Many mitral regurgitation (MR) patients are left untreated because of surgical ineligibility. For functional MR (FMR), geometric remodeling or dilation of the LV subsequently produces structural and functional changes in the MV, such as enlarged mitral annulus (MA), displaced papillary muscles (PPMs), tethered MV leaflets, and consequently incomplete MV closure. These combined complications further impose substantial challenges for surgical MV repair. The objective of this work is to develop a patient-specific computational model to simulate MV dynamics under diseased and repaired conditions. Methods: A patient-specific computational model of a 71 year-old male patient, who was diagnosed with severe MR due to tethered MV leaflets, was developed from multi-slice CT (MSCT) cardiac scans obtained from Hartford Hospital (with IRB approval). The model consists of the MV leaflets, mitral apparatus, and chordae tendineae, which were characterized by age-matched human cadaver MV material properties derived experimentally. MV closure dynamics was simulated and validated by comparing the leaflet geometries from the FE model output and the systolic CT MV images. The 
surgical placement of annuloplasty rings of different sizes and anterolateral papillary muscle reposition techniques were simulated to model MV repairs. Results: The model captured MV dynamics with close approximation of the posterior mitral leaflet (PML) restriction and apical tenting. Tethering of PML imposed higher stress on PML and chords. For this patient, reducing the MA by annuloplasty alone could not completely release tethering tension nor eliminate MR. Our simulation results demonstrated that combining the annuloplasty with the anterolateral papillary muscle reposition technique, i.e., repositioning several chordal origins, could effectively reduce MR. Conclusions: The computational model developed in this study can assist clinicians with patientspecific pre-operatively planning by providing virtual and detailed quantitative analysis of the valve dynamics and optimal treatment outcomes.

\section{Computational Simulation of in vivo Pulmonary Autograft Early After the Ross Procedure: Wall Stress Analysis}

Yue Xuan ${ }^{1}$, Hesam S. Moghaddam 1 , Ismail El-Hamamsy ${ }^{2}$, Francois-Pierre Mongeon ${ }^{2}$, Richard L. Leask ${ }^{3}$, Alexander Emmott ${ }^{3}$, Elaine E. Tseng ${ }^{1}$, Liang $\mathrm{Ge}^{1}$

${ }^{1}$ UCSF Medical Center and San Francisco VA, San Francisco, CA, USA; ${ }^{2}$ Montreal Heart Institute and Université de Montréal, Montreal, QC, ${ }^{3}$ McGill University, Montreal, QC, Canada

Objective: Compared to mechanical and bioprosthetic aortic valve replacements, the Ross operation provides excellent hemodynamics, freedom from oral anticoagulation, living tissue growth for children and young adults, and restoration of normal life expectancy. The Achilles heel of the Ross operation is the need for late reoperation due to aneurysmal dilatation and aortic insuffi- ciency. We previously demonstrated elevated autograft wall stress without appreciable dilatation immediately after surgery. Increased wall stress triggers biomechanical remodeling that can lead to autograft dilatation. In this study, we used a patient-specific model to investigate changes in autograft stress one year after surgery. Methods: After informed consent, one patient underwent Ross procedure as full root replacement; pulmonary and aortic tissue were removed for material properties testing. Cine magnetic resonance imaging (MRI) was performed for both preand post-operative geometry. MRI lumen geometry was used to create a numerical model of the aortic sinuses and pulmonary autograft. Material properties and wall thickness of autograft and aorta measured from biaxial stretching were incorporated for both baseline and follow-up models. Suturing between autograft and Dacron interposition graft, and between Dacron graft and aorta were simulated. Simulations of baseline and one-year follow-up models were performed using LS-DYNA to determine autograft wall stress. Results: Average tissue stress (von Mises) was 102,124 , and $109 \mathrm{kPa}$ at sinotubular junction (STJ), sinuses, and annulus, respectively, immediately after surgery at $80 \mathrm{~mm} \mathrm{Hg}$. In contrast, the corresponding average stress at 1 year follow-up was 97,85 , and $97 \mathrm{kPa}$, respectively (figure 1). Conclusions: To our knowledge, we created the first patient-specific computational model of the Ross procedure postoperatively using in vivo MRI imaging, autograft and aortic material properties. In this patient, average tissue stress was higher in autograft sinuses than annulus and STJ immediately after the operation. However, during the first year post-surgery, this autograft remodeled its shape to reduce stress differences among STJ, sinuses, and annulus. Future studies will further investigate this relationship between remodeling and stress.
Fig. 1. Stress distribution on one-year postoperative model at $80 \mathrm{~mm} \mathrm{Hg}$. Red section is interpositioned Dacron graft. The contours represent tissue stress (Von Mises) in $\mathrm{kPa}$ (for Abstract of Yue Xuan).

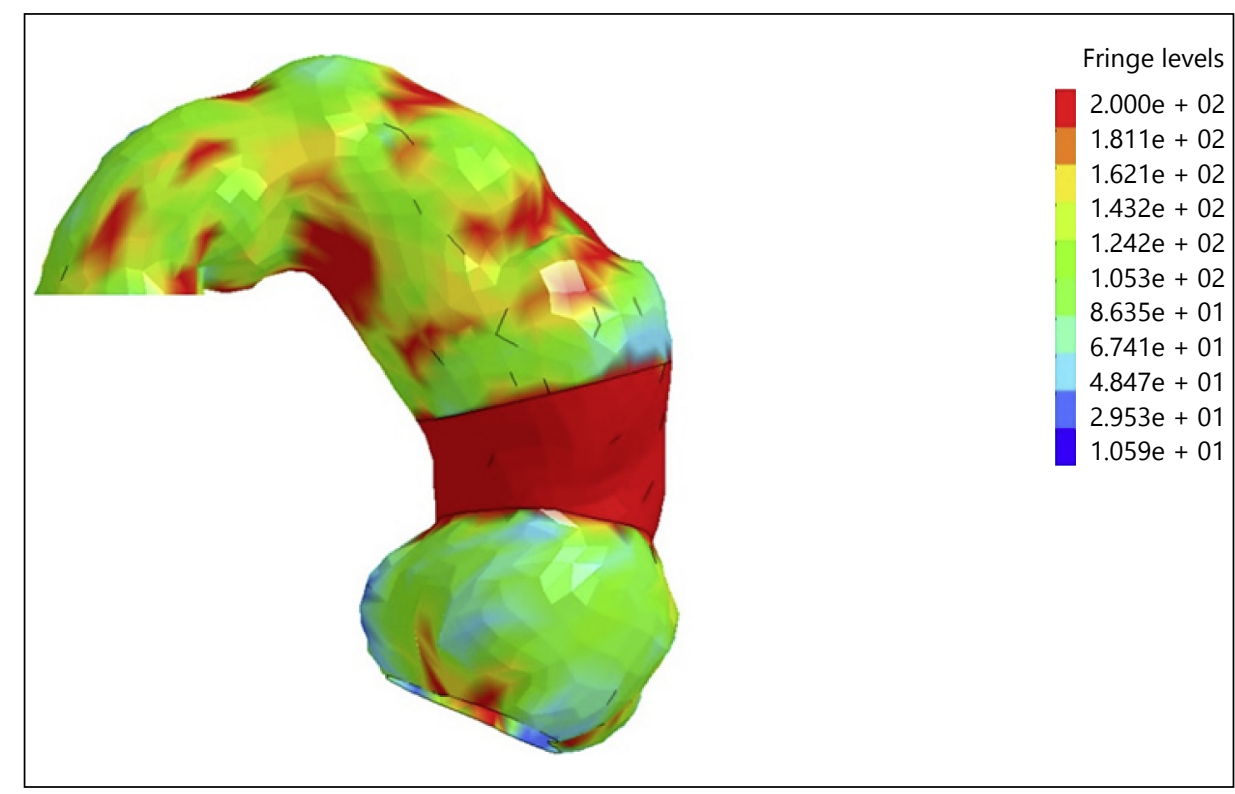




\section{Remodeling of Tissue-Engineered Heart Valves during a One-Year Follow-Up Period in Sheep - A Computational-Experimental Analysis}

Sandra Loerakker ${ }^{1}$, Bart Sanders ${ }^{1}$, Boris Schmitt ${ }^{2}$, Petra E. Dijkman ${ }^{3}$, Hendrik Spriestersbach ${ }^{2}$, Anita Driessen-Mol', Emanuela S. Fioretta', Marco Bartosch ${ }^{2}$, Laura Frese ${ }^{3}$, Simon P. Hoerstrup ${ }^{3}$, Felix Berger ${ }^{2}$, Frank P.T. Baaijens

${ }^{1}$ Eindhoven University of Technology, Eindhoven, Netherlands; ${ }^{2}$ Deutsches Herzzentrum Berlin, Berlin, Germany; ${ }^{3}$ University and University Hospital Zurich, Zurich, Switzerland

Objective: The functionality of tissue-engineered heart valves (TEHVs) often decreases over time due to leaflet retraction. Since mechanical factors play an important role in the remodeling process of cardiovascular tissues, understanding the interplay between mechanics and remodeling is crucial for developing TEHVs with long-term functionality. Previously, we suggested a valve design that could prevent or minimize leaflet shortening after implantation. The goal of the present study was to investigate the in vivo remodeling process with this new design and understand and predict the (robustness of the) remodeling process using computational models. Methods: TEHVs with the new design were implanted in the pulmonary position of sheep $(n=3)$ for a period of a full year. Valve functionality was monitored every four weeks via cMRI, and tissue composition and organization after remodeling were investigated in the explants. Based on the initial geometry and material properties of the valves before implantation, a computational model was used to predict and understand (the robustness of) the in vivo remodeling process. This model included cell contractility, cell-mediated collagen contraction, and strain-dependent collagen remodeling. Results: For the first time, all TEHVs preserved their functionality throughout the complete implantation period. The collagen fibers appeared to remodel from an initially random distribution towards a circumferentially oriented collagen network. Furthermore, the contractility of the cells in the valve appeared to be low. Our computational model was able to quantitatively predict the remodeling response of the valves when cell contractility was assumed to be low. Variations in cell contractility and initial leaflet thickness in the model demonstrated that valve functionality after remodeling is most sensitive to cell contractility, whereas the collagen architecture appeared to be quite insensitive to changes in cell contractility or leaflet thickness. Conclusions: Our combined computational-experimental efforts confirmed that TEHVs can maintain their functionality during a oneyear follow-up period when the initial valve design is chosen carefully. Computational models were essential in defining a rational design, and predicting the most important determinants in the remodeling process. We gratefully acknowledge the support of the EU ([FP7/2007-2013], grant agreement no. 242008), and the Netherlands CardioVascular Research Initiative (CVON2012-01).

\section{A New Fluid-Structure Interaction Model for Bicuspid Aortic Valves}

Karin Lavon ${ }^{1}$, Rotem Halevi', Gil Marom ${ }^{2}$, Sagit Ben Zekry', Rami Haj-Ali ${ }^{1}$, Hans Joachim Schäfers ${ }^{4}$, Ehud Raanani ${ }^{5}$

${ }^{1}$ School of Mechanical Engineering, Faculty of Engineering, Tel Aviv University, Tel Aviv, Israel; ${ }^{2}$ Biomedical Engineering Department, Stony Brook University, 11794, NY, USA;

${ }^{3}$ Echocardiography Laboratory, Chaim Sheba Medical

Center, Tel Hashomer, Israel; ${ }^{4}$ Department of Thoracic and Cardiovascular Surgery, University Hospitals of Saarland, Homburg, Germany; ${ }^{5}$ Department of Cardio-Thoracic Surgery, Chaim Sheba Medical Center, Tel Hashomer, Israel

Background: The Bicuspid aortic valve (BAV) is associated with valvular pathologies such as aortic regurgitation and aortic stenosis. A possible cause for these complications is related to a non-optimal geometry of the valve. The common configuration of BAV include one raphe with varying cusps angles. Surgical repair is often performed in BAVs with smaller non fused cusp (NFC) angle. The aim of this study is to provide a new Fluid-Structure Interaction (FSI) modeling approach of BAVs that can be used to investigate the influence of different geometrics parameters on its functionality. The effect on parameters such as effective orifice area (EOA), hemodynamic metrics and stresses magnitudes on the tissues are examined. Methods: Parametric FSI models of BAVs were generated, with four common BAV geometries. These include one raphe and different NFC angles from $120^{\circ}$ to $180^{\circ}$. The material properties of the structure and the applied pressures of the left ventricle and ascending aorta corresponded to physiologic response values. A 'dry' (without fluid) structural finite element (FE) BAV models were performed and compared to the FSI cases. Echocardiography (Echo) data from patients with BAV was used to correlate with the present FE results. Results: The simulated BAVs have significant geometrical differences leading to different mechanical responses and behavior. Increased and eccentric located effective orifice areas (EOAs) were found in the models having decreased NFC angles, leading to asymmetric blood flow jet. The measured echo results support this predicted pattern. During diastole and systole, high stresses and shear stresses were found, respectively, in the models of NFC with decreased angle. Conclusions: The predicted biomechanical behavior in the form of jet flow patterns (hemodynamics) and stresses can help assess the functionality and durability of BAVs. High stress regions on the leaflets explain the initiation of calcification, while asymmetric flow jet can be a major cause for eccentric aortic dilatation. This can explain early failure in BAVs with decreased NFC angle. The present computational modeling is a step forward towards patient specific simulation of future BAV repair. 


\section{Initial Impact of Bicuspid Aortic Valve Morphotype on Ascending Aorta Hemodynamics: An in vitro Study}

\section{Andrew McNally, Philippe Sucosky}

University of Notre Dame, South Bend, IN, USA

Objective: The heterogeneous bicuspid aortic valve (BAV) morphotypes (left-right-coronary cusp fusion (LR), right-noncoronary cusp fusion (RN), and left-non-coronary cusp fusion $(\mathrm{LN})$ ) are associated with different ascending aorta (AA) dilation patterns, suggesting hemodynamics effect BAV aortopathogenesis. Assessment of this theory is hampered by limited knowledge of the hemodynamic abnormalities generated by different BAV morphotypes. Therefore, the objective of this study was to compare experimentally hemodynamics in a normal (i.e., non-dilated) AA subjected to tricuspid aortic valve (TAV), LR-BAV, RN-BAV, and NL-BAV flow. Methods: Tissue BAV models were fabricated by suturing two leaflets of porcine TAVs and were mounted in a realistic aortic root connected to a compliant silicone AA model. Different BAV morphotypes were obtained by changing the orientation of the fused leaflet with respect to the sinuses. Physiologic pulsatile flow conditions were generated by a left-heart simulator. The flow was measured downstream of the valve and in the tubular AA using particle image velocimetry at 20 phases of the cardiac cycle. Mean and turbulent flow quantities were obtained by ensemble-averaging over 415 image pairs at each phase. Results: All three BAVs generated skewed and asymmetrical orifice jets, exposing the tubular AA to abnormal hemodynamics (figure 1). Viscous shear stress (VSS) captured $10 \mathrm{~mm}$ downstream of the sinotubular junction was oscillatory in the TAV AA but pulsatile in all three BAV AAs. While the maximum peak-systolic VSS was similar between the TAV and BAV morphotypes, (TAV: 8.5 Pa, LR-BAV: 9.9 Pa, RN-BAV: 5.1 Pa, NL-BAV: 7.3 Pa), the maximum peak- systolic Reynolds shear stress in LR-BAV and NL-BAV exhibited a 2.3-fold increase relative to TAV (TAV: $160 \mathrm{~Pa}, \mathrm{LR}-\mathrm{BAV}$ : $380 \mathrm{~Pa}$, RN-BAV: $44 \mathrm{~Pa}, \mathrm{NL}-\mathrm{BAV}$ : $370 \mathrm{~Pa})$. Conclusions: This in vitro study isolated flow abnormalities marked by increased jet skewness, altered flow directionality and increased turbulence in nondilated BAV AAs relative to the TAV AA, and their dependence on the BAV morphotype, with the LR-BAV showing the highest degree of jet skewness and shear stress abnormalities.

\section{Heart Valve Repair}

\section{Ten Year Single Center Experience with Robotic Assisted Mitral Valve Repair}

Alfredo Trento, Danny Ramzy, Michele De Robertis, James Mirocha, Wen Cheng, Lawrence Czer

Cedars-Sinai Medical Center, Los Angeles, CA, USA

Background: To compare the surgical outcomes in three eras over 10-years with robotic assisted mitral valve repair (R-MVr). Methods: From June 2005 to August 2015, 534 R-MVr were performed. We divided our experience into 3 Eras. Era-1 included first 120 cases using first generation DaVinci system, Era- 2 next 180 with newer system and Era-3 latest 234 cases adding a second robotic surgeon. Every patient needing isolated MVr underwent $\mathrm{R}-\mathrm{MVr}$ and received an annuloplasty band and included one or more of the following: leaflet resection, chordal transposition and/ or neochordal replacement. Results: All 534 patients had preoperative severe mitral regurgitation (MR) with no differences between Eras for preoperative characteristics, age (60.9 years), fe-

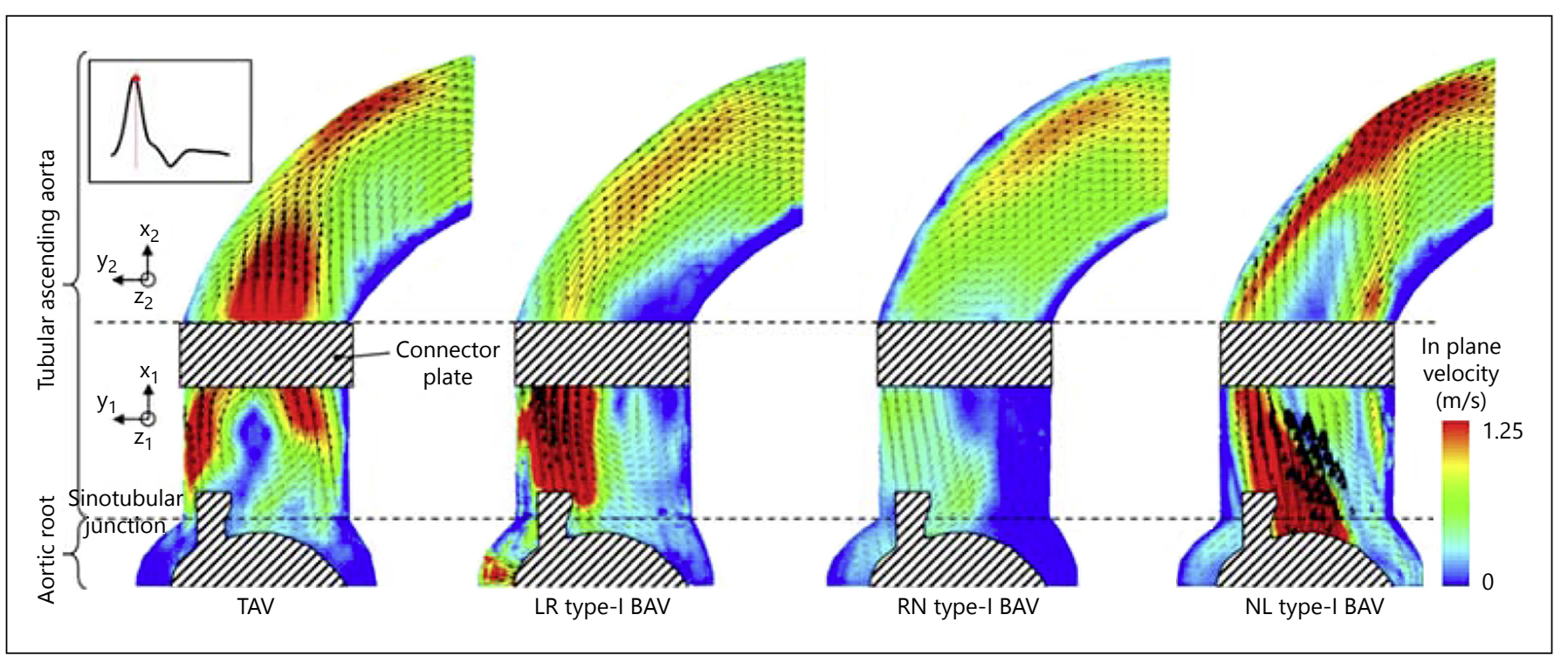

Fig. 1. Instantaneous velocity vector fields measured at peak systole in the four valve-AA models (for Abstract of Andrew McNally). 
males $(33 \%)$, ejection fraction $(61.7 \%)$, congestive heart failure $(27.3 \%)$, creatinine $(0.97 \mathrm{mg} / \mathrm{dl})$ and NYHA class. Anterior prolapse and Barlow syndrome was greater in later eras compared to Era-1 (5.8 vs. 27.8 vs. $14.1 \%)$. One hospital death ( $0.33 \%)$ occurred in Era-1 and none in Era-2 or Era-3. Overall, 8 (1.5\%) patients required subsequent MV replacement via sternotomy, 6 (5.0\%) Era1, 2 (1.1\%) Era-2 and none Era-3. One patient each in Era-1 and Era-2 had re-repair through right mini-thoracotomy and another in Era-1 had MV replacement through mini-thoracotomy during the original procedure. No re-repair or replacement was required in Era-3. Cross clamp times decreased from Era-1, 116 minutes to 91 and 79 in Era- 2 and Era-3 respectively despite training a junior associate doing part of the procedure in Era-2 progressing to 2 surgeons in Era-3. Overall $98.7 \%$ had post-pump none-mild MR (86.5\% with none-trace). Follow-up echo (1 month to 1 year) showed overall none-trace MR in $73.7 \%$, mild in $19.9 \%$ and $0.6 \%$ severe MR (Era-3 80.6\% had none-trace and no severe MR). Conclusions: Complications and reoperations occurred early in our experience. The newer system together with increased experience made R-MVr of all types of degenerative mitral valve pathology reproducible. Training robotic surgeons in high volume centers helps avoid complications during introduction of this technology while not affecting the crossclamp time. The addition of a fully trained surgeon to a robotic program as seen in Era-3 can further reduce OR time and improve outcomes.

\section{Remodeling Associated with Aortic Ring Annuloplasty: Long Term Results of a Physiological Approach to Valve Sparing}

\author{
Emmanuel LANSAC ${ }^{1}$, Isabelle Di Centa ${ }^{2}$, Ghassan Sleilaty ${ }^{1}$, \\ Stéphanie Lejeune ${ }^{1}$, Nizar Khelil', Alain Berrebi ${ }^{1}$, \\ Christelle Diakov' ${ }^{1}$, Leila Mankoubi ${ }^{1}$, Marie Christine Malergue ${ }^{1}$, \\ Milena Noghin ${ }^{1}$, Konstantinos Zannis ${ }^{1}$, Suzanna Salvi ${ }^{1}$, \\ Patrice Dervanian ${ }^{1}$, Mathieu Debauchez ${ }^{1}$
}

${ }^{1}$ Institut Mutualiste Montsouris, Paris, ${ }^{2}$ Hopital Foch, Suresnes, France

Objective: An untreated dilated aortic annulus $(>25 \mathrm{~mm})$ is a major risk-factor for failure of aortic valve sparing in either bicuspid or tricuspid valve. Aortic annuloplasty efficiently reduce the annulus, increasing the coaptation height thus protecting the repair. This study analyzes long term results of 149 consecutive patients operated on with a standardized and physiological approach to aortic valve repair associating Remodeling of the aortic root with a subvalvular external aortic ring annuloplasty. Methods: Data were collected into the multicentric international AVIATOR registry (AorticValve repair InternATiOnal Registry). Preoperative $\mathrm{AI} \geq$ grade III was present in $42.9 \%$ (64), valve was bicuspid in 47 (31.5\%). Results: Cusp repair was performed in $99(66.4 \%)$ patients. The thirty-day operative mortality was 2 (1.4\%). The aortic ring significantly reduced the native aortic annulus diameter of $-5.3 \pm 3.3 \mathrm{~mm}$. Mean follow-up was $47 \pm 39.4$ months $(0-145.5)$. Actuarial survival at 7 years was $89.9 \%$. Freedom from reoperation at 7 years was $90.5 \%$. Further breakdown by time period (20032008 «Eye balling» repair vs. 2009-1014 systematic cusp effective height assessment) shows that 7 -year freedom from reoperation increased, from $85.8 \% \pm 5.5 \%$ to $98.9 \% \pm 1.1 \%(\mathrm{p}=0.085)$. A total of 7 patients underwent valve-related reoperation for structural valve deterioration for recurrent $\mathrm{AI}$ in the root aneurysm group between 1 month and 6 years postoperatively. When analyzing by time periods, 7 -year freedom from AI $\geq 3$ tended to increase from $89.8 \% \pm 4.9 \%$ («Eye balling») to $100 \%$ (systematic cusp effective height assessment) ( $\mathrm{p}=0.107)$, while freedom from $\mathrm{AI} \geq$ grade 2 remained unaffected $(77.6 \% \pm 6.8 \%$ and $78.0 \% \pm 11.7 \%, \mathrm{p}=0.938)$. Freedom from Major Adverse Valve Related Events (MAVRE) at 7 years was $82.4 \%$, and freedom from thrombo-embolic events was $95.1 \%$. The type of valve was not identified as an independent predictor of MAVRE $(p=0.532)$. Pre operative AI grade, cusp repair, type of valve, Marfan syndrome, pre operative aortic annulus diameter had no influence on reoperation. Conclusions: External aortic ring annuloplasty provides a reproducible technique for aortic valve repair with satisfactory long results for aortic root aneurysm with bicuspid or tricuspid valve. Longer follow-up is ongoing with AVIATOR registry.

\section{Predictors of New-Onset Atrial Fibrillation in Preserved Ejection Fraction Patients Undergoing Isolated Mitral Valve Repairs}

\section{J. Trent Magruder, Sarah Collica, Stephen Belmustakov, Todd C. Crawford, Joshua C. Grimm, Duke E. Cameron, William A. Baumgartner, Kaushik Mandal}

Johns Hopkins, Baltimore, MD, USA

Objective: Among patients who present with mitral regurgitation but preserved ejection fractions, little is known about who will develop atrial fibrillation (AF) on long-term followup. We sought to determine the incidence of and risk factors for long-term atrial fibrillation (LTAF) in this cohort. Methods: We included all patients undergoing isolated mitral valve repair (MVR) between 1997-2014 at our institution with a preserved ejection fraction $(\geq 60 \%)$. Patients who had AF preoperatively were excluded from the final analysis. Our primary outcome, LTAF, was defined as AF occurring after discharge following MVR (at least seven days following MVR), and was determined via telephone interviews with patients as well as chart review (outpatient visits, EKGs). Results: 269 patients were included in the study with a mean followup of $3.1 \pm 3.8$ years. A total of 31 patients developed LTAF, and freedom from LTAF was $93.5 \%$ at one year and $85.2 \%$ at five years. Patients developing LTAF were of similar mean age ( $60 \mathrm{vs.} 57$ years in controls, $\mathrm{p}=0.23$ ) and had similar preoperative comorbidities, but did show a trend toward larger left atrial diameter $(5.0 \mathrm{vs} .4 .6 \mathrm{~cm}, \mathrm{p}=$ 0.06). After risk adjustment with multivariable Cox regression analysis, increasing left atrial size was associated with LTAF (HR $1.64, \mathrm{p}=0.03$ ), while male gender appeared protective (HR 0.41 , $\mathrm{p}=0.04)$. On followup, 29 patients $(10.8 \%)$ developed moderate or greater mitral regurgitation at a mean of 2.2 years following MVR. Using a longitudinal mixed-effects model, we were unable to detect an association between recurrent mitral regurgitation following MVR and LTAF (OR 1.44, p = 0.28). Conclusions: LTAF occurs in about $15 \%$ of preserved ejection fraction patients undergoing MVR by five years. Increasing left atrial diameter is an independent predictor of LTAF, while male gender appears to be inde- 
pendently protective; interestingly, age was not a significant predictor in our multivariable Cox model. Concomitant left atrial maze may be considered in patients with preserved ejection fraction and enlarged left atria undergoing MVR.

\section{Combined Mitral and Aortic Valve Reconstruction}

Alexander Wick, Diana Aicher, Christian Giebels, Takahashi Kunihara, Hans-Joachim Schäfers

Saarland University, Homburg, Germany

Objective: Isolated repair of the mitral (MVR) has become routine, repair of the aortic valve (AVR) is increasingly accepted with good mid-term stability. It is uncertain whether combined MVR and AVR will lead to acceptable risk and stability. We analyzed our experience with combined MVR and AVR. Methods: Between 03/1997-06/2014, 144 patients ( $\mathrm{f}=48 ; \mathrm{m}=96$; age: $60 \pm$ 16 years) underwent MVR and AVR for relevant mitral (MR) and aortic (AR) regurgitation. 11 patients had emergency operation for active endocarditis. Concomitant procedures were left atrial ablation $(\mathrm{n}=77 ; 54 \%)$, coronary artery bypass grafting $(\mathrm{n}=24 ; 17 \%)$ and replacement of the aortic arch (partial $n=11 ; 8 \%$ ) (total $n=7$; $5 \%) .53$ patients underwent additional tricuspid valve repair. Aortic valve morphology was bicuspid $(n=30)$, tricuspid $(n=112)$, or unicuspid $(\mathrm{n}=2)$. The majority $(\mathrm{n}=100)$ had isolated cusp repair by central plication or triangular resection. Pericardial patches were used to close perforations or fenestrations $(n=18)$. In 24 patients additional root repair (root remodeling) was performed, in 44 patients sinotubular junction remodeling. MVR involved an annuloplasty in all patients, in 59 as the only procedure. In 31 standard repair of posterior leaflet prolapse was performed, in 19 anterior and in 17 bileaflet prolapse was corrected. 2 patients had repositioning of the posterior papillary muscle for ischemic MR. 16 patients underwent mitral commissurotomy. All patients were followed (mean $68 \pm 49$ months, [12 to 217]). Results: Hospital mortality was $3.5 \%$. Five and 10 -year-survival was $80 \%$ and $73 \%$, freedom from reoperation at 5 and 10 years was $87 \%$ and $80 \%$. 7 patients had to be reoperated because of recurrent MR, 11 due to recurrent $A R, 13$ patients for both recurrence of $A R$ and $M R$. Freedom from mitral valve reoperation at 5 and 10 years was $89 \%$ and $87 \%$. Freedom from aortic valve reoperation at 5 and 10 years was $90 \%$ and $84 \%$. At reoperation in 11 patients the valve could be rerepaired. Overall freedom from valve replacement at 5 and 10 years was $91 \%$ and $86 \%$. During follow-up no bleeding complications occurred; 5 patients developed endocarditis and 3 had thrombembolic events. The 10-year freedom from all valve-related complications was $94 \%$. Conclusions: Long-term results after double valve repair are promising. Valve-related complications are rare.

\section{Mitral Stenosis after Mitral Valve Repair for Degenerative Disease May Limit Exercise Capacity}

Kiyoshi Doi, Hitoshi Yaku, Satoshi Numata, Sachiko Yamazaki, Keiichi Itatani, Suguru Ohira, Kaichiro Manabe, Kiminori Ikemoto, Tetsuhiro Yamano

Kyoto Prefectural University of Medicine, Kyoto, Japan

Objective: Mitral repair for degenerative mitral regurgitation, even following the use of a true-sized ring, is occasionally associated with high mitral pressure gradient (PG), and this association becomes more prominent during exercise. In this study, clinical impact of such exercise-induced mitral stenosis was assessed using cardiopulmonary exercise test (CPX). Methods: Between October 2004 and July 2015, 283 consecutive patients underwent mitral valve repair for degenerative mitral regurgitation at our institution. Of these patients, both exercise stress echocardiography (ESE) and CPX were performed in 36 patients more than 3 months after surgery. For annuloplasty, semi-rigid complete ring, semirigid partial band, and flexible partial band were used in 12, 20 and 4 patients, respectively. Results: All patients were in sinus rhythm at postoperative echocardiography. None of the patient had more than moderate mitral regurgitation. Postoperative ejection fraction was $60.2 \pm 8.5 \%$. Peak exercise intensity according to ESE ranged from 50 to 125 Watts $(90.6 \pm 28.0$ Watts $)$, which significantly increased mean mitral PG from $3.6 \pm 1.6 \mathrm{~mm} \mathrm{Hg}(1.6-9.5$ $\mathrm{mm} \mathrm{Hg}$ ) at rest to $10.4 \pm 4.8 \mathrm{~mm} \mathrm{Hg}(3-24.9 \mathrm{~mm} \mathrm{Hg})$ at peak exercise. All the 36 patients showed good correlation between the exercise intensity and mean mitral PG. Therefore, a linear regression line for each patient could be drawn, and the mean mitral PG, at a constant exercise intensity of 100 Watts, could be estimated from the line. The estimated mean mitral PG at 100 Watts was $11.5 \pm 6.0 \mathrm{~mm} \mathrm{Hg}(4.4-24.6 \mathrm{~mm} \mathrm{Hg})$. In CPX, the ratio of the oxygen pulse to the age and sex matched control (\%VO2 pulse), which is an indicator of functional capacity, was $93.7 \pm 17.5 \%$ (56$151 \%)$. The $\% \mathrm{VO} 2$ pulse was closely negatively correlated with the

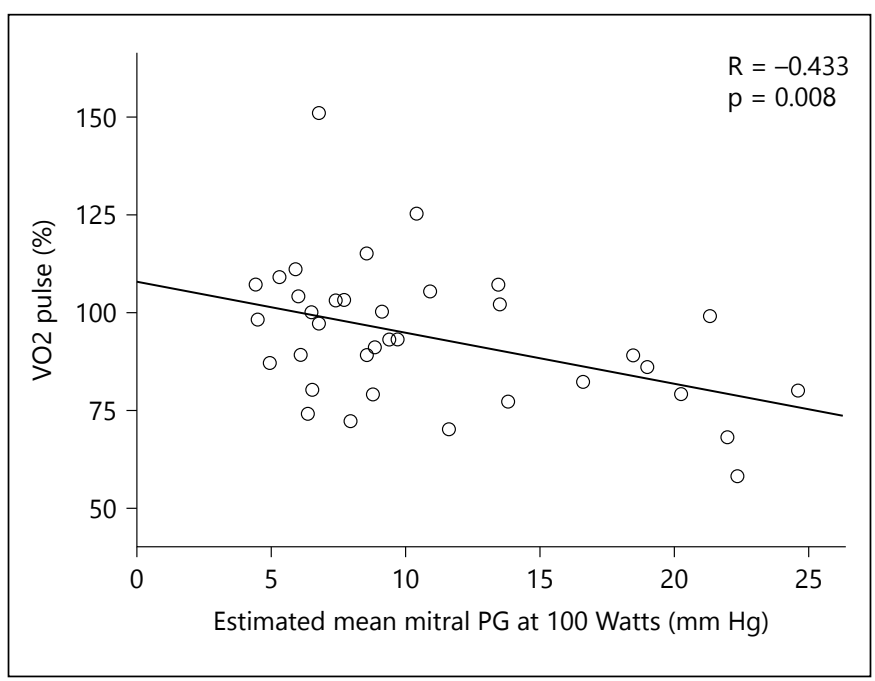

Fig. 1. (for Abstract of Kiyoshi Doi). 
estimated mean mitral PG at 100 Watts $(\mathrm{R}=-0.433, \mathrm{p}=0.008)$. Conclusion: Exercise-induced mitral stenosis after mitral valve repair for degenerative disease may be associated with a worse postoperative functional capacity.

\section{Tricuspid Valve Repair: What to Do When a Ring Is Not Enough}

\author{
Matteo Pettinari, Philip Bertrand, Herbert Gutermann, \\ Christiaan Van Kerrebroeck, Robert Dion
}

Ziekenhuis Oost Limburg, Genk, Belgium

Objective: Annuloplasty alone is not always successful, even in functional tricuspid regurgitation (TR), in presence of severe leaflets tenting. Other TR etiologies (congenital, organic, infective, traumatic or iatrogenic) also need additional techniques. We sought to present the clinical and echocardiographic outcome of these complex TR repairs. Methods: Between May 2008 and December 2014, 301 pts benefited from TV repair, and 41 (13.6\%) needed more than a ring annuloplasty. Mean age was $66.7 \pm 1,3$ pts $(73 \%)$ had severe renal failure, redo surgery was performed in 16 cases (39\%) and mean EUROscore II was $11.5 \%$. TR was due to severe leaflet tethering $(>8 \mathrm{~cm})$ in 23 , Ebstein's anomaly in 3, rheumatic valve disease in 2 , acute endocarditis in 3 , previous trauma in 1, and interference of PM lead in 9. Beside a semi-rigid ring annuloplasty, leaflet augmentation was used in 29 , neo-chordae in 8 , $\mathrm{PM}$ lead repositioning in 8 , triangular resection in 1 and resection of secondary chords in 2. Results: Median follow up was 1.7 y (0.16.8). $30 \mathrm{~d}$ and $4 \mathrm{y}$ survival were $85.4 \%$ and $75.5 \%$ respectively. $95.2 \%$ of the pts were in NYHA I. At the last echo restudy, freedom from TR $>2$ was $91.7 \%$ and the RVEDD significantly decreased from $6.1 \pm 0.8 \mathrm{~cm}$ to $3.8 \pm 0.8 \mathrm{~cm}(\mathrm{P}<0.01)$. Only one pt needed to be reoperated (freedom from reoperation $95.5 \pm 4.4 \%$ ). Conclusions: In functional severe leaflet tethering and other lesions likely not amenable to annuloplasty alone, complex TV repair is safe, effective, durable and associated with RV remodeling.

\section{Early Echocardiographic Outcomes of Mitral Valve Annuloplasty versus Annuloplasty Combined with a Papillary Muscle Sling for Moderate to Severe Functional Mitral Regurgitation}

\section{Christos G. Mihos' ${ }^{1}$, Andres M. Pineda ${ }^{2}$, Romain Capoulade ${ }^{1}$, Maiteder C. Larrauri-Reyes ${ }^{2}$, Serguei Melnitchouk' ${ }^{1}$ Judy Hung ${ }^{1}$, Orlando Santana ${ }^{2}$ \\ ${ }^{1}$ Massachusetts General Hospital, Boston, MA, ${ }^{2}$ Coulmbia University, Division of Cardiology, Mount Sinai Heart Institute, Miami Beach, FL, USA}

Background: Recurrent mitral regurgitation (MR) occurs in approximately $20-30 \%$ of patients with cardiomyopathy after mitral valve annuloplasty (Ring). A concomitant papillary muscle sling (Ring+Sling) may improve valve repair by promoting a more physiologic geometry of the mitral apparatus. Methods: Using 2:1

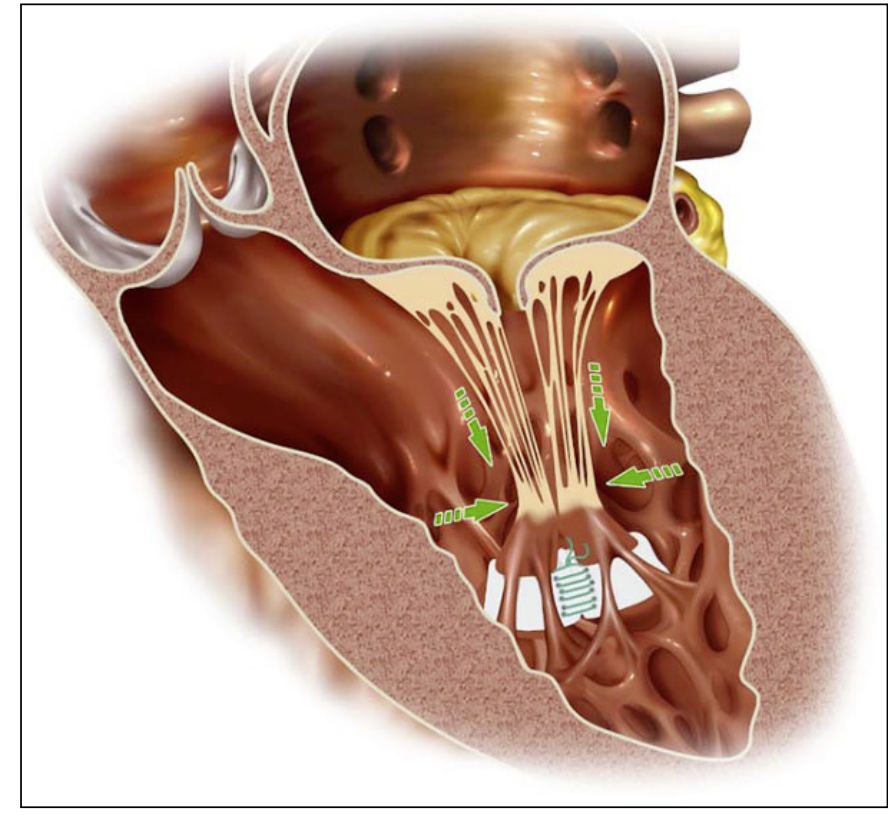

Fig. 1. (for Abstract of Christos G. Mihos).

propensity-score matching, the echocardiographic outcomes of 26 patients with moderate to severe functional MR and cardiomyopathy who underwent Ring+Sling (4-mm polytetrafluoroethylene graft placed around the base of the papillary muscles) were compared with 13 patients who underwent Ring only, between March 2008 and May 2015. Functional MR was defined as restricted systolic leaflet closure, with normal leaflet anatomy and pliability. The MR was graded semi-quantitatively as 0 (trace/none), 1+ (mild), 2+ (moderate), and 3-4+ (moderate to severe). Results: The baseline demographics were similar, except for a higher incidence of hypertension in the Ring+Sling group (100\% vs. $69 \%, \mathrm{p}<$ $0.001)$. There was no operative mortality in the Ring+Sling group, and 1 in patients undergoing Ring only $(\mathrm{p}=0.15)$. The mean time to follow-up echocardiogram was 10.9 months (range 0.1-42 months). At follow-up, a Ring+Sling repair was associated with a lower mitral valve tenting height ( $9 \mathrm{~mm}$ vs. $12 \mathrm{~mm}, \mathrm{p}=0.04)$ and interpapillary muscle distance ( $14 \mathrm{~mm}$ vs. $22 \mathrm{~mm}, \mathrm{p}<0.001)$, a smaller anterior leaflet tethering angle (30 degrees vs. 36 degrees, $\mathrm{p}=0.05)$ and posterior leaflet tethering angle (75 degrees vs. 94 degrees, $\mathrm{p}=0.02)$, and a greater leaflet coaptation length $(10 \mathrm{~mm}$ vs. $8 \mathrm{~mm}, \mathrm{p}=0.009)$, when compared with Ring only. The mean MR grade (0.7 vs. 0.9 ), and recurrence of moderate or greater MR (15\% vs. $31 \%$ ), did not reach a statistical difference between the groups at follow-up. Finally, actuarial survival at 3 years was $93 \%$ for Ring+Sling, and $85 \%$ for Ring only $(p=0.28)$. Conclusions: In patients with cardiomyopathy and moderate to severe functional MR, a Ring+Sling repair can be safely performed, and it improves the geometry of the mitral valve apparatus in the early post-operative period. Longer-term follow-up is needed to assess Ring+Sling durability and effects on left ventricular reverse remodeling. 


\section{Heart Valve Imaging}

\section{Assessment of Myocardial Fibrosis in Aortic Stenosis Using Cardiac Magnetic Resonance Imaging}

Calvin Chin ${ }^{1}$, Russell J. Everett ${ }^{2}$, Jacek Kwiecinski ${ }^{3}$, Alex Vesey ${ }^{2}$, Emily Yeung ${ }^{2}$, Gavin Esson'2, William Jenkins'2, Maria Koo², Saeed Mirsadraee ${ }^{2}$, Audrey White ${ }^{2}$, Alan Japp ${ }^{2}$, Sanjay Prasad ${ }^{4}$, Scott Semple ${ }^{5}$, David Newby ${ }^{2}$, Marc Dweck ${ }^{2}$

${ }^{1}$ Department of Cardiovascular Science, National Heart Center, Singapore, Singapore; 'University of Edinburgh, Edinburgh, United Kingdom; ${ }^{3}$ First Department of Cardiology, Poznan University of Medical Sciences, Poland, Poland; ${ }^{4}$ Royal Brompton Hospital, London, ${ }^{5} \mathrm{Clinical}$ Research Imaging Centre, Edinburgh, United Kingdom

Background: Myocardial fibrosis is a key process driving the transition from hypertrophy to heart failure in aortic stenosis and can be assessed using cardiac magnetic resonance (CMR) imaging. Methods: In a prospective observational cohort study, 203 subjects (166 patients with aortic stenosis (age 69, 69\% males) and 37 age and sex-matched healthy volunteers underwent comprehensive phenotypic characterisation with clinical, imaging and biomarker evaluation. The burden of diffuse and replacement fibrosis was quantified using CMR with T1-derived indexed fibrosis volume (IFV) and late gadolinium enhancement (LGE) respectively. Patients were followed up for 2.40 .7 years for all-cause mortality. Results: IFV demonstrated an excellent correlation with diffuse histological fibrosis on myocardial biopsies $(\mathrm{r}=0.87, \mathrm{P}<0.001, \mathrm{n}=$ 12) and offered good discrimination between disease states (aortic stenosis $22.4[18.7,26.8]$ versus healthy volunteers $15.2[13.4,18.2]$ $\left.\mathrm{ml} / \mathrm{m}^{2}, \mathrm{P}<0.001\right)$. The upper limit of normal from the control group was used to define diffuse fibrosis and categorize patients with aortic stenosis: compensated remodelling (IFV $<22.5 \mathrm{ml} / \mathrm{m}^{2}$, $51 \%$ of patients), diffuse myocardial fibrosis (IFV $\geq 22.5 \mathrm{ml} / \mathrm{m}^{2}$, $22 \%$ ) and replacement fibrosis (mid-wall LGE, 27\%). Across these groups there was evidence of increasing hypertrophy (LV mass index $73 \pm 11$ versus $96 \pm 11$ versus $107 \pm 25 \mathrm{~g} / \mathrm{m}^{2}$ ), myocardial injury (troponin I natural log $1.43 \pm 0.96$ versus $2.02 \pm 0.93$ versus $2.60 \pm 0.90)$ and left ventricular dysfunction (E/e' $13.1 \pm 7.7$ versus $13.2 \pm 4.8$ versus $16.5 \pm 6.5$; longitudinal systolic function $13.2 \pm$ 2.6 versus $12.5 \pm 2.4$ versus $11.2 \pm 3.1 \mathrm{~mm}$ ) consistent with progressive decompensation (all $\mathrm{P}<0.05$ ). Moreover this categorisation was of prognostic value with a step-wise increase in mortality rates (11 versus 45 versus 75 deaths/1000 patient-years respectively, $\mathrm{p}=$

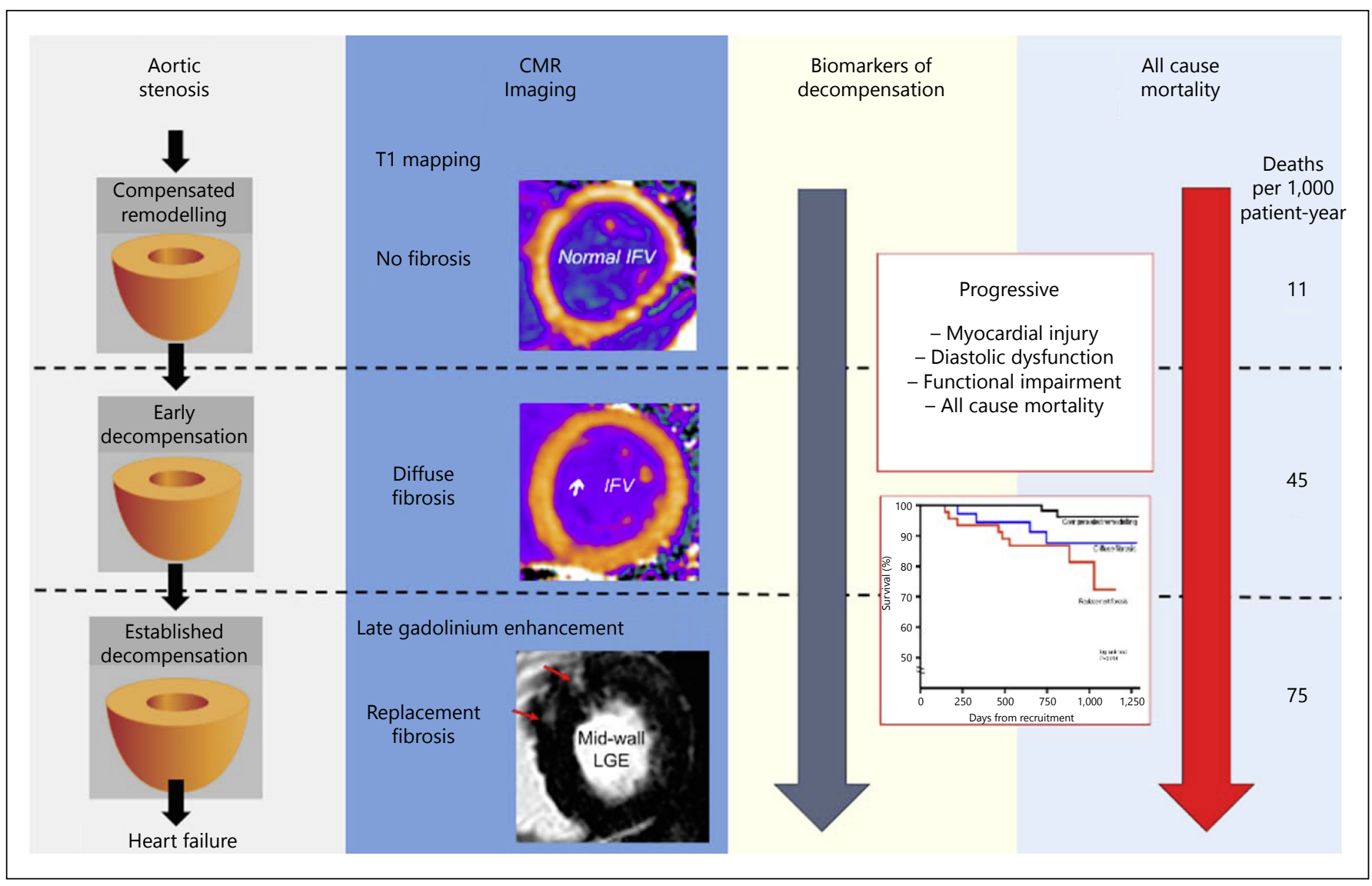

Fig. 1. (for Abstract of Russell J. Everett). 
0.014). Conclusion: CMR can monitor ventricular decompensation in aortic stenosis identifying the development of diffuse and then replacement fibrosis. This may be of major clinical value in tracking the health of the myocardium in heart valve disease and in optimizing timing of aortic valve replacement.

\section{Causes and Mechanisms of Isolated Mitral Regurgitation in the Community: Impact and Outcome}

Volha Dziadzko, Marie-Annick Clavel, Maurice Enriquez-Sarano

Mayo Clinic, Rochester, MN, USA

Background: Mitral regurgitation (MR) is frequent but mechanisms, etiology in the population and implication for outcome and management are unknown. Methods: We identified all cases of isolated (no other left-sided valvular disease or previous valvular surgery) moderate-severe MR in an entire community (Olmsted County, MN) in 2000-2010 using Doppler echocardiography. Among 29,390 patients with echoes 727 fulfilled eligibility criteria with electronic images for direct examination. Results: Overall age was $73 \pm 18$ years, $51 \%$ females, ejection fraction (EF) $49 \pm 17 \%$ with $65 \%$ functional MR (FMR), 32\% organic MR (OMR), 2\% mixed. Stratified by mechanism, FMR was due to LV remodeling (valve tenting) in $38 \%$ and to pure annular dilatation in $27 \%$, degenerative OMR with prolapse/flail in $23 \%$ and miscellaneous MR in $12 \%$. This isolated MR mechanism shows profound differences in clinical, echocardiographic characteristics and outcomes (table 1). Conclusions: Analysis of etiology and mechanism of isolated MR provides information of essential clinical value. FMR is the most common cause, most frequently with tenting and low EF. FMR due to isolated annular dilatation is often with atrial fibrillation/flutter. Degenerative MR predominates in organic MR. Mortality rate is considerably higher in FMR particularly with LV re- modeling. Standard surgical treatment is exceptionally used in FMR irrespective of EF and incompletely used in degenerative MR emphasizing MR unmet therapeutic need.

\section{Aortic Valve Stenosis Complicates the Hemodynamic Hypothesis for Regional Bicuspid Aortic Valve Aortopathy Development}

\author{
Alex J. Barker ${ }^{1}$, Pim van Ooij ${ }^{2}$, Emilie Bollache ${ }^{1}$, \\ S Chris Malaisrie ${ }^{1}$, Patrick M. McCarthy' ${ }^{1}$, Jeremy Collins ${ }^{1}$, \\ James Carr ${ }^{1}$, Paul W. M. Fedak ${ }^{3}$, Michael Markl ${ }^{1}$ \\ ${ }^{1}$ Northwestern University, Chicago, IL, USA; ${ }^{2}$ Amsterdam \\ Medical Center, Amsterdam, Netherlands; ${ }^{3}$ University of \\ Calgary, Calgary, AB, Canada
}

Objective: Bicuspid aortic valve (BAV) morphology will alter transvalvular blood flow patterns and vessel wall shear stress (WSS). These hemodynamic changes have been associated with the regional expression of BAV aortopathy. The purpose of this study was to use aortic WSS atlases to understand the role of aortic valve morphology and stenosis on the expression of WSS in the ascending aorta of a large BAV patient cohort. Methods: $256 \mathrm{pa}$ tients underwent MR angiography, 4D flow and bSSFP MRI for surveillance or diagnosis of BAV. Valve morphology was determined using bSSFP and recorded as right-left coronary $(\mathrm{RL}, \mathrm{n}=$ $199,47 \pm 14$ years $)$ or right-noncoronary leaflet fusion $(\mathrm{RN}, \mathrm{n}=$ $57,48 \pm 13$ years). $4 \mathrm{D}$ flow was used to determine significant stenosis (peak velocity $>3 \mathrm{~m} / \mathrm{s}$ ) and WSS was computed from the velocity field. WSS atlases (fig. 1) were created for each morphology group (RL or RN) stratified by presence of stenosis (+ or -). Confidence intervals were plotted using the voxelwise standard deviation (SD) of WSS. Results: Fig. 1 summarizes the regional WSS atlases and confidence intervals (SD maps) for the BAV patients. The outflow patterns were fairly consistent across the un-

Table 1. Clinical, echocardiographic parameters and outcomes of isolated MR classified by etiology and mechanism (mean \pm SD or \%) (for Abstract of Volha Dziadzko)

\begin{tabular}{|c|c|c|c|c|c|}
\hline & $\begin{array}{l}\text { FMR ventricular } \\
\text { remodeling }\end{array}$ & $\begin{array}{l}\text { FMR isolated } \\
\text { annular dilatation }\end{array}$ & $\begin{array}{l}\text { OMR } \\
\text { degenerative }\end{array}$ & $\begin{array}{l}\text { Miscellaneous } \\
\text { MR }\end{array}$ & $\mathrm{p}$ value \\
\hline $\mathrm{N}$ cases, $\%$ & $278(38)$ & $194(27)$ & $168(23)$ & $87(12)$ & \\
\hline Age at diagnosis, years & $73 \pm 14$ & $80 \pm 10$ & $71 \pm 16$ & $59 \pm 30$ & $<0.0001$ \\
\hline Gender, male, $\%$ & 59 & 32 & 60 & 34 & $<0.0001$ \\
\hline \multicolumn{6}{|l|}{ Echocardiographic parameters } \\
\hline MV regurgitant volume, $\mathrm{ml}$ & $38 \pm 13$ & $37 \pm 11$ & $54 \pm 26$ & $44 \pm 19$ & $<0.0001$ \\
\hline LV end-diastolic diameter, $\mathrm{mm}$ & $59 \pm 8$ & $48 \pm 5$ & $52 \pm 7$ & $46 \pm 10$ & $<0.0001$ \\
\hline Atrial fibrillation/flutter, \% & 29 & 54 & 13 & 10 & $<0.0001$ \\
\hline \multicolumn{6}{|l|}{ Outcomes } \\
\hline MV surgery 5 years, $\%$ & $3 \pm 1$ & $3 \pm 1$ & $44 \pm 4$ & $20 \pm 5$ & $<0.0001$ \\
\hline Survival 5 years, $\%$ & $46 \pm 3$ & $50 \pm 4$ & $74 \pm 3$ & $51 \pm 6$ & $<0.0001$ \\
\hline
\end{tabular}


Fig. 1. Wall shear stress (WSS) and WSS standard deviation (SD) atlases of 256 bicuspid aortic valve (BAV) patients broken into 4 groups by degree of stenosis and valve morphology: a, b right-left ( $\mathrm{RL}$ ) coronary leaflet fusion patients, and c, $\mathbf{d}$ rightnoncoronary leaflet fusion patients. Note the high standard deviation in the stenotic BAV patients, which indicates a large amount per patient WSS variability (as compared to the unobstructed BAV patients) (for Abstract of Alex J. Barker).

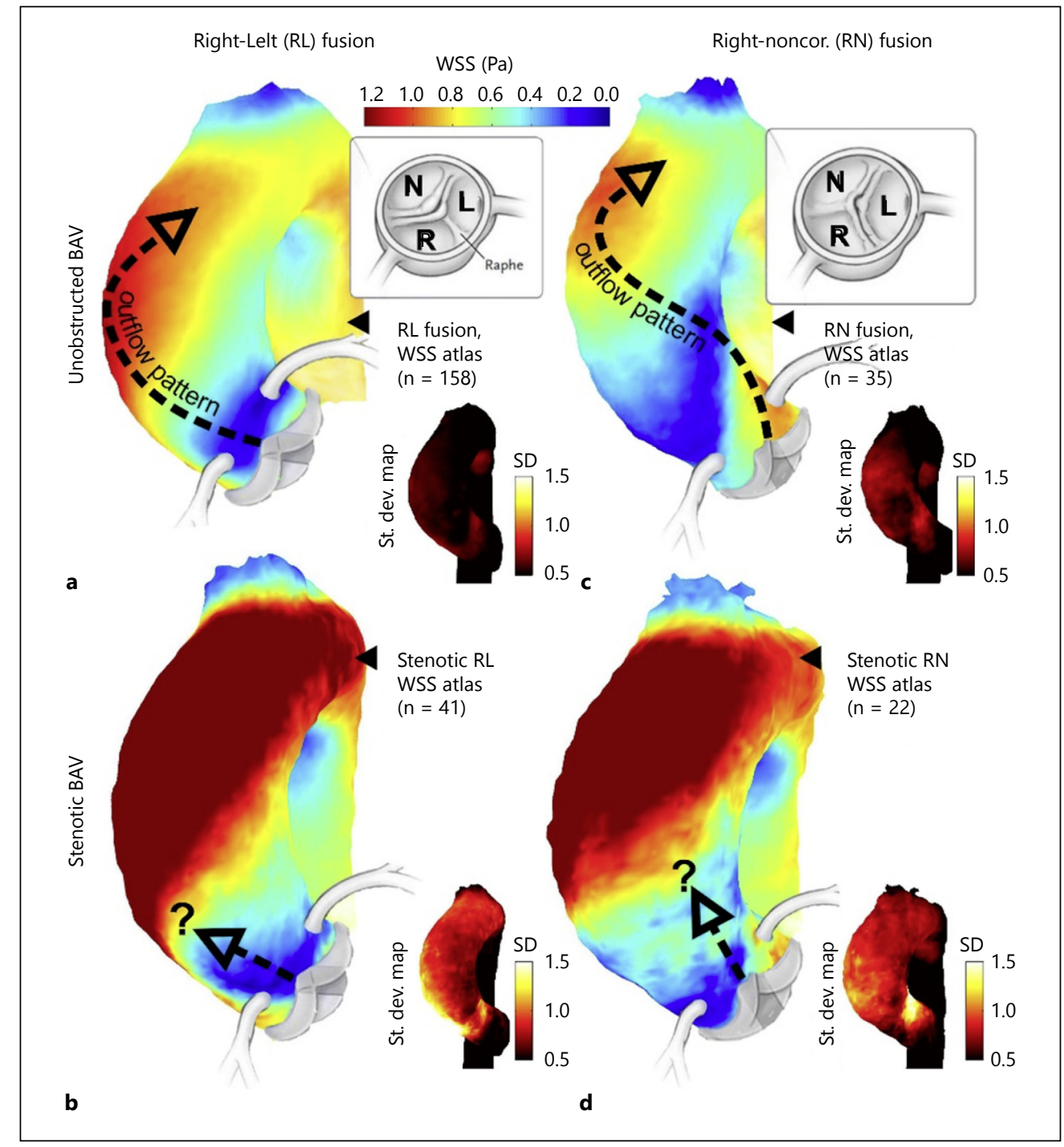

obstructed patients, as seen by the low magnitude of the SD maps. $41 \mathrm{RL}$ and $22 \mathrm{RN}$ patients were found to be significantly stenotic. In the stenotic group, the outflow patterns varied markedly as evaluated by the SD maps. Significantly higher WSS was observed in the stenosis cohorts. Aortic size was similar between all four groups for the sinus of valsalva (RL-, 39.6; RL+, 37.9; RN-, 38.6; $\mathrm{RN}+, 40.8 \mathrm{~mm}$ ) and mid-ascending aorta (RL-, 38.4; RL+, 38.8; $\mathrm{RN}-, 37.6 ; \mathrm{RN}+, 41.2 \mathrm{~mm}$ ). Conclusions: A consistent pattern of elevated WSS was found in the unobstructed RL (at the root and tubular ascending aorta) and RN patients (in the distal ascending aorta). Aortic valve stenosis was found to introduce more variability in the WSS maps.

\section{Low Ejection Fraction, Low-Flow, Low-Gradient Aortic Stenosis: Dobutamine Stress Echocardiographic Criteria to Assess Stenosis Severity}

Eden Touboul', Abdellaziz Dahou', Ian Burwash², Maurice Enriquez-Sarano ${ }^{3}$, Jutta Bergler-Klein ${ }^{4}$, Stefan Orwat ${ }^{5}$, Helmut Baumgartner ${ }^{5}$, Gerald Mundigler ${ }^{4}$, Eric Larose ${ }^{1}$, Philippe Pibarot ${ }^{1}$, Marie-Annick Clavel ${ }^{1}$

${ }^{1}$ IUCPQ, Quebec, QC, ${ }^{2}$ Ottawa Heart Institute, Ottawa, ON, Canada; ${ }^{3}$ Mayo Clinic, Rochester, MN, USA; ${ }^{4}$ Vienna General Hospital, Vienna, Austria; ${ }^{5}$ Muenster general hospital, Muenster, Germany

Objective: In patients with low-flow, low-gradient aortic stenosis (LFLGAS), dobutamine stress echocardiography (DSE) has been shown to assess stenosis severity. ACC/AHA guidelines con- 


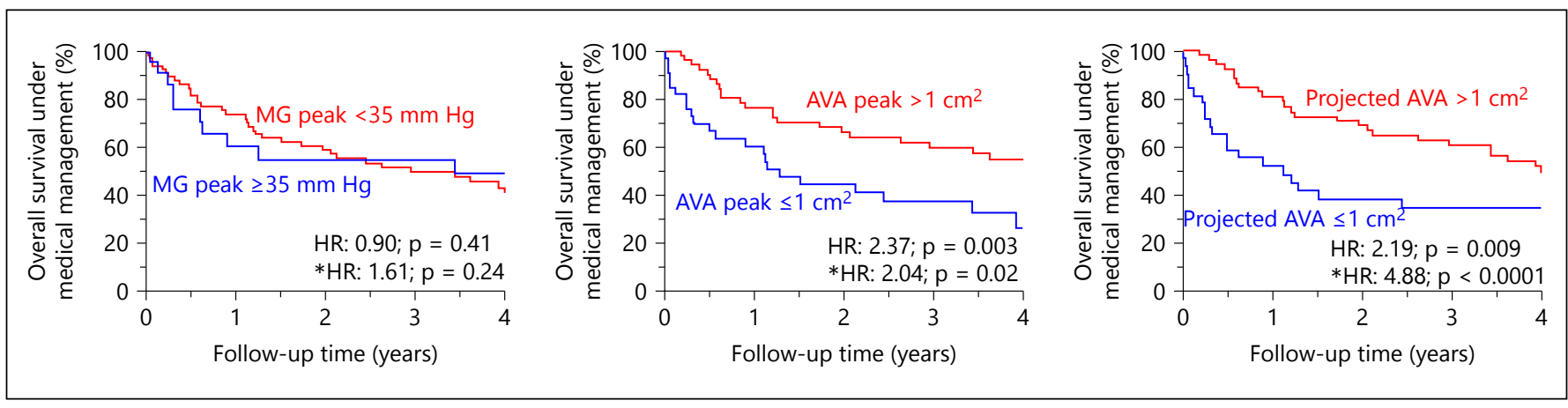

Fig. 1. Overall survival under medical management according to dobutamine stress echocardiographic parameters. * Adjusted for age, sex, coronary artery disease and chronic kidney failure (for Abstract of Eden Touboul).

sider patients to have true-severe stenosis when mean gradient $(\mathrm{MG}) \geq 40 \mathrm{~mm} \mathrm{Hg}$ with an aortic valve area $(\mathrm{AVA}) \leq 1 \mathrm{~cm}^{2}$ at any stage of DSE. However these criteria have not been previously validated. The aim of this study was to assess the value of these criteria to predict the presence of severe AS and the occurrence of death in patients with LFLGAS. Methods: 187 patients with LFLGAS were prospectively recruited in the TOPAS study and underwent a DSE with measurement of MG, AVA and the projected-AVA at normal flow rate. Actual severity of AS was corroborated by Computed Tomographic measurement of aortic valve calcium in 33 patients and by macroscopic evaluation (including valve weight) of the explanted valve in 54 patients. According to this assessment, 50/87 (57\%) of patients had true-severe stenosis. Results: Peakstress-MG or stress-AVA were not able to discriminate true vs. pseudo severe AS whereas projected-AVA was $(\mathrm{p}=0.01)$. Moreover, the composite of $\mathrm{MG} \geq 40 \mathrm{~mm} \mathrm{Hg}$ and AVA $\leq 1 \mathrm{~cm}^{2}$ allow correct classification of AS severity in $46 \%$ of patients versus $70 \%$ with projected-AVA $\leq 1 \mathrm{~cm}^{2}$. Interestingly, while the best threshold of stress-AVA and projected-AVA to predict the presence of true-severe stenosis was $1.0 \mathrm{~cm}^{2}$, the best cut-point for MG was $35 \mathrm{~mm} \mathrm{Hg}$. Among the subset of 91 patients with conservative management, 52 died during a follow-up of $2.8 \pm 2.5$ years. After adjustment for age, sex, coronary artery disease and chronic kidney failure, peak-stress-MG $\geq 35 \mathrm{~mm} \mathrm{Hg}$ was not a predictor of mortality in this subset, whereas peak-stress-AVA $\leq 1 \mathrm{~cm}^{2}$ and, to a larger extent, projected-AVA $\leq 1 \mathrm{~cm}^{2}$ were strong predictors of mortality (figure 1). Conclusions: The main DSE criteria proposed in the guidelines to identify true-severe AS and recommend valve replacement in LFLGAS has limited value to predict actual hemodynamic stenosis severity and outcomes. It is preferable to use the projected-AVA to identify true-severe AS in LFLGAS. Moreover, the projected-AVA is the best predictor of mortality under conservative management.

\section{The En-Face Quantitative Analysis of Valvular Calcification; Novel Physiological Insights into Aortic Stenosis Using 4-Dimensional Computed Tomography}

William S. Jenkins ${ }^{1}$, Louis Simard ${ }^{2}$, Jérome Hourdain ${ }^{2}$, Marie-Annick Clavel ${ }^{2}$, Maurice Enriquez-Sarano ${ }^{1}$

${ }^{1}$ Mayo Clinic, Rochester, MN, USA; ${ }^{2}$ Laval University, Quebec, QC, Canada

Objectives: Aortic Valve Calcification (AVC) is mechanistic to aortic stenosis (AS) with classical concepts assuming homogeneous calcifications leading to severe AS. Multidetector computed tomography (MDCT) accurately measures global AVC but AVC distribution remains elusive due to imaging obliquity. We developed en-face imaging by re-registration of 4D-MDCT to quantify AVC spatial distribution. Methods: In 200 AS patients $(79 \pm$ 8 years; mean gradient $39 \pm 13 \mathrm{~mm} \mathrm{Hg}$ ) 4D volume rendered MDCT datasets were oriented to enface view for AVC spatial scoring (figure 1) including cusp edge calcification and AVC asymmetry. Results: Despite high AVC load (528 [353-700] AU/ $\mathrm{cm}^{2}$ ), asymmetry was present in $33 \%$ of subjects, with maximum AVC at non-coronary cusp in $75 \%(\mathrm{p}<0.001)$. Cusp edge calcification was none-trivial in $11 \%$, mild-moderate in $73 \%$ and severe in $15 \%$. Multivariately, adjusting for AVC severe AS was likely with symmetrical AVC (odds ratio [OR] 2.18, $\mathrm{p}=0.02$ ) and with edge calcification mild-moderate (OR 9.25, $\mathrm{p}=0.007$ vs. none-trivial) to severe (OR 4.63, $\mathrm{p}=0.01$ ), improving AS severity models $(\mathrm{p}<$ 0.001 ). Conclusions: MDCT en-face quantitation provides new insight into AS pathophysiology. Contrary to classical concepts, AVC is often inhomogeneous and asymmetric. AS severity, mainly determined by AVC load, is also influenced by its distribution, particularly to cusp edges. The impact of quantitatively measured AVC asymmetry on transcutaneous aortic valve replacement should be evaluated. 
Fig. 1. a 4-D MDCT en-face view of $\mathrm{AV}$ with asymmetric AVC; b edge calcification versus AS severity (for Abstract of William S. Jenkins).

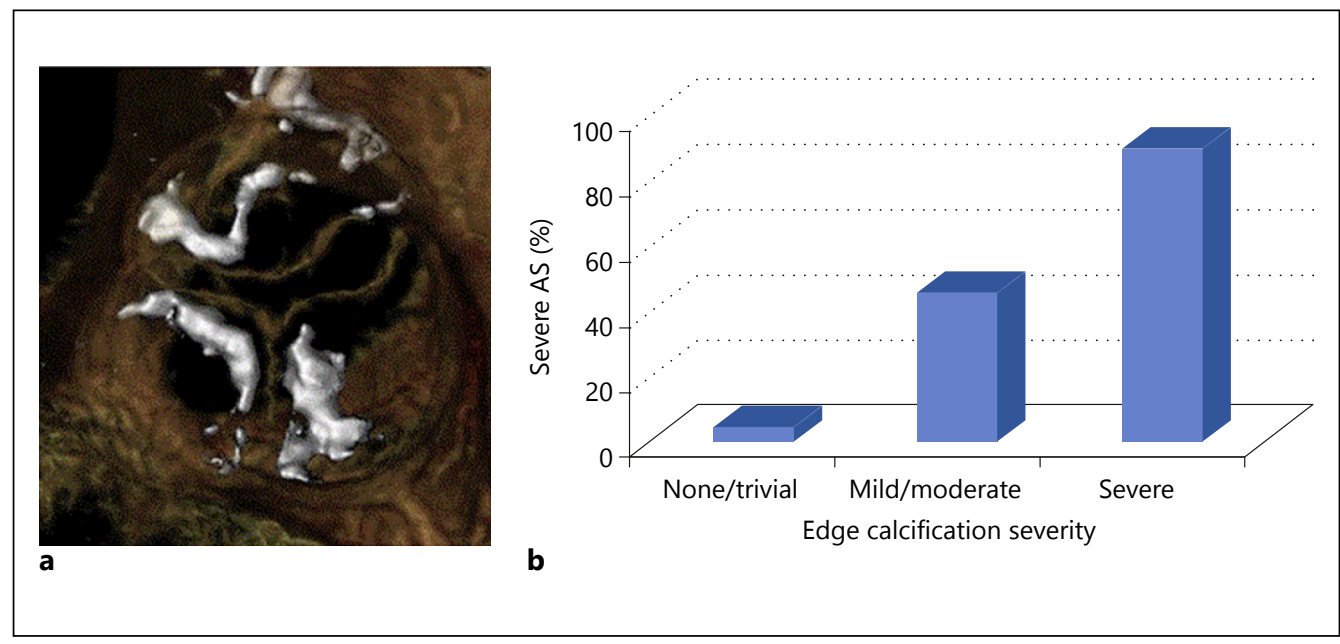

\section{Prognostic Value of Longitudinal Strain in Asymptomatic Aortic Stenosis and Preserved Left Ventricular Ejection Fraction - The COFRASA/ GENERAC Study}

Maria Melissopoulou, Virginia Nguyen, Tiffany Mathieu, David Attias, Julien Dreyfus, Isabelle Codogno, Alec Vahanian, David Messika-zeitoun

Bichat Claude Bernard Hospital, Paris, France

Background: It has been suggested that myocardial systolic deformation parameters may be a more sensitive marker of left ventricular (LV) systolic dysfunction than LV ejection fraction (LVEF). However, its prognostic value in patients with aortic stenosis (AS) remains debated. Methods: In an ongoing prospective cohort of asymptomatic patients with at least mild, pure, isolated AS, global longitudinal strain (GLS) and basal longitudinal strain (BLS) were measured at baseline using 2D speckle tracking imaging, and AS related events (occurrence of symptoms, congestive heart failure and sudden death) were prospectively collected. Results: We prospectively enrolled 160 patients $(72 \pm 10$ years, 112 males $(70 \%)$ ). Mean valve area (AVA) was $1.23 \pm 0.33 \mathrm{~cm}^{2}$, mean pressure gradient (MPG) $29 \pm 17 \mathrm{~mm} \mathrm{Hg}$ and mean peak velocity (PV) $3.4 \pm 0.8 \mathrm{~m} / \mathrm{sec}$. Overall, 58 patients had a severe AS (AVAi $\leq 0.6 \mathrm{~cm}^{2} / \mathrm{m}^{2}$ ). BLS but not GLS was correlated to AS severity (all $\mathrm{p}<0.05$ ). During a follow up period of 2 years, 21 events occurred. GLS was not predictive of outcome in univariate or multivariate analysis (all $\mathrm{p}>0.10$ ) whereas BLS was predictive of outcome in univariate analysis $(\mathrm{p}=0.007)$ but not in multivariate analysis after adjustment for AS severity (all $p>0.05$ ). In the subset of severe AS, BLS was predictive of outcome only after adjustment for AVAi $(\mathrm{p}=0.02)$ but not after adjustment for PV $(\mathrm{p}=0.27)$ or MPG $(\mathrm{p}=$ 0.24 ). Conclusions: Our data suggest that longitudinal strain assessed by $2 \mathrm{D}$ speckle tracking echocardiography, has a poor predictive value for future AS related events in asymptomatic patients with AS and preserved LVEF. Thus, in daily practice, the selection of patients who should undergo an early aortic valve replacement should not rely exclusively on this parameter.

\section{Non-Invasive Assessment of Paravalvular Aortic Regurgitation following Transcatheter Aortic Valve Replacement Based on Energy Loss}

\section{Ali N. Azadani ${ }^{1}$, Peyman N. Azadani ${ }^{2}$}

${ }^{1}$ University of Denver, Denver, CO, ${ }^{2}$ University of Utah School of Medicine, Salt Lake City, UT, USA

Objective: Paravalvular aortic regurgitation $(\mathrm{AR})$ is a frequent complication following transcatheter aortic valve replacement (TAVR). There is growing evidence indicating a significant association of paravalvular AR, even mild, with short- and long-term mortality. However, assessment of paravalvular AR remains imprecise and controversial. In this study, a novel echocardiographic index was introduced based on energy loss to quantify the severity of paravalvular AR post TAVR. The objective of this study was to evaluate the severity of AR based on energy loss and assess the impact of AR on left ventricular (LV) function. Our main hypothesis was that TAVR with mild AR imposes a significantly higher workload on the LV than surgical valve replacement of equivalent bioprosthetic size in which AR is minimal. Methods: $23 \mathrm{~mm}$ surgical Carpentier-Edwards Perimount bioprostheses were tested in-vitro in a pulse duplicator. To examine the impact of paravalvular AR on transvalvular energy loss, $23 \mathrm{~mm}$ TAVs were implanted within normal $23 \mathrm{~mm}$ Perimount bioprosthesis. We have previously shown that the valve-in-valve pressure gradient was not significantly different from normal $23 \mathrm{~mm}$ surgical bioprostheses; however, mild paravalvular AR was observed after TAV implantation. Transvalvular energy loss was measured through both invasive and non-invasive methods. Invasive measurements were achieved through measurement of instantaneous flow and pressure gradient, and non-invasive measurements were performed by Doppler echocardiography. Results: The total transvalvular energy loss of the $23 \mathrm{~mm}$ TAV measured by the echocardiographic method was $68 \%$ more than the $23 \mathrm{~mm}$ surgical bioprosthesis due to mild paravalvular leakage. During each cardiac cycle, $40 \%$ of TAV total energy loss was due to mild paravalvular AR. The novel echocardiographic method agreed well with invasive measurements with a 
maximum difference of $2.4 \%$. Conclusions: A novel echocardiographic method was developed to quantity transvalvular energy loss non-invasively. TAVR with mild AR imposed a significantly higher workload on the LV than surgical valve replacement of equivalent bioprosthetic size. Energy loss is an excellent method to obtain detailed information of TAV hemodynamic performance during the entire cardiac cycle. In addition, the new parameter may explain the significant association of paravalvular AR with shortand long-term mortality in TAVR patients.

\section{Heart Valve Outcomes}

\section{Early versus Delayed Surgery for Patients with Valvular Infective Endocarditis Complicated by Embolic Ischemic Cerebrovascular Events: A Meta-Analysis of 14 Studies}

\section{Christos G. Mihos ${ }^{1}$, Andres M. Pineda ${ }^{2}$, Orlando Santana ${ }^{2}$}

${ }^{1}$ Massachusetts General Hospital, Boston, MA, ${ }^{2}$ Coulmbia University, Division of Cardiology, Mount Sinai Heart Institute, Miami Beach, FL, USA

Background: An ischemic cerebrovascular accident (CVA) in the setting of valvular infective endocarditis occurs in $10 \%$ to $40 \%$ of patients, and is associated with significant morbidity. Given the increased risk of peri-operative cerebrovascular complications and mortality, the optimal timing of surgical intervention in this population is debated. Methods: Utilizing Pubmed, EMBASE, Ovid, and Cochrane databases, a systematic review was performed, and identified 14 studies published through September 2015, that compared early versus delayed valve surgery for patients with infective endocarditis complicated by an ischemic CVA. Early surgery was defined as $\leq 3$ days in $1, \leq 7$ days in 8 , and $\leq 14$ days in 5 studies, respectively. The main exclusion criteria were: 1) studies reporting on either early or delayed surgery only; and, 2) incomplete reporting of outcomes. The incidence of peri-operative CVA, operative mortality, and 1-year survival were selected as the end-points for the meta-analysis. Risk ratios (RR) were calculated by the MantelHaenszel method under a fixed or random effects model, as appropriate. Results: A total of 833 patients (early surgery $=330$, delayed surgery $=503$ ) were included in the studies. The majority of valve operations were performed for aortic and/or mitral valve infective endocarditis, with prosthetic valve disease present in $0 \%$ to $60 \%$. Infection caused by Staphylococcus Aureus ranged from $19 \%$ to $66 \%$, and the incidence of heart failure at the time of operation was $24 \%$ to $66 \%$. The strategy of early surgery was associated with an increased risk of operative mortality (RR 1.72, 95\% confidence interval [CI] 1.27-2.34, $\mathrm{p}=0.0005$ ), which was significant regardless of early surgical intervention within the first 7 days (RR 2.19, 95\% CI $1.45-3.31, \mathrm{p}=0.0002$ ) or 14 days (RR $1.72,95 \%$ CI $1.12-2.64, \mathrm{p}=0.01$ ) post-CVA. There was no effect of early surgical intervention on the risk of peri-operative ischemic (RR 0.90, 95\% CI 0.23-3.53, $\mathrm{p}=0.88$ ) or hemorrhagic CVA (RR 1.3, 95\% CI $0.70-2.43, \mathrm{p}=0.41$ ), or on 1 -year survival (RR $0.93,95 \%$ CI $0.78-$
$1.12, \mathrm{p}=0.44)$. Conclusions: In patients with valvular infective endocarditis complicated by ischemic CVA, an early surgical intervention may be associated with an increased risk of operative mortality, with no observed benefit in 1-year survival.

\section{Matched Comparison of Self Expanding Transcatheter Heart Valves for the Treatment of Failed Surgical Aortic Bioprosthesis: Insights from the Valve in Valve International Data (VIVID) Registry}

\begin{abstract}
Sami M. Alnasser ${ }^{1}$, Asim Cheema ${ }^{1}$, Ran Kornowski ${ }^{2}$, Matheus Simonato ${ }^{3}$, Thomas Walther ${ }^{4}$, Azeem Latib ${ }^{5}$, Luca Testa ${ }^{6}$, Jean Claude Laborde ${ }^{7}$, David Hildick Smith ${ }^{8}$, Marco Barbanti ${ }^{9}$, Won Keun Kim ${ }^{10}$, Francesco Bedogni ${ }^{11}$, Hafid Amrane ${ }^{12}$, A.J. van Boven ${ }^{13}$, Marc Pelletier ${ }^{14}$, Josep Rodes Cabau ${ }^{15}$, Joelle Kefer ${ }^{16}$, Christian Frerker ${ }^{17}$, Nicolas M. Van Mieghem ${ }^{18}$, Ganesh Manoharan ${ }^{19}$, Stephen G. Worthley ${ }^{20}$, Anita W. Asgar ${ }^{21}$, Carmelo Sgroi ${ }^{22}$, John Webb ${ }^{23}$, Danny Dvir ${ }^{23}$

${ }^{1}$ University of Toronto, Toronto, ON, Canada; ${ }^{2}$ Rabin Medical Center, Tel Aviv, Israel; ${ }^{3}$ Escola Paulista de Medicina, Sao Paulo, Brazil; ${ }^{4}$ Kerckhhoff Heart Center, Bad Nauheim, Germany; ${ }^{5}$ Ospedale San Raffaele, Milan, Italy; ${ }^{6}$ Istituto Clinico S. Ambrogio, Milan, Italy; ${ }^{7}$ St. George Hospital, London, Plumtree Nottingham, ${ }^{8}$ Royal Sussex County Hospital, Brighton, United Kingdom; ${ }^{9}$ Ferrarotto Hospital, University of Catania, Catania, Italy; ${ }^{10}$ Kerckhoff Heart Center, Bad Nauheim, Germany; ${ }^{11}$ Istituto Clinico S Ambrogio, Milan, Italy; ${ }^{12}$ Medisch Centrum, Leeuwarden, ${ }^{13}$ Medisch Centrum Leeuwarden, Leeuwarden, Netherlands; ${ }^{14} \mathrm{New}$ Brunswick Heart Center, Saint John, NB, ${ }^{15}$ Quebec Heart and Lung Institute, Laval, QC, Canada; ${ }^{16}$ Cliniques Saint Luc, Université Catholique de Louvain, Brussels, Belgium; ${ }^{17}$ Asclepios Clinic St. Georg, Hamburg, Germany; ${ }^{18}$ Erasmus MC, Rotterdam, Rotterdam, Netherlands; ${ }^{19}$ Royal Victoria Hospital, Belfast, United Kingdom; ${ }^{20}$ The University of Adelaide, Adelaide, Australia; ${ }^{21}$ Montreal Heart Institute, Montreal, QC, Canada; ${ }^{22}$ Ferrarotto's Hospital, Catania, Italy; ${ }^{23}$ St. Paul's Hospital, Vancouver, BC, Canada
\end{abstract}

Objective: Portico ${ }^{\circledR}$ (St. Jude Medical) and CoreValve ${ }^{\mathrm{TM}}$ (Medtronic) are both nitinol based self-expanding transcatheter heart valves with unique features enabling device retrievability for Portico and supra annular valve position for CoreValve systems. In this study, we sought to investigate the effect of the different self-expanding valve design on hemodynamic and clinical performance when implanted for degenerated aortic valve bioprosthesis (valve in valve, ViV). Methods: Patients undergoing Portico implantation for degenerated aortic bioprosthesis were compared to those undergoing CoreValve implantation after matching for differences in age, gender, STS score, procedural access, bioprosthesis valve design and label size in a 1:2 fashion. Blinded core lab analysis of post-implantation imaging data was conducted independently. Outcomes were defined as per Valve Academic Research Consortium II (VARC-II). Results: A total of 159 patients that underwent $\mathrm{ViV}$ using a self-expanding device were included in the analysis (age $79 \pm 8$ yrs, $61.5 \%$ female). The group included 53 Por- 
Table 1. (for Abstract of Sami M. Alnasser)

\begin{tabular}{|c|c|c|c|}
\hline & $\begin{array}{l}\text { Portico } \\
(\mathrm{n}=53)\end{array}$ & $\begin{array}{l}\text { CoreValve } \\
(n=106)\end{array}$ & $\begin{array}{l}\mathrm{p} \\
\text { value }\end{array}$ \\
\hline Age, years, mean \pm SD & $79 \pm 7$ & $78 \pm 9$ & 0.60 \\
\hline Females, n (\%) & $35(66)$ & $59(56)$ & 0.32 \\
\hline STS score, mean \pm SD & $6(6.5)$ & $6.7(5.5)$ & 0.92 \\
\hline \multicolumn{4}{|l|}{ Surgical valve characteristics } \\
\hline Surgical label size, mean \pm SD & $22.8 \pm 1.8$ & $22.8 \pm 2.1$ & 1 \\
\hline $\mathrm{AVA}, \mathrm{cm}^{2}$, mean $\pm \mathrm{SD}$ & $0.94 \pm 0.45$ & $0.86 \pm 0.4$ & 0.3 \\
\hline Mean gradient, $\mathrm{mm} \mathrm{HG}$, mean $\pm \mathrm{SD}$ & $35 \pm 17.5$ & $37 \pm 17.6$ & 0.52 \\
\hline Stentless, $\mathrm{n}(\%)$ & $5(9)$ & $9(9)$ & 1 \\
\hline \multicolumn{4}{|l|}{ Procedural details } \\
\hline Peripheral access, $\%$ & 94 & 95 & 0.84 \\
\hline Post-dilatation, $\%$ & 26 & 20 & 0.42 \\
\hline \multicolumn{4}{|l|}{ Outcomes } \\
\hline Death & $2(4)$ & $1(1)$ & 0.29 \\
\hline Stroke & $1(2)$ & $0(0)$ & 0.16 \\
\hline Major bleeding & $6(11)$ & $4(4)$ & 0.07 \\
\hline Major vascular bleeding & $1(2)$ & $3(3)$ & 0.55 \\
\hline New pacemaker, $\mathrm{n}(\%)$ & $1(2)$ & $6(7)$ & 0.43 \\
\hline Composite endpoint of (coronary obstruction, malposition, 2nd THV), $\mathrm{n}(\%)$ & $2(4)$ & $10(9)$ & 0.34 \\
\hline \multicolumn{4}{|l|}{ Post Implantation Hemodynamics } \\
\hline $\mathrm{EOA}, \mathrm{cm}^{2}$, mean $\pm \mathrm{SD}$ & $1.31 \pm 0.47$ & $1.62 \pm 0.55$ & 0.003 \\
\hline Prosthesis patient mismatch, any $\left(<0.85 \mathrm{~cm}^{2} / \mathrm{m}^{2}\right), \%$ & 77 & 55 & 0.02 \\
\hline $\mathrm{MG}$, mean $\pm \mathrm{SD}$ & $17.2 \pm 7.6$ & $15.5 \pm 7.4$ & 0.19 \\
\hline AI ( $\geq$ moderate $)$, core lab reported, $\mathrm{n}(\%)$ & $7 / 51(13.7)$ & $4 / 94(4.2)$ & 0.04 \\
\hline
\end{tabular}

tico implantations that were matched with 106 CoreValve patients having similar clinical and surgical valve characteristics. Baseline demographic and procedural characteristics, echocardiographic and clinical outcomes are detailed in the table. Clinical outcomes were comparable between the two groups; trend toward higher rate of major bleeding is noted among Portico group. Malpositioning, need for 2nd THV and coronary obstruction were higher among CoreValve but not statistically significant ( $4 \%$ vs. $9 \%, \mathrm{p}=$ $0.34)$. CoreValve is associated with larger effective orifice area $(1.31 \pm 0.47$ vs. $1.62 \pm 0.55, \mathrm{p}=0.003)$, lower rate of patient prosthesis mismatch ( $77 \%$ vs. $55 \%, \mathrm{p}=0.02)$, with comparable postoperative mean aortic gradient $(17.2 \pm 7.6$ Vs. $15.5 \pm 7.4, \mathrm{p}=0.19)$. Rate of $\geq$ moderate aortic insufficiency was observed more frequently among Portico compared to CoreValve group $(13.7 \%$ vs. $4.2 \%, \mathrm{p}=0.04)$. Conclusions: Design differences between the studied self-expanding devices are associated with variable post implantation THV hemodynamics, observed more favorably in the CoreValve group. Further analyses of implantation data (operators experience, THV depth etc.) \& longer term outcomes are planned to further understand the mechanism and significance of observed hemodynamic differences.

\section{Transcatheter Mitral Valve Implantation: One Year Outcomes of the First-in-Man Experience with an Apically Tethered Device}

\author{
Alison Duncan, Cesare Quarto, Simon Davies, \\ Anselm Uebing, Neil Moat \\ The Royal Brompton Hospital, London, United Kingdom
}

Background: Long-term outcome after Transcatheter Mitral Valve Implantation (TMVI) has received little attention. We report one-year outcomes of first-in-man implants with the TENDYNE Bioprosthetic Mitral Valve System. Methods: Four patients underwent TMVI using a TENDYNE system (tri-leaflet porcine pericardial valve in self-expanding nitinol frame, with an apical tether to stabilize the device). The device was implanted under 3-dimensional transoesophageal echocardiographic guidance using general anaesthesia via a transapical left mini-thoracotomy using a 34Fr sheath advanced over a wire into the left atrium. The device was extruded through the sheath, positioned by rotating into the correct anatomical position, and deployed. The apical pad was inserted and tension on the tether was adjusted to optimize device position. The apical pad facilitated LV apical closure. Results: TMVI procedures were performed between October 2014 and January 2015. Immediately after TMVI, no patient had paravalvular or transvalvular mitral regurgitation, or increased transmitral gradient (table 1). One patient (previous mitral annuloplas- 
Table 1. Patient outcome after TENDYNE TMVI (for Abstract of Alison Duncan)

\begin{tabular}{lllll}
\hline & Patient 1 & Patient 2 & Patient 3 & Patient 4 \\
\hline Age (years) & 68 & 75 & 87 & 75 \\
Alive 6-months after TMVI & Yes & Yes & Yes & Yes \\
NYHA class (baseline to 6 months) & From IV to I & From IV to I & From III to I & From III to II \\
Paravalvular MR on echo at 6 months & None & Trivial & Trivial \\
LVOT gradient (mm Hg) at 6 months & 5 & 4 & 7 & None \\
Pulmonary pressure (mm Hg) (baseline to 6 months) & From 104 to 65 & From 61 to 38 & From 75 to 37 \\
Six-minute walk (m) (baseline to 6 months) & From 240 to 320 & From 210 to 330 & Not done 45 to 20 \\
Brain natiuretic peptide (ng/dl) (baseline to 6 months) & From 347 to 156 & From 335 to 235 & From 470 to 682 From 143 to 216 \\
Alive 1-year after TMVI & Yes & Yes & No & Yes \\
\hline
\end{tabular}

ty) developed left ventricular outflow tract (LVOT) obstruction $(95 \mathrm{~mm} \mathrm{Hg})$ associated with systolic anterior motion of the mitral valve. Following LVOT stenting, LVOT gradient decreased to $47 \mathrm{~mm} \mathrm{Hg}$. All 4 patients were discharged home, independent of activities of daily living. At 6-months, all were alive, living independently at home, with no further heart failure hospital admissions. Functional capacity and 6-minute walk test increased in all. All valves were stable, with reduction in tricuspid regurgitation and pulmonary pressures. Two patients had evidence of trivial paravalvular MR but were asymptomatic; one had biochemical evidence of haemolysis but was not anaemic. At one year, one patient had died of an embolic stroke (on aspirin, declined anticoagulation). Conclusions: We report satisfactory 6-month outcomes of first-in-man implants with the TENDYNE Bioprosthetic Mitral Valve System. All were living independently at home at 6-months. At one-year, 1 patient had died of an embolic stroke. We envisage reporting 1-year echocardiographic, biochemical and functional data by January 2016.

\section{Conventional Mitral Valve Surgery in MitraClip Candidates}

Thilo Noack, Yamen Al Ghanam, Philipp Kiefer, Fabian Emrich, Philipp Lurz, David M. Holzhey, Farhad Bakhtiary, Martin Misfeld, Gerhard Schuler, Friedrich-Wilhelm Mohr, Joerg Seeburger

Heart Center Leipzig University, Leipzig, Germany

Objective: The aim of this study was to evaluate the surgical outcome in patients with mitral regurgitation (MR), who were assigned for percutaneous mitral valve repair (PMVR), but were declined for PMVR by the heart team and afterwards scheduled for conventional mitral valve (MV) surgery. The clinical outcome for this group has not been well described. Methods: The reasons for declination of PMVR, perioperative and operative data, 30day mortality and 1-year survival after conventional MV surgery were retrospectively analysed. Results: Between 08/2009 to 08/2013, 118 patients were assigned for PMVR. 29 patients (mean age $77 \pm 8$ years; $62 \%$ male) with high surgical risk (logistic EuroSCORE $17.3 \pm 10.8 \%$ ) were declined for PMVR by the heart team due to contraindication according EVEREST-criteria $(\mathrm{n}=$
$13 ; 42 \%)$, relevant tricuspid regurgitation $(\mathrm{n}=13 ; 42 \%)$, possibility for conventional MV repair (MVR) $(n=6 ; 19 \%)$, relevant coronary artery disease in 2 patients $(6 \%)$ or no indication for $\mathrm{MV}$ intervention in 1 patient $(3 \%)$. Two (6\%) patients had an indication for left ventricular assist device (LVAD) implantation. Isolated minimally invasive MVR were successfully performed in 6 patients (21\%), MVR with tricuspid valve repair (TVR) in 5 patients (17\%), isolated MV replacement in 6 patients (21\%), MV replacement with TVR in 7 patients $(24 \%)$ and isolated coronary artery bypass grafting in 1 patient (3\%). LVAD implantation was performed in 2 patients (7\%). The 30 -day mortality was $0 \%$, and the estimated overall 1 -year survival was $77.8 \%$. The observed mortality was significantly lower than predicted by EuroSCORE. Conclusions: Conventional MV surgery in MitraClip candidates can be performed without 30 -day mortality and promising 1-year survival. The conventional MV surgery could be considered in MitraClip candidates with surgical relevant co-morbidities and contraindication according EVEREST-criteria, performed in experienced centers. Furthermore, the data underline the importance of interdisciplinary heart team decision for each MitraClip candidate.

\section{Epidemiology of Valvular Heart Diseases: A 30-Year Retrospective of Hospitalizations and In-Hospital Mortality in New York State}

\author{
Phyllis G. Supino, Ofek Y. Hai, Jeffrey S. Borer \\ SUNY Downstate Medical Center, Beverly, MA, USA
}

Objective: The epidemiology of valvular heart disease (VHD) is inadequately defined. The study objectives were to define temporal trends in the volume of hospitalized patients, surgical procedures and mortality outcomes in a large geographic area during the past three decades. Methods: We conducted a longitudinal analysis of all inpatient hospital records $(79,689,879)$ obtained from the New York State (NYS) Statewide Planning and Research Cooperative System (SPARCS) database for years [yrs] 19832012. VHD cases $(2,720,313)$ were identified from principal or secondary ICD-9 codes for aortic, mitral, tricuspid or pulmonic VHD. Linear regression was used to evaluate trends over time for 
VHD hospitalizations, valve surgery (VS) and in-hospital deaths (all VHD, VS). Logistic regression was used to predict mortality risk factors. Results: From 1983-2012, total hospitalizations decreased by $\sim 500,000$ cases; simultaneously, VHD hospitalizations increased markedly (34,395 in 1983 to 125,139 in 2012). Rate of increase was linear across all VHD categories $=4,248$ new cases $(12.4 \%) / y r, r^{2}=0.99, \mathrm{p}<0.0001$ through 2006 (peak $=132,323$ cases), remaining at $\sim 130,000$ cases/yr through 2012. A parallel trend was found for performance of VS (2,582 cases in 1983 to 7,787 in 2012), linearized increase rate $=207 \mathrm{VS}(8.0 \%) / \mathrm{yr}, \mathrm{r}^{2}=$ $0.97, \mathrm{p}<0.001$. Both numbers of hospitalizations and performance of VS increased with patient age $(\mathrm{p}<0.001)$. Over the study interval, 123,787 patients with VHD died in the hospital, including 9,272 who died after VS; avg. case fatality rates were 4.6\% (all VHD) and 6.4\% (VS). Deaths were independently associated with advancing age, nonelective admission, and presence of associated HF ( $\mathrm{p}<0.0001$, all). Male gender predicted increased risk of death among the general VHD population; female gender predicted risk of death among those undergoing VS. Conclusions: The incidence of VHD hospitalization and VS in NYS has substantially risen since the early 1980s and can be expected to rise as the population ages. These findings suggest intensive planning for dealing with public health implications of these increases as we attempt to meet the growing needs of this patient population.

\section{Right Ventricular Function By Speckle Tracking and Outcomes in Low-Flow, Low Gradient Aortic Stenosis with Low LVEF}

\author{
Abdellaziz Dahou' ${ }^{1}$, Marie-Annick Clavel', Romain Capoulade ${ }^{2}$, \\ Philipp E. Bartko ${ }^{2}$, Julien Magne ${ }^{3}$, Gerald Mundigler ${ }^{4}$, \\ Jutta Bergler-Klein ${ }^{4}$, Ian Burwash ${ }^{5}$, Julia Mascherbauer4, \\ Henrique B. Ribeiro', Kim O'Connor' ', Helmut Baumgartner ${ }^{6}$, \\ Mario Senechal', Jean G. Dumesnil', Raphael Rosenhek ${ }^{4}$, \\ Josep Rodés-Cabau ${ }^{1}$, Philippe Pibarot ${ }^{1}$ \\ ${ }^{1}$ Québec Heart-Lung Institute, Québec, QC, Canada; \\ ${ }^{2}$ Massachusetts General Hospital, Boston, MA, USA; ${ }^{3}$ Centre \\ Hospitalier Universitaire Limoges, Limoges, France; ${ }^{4}$ Division \\ of Cardiology, Medical University of Vienna, Vienna, Austria; \\ ${ }^{5}$ University of Ottawa Heart Institute, Ottawa, ON, Canada; \\ ${ }^{6}$ Department of Cardiovascular Medicine, University Hospital \\ Muenster, Muenster, Germany; ${ }^{7}$ Laval University, Québec, QC, \\ Canada
}

Background: Left ventricular longitudinal strain (LVLS) is a powerful predictor of outcome in patients with low-flow, lowgradient aortic stenosis (LF-LG AS) and low LV ejection fraction (LVEF). However, the impact of right ventricular (RV) function on the outcome of these patients remains unknown. Objectives: The aim of this study was to examine the impact of RV function as evaluated by RV free wall longitudinal strain (RVLS) on mortality in patients with LF-LG AS and low LVEF. Methods: 211 patients with LF-LG AS (mean gradient $<40 \mathrm{~mm} \mathrm{Hg}$ and indexed aortic valve area [AVA] $\leq 0.6 \mathrm{~cm}^{2} / \mathrm{m}^{2}$ ) and low LVEF $[\leq 40 \%]$ were prospectively recruited in the True or Pseudo-severe Aortic Stenosis (TOPAS) study. AS severity was assessed using the pro- jected aortic valve area (AVAproj) at normal flow rate. Among the 211 patients, 128 had RVLS measurement available at rest and were included in this analysis. RVLS measurement under dobutamine stress echocardiography (DSE) was available in 58 of these 128 patients. Results: Two-year survival was lower in patients with RVLS $|13| \%(69 \pm 5 \%)(\mathrm{p}=0.04)$. In multivariable Cox analysis stratified for the type of treatment (AVR vs. conservative) and adjusted for age, AS severity, previous myocardial infarction and LVLS, rest $\mathrm{RVLS}<|13| \%(\mathrm{HR}=2.70$; $95 \% \mathrm{CI}=$ $1.19-6.11 ; \mathrm{p}=0.018)$ was independently associated with all-cause mortality. RVLS had incremental prognostic value over baseline risk factors and LVLS (likelihood ratio; $\mathrm{p}=0.01$ ). Reduced stress RVLS was also associated with increased risk of mortality (stress $\mathrm{RVLS}<|14| \%$ : $\mathrm{HR}=2.98 ; 95 \% \mathrm{CI}=1.30-6.52 ; \mathrm{p}=0.01$ ). In multivariable Cox analysis, stress RVLS $<|14| \%$ remained independently associated with mortality $(\mathrm{HR}=2.94 ; 95 \% \mathrm{CI}=1.23-7.02$; $\mathrm{p}=0.015)$. After further adjustment for rest RVLS, stress RVLS $<|14| \%$ remained independently associated with mortality (HR = 3.29; 95\% CI = 1.17-9.25; $\mathrm{p}=0.024)$, whereas rest RVLS was not $(p>0.05)$. Conclusion: In this series of patients with LFLG AS and low LVEF, reduced RVLS was independently associated with increased risk of mortality. Furthermore, stress RVLS provided incremental prognostic value beyond that obtained from rest RVLS. Thus, RVLS measurement at rest and under DSE may be helpful to enhance risk stratification in this high risk population.

\section{Does the Use of a Decision Aid Improve Decision Making in Prosthetic Heart Valve Selection? A Multicenter Randomized Clinical Trial}

\author{
Nelleke M. Korteland ${ }^{1}$, Yunus Ahmed ${ }^{2}$, David R. Koolbergen ${ }^{2}$, \\ Marjan Brouwer ${ }^{3}$, Frederiek de Heer ${ }^{3}$, Jolanda Kluin ${ }^{3}$, \\ Eline F. Bruggemans ${ }^{4}$, Robert J.M. Klautz ${ }^{4}$, Anne M. Stiggelbout ${ }^{4}$, \\ Jeroen J.J. Bucx ${ }^{5}$, Ad J.J.C. Bogers ${ }^{1}$, Johanna J.M. Takkenberg ${ }^{1}$ \\ ${ }^{1}$ Erasmus MC, Rotterdam, ${ }^{2} \mathrm{AMC}$, Amsterdam, ${ }^{3} \mathrm{UMC}$ Utrecht, \\ Utrecht, ${ }^{4}$ LUMC, Leiden, ${ }^{5}$ Diakonessenhuis, Utrecht, \\ Netherlands
}

Objective: Current clinical practice guidelines recommend shared decision-making (SDM) and explicit consideration of patient preferences in prosthetic heart valve selection, but no tools to support SDM are available. In the Netherlands a patient decision aid (PDA) to support prosthetic heart valve selection was recently developed. A multicenter randomized controlled trial was conducted to assess whether use of the PDA results in optimization of SDM in prosthetic heart valve selection. Methods: Patients were stratified by center and randomized in a 1:1 fashion to standard preoperative care (control group) or standard preoperative care including use of the PDA (intervention group). $\mathrm{Pa}$ tients completed two questionnaires (one preoperative after surgical workup and one 3 months postoperative). Primary endpoint was decisional conflict (DCS), secondary endpoints included patient knowledge, participation in decision-making, emotional distress (HADS), (valve-specific) quality of life (SF-36), and regret (DRS). Results: 155 patients (114 males/41 females; median age 66 years (range $21-84)$ ) were randomized (78 control, 77 inter- 
vention). Preoperatively, 50\% of patients experienced decisional conflict (score $\geq 25$ ), and $25 \%$ had scores $>37.5$ which are associated with decision delay or feeling unsure about implementation. There was no difference in total DCS score between intervention and control patients, but intervention patients felt more informed (median DCS informed subscore: 8 versus 16 respectively; $\mathrm{p}=$ $0.046)$ and had a better basic understanding of prosthetic valves than control patients ( $88 \%$ versus $67 \%$ respectively; $\mathrm{p}=0.004$ ). Seventy-five percent of patients wanted to be involved in decision-making, whereas $53 \%$ agreed that they actually were involved, with no difference between intervention and control patients. Intervention patients experienced less emotional distress (median HADS score: 6 versus 9 respectively; $\mathrm{p}=0.015$ ) and experienced better mental well-being than control patients (mean SF-36 score: 54 versus 50 respectively; $\mathrm{p}=0.032$ ). Three months postoperative there were no differences in valve-specific quality of life and regret between intervention and control patients. Conclusions: A PDA to support SDM in the setting of prosthetic heart valve selection results in more knowledgeable, better informed and less distressed patients and better patient mental well-being. To lower overall decisional conflict, other factors such as adequate implementation are important to consider in conjunction with the PDA.

\section{Minimally Invasive Valve Surgery}

\section{Minimal Invasive and Sutureless Technology: Are These Advantages for Patients Undergoing Aortic Valve Replacement?}

Steffen Pfeiffer, Joachim Sirch, Jurij M. Kalisnik, Ferdinand Vogt, Theodor Fischlein, Giuseppe Santarpino

Klinikum Nürnberg, Paracelsus Medical University, Nuremberg, Germany

Objective: Minimally invasive (MI) aortic valve replacement (AVR) could cause less morbidity than conventional surgery. But up to now, strong clinical advantages have not yet been demonstrated. The use of sutureless aortic valve prostheses in addition reduces the surgical time. However, whether shorter surgical time results in improved patient outcome remains to be determined. Methods: From June 2007 to June 2015, 627 patients underwent elective isolated aortic valve replacement through upper ministernotomy either with a sutureless valve (group A, $\mathrm{n}=206$ ) or a stented aortic bioprosthesis (group B, $\mathrm{n}=247$ ). 174 patients underwent isolated aortic valve replacement through full sternotomy with a stented bioprosthesis (group C). An intra hospital clinical outcome comparison between these 3 groups was performed. Results: The group A was the eldest (table 1). Aortic X-clamp, cardiopulmonary bypass and operation times were shorter in group A than in B and C. As expected, $\mathrm{X}$-clamp time in minimal invasive

Table 1. (for Abstract of Steffen Pfeiffer)

\begin{tabular}{|c|c|c|c|c|c|c|}
\hline & $\begin{array}{l}\text { Group A } \\
\text { J-sternotomy + } \\
\text { sutureless } \\
\mathrm{n}=206\end{array}$ & $\begin{array}{l}\text { Group B } \\
\text { J-sternotomy + } \\
\text { stented } \\
\mathrm{n}=247\end{array}$ & $\begin{array}{l}\text { Group C } \\
\text { Full-sternotomy + } \\
\text { stented } \\
\mathrm{n}=174\end{array}$ & \multicolumn{3}{|l|}{$\mathrm{p}$} \\
\hline Age, years old & $77 \pm 5$ & $70 \pm 8$ & $74 \pm 7$ & $<0.001$ & $<0.001$ & $<0.001$ \\
\hline $\mathrm{X}$-Clamp time, $\min$ & $36 \pm 10$ & $60 \pm 18$ & $54 \pm 16$ & $<0.001$ & $<0.001$ & $<0.001$ \\
\hline Drainage, $\mathrm{ml}$ & $385 \pm 287$ & $403 \pm 306$ & $500 \pm 338$ & 1.0 & 0.001 & 0.006 \\
\hline Transfusions, units & $1.3 \pm 2.1$ & $1.0 \pm 1.9$ & $1.8 \pm 2.6$ & 0.33 & 0.05 & 0.001 \\
\hline Troponin, ng/ml & $0.4 \pm 0.3$ & $0.5 \pm 0.5$ & $2.2 \pm 12$ & 1.0 & 0.13 & 0.16 \\
\hline $\mathrm{CK}, \mathrm{U} / \mathrm{l}$ & $667 \pm 853$ & $768 \pm 738$ & $767 \pm 877$ & 0.9 & 1.0 & 1.0 \\
\hline $\mathrm{CK}-\mathrm{MB}, \mathrm{ng} / \mathrm{ml}$ & $23 \pm 37$ & $38 \pm 89$ & $65 \pm 347$ & 1.0 & 0.3 & 0.8 \\
\hline Creatinine, mg/dl & $1.1 \pm 0.5$ & $1.1 \pm 0.4$ & $1.2 \pm 0.6$ & 0.8 & 0.005 & 0.07 \\
\hline Stroke, \% & $7(3.4)$ & $3(1.2)$ & $4(2.3)$ & 0.1 & 0.37 & 0.31 \\
\hline Wound infection, $\%$ & $2(1.0)$ & $4(1.6)$ & $4(2.3)$ & 0.43 & 0.27 & 0.44 \\
\hline Pacemakers, \% & $21(10)$ & $13(5.3)$ & $9(5.2)$ & 0.036 & 0.05 & 0.58 \\
\hline
\end{tabular}


approach without sutureless was prolonged (table 1). MI approach enables an advantage for bleeding complications in terms of postoperative drainage and need for transfusions (table 1). MISutureless showed a protective effect on renal function but a higher incidence of permanent pacemaker implantation (table 1). We recorded no difference in terms of postoperative cardiac enzymes, no difference in oro-tracheal intubation time as well as hospital/ ICU stay (table 1). There was no statistical difference in terms of mortality, stroke and wound infection (table 1). Conclusions: MI approach alone allowed a protective effect on bleeding complication, but is time demanding. MI sutureless AVR was associated with significant shorter surgical times compared with stented bioprostheses. Although sutureless-AVR patients were significantly older, mortality and renal outcome were comparable with stented AVR groups.

\section{Minimally Invasive versus Conventional Extracorporeal Circulation in Minimally Invasive Mitral Valve Surgery}

\section{Christian J. Rustenbach, Ulrich F.W. Franke, Ragi Nagib, Samir Ahad, Adrian Ursulescu, Hardy Baumbach}

Robert-Bosch-Hospital, Stuttgart, Germany

Background: The minimally-invasive extracorporeal circulation (MECC) technology is introduced and mainly used in coronary bypass surgery. This closed circuit system aims to reduce the foreign surface as well as the priming volume to minimize perioperative inflammation. In our hospital we routinely perform mitral valve procedures trough a minimally-invasive access. According to this attitude we logically had to combine both approaches. Methods: In this 1:2 prospectively randomized single-center study we analyzed patients $(n=65)$ undergoing an isolated minimally-invasive mitral valve procedure. A conventional open extracorporeal circulation was applied in the CECC-group $(n=20)$ and a minimalized (closed circuit) extracorporeal circulation system in patients in the MECC-group $(n=45)$. Leukocytes, C-reactive protein (pre- and postoperatively) as well as the incidence of organic dysfunction (e.g. renal failure), myocardial damage, and clinical outcome (duration of ventilation, length of intensive care unit and total hospital stay) were measured. Results: Demographic data were comparable between both groups. The postoperative data are summarized in table 1 . The levels of leukocytes were not significant lower in the MECC than in CECC group. Renal function was better preserved. The decrease of C-reactive protein and interleukin- 6 was significant between both groups. We were able to validate significant higher postoperative hemoglobin levels as well as a shorter ventilation time and a shorter ICU stay. Conclusion: This pilot study demonstrates the feasibility of MECC in minimally-invasive mitral valve procedures. The results suggest that the use of a MECC might lead to reduced inflammatory response and decreased platelet consumption, less hemodilution, a lower C-reactive protein as well as procalcitonin and interleukin-6 levels.
Table 1. Demographic and postoperative data (for Abstract of Christian J. Rustenbach)

\begin{tabular}{|c|c|c|c|}
\hline & $\begin{array}{l}\text { CECC } \\
(\mathrm{n}=20)\end{array}$ & $\begin{array}{l}\text { MECC } \\
(\mathrm{n}=45)\end{array}$ & $\begin{array}{l}\mathrm{p} \\
\text { value }\end{array}$ \\
\hline \multicolumn{4}{|l|}{ Demographic data } \\
\hline Age, years & $67.7 \pm 10.9$ & $67.9 \pm 10.9$ & 0.835 \\
\hline $\mathrm{BMI}, \mathrm{kg} / \mathrm{m}^{2}$ & $27.2 \pm 4.5$ & $24.8 \pm 3.6$ & 0.194 \\
\hline EuroSCORE $[\log ]$ & $5.8 \pm 2.5$ & $6.0 \pm 3.0$ & 0.764 \\
\hline LVEF, \% & $60.1 \pm 13.2$ & $60.8 \pm 10.7$ & 0.540 \\
\hline \multicolumn{4}{|l|}{ Postoperative data } \\
\hline Ventilation, h & $6.7 \pm 2.4$ & $5.6 \pm 1.8$ & 0.011 \\
\hline ICU stay, days & $2.3 \pm 1.8$ & $1.4 \pm 0.7$ & 0.001 \\
\hline $\mathrm{X}$-Clamp, min & $80.91 \pm 13.78$ & $93.74 \pm 17.44$ & 0.326 \\
\hline $\mathrm{Hb}$, preOP, g/l & $122.8 \pm 13.7$ & $129.8 \pm 15.5$ & 0.611 \\
\hline $\mathrm{Hb}, 6 \mathrm{~h}$ postOP, $\mathrm{g} / \mathrm{l}$ & $102.7 \pm 9.2$ & $116.2 \pm 23.2$ & 0.050 \\
\hline $\mathrm{Hb}, 48 \mathrm{~h}$ postOP, $\mathrm{g} / \mathrm{l}$ & $99.4 \pm 10.9$ & $103.4 \pm 25.4$ & 0.123 \\
\hline Thrombocytes, preOP, GIGA/l & $195.3 \pm 57.7$ & $179.8 \pm 15.5$ & 0.386 \\
\hline Thrombocytes, 6 h postOP, GIGA/l & $125.1 \pm 46.3$ & $130.4 \pm 39.9$ & 0.114 \\
\hline Thrombocytes, $48 \mathrm{~h}$ postOP, GIGA/l & $125.5 \pm 51.2$ & $143.0 \pm 51.6$ & 0.058 \\
\hline Leukocytes, preOP, GIGA/l & $5.4 \pm 1.8$ & $4.9 \pm 1.2$ & 0.710 \\
\hline Leukocytes, 6 h postOP, GIGA/l & $11.8 \pm 3.5$ & $11.2 \pm 3.8$ & 0.608 \\
\hline Leukocytes, $48 \mathrm{~h}$ postOP, GIGA/l & $10.8 \pm 4.3$ & $10.2 \pm 2.4$ & 0.126 \\
\hline Procalcitonin, preOP, ng/ml & $0.05 \pm 0.1$ & $0.07 \pm 0.1$ & 0.767 \\
\hline Procalcitonin, $6 \mathrm{~h}$ postOP, ng/ml & $1.40 \pm 0.9$ & $0.71 \pm 0.08$ & 0.043 \\
\hline Procalcitonin, $48 \mathrm{~h}$ postOP, $\mathrm{ng} / \mathrm{ml}$ & $1.58 \pm 2.3$ & $1.34 \pm 1.6$ & 0.399 \\
\hline CRP, preOP, mg/dl & $0.32 \pm 0.2$ & $0.35 \pm 0.3$ & 0.818 \\
\hline CRP, $12 \mathrm{~h}$ postOP, $\mathrm{mg} / \mathrm{dl}$ & $4.6 \pm 1.0$ & $3.8 \pm 1.1$ & 0.001 \\
\hline CRP, $48 \mathrm{~h}$ postOP, mg/dl & $17.1 \pm 6.9$ & $17.5 \pm 5.4$ & 0.210 \\
\hline Interleukin-6, preOP, pg/ml & $3.2 \pm 2.2$ & $2.8 \pm 2.2$ & 0.769 \\
\hline Interleukin- $6,6 \mathrm{~h}$ postOP, $\mathrm{pg} / \mathrm{ml}$ & $287.9 \pm 41.5$ & $264.1 \pm 58.77$ & 0.513 \\
\hline Interleukin-6, $24 \mathrm{~h}$ postOP, $\mathrm{pg} / \mathrm{ml}$ & $366.9 \pm 34.7$ & $177.1 \pm 41.8$ & 0.047 \\
\hline Creatinine, $\mathrm{preOP}, \mathrm{mg} / \mathrm{dl}$ & $1.2 \pm 1.4$ & $0.9 \pm 0.2$ & 0.036 \\
\hline Creatinine, $6 \mathrm{~h}$ postOP, $\mathrm{mg} / \mathrm{dl}$ & $0.94 \pm 0.2$ & $0.92 \pm 0.2$ & 0.760 \\
\hline Creatinine, $48 \mathrm{~h}$ postOP, $\mathrm{mg} / \mathrm{dl}$ & $1.32 \pm 0.6$ & $0.9 \pm 0.3$ & 0.010 \\
\hline
\end{tabular}

\section{Minimally Invasive Mitral Valve Surgery: Technique of Choice for Cardiac Reoperations}

\section{Davide Ricci, Cristina Barbero, Giovanni Marchetto, Suad ElQarra, Massimo Boffini, Mauro Rinaldi}

AOU Città della Salute e della Scienza di Torino - University of Turin, Turin, Italy

Objectives: Re-do mitral valve procedures performed through median sternotomy carry substantial mortality and morbidity. To avoid complications of sternal re-entry and to provide adequate mitral valve exposure, antero-lateral thoracotomy has been suggested by some authors. Methods: From July 2005 to July 2015, 850 mitral valve operations have been performed in our institution with a mini-invasive video-assisted approach through a right mini-thoracotomy. Among these, 198 (23.3\%) were performed on patients who had undergone one or more previous cardiac surgery procedures. Results: Mean cardio-pulmonary bypass time and clamp time were $136 \pm 49 \mathrm{~min}$ and $86 \pm 29 \mathrm{~min}$, respectively. Arte- 
rial cannulation was performed either on the ascending aorta, with the endo-direct cannula (17 patients, $8.6 \%$ ), or peripherally with a femoral artery approach (13 patients, $6.6 \%$ with a standard femoral cannula and 158 patients, $79.8 \%$, with the endoreturn kit). In 9 cases $(4.5 \%)$ surgery was performed under ventricular fibrillation. Conversion to median sternotomy was necessary in only 1 patient (0.5\%). Median ICU stay was $14 \mathrm{~h}$, median mechanical ventilation time was $24 \mathrm{~h}$; median hospital stay was 8 days. Bleeding requiring surgical revision occurred in 12 patients (6.0\%). Hospital mortality was $5.0 \%$ (10/198 patients). Conclusions: Port-access videoassisted right mini-thoracotomy allows good results in a difficult subset of patients; it allows minimal adhesion dissection, short ICU and hospital stay. In our practice, this technique has become the treatment of choice for mitral valve re-do surgery.

\section{Transcatheter Aortic Valve Replacement in Patients with Pulmonary Hypertension}

Mohammad Rajaei ${ }^{1}$, Jose Condado ${ }^{2}$, Hanna Jensen ${ }^{1}$, Jose Binongo ${ }^{3}$, Chandan Devireddy ${ }^{2}$, Bradley Leshnower ${ }^{1}$, Jessica Forcillo ${ }^{1}$, Stamatios Lerakis ${ }^{2}$, Eric Sarin ${ }^{1}$, Kreton Mavromati ${ }^{2}$, Robert Guyton ${ }^{1}$, James Stewart ${ }^{2}$, Peter Block ${ }^{2}$, Lillian Tsai ${ }^{1}$, Amy Simone ${ }^{1}$, Patricia Keegan ${ }^{1}$, Vasilis Babaliaros ${ }^{2}$, Vinod Thourani ${ }^{1}$

${ }^{1}$ Emory Structural Heart and Valve Center, Joseph B. Whitehead Department of Surgery, Division of Cardiothoracic Surgery, Atlanta, GA, ${ }^{2}$ Emory Structural Heart and Valve Center, Department of Medicine, Division of Cardiology, Atlanta, GA, ${ }^{3}$ Emory University Rollins School of Public Health, Atlanta, GA, USA

Objective: Pulmonary hypertension (PHTN) is associated with poor outcomes in patients with severe aortic stenosis (AS) undergoing surgical aortic valve replacement (SAVR). In this study, we explore PHTN in patients undergoing transcatheter aortic valve replacement (TAVR), evaluate the post-operative trajectory of PHTN, and assess its effects on clinical outcomes. Methods: A retrospective review of 356 patients undergoing TAVR from 09/2007 to $05 / 2013$ in a US academic institution was performed. PHTN was determined by preoperative echocardiographic right ventricular systolic pressure (RVSP). Patients were categorized by the degree of pre-existing PHTN to none/mild ( $<35 \mathrm{~mm} \mathrm{Hg}, \mathrm{n}=90)$, moder-

Table 1. Characteristics and clinical outcomes by PHTN severity (for Abstract of Mohammad Rajaei)

\begin{tabular}{|c|c|c|c|c|}
\hline & $\begin{array}{l}\text { None/mild PHTN } \\
(\mathrm{n}=90)\end{array}$ & $\begin{array}{l}\text { Moderate PHTN } \\
(\mathrm{n}=129)\end{array}$ & $\begin{array}{l}\text { Severe PHTN } \\
(\mathrm{n}=137)\end{array}$ & $\mathrm{p}$ value \\
\hline \multicolumn{5}{|l|}{ Preoperative characteristics } \\
\hline Age (years) & $82.3 \pm 7.8$ & $82.8 \pm 7.7$ & $81.3 \pm 7.7$ & 0.29 \\
\hline Female & $41(46)$ & $61(47)$ & $66(48)$ & 0.93 \\
\hline STS PROM score & $9.9 \pm 5$ & $11.2 \pm 7.2$ & $12 \pm 5.4$ & 0.04 \\
\hline Moderate/severe COPD & $16(18)$ & $29(22)$ & $52(38)$ & $<0.01$ \\
\hline Moderate/severe MR & $17(19)$ & $42(33)$ & $73(54)$ & $<0.01$ \\
\hline \multicolumn{5}{|l|}{ 30-day outcomes } \\
\hline Mortality & $2(2)$ & $4(3)$ & $7(5)$ & 0.56 \\
\hline Stroke & $1(1)$ & $4(3)$ & $3(2)$ & 0.75 \\
\hline Renal failure & $1(1)$ & $4(3)$ & $3(2)$ & 0.62 \\
\hline ICU stay (median hours) & $26.2(23.0-49.7)$ & $29.7(24.0-55.5)$ & $29(24.5-89.8)$ & $<0.01$ \\
\hline Prolonged ventilation & $4(4)$ & $13(10)$ & $23(17)$ & 0.01 \\
\hline New pacemaker & $0(0)$ & $4(3)$ & $11(8)$ & $<0.01$ \\
\hline Post-operative LOS (mean, days) & $5.6 \pm 5.0$ & $6.7 \pm 6.5$ & $8.2 \pm 7.50$ & 0.01 \\
\hline Hospital readmission required & $11(12)$ & $11(9)$ & $13(10)$ & 0.66 \\
\hline Moderate paravalvular leak & $4(4)$ & $6(5)$ & $4(4)$ & 0.79 \\
\hline Moderate/severe MR & $8(8)$ & $18(15)$ & $32(25)$ & 0.01 \\
\hline
\end{tabular}

Data is presented as $\mathrm{n}(\%)$ for categorical variables, as mean \pm standard deviation for continuous variables that are normally distributed, and as median (interquartile range IQ1, IQ3) for continuous variables that are not normally distributed.

STS PROM = Society of Thoracic Surgeons Predicted Risk of Mortality; COPD = chronic obstructive pulmonary disease; $\mathrm{DLCO}=$ diffusing capacity of the lung for carbon monoxide; $\mathrm{MR}=$ mitral regurgitation; $\mathrm{ICU}=$ intensive care unit; LOS = length of stay. 
ate $(35-50 \mathrm{~mm} \mathrm{Hg}, \mathrm{n}=129)$ or severe ( $>50 \mathrm{~mm} \mathrm{Hg}, \mathrm{n}=137)$. Preoperative characteristics, operative parameters, and clinical outcomes were explored. Follow-up survival and echocardiographic data was gathered at 6 months after TAVR. Postoperative trajectory of RVSP was adjusted for age, left ventricular volume, left atrial dimension and the severity of preoperative mitral regurgitation. Results: A total of 356 patients with complete echocardiographic data were included in the study. The mean STS predicted risk of mortality (STS PROM) score was $11.2 \pm 6.1 \%$ for the study cohort and was highest in patients with severe PHTN $(12 \pm 5.4, \mathrm{p}=0.04)$. While patients with moderate and severe PHTN demonstrated longer ICU and hospital length of stay; there were no significant differences in 30-day or 1-year mortality, stroke, renal failure or hospital re-admission between study groups (table 1). In patients with severe PHTN, RVSP and the degree of mitral regurgitation decreased significantly following TAVR and all study groups demonstrated a significant improvement in NYHA class at six months following the procedure. Conclusions: Preoperative PHTN was not associated with increased mortality or morbidity after TAVR. Patients with severe PHTN demonstrated a decrease in RSVP after TAVR and all study groups demonstrated symptomatic relief. TAVR can be performed in patients with PHTN with comparable outcomes to patients with normal pulmonary pressures.

\section{Is There an Impact on Postoperative Morbidity and Long Term Survival after Minimal Access Aortic Valve Replacement}

Sven Lehmann, Denis R. Merk, Sergey Leontyev, Christian Etz, Martin Misfeld, Martin Haensig, Anna K. Funkat, Jens Garbade, Friedrich W. Mohr

Heartcenter Leipzig, Leipzig, Germany

Objective: Minimally invasive techniques are progressively challenging traditional approaches in cardiothoracic surgery. The aim of this study was to compare outcomes in patients undergoing minimal (MIS) versus conventional (CON) access aortic valve re- placement. Methods: 7290 consecutive patients undergoing primary aortic valve replacement at our institution from 11/94 to 06/15 were reviewed: 1776 patients had a MIS access and 5515 a CON access. Results: Patients in the CON group were significantly older than MIS patients ( $67 \pm 11$ vs. $66 \pm 13$ years, $\mathrm{p}<0.01$ ), had a lower EF (57 \pm 15 vs. $60 \pm 12 \%, p<0.01)$ and a higher logEuroscore $(8.3 \pm 10$ vs. $5.5 \pm 5.5, \mathrm{p}<0.01)$. There is no difference in crossclamp time ( $50 \pm 23$ vs. $52 \pm 23 \mathrm{~min}, \mathrm{p}=0.15)$ between two groups. 30-day survival rate was $98.2 \pm 0.3$ (MIS) and $94.4 \pm 0.3$ (CON; $\mathrm{p}<$ $0.01)$. The Survival rate after 15 years was $70.9 \pm 1.8 \%$ (MIS) vs. $53.1 \pm 1.0 \%(\mathrm{CON} ; \mathrm{p}<0.01)$. The multivariate analysis revealed COPD $(\mathrm{p}<0.01, \mathrm{OR}=2.2)$, age over 70 years $(\mathrm{p}<0.01$, OR 1.8$)$, pre-operative i.v. inotropic ( $\mathrm{p}<0.01$, OR 6.5$)$, dialysis $(\mathrm{p}<0.01$, OR 3.7), emergency operation ( $\mathrm{p}<0.01$, OR 4.5), preoperative reanimation ( $\mathrm{p}=0.02$, OR 3.2$)$, preoperative cardiac shock $(\mathrm{p}<0.01$, OR 4.1), prior cardiac surgery ( $\mathrm{p}<0.01$, OR 2.3$)$, active endorcarditis $(\mathrm{p}<0.01$, OR 2.1) and female gender $(\mathrm{p}=0.01, \mathrm{OR}=1.5)$ as independent risk factors for 30 day mortality. Conclusions: Although patient selection may have influenced some of the observed differences between our patient groups, minimal access surgery appears to be associated with significantly lower postoperative morbidity and superior long term survival.

\section{New Transcervical Approach to Transcatheter Mitral Valve Replacement (TMVR) Using CoreVista ${ }^{\mathrm{TM}}$ System}

\section{Cristiano Spadaccio, Fraser W. Sutherland}

Golden Jubilee National Hospital, Glasgow, United Kingdom

Background: Transcatheter mitral valve replacement (TMVR) is emerging as an attractive alternative to mitral surgery. Current access routes have significant shortcomings. Transapical (TA) entails a retrograde approach to the mitral valve with possible entanglement with subvalvular apparatus and is limited by the drawbacks associated with left ventricular puncture and thoracotomy access. Transfemoral (TF) is harnessed by the difficulty of manip-

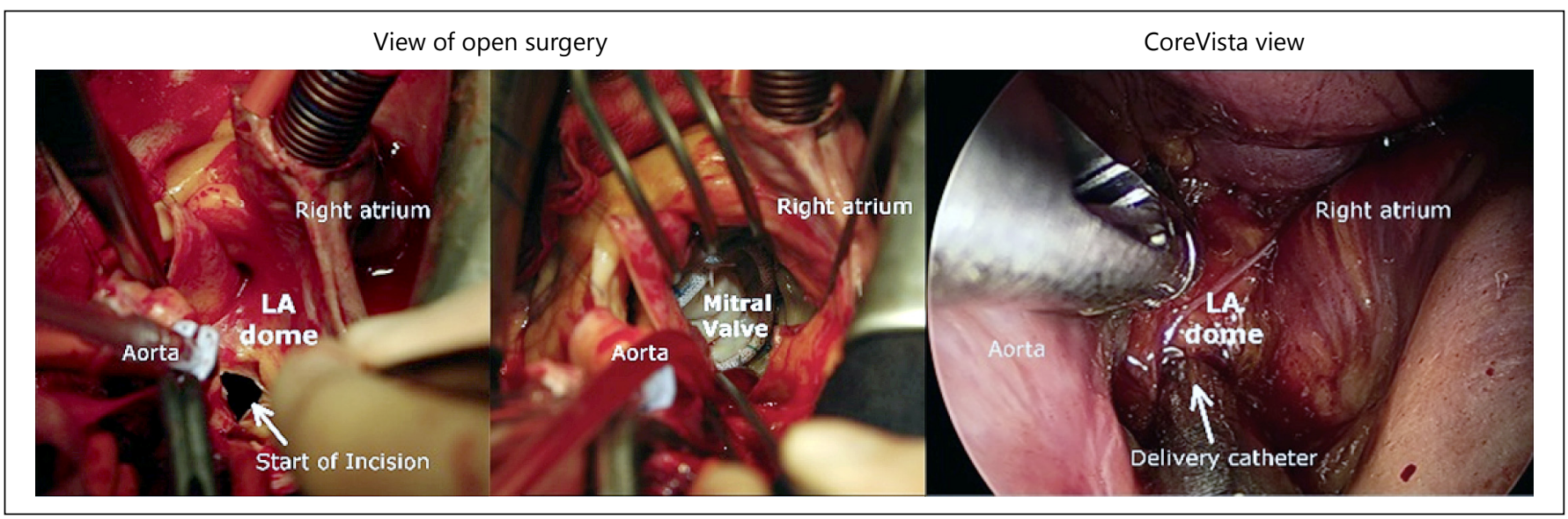

Fig. 1. (for Abstract of Fraser W. Sutherland). 
ulating the sizable delivery devices currently available for TMVR through a non-physiological trajectory across an atrial septal defect. We recently developed a device system (CoreVista ${ }^{\mathrm{TM}}$, CardioPrecision Ltd) to provide a transcervical access to cardiac structures through a short incision in the neck and successfully performed first-in-man cases of aortic valve replacement via both transcatheter and standard surgical approaches. We now sought to demonstrate the feasibility of this route for TMVR as the CoreVista system allows for visualization and exposure of the dome of the left atrium (LA) similar to routinely used open approaches for MV surgery. Methods: Thiel-embalmed human cadavers were used. Transcervical access was obtained with the CoreVista ${ }^{\mathrm{TM}}$ retraction system, LA exposed and purse string suture in the dome of the LA readily placed. A $36 \mathrm{Fr}$ delivery catheter was introduced and manipulated through the MV under echocardiographic guidance. The catheter was then removed and atrium closed. Results: The CoreVista ${ }^{\mathrm{TM}}$ system provided exposure of dome of LA similar to the standard surgical view (figure 1). The system allowed insertion of a standard TMVR delivery catheter, manipulation through the $\mathrm{MV}$, catheter removal and secure closure. Catheter was noted to follow a smooth curve from neck to valve. Conclusions: Although preliminary the results of this study prove the feasibility of TMVR through a transcervical access. The approach overcomes problems with TA and TF access routes, facilitating delivery of large devices to the MV. The procedure could be performed through a minor incision in the neck avoiding chest disruption and promises significant future benefits in terms of rapid recovery, reduction in complications and early discharge, similar to experience with transcervical aortic valve replacement.

\section{Aortic Valve Replacement via Right Mini-Thoracotomy: Is It Really Minimally Invasive?}

\author{
Mauro Del Giglio, Simone Calvi, Simone Turci, Marco Pagliaro, \\ Marco Panzavolta, Elisa Mikus
}

Maria Cecilia Hospital, GVM for Care and Research, Cotignola (Ra), Italy

Objective: Minimally invasive techniques in cardiac surgery require, at least, to obtain the same level of quality as compared to the traditional procedure while achieving a better aesthetic result. Minimally invasive aortic valve replacement through a right anterior mini-thoracotomy is a procedure developed during the last five years; it's use is not widespread because the surgical approach limits the surgeon's view to a very tiny operating field, resulting in a more challenging procedure. Nowadays the limits of this technique, as described in literature, are the longer cardiopulmonary bypass time compared to the standard approach and the need of peripherical cannulation. Methods: Between January 2010 and October 2015, 457 adult patients underwent isolated aortic valve replacement through a right anterior minithoracotomy in two Cardiosurgical centres managed by one single team of Cardiac Surgeons. Mean age was $71.6 \pm 12.1$ years (range: $29-93$ years) and 202 were males $(55.8 \%)$. At the beginning of our experience we prefer a peripherical cannulation, by time a total central arterial and venous cannulation was adopted. Results: Right mini-thoracotomy was successfully performed through a 4 to $6 \mathrm{~cm}$ skin inci- sion at the third intercostal space. Overall cardiopulmonary bypass time was $65.2 \pm 25.3$ minutes and aortic cross clamp time was $51.7 \pm 20.4$ minutes. In 435 patients $(95.2 \%)$ a biological prosthesis was implanted using running sutures. Median prosthesis size was $25 \mathrm{~mm}$. Median intensive care was 1.8 days. In-hospital mortality was $1.7 \%$ (8/457). Wound complication were observed in $5 \mathrm{~Pa}$ tients. Finally all patients were satisfied of the cosmetic results. Conclusions: Our initial series confirms that aortic valve replacement through a right mini-thoracotomy is a safe procedure with excellent results. This technique may be more complex, but using running sutures and others technical details, cardiopulmonary bypass and cross-clamping times comparable with the standard ones can be obtained. The central cannulation can be easily performed without increasing the surgical time and avoiding groin incisions. In our experience the advantages of this technique include early mobilization and rehabilitation, excellent aesthetic result and lower risk of wound complications for this reason we consider it a really minimally invasive approach.

\section{Basic Research - Valve Science and Diseases}

\section{Cadherin-11 Coordinates Cellular Migration and Extracellular Matrix Remodeling during Aortic Valve Development and Promotes Calcification in Mature Aortic Valves}

\author{
Derek C. Sung, Caitlin J. Bowen, Kiran A. Vaidya, \\ Jonathan T. Butcher
}

Cornell University, Meinig School of Biomedical Engineering, Ithaca, NY, USA

Objective: Proper remodeling of the endocardial cushions into thin fibrous valves is essential for gestational progression and longterm function. This process involves dynamic interactions between resident cells and their local environment, much of which is not understood. Furthermore, homeostatic maintenance of adult aortic valves may be compromised as a result of aberrant cell interactions. This study aims to elucidate the specific function of the cell-cell adhesion protein Cadherin-11 (Cad-11) in valvulogenesis and implicate a role for Cad-11 in adult valve pathogenesis. Methods: We utilized a Cad-11 null (Cad- $\left.11^{-/-}\right)$mouse and profiled the effects of Cad-11 deficiency on embryonic valve morphology using histological and immunohistochemical staining. Additionally, we conditionally overexpressed Cad-11 in heart valves using a novel double transgenic NFATC1 ${ }^{\mathrm{Cre}}$; Cad-11 $\mathrm{Tg}^{\mathrm{fl} / \mathrm{fl}}$ mouse model to study Cad-11 in adult valve homeostasis. Cardiac performance was evaluated with transthoracic echocardiography using Doppler ultrasound. Porcine aortic valve interstitial cells (VICs) were subjected to Cad-11 siRNA knockdown to study the role of Cad-11 on individual cell morphology, migration, and compaction, and gene expression was examined with qRT-PCR. Results: We show that Cad-11 deficiency results in significant embryonic and perinatal lethality due to valve related cardiac dysfunction. While endocar- 
dial to mesenchymal transformation is not abrogated, mesenchymal cells do not homogeneously cellularize the cushions. These cushions remain thickened with disorganized extracellular matrix (ECM), resulting in pronounced aortic valve insufficiency. Mice that survive to adulthood maintain thickened and stenotic valves, but interestingly do not develop calcification. Cad-11 $1^{-/-}$aortic valve leaflets contained reduced Sox 9 activity, $\beta 1$ integrin expression, and RhoA-GTP activity, suggesting that remodeling defects are due to improper migration and/or cellular contraction. Cad-11 deletion or siRNA knockdown reduced migration, eliminated collective migration, and impaired 3D matrix compaction by aortic VICs. Cad-11 depleted cells in culture contained few filopodia, stress fibers, or contact inhibited locomotion. Transfection of Cad11 depleted cells with constitutively active RhoA restored cell phenotypes. Overexpression of Cad-11 in the heart valves led to pathological ECM remodeling, calcification, and stenosis. Conclusions: Together, these results identify Cadherin-11 mediated adhesive signaling for proper remodeling of the embryonic semilunar valves, and implicate a role for Cad-11 in adult aortic valve calcification.

\section{Overexpression of Long Non-Coding RNA during Calcific Aortic Valve Stenosis Promotes an Osteogenic Program by Modifying the Epigenetic Signature of NOTCH1}

\author{
Fayez Hadji' , Marie-Chloé Boulanger ${ }^{1}$, Émilie Lavoie-Charland ${ }^{1}$, \\ Nathalie Gaudreault ${ }^{1}$, Sandra Guauque-Olarte ${ }^{1}$, \\ Rihab Bouchareb ${ }^{1}$, Mohamed Nsaibia', Luigi Bouchard², \\ Yohan Bossé ${ }^{1}$, Patrick Mathieu ${ }^{1}$ \\ ${ }^{1}$ Quebec Heart and Lung Institute, Quebec, QC, ${ }^{2}$ Department \\ of biochemistry, Faculty of Medicine and Health Sciences, \\ Université de Sherbrooke, Sherbrooke, QC, Canada
}

Objective: Calcific aortic valve stenosis (CAVS) is the most frequent valvular heart disease. Transdifferentiation of aortic valve interstitial cells (VICs) into osteogenic cells is an important process during the development ofCAVS. However, the molecular mechanisms involved during the osteogenic transition are poorly understood. Long non-coding RNAs (LncRNAs) may have an impact on the gene expression profile and may be involved in the osteogenic transdifferentiation during the development of the CAVS. Methods: Genome-wide transcriptomic by RNA sequencing (RNA-seq) and DNA genome-wide methylation studies were performed in human aortic valves in order to examine the differential expression of LncRNAs. In addition, the role of LncRNAs on the mineralization was explored and the molecular mechanisms by which it promotes osteogenic transdifferentiation was documented. Results: Transcriptomic profiling of CAVS and control valves revealed that $5 \mathrm{LncRNAs}$ were highly expressed in mineralized aortic valves. Multidimensional gene set analyses revealed that the CpG islands in the promoter region of one LncRNA were hypomethylated $(n=23)$ and associated with expression levels. These data were confirmed by q-PCR $(n=152)$, fluorescence in situ hybridization (FISH) and pyrosequencing $(n=60)$. Methylation status of the LncRNA promoter was inversely related to expression level and in isolated VICs a treatment with 5-aza-2'-de- oxycytidine, which inducesDNA demethylation, increased the expression of LncRNA by 3.0-fold. Knockdown or overexpression experiments showed that LncRNA play an important role in the osteogenic transdifferentiation via the Notch1 pathway. Promoter studies coupled with luciferase and chromatin immunoprecipitation (ChIP) revealed that this LncRNA interferes with the activity of NOTCH1 by physically interacting with the promoter and modifying its epigenetic signature. LncRNA-induced mineralization of VICs was rescued by a co-transfection of a vector encoding for the Notch intracellular domain (NICD). Conclusions: During CAVS the hypomethylation in the promoter region promotes the overexpression of LncRNA, which instructs an osteogenic program in VICs by modifying the epigenetic signature of NOTCH1. Thus, these data suggest that targeting LncRNA and or epigenetic mechanisms may reprogram VICs to a quiescent phenotype and may hold promise in the treatment of CAVS.

\section{Implication of Leptin in the Development of Calcific Aortic Valve Disease: Induction of Osteoblast Differentiation of Human Valvular Interstitial Cells via the Akt and Erk Pathways}

\author{
Mickael Rosa ${ }^{1}$, Rodrigo Lorenzi ${ }^{1}$, Ahmed Elkalioubie ${ }^{2}$, \\ Madjid Tagzirt ${ }^{1}$, Ramadan Jashari ${ }^{3}$, Francis Juthier ${ }^{2}$, \\ Antoine Rauch ${ }^{2}$, Anaïs Arbesu Y. Miar ${ }^{1}$, Delphine Corseaux ${ }^{1}$, \\ André Vincentelli ${ }^{2}$, Bart Staels ${ }^{1}$, Eric Van Belle ${ }^{2}$, Sophie Susen ${ }^{2}$, \\ Annabelle Dupont ${ }^{2}$ \\ ${ }^{1}$ European Genomic Institute for Diabetes (E.G.I.D.), FR 3508, \\ UNIV LILLE, Inserm UMR 1011; Institut Pasteur de Lille, F-59000, \\ Lille, ${ }^{2}$ European Genomic Institute for Diabetes (E.G.I.D.), FR \\ 3508, UNIV LILLE, Inserm UMR 1011; Institut Pasteur de Lille, \\ F-59000, Cardiovascular and Pulmonary, and Haematology \\ Department, CHRU de Lille, Lille, France; ${ }^{3}$ European Homograft \\ Bank, c/o Military Hospital Queen Astrid, Brussels, Belgium
}

Objective: Calcific aortic valve disease (CAVD) affects $2 \%$ to $6 \%$ of the population over 65 years old in industrialized countries. CAVD is now considered as an «atherosclerosis-like» disease of the aortic valve resulting from dysregulated processes such as calcification. This process is supported in part by the osteoblastic differentiation of valvular interstitial cells (VICs), the most prevalent cell type in aortic valves. Leptin was previously linked to vascular calcification. Our hypothesis is that leptin could play an important role in the calcifying processes implicated in CAVD via direct effects on human VICs. Methods: Patients with CAVD or with severe coronary artery disease (CAD) were included in the study. Presence of leptin was analyzed by immunohistochemistry and ELISA in 43 human explanted calcified aortic valves and blood samples of all included patients. Leptin receptors (ObR-L and ObR-S) expression was analyzed in aortic valves and in VICs isolated from calcific aortic valves. The effects of leptin on osteoblastic differentiation of cultured VICs in presence or not of Akt and ERK inhibitors were investigated by alizarin red staining, alkaline phosphatase (ALP) activity and RT-qPCR analysis for the osteoblastic markers osteopontin, ALP, bone morphogenetic protein 2 (BMP-2), and RUNX2. Results: Serum leptin concentration were higher in patients with CAVD compared to CAD patients $(27.7 \pm$ 
$5 \mathrm{ng} / \mathrm{ml}$ vs. $15.7 \pm 1.5 \mathrm{ng} / \mathrm{ml}, \mathrm{p}=0.002)$. Leptin was present in human calcific aortic valves, with higher concentrations in calcified vs. non-calcified valvular zones $(230 \pm 38 \mathrm{pg} / \mathrm{mg}$ of proteins vs. $122 \pm 20 \mathrm{pg} / \mathrm{mg}$ of proteins $\mathrm{p}=0.01)$. Both short and long leptin receptor isoform were expressed in VICs isolated from stenotic valves. Chronic leptin stimulation of VICs enhanced expression of ALP, BMP-2 and RUNX2 and decreased expression of osteopontin. This treatment led to higher ALP activity and calcium deposition in human VICs in a dose-dependent manner. Inhibiting Akt or ERK during leptin stimulation led to a reduced calcification by bringing the expression of calcification genes to the control levels. Conclusions: These novel findings underline the potential role of leptin in the progression of CAVD by enhancing calcification processes in human aortic VICs.

\section{Shear-Sensitive miRNA-181b Impairs Anti-Inflammatory Signaling in the Aortic Valve Endothelium}

\section{Jack Heath, Joan Fernandez Esmerats, Rachel Simmons, Sandeep Kumar, Hanjoong Jo}

Emory University, Atlanta, GA, USA

Background: Aortic valve (AV) disease is a significant contributor to cardiovascular mortality. The process is regulated by both endothelial cells (ECs) at the valve surface and interstitial cells within the tissue. Tissue dysfunction occurs preferentially on the valve's aortic side, where ECs are subjected to disturbed blood flow and oscillatory shear stress (OS). These ECs exhibit an inflammatory phenotype, whereas ECs on the opposite (ventricular) side experience laminar shear stress (LS) and an anti-inflammatory phenotype. Our research seeks to determine mechanisms responsible for differential endothelial responses to shear stress and potential therapeutic targets for AV disease. Methods: In vitro shear stress experiments were carried out using our cone-and-plate shear system, as pictured above. LS was induced by parallel force of 20 dynes $/ \mathrm{cm}^{2}$, and OS was induced at \pm 5 dynes $/ \mathrm{cm}^{2}$ for 24 hours. Gene knockdown, immunoprecipitation, and quantitative gene expression analysis were performed as previously described by our group. Results: We have performed microarray studies revealing several endothelial shear-sensitive microRNAs (miRNAs), small molecules which repress messenger RNA (mRNA) translation by targeting the 3' untranslated region matching the mRNA seed sequence. We found one miRNA, miRNA-181b, is upregulated in OS, correlating with increased expression of inflammatory adhesion molecules and decreased anti-inflammatory marker Klf2. OS also decreases expression of a predicted target of miRNA$181 \mathrm{~b}, \mathrm{O}-\mathrm{GlcNAc}$ transferase (OGT), which adds the O-GlcNAc signaling moiety onto key amino acids of target proteins. Knockdown of OGT resulted in increased inflammation even in response to normally protective LS, suggesting a link between OGT and inflammation. Via immunoprecipitation studies, we discovered OGT binds specifically to MEF2C, a key anti-inflammatory transcription factor, and this binding is impaired in OS. The decreased binding of OGT to MEF2C results in an inhibition of MEF2C OGlcNAc modification, which may affect the protein's transcriptional activity. Conclusions: Our results show an important path- way of transcriptional regulation of post-translational modification. Future studies are focused on mechanisms linking miRNA-181b to inflammatory signaling, and ex vivo/in vivo methods to determine therapeutic potential of inhibiting miRNA$181 \mathrm{~b}$. These studies may lead to new targets for AV disease treatment without surgical intervention.

\section{A Role for the Retinoblastoma Pathway in Aortic Valve Disease}

Marina Freytsis ${ }^{1}$, Lauren Baugh ${ }^{2}$, Phil Hinds ${ }^{1}$, Lauren Black ${ }^{2}$, Gordon Huggins ${ }^{3}$

${ }^{1}$ Tufts University, Boston, MA, ${ }^{2}$ Tufts University, Medford, MA,

${ }^{3}$ Tufts Medical Center, Boston, MA, USA

Objective: The pathological process of calcific aortic valve disease (CAVD) shares phenotypic and transcriptional similarities with the physiological process of osteogenesis. Since the retinoblastoma protein $(\mathrm{Rb})$ pathway regulates proper bone development through control of osteoblast differentiation, we hypothesized that the $\mathrm{Rb}$ pathway also regulates development and progression of CAVD. Method: We generated a mouse model of conditional $\mathrm{Rb}$ knockout ( $\mathrm{cKO}$ ) in the aortic valve regulated by Tie2-Cre mediated excision of floxed Rb alleles. RbFlox/ Flox;Tie2Cre+ were compared with control Rb+/Flox;Tie2Cre+ and mice with a loss of CDK6 kinase activity (CDK6 K43M; effectively a gain of $\mathrm{Rb}$ function) by echocardiography. Fixed and sectioned aortic valve samples were analyzed by histological staining and immunohistochemistry. Results: $\mathrm{Rb} \mathrm{cKO}$ animals were born in expected Mendelian ratio; however, they showed decreased viability at 12 months and showed significantly more aortic valve regurgitation by echocardiography compared to $\mathrm{Rb}+/ \mathrm{Flox}$; Tie2Cre+ control animals (figure $1 \mathrm{a}, \mathrm{b} ; 18 / 25$ vs. 2/37, P-value $<0.0001$ ). By comparison, CDK6 K43M mice had normal aortic valve function. The aortic valves of experimental $\mathrm{cKO}$ animals were observed to have increased leaflet thickness, altered collagen deposition by Masson's Trichrome and Movat pentachrome stains, and more intense a-SMA staining compared to controls (figure 1c). Alizarin red and Von Kossa staining did not demonstrate calcific lesions in cKO valves. Given the role of $\mathrm{Rb}$ as a master regulator of the cell cycle, we examined the effect of Rb loss on proliferation (phospho Histone H3) and apoptosis (TUNEL staining), however these parameters were comparable in control and $\mathrm{Rb}$ cKO animals. Conclusion: These studies demonstrate that maintenance of the $\mathrm{Rb}$ pathway is required for aortic valve function, aortic valve cell differentiation and maintenance of extracellular matrix with aging. These pathological changes may reflect an important role for the Rb-CDK6 pathway dependent regulation of valve cell differentiation for the prevention of CAVD-like disease. 


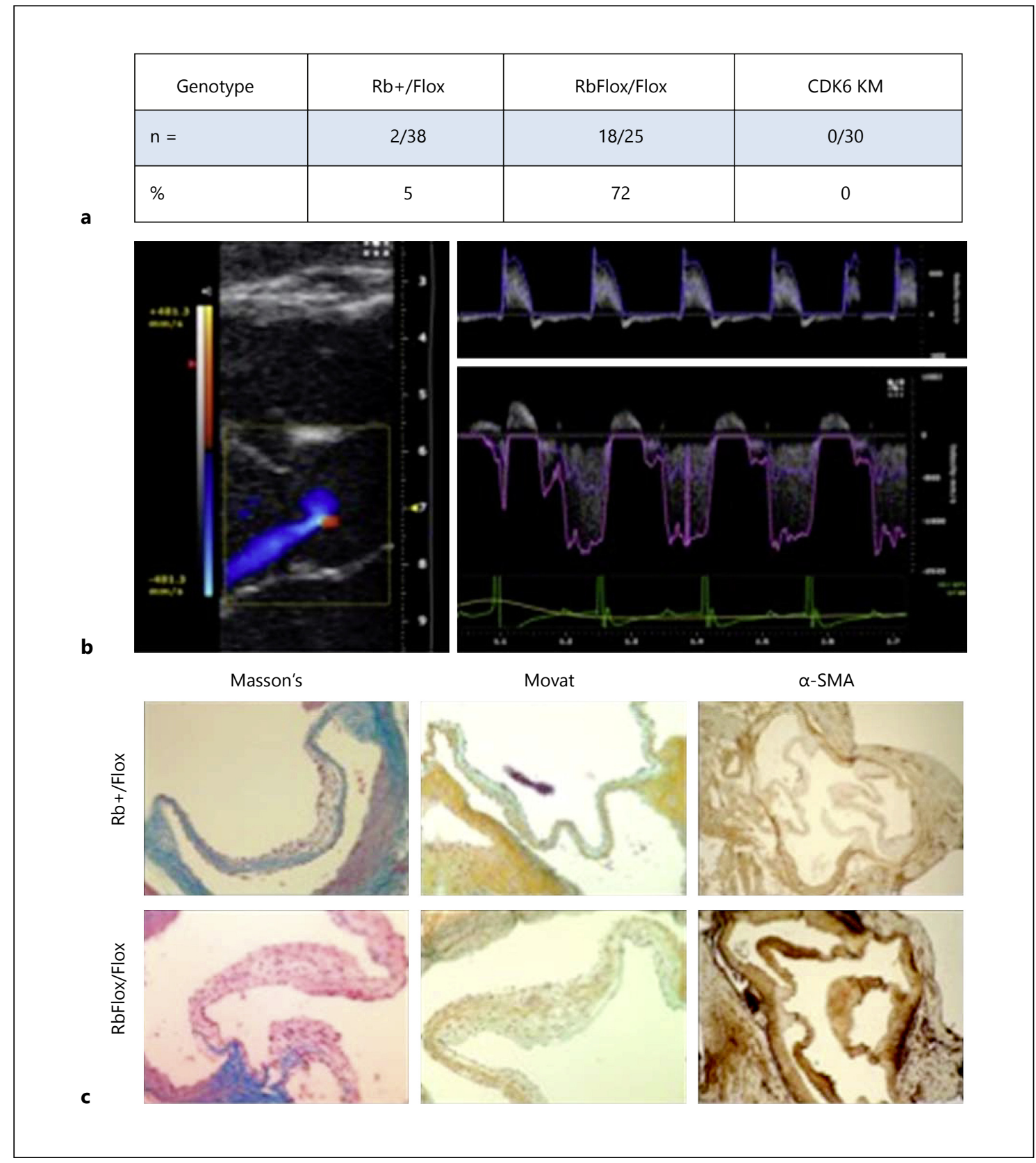

Fig. 1. a Incidence of regurgitation in studied mice. b Representative echocardiogram from Rb cKO mouse (right: color Doppler; left top: aortic side Doppler tracing; left bottom: ventricular side Doppler tracing). c Representative histological statins showing extracellular matrix composition by Masson's and Movat stains and aortic valve cell activation by IHC for a-SMA (for Abstract of Marina Freytsis). 


\section{GWAS of Aortic Stenosis (AS) in Patients with Bicuspid Aortic Valve (BAV) Disease}

\begin{abstract}
Mahyar Heydarpour ${ }^{1}$, Vanessa Monteiro-Vieira ${ }^{1}$, Karam Habchi ${ }^{1}$, Jan Sliwa ${ }^{2}$, Martin Sigurdsson ${ }^{1}$, Jochen Muehlschlegel ${ }^{1}$, Prem Shekar ${ }^{3}$, Eric Isselbacher ${ }^{4}$, Thoralf Sundt ${ }^{4}$, Simon Body

${ }^{1}$ Brigham \& Women's Hospital, Perioperative Genomics Center, Boston, MA, ${ }^{2}$ Brigham \& Women's Hospital, Cardiothoracic Anaesthesia, Boston, MA, 3Brigham \& Women's Hospital, Heart \& Vascular Center, Boston, MA, ${ }^{4}$ Massachusetts General Hospital, Corrigan Minehan Heart Center, Boston, MA, USA
\end{abstract}

Backgrounds: Aortic stenosis (AS) is the most common form of valve disease and the most common reason for valve replacement in Europe and North America. The presence of aortic valve (BAV) expedites the pathogenesis of calcific aortic valve disease (CAVD), the most common cause of AS. While CAVD shares several risk factors with atherosclerosis, it is still unknown which genetic factors can modify the risk of CAVD. In this study we described the association of common genetic variants with AS in patients with BAV. Objective: The objective was to determine if genetic variants are associated with CAVD in patients with BAV. Methods: 452 Caucasian BAV patients with available echocardiographic and genetic data were collected. The presence of AS was categorized by ASE criteria to two classes (No AS: none to mild AS, $\mathrm{N}=211$; AS: moderate to severe, AS N =224). AS was further classified into three grades: none (none to mild AS, $\mathrm{N}=211$ ), moderate (mild-moderate to moderate AS, $\mathrm{N}=38$ ), severe (moderatelysevere to severe AS, $\mathrm{N}=186$ ). After quality control $1,275,952$ genetic markers genotyped with the HumanOmni2.5 bead chip were compared between the patient groups. We performed two complementary analyses to derive association genetic markers (SNPs). An additive logistic regression model was performed for association analysis of AS (case-control). A logistic additive regression model was also performed for categorical grades of AS. Both analyses were adjusted for age and gender. A replication threshold was determined at Pvalue of $10^{-4}$. Quality control and genetic associations were done with PLINK. Results: We identified 1126 genetic markers associated with AS and AS-grades in 450 regions $(\mathrm{P}<$ $10^{-4)}$. After integration of results between the two phenotypes, we identified eight regions associated with AS and AS-grades $(\mathrm{P}<$ $\left.10^{-5}\right)$. The most significant markers were identified in or within $100 \mathrm{kbp}$ of BARHL2 (1p22.2), CREB5 (7p15.1), DIP2C (10p15.3), VEZT (12q22), TRA (14q11.2), CDH19(18q22.1), ISM1 (20p12.1), FRMPD4 (Xp22.2). SNPs in each region were in high linkage disequilibrium (LD). Conclusion: We present a comprehensive GWAS analysis for aortic stenosis in patients with BAV. These observations may help establish mechanisms for CAVD. Replication results will be presented along with potential mechanisms of CAVD in BAV.

\section{Genetic Association and Functional Studies Identifies GLIS1 As a Risk Locus for Mitral Valve Prolapse}

Christian Dina ${ }^{1}$, Nathan Tucker ${ }^{2}$, Francescaca Delling ${ }^{3}$, Susan Slaugenhaupt ${ }^{4}$, Robert A. Levine ${ }^{5}$, Albert A. Hagège 6 , Jean-Jacques Schott ${ }^{7}$, Xavier Jeunemaitre ${ }^{8}$, David Milan', Russell Norris ${ }^{9}$, Nabila Bouatia-Naji ${ }^{10}$

${ }^{1}$ Inserm UMR1087, CNRS UMR 6291, Institut du Thorax, Centre Hospitalier Universitaire (CHU), Nantes, France; ${ }^{2}$ Cardiovascular Research Center, Massachusetts General Hospital, Charlestown, MA, ${ }^{3}$ Framingham Heart Study, Framingham, MA, ${ }^{4}$ Center for Human Genetic Research, Massachusetts General Hospital and Harvard Medical School, Boston, MA, ${ }^{5}$ Cardiac Ultrasound Laboratory, Massachusetts General Hospital and Harvard Medical School, Boston, MA, USA $;{ }^{6}$ AP-HP, Department of Cardiology, Hôpital Européen Georges Pompidou, Inserm UMR970l Européen Georges Pompidou, Inserm UMR970, Paris, ${ }^{7}$ Inserm UMR1087, CNRS UMR 6291, Institut du Thorax, Centre Hospitalier Universitaire (CHU) Nantes, Nantes, Nantes, ${ }^{8}$ AP-HP, Departement of Genetics, Hopital Europeen Georges Pompidou, Inserm U970 Paris Cardiovascular Research Center, Paris, France; ${ }^{9}$ Cardiovascular Developmental Biology Center, Children's Research Institute, Medical University of South Carolinach Institute, Medical University of South Carolina, Charleston, SC, USA; ${ }^{10}$ Inserm UMR970 Paris Cardiovascular Research Center, Paris, France

Objective: Nonsyndromic mitral valve prolapse (MVP) is the most frequent indication for surgical repair of mitral regurgitation, and causes heart failure and arrhythmia. Our recent genome-wide association study (GWAS) identified six first susceptibility loci for MVP. Here we investigate the genetic and functional follow-up of a 7th risk locus that includes GLIS1, a zinc finger transcription factor (TF) recently showed to reprogram the mesenthymal-epithelial transition. Methods: We analyzed genotypes at GLIS1 locus on Chr1 in 2800 cases and $~ 9200$ controls from Europe and USA, all of European ancestry. We investigated the protein expression of Gils-1 by immunohistochemistry (IHC) during valve development (E.13 and E17) and in adult mice. We assessed atrioventricular regurgitation function in zebrafish after knockdown for GLIS1 by morpholino oligonucleotides. Results: The meta-analysis of GWAS conducted in 1412 cases and 2439 controls provided suggestive association at Chrl with top associated variant in the first intron of GLIS1 $\left(\mathrm{OR}=1.30, \mathrm{P}=7.1 \times 10^{-6}\right)$. Follow-up analyses indicate positive replication in two large case control studies: $\mathrm{MGH} /$ FHS cases $(\mathrm{N}=699)$ vs. FHS controls $(\mathrm{N}=5575), \mathrm{P}=1.4 \times 10^{-4}$ and HEGP cases $(\mathrm{N}=450)$ vs. DESIR 2 controls $(\mathrm{N}=820, \mathrm{P}=0.037)$. The global analysis including the discovery and the follow-up samples showed an overall significant association with MVP (OR = $\left.1.23, \mathrm{P}=1.2 \times 10^{-7}\right)$ and no evidence for heterogeneity $(\mathrm{P}=0.41)$. We found that Glis1 is expressed during valve morphogenesis in mice and maintained in the adult. Glis-1 is detected in nucleus from endothelial and valvular interstitial cells. Knockdown of Glis1 in zebrafish resulted in significant atrioventricular (AV) valve defect with regurgitation. Conclusions: Our study provides genetic and functional evidence for the implication of GLIS1 in MVP, a member of the Gli-family of TFs, effectors of the hedgehog 
(Hh) signaling, which play a central role in heart development. The association analyses in additional studies to attempt to reach genome-wide significance and the genetic and functional investigation of additional genes from the Gli-family is under way. This will provide further support for the etiological role of the Gli and $\mathrm{Hh}$ pathway in mitral valve function and disease.

\section{Percutaneous Heart Valve Therapy}

\section{Transcatheter Tricuspid Valve Repair: Initial Results from the Multicentre First-in-Man Feasibility Trial}

\author{
Alec Vahanian' ${ }^{1}$,Francesco Maisano², Jean-Michel Juliard', \\ Ottavio Alfieri ${ }^{3}$, Antonio Colombo ${ }^{3}$, Antonio L. Bartorelli ${ }^{4}$, \\ Francesco Alamanni ${ }^{4}$, Azeem Latib ${ }^{3}$ \\ ${ }^{1}$ Hôpital Bichat, Université Paris-Diderot, Paris, France; \\ ${ }^{2}$ University Hospital Zurich, Zurich, Switzerland; ${ }^{3}$ San Raffaele \\ University Hospital, Milan, ${ }^{4}$ Centro Cardiologico Monzino, \\ I.R.C.C.S., University of Milan, Milan, Italy
}

Objective: The TriCinch system percutaneously remodels the tricuspid valve to reduce annular dilatation in patients with functional tricuspid regurgitation (FTR). We report the early results of the first-in-man safety and feasibility multicentre study. Methods: The study enrolled high-risk patients with symptomatic functional tricuspid valve regurgitation (FTR), despite optimal medical therapy, in 3 European centres. Patients with concomitant and significant left side pathology, or considered unsuitable by local heart team judgement, were excluded. All patients were screened by echocardiography and cardiac CT to assess feasibility. Results: The study group included nine consecutive enrolled patients. Mean age $71.9 \pm 7.3$ years. Mean logistic EuroScore was $12.01 \pm$ 7.29. At baseline $78 \%$ of patients were in NYHA functional class III or IV, and $44 \%$ had diagnoses of moderate to severe right ventricular dysfunction. Device implantation was feasible in 5 patients. Overall procedural and 30 -days mortality was $0 \%$. One patient received a $27 \mathrm{~mm}$, one patient a $32 \mathrm{~mm}$ and three patients received a $43 \mathrm{~mm}$ TriCinch device. Mean procedural time was $72 \pm$ 12 minutes. Significant reduction in Septo-Lateral TV diameter after system tensioning was measured in all subjects, with an overall remodelling of $17 \%$ compared to baseline, remained consistent at latest follow-up. Improvement in TR severity of at least one grade in the postprocedural period occurred in all subjects, and was maintained at the most recent follow-up. At 6-months followup $(\mathrm{N}=3)$ no incidence of death or major adverse event was reported. $100 \%$ of patients presented NYHA class I-II, with positive improvement in quality of life observed in all subjects. Conclusions: Early outcomes suggest the feasibility and the safety of transcatheter tricuspid remodelling with the TriCinch therapy, in patients with severe and symptomatic FTR and who are a high surgical risk, showing encouraging improvements to quality of life. Long-term follow-up is ongoing and the effect on the treatment FTR will be validated in this trial.

\section{Crimp Induced Leaflet Damage and Calcification of Transcatheter Heart Valves}

\author{
Aditi Sinha ${ }^{1}$, Oleksandr Barannyk ${ }^{2}$, Arash Kheradvar ${ }^{1}$ \\ ${ }^{1}$ University of California, Irvine, Irvine, CA, USA; \\ ${ }^{2}$ ViVitro Labs Inc., Victoria, BC, Canada
}

Objective: There is increasing evidence regarding different complications arising from clinical implantation of transcatheter aortic heart valves (THV). However, little is known if crimp-induced damage increases tissue calcification thereby reducing the durability of THRs. We are addressing the association between tissue micro-damage and calcification under accelerated wear conditions. Methods: Self-expandable THVs with glutaraldehyde-fixed bovine pericardial leaflet were used. The valves were divided into (1) uncrimped, (2) 18-Fr crimped, and (3) 14-Fr crimped. The six THVs were tested in an accelerated wear testing (AWT) system for 50 million cycles, at $37^{\circ} \mathrm{C}$, and 800 cycles/minute. A calcifying solution was used with a final concentration of $55 \mathrm{mM}(\mathrm{KCl}), 1.5 \mathrm{mM}$ $\mathrm{CaCl}_{2}, 1.25 \mathrm{KH}_{2} \mathrm{PO}_{4}$ and $20 \mathrm{mM}$ Barbital. The solution was monitored for consistent $\mathrm{pH} 7.5$ and was changed weekly within the test period to minimize fluctuation of $\mathrm{Ca}++$ and $\mathrm{HPO} 4$. The pressure difference across the valves was adjusted at $120 \mathrm{~mm} \mathrm{Hg}$. After completion of the tests, the valves were retrieved, washed, photographed, imaged using micro-CT scan, and tissues were embedded for histological analyses including stains for calcium (Von Kossa), collagen (Trichrome), and elastin (VVG). Results: Our results indicate that, all the valves, regardless of crimping, exhibited severe tissue calcific lesions around the stent commissures. However, micro-CT revealed that only crimped samples had discrete regions of leaflet calcification in the central region (figure 1e and 1f). Furthermore, histological stains of the tissue segments demonstrated severe and permanent microstructural damage to the tissue including fragmentation of collagen fibers (figures $1 \mathrm{~h}$ and $1 \mathrm{i}$ ). Conclusions: Our results indicate severe crimp-induced structural damage to leaflets and hydroxyapatite deposition in the belly region of crimped leaflets; a finding that was not obvious in uncrimped groups. Further analysis is underway to determine the quantity of hydroxyapatite deposition on tissues. 

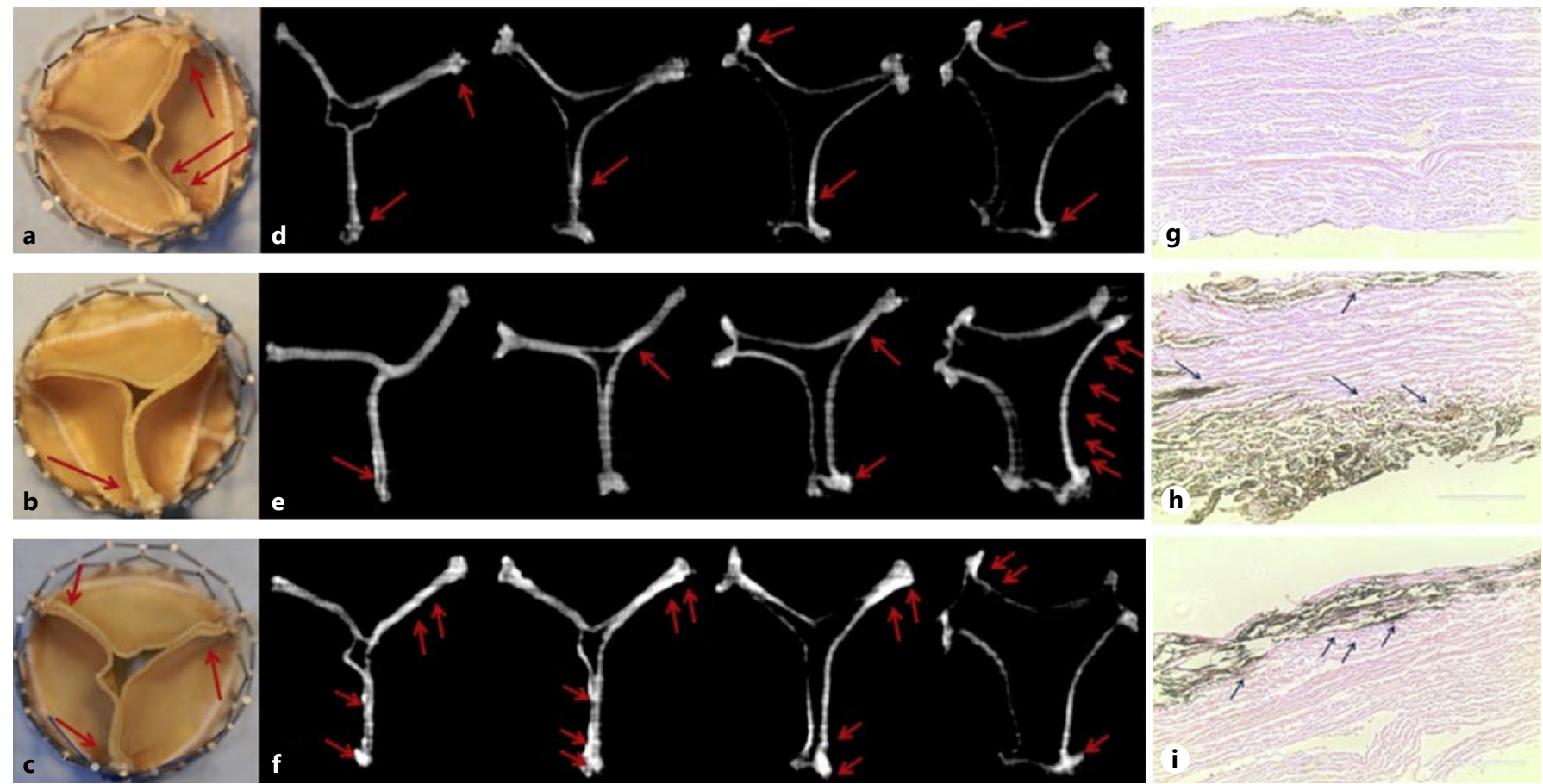

Fig. 1. a-c show photograph of uncrimped, 18 Fr crimped and 14 Fr crimped valves, respectively.d-f show micro-CT scan of uncrimped, 18 Fr crimped valves, respectively. Red arrows indicate calcific deposits. g-i show uncrimped, 18 Fr crimped and 14 Fr crimped valves, respectively with Von Kossa staining of the valve tissue segments. Black arrows indicate calcification (for Abstract of Arash Kheradvar).

\section{Transcatheter Replacement of Failed Bioprosthetic Valves: Comprehensive in-vitro Bench Testing and Large in-vivo Clinical Assessment of the Effect of Implantation Depth on Hemodynamics After Valve-in-Valve}

\section{Matheus Simonato ${ }^{1}$, Danny Dvir ${ }^{2}$}

${ }^{1}$ Escola Paulista de Medicina - UNIFESP, Sao Paulo, Brazil;

${ }^{2}$ St. Paul's Hospital, Vancouver, BC, Canada

Objective: A major limitation of valve-in-valve (ViV) implantations is transcatheter heart valve (THV) underexpansion, which is associated with elevated post procedural gradients. Supra-annular THV device positioning may be advantageous in achieving lower gradients following ViV. Our objective was to utilize large clinical data and in-vitro bench testings in order to examine whether higher THV implantation would be associated with improved hemodynamics and to define optimal depth targets that would allow for ideal hemodynamics after ViV using different THV devices. Methods: In-vitro bench testing using pulse duplicators with three different THV devices were performed, having up to 13 different depths of implantation for each THV (CoreValve Evolut, CVE, Medtronic; SAPIEN XT, SXT, Edwards Lifesciences; Portico, St. Jude Medical). In addition, clinical cases from the Valve-in-Valve
International Data (VIVID) registry were analyzed. Images underwent blinded corelab analysis for the establishment of optimal implantation depth. Results: In-vitro analysis for the CVE, SXT and Portico suggested as well that higher implantation is associated with lower gradients. A total of 289 patients (159 CVE and 130 SXT) were included in the analysis. High implantation had significantly smaller incidence of elevated gradients when compared to deep implantation (CVE: $15 \%$ vs. $34.5 \%, p=0.03$, SXT: $20.8 \%$ vs. $44.3 \%, \mathrm{p}=0.04)$. Optimal implantation depth were defined: CVE, 0 to $5 \mathrm{~mm}$ depth; SXT, 0 to $10 \%$ frame height ( 0 to $2 \mathrm{~mm}$ ); Portico, 0 to $2 \mathrm{~mm}$. Conclusions: Optimal implantation of THV devices in the context of ViV involes higher positioning. Specific targets for positioning can be offered for each major THV device. 


\section{Mitral Valve Reconstruction with the Cardioband System: Results of the Multicentre Feasibility Trial}

Francesco Maisano ${ }^{1}$, Karl-Heinz Kuck ${ }^{2}$, Ottavio Alfieri ${ }^{3}$, Antonio Colombo ${ }^{3}$, Stephan Baldus ${ }^{4}$, Alec Vahanian ${ }^{5}$, Georg Nickenig ${ }^{6}$

${ }^{1}$ University Hospital Zurich, Zurich, Switzerland; ${ }^{2}$ Asklepios Klinik St. Georg Hospital, Hamburg, Germany; ${ }^{3}$ San Raffaele University Hospital, Milan, Italy; ${ }^{4}$ University Hospital Köln, Cologne, Germany; ${ }^{5}$ Assistance Publique-Hôpitaux de Paris, Bichat Hospital, Paris, France; ${ }^{6}$ University Hospital Bonn, Bonn, Germany

Objective: Cardioband system enables percutaneous implantation of an adjustable surgical-like device for mitral reconstruction and mitral regurgitation (MR) treatment using a transseptal approach. The aim of this multi-center study was to evaluate the feasibility, safety and up to 12 months outcome of Cardioband in patients with secondary mitral regurgitation MR. Methods: Between February 2013 and August 2015, 45 high-risk patients with significant Secondary MR were enrolled at 6 sites in Europe. All patients were screened by echocardiography and cardiac CT to as-

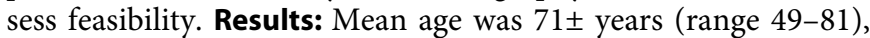
thirty four patients were males (76\%). Mean EuroScore II was $7.7 \% \pm 6.7 \%$ and median STS score 7.2\% (1.0\%-34.0\%). At baseline $87 \%$ of patients were in NYHA class III-IV with mean left ventricular ejection fraction of $32 \pm 11 \%$ (15\%-59\%). Device implantation was feasible in $100 \%$ patients. Procedural success, defined as device successfully implanted with reduction of MR $\leq 2+$ at discharge, was achieved in $95.6 \%$ of patients (43/45). After device cinching, an average $20 \%$ reduction of the septo-lateral diameter was observed (from $39 \pm 6 \mathrm{~mm}$ to $34 \pm 7 \mathrm{~mm}$; $<<0.01$ ). Thirty-day mortality was $4.4 \%$ (adjudicated as device unrelated). At 12 months follow up $(\mathrm{N}=20), 71 \%$ of patients presented NYHA class I-II with improvement in quality of life (MLWHFQ from 38 to 19; $p<0.05$ and $6 \mathrm{MWT}$ from 256 to 386 meters; $\mathrm{p}<0.05) .95 \%$ of patients $(\mathrm{N}=$ 19) had $M R \leq 2+$. Conclusions: Percutaneous transseptal mitral valve reconstruction with the Cardioband device is feasible, with sustained SL diameter and MR reduction. Safety profile is comparable to other transcatheter mitral procedures. MR severity reduction is stable and consistent up to 12 months, showing clinical benefit and encouraging improvements to quality of life.

\section{Frame Flexibility Effects on Leaflet Stress Distribution: Comparison of Transcatheter versus Surgical Aortic Valve Bioprostheses}

\section{Mostafa Abbasi, Mohammed Barakat, Koohyar Vahidkhah, Ali Azadani}

University of Denver, Denver, CO, USA

Objective: Sufficient stent radial expansion force is necessary in transcatheter aortic valve replacement (TAVR) to ensure proper valve anchoring. Due to the relatively high stent radial force, TAV leaflets are essentially mounted on a rigid frame. On the other hand, surgical bioprostheses are typically made from flexible stents to reduce stress at the commissures and improve valve longevity. The aim of this study was to compare leaflet stress distribution of a flexible frame surgical bioprosthesis with a rigid frame TAV using dynamic finite element analysis, considering tissue anisotropy. Methods: Since $23 \mathrm{~mm}$ Edwards-SAPIEN XT leaflet geometry is similar to $23 \mathrm{~mm}$ Carpentier-Edwards surgical biopros-
Fig. 1. Maximum principal stress distributions at the peak systolic and diastolic pressures on the TAV with rigid stent (top) and surgical bioprosthesis with flexible frame (bottom) (for Abstract of Ali Azadani).

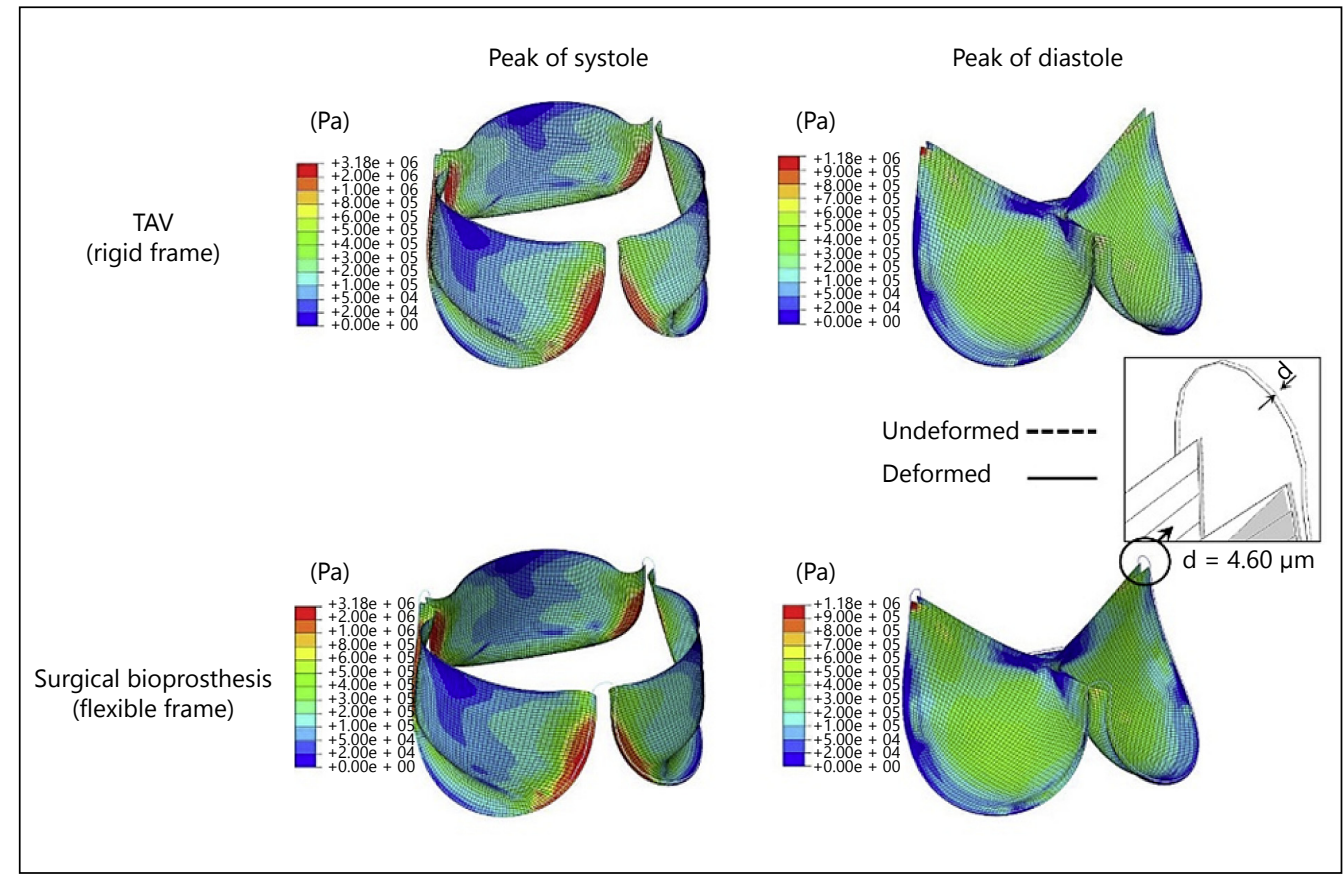


thesis with flexible frame, $3 \mathrm{D}$ geometries of the valves were created for finite element simulations in ABAQUS/Explicit. An Elgiloy wire with a thickness of $0.75 \mathrm{~mm}$ was considered as the frame of the surgical bioprosthesis. TAV frame was considered to be rigid. Leaflets were modeled as an anisotropic hyperelastic material based on the Fung's strain energy. Furthermore for consistency, identical leaflet thickness of $0.5 \mathrm{~mm}$ was considered for the TAV and the surgical bioprosthesis. Results: The maximum principal stress distributions of the $23 \mathrm{~mm}$ TAV and surgical bioprosthesis in the peak of systole and diastole are shown in figure 1. During systole, high stress regions were primarily observed in the boundary edge. The maximum principal stress reached to 3.174 and 3.174 $\mathrm{MPa}$ in the TAV and surgical bioprosthesis, respectively. During diastole, however, high stress regions were primarily observed at the commissures. The peak of stress reached to 1.172 and 1.173 $\mathrm{MPa}$ in the TAV and bioprosthesis, respectively. Conclusions: Flexibility of surgical bioprosthetic frame was insignificant under physiological loading in contrary to what is being claimed. Our results indicate that leaflet stress distribution of the TAV with rigid frame was comparable to the same size surgical bioprostheses, considering identical tissue thickness. However, by reducing TAV leaflet thickness to minimize the cross-sectional area of crimped TAVs, higher leaflet stress distribution is expected which may lead to diminished long-term valve durability.

\section{Sinus Flow Stasis Occurs in Valve in Valve Transcatheter Aortic Valve Implantation}

\author{
Lakshmi P. Dasi ${ }^{1}$, Hoda Hatoum ${ }^{1}$, Brandon Moore ${ }^{1}$, \\ Pablo Maureira ${ }^{2}$, Juan Crestanello ${ }^{1}$ \\ ${ }^{1}$ The Ohio State University, Columbus, $\mathrm{OH}, \mathrm{USA}$; \\ ${ }^{2}$ Lorraine University Hospital of Nancy, Nancy, France
}

Objective: Valve in valve (VIV) procedures using transcatheter aortic valves (TAV) are increasingly performed in heart valve surgeries as they provide a less invasive option than conventional surgeries. This study seeks to provide a detailed description of the sinus hemodynamics in the case of the VIV implantation by deploying a TAV within a porcine bioprosthetic valve (PBV). Knowledge of the aortic sinus blood flow dynamics in the coronary and non-coronary aortic sinus can provide insight into some causes of complications that happen in patients after implantation. Methods: A Medtronic Hancock II T505 valve was used as the PBV and a corresponding Medtronic CoreValve was used as the TAV in a way to match the annular size of the PBV. 2D High resolution Particle Image Velocimetry (PIV) was employed to visualize and quantitatively compare the sinus flow patterns, velocity, and shear stress fields from these two valves within both the coronary and the non-coronary sinuses in vitro. Leaflet kinematics were tracked for these different valve cases noting that the TAV leaflets extend superior (downstream) of the open PBV leaflets. Results: This study shows that the velocity and the vorticity within the PBV sinuses reached peak values of $0.7 \mathrm{~m} / \mathrm{s}$ and $1000 \mathrm{~s}-1$ with a $70 \%$ decrease in peak fluid shear stress near the aortic side of the leaflet in the non-coronary sinus. With the introduction of VIV, peak velocity and vorticity were reduced to around $0.4 \mathrm{~m} / \mathrm{s}$ and $550 \mathrm{~s}-1$, while peak shear stress was $38 \%$ higher along the aortic side of the coro- nary compared to the non-coronary VIV leaflet. Conclusions: Decreased flow near the base of the leaflet in the BPV non-coronary sinus may be a first step in assessing the risk of thrombosis and sub-clinical thrombosis in bioprosthetic aortic valves. Further, the lowered velocities and shear stress magnitudes in the presence of the TAV may indicate a higher risk of stroke and leaflet mobility issue.

\section{Systematic Transoesophageal Echocardiography after Mitral Valve Replacement - Prevalence and Determinants of Periprosthetic Leakage}

\section{Verdonk Constance}

Hopital Bichat, Paris, France

Background: Paravalvular complication is a well-known complication of transcatheter aortic valve implantation. Transcatheter mitral valve implantation (TMVI) is an emerging technology but contemporary series assessing the overall incidence and determinants of paravalvular regurgitation (PVR) after surgical mitral valve replacement (MVR) that TMVI could be compared with are seldom. At our institution, transoesophageal echocardiography (TEE) is performed as part of the systematic evaluation before discharge. Methods: We retrospectively enrolled all patients who underwent a MVR during the past two years. Medical history, indication and type of surgery and short-term outcome were collected. PVR was semi-quantitatively assessed using TEE performed before discharge. Results: We enrolled 399 patients (61 \pm 16 years, $58 \%$ female, $27 \%$ prior cardiac surgery). MVR was mainly performed for rheumatic disease (43\%). Most patients were severely symptomatic (60\% in NYHA class III/IV). A mechanical valve was implanted in $60 \%$ and a tissue valve in $40 \%$. In-hospital mortality was $10 \%$. Supraventricular arrhythmia occurred in 55\%, prolonged ( $>24$ hours) inotropic support in $46 \%$ and tamponade in $18 \%$. A TEE was performed in 310 patients $(77 \%)$; main reasons for not performing TEE were frailty $(\mathrm{N}=40 ; 10 \%)$, early death $(\mathrm{N}=19,5 \%)$ and contra-indication for TEE $(\mathrm{N}=10,3 \%)$. Overall incidence of PVR was $8 \%(\mathrm{~N}=25)$. A grade $\geq 2$ was observed in 14 patients (5\%) and 2 patients had to be re-operated. Mitral valve calcification was the only factor associated with PVR $(p=0.04)$. Conclusion: Surgical MVR is associated with a significant in-hospital mortality and morbidity. PVR is not uncommon but clinically significant (grade 2 or greater) are rare. The present study provides a reference emerging TMVI could be compared with. 


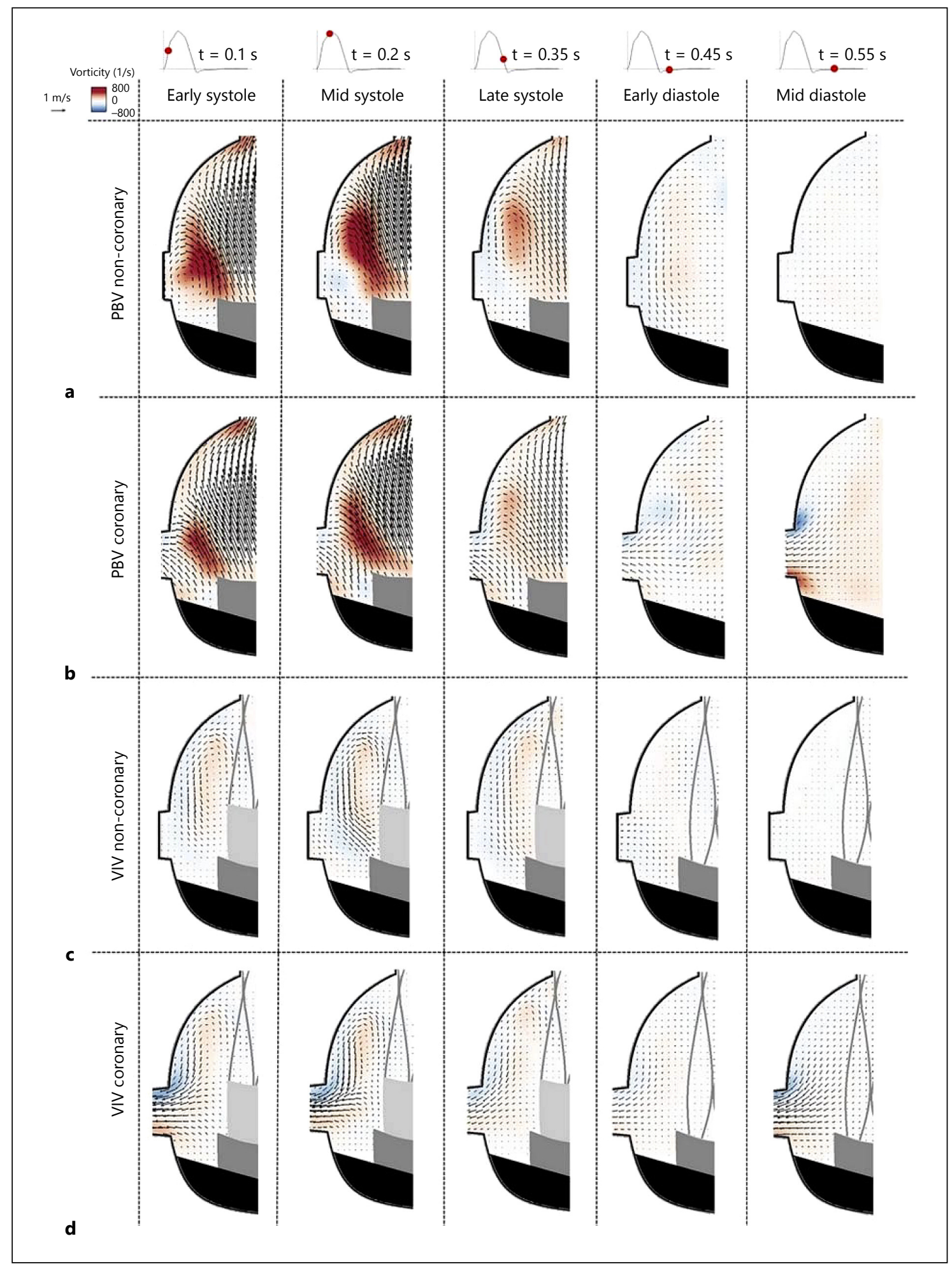

Fig. 1. Velocity vectors and vorticity contours within the sinus for BPV and VIV case at selected time points throughout the cardiac cycle (for Abstract of Lakshmi P. Dasi). 


\section{Poster Competition}

\section{Basic Research - Valve Science and Diseases}

\section{NPR2 Regulates Development and Homeostasis of the Mouse Aortic Valve}

\section{Mark Blaser ${ }^{1}$, Yu-Qing Zhou ${ }^{2}$, Hangjun Zhang ${ }^{1}$, Scott Heximer ${ }^{1}$, R. Mark Henkelman ${ }^{2}$, Craig Simmons ${ }^{1}$}

${ }^{1}$ University of Toronto, Toronto, ON, ${ }^{2}$ Toronto Centre for Phenogenomics, Toronto, ON, Canada

Background: C-type natriuretic peptide (CNP), which signals through the guanylate cyclase receptor NPR2, is shear-sensitive, elevated in the disease-protected ventricularis of the aortic valve, and reduced in stenotic valves. CNP/NPR2 signaling inhibits myofibrogenesis and osteogenesis of aortic valve interstitial cells in vitro, yet it is unknown whether this pathway has a functional and protective role in vivo. Methods: Homozygous knockout of CNP or NPR2 causes high levels of neonatal mortality ( $>95 \%)$, so male $\mathrm{Npr} 2+/-$; Ldlr-/- and control Npr2+/+; Ldlr-/- mice were fed a high-fat, high-cholesterol Western diet (TD.88137) for 10 months $(n=13-$ 18 /group). Valve/cardiac/vascular function was assessed by highresolution ultrasound, and valve matrix composition/morphology was examined by Movat's pentachrome, picrosirius red, and alizarin red. Results: Cardiac dysfunction arose in Npr2+/- mice: ejection fraction and fractional shortening were reduced by $24.1 \%$ and $29.7 \%$ respectively vs. controls $(\mathrm{p}<0.001)$. $N$ pr $2+/-$ mice were $3.5 \mathrm{x}$ less likely than controls to develop aortic regurgitation $(11.1 \%$ vs. $38.5 \%, \mathrm{p}<0.05)$. In contrast, aortic stenosis was observed in $\mathrm{Npr} 2+/-$ mice, whose aortic valve velocity ratio (transvalvular:left ventricular outflow tract velocity) was increased by $53.6 \%$ vs. controls with regurgitation $(\mathrm{p}<0.001)$, and by $134.8 \%$ vs. controls without regurgitation ( $\mathrm{p}<0.001)$. Proteoglycan-rich thickening, fibrotic collagen accumulation, and microcalcification occurred in Npr2+/- leaflets, but not in controls. $9.8 \%$ of $N p r 2+1-$ mice had bicuspid aortic valves (BAVs; incidence: $\mathrm{p}<0.05 \mathrm{vs}$. none in controls), with the worst valve function and matrix alterations. Npr2+/- mice also developed ascending aortic dilatations: diameter increased by $21.7 \%$ vs. all controls $(\mathrm{p}<0.001)$, while those Npr $2+/-$ mice with BAVs had larger dilatations than Npr2+/- mice with tricuspid aortic valves $(\mathrm{p}<$ $0.01)$. Conclusions: We identified three distinct phenotypes: 1) no regurgitation/stenosis in $61.5 \%$ of control Npr2+/+;Ldlr-/-, 2) regurgitation and mild stenosis in $38.5 \%$ of control Npr2+/+;Ldlr-/-, 3) severe stenosis with little regurgitation, increased BAV incidence, impaired cardiac function, and aortic dilatation in Npr2+/-;Ldlr-/-. Thus, NPR2 and downstream cGMP signaling are implicated in valve health/development, while progression of stenosis may preempt initiation of regurgitation. These findings reveal a protective role for CNP signaling in the aortic valve, suggest therapeutic strategies, and establish a novel BAV model. They also broadly implicate guanylate cyclase signaling in valvular development.

\section{The Role of Follistatin Like 1 in Postnatal Valve Growth}

\section{Stuti Prakash}

Academic Medical Center, Amsterdam, Netherlands

Objective: In this study we investigate the role of Follistatinlike 1 (Fstl1) in the development of the atrioventricular (AV) valves after birth. AV valves are conserved and dynamic structures composed of cells, derived from different lineages, and a highly organized extracellular matrix (ECM). Dysregulation of the cellular contribution and ECM organization leads to valve disease which is a leading cause of morbidity and mortality in adults. Fstl1 is a secreted glycoprotein expressed mostly in the non-myocardial component of the heart and is induced in response to injuries that promote myocardial hypertrophy and heart failure. Fstll is thought to be an extracellular regulator of BMP signalling. Methods: We have conditionally removed Fstl1 from the endocardial/endothelial cell lineage using the Tie2Cre deletor mouse line. We have performed echocardiographic analysis using the Visual Sonics Vevo 2100 imaging system to establish the hemodynamic changes in these Fstll conditional knock-out mice from the first until the 23rd day after birth. Afterwards the mice were sacrificed and analyzed by immunofluorescence and in situ hybridization. Results: The echocardiographic analysis showed that cardiac function was normal up to 10 days after birth. From 14 days onwards the heart was observed to be enlarged and mitral valve regurgitation was identified. E/A data showed diastolic dysfunction in mutants. Autopsy showed the hearts to be enlarged with hypertrophic ventricular walls and long, thick and billowing AV valves. Histological analysis showed an upregulation of extracellular matrix proteins and embryonic valvular genes. Evaluating the local activity of BMP and TGF $\beta$ signaling using antibodies directed to P-Smad1/5 and PSmad2/3, respectively, showed an upregulation in the endocardial and mesenchymal cells of the valves. Conclusions: Taken together, our data suggest that absence of Fstl1 from the endothelial/endocardial lineage results in a dysregulation of TGF $\beta / B M P$ signalling and in turn in very long and thick incompetent $\mathrm{AV}$ valves. The analysis, furthermore, suggest that these valves can be considered to be myxomatous valves.

\section{NF-kappaß P65 Phosphorylation at Serine 536 Mediates Lysophosphatidic Acid-Induced BMP-2 Gene through Rho-Kinase Pathway: Role in Calcific Aortic Valve Disease}

Mohamed Jalloul Nsaibia, Marie-Chloé Boulanger, Patrick Mathieu

IUCPQ, Québec, QC, Canada

Background: Calcific aortic valve stenosis (CAVS) is the most frequent heart valve disorder. There is so far no medical treatment for CAVS that could prevent its progression. Studies indicate that mineralization of the aortic valve may be related to the inflammatory process. Recently, we documented that lysophosphatidic acid 
(LPA), which is generated by autotaxine (ATX), was present in the aortic valve and was an important driver of aortic valve mineralization. The main objective of this work was to understand the signaling pathways by which LPA promotes the mineralization of the aortic valve. Methods: By using different approaches we have investigated the effect of LPA on the osteogenic phenotype. We have also documented the mechanisms whereby the NF- $\kappa B$ pathway is activated by LPA and promotes the osteogenic transition of cells. Results: In cell culture we found that the expression of osteogenic genes (BMP2, RUNX2, BGLAP, and COL1A1), alkaline phosphatase (ALP) activity and valve interstitial cells (VICs) mineralization were increased by several-fold after a treatment of cells with LPA. Also, we have shown that LPA-induced osteogenic response relied on RhoA pathway downstream of the LPA receptor-1 (LPAR1). In this regard, we found that RhoA is a regulator of the $\mathrm{NF}-\kappa \mathrm{B}$ pathway and promotes BMP2 expression. In addition, by using promoter luciferase assay we documented that NF- $\kappa B-p 65$ phosphorylation on serine 536 (p65 phospho S536) activates BMP2 promoter following a treatment with LPA. We next showed by using chromatin immunoprecipitation assays (ChIP) that the binding of p65 phospho S536 to BMP2 promoter is not reversed by the

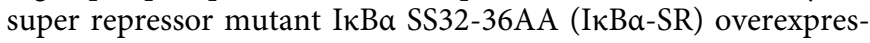
sion. LPA-induced mineralization of VICs was not inhibited by $\mathrm{I} \kappa \mathrm{Ba}-\mathrm{SR}$, whereas it was significantly reduced following the transfection of a vector encoding for a mutant p65 S536A, which cannot be phosphorylated at this site. Conclusion: Together, these results suggest that LPA-induced VICs mineralization is dependent on p65 phosphorylated serine 536 pathway. Hence, we documented a novel mechanism whereby LPAR 1 and RhoA modulate the NF- $\kappa B$ pathway and its downstream target BMP-2, which is a strong promoter of VICs mineralization and thus could represent a novel therapeutic target in CAVS.

\section{Aortic Valve Knockout of pRb Increases Calcific Aortic Valve Disease Characteristics}

\author{
Lauren Baugh ${ }^{1}$, Marina Freytsis ${ }^{2}$, Irene Georgakoudi ${ }^{1}$, \\ Philip Hinds ${ }^{3}$, Gordon Huggins ${ }^{4}$, Lauren D. Black, III \\ ${ }^{1}$ Tufts University, Medford, MA, ${ }^{2}$ Tufts University, Boston, MA, \\ ${ }^{3}$ Tufts Medical Center, Boston, MA, ${ }^{4}$ Tufts Medical Center, \\ Bostom, MA, USA
}

Objective: The retinoblastoma protein $(\mathrm{pRb})$ functions as a direct transcriptional coactivator required for osteogenic differentiation and bone formation. Because mechanisms that contribute to bone formation are relevant to calcific aortic valve disease (CAVD) we tested the role of $\mathrm{pRb}$ in mice fed a high fat diet. Methods: Conditional $\mathrm{Rb}$ knockout mice were created using floxed $\mathrm{Rb}$ alleles and Tie2-Cre to facilitate gene excision in the aortic valve. Control $(\mathrm{Rb} \mathrm{fl} /+)$ and knockout $(\mathrm{Rb} \mathrm{fl} / \mathrm{fl})$ mice were fed either a high fat (HF) or normal (norm) diet. Mice (up to 12 months of age)

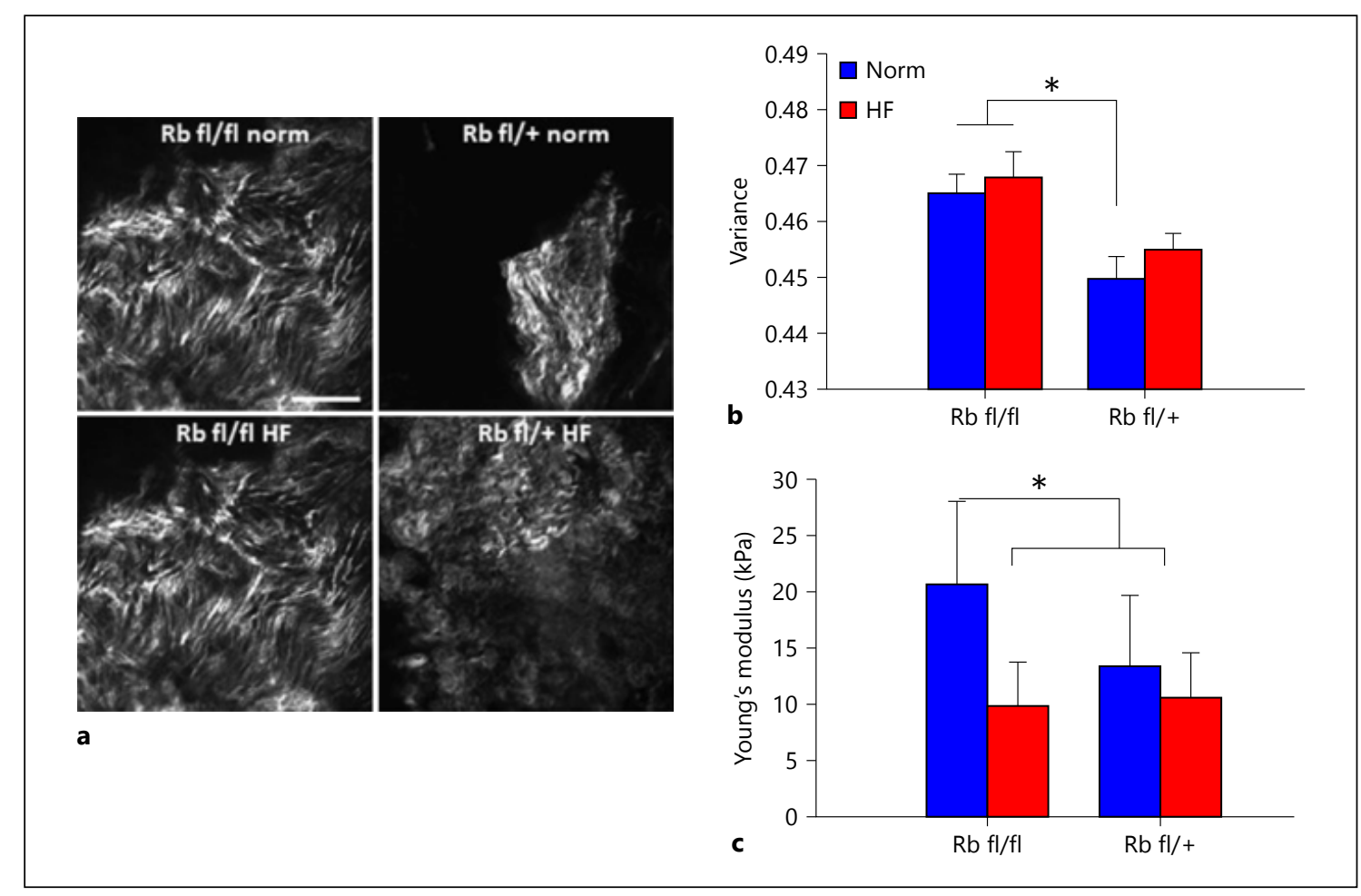

Fig. 1. a Representative image of the 4 sample groups showing SHG signal from collagen. $\mathbf{b}$ The variance of collage fiber organization in each sample group based on SHG images. c The Young's Moduli, measured by AFM, of each sample group. Scale bar is $50 \mu \mathrm{m} ; \mathrm{p}<0.05$ (for Abstract of Lauren Baugh). 
were imaged for aortic regurgitation and sacrificed for characterization via multiphoton microscopy. Second Harmonic Generation (SHG) signal from collagen was collected for 3 samples in each of the four groups through the entire depth of the valve leaflets (fig. 1a). Samples were also imaged using atomic force microscopy (AFM) to determine the valve mechanical properties. Results: After 12 months, $\mathrm{Rb}$ fl/fl mice showed an increase in aortic valve regurgitation $(\mathrm{p}<0.0001)$ and decreased survival compared to $\mathrm{Rb}$ $\mathrm{fl} /+$ mice. After sacrifice, SHG analysis of variance in collagen fiber orientation demonstrated a significant decrease in collagen fiber organization in $\mathrm{Rb} f l / f l$ mice on a normal $(\mathrm{p}=0.0068)$ and $\mathrm{HF}$ diet $(\mathrm{p}=0.0274)$ compared to $\mathrm{Rb} \mathrm{fl} /+$ mice on a normal diet (fig. $\mathrm{lb}$ ). $\mathrm{The} \mathrm{Rb}$ fl/fl normal diet mice also had a significantly higher valve leaflet elastic modulus as compared to $\mathrm{Rb} \mathrm{fl} / \mathrm{fl} \mathrm{HF}$ and $\mathrm{Rb} \mathrm{fl} /+\mathrm{HF}$ mice ( $\mathrm{p}<0.001$; fig. 1c). Conclusion: Absence of $\mathrm{pRb}$ increases CAVD characteristics in transgenic mice as demonstrated by increased aortic regurgitation, increased morbidity, decreased valve leaflet collagen fiber organization, and increased leaflet stiffness. Taken together our data demonstrate that $\mathrm{pRb}$ is required for regulation of collagen fiber organization to prevent the development of changes in the aortic valve characteristic of CAVD.

\section{Fibrocalcific Commitment of Aortic Valve Interstitial Cells via Tissue Factor Expression and Signaling}

\author{
Rodrigo Lorenzi ${ }^{1}$, Ahmed Elkalioubie ${ }^{1}$, Arbesu Y. Miar Anaïs ${ }^{1}$, \\ Ramadan Jashari ${ }^{2}$, Delphine Corseaux ${ }^{1}$, Annabelle Dupont ${ }^{1}$, \\ Francis Juthier ${ }^{1}$, Andre Vincentelli ${ }^{1}$, Bart Staels ${ }^{1}$, Eric Van Belle ${ }^{1}$, \\ Sophie Susen ${ }^{1}$ \\ 'University Lille, Inserm, CHU Lille, Institut Pasteur de Lille, \\ U1011, EGID, Lille, France; ${ }^{2}$ European Homograft Bank, \\ Brussels, Belgium
}

Objectives: Aortic valve stenosis (AVS) is an atherosclerosislike process characterized by valve interstitial cell (VIC) commitment to fibrocalcification. Tissue factor (TF) expression was shown to be associated with calcification in human stenotic aortic valves. Therefore we investigated the potential role of TF in the fibrocalcification commitment of VIC. Methods: TF expression in control and fibrocalcific human aortic valves was assessed by immunohistochemistry, western blot (antigen) and colorimetry (activity). Constitutive TF expression in VIC isolated from these valves and grown in culture, as well as after osteoblastic differentiation, was likewise assessed. Inducible TF expression in IL-1 $\beta$-stimulated (10 ng/mL) VIC isolated from control valves was also assessed. Downstream signaling pathways of FVIIa/TF complex in IL- $1 \beta$-stimulated VIC were assessed by western blot. Results: TF expression was higher in fibrocalcific vs. control aortic valves (TF/ $\beta$-actin: $0.6 \pm 0.1$ vs. $0.3 \pm 0.08$; activity: $253 \pm 25$ vs. $131 \pm 20 \mathrm{mU} /$ mg protein, respectively). TF expression in cultured VIC was higher in VIC isolated from fibrocalcific vs. control valves (antigen TF/ $\beta$-actin: $3.4 \pm 2.0$ vs. $0.7 \pm 0.1$; activity: $37 \pm 6$ vs. $19 \pm 4 \mathrm{mU} / \mathrm{mg}$ protein, respectively) and increased more than 3000 -fold after osteoblastic differentiation. Following IL- $1 \beta$ stimulation $(10 \mathrm{ng} / \mathrm{mL})$ of VIC isolated from control valves, TF expression was significantly upregulated compared to unstimulated VIC (antigen TF/ $\beta$ actin: $1.8 \pm 0.4$ vs. $0.7 \pm 0.1$; activity: $76 \pm 35$ vs. $19 \pm 4 \mathrm{mU} / \mathrm{mg}$ protein, respectively). In a TF-dependent way, FVIIa stimulation of TF-overexpressing VIC induced, when compared to unstimulated VIC, a significant activation of the ERK (pERK/ERK $0.8 \pm 0.2$ vs. $0.2 \pm 0.1$ respectively, $0.3 \pm 0.1$ with anti-TF antibody) pathway. This stimulation also induced, when compared to unstimulated VIC, the nuclear translocation of Smad2/3 (pSmad2/Smad 2/3 $0.2 \pm 0.02$ vs. $0.09 \pm 0.0 .01$ respectively, $0.08 \pm 0.006$ with anti-TF antibody) and of $\beta$-catenin ( $\beta$-catenin/ $\beta$-actin $1.2 \pm 0.07$ vs. $0.3 \pm$ 0.1 respectively, $0.3 \pm 0.1$ with anti-TF antibody) signaling pathways. In addition, fibronectin and RUNX2 expression were increased $48 \mathrm{~h}$ after FVIIa stimulation ( 3 and 2 -fold, respectively). Conclusions: These results suggest an implication of TF/FVIIa axis in VIC commitment to AVS. Modulation of this pathway may represent a new therapeutic target in AVS treatment.

\section{Cyclic Stretch Modulates Calcific Lesion Formation in 3D Co-Cultured Valve Cells}

\section{Jennifer Richards, Alexa Wnorowski, Jonathan Butcher}

Cornell University, Ithaca, NY, USA

Objective: Calcific aortic valve disease (CAVD) is characterized by compromised endothelial and activated interstitial populations. While mechanical tension in valve interstitial cell phenotype has been well studied in $2 \mathrm{D}$, the role of mechanical stress on valve endothelial-interstitial interactions in 3D are poorly understood. We here determined how mechanical tension and cyclic strain regulate interstitial (VIC) and endothelial (VEC) interactions and phenotypes in 3D osteogenic environments. Methods: We seeded collagen hydrogels inside stainless steel compression springs so there was equal constraint around the circumference of the gel. Using a custom bioreactor system, we applied cyclic strain over a period of 7 days in both standard and osteogenic media conditions, comparing additionally against mechanically stressed (anchored) but non-stretched controls. Results: We found that VIC-only cultures calcified in anchored 3D gels under osteogenic media conditions, but created no lesions. VEC do not calcify in 3D culture. Interestingly, we found that anchored VIC+VEC co-cultures in osteogenic environments develop raised 3D calcific nodules ( $25-$ $50 \%$ of gel thickness) similar to valve lesions seen in vivo. These lesions contained radially reoriented collagen fibers with similarly aligned VIC with increased VEC density and decreased VEC cell area, while non-lesion areas remained similar to control conditions. Interestingly, cyclic stretch increased lesion number, but decreased lesion size. Cyclic strain enhanced apoptosis in co-cultures while decreasing proliferation, suggesting an active remodeling of the collagen hydrogels in strained conditions. Overall calcium deposition was unchanged between static and stretched conditions. Co-culture gels cultured statically in OGM increased in aSMA expression, supporting myofibroblastic activation, which was decreased with cyclic stretch. However, cyclic strain increased expression of Runx2 and osteopontin, supporting osteoblastic differentiation. Furthermore, cyclic stretch increased VEC apoptosis increased concomitant with a decrease in eNOS expression. Finally, blocking cell contractility through ROCK inhibition abrogated calcium deposition, nodule formation, and osteogenic differentiation. Conclusions: These results demonstrate the need for 
cellular tension for calcification, nodule formation and myofibroblastic activation. Furthermore, the mechanical tension state of the VEC is an important contributor for aortic valve homeostasis and calcific susceptibility. 3D in vitro co-culture platforms are advantageous to identify and dissect emergent mechanobiological mechanisms of CAVD.

\section{Role of miRNA-483-3p in Valvular Endothelial Dysfunction} Joan Fernandez Esmerats, Jack Heath, Sandeep Kumar,
Hanjoong Jo

Georgia Institute of Technology and Emory University, Atlanta, GA, USA

Introduction: Calcific Aortic Valve Disease (CAVD) is a significant cause of mortality in the aging population. Currently, the only treatment is surgical valve replacement. CAVD is associated with endothelial cell dysfunction, followed by valve interstitial cell differentiation and calcification. This process can be mediated by miRNAs, small nucleotide sequences that degrades or inhibit target gene translation. Several studies have found that calcification is preferentially located on the fibrosa, the aortic side of the valve which is subjected to disturbed flow (low and oscillatory shear stress). Here we tested the hypothesis that flow disturbance in the fibrosa endothelium plays a key role in the onset and progression of CAVD by regulating miRNA expression. Materials and Methods: Human aortic valve endothelial cells (HAVECs) from nonstenosed aortic valves were subjected to oscillatory shear stress $\left(\mathrm{OS}, \pm 5\right.$ dynes $\left./ \mathrm{cm}^{2}\right)$ or high and unidirectional shear stress (LS, 20 dynes $/ \mathrm{cm}^{2}$ ) in our cone-and-plate viscometer. We performed mRNA and miRNA array studies. We also validated expression of selected mechanosensitive genes and miRNAs by qPCR. Further study of gene function was done using siRNAs and miRNA overexpression was accomplished with pre-miRNAs to identify and validate the target genes. Results: miRNA-483-3p was one of the miRNAs that showed a consistent differential expression between OS and LS. We found that miR-483-3p was consistently downregulated in OS conditions in HAVECs. Through a systems biological analysis, we identified and validated several target genes including Ash2L (a critical component of the p53-dependent apoptosis pathway), which was robustly regulated by miR-483p and shear stress. Overexpression (with pre-miR-483) and inhibition (with anti-miR-483) of miR-483-3p in HAVECs decreased and increased Ash2l gene and protein expression, respectively. Conclusions: miRNA-483-3p is a mechanosensitive miRNA upregulated in HAVECs under LS conditions. Ash2L is a direct target of miR-483-3p and is downregulated by LS. Apoptosis is known to be upregulated in calcified valves but the exact mechanism is unknown, but our data proposes a possible mechanism by which shear stress regulates apoptosis in the valve endothelium. This novel finding may shed novel insights into the CAVD mechanisms and serve as new therapeutic targets for the disease.

\section{Fully-Coupled FSI Simulation of Bioprosthetic Heart Valve Using Smoothed Particle Hydrodynamics}

\author{
Wenbin Mao ${ }^{1}$, Kewei Li ${ }^{2}$, Andres Caballero ${ }^{1}$, Wei Sun ${ }^{1}$ \\ ${ }^{1}$ Georgia Institute of Technology, Atlanta, GA, \\ ${ }^{2}$ University of Connecticut, Storrs, CT, USA
}

Objective: Computational modeling of heart valve dynamics, with both fluid dynamics and valve structural responses, is challenging. In this study, our objective is to develop a fully-coupled fluid-structure interaction (FSI) method, using smoothed particle hydrodynamics (SPH), to simulate the dynamic motion of a bioprosthetic heart valve (BHV). Methods: Explicit finite element (FE) method in ABAQUS was used to simulate the structural deformation of the BHV, which was discretized with 7896 hexahedral elements (C3D8R). Mechanical properties of BHV leaflets were modeled using the anisotropic hyperelastic Holzapfel-Gasser-Ogden model. To elicit the impact of tissue anisotropy, the incompressible Ogden hyperelastic model was used as well in our simulations. The blood flow passing through the BHV was modeled by the SPH method, which is a meshless, Lagrangian particle-based method. The FE and SPH are fully coupled in ABAQUS at the fluid and structure interface, i.e., the fluid pressure induced by the fluid particles deforms the leaflets of the BHV. The Lagrangian nature associated with no fixed mesh for the fluid makes this method well suitable for modeling complex flows and large deformations. Results: The dynamics motion of BHV leaflets was simulated with pulsatile flows in accordance with conditions specified in the ISO-5840:2005. Overall, the FSI model resulted in realistic deformations of leaflets. Hemodynamic parameters, e.g., transvalvular pressure gradient: $8 \mathrm{~mm} \mathrm{Hg}$, peak velocity: $1.5 \mathrm{~m} / \mathrm{s}$, and effective orifice area: $1.8 \mathrm{~cm}^{2}$, were comparable to experiment data. The peak strains of the leaflets were about 0.12 and 0.2 at systole and diastole phases, respectively. We found that material anisotropy has significant impact on the valve opening and the stress distribution at the closure. A structure-only model cannot correctly capture the valve dynamics. The approach was then extended to simulate the aortic valve deformation in a patient-specific left ventricle (LV) outflow tract model, coupled with the dynamic motion of the LV. Conclusions: The approach using the SPH method to model the BHV FSI is promising. This approach may be extended to model the whole heart FSI dynamics. 


\section{Experimental and Computational Valve Tissue Studies}

\section{Computational Characterization of Bioprosthetic Heart Valve Positioning to Enhance Long Term Performance}

\section{Orla M. McGee, Paul S. Gunning, Laoise M. McNamara}

NUI Galway, Galway, Ireland

Background: Incorrect positioning of the Transcatheter Aortic Valve's (TAV's) can cause adverse effects such as mitral insufficiency, arrhythmias or aortic injury [1]. Positioning of TAV's could have an effect on the stresses and strains in both the stent and arterial tissue. Improved knowledge on the effect of positioning could enhance the long term mechanical performance. Computational models have been applied to model the performance of TAV's $[2,3]$. However such methods have not been applied to predict the role of valve positioning. The objective of this research is to develop computational models to predict the role of positioning on the mechanical performance of TAV's. Methods: A solid model of the aortic root was generated from MRI images. A computational model was created in ABAQUS to model the crimping and deployment of a self-expanding Nitinol stent into the aortic root. The aortic tissue was modeled as isotropic hyperelastic. Nitinol was modeled as superelastic. The stent was deployed in three different positions; on the aortic annulus, $5 \mathrm{~mm}$ above the annulus and $5 \mathrm{~mm}$ below the annulus. Results: The highest stresses in both the aortic tissue and the stent were observed when the stent was deployed at the aortic annulus. Interestingly, the lowest stresses in both the aortic tissue and the stent were found when the stent was deployed at the $5 \mathrm{~mm}$ sub annular position. However results showed the maximum Von Mises stresses predicted for all three deployment positions are well below the rupture stress of the aortic tissue (1.75 MPa [4]). Conclusion: These results show that the positioning of the valve does have an effect on the stresses seen both in the aortic tissue and in TAV stent.

\section{References}

1 Masson J-B, et al: JACC: Cardiovasc Interv 2009;2:811-820.

2 Wang Q, et al: J Biomech 2012;45;1965-1971.

3 Gunning PS, et al: Ann Biomed Eng 2014;42:1989-2001.

4 Vorp DA, et al: Ann Thorac Surg 2003;75:1210-1214.

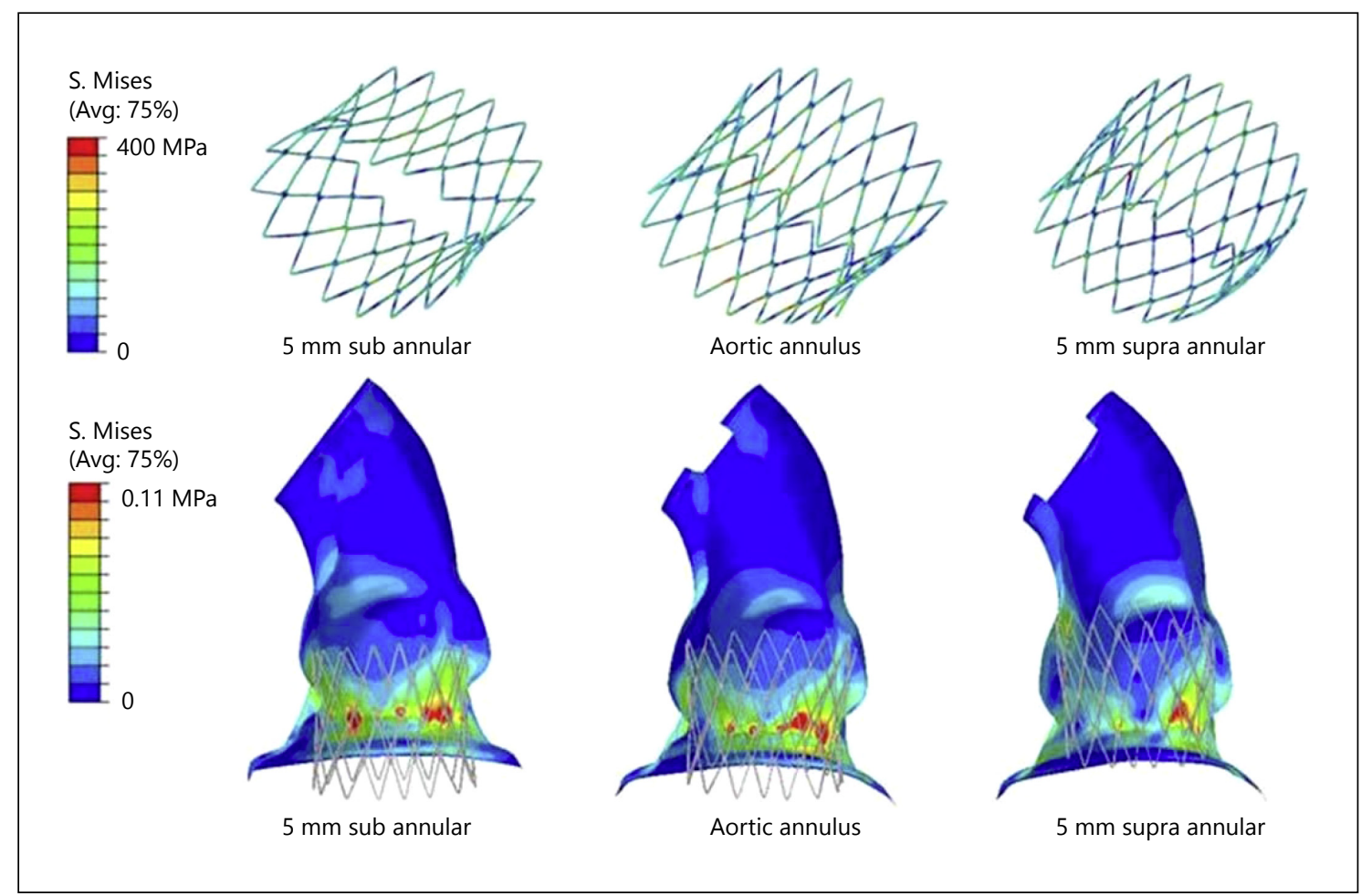

Fig. 1. Contour plots of Von Mises stresses (MPa) (for Abstract of Orla M. McGee). 


\section{Improved Bioprosthetic Heart Valve Design: A 3D Computational Model to Assess the Effect of Geometrical Parameters on Structural Deterioration}

Yaghoub Dabiri, Kent Paulson, Imtiaz Ali, Janet Ronsky, Kishan Narine

University of Calgary, Calgary, AB, Canada

Objective: Stress distribution in bioprosthetic aortic valves (BAVs) leaflets determines structural valve deterioration (SVD). The development of stresses in the leaflets is a multifactorial process involving the material of the leaflets, the loading conditions, the flow pattern, and the geometrical parameters. Geometrical parameters are particularly important because they can be altered during valve design to reduce SVD. Computational studies are becoming a regulatory requirement. This study has developed a computational package to assess alterations in BAV leaflets due to geometrical parameters. Methods: A Fung transverse hyperelastic model was used for the leaflets. The constant parameters were determined by fitting the experimental data obtained from biaxial tests to the results obtained from the Fung model. The three-dimensional leaflet geometries were developed based on data from literature. In a closed configuration a $12 \mathrm{KPa}$ pressure was applied on the aortic side of the leaflets. ABAQUS (Simulia, Providence, USA) software package was used for finite element calculations. Three case studies A, B and C with different geometrical specifications were considered: leaflets inclination toward the annulus wall: C $>$ B $>$ A, the circumferential curvature B $>$ A = C. Results: Regardless of the geometrical specifications, the maximum values of von Mises stress as well as the minimum principal stress occurred in the fixed edge area and close to the coaptation area. However, the geometrical specifications had profound effects on the values of stresses. The maximum von Mises stress increased by 10 and $29 \%$ in B and A compared to C. As well, the minimum principal stresses was larger by 11 and $21 \%$ in B and A compared to C. As such, in case study C, SVD propensity is demonstrated to be significantly lower compared to B and A. Conclusions: The effects of geometrical parameters on BAV deterioration can be computed in a 3 dimensional model. Geometrical parameters have a significant effect on SVD but can be optimised in the design process to reduce SVD or used to assess durability in new or existing BAVs.

\section{The Role of the Host Cellular Response on Bioprosthetic Heart Valve Calcification}

\section{Hobey Tam, Nathaniel Parchment, Josh Van Swol,} Daniel Infante, Naren Vyavahare

Clemson University, Clemson, SC, USA

Objective: A common failure mode of $\mathrm{BHVs}$ is calcification and the underlying factors driving calcification remain poorly understood. The objective of this research was to discern the role of host cellular response during bioprosthetic heart valve (BHV) calcification in a rat subdermal model. Methods: Bovine pericardium (BP) patches were treated in a $0.6 \%$ glutaraldehyde (GLUT) solution for 24 hours and stored in $0.2 \%$ GLUT thereafter. These patches were cut into $1.0 \times 1.0 \mathrm{~cm}$ squares then sealed in dialysis bags (M.W. Cutoff $12.5 \mathrm{kD}$ ). Both the dialysis bags and unenclosed GLUT patches of biomaterial were subcutaneously implanted in rats. TRI crosslinked leaflets served as a negative control for calcification in our animal studies as these tissues do not calcify as shown in previous

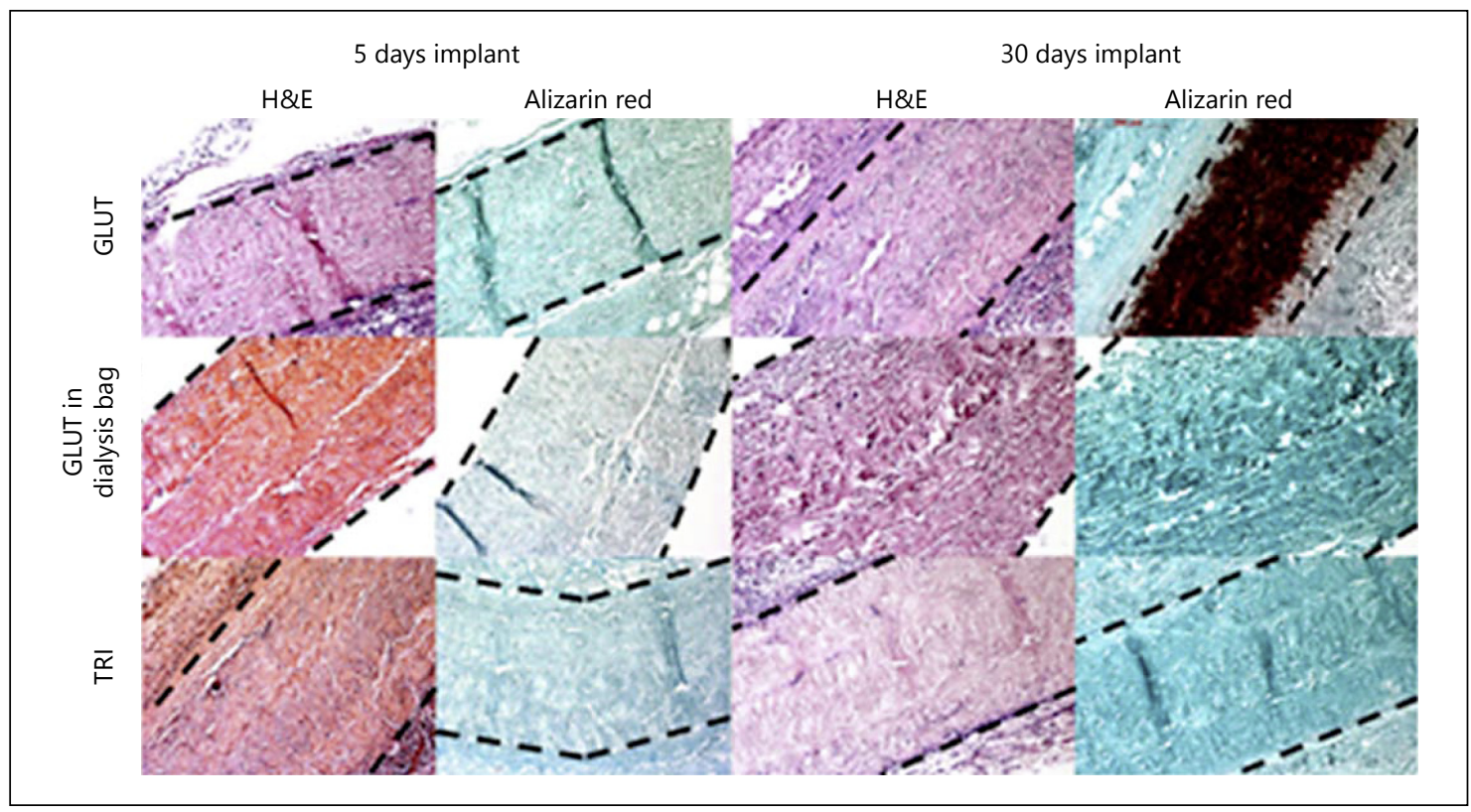

Fig. 1. Histology of bovine pericardium samples subcutaneously implanted in rats at 5 or 30 days (for Abstract of Hobey Tam). 
studies. After periods of 5 and 30 days, rats from each treatment were sacrificed and the retrieved implants were analyzed with alizarin red, H\&E, Masson's trichrome, and IHC as well as for quantitative calcium and phosphorus content by ICP. Results: At 30 days after implantation, unenclosed GLUT samples were calcified heavily; however, samples enclosed in dialysis bags did not calcify (figure 1). TRI treated samples did not calcify. Further analysis via IHC showed infiltration of macrophages into all implants except those isolated inside dialysis bags. There was a noted difference between the infiltration behavior of macrophages in GLUT vs. TRI samples. Macrophages penetrated deep into the tissue of GLUT, while at both 5 and 30 day time-point, TRI samples exhibited little infiltration restricted to the periphery of the implant. Conclusions: The current study suggests that the host cellular response may play a role in calcification. It merits further investigation into the biological triggers that elicit this response in the hopes of suppressing these triggers and attenuating calcification. This would extend $\mathrm{BHV}$ implant life and reduce risk to patient significantly.

Acknowledgements: NIH R01HL108330 and NSF GRFP.

\section{Undersized Ring Annuloplasty Increases Strain in the Left Ventricle: Finite Element Analysis}

\begin{abstract}
Joe L. Pantoja', Ashley E. Morgan' ${ }^{2}$, Liang Ge2, Eugene A. Grossi', Jonathan W. Weinsaft ${ }^{4}$, Morton O. Jensen ${ }^{5}$, Robert A. Levine ${ }^{6}$, Mark B. Ratcliffe ${ }^{2}$

${ }^{1}$ UCSF School of Medicine, San Francisco, CA, ${ }^{2}$ San Francisco VA Medical Center, San Francisco, CA, ${ }^{3}$ New York University, New York, NY, ${ }^{4}$ Cornell University, New York, NY, ${ }^{5}$ University of Arkansas, Fayetteville, AR, ${ }^{6}$ Massachusetts General Hospital, Boston, MA, USA
\end{abstract}

Objective: Ischemic mitral regurgitation (MR) is difficult to treat. Standard therapy is coronary revascularization, combined with mitral valve repair using undersized ring annuloplasty (RA). Unfortunately, recurrence rates after this procedure are high, up to $40 \%$ in some series. This high recurrence rate is likely due to con- tinued dilation of the left ventricle (LV). To better understand the causes of continued LV dilation and recurrent MR, we studied the effect of undersized RA on strain in the wall of the LV. We hypothesize that the acute change in ventricular shape induced by RA will cause increased strain, especially in the regions of the LV nearest the mitral valve. Methods: Finite element models were previously created, based on cardiac magnetic resonance images of five sheep with mild to moderate ischemic MR. A $24 \mathrm{~mm}$ rigid annuloplasty ring was modeled and used to simulate virtual RA. Longitudinal and myofiber strains were calculated at end diastole and end systole, with pre-operative early diastolic geometry as the reference state. Results were compared using mixed-model regression analysis. Results: Undersized RA caused significant increases in longitudinal strain at end diastole (figure 1). This effect was greatest in the proximal-lateral LV wall, where longitudinal strain after RA was approximately double the pre-operative strain $(0.047 \pm 0.018$ vs. $0.082 \pm 0.028 \mathrm{p}=0.017)$. There were no significant changes in myofiber strains at end diastole, and no significant changes in either strain type at end systole. Conclusion: Undersized RA causes a substantial increase in longitudinal strain in the proximal lateral LV wall. We suspect that this 'stretching' of the proximal-lateral LV wall may stimulate continued LV enlargement and recurrence of mitral regurgitation. Further studies will repeat this experiment in a human model of ischemic mitral regurgitation and investigate the effect of varying ring size and shape on LV strain.

\section{Computational Assessment of the Effects of Bicuspid Aortic Valve Morphotype on Ascending Aorta Hemodynamics}

\author{
Kai Cao ${ }^{1}$, Philippe Sucosky ${ }^{2}$ \\ ${ }^{1}$ University of Notre Dame, Notre Dame, IN, ${ }^{2}$ Wright State \\ University, Dayton, $\mathrm{OH}$, USA
}

Objective: The bicuspid aortic valve (BAV) is associated with a high prevalence of aortic dilation, whose expression depends on the type of leaflet fusion. While hemodynamics is presumed to be a po-

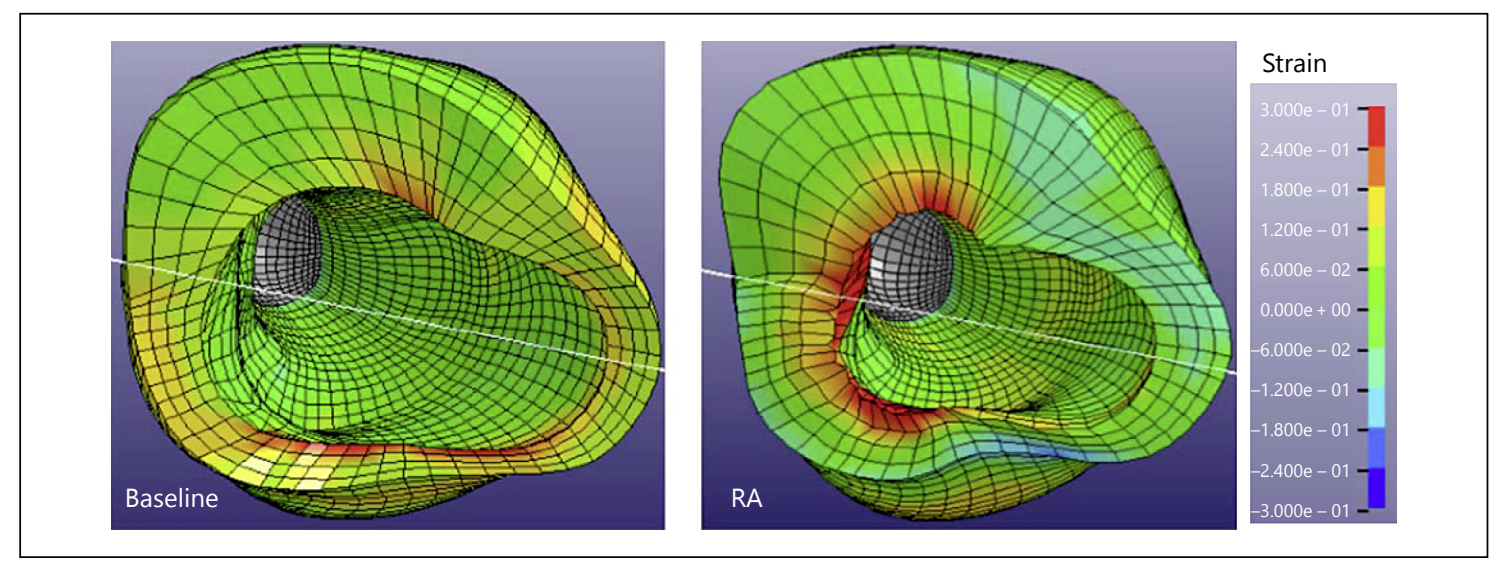

Fig. 1. Baseline vs. undersized ring annuloplasty (RA): longitudinal strain at end diastole in one sheep (for Abstract of Ashley E. Morgan). 


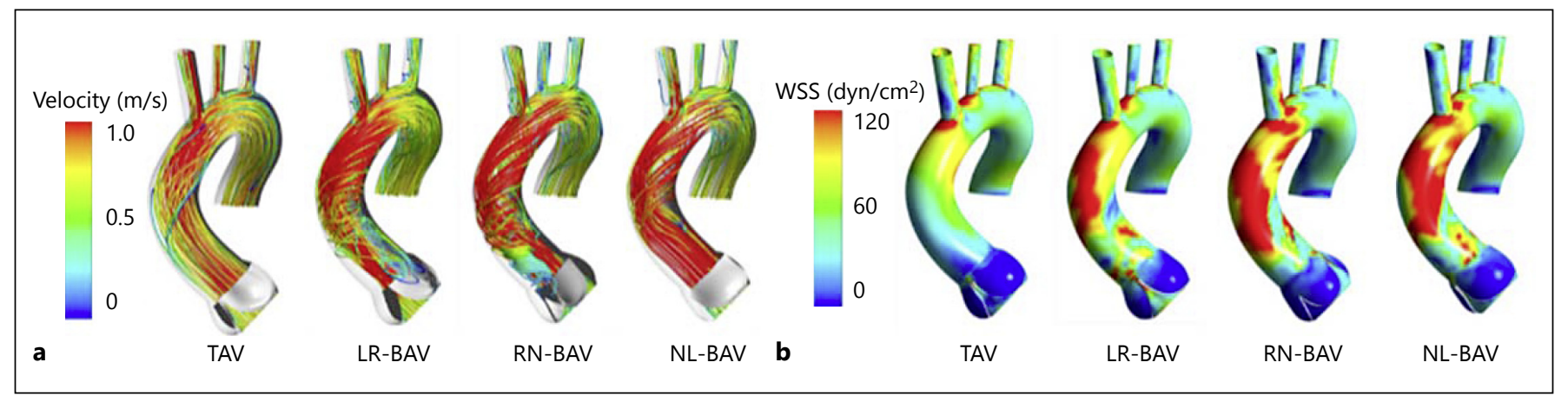

Fig. 1. Streamlines (a) and total WSS (b) fields at peak systole (for Abstract of Kai Cao).

tential contributor to BAV aortopathy, the initial impact (i.e., predilation) of the BAV anatomy on ascending aorta (AA) hemodynamic stresses remains largely unknown. The objective of this study is to quantify computationally the initial impact of different type-I BAV morphotypes (i.e., left-right (LR), right-non (RN), non-left (NL) coronary cusp fusion) on AA hemodynamics and wall shear stresses (WSS) using three-dimensional (3D) unified valve-aorta fluid-structure interaction (FSI) models. Methods: 3D unified anatomies of TAV, LR-BAV, RN-BAV and NL-BAV along with non-dilated AAs were created in SolidWorks based on published human aortic valve and aorta data. Unsteady FSI simulations were performed using an arbitrary Lagrangian-Eulerian approach in the commercial software ANSYS 15.0. Aortic flow was simulated by imposing a ventricular pressure waveform at the aortic sinus and aortic pressure waveforms at the aorta outlets. Results: The AA streamlines and WSS distributions at peak systole are shown in fig. 1. In the TAV AA, the streamlines are essentially parallel to the aortic wall but a few peripheral streamlines wrap around the main flow. In contrast, all BAV AAs exhibit extensive right-handed helical streamlines, which deflect backward toward the valve. The intense flow helicity results in WSS overload in all BAV AAs (LRBAV AA: $50.2 \mathrm{dyn} / \mathrm{cm}^{2}$; RN-BAV AA: $56.6 \mathrm{dyn} / \mathrm{cm}^{2}$; NL-BAV AA: $\left.58.3 \mathrm{dyn} / \mathrm{cm}^{2}\right)$ relative to the TAV-AA $\left(39.7 \mathrm{dyn} / \mathrm{cm}^{2}\right)$. While those high WSS levels are localized to the distal segment of the LR-BAV AA, they diffuse over the entire wall of the RN-BAV and NL-BAV AAs. Conclusion: This computational study indicates the existence of strong hemodynamic abnormalities in type-I BAV AAs, their dependence on BAV morphotype and their colocalization with sites vulnerable to dilation. These results provide new insights into BAV flow and its possible role in secondary aortopathy.

\section{Matrix Stability and Composition of Cryopreserved and Decellularized Heart Valves}

\section{Karolina Theodoridis, Jannina Müller, Robert Ramm, Tobias Goecke, Sotiris Korossis, Igor Tudorache, Serghei Cebotari, Axel Haverich, Andres Hilfiker}

\section{Hannover Medical School, Division of Thoracic and Cardiovascular Surgery, Hannover, Germany}

Objective: For heart valve replacement therapy, non-fixed decellularized homogeneic heart valves seem to be grafts of choice. For an optimal patient-matched use a method for long-term storage is needed. Thus, we analyzed the impact of cryopreservation, either before or after decellularization, on the structure of porcine pulmonary valves (pPV). Methods: $\mathrm{pPVs}$ were either cryopreserved $\left(-150^{\circ} \mathrm{C}\right)$ and then decellularized $(0.5 \%$ TritonX-100 for 24 $\mathrm{h}$, followed by $0.5 \%$ SDS for $24 \mathrm{~h}$ ) (CD) or first decellularized and afterwards cryopreserved (DC). Material integrity and composition was analyzed histologically, by DNA-, collagen-, and GAGquantification and by uniaxial stress tests. Residual SDS in the matrix, which is potentially harmful for invasive cells, was quantified by a methylene blue assay. Decellularized cusps were tested for cytotoxicity with porcine arterial endothelial cells (paEC). CD and DC valves were compared to native $(\mathrm{N})$, native cryopreserved $(\mathrm{C})$, and fresh decellularized (D) valves. Results: Only D valves showed a significant increase in the E-modulus and the tensile strength. In all valves, decellularization resulted to a reduction of DNA and GAGs, while no effect was seen on the collagen-content. CD valves demonstrated a significantly higher DNA and a non-significant higher SDS content in comparison to DC valves. Despite residual SDS present in all decellularized valves, paECs were able to form a confluent layer on CD and DC cusps. Conclusions: Whether CD valves truly bear a potentially higher immunogenicity has to be tested, but cryopreserving decellularized grafts seems to be an appropriate method for long-term storage. 


\section{Tissue Engineered Heart Valves}

\section{Manipulating Actin Signaling in Valve Endothelial Cells with Varied Lacunarity Geometric Photopatterned Vascular Networks, An Outside-In Approach}

\section{Christopher A. Arevalos, Elisabeth Godfrey,} Kathryn Jane Grande-Allen

Rice University, Houston, TX, USA

Objective: The development of complex engineered tissues is currently limited by the ability to incorporate functional microvasculature to encourage survival and growth. This issue is particularly relevant to pediatric tissue engineered heart valves. Vascular networks of varying complexity can be designed within engineered tissues, but the amount of biological complexity necessary for proper biological functionality is unclear. Prior work has identified that the geometric lacunarity of a network of valve endothelial cells (VECs) in an in vitro angiogenesis model can be tuned by manipulating the activity of the actin regulator ROCK with small molecule inhibitors. To examine the fundamental relationship between network structure and VEC biology, this work cultured VECs atop varied lacunarity patterns using biomaterial patterning strategies, then assessed the expression of several angiogenesis related markers. Methods: Polyethylene glycol diacrylate hydrogels were patterned with RGDS into $15 \mu \mathrm{m}$ deep tubule networks using photolithography. VECs were then seeded on a vessel-mimicking pattern, a higher lacunarity hexagon-based pattern, or a lower lacunarity triangle-based pattern. After 24 hours of culture, expression of pMLC, pAKT, pMAPK, beta-catenin, VEGFR2, CD31, aSMA, NCadherin, VECadherin, and perlecan was investigated using immunocytochemistry. Changes in tubule circularity and actin alignment were quantified after F-actin staining. Results: Notable differences in several angiogenesis related markers were found as function of the pattern the cells were seeded on. VEC pMLC activity was lowest when seeded in the triangle pattern, while pMAPK was highest in the hexagon pattern. Actin organization and circularity was highest in the vessel mimicking tubules. No significant differences were apparent in the other markers. Conclusions: Controlling the geometric pattern of VEC networks mimicked changes in signaling previously observed in angiogenesis models with VECs. These results suggest a more biomimetic vessel pattern in a tissue engineered valve would stimulate a more functional endothelial response. Addition of further valve-specific elements will enable the creation of structured biomaterial scaffolds for application to pediatric TEHV development as well as models of calcific aortic valve disease, in which angiogenesis plays a role.

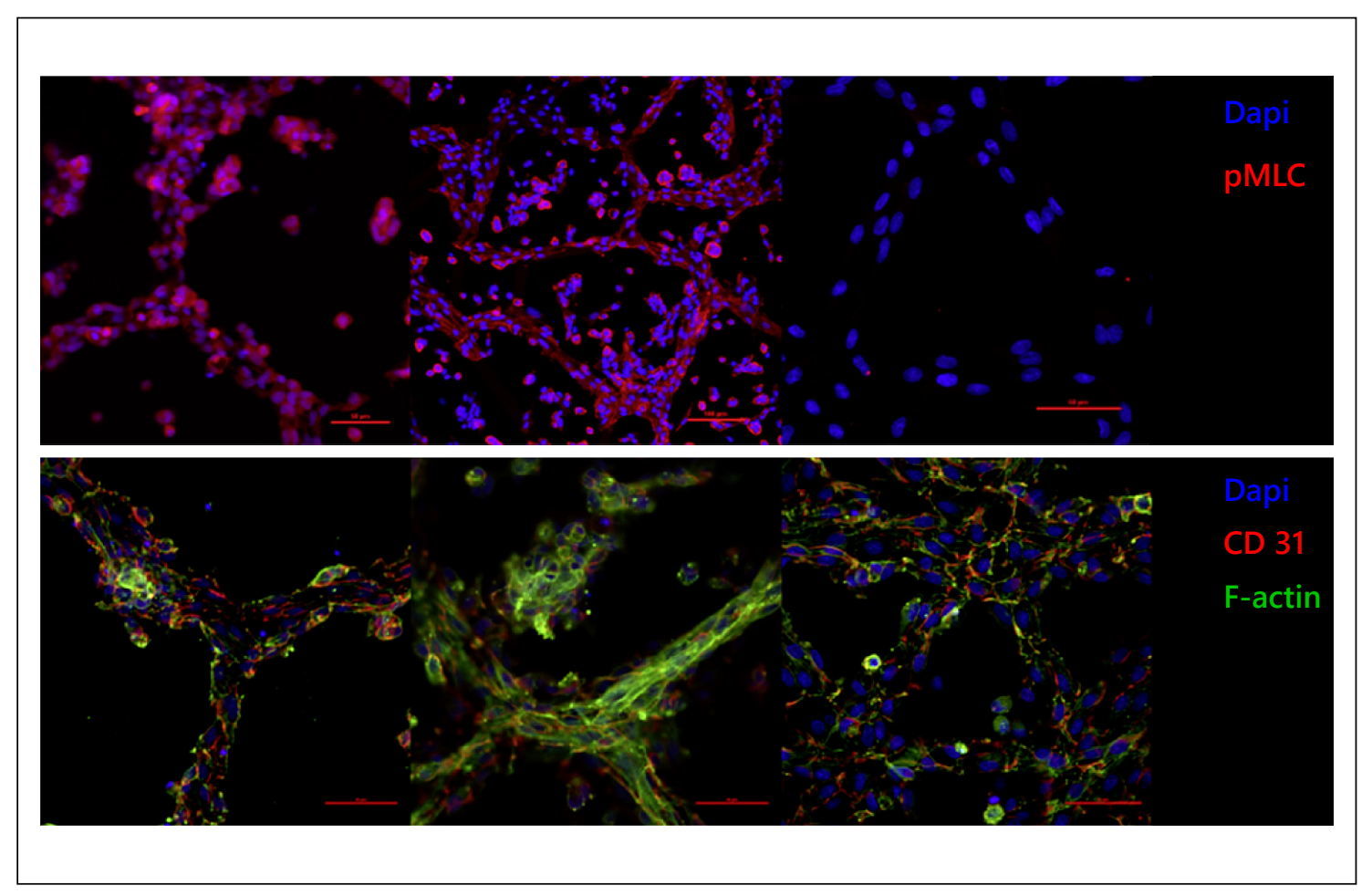

Fig. 1. (for Abstract of Christopher A. Arevalos). 


\section{Automated Bioreactors Used to Grow Tissue Engineered Heart Valves}

Leslie Sierad ${ }^{1}$, Nicolas Hanks' ${ }^{2}$, Allison Kennamer ${ }^{3}$, Nicholas Rierson ${ }^{3}$, Dan Simionescu ${ }^{3}$

${ }^{1}$ Aptus, LLC, Clemson, SC, ${ }^{2}$ Hanks Instrumentation, LLC, Pendleton, SC, ${ }^{3}$ Clemson University, Clemson, SC, USA

Objective: A tissue-engineered heart valve (TEHV) replacement could change the prognosis for the 60,000 children born with bicuspid aortic heart valve disease every year who will likely need a new heart valve as a child or young adult. Successful development of TEHVs depends on sufficient preconditioning, but current commercially-available bioreactor systems do not maintain sterile conditions or fail to mimic bodily conditions. A bioreactor is needed that can reach and maintain those conditions. Methods: A bioreactor was developed to reach physiologically-relevant conditions while maintaining sterility. Hardware and software was incorporated to provide automation of select conditions and challenged to test automated correction to set conditions. In experiments to develop a tissue-engineered aortic valve root (AVR), porcine AVRs were mounted into universal tissue-holding devices, decellularized, and repopulated with human adipose derived stem cells (ADSCs). The cell-seeded AVRs were subjected to progressive conditioning in rotating chambers and heart valve bioreactors until reaching pulmonary pressures. Results: In addition to physiological pulmonic and aortic conditions, the bioreactor system is capable of at least 150 beats per minute, systolic pressures over $200 \mathrm{~mm} \mathrm{Hg}$, flow rates over $100 \mathrm{~mL}$ per stroke, and adjustable media viscosity. With an internal volume of less than 1 liter and a footprint of less than $30 \times 13 \mathrm{~cm}$, four systems can independently operate inside one standard cell culture incubator and be monitored via webcam. Challenging the system by increasing constriction to raise pressure resulted in an automated adjustment back to the systolic pressure set point, but influenced diastolic pressure and stroke volume. ADSCs seeded within aortic cusps exhibited valvular interstitial cell markers after rotational preconditioning. Progressive bioreactor conditioning of valves up to pulmonary pressures facilitated roughly $25 \%$ cellular retention and increased spreading and alignment over static controls. Conclusions: To date, these patent-pending bioreactors are the only commercially available systems that simulate the human in vivo environment and meet rigorous consumer demands. By providing this equipment to researchers, developing clinical collaborations, and assisting with tissue-engineered product commercialization, we hope to achieve the vision of 'One Surgery, for Life' so children and young adults can have the extended life their bodies were designed to live.

\section{A Novel Autologous Heart Valve with Growth Potential}

Yoshiaki Takewa, Yasuhide Nakayama, Hirohito Sumikura, Noritsugu Naito, Eisuke Tatsumi

National Cerebral and Cardiovascular Center, Osaka, Japan

Objective: We are developing a novel autologous heart valve (Biovalve) with a unique in-body tissue engineering method. This may provide more biocompatible heart valves for the valvular disease. In this study, we evaluated the feasibility of them in a large animal experiment. Methods: We created many kinds and sizes of plastic molds for Biovalves with 3D printer easily and quickly, and embedded them in the subcutaneous spaces of adult goats for 2 months. After confirming encapsulation of membranous tissue around the molds, we extracted them with the tissue and removing the molds only, and finally got beautiful tri-leaflet valves made by completely autologous connective tissues and fibroblasts. We selected 3 types of Biovalve (a conventional type, a full-root type and a valve with a metallic stent for transcatheter implantation) in this study. Five cases of conventional Biovalves were implanted in the aorta under cardiopulmonary bypass, 8 cases of full-root type were implanted in the apico-aortic bypass, and 22 cases of stent valve type were implanted with transcatheter technique into in situ the aortic or pulmonary valve. Results: In each type, Biovalves were successfully implanted and showed smooth movement of the leaflets with a little regurgitation for maximum 12 months. Histological examination showed the autologous cells covering the laminar surface of the Biovalve leaflets and also getting into the connective tissues. Conclusions: The Biovalves have a potential to be used for valve surgery and satisfy the higher requirements of the systemic and pulmonary circulation maintaining the histological character as autologous tissues.

\section{Biovalve Type SC: in vivo, Tissue-Engineered, Autologous, 3D Heart Valvular Tissue. Mold Design, Excellent Mechanical Properties, Valular Functions, and in vivo Performances}

\author{
Yasuhide Nakayama, Yoshiaki Takewa, Hirohito Sumikura, \\ Takeshi Moriwaki, Maya Furukoshi, Marina Funayama, \\ Eisuke Tatsumi
}

National Cerebral and Cardiovascular Center, Osaka, Japan

Objective: Autologous pericardium is widely used for the reconstruction of intracardiac structures and pulmonary artery, and for aortic valve leaflets after fixation with glutaraldehyde for mechanical strengthening. We developed autologous prosthetic tissues by using in-body tissue architecture (IBTA) technology, which is a novel and practical approach of regenerative medicine based on the tissue encapsulation phenomenon of foreign materials in living bodies. In this study, IBTA-based heart valves with robust tissue wall [Biovalve (BV) Type SC] were prepared by using a novel caged mold. The mechanical properties of the valves were compared with human pericardium, and in vivo durability 
was evaluated in animal implantation study after in vitro evaluation using a pulsatile circulation circuit. Methods and Results: The BV preparation molds could be produced rapidly by using a $3 \mathrm{D}$ printing machine, based on the designed 3D-shape and size of native heart valves regardless of their type. Upon 1- or 2 -month goat subcutaneous embedding of the molds aortic or pulmonary valve-shaped BVs (diameter 5-30 $\mathrm{mm}$ ) were formed as type SC from completely autologous connective tissue with collagen and fibroblasts. All surfaces of the BVs that were in contact with blood were highly smooth and flat. The three leaflet tissues were seamlessly connected with the surrounding conduit tissue, and showed homogeneous wall thickness and excellent mechanical strength that was equivalent to or more than that of the human pericardium, with individual or regional differences. As an aortic valve, in vitro evaluation of the BV showed excellent valvular functions (regurgitation ratio $5.7 \%$ at 70 beats per minute). In a goat model, postoperative echocardiography after surgical replacement of the biovalves with stents showed smooth movement of the leaflets with little regurgitation under systemic circulation. In all implantation studies, the luminal surface after implantation was very smooth and fully covered with thin neointima, including endothelial cells without thrombus formation. Conclusions: Functional, completely autologous, robust, 3D aortic or pulmonary BVs showing the potential for clinical application were developed by in-body embedding of specially designed molds.

\section{Development and Revitalization of a Mitral Valve Scaffold for Tissue-Engineered Regeneration}

\author{
Chris deBorde ${ }^{1}$, Leslie Sierad ${ }^{1}$, Christopher Wright ${ }^{2}$, Jun Liao ${ }^{3}$, \\ Agneta Simionescu ${ }^{1}$
}

${ }^{1}$ Clemson University, Clemson, SC, ${ }^{2}$ Greenville Hosptial System, Greenville, SC, ${ }^{3}$ Mississippi State University, Starkville, MS, USA

Objective: Pathologies of the mitral valve are alarmingly common. Band annuloplasty and glutaraldehyde-fixed valves are current treatments, but their use if often associated with thromboembolic complications, calcification, and the risk of additional reoperations. A tissue engineered option is feasible and holds great potential. The aim of this study is to develop an acellular, noncytotoxic mitral valve scaffold, which can be recellularized with human adipose tissue-derived stem cells (ASCs). We will investigate how the scaffold's niche microstructure together with biomechanical and biochemical cues provided by bioreactor conditioning encourage the differentiation of seeded stem cells into valve specific cells. Methods: Porcine mitral valves were treated with detergent-based solutions to remove cells, while leaving a well-preserved extracellular matrix scaffolds. Scaffold characterization included biaxial mechanical testing of the annulus and leaflets, thermal denaturation evaluation, histological analysis, as well as resistance to collagenase and elastase. Scaffolds were treated with penta-galloyl glucose (PGG) for stabilization and injected with human adipose derived stem cells. The annulus and leaflet structures were also covered with ASCs. The constructs were mounted in a bioreactor for three weeks and their conditioning was analyzed using immunohistochem- istry. Results: Scaffold characterization results indicate a complete removal of cellular and nucleic acids, while maintaining extracellular matrix integrity and collagen and elastin microstructure were not lost (figure 1). Biaxial mechanical testing of the scaffold indicates that after treatment with PGG, mechanical properties of the scaffold were restored from decellularization. Internal and external seeding of our scaffolds were successful. A cytotoxicity assay indicates living cells after seeding indicating our scaffold as noncytotoxic. Our bioreactor allows for coaptation of the leaflets and chordae when physiological pressures and pulsatile flow were applied. Conclusions: Our PGG-treated mitral valve scaffolds are void of any xenogeneic cellular components and have ideal mechanical properties. We have retained basal lamina proteins essential for cellular attachment and preserved the integrity of our extracellular matrix. Our scaffold is noncytotoxic, as seeded cells were alive after three weeks in a bioreactor study.

\section{Tissue Engineered Tricuspid Valve Tube Graft: Histology and Biomechanics}

Diana M. Ropcke ${ }^{1}$, Jonas Rasmussen ${ }^{1}$, Christine Ilkjaer ${ }^{1}$, Soren N. Skov' ${ }^{1}$, Marcell J. Tjornild' ${ }^{1}$, Ulrik T. Baandrup', Carl Christian Danielsen ${ }^{3}$, Sten L. Nielsen ${ }^{1}$

${ }^{1}$ Aarhus University Hospital, Aarhus $\mathrm{N},{ }^{2}$ Aalborg University Hospital, Aalborg, ${ }^{3}$ Aarhus University, Aarhus C, Denmark

Objective: Tricuspid valve surgery using small intestinal submucosal porcine extracellular matrix (ECM) designed as a tube graft represents a promising alternative to conventional valve replacement. Histological examination and uni-axial tensile tests were carried out in valves before and six months after implantation in pigs. Methods: Twenty-five $60 \mathrm{~kg}$ pigs were operated and received a tricuspid valve tube graft. Five animals survived for the scheduled six months. Anterior and septal tricuspid leaflets were explanted. Endothelialization, collagen content, mineralization, neovascularization, burst strength and tensile strength were determined for native leaflets $(n=5), E C M$ material before implantation $(n=5)$, and ECM leaflets after six months $(n=5)$. Results: ECM leaflets had a significantly larger cross sectional area, both at implantation $\left(0.5 \pm 0.03 \mathrm{~mm}^{2}\right)$ and after six months $\left(4.9 \pm 5.4 \mathrm{~mm}^{2}\right)$ compared to native anterior leaflets $\left(0.6 \pm 0.1 \mathrm{~mm}^{2}\right), \mathrm{p}<0.05$. The collagen content was increasing over time and was significantly larger in ECM tissue compared to native leaflet tissue (native/ECM baseline/ECM six months: $0.08 \pm 0.04 \mathrm{mg} / \mathrm{mm}, 0.1 \pm 0.008 \mathrm{mg} / \mathrm{mm}, 0.6 \pm 0.7 \mathrm{mg} /$ $\mathrm{mm}), \mathrm{p}<0.05$. Maximum strength was higher in ECM than in native leaflets, but tended to reach native leaflet values over time after implantation (native/ECM baseline/ECM six months: $5.3 \pm 2.5 \mathrm{MPa}, 17.1 \pm 4.4 \mathrm{MPa}, 3.4 \pm 3.4 \mathrm{MPa}), \mathrm{p}>0.05$. Histologically, ECM valves showed endothelialization, host cell infiltration and structural collagen organization together with elastin generation after six months, indicating tissue engineering and gradual development of a close-to-native leaflet structure without foreign body response (fig. 1). Conclusion: ECM tricuspid tube grafts are stronger and thicker than native leaflet tissue. Over time, collagen amount increases. Maximum 


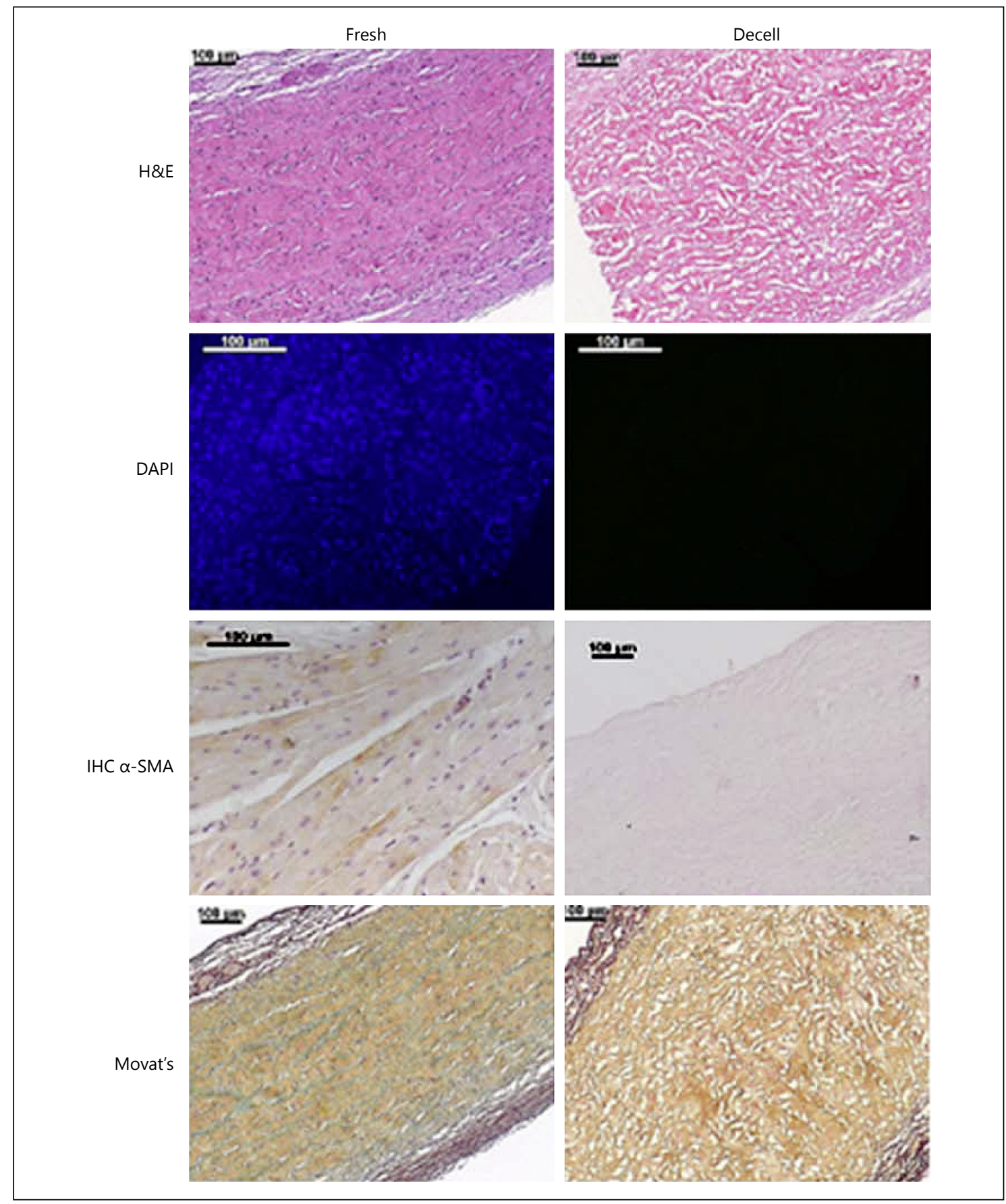

Fig. 1. An H\&E and DAPI stain were completed to show removal of cellular components. Immunohistochemistry for a-SMA, a common cellular protein, was used to show removal of proteins from the tissue. Lastly, a Movat's Pentachrome stain was used to show preservation of ECM integrity (for Abstract of Chris deBorde).

strength reaches levels comparable to native leaflets. Histologically, the acellular ECM tube grafts showed evidence of constructive tissue remodeling with endothelialization and connective tissue organization, leading to a close-to-normal leaflet appearance. 


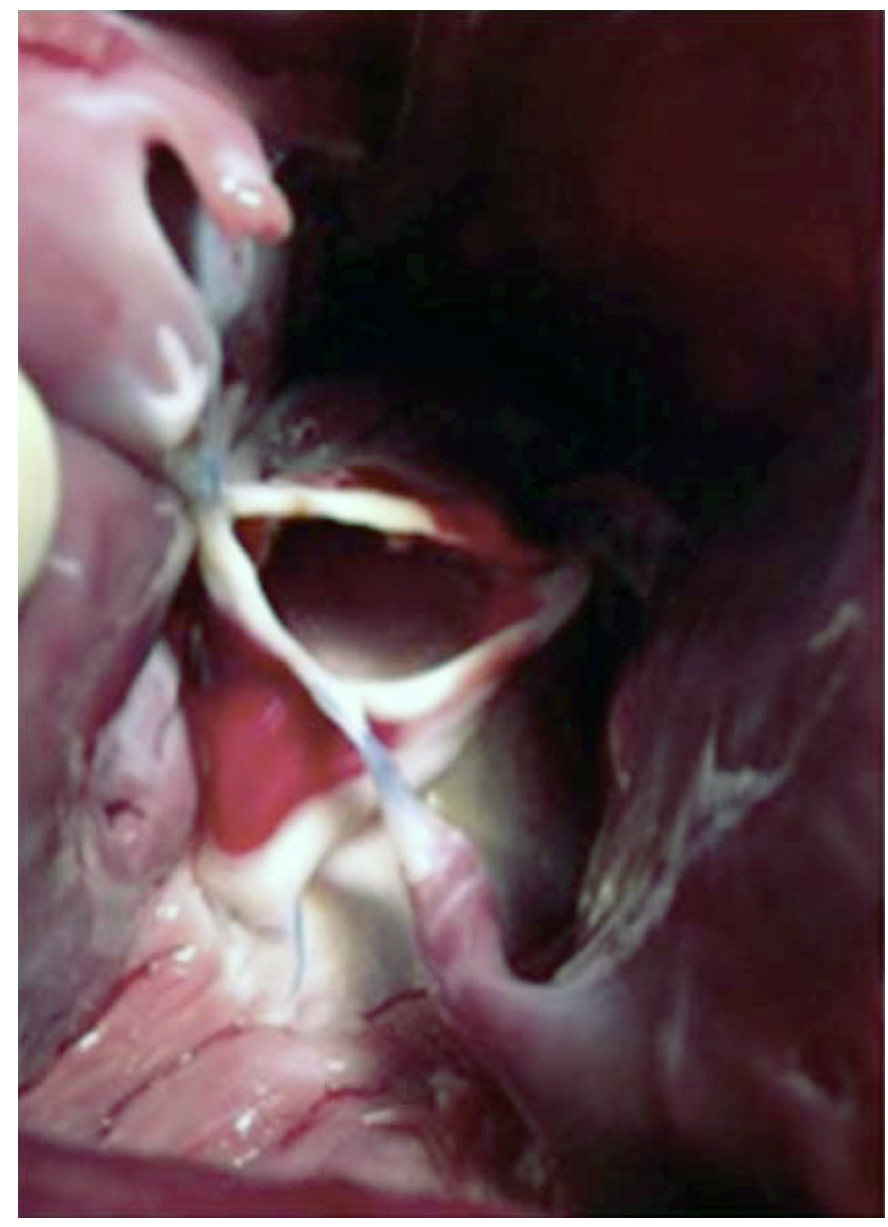

Fig. 1. ECM tricuspid valve from ventricular side after six months (for Abstract of Diana M. Ropcke).

\section{Heart Valve Imaging}

\section{Echocardiographic Follow-Up of Children with Valvular Lesions Secondary to Rheumatic Heart Disease: Data from a Prospective Registry}

\section{Anita Saxena, S. Ramakrishnan, S.K. Gupta, R. Juneja}

All India Institute of Medical Sciences, New Delhi, India

Background: Rheumatic heart disease (RHD) is estimated to affect more than 20 million people worldwide. Vast majority of those affected with RHD are children and adolescent from developing countries. Few studies have reported echocardiographic follow up of children affected by RHD. We report follow the cohort of children with RHD recruited in a prospective registry, using cross sectional and Doppler echocardiography. Methods: All consecutive children with RHD $<15$ years of age attending the outpatient or inpatient services of a tertiary referral hospital and recruited in a registry were included. Data on detailed clinical assessment and echocardiography was analyzed to look for change in severity of valve lesions on follow up. Results: A total of 374 children were recruited in the registry. 223 of these had follow up data and are included in this analysis. Their aged ranged from $5-15$ years (mean $11.8+2.9$ years), $64 \%$ were boys. Only $14 \%$ were from urban or semi-urban areas. $70 \%$ of children belonged to lower socio-economic strata. One fourth had advanced valvular lesions and presented in NYHA class III or IV. Echocardiography revealed mitral regurgitation (MR) in $85 \%$, it was severe in $58 \%$. Mitral stenosis (MS) with or without MR was present in 18\%. Aortic regurgitation (AR) was present in 90 (40\%), in $67(74 \%)$ of these, it was associated with MR or MR and MS. At a follow up of $11.9+5.9$ months, MR regressed in 22/164 patients (13.4\%), by one grade in 21 and by two grades in one. Out of 90 cases with AR, two showed regression, two progressed by one grade. MS progressed in one case. All but 6 patients were advised intramuscular long acting penicillin for secondary prophylaxis; these 6 patients were advised oral prophylaxis. Compliance with secondary prophylaxis improved from $63 \%$ at the time of recruitment to $92 \%$ at last follow up. $14 \%$ have had one or more events. Conclusion: RHD continues to be prevalent in children; most patients are from rural and poor background. With good secondary prophylaxis, valve lesions tend to remain stationary or improve over time as assessed by echocardiography.

\section{Functional Anatomy of the Mitral Valve and Left Ventricle in Ischemic Mitral Regurgitation Assessed with Multislice Computed Tomography}

\author{
Natalia Solowjowa ${ }^{1}$, Leyla Musayeva ${ }^{2}$, Yuriy Hrytsyna ${ }^{1}$, \\ Christoph Knosalla ${ }^{1}$, Volkmar Falk ${ }^{1}$ \\ ${ }^{1}$ Deutsches Herzzentrum Berlin, Berlin, Germany; ${ }^{2} \mathrm{~N}$. Tusi \\ Clinic, Baku, Azerbaijan
}

Objective: Functional mitral regurgitation (FMR) remains an important prognostic determinant in ischemic cardiomyopathy (ICMP). A differentiated surgical approach based on parameters of left ventricular (LV) and mitral valve (MV) remodeling is essential. We evaluated volumetric and geometric parameters of the MV-LV complex with multislice computed tomography (MSCT) in ICMP, and their relation to moderate and severe FMR. Methods: Between 09/09 and 06/15, 121 patients (m:w = 101:20, age 37-79, median 66.0 years; mean NYHA class 2.97 ) with echocardiographic LV ejection fraction (LVEF) $<40 \%$ underwent MSCT for LV functional assessment. Indexed left atrial and LV enddiastolic and endsystolic volumes (LAVI, LVEDVI, LVESVI), LV volumetric sphericity index (SI), LVEF, cardiac output (CO) and cardiac index (CI) were calculated and analyzed retrospectively. Regional wall motion abnormalities were assessed semiquantitatively. MV apparatus was characterized by intercommissural and anteroposterior $\mathrm{MV}$ annulus diameter (ICD, AMD), MV annulus area and posterior circumference (MVAA, MVAPC), coaptation distance, tenting area, anterior and posterior leaflet to annulus angle (CD, TA, ALA, PLA) in segments 1-3 and interpapillary muscle distance (IMD), mea- 
sured in midsystole. These parameters were compared in patients $(\mathrm{n}=87)$ with moderate MR $(<2.0)$ and patients $(\mathrm{n}=34)$ with severe $(\geq 2.0)$ MR. Results: The differences in LVEDVI, LVESVI and LVEF between the two groups were not significant, whereas LAVI and SI were significantly higher in patient with severe FMR (LAVI $73.3 \pm 24.8$ vs. $56.3 \pm 17.1 \mathrm{ml} / \mathrm{sqm}, \mathrm{p}=0.001$; SI $0.48 \pm 0.1$ vs. $0.42 \pm 0.09, \mathrm{p}=0.009)$. Patients with severe FMR showed significantly longer CD (CD A2-P2 $10.4 \pm 2.3$ vs.8.6 $\pm 2.0 \mathrm{~mm}, \mathrm{p}=$ 0.000; CD A3-P3 $8.7 \pm 2.3$ vs.7.6 $\pm 2.0 \mathrm{~mm}, \mathrm{p}=0.011$ ) and larger TA (TA A2-P2 $2.26 \pm 0.9$ vs. $1.64 \pm 0.6 \mathrm{sqcm}, \mathrm{p}=0.001$ ), demonstrating more advanced MV remodeling. Motion abnormalities of posterior papillary muscle and posterior load bearing LV wall were found significantly more often $(p=0.004)$ in severe FMR. The differences in MV diameter, circumference and area, IMD and segmental leaflet to annulus angles did not reach significance. Conclusions: MSCT represents an appropriate tool for evaluation of FMR. Our data show more pronounced tethering of the mitral leaflets at the central and posteromedial segments, higher prevalence of motion abnormalities of posterior papillary muscle and more advanced LA and LV remodeling in patients with severe FMR.

\section{Assessment of Left Ventricular Mechanics in Mitral Valve Prolapse}

Maxime Poilane, Caroline Cueff, Antoine Jobbe-Duval, Aurélie Dussauge, Philippe Jaafar, Damien Guijarro, Nicolas Piriou, Jean Noel Trochu, Jean-Christian Roussel, Thierry Le Tourneau

Institut du Thorax, Nantes, France

Objective: Valve apparatus alterations may influence left ventricular (LV) mechanics in mitral valve prolapse (MVP). To assess LV mechanics and ventriculo-valvular interaction in MVP. Methods: We prospectively enrolled patients with MVP (leaflet displacement $>2 \mathrm{~mm}$ above the annulus) and controls without MVP. A comprehensive echocardiography was carried out with 2D speckle tracking myocardial longitudinal strain assessment, $2 \mathrm{D}$ mitral valve apparatus analysis, mitral regurgitation (MR) quantification, ventriculo-atrial disjunction and posterior wall bulging assessment. Global and regional longitudinal strain were compared between the two groups and the determinants of myocardial deformation alteration were sought. Results: 194 adult patients with MVP and 30 matched controls were included. In MVP group MR was not significant in 76 (39\%) patients (NoMRMVP, effective regurgitant orifice $<10 \mathrm{~mm}^{2}$ ), mild to moderate in $53(27 \%)$ and severe in $65(34 \%)$ patients. Posterior (PML) and/ or anterior (AML) mitral leaflet prolapse were present in 188 (93\%) and 85 (42\%) patients, respectively. PML and AML positions averaged $-6.0 \pm 3.4 \mathrm{~mm}$ and $-1.6 \pm 3.5 \mathrm{~mm}$, respectively. Ventriculo-atrial disjunction was found in $82(41 \%)$ patients and averaged $5.7 \pm 1.6 \mathrm{~mm}$. Posterior wall thickening was clearly increased in MVP groups with frank wall bulging in 82 patients (41\%). Despite the third left chamber (between mitral annulus and prolapsed leaflets) forward stroke volume was preserved in MVP groups, and even increased in NoMR-MVP $\left(44 \pm 9 \mathrm{ml} / \mathrm{m}^{2}\right.$ vs. $39 \pm 9 \mathrm{ml} / \mathrm{m}^{2}$ in Controls, $\left.\mathrm{p}<0.001\right)$. In addition LV EF was improved in NoMR-MVP ( $64 \pm 7 \%$ vs. $61 \pm 6 \%$, P = 0.01). Global Longitudinal Strain (GLS) was also improved in NoMR-MVP $(-21.5 \pm 2.8 \%)$ and in the overall MVP group $(-21.7 \pm 3.0 \%)$ compared with Controls $(-19.7 \pm 1.7 \%$, both $\mathrm{P}<0.001)$. In multivariate analysis GLS improvement was associated with the magnitude of PML prolapse $(\beta=0.14, \mathrm{P}=0.04)$, posterior wall thickening $(\beta=-0.16, P=0.004)$, posterior papillary muscle displacement in systole $(\beta=-0.21, P=0.002)$ and posterior wall bulging $(\beta=$ $-0.23, \mathrm{P}<0.001)$. Conclusions: MVP and associated abnormalities have a significant impact on LV mechanics. LV systolic function is improved in MVP without MR as assessed by EF and GLS and preserves forward stroke volume. These changes might have deleterious effect at long-term on LV myocardium.

\section{Deformation Imaging and Long Term Outcome after Mitral Valve Reconstruction: Preoperative Longitudinal Strain Rate Is a Strong Independent Predictor of Cardiac Related Morbidity and Mortality}

\author{
Aleksandra Miskovic ${ }^{1}$, Tudor Poerner ${ }^{2}$, Björn Göbel ${ }^{3}$, \\ Anton Moritz ${ }^{1}$ \\ ${ }^{1}$ University Hospital Frankfurt, Frankfurt am Main, ${ }^{2}$ University \\ Hospital Jena, Department of Internal Medicine I, Jena, \\ ${ }^{3}$ University Hospital Jena, Department of Internal Medicine I, \\ Jena, Germany
}

Objective: Strain rate has been shown to correlate very well with contractility and the assessment of left ventricular systolic function is one of the most important predictive factors for postoperative survival in mitral valve surgery. In this prospective cohort analysis, we studied the association between preoperative echocardiographic LV measures including longitudinal deformation parameters Strain(S) and peak Strain Rate (pSR) and occurrence of LV dysfunction or cardiac related death after mitral valve repair. Methods: Between 2002 and 2004, 95 consecutive patients (mean age $63 \pm 13 \mathrm{y}$ ) underwent MVR for severe mitral regurgitation in our institution. Echocardiography and tissue Doppler derived strain rate imaging (SRI) was performed preoperatively and 7 days after surgery. Off-line analysis in SRI included measurements of peak systolic Strain(S) and peak Strain Rate (pSR), calculated for a 16 segment model and expressed as mean values for the whole LV. Cardiac events and LV ejection fraction (EF) were recorded during a median follow-up of 10.5 years (5.2-11.6). Results: 21 patients died, four patients were lost to follow up. Cardiac events were defined as cardiac related or sudden death, incidence of LV dysfunction or decompensation. Echocardiographic measurements included the following conventional and deformation parameters: LV enddiastolic diameter $60.5 \pm 8.2(\mathrm{~mm}) ; \mathrm{LV}$ endsystolic diameter $41.3 \pm 9.4$ $(\mathrm{mm})$, LA diam. $54.6 \pm 10.4(\mathrm{~mm})$, EF $56.3 \pm 14.3 \%$, pSR $\left(-^{1}\right)$ $-1.19 \pm 0.29$, pS $(\%)-18 \pm 0.05$. After adjustment for important clinical and echocardiographic variables at multivariate Cox regression analysis pSR emerged as a strong independent predictor of cardiac events $(\mathrm{p}<0.0001)$. ROC analysis of $\mathrm{pSR}$ provided a cut-off of $-1.26\left(\mathrm{~s}^{-1}\right)$ for developing cardiac morbidity and mortality with $97 \%$ sensitivity and $70 \%$ specificity (AUC $0.86 \mathrm{p}<$ 0.001, CI 0.77-0.92). The Hazard Ratio for major cardiac events 
with low global Strain Rate (<-1.2) was 8.4 (95\% CI: 4.02-17.05, $\mathrm{p}<0.001$. Conclusion: The association between global longitudinal strain rate and long term cardiac outcome after MVR was independent of existing risk stratification variables validated by guidelines. A low pSR $(<-1.26)$ was associate to significantly higher risk of cardiac events during long term follow up, indicating a possible threshold for early operation in patients with uncertain or absent symptoms.

\section{Relationship between Aortic Valve Calcification and Hemodynamic Severity of Aortic Stenosis: Effect of Aortic Valve Phenotype and Age - Progressa Study}

Mylène Shen ${ }^{1}$, Lionel Tastet ${ }^{1}$, Romain Capoulade ${ }^{1}$, Éric Larose ${ }^{1}$ Élisabeth Bédard ${ }^{1}$, Marie Arsenault ${ }^{1}$, Philippe Chetaille ${ }^{2}$, Jean-Gaston Dumesnil ${ }^{1}$, Patrick Mathieu ${ }^{1}$, Marie-Annick Clavel ${ }^{1}$, Philippe Pibarot ${ }^{1}$

${ }^{1}$ Quebec Heart and Lung Institute, Quebec City, QC, ${ }^{2} \mathrm{CHUQ}$, CHUL, Centre Mère-Enfant Soleil, Quebec City, QC, Canada

Background: Aortic valve calcification (AVC) measured by computed tomography (CT) is useful to assess actual severity (anatomic severity) of AS in patients with discordant hemodynamic parameters or uncertain grading at echocardiography. However, impact of aortic valve phenotype (tricuspid vs. bicuspid) and age on the relationship between AVC and hemodynamic severity remains unknown. We hypothesized that the relationship between AVC and hemodynamic severity of AS is influenced and modulated by aortic valve phenotype and age. Methods: 200 patients with AS and preserved LVEF underwent a comprehensive Doppler-echocardiography and CT in the context of the prospective PROGRESSA study. Mean transvalvular gradient (MG) measured by Doppler was used to assess AS hemodynamic severity. AVC was determined by the Agatston method and indexed to the LV outflow tract area to obtain AVC density (AVCd). AVCd was used to assess AS anatomic severity. Since it has been previously shown that relationship between AVC and hemodynamic sever- ity is influenced by sex, statistical analyses were all stratified according to sex. Results: 39 patients had a bicuspid aortic valve (BAV). The correlation between MG and AVCd was modest $(\mathrm{r}=$ $0.44, \mathrm{p}<0.0001)$ in the whole cohort. Analyzed by valve phenotype, correlation was good between AVCd and MG in the TAV group $(\mathrm{r}=0.63, \mathrm{p}<0.0001)$ but non-significant in the BAV group $(\mathrm{r}=0.24, \mathrm{p}=0.14)$. Median age was 51 and 72 years for BAV and TAV patients respectively. In the TAV group, the strength of the AVCd-MG correlation was similar in younger $(<72$ y.o.; $r=0.63$, $\mathrm{p}<0.0001)$ vs. older $(\geq 72$ y.o.; $\mathrm{r}=0.60, \mathrm{p}<0.0001)$ patients. In the BAV group, there was no correlation between AVCd and MG in the younger patients $(<51$ y.o.; $r=-0.005, p=0.99)$, whereas there was a strong correlation in the older patients $(\geq 51$ y.o.; $r=$ $0.67, p=0.0009)$. Conclusions: AVC appears to be the main factor determining the hemodynamic severity of AS in TAV and in older BAV patients. Hence in these patients, AVC or AVCd measured by CT is a reliable parameter to corroborate AS severity in patients who have uncertain or discordant findings at echocardiography. However, among younger BAV patients, some may have a hemodynamically significant stenosis with no/minimal AVC.

\section{Systolic Hypertension and Discrepancies in the Progression of Calcific Valve Stenosis - Results from the PROGRESSA Study}

\section{Lionel L. Tastet, Romain Capoulade, Marie-Annick Clavel, Éric Larose, Mylène Shen, Abdellaziz Dahou, Marie Arsenault, Patrick Mathieu, Élisabeth Bédard, Jean G. Dumesnil, Alexe Tremblay, Yohan Bossé, Jean-Pierre Després, Philippe Pibarot \\ Institut Universitaire de Cardiologie et Pneumologie de Québec, Québec, QC, Canada}

Objective: Systolic hypertension (SHPT, i.e. systolic blood pressure $\geq 140 \mathrm{~mm} \mathrm{Hg}$ ) is one of the most frequent comorbidities encountered in patients with aortic stenosis (AS). This prospec-

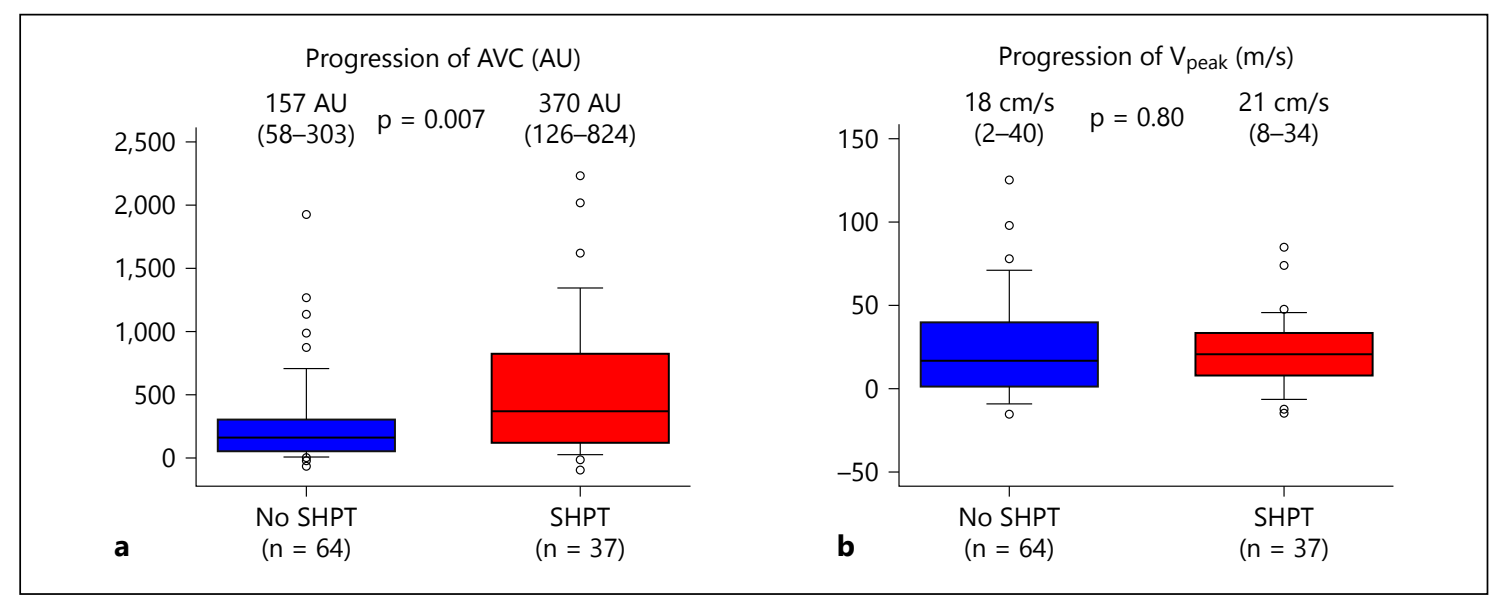

Fig. 1. (for Abstract of Lionel L. Tastet). 
tive study sought to examine the impact of SHPT both on the progression of aortic valve calcification (AVC) and hemodynamic progression of AS. Methods: One hundred and one patients with AS were prospectively recruited in the PROGRESSA study. AVC progression was measured by multidetector computed tomography using the Agatston method and hemodynamic progression was assessed by increase in peak aortic jet velocity (Vpeak) measured by Doppler Echocardiography, both at baseline and at 2 -year follow-up. Results: The mean age was $65 \pm 13$ years and $74 \%$ were male. During the 2 -year follow-up, patients with SHPT $(\mathrm{n}=37)$ at baseline had faster progression of AVC compared to those without SHPT (AVC: +370[126-824] vs. +157[58-303] AU; $\mathrm{p}=0.007$, figure $1 \mathrm{a})$. In contrast, there was no significant difference in hemodynamic progression between two groups (Vpeak: $+21[8-34]$ vs. $+18[2-40] \mathrm{cm} / \mathrm{s} ; \mathrm{p}=0.80$, figure $1 \mathrm{~b})$. In multivariable analysis adjusted for age, sex, dyslipidemia, diabetes, metabolic syndrome, antihypertensive medication, creatinine level, baseline Vpeak and baseline AVC, SHPT remained significantly associated with faster progression of AVC $(\mathrm{p}=0.001)$. Interestingly, there was a significant interaction between SHPT and AVC progression on hemodynamic AS progression (pinteraction $<0.0001$ ). In patients with SHPT, AVC progression was not associated with Vpeak progression $(\mathrm{p}=0.35)$, whereas in those without SHPT, there was a significant association $(\mathrm{p}<0.0001)$. Conclusions: This prospective study shows that SHPT is a powerful and independent predictor of faster progression of AVC but not of hemodynamic progression in AS patients. These findings suggest that hemodynamic progression of AS may be underestimated in presence of SHPT. Future clinical trials are needed to assess the efficacy of antihypertensive treatment on the progression of valve stenosis.

\section{Mitral Butterfly First in vitro Tests - One Step Ahead in Percutaneous Mitral Interventions}

\author{
Birgit Opitz ${ }^{1}$, Marie Elisabeth Stelzmueller ${ }^{1}$, Wilfried Wisser ${ }^{1}$, \\ Werner Reichenfelser ${ }^{2}$, Carla Marotta ${ }^{3}$, Denisa Muraru', \\ Luigi Paolo Badano ${ }^{3}$, Werner Mohl ${ }^{1}$ \\ ${ }^{1}$ Medical University Vienna/Department of Cardiac Surgery, \\ Vienna, ${ }^{2}$ Technical University Vienna, Vienna, Austria; \\ ${ }^{3}$ University of Padua/Department of Cardiac, Thoracic and \\ Vascular Sciences, Padua, Italy
}

Background: The emerging trends in mitral valve (MV) disease towards minimal-invasive treatment options demand sophisticated analysis and sound comprehension of the valve's pathophysiology for development of feasible treatment options. We aim to deliver a novel transcatheter approach in mitral regurgitation (MR) correcting malcoaptation on basis of personalized 3D transesophageal echocardiography (TEE) having the ambition to achieve the best possible outcome for patients. Methods: In this procedure a device for the posterior mitral leaflet (PML) is developed via 3D TEE analysis. Since most of the pathologies in MR result from PML, the wing-like structure slides over this vey leaflet while leaving the anterior mitral leaflet (AML) unaffected in both structure and function allowing normal leaflet motion and thus re-establishing a sufficient closing plane by posterior leaflet extension. Device construction is done through assessment of the subvalvular apparatus, determination of point of coaptation and most importantly, exact definition of the annulus. Additionally, optimal length of artificial chords is measured in order to prevent prolapse. Implantation is done transapically and may be extended to a transseptal and transaortal approach. The widget is out of a semi-flexible material squeezing the three cover parts of the diseased leaflet in between resembling the natural subdomains known as P1, P2, $\mathrm{P} 3$. This cover is anchored by device parts pressing against mitral annulus and interacting with a standoff pressing from the ventricular aspect of the leaflet. Results: Prototypes of 73 patients' mea-

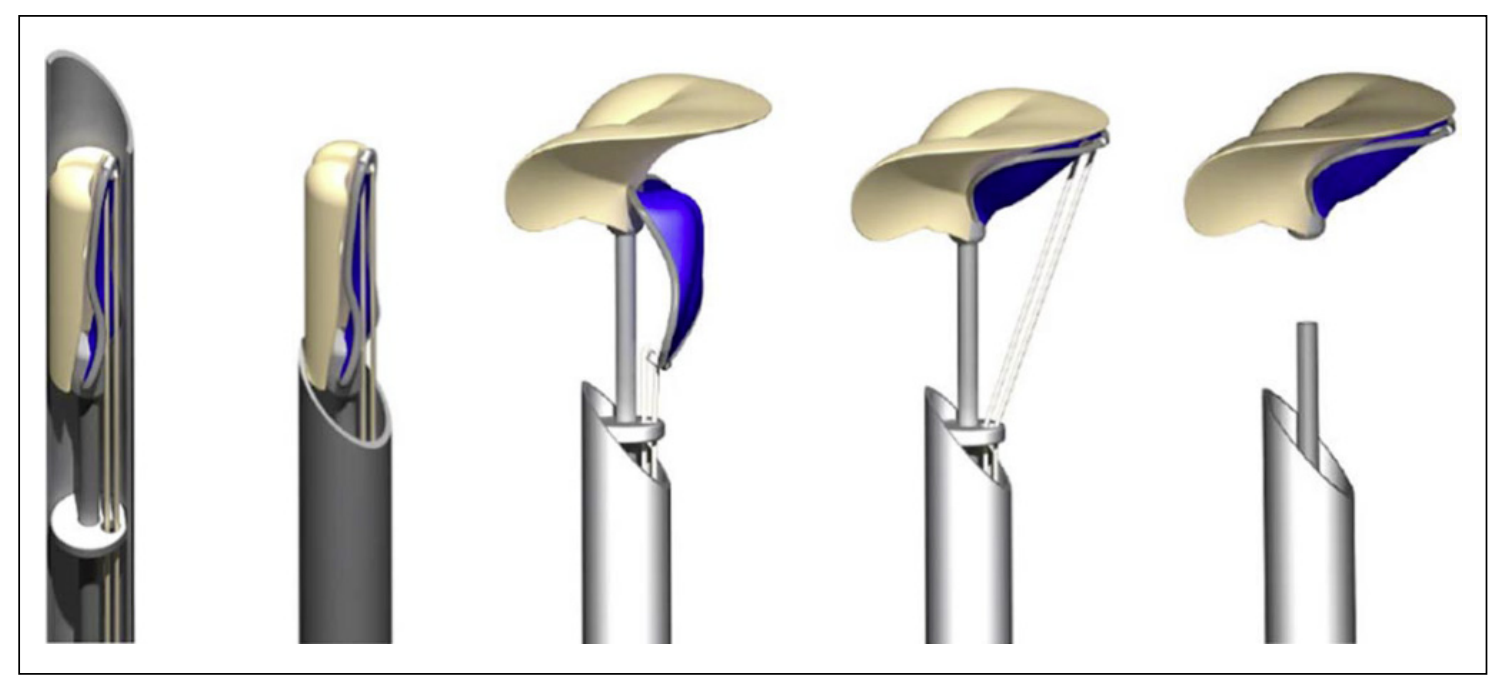

Fig. 1. (for Abstract of Birgit Opitz). 
surements are being developed. Device parts correct closure plane individually by $3 \mathrm{D}$ quantitation of MV. First in vitro testing shows the feasibility of the device. Conclusions: To conclude, 3D TEE adds substantial information in designing and guiding MV interventions as it improves visualization of function and disease localization. At present, the concept is in the prototype phase. Further aspects of device construction have to be considered and amendment of the semi-automatic software seems to be necessary.

\section{Heart Valve Outcomes}

\section{Gender Differences in the Progression of Aortic Valve Stenosis-Prognostic Implication}

\author{
Virginia Nguyen, Tiffany Mathieu, Maria Melissopoulou, \\ Claire Cimadevilla, Isabelle Codogno, Virginie Huart, \\ Xavier Duval, Alec Vahanian, David Messika-Zeitoun \\ Bichat Hospital, Paris, France
}

Objective: Female displays a lower degree of aortic valve calcification (AVC) than male for the same hemodynamic severity of valve stenosis (AS) and AS progresses faster with increasing baseline severity. However, the impact of gender on AS progression and outcome has not been specifically evaluated. Methods: In 203 patients with at least 2 years of follow-up (96 mild AS, 89 moderate AS and 18 severe AS), enrolled in a prospective cohort, we evaluated at baseline and yearly thereafter AS hemodynamic severity using echocardiography (mean pressure gradient (MPG)) and degree of AVC using computed tomography. The occurrence of AS related events (sudden death, congestive heart failure, or new onset of symptoms) was prospectively recorded. Results: After a mean follow-up of $3.2 \pm 1.2$ years, mean MGP increased from $23 \pm 11$ to $33 \pm 17 \mathrm{~mm}$ $\mathrm{Hg}(+3 \pm 4 \mathrm{~mm} \mathrm{Hg} /$ year $)$ and mean AVC from $1168 \pm 984$ to $1852 \pm$ $1502 \mathrm{AU}(+218 \pm 226 \mathrm{AU} /$ year). Baseline AS hemodynamic severity was similar in female and in male ( $23 \pm 13$ vs. $23 \pm 11 \mathrm{~mm} \mathrm{Hg}, \mathrm{p}=$ 0.29 ) but hemodynamic progression was higher in female ( $5 \pm 5$ vs. $3 \pm 3 \mathrm{~mm} \mathrm{Hg}, \mathrm{p}=0.04)$ and in multivariate analysis, after adjustment for age, valve anatomy and baseline MPG, gender was an independent predictor of hemodynamic progression $(\mathrm{p}=0.0005)$. AVC progression was not different between female and male (228 \pm 307 vs. $215 \pm 193$ AU/year, $\mathrm{p}=0.30$ ) but baseline AVC was significantly lower in female $(906 \pm 989$ vs. $1256 \pm 969 \mathrm{AU}, \mathrm{p}=0.0002)$ and in multivariate analysis after adjustment for age, valve anatomy and baseline AVC, gender was also an independent predictor of AVC progression $(\mathrm{p}=0.002)$. Female presented also with a lower eventfree survival than male $(56 \%$ vs. $77 \%$ at 5 years, $p=0.03)$ and in multivariate analysis, gender was an independent predictor of outcome $(p=0.007)$. Conclusion: Degree of AVC is closely related to hemodynamic severity but female presented lower AVC load than male for similar AS hemodynamic severity. In addition to baseline severity, gender was an independently associated with both anatomic and hemodynamic progression which translated into a higher ASrelated events rate. These results strongly suggest that AS pathophysiology may be different in female and in male and that a closer follow-up may be advised in female.

\section{Is the Euroscore II Suited to Predict Mortality after Surgical Aortic Valve Replacement in Octogenarians?}

Thomas Senage, Thierry Le Tourneau, Caroline Cueff, Magali Michel, Bertrand Rozec, Christian Périgaud, Antoine Mugniot, Roussel Jean-Christian

Nantes University Hospital, St-Herblain, France

Objective: Since 2011, the Euroscore II is available and can be used to predict mortality after cardiac surgery. The aim of this study was to assess the current hospital mortality rates and to validate this score in a large monocentric cohort of octogenarian patients operated for aortic valve replacement (AVR). Methods: Between January 2002 and December 2014, 1240 consecutives octogenarian patients ( 573 females/667 males) with a mean age of $82.6 \pm 2.3$ years [ $80-94$ ] underwent surgical isolated AVR ( $\mathrm{n}=769-62.0 \%)$ or combined procedure (AVR + Coronary Artery Bypass Grafting CABG: $\mathrm{n}=$ 471-38.0\%) in the same center. Global mean Log Euroscore and Euroscore II were respectively $10.5 \pm 6.5 \%$ [5.5\%-67.9\%] and $3.4 \pm$ $4.0 \%$ [0.9\%-51.4\%]. Results: The hospital mortality rate was $2.3 \%$ $(\mathrm{n}=28)$. Median ICU stay was 2 days [Min-Max: 0-60] for a median hospital length of stay of 12 days [Min-Max: 6-112]. In multivariate analysis, the independent risk factors for hospital mortality were preoperative cardiogenic shock $(\mathrm{OR}=12.60$; $95 \%$ CI $[3.71-$ $37.21])$ and cardiopulmonary bypass time (OR $=1.02 ; 95 \% \mathrm{CI}$ [1.01-1.03]). Associated bypass grafting was not associated with a significant increase of mortality (hospital mortality of $2.0 \%(\mathrm{n}=15)$ and $2.8 \%(\mathrm{n}=13)$ for isolated and combined AVR respectively). Neither the Logistic Euroscore nor the new Euroscore II were correlated with mortality, with poor discriminative powers (Areas under the ROC curve of $0.6095 \%$ CI [0.57-0.62] and $0.5895 \%$ CI [0.55-0.61] respectively). Conclusions: Isolated AVR in octogenarians is currently safe and associated with a very low mortality rate. Associated CABG can safely be performed in selected octogenarian patients. Although closer to the observed mortality, the new Euroscore II does not allow to predict the individual risk of death of octogenarian patients requiring AVR.

\section{Long Term Predictors of Recurrent Mitral Regurgitation after Mitral Valve Plasty Evaluated by Three Dimensional Transesophageal Echocardiography}

Keitaro Mahara, Haruka Fujimaki, Syuichiro Takanashi

Sakakibara Heart Institute, Tokyo, Japan

Objective: We sought to determine the prognostic factors of recurrent mitral regurgitation (MR) during long term follow up after mitral valve plasty (MVP) by three dimensional transesophageal echocardiography (3DTEE). Methods: We investigated consecutive 339 patients (age $60 \pm 15,215$ male) who scheduled MVP for mitral valve prolapse from January 2012 to July 2014. Eight patients who were converted to mitral valve replacement and four patients who experienced recurrent MR caused by infective endo- 
carditis $(n=3)$ or ring detachment $(n=1)$ were excluded. Of these 327 patients, 207 patients who underwent MVP for A2 or P2 prolapse were enrolled. We performed transthoracic echocardiography (TTE) and 3DTEE before and within 2 weeks after surgery. TTE was also undergone 6 months, 1 year after surgery and annually after 1 year. We defined recurrent MR as more than or equal to moderate regurgitation by TTE. Results: During a mean followup of $17.7 \pm 9.9$ months after MVP, 11 patients (5.2\%) experienced recurrent MR and 6 patients $(2.8 \%)$ needed reoperation. Ten out of 11 patients had less than moderate degree of MR before discharge. TTE parameters before surgery including left ventricular ejection fraction, left ventricular end systolic and diastolic diameters, and severity of MR (effective orifice area, regurgitant volume, regurgitant fraction) were similar in with or without recurrent MR. The preoperative 3DTEE parameters including A2 height, P2 height and mitral annulus circumference, mitral valve annulus sphericity were similar in with or without recurrent MR. Three dimensional TEE after surgery revealed that the coaptation heights at repaired lesions in patients with recurrent MR were significantly shorter than those without recurrent MR. (5.1 \pm 1.6 vs. $7.3 \pm 1.6 \mathrm{~mm}, \mathrm{p}<0.01, \mathrm{n}=11,196)$. Conclusions: Coaptation heights measured by postoperative 3DTEE can predict the tendency of recurrent MR. When postoperative TEE revealed short coaptation length, the patients need to receive a careful follow up, even though there is no significant recurrent MR before discharge.

\section{Prognostic Value of Exercise-Stress Echocardiography in Asymptomatic Patients with Aortic Valve Stenosis}

\author{
Goublaire Coppelia, Jr., Melissopoulou Maria, Jr., \\ Nguyen Virginia, Brochet Eric, Cimadevilla Claire, \\ Codogno Isabelle, Vahanian Alec, Messika-Zeitoun David \\ Bichat Hospital, Paris, France
}

Aims: The usefulness of exercise-stress echocardiography for the evaluation of asymptomatic patients with aortic stenosis (AS) is not well defined. The value of exercise-induced mean pressure gradient (MPG) increase for predicting the occurrence of symptoms is still debated. The purpose of this study was to evaluate the additional prognostic value of exercise-stress echocardiography in predicting the occurrence of symptoms in patients having a normal standard exercise test. Methods: In this observational retrospective study, asymptomatic patients with moderate or severe AS and $\mathrm{LVEF}>50 \%$, for whom an exercise-stress echocardiography was performed, were included. The clinical and echocardiographic data at rest and at peak exercise were collected. The composite primary outcome variable was the occurrence of symptoms or heart failure related to the AS or death from cardiovascular causes in the follow-up period. Results: Among the 148 included patients, 36 (24\%) underwent an aortic valve replacement after the exercise test. Among the 112 patients with a normal exercise test, 30 (28\%) reached a clinical endpoint during follow-up, with a mean followup time of $14.3 \pm 7.9$ months. On multivariate analysis, MPG at rest $(\mathrm{p}=0.001$; HR 1.061 [1.025-1.097]) was the only parameter associated with clinical outcome. Exercise-induced MPG increase ( $\mathrm{p}=0.273$; HR 0.974 [0.93-1.021] was not correlated with the clin- ical outcome. Conclusion: Exercise-induced MPG increase did not improve the risk stratification in our study. Thus, our results do not confirm the prognostic value of exercise-stress echocardiography in asymptomatic patients with AS.

\section{Mechanisms and Predictors for Recurrent MR after Mitral Valve Repair}

Hillit Cohen, Micha Feinberg, Michael Stein, Leonid Sternik,
Ehud Raanani

Sheba Medical Center, Ramat Gan, Israel

Objectives: Mitral Valve repair has become the standard of care for patients with severe degenerative MR. Most large series report recurrent significant regurgitation in the range of $10-20 \%$ at 10 years. Probably some of the failures can be avoided by correct technique in the initial operation. Understanding the mechanisms of repair failure is crucial in optimizing surgical technique and improving long term durability of mitral valve repair. Methods: 565 patients with myxomatous mitral valve disease underwent primary mitral valve repair in our department between 2004 and 2014. All patients were followed prospectively. Follow up (FU) ranged a mean of $36 \pm 30$ clinical outcomes, re-operation reports and echocardiographic exams were analyzed and timing and mechanisms of repair failure were defined. Results: Overall, there was $1(0.2 \%)$ inhospital death and $20(3.5 \%)$ late deaths. Freedom from reoperation on the mitral valve was $96 \%$. At FU, 58 patients (10.2\%) were found to have recurrent moderate or severe MR, of these patients, 27 patients (47\%) needed reoperation on the mitral valve. Mechanisms of repair failure were: recurrent prolapse or flail of the same segment $42 \%$, prolapse or flail of a different segment $12 \%$, SAM $14 \%$, restriction $10 \%$, SBE $5 \%$, technical $3 \%$ and in $14 \%$ of cases, no definite failure mechanism was determined. Independent predictors of failure were Pre-LVEDD and SPAP. Pre-operative larger LV indices (LVEDD, LVESD, LV volume index) were associated with recurrent prolapse as failure mechanism. Conclusions: Rates of recurrent MR after repair are significant. Pre-operative larger LV seems to be associated with a higher risk of recurrent prolapse.

\section{Outcomes after Surgical Aortic Valve Replacement with Bioprostheses and Allografts in the Last Decades: What Do We Know?}

\author{
Simone A. Huygens' ${ }^{1}$ Mostafa M. Mokhles', \\ Milad Hanif', Jos A. Bekkers' ${ }^{1}$, Ad J.J.C. Bogers ${ }^{1}$, \\ Maureen P.M.H. Rutten-van Mölken' ${ }^{2}$ Johanna J.M. Takkenberg ${ }^{1}$ \\ ${ }^{1}$ Erasmus MC, Rotterdam, ${ }^{2}$ Institute of Medical Technology \\ Assessment/Department of Health Policy and Management, \\ Erasmus University Rotterdam, Rotterdam, Netherlands
}

Objective: Many observational studies have reported outcomes after surgical aortic valve replacement (AVR), but there are no recent systematic reviews and meta-analyses including all available bioprostheses and allografts. The objective of this study is to 
Table 1. Study characteristics and pooled estimates of outcome measures after AVR with bioprostheses and allografts (for Abstract of Simone A. Huygens)

\begin{tabular}{lcc}
\hline & Bioprotheses & Allografts \\
\hline Study characteristics & & \\
Follow-up, years, mean \pm SD & $6.7 \pm 4.7$ & $8.5 \pm 3.0$ \\
Age, years, mean \pm SD & $71.8 \pm 9.3$ & $48.8 \pm 13.0$ \\
Male, \% & 61.0 & 69.4 \\
Concomitant procedures, \% & 50.2 & 26.8 \\
\hline Pooled estimates of outcome measures, \%/years (95\% CI) & & \\
Early mortality & $4.99(4.44-5.62)$ & $5.03(3.61-7.01)$ \\
Late mortality & $5.70(4.99-6.53)$ & $1.68(1.23-2.28)$ \\
Structural valve deterioration & $0.60(0.47-0.76)$ & $2.26(1.02-4.97)$ \\
Nonstructural valve dysfunction & $0.20(0.13-0.32)$ & - \\
Valve thrombosis & $0.04(0.03-0.07)$ & - \\
Thromboembolism & $1.10(0.83-1.47)$ & $0.46(0.20-1.08)$ \\
Bleeding & $0.44(0.30-0.65)$ & $0.15(0.09-0.25)$ \\
Endocarditis & $0.38(0.32-0.44)$ & $0.42(0.31-0.58)$ \\
Re-interventions & $0.75(0.61-0.91)$ & $1.87(1.52-2.31)$ \\
\hline
\end{tabular}

$\mathrm{SD}=$ Standard deviation; $\mathrm{CI}=$ confidence interval.

provide a comprehensive overview of the outcomes after AVR with bioprostheses and allografts reported in the last 15 years. Methods: We conducted a systematic literature review of studies published between 2000-2015. Inclusion criteria were observational studies or RCTs reporting on outcomes of AVR with bioprostheses (stented or stentless) or allografts, with or without coronary artery bypass grafting (CABG) or valve repair procedure with study population size $n \geq 30$ and mean follow-up length $\geq 5$ years. Fifty-four bioprostheses studies and fourteen allografts studies were included, encompassing 55,712 and 3,872 patients and 349,840 and 32,419 follow-up patient-years, respectively. We pooled early mortality risk and linearized occurrence rates of valve-related events, re-intervention and late mortality in a random effects model. Sensitivity, meta-regression and subgroup analyses were performed to investigate the influence of outliers on the pooled estimates and to explore sources of heterogeneity. Funnel plots were used to investigate publication bias. Results: Study characteristics and pooled estimates of outcome measures are reported in the Table. There was substantial heterogeneity in most outcome measures. Meta-regression analyses identified covariates that could explain the heterogeneity: implantation period, valve type, patient age, gender, pre-NYHA class III/IV, concomitant CABG, study design, and follow-up length. There is possible publication bias in all outcome measures. Conclusions: This comprehensive systematic review and meta-analysis provided an overview of outcomes after AVR with bioprostheses and allografts reported in the last 15 years. Our results can support patients and doctors in the prosthetic valve choice and can be used in microsimulation models to predict patient outcomes and estimate cost-effectiveness of AVR with bioprostheses or allografts compared with other heart valve prostheses.

\section{Natural History of Chronic Severe Aortic Regurgitation: A 30-Year Prospective Study}

\author{
Antonio S. Santis, Guilherme Spina, Roney Sampaio, \\ Tarso Accorsi, Joao Fernandes, Vitor Rosa, Milena Paixão, \\ Carlos Manoel Brandão, Flavio Tarasoutchi
}

Instituto do Coração (Heart Institute) - Incor, São Paulo, Brazil

Objectives: Chronic Aortic regurgitation (AR) still presents a considerable prevalence among heart valve diseases. Surgical intervention based exclusively on ventricular diameters still remains controversial. The aim of this study is to verify the evolution and overall mortality of AR based on the longest period of follow-up already reported for patients with AR. Methods: This was a prospective study of 73 patients with chronic severe AR, followed during a mean period of $28.8 \pm 2.6$ years. Surgical treatment was determined by the appearance of symptoms and/or systolic dysfunction of the left ventricle (LV). Continuous variables were expressed as mean \pm standard deviation. Categorical variables were expressed as proportions. Chi-square test and Students t test were used in the univariated analysis, according to the pattern of variables. Survival projections were determined by Kaplan-Meier curves. Results: The main cause of AR was rheumatic cardiac disease (94\%), with male predominance (73\%). At the beginning of follow-up, $30 \%$ of patients were symptomatic. Surgical treatment was performed in $52 \%$ of patients and consisted mainly in aortic valve replacement (92\% of the procedures). The overall mortality was $15 \%$. The study showed a higher long-term mortality in the surgical group than in the clinical group (26\% versus $2.9 \%$, p 0.007 . HR $=9.2$, CI $1.35-$ 191.8), mainly due to late prosthesis dysfunction and postoperative complications in prosthesis re-replacement surgery (cardiogenic shock, bleeding, infection). Patients that remained in clinical

Cardiology 2016;134:136-310 
treatment, mainly due to the absence of symptoms or ventricular dysfunction, exhibited a low long-term mortality rate $(2.9 \%)$, despite of LV remodeling (mean LV diastolic diameter of $65 \pm 6.9$ $\mathrm{mm}$, mean LV systolic diameter of $45.3 \pm 9.6 \mathrm{~mm}$ ). Reoperation was performed in $40 \%$ of patients and was a mortality predictor $(\mathrm{HR}=6.1, \mathrm{p}=0.006)$. Conclusions: The present study, based on the longest follow-up period reported for rheumatic AR, found no mortality registry in the asymptomatic AR, demonstrating a favorable prognosis for this subgroup of patients, even with LV remodeling. Surgical treatment, although necessary for symptomatic patients or with left ventricular dysfunction, is associated with higher long-term morbidity and mortality related to prosthetic dysfunction.

\section{Percutaneous Heart Valve Therapy}

\section{Ketoisocaproic Acid Predicts Acute Kidney Injury and Mortality after Transcatheter Aortic Valve Replacement: A Metabolomic Profiling Study}

\author{
Sammy Elmariah ${ }^{1}$, Laurie A. Farrell ${ }^{1}$, Maureen Daher ${ }^{1}$, Xu Shi ${ }^{1}$, \\ Jordan E. Morningstar ${ }^{1}$, Michelle J. Keyes ${ }^{1}$, Gus J. Vlahakes ${ }^{1}$, \\ Ignacio Inglessis ${ }^{1}$, Jonathan J. Passeri ${ }^{1}$, Igor F. Palacios ${ }^{1}$, \\ Caroline S. Fox ${ }^{2}$, Eugene P. Rhee ${ }^{1}$, Robert E. Gerszten ${ }^{1}$ \\ ${ }^{1}$ Massachusetts General Hospital, Harvard Medical School, \\ Boston, MA, ${ }^{2}$ Framingham Heart Study, National Heart, Lung, \\ and Blood Institute, Framingham, MA, USA
}

Background: Acute kidney injury (AKI) occurs commonly after transcatheter aortic valve replacement (TAVR) and is associated with markedly increased post-operative mortality. Predictors of AKI would be of tremendous clinical value by allowing for accurate assessment of procedural risk and investigation and eventual implementation of preventative measures. We applied novel metabolomic profiling techniques to identify biomarkers predictive of AKI after TAVR. Methods: We performed liquid chromatography-mass spectrometry based metabolite profiling of negative ions in patients undergoing TAVR and subjects from the community based Framingham Heart Study (FHS; $\mathrm{N}=2164$ ). AKI was defined using the Valve Academic Research Consortium-2 criteria. We examined the associations of plasma metabolites with chronic kidney disease (CKD) within the TAVR and FHS cohorts and with incident AKI after TAVR. Results: Of 44 patients (mean age $82 \pm 9$ yrs, 52\% female) undergoing TAVR, 22 (50\%) had CKD and $9(20 \%)$ developed AKI. We detected markedly concordant cross-sectional metabolic changes associated with CKD in the hospital-based TAVR and FHS cohorts. Of 71 metabolites, baseline ketoisocaproic acid (ratio AKI/no AKI $=1.44, P=6.0 \mathrm{E}-4$ ) was differentially detected in patients that subsequently developed AKI. Correlation between ketoisocaproic acid and estimated glomerular filtration rate (eGFR) was weak. After adjustment for age, gender, diabetes status, and estimated glomerular filtration rate (eGFR), ketoisocaproic acid (OR per SD increase $=7.34,95 \% \mathrm{CI}$ $1.77-30.45 ; P=0.006)$ remained predictive of $\mathrm{AKI}$ and was in fact

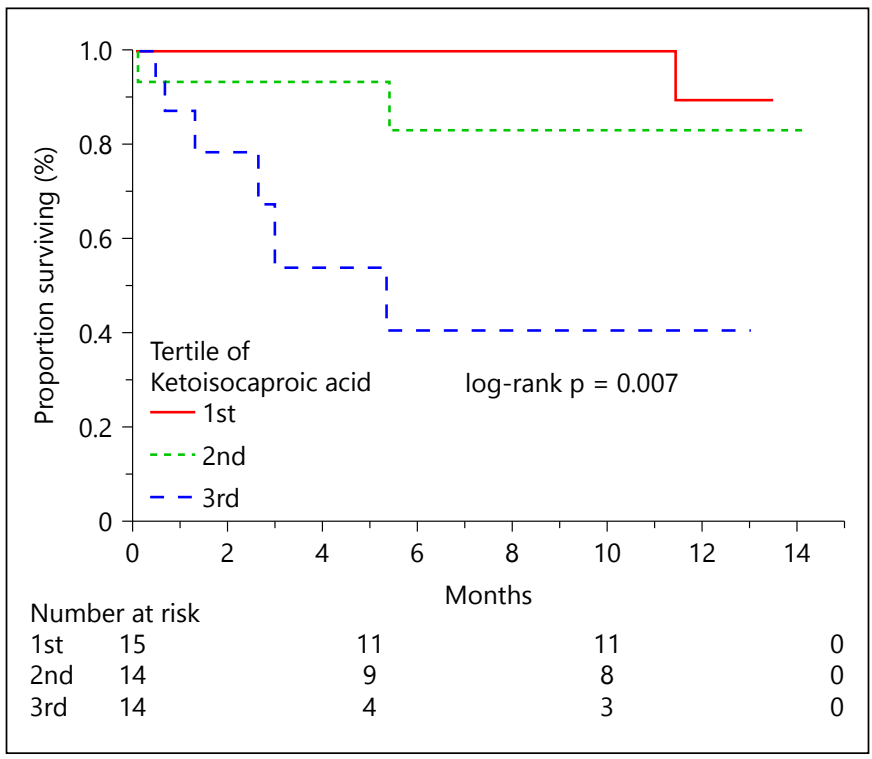

Fig. 1. (for Abstract of Sammy Elmariah).

more predictive than baseline eGFR. Of the patients that suffered AKI, 6 (66.7\%) subsequently died, compared to $3(8.6 \%)$ deaths among those patients that did not develop AKI $(P=0.0008)$. Ketoisocaproic acid (HR per SD increase $=3.25,95 \%$ CI 1.46-7.23; $P=0.004)$ was predictive of all-cause mortality after TAVR, independent of age, gender, diabetes status, and baseline eGFR (figure 1). Conclusion: In an elderly population with severe aortic stenosis undergoing TAVR, ketoisocaproic acid improves the prediction of acute kidney injury and mortality. Future efforts in larger TAVR cohorts are required to validate this novel biomarker.

\section{The Effect of Valve Positioning on Coronary Perfusion and the Flow Field Downstream of a Medtronic CoreValve Prosthesis: An in-vitro Study}

Desiree Pott, Martin Buesen, Christoph Schmitz, Simon Sonntag, Thomas Schmitz-Rode, Ulrich Steinseifer

Department of Cardiovascular Engineering, Helmholtz Institute, RWTH Aachen University, Aachen, Germany

Objective: The flow field behind a Medtronic Corevalve TAVI prosthesis was investigated in three different positions regarding the implantation heights (normal, high, low) in order to analyze the impact of the implantation height on the flow field and on the coronary perfusion. Methods: A silicon model of the aorta, including the sinuses of Valsalva and both coronary arteries was manufactured. The prosthesis was inserted in the correct implantation position and then approximately $8 \mathrm{~mm}$ higher and lower. For the flow measurements, the CVE pulse duplicator was used to produce physiological flow and pressure curves $(5 \mathrm{l} / \mathrm{min}$ and $120 / 80 \mathrm{~mm}$ $\mathrm{Hg}$ ) through the prosthesis. The flow field was investigated by particle image velocimetry technique. Two high-speed cameras re- 
corded the particles (ILA GmbH, Jülich, Germany) in the fluid (water/glycerine mixture with a viscosity of $3.6 \mathrm{mPas}$ at $37^{\circ} \mathrm{C}$ ), illuminated by a laser (Nd:YAG, Pegasus, New Wave Research Inc.). The flow field was divided into six planes with $5 \mathrm{~mm}$ distance to each other. The recorded data was post-processed using the software dynamic studio (Dantec, Denmark) and Tecplot (USA). Results: The analysis showed that for each position a central jet of the same maximum velocity developed in early systole. Depending on the implantation height, a different amount of fluid passed through the coronary arteries over one heart cycle. Furthermore, the inflow direction of the fluid in the near proximity of the pulmonary arteries inside the sinuses of Valsalva varied depending on the implantation height. Conclusions: The amount of coronary perfusion flow and the inflow direction highly depends on the implantation height of the prosthesis.

\section{St. Jude Trifecta versus Edwards Perimount Magna Ease Bioprosthesis: No Difference in Coronary Flow after Transcatheter Aortic Valve-in-Valve Implantation: An in vitro Investigation}

\author{
Sina Stock ${ }^{1}$, Michael Scharfschwerdt ${ }^{1}$, Roza Meyer-Saraei ${ }^{2}$, \\ Doreen Richardt ${ }^{1}$, Efstratios I. Charitos ${ }^{3}$, Hans-Hinrich Sievers ${ }^{1}$, \\ Thorsten Hanke ${ }^{1}$ \\ ${ }^{1}$ Department of Cardiac and Thoracic Vascular Surgery, \\ University of Luebeck, Luebeck, ${ }^{2}$ Department of Cardiology, \\ Angiology and Intensive Care Medicine, University of Luebeck, \\ Luebeck, ${ }^{3}$ Department of Cardiac and Thoracic Surgery, \\ University of Halle, Halle, Germany
}

Background: The Transcatheter Aortic Valve-in-Valve Implantation (TAViVI) is an evolving treatment strategy for degenerated surgical aortic valve bioprostheses (SAVB). Despite excellent hemodynamics, there is some concern regarding coronary obstruction, especially after TAViVI in calcified SAVB with externally mounted leaflets. We sought to determine differences in coronary flow after TAViVI in non-calcified and 'calcified' SAVB with externally and internally mounted leaflets in an in vitro investigation. Methods: We constructed aortic root models incorporating geometric dimensions known as risk factors for coronary obstruction and inserted SAVB with externally (Trifecta ${ }^{\mathrm{TM}}$, TRI, size 25) respectively internally (Perimount ${ }^{\circledR}$ Magna Ease, PERI, size 25) mounted leaflets. Hemodynamic performance (transvalvular gradients and geometric orifice area) was measured before and after TAViVI (Sapien XT ${ }^{\mathrm{TM}}$, SAP, size 23). Left and right coronary flow (LCF, RCF) were examined at two different coronary heights $(\mathrm{CH}, 8 \mathrm{~mm}$ and $10 \mathrm{~mm})$. After the first experimental run, the SAVB leaflets were artificially 'calcified' with glue and the measurements repeated. Results: In both models, we found no significant reduction in coronary flow, neither when testing TRI nor PERI (all p-values non-significant). Non-calcified model: TAViVI in TRI reduced RCF from $0.64 \pm 0.06 \mathrm{ml} /$ stroke to $0.6 \pm 0.07 \mathrm{ml} /$ stroke $(\mathrm{CH} 8 \mathrm{~mm})$ respectively from $0.62 \pm 0.06 \mathrm{ml} /$ stroke to $0.58 \pm$ $0.04 \mathrm{ml} /$ stroke $(\mathrm{CH} 10 \mathrm{~mm})$. LCF dropped from $0.9 \pm 0.08 \mathrm{ml} /$ stroke to $0.87 \pm 0.06 \mathrm{ml} /$ stroke $(\mathrm{CH} 8 \mathrm{~mm})$ respectively from $0.89 \pm$ $0.05 \mathrm{ml} /$ stroke to $0.82 \pm 0.07 \mathrm{ml} /$ stroke $(\mathrm{CH} 10 \mathrm{~mm})$. TAViVI in PERI reduced RCF from $0.62 \pm 0.08 \mathrm{ml} /$ stroke to $0.61 \pm 0.07 \mathrm{ml} /$ stroke $(\mathrm{CH} 8 \mathrm{~mm})$ respectively from $0.62 \pm 0.04 \mathrm{ml} /$ stroke to $0.6 \pm$ $0.06 \mathrm{ml} /$ stroke $(\mathrm{CH} 10 \mathrm{~mm})$. LCF remained $0.89 \mathrm{ml} /$ stroke $(\mathrm{CH}$ $8 \mathrm{~mm}$ ) respectively dropped from $0.87 \pm 0.04 \mathrm{ml} /$ stroke to $0.83 \pm$ $0.03 \mathrm{ml} /$ stroke (CH $10 \mathrm{~mm})$. 'Calcified' model: TAViVI in TRI reduced RCF from $0.67 \pm 0.05 \mathrm{ml} /$ stroke to $0.62 \pm 0.04 \mathrm{ml} /$ stroke $(\mathrm{CH} 8 \mathrm{~mm})$ respectively from $0.64 \pm 0.08 \mathrm{ml} /$ stroke to $0.58 \pm$ $0.06 \mathrm{ml} /$ stroke $(\mathrm{CH} 10 \mathrm{~mm})$. LCF dropped from $0.85 \pm 0.06 \mathrm{ml} /$ stroke to $0.79 \pm 0.08 \mathrm{ml} /$ stroke $(\mathrm{CH} 8 \mathrm{~mm})$ respectively from $0.79 \pm$ $0.1 \mathrm{ml} /$ stroke to $0.76 \pm 0.04 \mathrm{ml} /$ stroke $(\mathrm{CH} 10 \mathrm{~mm})$. TAViVI in PERI increased RCF from $0.65 \pm 0.06 \mathrm{ml} /$ stroke to $0.69 \pm 0.17 \mathrm{ml} /$ stroke $(\mathrm{CH} 8 \mathrm{~mm})$ respectively remained $0.58 \mathrm{ml} /$ stroke $(\mathrm{CH}$ $10 \mathrm{~mm}$ ). LCF dropped from $0.83 \pm 0.04 \mathrm{ml} /$ stroke to $0.77 \pm 0.12 \mathrm{ml} /$ stroke $(\mathrm{CH} 8 \mathrm{~mm})$ respectively increased from $0.76 \pm 0.13 \mathrm{ml} /$ stroke to $0.8 \pm 0.04 \mathrm{ml} /$ stroke $(\mathrm{CH} 10 \mathrm{~mm})$. Conclusions: TAViVI seems to be feasible in both SAVB types, non-calcified as well as 'calcified', the risk of coronary obstruction is similar in patients with an anatomy like the aortic root models used in this study.

\section{Novel Predictor of Mortality in Patients Undergoing Transcatheter Aortic Valve Implantation}

\section{Silvana Hamati, Mirko Doss, Christoph Liebetrau, Thomas Walther}

Kerckhoff Heart Center Bad Nauheim, Bad Nauheim, Germany

Objective: There are no reliable biomarkers for short- or longterm outcome prediction in patients undergoing transcatheter aortic valve implantation (TAVI). Our goal was to investigate whether presepsin is also suited for risk assessment in TAVI patients. The aim of this study was to assess whether Presepsin, a novel marker indicative for sepsis, has prognostic value in the postoperative outcome in patients after transcatheter aortic valve implantation (TAVI). Methods: We included 72 patients undergoing TAVI in this single-center, observational study. We matched 31 patients who had died within 12 months after TAVI with 41 surviving patients with regards to age and major risk factors. Baseline presepsin levels were correlated to the hsTNT, the NT-proBNP, the ES II as well as to mortality after one year. Patients were selected in a non-randomised fashion. From all patients clinical data was reported and blood assessment was performed before and immediately after TAVI. From all patients informed consent is obtained and approval by the local ethics committee was achieved. Results: The Receiver operating characteristic curve (ROC) of presepsin and the various risk scores for the prediction of mortality after TAVI are presented in figure 1. Presepsin provided the best result, with an area under the ROC curve of 0.703 vs. ES II with an AUC of 0.687. Comparing the predictive power of prespsin in addition to ES II showed the best predicitve result with an AUC of 0.811 . Presepsin provided the best result, with an area under the ROC curve of 0.703 vs. ES II with an AUC of 0.687. Comparing the predictive power of prespsin in Addition to ES II showed the best predicitve result with an AUC of 0.811. Conclusions: In summary, elevated presepsin levels are associated with significantly increased mortality after TAVI. Combining presepsin with ES II provides additive predictive value of risk stratification. 


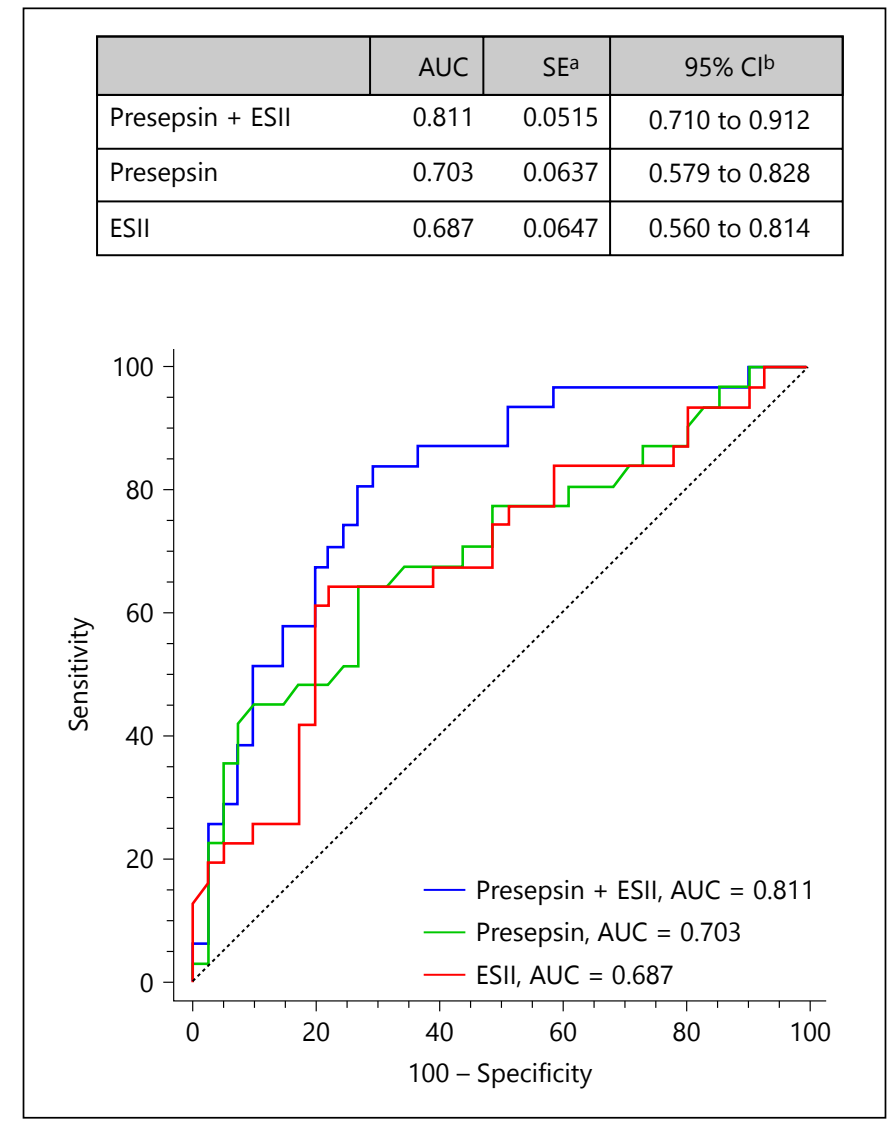

Fig. 1. (for Abstract of Silvana Hamati).

\section{Transcarotid TAVR Utilizing EEG Guided Selective Cerebral Perfusion}

Keith B. Allen, David J. Cohen, Adnan Chhatriwalla, Sanjeev Aggarwal, Anthony J. Hart, Suzanne Baron, J. Russell Davis, Haley Haines, A. Michael Borkon

St. Luke's Mid America Heart Institute, Kansas City, MO, USA

Objective: Technical advances in transcatheter valve technology have led to progressively smaller devices and increased use of transfemoral access; however, approximately $30 \%$ of patients continue to require use of alternative access sites. Traditional approaches to alternate access such as transapical, direct aortic or subclavian are often feasible but may not be ideal in patients with reduced ejection fraction, COPD or patent mammary grafts. Transcarotid delivery of transcatheter valve may circumvent these limitations. Methods: Between February and August 2015, 14 patients whose anatomy precluded femoral or safe transapical, transaortic, or subclavian access underwent transcather aortic valve replacement utilizing transcarotid access. Surgical technique included direct exposure of the proximal common carotid artery (fig. 1a); modification/shortening of the device delivery sheath (fig. 1b, c); and intraoperative EEG monitoring to guide the need for femoral to carotid shunting during device delivery (fig. 1d). Results: $\mathrm{Me}$ dian STS predicted surgical mortality was $11 \%$ (range 3-21) with a mean age of 83.9 (range 62-90). Both right (10) and left (4) carotid access was utilized for delivery of either balloon-expandable ( $\mathrm{n}=13$ ) or self-expanding $(\mathrm{n}=1)$ commercially available valves. Procedural success was $100 \%$. No patient required femoral to carotid shunting and in $93 \%(13 / 14)$ a transverse carotid arteriotomy with primary repair was utilized (fig. 1e). There were no in hospital/30 day deaths or strokes. Complications included pericardial effusion that required a surgical subxiphoid window $(n=1)$, pacemaker $(n=2)$, valve in valve procedure to treat significant perivalvular regurgitation $(\mathrm{n}=1)$, and a carotid conduit constructed following retrieval of a malfunctioning valve $(n=1)$. Median length of stay was 3 days (range 1-9) and all patients were discharged to home. With a median follow up of four months (range 2-7 months) survival remains $100 \%$ with no late carotid access complications or strokes (fig. 1f). Conclusions: Transcarotid transcatheter aortic valve replacement incorporating a cerebral protection strategy guided by intraoperative EEG monitoring is safe and may be preferable to more invasive alternative access options such as the direct aortic or transapical approach.

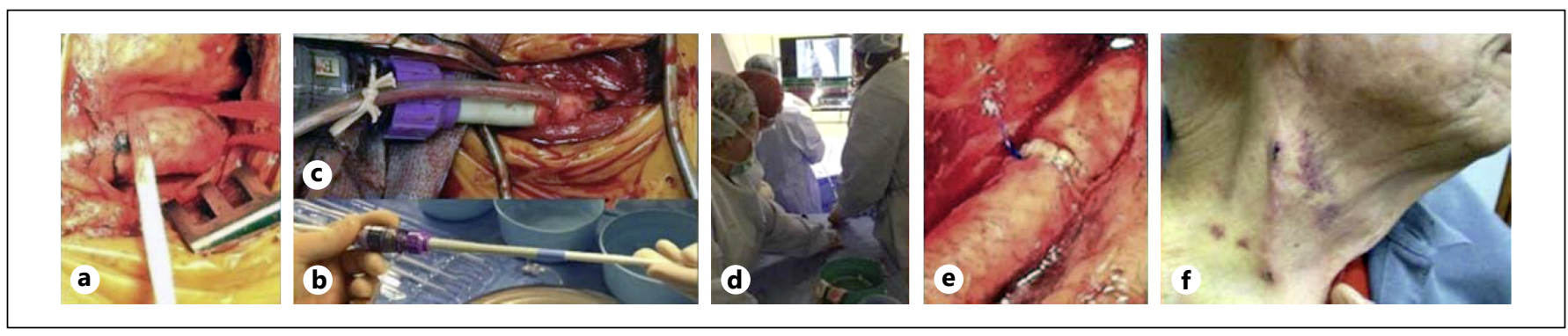

Fig. 1. (for Abstract of Keith B. Allen). 


\section{Novel Apical Closure Device for Transapical Procedures}

\section{Ziv Beckerman, Sr. ${ }^{1}$, David Taggart ${ }^{2}$, Gil Bolotin ${ }^{1}$}

${ }^{1}$ Rambam Health Care Campus, Haifa, Israel; ${ }^{2}$ University of Oxford, Department of Cardiovascular Surgery, Oxford, United Kingdom

Background: Trans-apical access is common and safe for aortic and mitral valve interventions. Apical closure is based on pursestring sutures, requiring general anesthesia, thoracotomy and rib spreading. Several complications have been reported with this technique, namely acute bleeding, delayed apical rupture and development of pseudoaneurysms. We describe a novel apical closure technology, aiming at allowing an easy, safe, durable closure and future percutaneous apical access. Methods: The closure device (Wisefix) is a spiked $20 \mathrm{~mm}$ diameter ring of medical-grade Titanium. The ring is placed on the apex prior to penetration and closed upon completion of the procedure. Upon closure, the associated myocardium undergoes an internal directed movement and the central hole is closed by tissue recruitment. In the current prospective chronic animal study, 4 -Wisefix ring models were tested on 4 sheep. All together 11 devices were tested. Aspirin was given 2 days preoperatively. Heparin was given after thoracotomy to achieve ACT $>500$. The devices were implanted on the LV apical, lateral and anterior walls, a 26F catheter was inserted and extracted, followed by closure of the device. All animals underwent preoperative and follow-up echocardiography at 5 days, 1 month, and prior to sacrifice (3-6 months). Cardiac tissue was sent for histopathological examination. Results: Ring models varied in

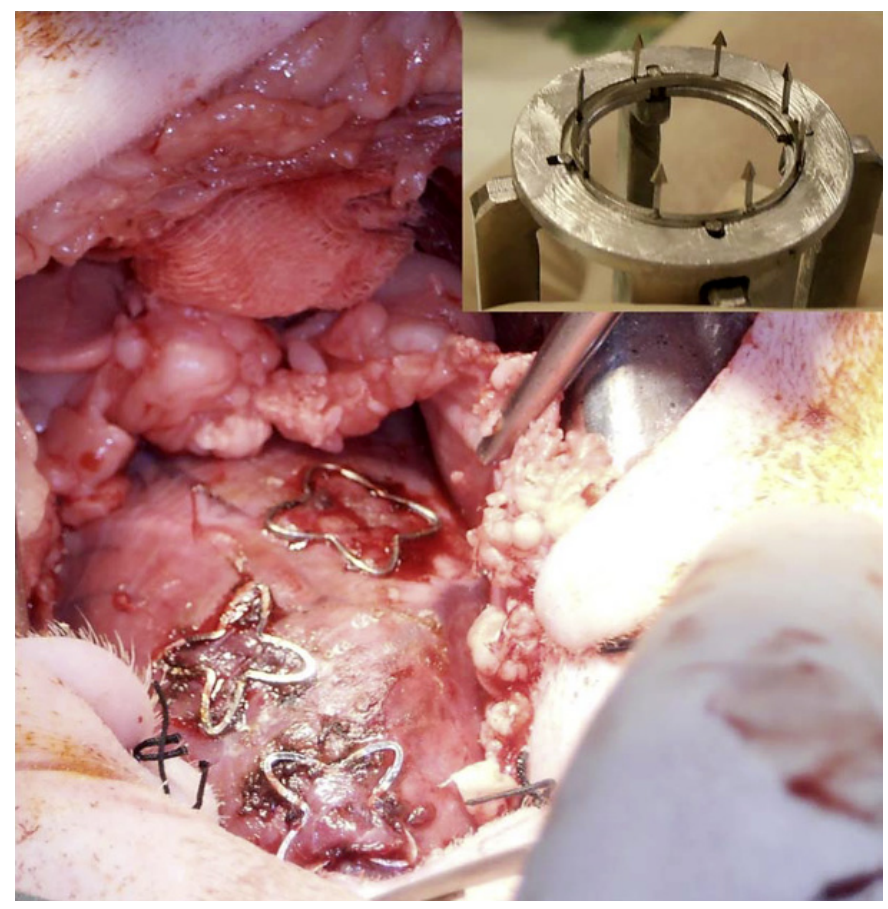

Fig. 1. (for Abstract of Ziv Beckerman, Sr.). prong length ( $7 \mathrm{~mm}$ and $5 \mathrm{~mm})$, prong-ring angle $\left(90^{\circ}\right.$ and $\left.80^{\circ}\right)$, and prong>s tip (straight and arrowhead). A $7 \mathrm{~mm}$ straight Wisefix was tested twice and yielded moderate hemostasis. A $5 \mathrm{~mm}$ straight Wisefix did not result in good hemostasis. A $5 \mathrm{~mm} 80^{\circ}$ straight Wisefix was tested 4 times and yielded good attachment and hemostasis. A $5 \mathrm{~mm} 90^{\circ}$ arrowhead Wisefix was tested 4 times, yielding the best attachment and hemostasis (figure 1). All echocardiography exams demonstrated preserved ventricular function and no pericardial effusions. Pathology demonstrated intact devices. Histologically tissue reaction was within expected limits. Conclusions: Wisefix closure device demonstrated easy applicability and hemostatic effect in sealing the LV puncture site. Human studies are required to ensure safety before percutaneous application of this novel technology.

\section{Heart Valve Repair}

\section{Successful Repair of Functional Mitral Regurgitation with Severe Leaflet Tethering in Patients with Dilative Cardiomypathy: Experience with the Double-Sutured-Ring and Double-String-Technique}

Frank Langer, Annika Klär, Behnaz Baschi, Maria Blümel, Takashi Kunihara, Diana Aicher, H.-J. Schäfers

University Hospital Homburg, Homburg, Germany

Objective: Functional mitral regurgitation (MR) is frequently observed in patients with dilative cardiomyopathy and contributes significantly to heart failure. Aggressively undersized ring annuloplasty has been proposed as surgical treatment option. Repair failure may however may occur in patients with marked LV-dilatation either as ring dehiscence or persistent leaflet thethering. We have developed a repair technique, which prevents ring dehiscence and relocates the both papillary muscle towards the aorto-mitral continuity using two transventricular PTFE-sutures (doubleSTRING). Methods: Between 11/2005 and 10/2015 87 patients with dilative cardiomyopathy with a tenting height $>10 \mathrm{~mm}$ (48 male, age $66 \pm$ 16 years, EF $31 \pm 7 \%$, MPAP $34 \pm 10 \mathrm{~mm} \mathrm{Hg}$, LVEDD $66 \pm 10 \mathrm{~mm}$ ) underwent moderately undersized annuloplasty (band or ring, median size $30 \mathrm{~mm}$ ) combined with doubleSTRING. In patients with significant LV-dilatation (LVEDD $>65 \mathrm{~mm}$ ) the annuloplasty device was secured with a second bite after initial tying (doublesutured-RING) since $02 / 2008$. CPB time was $79 \pm 22 \mathrm{~min}$, myocardial ischemic time was $54 \pm 16$ min. Results: Hospital mortality was $7 \%, 5$-year survival $71 \%$. Freedom from MR > II at 5 years was $89 \%$, freedom from reoperation at 5 years 95\%. LVEDD (66 \pm 10 to $61 \pm 13 \mathrm{~mm}$, p65 mm-subgroup had lower EF ( $29 \pm 10 \%$ vs. $33 \pm$ $8 \%, \mathrm{p}=0.04$ ), reverse remodeling was observed (LVEDD $73 \pm 9$ to $67 \pm 13, \mathrm{p}<0.001 ; \operatorname{LVESD} 59 \pm 14 \mathrm{~mm}$ to $56 \pm 12 \mathrm{~mm}, \mathrm{p}=0.03)$ in this high-risk-group. Conclusions: The combination of secured moderately undersized annuloplasty and repositioning of both papillary muscles leads to low rate of recurrent MR in high-riskpatients with significant LV-dilatation and severe leaflet tethering. 


\section{Follow-Up of the Mid-Term Results for Mitral Valve Repair for Rheumatic Mitral Regurgitation}

\section{Samy M. Amin, Yasser Mohamed Menaissy}

Kasr el Eini, Faculty of Medicine, Cairo, Egypt

Objective: Rheumatic mitral valve disease is considerably less common in North America and European countries than in developing countries, where rheumatic heart disease remains by far the leading cause of valvular diseases. This study was done to evaluate the midterm results over $10 \mathrm{y}$ of mitral valve repair for rheumatic mitral regurgitation in term of survival rate and late valve failure and need of redo surgery. Methods: This is a retrospective study from January 2004 to January 2014, one hundred and twenty patients with rheumatic mitral valve disease underwent mitral valve repair in our hospital. Age ranged from 15 to 53 years, $80 \%$ patients were female. The lesions were pure mitral regurgitation in 95 (79.1\%) patients, predominant mitral regurgitation with stenosis in $12(10 \%)$, and predominant mitral stenosis with regurgitation in $13(10.8 \%)$. Ninty patients (75\%) patients were in normal sinus rhythm. Results: Follow-up time ranged from 6 to 120 months, mean $55.4 \pm 3.2$ months. There were 10 late deaths. Survival at 5 and 10 years was $96.5 \%$ and $91.2 \%$, respectively. 25 patients $(20.8 \%)$ patients had mitral regurgitation during follow-up, and 8 underwent reoperation with no hospital mortality. Freedom from reoperation at 5 and 10 years was $93.5 \%$ and $82.7 \%$, respectively. Progression of mitral regurgitation at 5 and 10 years was $71.4 \%$ and $59.3 \%$, respectively. Freedom from all late events at 5 and 10 years was $72.6 \%$ and $54.2 \%$, respectively. Conclusions: Mitral valve repair for rheumatic mitral regurgitation is associated with a significant rate of valve failure and reoperation. However, it has a satisfactory survival rate and is a good alternative to valve replacement, especially for young female patients in child bearing period to avoid the life-long risks of a prosthetic valve and anticoagulation related problems.

\section{Sinus Plication to Improve Valve Configuration in Bicuspid Aortic Valve Repair}

\begin{abstract}
Ulrich Schneider, Wolfram Schmied, Dipl-Psych, Diana Aicher Christian Giebels, Lena Winter, Hans-Joachim Schäfers
\end{abstract}

Department of Thoracic and Cardiovascular Surgery, Saarland University Medical Center, Homburg, Germany

Objective: Previous experience with repair of the regurgitant bicuspid aortic valve (BAV) has shown a strong influence of commissural orientation on repair durability, with an orientation of less than $150^{\circ}$ yielding suboptimal results. We have modified commissural orientation by asymmetric plication of the fused sinuses and adding a circumferential annuloplasty to the standard cusp repair. We compared the results of the modified technique with the old approach. Methods: Between 2009 and 2014, 35 patients with BAV and commissural orientation $150^{\circ}$ or less underwent aortic valve repair for severe regurgitation including plication of the fused sinuses and circular annuloplasty. The control group consisted of 21 historic controls operated between 2000 and 2008 using similar concepts, but without sinus plication; subcommissural plication was used as annuloplasty. The groups were similar in most respects, differences existed in preoperative annular diameter, prevalence of complete fusion, use of a pericardial patch, plication of the fused cusp. Results: At discharge the degree of AR was significantly lower in the study group $(\mathrm{p}=0.006)$. Survival after 5 years was $100 \%$ in the study group and $95.2 \%$ in the control group after 5 and 10 years. Five-year freedom from reoperation in the study group was $93.0 \%$ and $57.1 \%$ in the control group $(\mathrm{p}=$ 0.0013 ), freedom from valve replacement was $95.8 \%$ and $79.3 \%$ $(\mathrm{p}=0.0355)$. Freedom from recurrent AR grade II or greater was $57.1 \%$ in the study group and $33.3 \%$ in the control group ( $\mathrm{p}=$ $0.0024)$. Mean postoperative transvalvular gradient was significantly lower in the study group $(14.3 \pm 6.5 \mathrm{~mm} \mathrm{Hg}$ vs. $28.9 \pm 18.5$ $\mathrm{mm} \mathrm{Hg}, \mathrm{p}=0.003$ ). Conclusions: Plicating the fused sinuses and thus reducing root circumference in the fused part improves commissural orientation of the BAV. This leads to better short- and midterm stability in BAV repair and improves systolic aortic valve function.

\section{Remodelling Forces of a Rigid Mitral Annuloplasty Ring - A Potential Risk Factor for Ring Dehiscence in Mitral Valve Repair?}

\author{
Soeren N. Skov' ${ }^{1}$, Diana M. Roepcke ${ }^{1}$, Christine Ilkjaer ${ }^{1}$, \\ Jonas Rasmussen ${ }^{1}$, Marcell J. Tjoernild ${ }^{1}$, Hans Nygaard ${ }^{1}$, \\ Morten O. Jensen ${ }^{2}$, Sten L. Nielsen ${ }^{1}$
}

${ }^{1}$ Aarhus University Hospital, Aarhus N, Denmark; ${ }^{2}$ University of Arkansas, Fayetteville, AR, USA

Objective: Mitral annulus reconstruction with a remodelling annuloplasty ring is an essential part of the surgical treatment for mitral regurgitation. Despite very good overall repair durability in degenerative mitral valve disease, force accumulation at the attachment of the annuloplasty rings may be a potential risk factor for ring dehiscence and repair failure, especially in patients with severe type I annular dilatation of either degenerative or ischemic etiology. The aim of this study was to conduct an in-vivo assessment of the remodelling forces of a rigid mitral annuloplasty ring compared to the native mitral valve. Methods: Measurements were performed in-vivo in an $80 \mathrm{~kg}$ porcine model. Seven animals received a rigid normal-sized annuloplasty ring (Carpentier-Edwards Classic Annuloplasty Ring, size 32) and 7 animals were used as controls in a no ring group. Mitral annular forces were obtained with a novel annular force transducer optimized for simultaneous in- and out of mitral annulus plane measurements. Geometry was measured with a sonomicrometry ultrasound technique based on 11 implanted crystals. Results: Rigid ring implantation significantly reduced mitral annular motion in the posterior segment, both commissural segments and the septal-lateral direction compared to the no ring group, see figure 1 . This resulted in a significant reduction of deformational forces in the mitral annulus anterior commissural segment and the commissure-to-commissure direction, which reflected the remodelling forces accumulated in the rigid ring. Conclusions: Rigid ring annuloplasty significantly restricted cyclic annular dynamics and reduced force transmission from the mitral annulus compared to the native mitral valve. We 


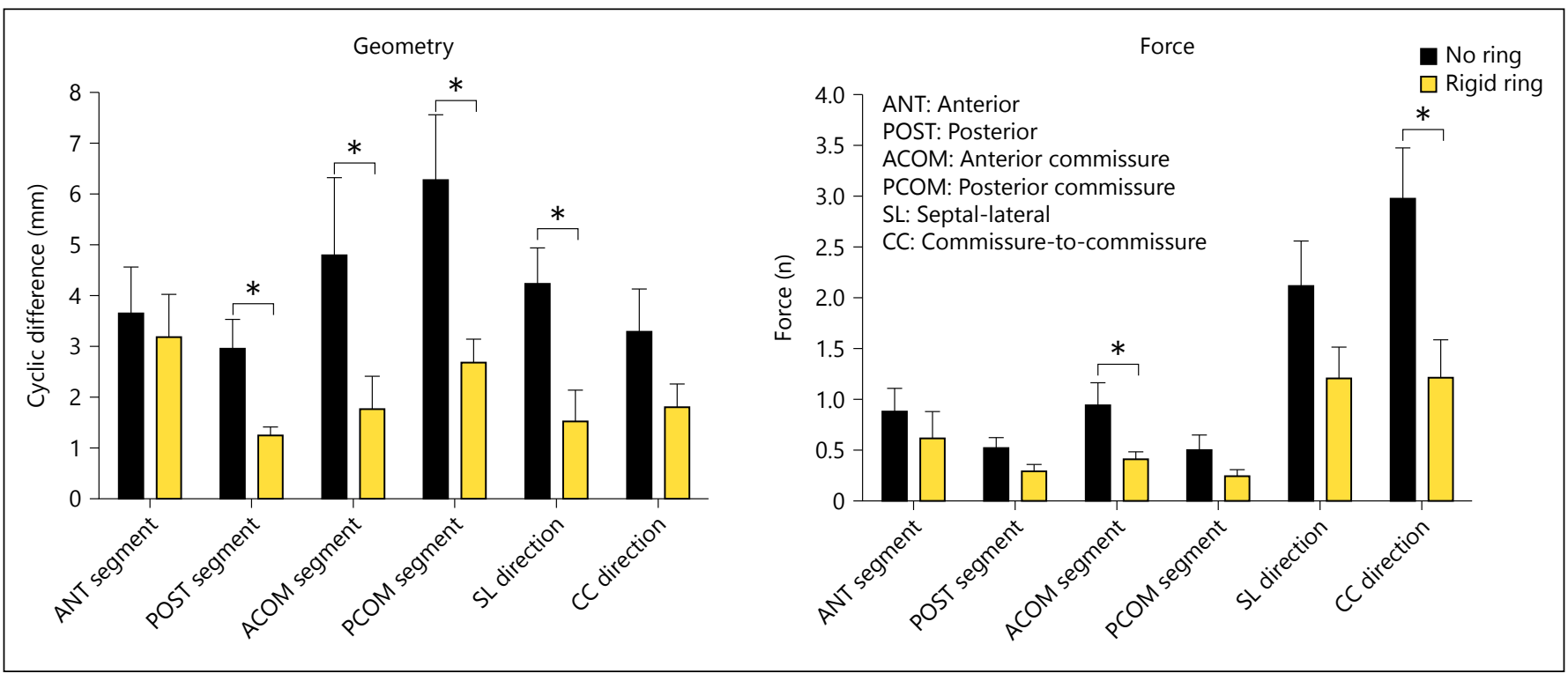

Fig. 1. (for Abstract of Soeren N. Skov).

anticipate that this difference in force transmission corresponded to the remodelling forces accumulated in each segment of the mitral annuloplasty ring. Mitral annular stabilization with a rigid ring may act intentionally for mitral valve repair. However, a significant force accumulation in the ring itself and suture attachment might potentially lead to ring dehiscence and repair failure.

\section{Mitral Valve Repair without Leaflet Resection in Right Minithoracotomy Approach}

\section{Kazuma Okamoto, Mikihiko Kudo, Akihiro Yoshitake, Hiroto Kitahara, Ichiro Kashima, Yuta Akamatsu, Hidetoshi Oka, Akinori Hirano, Mio Kasai, Shinji Kawaguchi, Ryo Aeba, Hideyuki Shimizu}

Keio University, Tokyo, Japan

Objective: Although the resection-and-suture technique has been the gold standard in mitral valve repair, decision of resection area is sometimes difficult and if the leaflet is resected too much, repair changes to be very difficult. To hand mitral valve repair from experienced masters to general cardiac surgeons and to adopt it in right minithoracotomy approach, mitral valve repair should be modified to redoable procedures. A non-resection repair using McGoon technique combined with neochordae creations and ring annuoplasty was applied as a standard strategy in our institute. Methods: Surgical result and long-term result of consecutive mitral valve repair using a non-resection technique through right minithoracotomy in a single center between October 2005 and 2015 were verified. Results: In 356 cases minithoracotomy mitral valve repair, non-resection technique was applied to 253 cases (71.1\%). The number of created neochordae was 3.6. Among cases using loop technique (219 cases $(86.6 \%)), 125$ cases were treated without additional leaflet manipulation (plication, McGoon). In pure posterior mitral leaflet prolapse cases (118 cases), loop technique was applied in 103 cases. McGoon leaflet folding was applied in 12 cases. Recurrent mitral valve regurgitation greater than moderate occurred in 22 cases (8.6\%) and freedom from recurrence at 5 years was $86.2 \%$. Reintervention for mitral valve has occurred in 6 cases $(2.4 \%)$. Conclusions: The result of the non-resection mitral valve repair using right minithoracotomy approach was acceptable but not excellent. However, further investigation of reason of recurrence and modification of surgical technique will diminish the recurrence rate.

\section{Flexible Posterior Annuloplasty Band vs. Semirigid Complete Ring in Patients with Degenerative Mitral Valve Disease}

\section{Alexander Bogachev-Prokophiev, Alexander Afanasyev, Sergei Zheleznev, Vladimir Nazarov}

Novosibirsk State Research Institute of Circulation Pathology, Novosibirsk, Russian Federation

Objective: To compare flexible band and complete ring in patients undergoing nitrate valve repair due to Barlow disease or FED. Methods: From September 2011 through September 2014 171 patients undergone mitral valve repair with flexible band or semirigid annuloplasty ring. Mean age was $57(42 ; 65)$ and 54 $(41 ; 63)$ years for the semirigid and flexible ring groups, respectively. There was no significant difference between the two groups in baseline characteristics and preoperative echocardiography. $\mathbf{R e}-$ sults: There were no early mortalities. The left ventricular enddiastolic diameter, left ventricular end-systolic diameter, and left atrial size were significantly decreased in both groups. The 2-year 


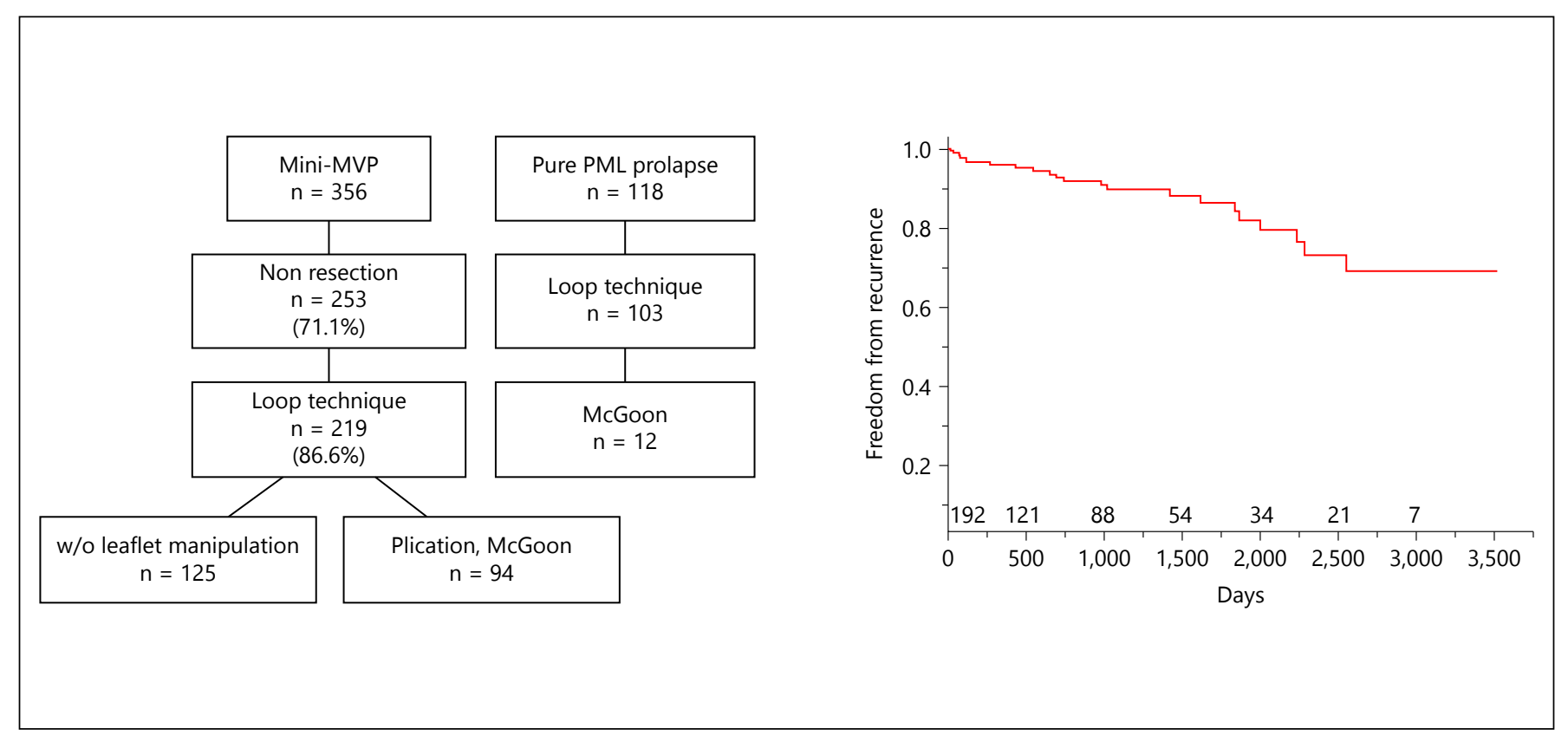

Fig. 1. (for Abstract of Kazuma Okamoto).

freedom from recurrence of significant mitral regurgitation (grade $2+$ ) was $80.8 \pm 6.5 \%$ in the semirigid group and $92.8 \pm 3.1 \%$ in the flexible group (log rank test 0.002 ). Independent prognostic factor for recurrence of mitral regurgitation in Cox regression analysis was residual mild to moderate mitral regurgitation revealed by intraoperative transesophageal echocardiography. The 3-year freedom from reoperation was $90.3 \pm 6.8 \%$ and $100 \%$ respectively (log rank test 0.044 ). Conclusions: Mitral annuloplasty using flexible posterior annuloplasty band favors better mid-term results in our prospective randomized study. There was no difference between semirigid and flexible rings in term of left ventricle remodeling.

\section{Impact of a Tailored Surgical Approach on Early Changes in Autograft Root Dimensions Following the Ross Procedure}

\section{Ismail Bouhout, Trevor Semplonius, Raymond Cartier, Nancy Poirier, Ismail El-Hamamsy}

Montreal Heart Institute, Montreal, QC, Canada

Objective: Late pulmonary autograft dilatation after the Ross procedure represents one of its main limitations. Previous studies have shown that early dilatation ( $<1$ year) is a predictor of late reoperation, highlighting the importance of surgical technique. The aim of this study was to evaluate the impact of a tailored surgical approach on early changes in autograft root dimensions following the Ross procedure. Methods: From 2011 to 2014, 100 consecutive patients underwent a Ross procedure using a total root technique ( $44 \pm 12$ years; $71 \%$ male). The main indication was aortic stenosis in $63(63 \%)$, aortic regurgitation in $15(15 \%)$, mixed disease in 20 (20\%). Specific measures included: replacement of the ascending aorta (57\%), trimming of the autograft at the sinotubular junction (100\%) and a concomitant extra-aortic annuloplasty ring (18\%). Strict blood pressure control was maintained for the first 6 months $(<110 \mathrm{~mm} \mathrm{Hg}$ systolic BP). Aortic root dimensions were obtained from follow-up serial echocardiograms. Mean follow-up was $1.3 \pm$ 0.8 years. Aortic dimensions were analyzed using mixed model effects. Results: Autograft annular dimensions did not change between discharge and 1 year postoperatively $(p=N S)$. A statistically significant increase in sinus of Valsalva dimensions from $31 \pm$ $2 \mathrm{~mm}$ at discharge to $33 \pm 3 \mathrm{~mm}$ at 3 months was observed ( $\mathrm{p}=$ $0.001)$, and in sinotubular junction diameter $(27 \pm 2 \mathrm{~mm}$ at discharge vs. $29 \pm 2 \mathrm{~mm}$ at 3 months; $\mathrm{p}<0.001$ ). These clinically normal dimensions remained stable thereafter up to 1 year $(p=N S)$. Aortic regurgitation and ascending aorta replacement were associated with changes in sinus of Valsalva and sinotubular junction dimensions $(\mathrm{p}<0.001$ and $\mathrm{p}=0.001)$. In contrast, patients with aortic stenosis had no changes in autograft root dimensions up to 1 year after surgery at the sinus of Valsalva $(p>0.99)$ and sinotubular junction ( $p>0.99$ ). Conclusions: Use of a tailored surgical approach appears to mitigate clinically significant early dilatation of the autograft root following a Ross procedure. Nevertheless, patients with preoperative aortic dilatation and aortic regurgitation should be closely monitored to determine whether the observed changes represent early adaptation or dilatation. 


\section{Minimally Invasive Valve Surgery}

\section{Comparison of Conventional Blood Cardioplegia vs. Custodiol ${ }^{\circledR}$ Cardioplegia in Minimally Invasive Mitral Valve Surgery}

David Heimansohn, Peter Walts, Robert Robison, Sina Moainie, Doug Kennett, Meghan Honerlaw, Sherrie Steckley

St. Vincent Heart Center of Indiana, Indianapolis, IN, USA

Objective: Minimally invasive mitral valve repair can be performed on an arrested heart using cold blood potassium cardioplegia (BC) as a standard approach. Histidine-tryptophan-ketoglutarate (HTK) cardioplegia can also be used for minimally invasive mitral valve surgery, and we hypothesize HTK cardioplegia is as safe and effective as BC in myocardial protection. Methods: We compared a post hoc analysis of 205 patients undergoing a minimally invasive mitral valve procedure by a single surgeon between 2007-2013. All operations were performed using a small right thoracotomy approach with femoral arterial and venous cannulation and the use of an aortic crossclamp. All patients were delivered antegrade HTK cardioplegia or antegrade/retrograde BC. Results: One hundred and five patients underwent mitral valve surgery in the HTK group and 100 patients in the BC group. The HTK group was older (64.6 yrs vs. $58.5 \mathrm{yrs}, \mathrm{p}=0.001)$, had more diabetes $(16 \%$ vs. $7 \%, \mathrm{p}=0.046)$, and higher preoperative creatinine ( 0.98 vs. 0.90 , $\mathrm{p}=0.039)$. Weight (78 kg vs. $77 \mathrm{~kg}, \mathrm{p}=0.69)$, ejection fraction $(52 \%$ vs. $54 \%, \mathrm{p}=0.13)$, previous operation $(13 \%$ vs. $12 \%, \mathrm{p}=0.83)$, and cerebrovascular disease ( $15 \%$ vs. $10 \%, \mathrm{p}=0.28)$ were all similar. A concomitant maze procedure was performed in 32 patients $(30 \%)$ of the HTK group and 23 patients (23\%) of the BC group. Cardiopulmonary bypass times were identical between HTK and BC groups (138 minutes both groups), and cross clamp times were similar (96.4 minutes vs. 99.7 minutes, $\mathrm{p}=0.43$ ). No differences were found with postoperative outcomes between HTK and BC groups when comparing bleeding ( $3 \%$ vs. $1 \%, \mathrm{p}=0.31$ ), renal failure $(1 \%$ vs. $1.4 \%, \mathrm{p}=0.83)$, prolonged ventilation $(8.4 \%$ vs. $9.8 \%$, $\mathrm{p}=0.75$ ), and any postoperative complications ( $13.6 \mathrm{vs.} 12.6 \%, \mathrm{p}=$ 0.85 ). Total postoperative inotrope time was not significantly dif-

Table 1. Postoperative variables (for Abstract of David Heimansohn)

\begin{tabular}{|c|c|c|c|}
\hline Postoperative variable & $\begin{array}{l}\text { CB } \\
\text { cardioplegia } \\
\text { mean (SD) }\end{array}$ & $\begin{array}{l}\text { HTK } \\
\text { cardioplegia } \\
\text { mean (SD) }\end{array}$ & $\mathrm{p}$ value \\
\hline Complication, $\%$ & $12.7(0.335)$ & $13.7(0.346)$ & 0.851 \\
\hline Bleeding, \% & $1(0.1)$ & $3(0.171)$ & 0.3148 \\
\hline Inotrope total, $\mathrm{h}$ & $19.65(75.35)$ & $23.75(53.08)$ & 0.656 \\
\hline Prolonged ventilation, $\mathrm{h}$ & $9.86(0.300)$ & $8.42(0.279)$ & 0.7510 \\
\hline Renal failure, $\%$ & $1.41(0.014)$ & $1.05(0.103)$ & 0.8365 \\
\hline Cross clamp time, $\min$ & $99.69(29.622)$ & $96.39(29.52)$ & 0.429 \\
\hline Pump time, $\min$ & $138.0(36.95)$ & $138.1(39.40)$ & 0.993 \\
\hline
\end{tabular}

Abstracts ferent between the two groups $(\mathrm{HTK}=23.75$ hours vs. $\mathrm{CB}=19.65$ hours, $\mathrm{p}=0.65$ ). Conclusions: HTK crystalloid cardioplegia is safe and as effective in minimally invasive mitral valve surgery as conventional cold blood cardioplegia.

\section{Left Atrial Appendage Clipping during Minimally Invasive Mitral Valve Surgery}

\author{
Walid Elkhalili, Michael Grinn, Walid Elzomor, \\ Fayez E. Shamoon, Aiman Hamdan, Vincent A. DeBari, \\ Robert Cosenza, Chirag Badami, Mark Connolly, \\ Kourosh Asgarian
}

SJRMC, Paterson, NJ, USA

Objective: Closure of the Left Atrial Appendage (LAA) is a treatment employed to decrease the risk of thrombosis and embolic events in the dilated, fibrillating left atrium. At least $90 \%$ of thrombi are found in the LAA. We examine the safety and feasibility of clipping the LAA via mini-thoracotomy approach during minimally invasive mitral valve surgery. Methods: A retrospective cohort study was conducted at a tertiary care hospital, including 50 patients, 22 which received Atrial Clipping (AC), and 28 which had no atrial clipping (NAC). All patients underwent minimally invasive mitral valve surgery and had no prior history of cardiac surgery. Patient demographics, clinical characteristics, intra-operative complications, and in-hospital course were collected by review of the medical records. Transesophageal Echocardiogram (TEE) was used intraoperatively to exclude the presence of left atrial thrombus. Continuous variables were evaluated by the D'Agostino-Pearson omnibus normality test. Categorical variables were subjected to analysis by Fisher's exact test. For this study $\alpha$ was set at 0.05 ; p-values (two-tailed) $<0.05$ were required for statistical significance. Data was analyzed using SPSS Statistics. Results: The median ages were 66.0 years (IQR: 50.8 to 81.3 ) and 56.0 years (IQR: 46.0 to 66.0) for the AC and NAC groups, respectively. In the NAC group, 2 patients had severe mitral stenosis as the indication for the mitral valve surgery, 1 patient had infective endocarditis, and 25 patients had severe mitral regurgitation. The in hospital mortality was one patient in the NAC group; the patient was a female who had mitral valve replacement. There were no mortalities in the AC group. All demographic variables between the two groups were similar, and the D'Agostino-Pearson omnibus normality test showed no difference from normal. Conclusions: We are reporting the first human application of the atrial clip through direct vision right-sided minithoracotomy as a concomitant procedure in patients undergoing mitral valve surgery. The clipping was achieved rapidly and more safely with no intraoperative complications. When compared to the control group there was no increase in clip-related bleeding and the operation time was not significantly increased. Postoperative in-hospital mortality did not differ significantly between the groups. 


\section{Feasibility of Partial Sternotomy as Approach for Aortic Valve Surgery in Obese Patients}

\author{
Udo Boeken, Sudharson Rajah, Jan Philipp Minol, \\ Hildegard Gramsch-Zabel, Payam Akhyari, Artur Lichtenberg \\ University Hospital, Duesseldorf, Germany
}

Objective: Minimally invasive access to the heart via partial sternotomy (PS) represents an increasingly used technique. We report our experience with PS for aortic valve replacement (AVR) and more complex procedures and particularly aimed to analyse the impact of BMI on morbidity and mortality. Methods: 323 patients underwent cardiac surgery via PS between $8 / 2009$ and $05 / 2013$. Mean age was $73 \pm 10$ years. AVR was performed in 275 patients, in further 35 patients in combination with surgery on the ascending aorta (AA). Isolated AA-procedures were found in 13 patients. According to BMI patients could be divided into 5 groups: $3.4 \%$ underweight (uw) (BMI below $20 \mathrm{~kg} / \mathrm{m}^{2}$ ), 24.8\% normal (BMI $\left.20-24.99 \mathrm{~kg} / \mathrm{m}^{2}\right), 46.4 \%$ overweight (BMI $25-29.99 \mathrm{~kg} / \mathrm{m}^{2}$ ), 20.7\% obese (BMI 30-34.99 $\mathrm{kg} / \mathrm{m}^{2}$ ), and $4.6 \%$ severely obese (so) $\left(\mathrm{BMI}>35 \mathrm{~kg} / \mathrm{m}^{2}\right)$. Results: ICU- and hospital stay were significantly prolonged in the severely obese patients and in the underweight group (36 \pm 10 and $35 \pm 11$, respectively vs. $26 \pm 7$ hours in all other patients; $17.5 \pm$ 7.2 and $18.4 \pm 8.2$, respectively vs. $12.1 \pm 5.3$ days, $\mathrm{p}<0.05)$ after PS. In-hospital mortality was also increased in these 2 groups (9.1\%, 6.7\% compared to $1.7 \%)$. Duration of operation, of extracorporeal circulation, and of aortic cross-clamping was prolonged by trend in obese and severely obese patients. In these 2 groups we also found an increased incidence of deep sternal wound infections with 4.5 and $13.3 \%$ compared to 1.2 in all other groups $(\mathrm{p}<0.05)$. Altogether, 7 patients needed a conversion to full sternotomy $(2.2 \%$, gr. so $13.3 \%)$, in 8 patients a re-operation due to bleeding was necessary (2.5\%, gr. uw $18.2 \%)$. At follow-up, survival was $93.8 \%$ in all patients, without significant differences between the groups. Conclusions: Minimally invasive access represents a good option for aortic valve surgery and further procedures on the AA, likewise for underweight and severely obese patients. However, particularly these 2 groups displayed a complicated perioperative course and a higher mortality. Obese patients most notably suffered from an impaired wound healing. In our patients, BMI only influenced short-term outcome whereas it did not affect midterm results.

\section{Minimally Invasive Aortic Valve Replacement via Right Anterior Mini-Thoracotomy: Initial Experience with 200 Cases}

Francesco Musumeci ${ }^{1}$, Giovanni Mariscalco ${ }^{2}$, Riccardo Gherli ${ }^{1}$, Brenno Fiorani ${ }^{1}$, Marcello Bergonzini ${ }^{1}$, Mrco Bega ${ }^{1}$

${ }^{1}$ S. Camillo Hospital, Rome, Italy; ${ }^{2}$ Glenfield Hospital, Leicester, United Kingdom

Objective: Aortic valve replacement (AVR) through right anterior minithoracotomy (MT) is increasingly performed, becoming a feasible and popular alternative to conventional full sternotomy (FS). AVR through right anterior MT has been proved to have outstanding outcomes, minimizing patient trauma, improving recovery, and reducing hospital expenditures. We report our experience with the first 200 case of AVR through right anterior MT. Methods: From August 2012 to September 2015, 200 elective consecutive patients were operated on AVR through right anterior MT (4- to $6-\mathrm{cm}$ incision) by a single surgeon. The population had an average age of $69.9 \pm 11.9$ years (range, 31 to 86), contained 108 (54\%) men, and revealed a mean EuroSCORE II of $10.4 \pm 7.2$. Indication for AVR included stenosis in 90 patients (45\%), mixed lesions in $82(41 \%)$, and predominant regurgitation in $28(14 \%)$. Multislice computed tomography (MSCT) imaging was used for surgical planning in all subjects. Aortic cannulation/clamping were performed through a right-sided minithoracotomy and venous cannulation percutaneously through the groin. For obtaining optimal intercostal space (ICS) distances between the incision to the aorta and cardiac structures, 2- and 3-dimensional MSCT images were evaluated. Results: Hospital mortality accounted for 4 (2\%) patients, $2(1 \%)$ patients required a conversion to full sternotomy and other 13 (6.5\%) a re-exploration for bleeding. Overall, cardiopulmonary bypass (CPB) was $108 \pm 25$ min and cross-clamp (ACC) time was $74 \pm 14 \mathrm{~min}$. Although not significant, a decrease in $\mathrm{CPB}$ time was observed with surgeon experience $\left(\mathrm{R}^{2}=0.2, \mathrm{p}=\right.$ $0.255)$. Finally, postoperative stroke was registered in $1(0.5 \%)$ patients and atrial fibrillation in 55 (28\%). Mean hospital length of stay was $11.8 \pm 8.4$ days. Conclusions: Our initial experience on AVR through anterior MT demonstrated excellent results with reduced complication rate.

\section{Bio-Bentall Procedure in Aortic Root Pathology: A 9-Year Single-Center Experience}

\author{
Mauro Del Giglio, Elisa Mikus, Alberto Tripodi, Marco Paris, \\ Alberto Albertini, Giorgio Noera \\ Maria Cecilia Hospital, GVM for Care and Research, Cotignola \\ (Ra), Italy
}

Objective: Since 1968 Bentall and De Bono technique is the standard surgical approach to treat aortic root pathology. Other less common alternative procedures have been proposed to avoid anticoagulation therapy. The aim of this article is to describe our experience to treat aortic root pathology in patients who need or want a biological conduit. Methods: Between January 2010 and January 2015,151 patients $(M=122,80.8 \%)$ elected to undergo 
the Bio-Bentall procedure at our Institute. Mean age was $67.4 \pm$ 10.2 years. Indications for aortic root surgery included chronic aneurysm due to calcified degenerative disease in 48 patients (31.8\%), annuloaortic ectasia in 100 (66.3\%), and infective endocarditis in the remaining 3 . Eleven (7.3\%) patients had previously undergone cardiac surgery. Results: A full sternotomy was adopted in $115(76.2 \%)$ patients, while the remaining $36(23.8 \%)$ underwent a minimally invasive approach through a ministernotomy. In-hospital mortality was $4 / 151$ (2.6\%). No patient died in the minimally invasive group. Otherwise, no significant differences were found between the groups as regards early postoperative outcome. The groups did not differ with regard to the need for tracheotomy due to respiratory failure (3.4 in FS vs. $2.8 \%$ in JS), incidence of atrial fibrillation ( $31.3 \%$ in FS vs. 30.6 in JS) or other postoperative complications. Median intensive care unit (ICU) stay was 46 (IQR 51.1) hours and median total length-ofstay was 9 (IQR 6.5) days. Where a minimally invasive approach was used, no conversion to full sternotomy was necessary. Three patients (1.9\%) underwent a redo procedure for prosthesis dysfunction. Conclusions: Our experience shows that biological prostheses inserted into the Dacron graft have a good durability; in the case of redo surgery, the double sewing technique can simplify it and the transcatheter aortic valve may represent an alternative to traditional surgery. The mortality rate is admissible. Finally, in a selective population, minimally invasive surgery can play a key role.

\section{Robotic Mitral Repair: The Ring Issue \\ Emiliano Navarra ${ }^{1}$, Philippe Noirhomme ${ }^{1}$, Laurent De Kerchove ${ }^{1}$, David Glineur ${ }^{2}$, Stefano Mastrobuoni ${ }^{1}$, Michel Van Dyck' ${ }^{1}$ Gebrine El Khoury ${ }^{1}$ \\ ${ }^{1}$ Cliniques Universitaires St. Luc - UcL - Brussels, Belgium, Brussels, Belgium; ${ }^{2}$ University of Ottawa Heart Institute, Ottawa, ON, Canada}

Background: Robotic mitral valve repair is increasingly accepted as an effective treatment for degenerative mitral regurgitation. To respect the basic principles of mitral valve repair and the policy of our institution, we introduced the use of complete ring, instead of a posterior band for annuloplasty. Aim of this work is to evaluate the feasibility and the early results in robotic mitral valve repair with the use of complete annuloplasty ring. Methods: Between Febrary 2012 and October 2015, 120 patients (mean age $56 \pm 12$ years old) underwent robotic mitral valve repair with the Da Vinci system: 14 had a posterior band and 106 a complete ring. In most of cases, the complete annuloplasty was performed using 9 to 12 separeted U-stichs. In few cases the ring was inserted by using 3 running sutures. Leaflet procedures include posterior triangular resection in 30 patients (25\%), quadrangular resection in one patients $(0.8 \%)$, chordae replacement in 87 patients (72.5\%). Commissural repair was performed in 18 patients (15\%). Results: Mean aortic cross clamp time was $109 \pm$ 26 minutes for the entire population and $108 \pm 27$ minutes in complete ring $(\mathrm{p}=0.77)$. In four patients $(3.3 \%)$ initial repair was not satisfactory and a second run of $\mathrm{CPB}$, without conversion to sternotomy, was necessary to achieve a good result. There was no perioperative mortality. Tree patients underwent surgical revision for bleeding (2.5\%). Discharge echocardiography showed no or trivial MR in all patients. Median follow up was 15 months and was $100 \%$ complete. During the follow-up two patients needed re-repair due to MR recurrence. Conclusions: The use of complete annuloplasty ring is safe and reproducible in robotic mitral valve repair. Early results are promising and show that the use of complete ring can be a good alternative to the posterior band.

\section{Preserved Mobility of Posterior Mitral Leaflet after Mitral Valve Repair with Loop Technique}

\author{
Hiroto Kitahara, Mitsuru Murata, Kazuma Okamoto, \\ Mikihiko Kudo, Akihiro Yoshitake, Tsuruta Hikaru, Yuji Itabashi, \\ Keiichi Fukuda, Hideyuki Shimizu \\ Keio University School of Medicine, Shinjyuku, Tokyo, Japan
}

Objective: Recently, loop technique has been standardized in mitral valve repair with an excellent long-term outcome. The aim of this study was to analyze whether the loop technique could preserve the leaflet mobility using trans-thoracic echocardiography. Methods: Among the concomitant 390 patients underwent mitral valve repair at Keio University Hospital between January 2007 and August 2015, 326 patients had a prolapse of posterior leaflet. Of these, 95 patients who analyzed by echocardiography were retrospectively assessed. These patients were divided into four groups, (i) group L1 $(n=30)$ consisted of patients who underwent loop technique alone, (ii) group L2 $(n=14)$ loop technique with resection and suture, (iii) group L3 $(n=40)$ loop technique with plication of indentation, (iv) group $\mathrm{R}(\mathrm{n}=11)$ resection and suture alone. Results: The mean postoperative mobile PML angle of the group L1 and L2 $\left(38.4 \pm 15.8^{\circ}, 37.3 \pm 16.0^{\circ}\right)$ were significantly larger than the group L3 and R $\left(17.3 \pm 14.8^{\circ}\right.$, $\left.13.6 \pm 14.7^{\circ}\right)$, respectively $(\mathrm{P}<0.01)$. Ring size of the group L1 and $\mathrm{L} 2(32.1 \pm 3.2 \mathrm{~mm}, 33.0 \pm 2.5 \mathrm{~mm})$ were significantly larger than the group $\mathrm{R}(28.9 \pm 1.9 \mathrm{~mm})(\mathrm{P}<0.01)$. Conclusions: The loop technique could preserve the mobility of PML and enable to implant larger ring, resulting in the reduction of mean mitral valve pressure gradient. 


\section{Poster Display (Abstracts and Cases)}

\section{Basic Research - Valve Science and Diseases}

\section{Different Bicuspid Aortic Valve Morphotypes Show Distinct Mechanisms of Gene Expression Alteration in the Aorta: Relevance to the Identification of Potential Circulating Biomarkers}

\author{
Alessandro Della Corte, Ciro Bancone, Marianna Buonocore, \\ Gilda Cobellis, Marilena Cipollaro, Umberto Galderisi, \\ Marisa De Feo, Giuseppe Limongelli, Amalia Forte \\ Second University of Naples, Naples, Italy
}

Objective: The pathogenesis of the aortopathy underlying the increased risk of aortic dilatation and dissection in bicuspid aortic valve (BAV) patients is currently poorly understood. Moreover, no available circulating biomarker, related to the risk or severity of the aortopathy, has ever been identified. We aimed to do so by coupling a differential gene expression study with serum assay of the respective products. Methods: According to state-of-the-art knowledge, the genes whose expression were investigated by RT-PCR in aortic wall samples (convexity and concavity) of BAV stenosis patients with and without ascending dilatation belonged to the TGF- $\beta$ pathway, to the MMP family, and to the players of anti-oxidant protection. BAV patients were also distinguished according to valve morphotype, i.e. right-left coronary fusion (RL) and right-noncoronary fusion (RN). Aortic wall samples from TAV stenosis patients and organ donors were also included in study for comparison. Serum samples for ELISA quantification of gene product concentrations were taken from the same groups of patients and from healthy subjects. Correlations were analysed between pairs of RT-PCR results and between gene expression data and ELISA results. The associations with age, hypertension, medications were also investigated. Results: Even in BAV stenosis patients without overt aortic dilatation, significant changes of gene expression compared to control aorta were observed, including increased expression of TGF- $\beta 1$ and MMP-2, decreased expression of MMP-14 and endoglin, and lack of the increase in superoxyde-dismutase-3 (SOD3) expression that was observed in TAV stenosis patients. The expression levels of different genes showed morphotypespecific correlations. Similarly, RL-BAV patients and RN-BAV patients showed distinctive correlations between expression levels of some genes and serum concentration of the respective products: this included a significant correlation of both MMP-2 and TGF- $\beta 1$ gene expression with serum concentration of MMP-2 in the RL-BAV group, and with serum SOD3 in RN$\mathrm{BAV}$. Of note, only in RL-BAV patients the use of angiotensin receptor blockers was associated with significantly lower concentration of serum TGF- $\beta 1$. Conclusions: This study suggested differences between valve morphotypes in the pathogenetic mechanisms underlying aortic wall maladaptive remodelling. Different molecules could be tested as possible biomarkers in RL and $\mathrm{RN}$ types of BAV.

\section{Calcific Aortic Valve Disease after Thoracic Radiation Therapy: An Immunohistochemical Study}

\author{
J.W. van Rijswijk ${ }^{1}$, E.S. Farag ${ }^{1}$, O.J. de Boer ${ }^{2}$, A.C. van der Wal ${ }^{2}$, \\ B.A.J.M. de $\mathrm{Mol}^{1}$, J. Kluin ${ }^{1}$ \\ ${ }^{1}$ Department of Cardiothoracic Surgery, Academic Medical \\ Center, Amsterdam, ${ }^{2}$ Department of Pathology, Academic \\ Medical Center, Amsterdam, Netherlands
}

Objective: Radiation-associated aortic valve stenosis is frequently seen as a late sequela after thoracic radiotherapy. Although the clinical relationship between thoracic radiotherapy and valvular dysfunction has been established, the mechanism behind the accelerated aortic valve stenosis remains unclear. The aim of this study was to determine whether increased inflammatory cell infiltration is present in aortic valves after radiotherapy and whether radiotherapy induces leaflet fibrosis and calcification. Methods: Valve specimens from 44 patients were obtained after surgical aortic valve replacement. 28 patients had previously undergone thoracic or mediastinal radiation therapy for breast cancer $(n=22)$ or (non) Hodgkin lymphoma $(\mathrm{n}=6)$, respectively. 16 patients who had never undergone thoracic radiotherapy were included as control. The cellular composition of the valve leaflets was characterized by immunohistochemistry for (CD3-positive) T-cells, (CD20positive) B-cells and (CD68 or CD163-positive) macrophages. Histological stainings were used to estimate the amount of collagen and mineralization. Results: Mean age at valve replacement was lower in the mediastinal radiation group $(61.7 \pm 7.3)$ than in the control $(74.1 \pm 8.0)$ and thoracic-radiation group (73.0 \pm $7.2 \mathrm{yrs}, \mathrm{p}<0.01)$. Mean time from radiotherapy to valve replacement was longer after mediastinal radiotherapy $(31.5 \pm 4.6 \mathrm{yrs})$ than after thoracic radiotherapy $(18.1 \pm 9.3$ yrs, $\mathrm{p}=0.0023)$. Expression of CD68-positive macrophages was significantly lower in the thoracic-radiation group ( $\mathrm{p}=0.0064$ ) when compared to the controls. For CD3, CD20 and CD163, no changes in staining were detected between the groups. Relative collagen content was increased in the mediastinal $(\mathrm{p}<0.001)$ and the thoracic groups $(\mathrm{p}=$ $0.014)$. Aortic valves exposed to mediastinal radiotherapy contain $12 \pm 8 \%$ calcified tissue, compared to $20 \pm 8 \%$ after thoracic radiotherapy ( $p=0.034)$ and $24 \pm 14 \%$ in the control group ( $p=0.07)$. Conclusions: The present study is the first to demonstrate the composition of the inflammatory infiltrate and extracellular matrix in the irradiated aortic valve. Thoracic radiotherapy does not result in long-term increases in inflammatory response in human aortic valves. Irradiated aortic valves show the same degree of late $\mathrm{T}$ and $\mathrm{B}$ lymphocyte infiltration as control valves, while macrophages were found decreased after thoracic radiation. Furthermore, patients having undergone mediastinal radiotherapy develop aortic valve stenosis earlier in life, but their valves contain more collagen and less calcified tissue than stenotic aortic valves after thoracic radiotherapy. 


\section{A Role for Iron in Aortic Valve Stenosis}

Andres Laguna-Fernandez ${ }^{1}$, Miguel Carracedo ${ }^{1}$, Gregoire Jeanson ${ }^{1}$, Edit Nagy², Per Eriksson', Giuseppina Caligiuri ${ }^{3}$, Anders Franco-Cereceda ${ }^{1}$, Magnus Back ${ }^{1}$

${ }^{1}$ Karolinska Institutet, Stockholm, ${ }^{2}$ Karolinska University Hospital, Stockholm, Sweden; ${ }^{3}$ Institut National de la Santé et de la Recherche Médicale U1148, Paris, France

Objective: Aortic valve stenosis (AS) is the most common valvulopathy and a leading cause of cardiovascular mortality. Inflammation, extracellular matrix (ECM) remodeling and calcification are necessary steps for the narrowing of the valve and the consequential obstruction in the cardiac outflow. Although it has been recently shown that intraleaflet hemorrhage is associated with disease progression, the possible pathological role of iron accumulation in the valve is not known. This study aimed to identify mechanisms linking the iron content in human aortic valves with pathological changes associated with AS and to investigate the role that valve interstitial cells (VIC) play in this context. Methods: Human aortic valves were obtained from 51 patients undergoing aortic valve replacement surgery. Histological characterization of iron positivity was analyzed in relation to gene expression and clinical parameters. In addition, primary VIC cultures were established and used for iron detection, gene and protein expression studies, and the evaluation of VIC proliferation and elastin production. Results: A larger proportion of calcified tissue stained positive for iron compared with non-calcified tissue $(60.5 \%$ vs. 27.6 ; $\mathrm{P}<0.05)$. Multivariate analyses showed that the degree of iron accumulation was associated with the percentage of valve calcification and with an increased expression of RANK ligand, Osteopontin and MMP9; genes that promote ECM remodeling and calcification. In addition, we demonstrated that pathways with a major impact on AS (TNF- $\alpha$ and TGF- $\beta$ pathways), regulate the expression of the iron transporters DMT1 and FPN1 in VIC. Quantitative iron measurements revealed that the intracellular iron concentration of VIC increased progressively $-34 \pm 1.2,43 \pm 2.5$ and $52 \pm 6.1 \mathrm{nmol} \mathrm{Fe} / \mathrm{mg}$ protein $(\mathrm{P}<0.05)$ - after 3, 5 and 7 days of incubation in an iron-enriched environment. Finally, we showed that iron promoted an increase in VIC proliferation $(132 \pm 25.8 \% ; \mathrm{P}<0.05)$ and a decrease in elastin production $(47 \pm 18.7 \%$; $\mathrm{P}<0.05)$. Conclusions: Iron, which may accumulate in the aortic valve by means of intraleaflet hemorrhages, may be taken up by VIC in a pro-inflammatory environment and actively contribute to VIC proliferation, ECM remodeling and calcification. These findings suggest that iron uptake by VIC may be an important factor favoring AS progression.

\section{Consequences of Elevated Serotonin in Angiotensin-II-Induced Hypertensive Mice}

\section{Jessica Morales, Nancy Diaz, Kartik Balachandran}

University of Arkansas, Fayetteville, AR, USA

Objective: Elevated serotonin is known to cause valvulopathy. The interaction between serotonin and elevated blood pressure has not been explored in depth in the context of valvular disease. Our earlier work pointed to several serotonin receptor subtypes as sensitive to mechanical stress. We thus hypothesized that increased serotonin would result in accelerated progression toward valve dysfunction when blood pressure was elevated. Methods: C57/ BL6J wildtype mice were divided into four groups ( $\mathrm{n}=8$ per group) and subcutaneously implanted with osmotic pumps containing: PBS (control), 5HT (2.5 ng/kg/min), Ang-II (400 ng/kg/min), and $5 \mathrm{HT}+$ Ang-II (combination). Blood pressure of the mice was monitored using the tail cuff method. Echocardiography was performed on the mice before surgery and every week thereafter to assess fractional shortening and ejection fraction. After three weeks, the mice were sacrificed and their hearts excised, embedded and sectioned for analysis via histology and immunohistochemistry. Blood serum was collected for further analysis. In separate experiments, valve interstitial cells (VICs) were directly stimulated with $5 \mathrm{HT}$, Ang-II or both and assayed for cellular contractility and collagen remodeling. Results: See table 1 . After three weeks, average systolic blood pressure was significantly increased in the 5HT, Ang-II and combination groups compared to control. Analysis of blood serum verified elevated systemic levels of 5HT and Ang-II as expected based on treatment group. H\&E staining demonstrated thicker leaflets in the combination groups, suggesting a more aggressive remodeling process. Picrosirius red image analysis showed that the Ang-II and combination groups had the largest proportion of thick collagen fibers. Echocardiographic analysis demonstrated significantly increased ejection fraction and fractional shortening in Ang-II and the combination groups. VIC cellular contractility and collagen gene expression was highest for the combination treatment compared to all other groups, suggesting increased VIC activation and remodeling. Conclusions: Our results suggest that the combination of Ang-II and 5HT had the greatest effect on cardiac function and valve remodeling. We are currently analyzing markers for $5 \mathrm{HT}$ receptor signaling to understand the dominant mechanisms for these reported observations.

Table 1. (for Abstract of Kartik Balachandran)

\begin{tabular}{lcccc}
\hline & PBS (control) & 5HT & Ang-II & 5HT + Ang-II \\
\hline Systolic bp, mm Hg & $120.6 \pm 10.3$ & $140.2 \pm 18.2^{*}$ & $142.6 \pm 25.9^{*}$ & $145.1 \pm 20.4^{*}$ \\
Leaflet thickness, mm & $0.043 \pm 0.008$ & $0.047 \pm 0.011$ & $0.051 \pm 0.031^{*}$ & $0.059 \pm 0.023^{*}$ \\
Ejection fraction (3 weeks) & $57.2 \pm 6.2$ & $59.1 \pm 7.5$ & $42.0 \pm 7.9^{*}$ & $40.8 \pm 6.2^{*}$ \\
\% thickest collagen fibers (from P-Red stains) & $6.8 \pm 1.5$ & $4.5 \pm 2.3$ & $8.8 \pm 1.8^{*}$ & $11.9 \pm 2.5^{*}$ \\
\hline
\end{tabular}

$* \mathrm{p}<0.05$ compared to control. 


\section{FHL2-RhoA Signalling Mediates Mechanical Regulation of Aortic Valve Interstitial Cell Osteogenic Differentiation}

\author{
Alan Y.L. Lam, Jan-Hung Chen, Zahra Mirzaei, Kuiru Wei, \\ Craig A. Simmons \\ University of Toronto, Toronto, ON, Canada
}

Objective: Calcified aortic valve lesions often include osteoblasts and form preferentially in the natively stiffer fibrosa side of the leaflet, suggesting roles for valve interstitial cell (VIC) osteogenesis and matrix biomechanics in valve disease. Osteoprogenitor differentiation is mediated by FHL2 and modulated by matrix stiffness via RhoA. However, the roles of FHL2 and RhoA in mechanically-regulated VIC osteogenesis and valve disease are unknown. We hypothesize that matrix stiffness regulates RhoA-dependent FHL2 expression to direct VIC osteogenesis. Methods: The relevance of FHL2 in disease in vivo was investigated by immunostaining for FHL2 and the osteogenic transcription factor Runx 2 in pigs fed an atherogenic or normal chow diet for 6 months. In vitro experiments utilized primary porcine aortic VICs grown in osteogenic media on collagen-coated silicone substrates with elastic moduli mimicking soft disease-protected $(7 \mathrm{kPa})$ and stiff disease-prone $(23 \mathrm{kPa})$ valve regions. VIC osteogenesis was characterized by alkaline phosphatase activity and qPCR for Runx2. RhoA activation was measured by ELISA and manipulated pharmacologically or with adenovirus to examine the effect on FHL2 nuclear localization (assessed by immunostaining) and VIC osteogenesis. The role of FHL2 expression was probed with siRNA. Results: FHL2 and Runx2 were upregulated and co-localized in immunostained sclerotic aortic valves suggesting pathological involvement of FHL2 in vivo. In vitro, VIC osteogenesis was phenotypically characterized by the formation of ALP-positive aggregates. VICs grown on the stiff substrate formed more ALP-positive aggregates, had increased RhoA activation, and increased FHL2 nuclear localization (all p < 0.05). Exogenously increasing RhoA activation enhanced osteogenic aggregate formation and up-regulated Runx2 expression, while suppression of RhoA activity or siRNA knockdown of FHL2 each independently reduced osteogenic aggregate formation (all $\mathrm{p}<0.05$ ), confirming roles for RhoA and FHL2 in VIC calcification. Increasing or decreasing RhoA activation resulted in increased or decreased FHL2 nuclear localization, respectively, while simultaneous RhoA activator and FHL2 siRNA treatment reduced osteogenic aggregate formation compared to RhoA activator alone (all $\mathrm{p}<0.05)$, indicating FHL2 operates downstream of RhoA. Conclusions: VIC osteogenic differentiation in vitro is mechanically modulated via a pathway involving FHL2 operating downstream of RhoA. This signalling axis may be a target to disrupt valve calcification.

\section{Deciphering the Role of Oxidized Lipoproteins in Regulating CAVD Pathobiology}

Ana M. Porras, Kristyn S. Masters

University of Wisconsin-Madison, Madison, WI, USA

Objective: The deposition of oxidized lipids is a hallmark of calcific aortic valve disease (CAVD), but its relationship to other CAVD features remains unclear. For example, while proteoglycan (PG) enrichment and oxLDL deposition occur early in CAVD, it is not known whether this is stimulated by PGs encouraging the retention of oxLDL or high levels of circulating oxLDL causing VICs to upregulate PG secretion (or a mixture of both). This study sought to examine the role of oxLDL in regulating cellular events related to not only PG enrichment, but also subsequent CAVD features such as angiogenesis. Methods: Porcine VICs were cultured in media containing $0.1 \%$ delipidated serum supplemented with LDL or oxLDL and then assessed for proliferation, apoptosis, myofibroblastic gene expression, extracellular matrix deposition, and production of angiogenic factors; conditioned media from the LDL/oxLDL-treated VIC cultures were applied to endothelial cell (EC) cultures to examine angiogenic sprouting. The ability of VICs to oxidize LDL was also assessed, as was oxLDL binding to substrates presenting different PG compositions. Results: Compared to the untreated control, oxLDL treatment did not alter VIC proliferation or apoptosis, but did reduce aSMA gene expression by VICs. Moreover, VICs treated with oxLDL deposited more heparan sulfate proteoglycans and collagen type I relative to the control. Likewise, an increase in the secretion of fibroblast growth factor-2 and vascular endothelial growth factor was observed following exposure to oxLDL, while the production of platelet-derived growth factor was unaffected. Media conditioned by VICs exposed to oxLDL induced extensive EC spheroid sprouting, while VIC treatment with unmodified LDL had no effect on the production of any of these proteins or EC sprouting. VICs exhibited only a modest ability to perform LDL oxidation themselves. Conclusions: The results of this study indicate that oxLDL is capable of inducing many of the phenotypic changes associated with CAVD progression. Specifically, these findings suggest that deposition of circulating oxLDL may fuel both pathological extracellular matrix remodeling and angiogenesis in the valve. Deciphering the sequence of pathological events in CAVD will help us to not only better understand disease etiology, but also refine approaches to develop targeted treatments. 


\section{Mutations in DZIP Cause Mitral Valve Prolapse}

David Milan ${ }^{1}$, Katelin Toomer ${ }^{2}$, Dan Fraher ${ }^{1}$, Michael Talkowski ${ }^{1}$, Ronen Durst ${ }^{3}$, Francesca Delling ${ }^{4}$, Robert Levine ${ }^{1}$, Susan Slaugenhaupt ${ }^{1}$, Russell Norris ${ }^{2}$

${ }^{1}$ Massachusetts General Hospital, Boston, MA, ${ }^{2}$ Medical University of South Carolina, Charleston, SC, USA; ${ }^{3}$ Hadassah Hebrew University Medical Center, Jerusalem, Israel; ${ }^{4}$ Beth Israel Deaconess Medical Center, Boston, MA, USA

Objective: Mitral valve prolapse (MVP) affects $2.5 \%$ of the population, predisposing to endocarditis, congestive heart failure, and need for valve surgery. Despite clear heritability, the details of MVP genetics remain largely unknown. We studied a large family segregating MVP in order to better understand the genetics of MVP. Methods: We used Sanger sequencing of genomic DNA from family members, Western blot from immortalized lymphblast lines to examine Dzip protein levels, immunohistochemistry of acetylated and gamma tubulin to identify cilia, zebrafish morpholino knockdown and assessment of cardiac AV valve function with high speed videography, murine knockout with immunohistochemistry and microscopic examination of the heart valves. Results: The MVP family had been previously mapped to the long arm of chromosome 13, but capture sequencing of all coding regions in the linked interval had been unrevealing. Immunohistochemistry of the recently described DCHS1 heterozygous knockout model of MVP revealed loss of cilia in valve interstitial cells. Examination of the linked Chromosome 13 interval revealed DZIP as a cilia pathway candidate gene. Sanger sequencing of affected individuals in this cohort revealed a mutation DZIP1 S70R that segregates with MVP. Expression analysis using Western blots in lymphoblast lines revealed reduced protein expression of Dzip1 in affected individuals compared to phenotype negative family members. Zebrafish knockdown of dzip showed increased atrioventricular regurgitation, and the valve specific knockout mouse shows reduced cilia length and mitral valve specific defects including increased leaflet thickness and length. Conclusions: These data support a role for DZIP in ciliogenesis in the heart valve during development and demonstrate that loss of DZIP function leads to mitral valve prolapse.

\section{A Latent TGF- $\beta$ Binding Protein 2 Mutation Segregates with Mitral Valve Prolapse in a Large Pedigree}

Amichai Gutgold, Ronen Durst, Shoshana Shpitzen, Dona Zfat, Gil Leibowitz, Eran Leitersdorf, Ronen Beeri, Chaim Lotan, Dan Gilon

Hadassah Medical center, Jerusalem, Israel

Objective: MVP (Mitral Valve Prolapse) is a prevalent valve defect, but the pathophysiolical mechanisms leading to the development of the disease are not well known. As MVP has a familial association, we aimed to find a gene linked to MVP phenotype in a family with a presumed autosomal-dominant inheritance. Methods: In a 3 generations Ashkenazi-Jewish family, 6 out of 25 examined family members had echocardiographic criteria for MVP, and 2 others had a thicken mitral valve not fulfilling these criteria. We used Next-generation sequencing, on 4 affected family members, and identified coding sequence variations shared by all. Next, we tested these in the rest of the family members for segregation with the trait. Results: Next generation sequencing raised 300 gene mutations common to all 4 affected. Out of these, 9 most probable genes, according to several factors, as type of mutation and mutation frequency were sequenced for all family members. 7 genes didn`t show segregation, and one more seemed improbable because the mutation was found in all the tested DNA samples. A single $\mathrm{C}$ to $\mathrm{T}$ nucleotide substitution mutation in LTBP2 (Latent TGF- $\beta$ binding protein 2 ) gene, located in chromosome 14, was identified as a probable and suitable gene in linkage analysis tests. Conclusion: We found that a mutation in LTBP2 gene might be responsible for a non-syndromic MVP in an Ashkenazi-Jewish family. LTBP2 is a protein similar to the Fibrillin 1 protein, which was identified to be the defective protein in Marphan syndrome. The LTBP2 protein is assumed to have mainly structural properties, along with TGF- $\beta$ regulation activity. A defect in both of these functions, might lead to a defect in connective tissue development and regeneration, underlying the basic pathology of MVP. Further research of prevalence of this mutation in familial MVP, and the functional significance of the identified mutation must be carried out in order to understand its exact role.

\section{Severe Aortopathy Is Specific to Regions of Elevated Wall Shear Stress in the Human Bicuspid Aorta}

\author{
David G. Guzzardi ${ }^{1}$, Alex J. Barker ${ }^{2}$, Pim van Ooij ${ }^{3}$, Sean Kang ${ }^{1}$, \\ S. Chris Malaisrie ${ }^{2}$, Jyothy J. Puthumana ${ }^{2}$, Darrell D. Belke ${ }^{1}$, \\ Holly E. Mewhort ${ }^{1}$, Daniyil A. Svystonyuk', Subodh Verma ${ }^{4}$, \\ Jeremy Collins'2, James Carr' ${ }^{2}$, Robert O. Bonow ${ }^{2}$, Michael MarkP ${ }^{2}$, \\ James D. Thomas ${ }^{2}$, Patrick M. McCarthy ${ }^{2}$, Paul W. Fedak ${ }^{1}$
}

${ }^{1}$ University of Calgary, Calgary, $A B$, Canada; ${ }^{2}$ Northwestern University, Chicago, IL, USA; ${ }^{3}$ Academic Medical Center, Amsterdam, Netherlands; ${ }^{4}$ University of Toronto, Toronto, ON, Canada

Objective: Bicuspid aortic valve (BAV) disease predisposes patients to medial elastic fiber degeneration, weakening and aneurysm formation of the ascending aorta, collectively known as bicuspid aortopathy. Although previous work has demonstrated severe aortopathy in aortic wall subjected to elevated wall shear stress (WSS), whether these changes are specific to these regions of elevated WSS is unclear. This study builds on previous work by comparing tissue from multiple regions of normal WSS throughout the human BAV aorta to regions of elevated WSS as determined by $4 \mathrm{D}$ flow MRI. Methods: Aortic tissue (mean aortic diameter $4.6 \pm 0.3$ $\mathrm{cm}$; range: $4.0-5.2 \mathrm{~cm})$ was collected from BAV patients $(\mathrm{N}=17)$ undergoing ascending aortic surgery and who received pre-operative 4D flow MRI. Regions of 'normal' and 'elevated' WSS were generated relative to the $95 \%$ confidence interval for normal physiological WSS values of a tricuspid aortic valve population. Tissue was evaluated for elastin fiber content and architecture (fiber thickness and fiber-to-fiber distance), and mediators of extracel- 
lular matrix degradation (TGF $\beta$-1, MMPs and TIMPs). Multiple regions of normal WSS were averaged within each aorta and compared to regions of elevated WSS. Results: Mean elastin fiber content $(\mathrm{p}=0.018)$ and thickness $(\mathrm{p}=0.002)$ were significantly decreased, and TGF $\beta-1$ concentrations increased $(\mathrm{p}=0.083)$ in regions of elevated WSS compared to an average of all regions of normal WSS in the same aortas. Importantly, TGF $\beta-1$ concentrations negatively correlated to elastic fiber content only in regions of elevated WSS $(\mathrm{r}=-0.548 ; \mathrm{p}=0.053)$ and not in adjacent tissue regions subjected to normal WSS. Conclusions: For the first time, we show that the aortopathy (elastic fiber degeneration and molecular mediators) in BAV aortas is specific to regions of elevated WSS compared to multiple regions of normal WSS in the same BAV aortas. Importantly, these results extend the observation that non-invasive hemodynamic mapping using 4D flow MRI may be a useful tool in designing patient-specific resection strategies, and further implicate hemodynamics in the expression of human bicuspid aortopathy.

\section{Warfarin Induces Calcification of the Aortic Valve via ERK1/2}

\section{Neil Venardos, Michael J. Weyant, Thomas B. Reece, Xianzhong Meng, David A. Fullerton}

University of Colorado, Aurora, CO, USA

Background: Approximately 3 million people in the United States currently take warfarin. This drug has been identified as an instigator of calcification in blood vessels, and recent clinical evidence associates warfarin usage with calcification of the aortic valve. However, potential mechanisms for this phenomenon are poorly understood. Aortic valve interstitial cells (AVICs) have been implicated in the pathogenesis of aortic stenosis. Upon appropriate stimulation, these cells adopt an osteoblast-like phenotype, producing bone-forming proteins. We therefore hypothesized that warfarin induces an osteogenic phenotypic change in AVICs. Our purposes were to determine: (1) the effect of warfarin on AVIC osteogenic protein expression and (2) the signaling pathway by which this effect is mediated. Methods: Human AVICs were isolated from normal aortic valves from explanted hearts of four patients undergoing cardiac transplantation. Cells were treated with warfarin $(10 \mu \mathrm{M})$, heparin $(0.1,1.0,10 \mathrm{U} / \mathrm{mL})$, and dabigatran $(10,500,1000 \mathrm{ng} / \mathrm{mL})$ for 48 hours. In separate experiments, cells were treated with warfarin for 48 hours with or without extracellular signal-related kinase $1 / 2$ (ERK1/2) inhibitor PD98059 $(5,10$, and $25 \mu \mathrm{M})$. After these treatments, cell lysates were analyzed for levels of osteogenic protein runt-related transcription factor-2 (Runx-2) (immunoblotting); Runx-2 is necessary for osteogenesis. $\beta$-actin served as a loading control. Statistics were by Mann-Whitney U test $(\mathrm{p}<0.05)$. Results: Warfarin, but not dabigatran or heparin, significantly increased Runx-2 expression in isolated human AVICs $(* \mathrm{p}<0.05)$. Importantly, this increase was prevented by inhibition of ERK $1 / 2$ signaling (see figure $1,{ }^{*} \mathrm{p}<0.05$ ). Conclusions: Warfarin induces an osteogenic transformation in isolated human AVICs, which is mediated by ERK1/2 signaling. These osteogenic changes were not observed with other anticoagulants such as dabigatran or

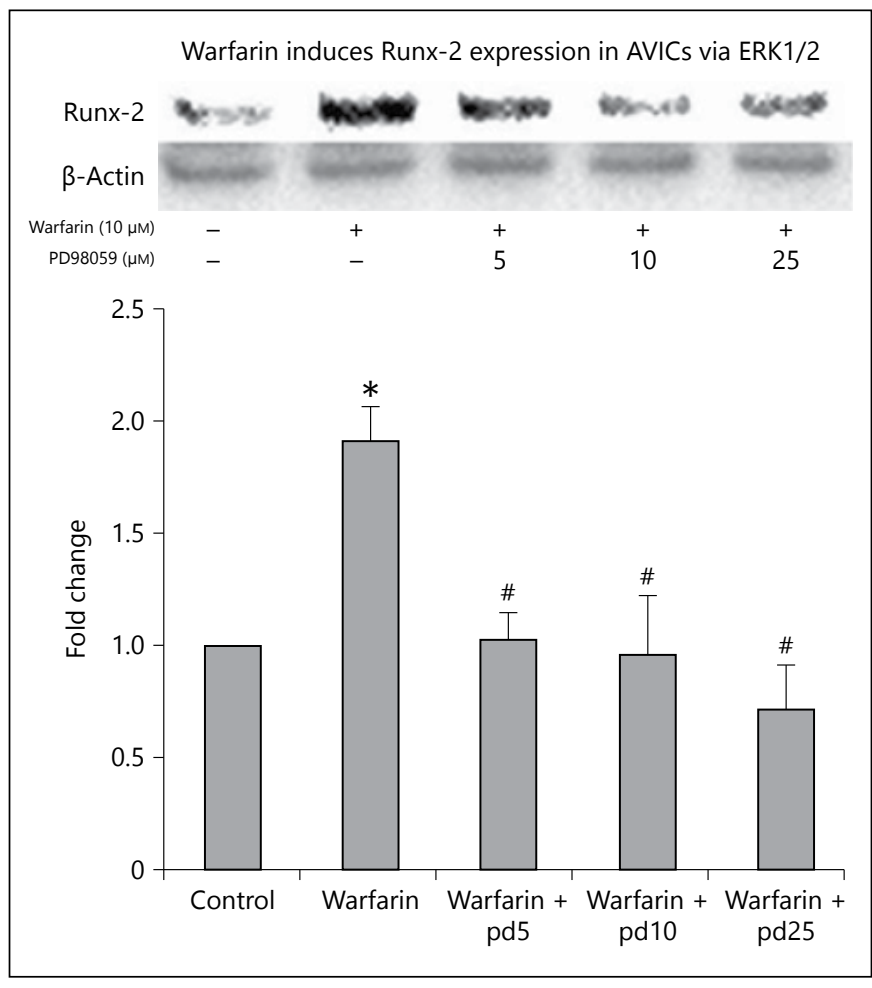

Fig. 1. (for Abstract of Neil Venardos).

heparin. These results implicate warfarin in aortic valve calcification and highlight a potential mechanism for warfarin-induced aortic stenosis.

\section{Altered Valve Interstitial Cell Architecture Results in Pathological Cell Function and Phenotype}

\section{Ishita Tandon, Atefeh Razavi, Prashanth Ravishankar, Kartik Balachandran}

University of Arkansas, Fayetteville, AR, USA

Objective: It is generally known that cell architecture and shape is altered in diseased tissues compared to healthy ones. However, the role of valve interstitial cell (VIC) structure, shape and architecture in regulating or predicting overall balance between health and disease in the context of the valve is not well understood. The aim of this study was thus to elucidate this relationship between cell shape, function and pathobiology, in terms of VIC contractile output, metabolism and phenotype. Methods: Valve interstitial cell (VIC) monolayers with defined cellular architectures were engineered via constraining cells to micropatterned fibronectin lines $(10,20,40,60$ and $80 \mu \mathrm{m}$ wide) (fig. 1a). Samples were cultured for 2 days in either normal DMEM or an osteogenic DMEM (containing added ascorbic acid, $\beta$-glycerophosphate and dexamethasone). Cellular contractility was quantified using a valve thin film assay and phenotype analyzed via western blotting 


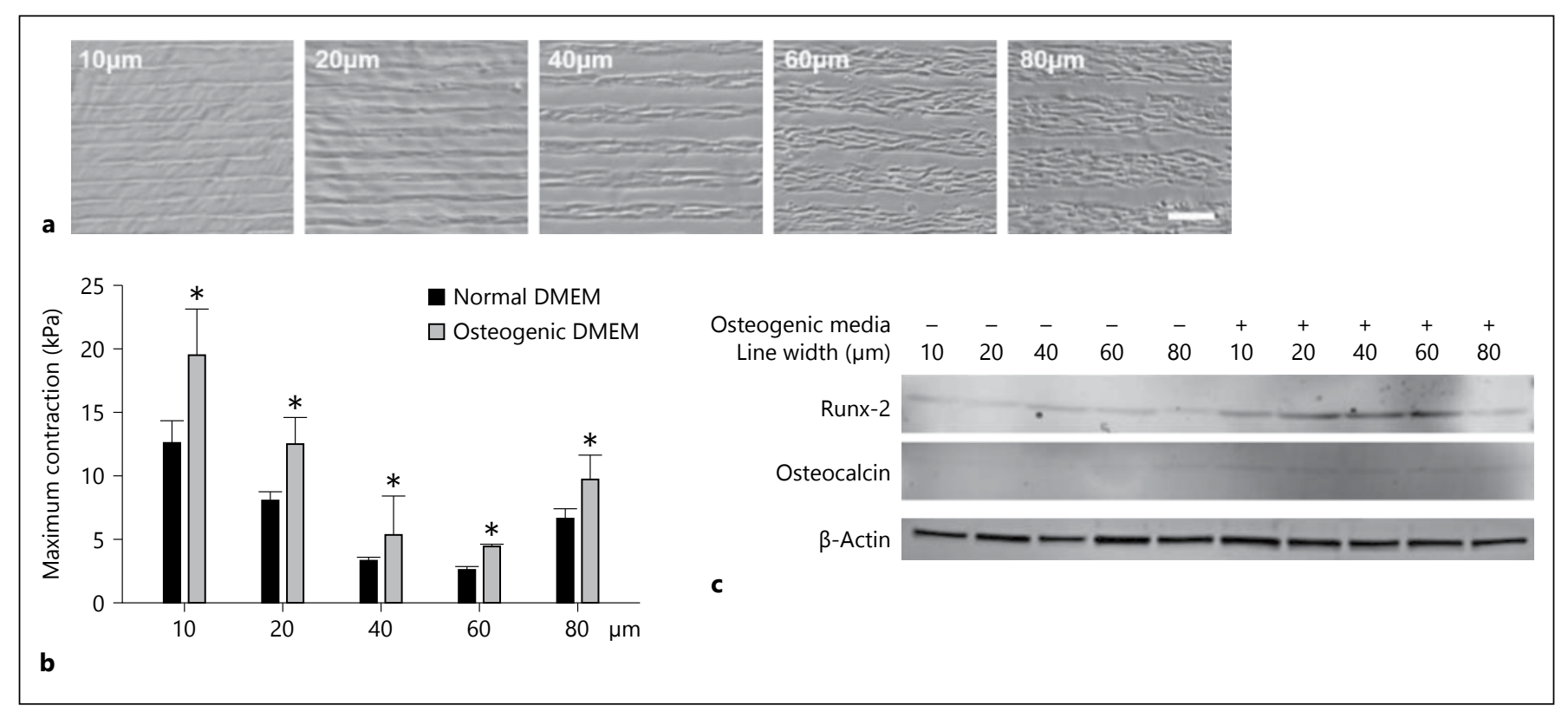

Fig. 1. (for Abstract of Kartik Balachandran).

and alkaline phosphatase activity assay. Imaging of endogenously fluorescent NADH and FAD was used to quantify cellular metabolic activity. Results: In all pattern widths, cells displayed wellaligned actin architecture, with maximum cell and nuclear elongation occurring for the $10 \mu \mathrm{m}$ pattern width. Cell elongation and actin alignment was increased in the osteogenic DMEM. Contractility was highest for the most elongated cells $(10 \mu \mathrm{m})$, but was also increased in cells on the widest pattern $(80 \mu \mathrm{m})$, suggesting a role for both cell elongation and cell-cell contact in regulating contractility (fig. 1b). Contractility was significantly higher in samples treated with osteogenic DMEM (fig. 1b), and was correlated with increased Runx2 and osteocalcin expression (fig. 1c) in the more elongated cells. FAD/NADH redox ratio was statistically unchanged in all pattern widths that were cultured in normal DMEM, but was significantly reduced in the most elongated cells $(10 \mu \mathrm{m})$ in samples cultured in osteogenic medium, suggesting lower oxidative phosphorylation and a likely switch toward cell differentiation. Conclusions: Overall, our results suggest that altered cellular architecture altered VIC contractility, metabolism and phenotype. We also report that cell elongation predicted accelerated progression toward disease.

\section{Role of Tissue Factor Pathway Inhibitor-2 (TFPI-2) in Aortic Valve Calcification}

\begin{abstract}
Rena Frank ${ }^{1}$, Maxime Bucci ${ }^{1}$, Antoine Rauch ${ }^{2}$, Rodrigo Lorenzi ${ }^{1}$, Mickael Rosa ${ }^{1}$, Anais Arbesu Y. Miar ${ }^{1}$, Madjid Tagzirt ${ }^{1}$, Francis Juthier ${ }^{2}$, Ramadan Jashari ${ }^{3}$, Andre Vincentelli ${ }^{2}$, Eric Van Belle ${ }^{2}$, Bart Staels', Delphine Corseaux ${ }^{1}$, Annabelle Dupont ${ }^{2}$, Sophie Susen ${ }^{2}$, Christophe Zawadzki ${ }^{2}$

${ }^{1}$ European Genomic Institute for Diabetes (EGID), FR3508, UNIV LILLE, Inserm UMR 1011, Institut Pasteur de Lille, F-59000, Lille, ${ }^{2}$ European Genomic Institute for Diabetes (EGID), FR3508, UNIV LILLE, Inserm UMR 1011, Institut Pasteur de Lille, F-59000, Cardiovascular and Pulmonary, and Haematology Department, CHRU de Lille, Lille, France; ${ }^{3}$ European Homograft Bank, c/o Military Hospital Queen Astrid, Brussels, Belgium
\end{abstract}

Objective: Calcific aortic valve disease (CAVD) is widely accepted as an " atherosclerostic-like process » characterized by deregulated fibrosis of the valve leaflets, angiogenesis and calcification involving valve interstitial cells (VIC). Tissue Factor Pathway Inhibitor-2 (TFPI-2), a Kunitz-type serine proteinase inhibitor expressed in atherosclerotic plaques, presents anti-inflammatory properties resulting of metalloproteinases (MMPs) inhibition. The aim of this work was to study the expression of TFPI- 2 in valves and determine its relationships with MMPs and osteogenic markers in the process of calcification. Methods: The expression of TFPI- 2 was determined in 3 control and 6 fibrocalcific aortic valves by histology to determine its location with calcifications. Its association with MMPs (MMP-1, 2, and their inhibitor TIMP-1) and osteogenic markers (alkaline phosphatase (ALP), Runx2, osteonectin (SPARC) and osteopontin (OPN)) expression was studied by qPCR/ELISA in VIC obtained from 18 fibrocalcific valves in standard (SM) vs. procalcifying medium culture (PM). These 
markers were also studied in VIC overexpressing TFPI-2 after plasmid transfection vs. non-transfected VIC. Results: TFPI- 2 immunostaining was weak on the ventricular side of control valves in comparison to fibrocalcific ones in which TFPI- 2 expression was heterogeneous but strong around calcifications. TFPI-2 expression was significantly higher in VIC obtained from fibrocalcific valves in comparison to control ones $(p<0.05)$. In VIC culture, TFPI-2 expression was significantly increased in PM vs. SM and was time-dependent. The PM condition led to a decrease in MMP$1,-2$ and OPN expression, while ALP mRNA levels increased. The expression of TFPI-2 mRNA was strongly induced in transfected VIC vs. non-transfected ones (x4500-fold in SM and x500-fold in PM). The same trend was observed with TFPI- 2 antigen. TFPI2-overexpressing VIC expressed lower levels of MMP-1, -2, TIMP1 and all osteogenic markers mRNA in SM. In PM, VIC transfection induced a decrease in ALP and SPARC expression. Assessment of antigen/activity by ELISA confirmed these findings at the protein level. Conclusion: TFPI-2 expression could be increased by procalcifying stimuli in fibrocalcific valves and interact in the calcification process by modulating the expression of MMPs and osteogenic markers in CAVD.

\section{Cyclic Strain Inhibits Valve Endothelial Cell Vasculogenic Network Formation Independent of Piezo 1}

\section{Christopher A. Arevalos, Claudia Iriondo, Jonathan Berg, Kathryn Jane Grande-Allen}

Rice University, Houston, TX, USA

Objective: Valve endothelial cells (VECs) undergo unique mechanical stimulation compared to peripheral vascular endothelial cells. During the progression of CAVD, thickening and hardening of the aortic valve alters the mechanical environment of the VECs.
Angiogenesis is a known important contributor to the progression of CAVD in the normally avascular aortic valve. However, it is unknown how mechanical strain contributes to maintain the avascularity of the aortic valve or how disturbance of the normal mechanical strain contributes to the progression of CAVD. Therefore, this study examined the effect of mechanical stimulation, and the role of Piezol, a mechanically regulated $\mathrm{Ca}+$ importer, in regulating the response of valve endothelial cells to uniaxial strain in an angiogenesis matrigel model. Methods: CD31+ porcine aortic VECs were uniaxially stretched at $0.5 \mathrm{~Hz}$ at $0 \%, 5 \%$, or $10 \%$ strain for 24 hours using the Flexcell unixaxial strain system. The VECs were then seeded on matrigel, and the resultant vasculogenic tubule like networks were then quantified after 7 hours. Piezol was silenced using an siRNA treatment for 48 hours, cyclically stretched for 24 hours, and tested in the matrigel model. Results: Network analysis revealed a strong pattern of strain to decrease the propensity of VECs to form networks. Quantification showed a significant decrease in junction and vessel segment density when strained at $5 \%$ or $10 \%$. Lastly, silencing of Piezo 1 significantly decreased network formation compared to a scrambled siRNA treatment, but showed no significant effect between cyclic stretch or static cultures. Conclusions: These results indicate that a mechanically disrupted valve, such as those calcified by CAVD, are more prone to worsening disease conditions through increased angiogenesis. These results suggest that Piezo1 has a role in regulating angiogenesis signaling in VECs, but this effect was independent of stretching in this study. This additional understanding of the mechanisms underlying VEC vessel formation and CAVD development can help pave the way for the development of an interventional treatments that target these valve specific mechanisms.

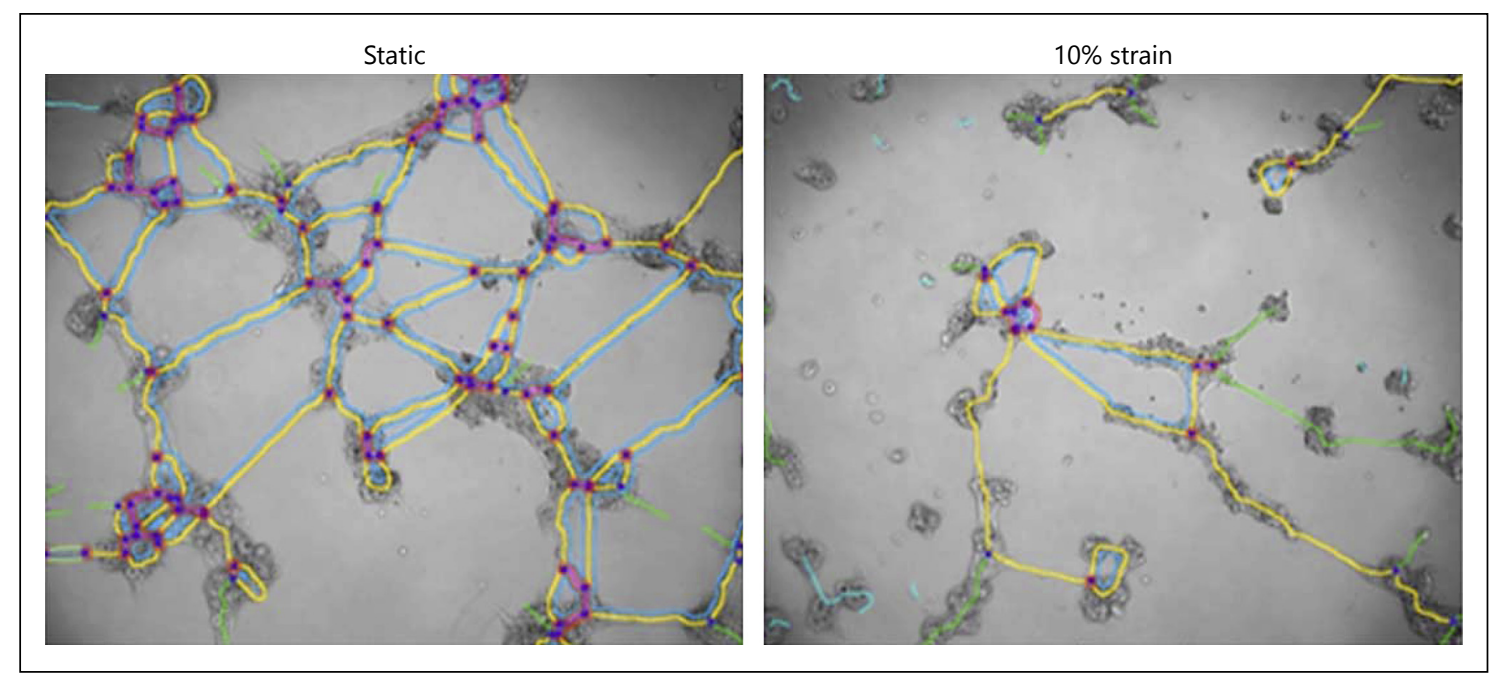

Fig. 1. (for Abstract of Christopher A. Arevalos). 


\section{Decreased Systolic Load Suppresses Key Genes and Regulators Involved in Fetal Tricuspid Valve Development}

Sarah Walcott-Sapp ${ }^{1}$, Herbert M. Espinoza' , Isa Lindgren', Samantha Louey', Sonnet Jonker', George D. Giraud ${ }^{2}$, Kent L. Thornbug ${ }^{1}$, Frederick Tibayan ${ }^{1}$

${ }^{1}$ Oregon Health and Science University, Portland, OR, ${ }^{2}$ Portland VA Health Care System, Portland, OR, USA

Background: Adult cardiovascular disease has roots in fetal and neonatal life, but the developmental origins of valve disease remain unexplored. Inadequate deposition of structural matrix components creates vulnerability for valve degradation in adults, and the hemodynamic environment in utero seems to be critical for establishing normal valve composition. Increased systolic load during late gestation suppresses critical signaling molecules and extracellular matrix genes in aortic and mitral valves. The impact of decreased systolic load has not been determined. The aim of the current study was to investigate decreased systolic load and tricuspid valve development. Methods: Chronically instrumented fetal sheep (124 \pm 1 days gestation, term $=147$ days) received continuous intravenous infusions of enalaprilat $(345 \mu \mathrm{g} /$ day, 48 hours, $\mathrm{n}=$ 5 ) to decrease blood pressure; controls received an equivalent volume of Lactated Ringers $(n=5)$. Tricuspid valve tissue was harvested at post-mortem, and expression of signaling and extracellular matrix genes was measured with quantitative PCR and normalized to beta-actin. Results: Fetal blood pressure was lower in the enalaprilat group. Expression of microRNA-21, a mediator of shear stress effects, was suppressed in the enalaprilat group as were shear stress response genes endothelin-1 and klf-2. Cell proliferation and extracellular matrix genes BMP-4, twist, sox 9, collagen $1 a 1$, collagen 1a2, and collagen $2 a 1$ were also suppressed in the enalaprilat group. Conclusions: Reduced systolic load decreases the expression of regulators of tricuspid valve development in the fetus. These changes impact the structural composition of fetal cardiac valves and may have long term implications for valve compo-

Table 1. (for Abstract of Sarah Walcott-Sapp)

\begin{tabular}{lcc}
\hline & $\begin{array}{l}\text { Control, } \\
\text { mean } \pm \text { SEM }\end{array}$ & $\begin{array}{c}\text { Enalaprilat, } \\
\text { mean } \pm \text { SEM }\end{array}$ \\
\hline Day 0 aortic pressure & $41 \pm 1$ & $41 \pm 1$ \\
Day 2 aortic pressure & $40 \pm 1$ & $34 \pm 2^{*}$ \\
MicroRNA-21 & $1.1 \pm 0.2$ & $0.3 \pm 0.5^{*}$ \\
Endothelin-1 & $0.003 \pm 0.0006$ & $0.0015 \pm 0.0001^{*}$ \\
Klf-2 & $0.05 \pm 0.02$ & $0.01 \pm 0.001^{*}$ \\
BMP-4 & $0.05 \pm 0.009$ & $0.02 \pm 0.002^{*}$ \\
Sox 9 & $0.8 \pm 0.1$ & $0.3 \pm 0.04^{*}$ \\
Twist & $0.09 \pm 0.009$ & $0.05 \pm 0.01^{*}$ \\
Collagen 1a1 & $1.7 \pm 0.3$ & $0.8 \pm 0.2^{*}$ \\
Collagen 1a2 & $6.6 \pm 0.6$ & $3.4 \pm 0.4^{*}$ \\
Collagen 2a1 & $3.0 \pm 0.5$ & $1.7 \pm 0.3^{*}$ \\
\hline
\end{tabular}

$* \mathrm{p}<0.05$ versus control. sition and function. These data lend insight into the role of development in the pathogenesis of tricuspid valve disease in adulthood and suggest targets for pharmaceutical and tissue engineering interventions.

\section{Involvement of the Receptor-Type Tyrosine-Protein Phosphatase F Gene PTPRF, a Cell Adhesion-Like Molecule, in Mitral Valve Prolapse (MVP)}

Pauline Labbé, Antoine Rimbert, Antoine Jobbe-Duval, Simon Lecointe, Solena Le Scouarnec, Florence Kyndt, Thierry Le Tourneau, Jean-Jacques Schott, Jean Merot

Inserm U1087, Nantes, France

Objective: Our objectives were to identify MVP causative genes using familial approach, to assess MVP phenotype and to determine the molecular and cellular mechanisms involved in the pathology. Methods: Exome sequencing and Identity by Descent analysis (IBD) were used to identify shared genetic variants in the family. Phenotype was assessed by echocardiography. Expression profile of PTPRF was analyzed by RT-PCR in normal and pathological mitral valve tissue and PTPRF protein stability evaluated by western blotting after cycloheximide treatment in Hek293 cells transfected with PTPRF cDNA. Results: The family was identified after one member underwent valve surgery for Barlow-type MVP. Exome sequencing in 3 affected coupled to IBD analysis identified a rare variant MAFG (p.Ile328Met) in PTPRF gene. Out of $16 \mathrm{mu}-$ tated patients, $12(75 \%)$ had a MVP (7 bileaflet and 5 posterior leaflet MVP) and 3 (19\%) abnormal anterior coaptation. Mitral leaflets were elongated and significantly thickened. PTPRF alternative splicing gives rise to several isoforms in humans. The longest isoform encodes the LAR receptor involved in neuronal development and axon guidance. LAR is a repressor of cell migration and proliferation, participates in adherens junctions, modulates small GTPases activity by interacting with the Rho/Rac regulator Trio, inhibits EGF/FGF signaling and interacts with Heparan Sulfates Proteoglycans (HSPGs) involved in cell-cell and cell-matrix adhesion. PTPRF thus appeared as a good MVP causing candidate gene. However, the identified mutation (I328M) only targets the short isoform (sPTPRF) whose structure, expression profile and functions remain unknown. We showed here by RT-PCR that SPTPRF is expressed in human mitral valve together with the longest PTPRF isoform encoding LAR. Re-expression study in Hek293 cells revealed the sPTPRF isoform interacts with HSPGs and that the I328M mutation decreases the protein stability. Conclusions: Our results suggest a potential loss of function mutation in PTPRF-short isoform in autosomal dominant Barlow's disease. The precise role of the mutation on cell proliferation, cytokine (EGF-TGF $\beta$ ) signaling and cell matrix interaction remains to be elucidated. 


\section{Layer-Specific Changes in Collagen Fiber Architecture in Calcific Aortic Valve Disease}

\section{Heather N. Hutson, Paul Campagnola, Kristyn S. Masters}

University of Wisconsin-Madison, Madison, WI, USA

Objective: The aortic heart valve is composed of three distinct layers: the fibrosa, spongosa, and ventricularis. Disarray of the extracellular matrix (ECM) components comprising these layers is believed to be one of the earliest events in the development of calcific aortic valve disease (CAVD), and dysregulated ECM remodeling continues throughout disease progression. The current study aimed to characterize collagen dynamics in CAVD by quantifying collagen fiber organization and crosslinking across the individual ECM layers in healthy and diseased human valves. Such efforts to characterize ECM changes in CAVD are important for understanding disease-related changes in valve architecture, their impact on valve mechanics, and their relationship to other CAVD events. Methods: Human aortic valves were obtained from individuals between 51 and 77 years old with no evidence of valvular disease (healthy) and individuals who underwent valve replacement surgery due to severe stenosis (diseased). Second harmonic generation (SHG) images of valve sections were acquired and collagen fiber characteristics analyzed through the use of ctFIRE and CurveAlign software. Immunohistological staining and qRT-PCR were used to determine the expression of proteins known to crosslink collagen, such as PLOD1 and lysyl oxidase (LOX). Results: Analysis of collagen fibers revealed layer-specific differences in collagen fiber features between healthy and diseased tissues. Specifically, in the fibrosa, collagen fiber thickness did not change with disease, but the fibers became significantly shorter and less aligned. Meanwhile, in the diseased spongiosa, collagen fibers were significantly wider, as well as less aligned and more densely packed compared to collagen fibers in the healthy leaflet. Quantification of LOX gene expression indicated an increase in LOX in diseased tissues, and staining revealed that this increase was primarily localized to the fibrosa. Conclusions: This study reports layer-specific alterations to collagen fiber architecture during CAVD, with evidence of localized LOX expression. These ECM alterations likely contribute to leaflet stiffening in CAVD and may act as potential therapeutic targets.

\section{Calcification and Fibrosis Ratio in Stenosed Aortic Valve: The Impact of Patient Sex}

\author{
Louis Simard, François Dagenais, Philippe Pibarot, \\ Patrick Mathieu, Christian Couture, Siamak Mohammadi, \\ Sylvain Trahan, Sylvain Pagé, Philippe Joubert, \\ Marie-Annick Clavel
}

Quebec Heart and Lung Institute, Quebec, QC, Canada

Objective: Previous studies showed that, in aortic stenosis (AS), women present lower aortic valve calcium (AVC) load than men for the same hemodynamic AS severity. Since the major mechanisms involved in AS pathophysiology are calcification and fibrosis of the valve, we hypothesized that women would develop a more fibrotic pattern compared to men. Methods: We recruited 114 patients with preserved left ventricular ejection fraction (LVEF $\geq 50 \%$ ) who underwent a comprehensive Doppler-echocardiography and computed tomography (MDCT) within 3 months prior to surgical aortic valve replacement. Valvular fibrosis degree was determined using a semi-quantitative score obtained by hematoxylin-eosin staining on anatomic specimen. AVC was quantified both by Agatston method (MDCT) and Warren-Yong score. Men $(n=62)$ and Women $(n=36)$ were frequency-matched for age, body mass index (BMI), hypertension, renal disease, diabetes, and AS severity (peak jet velocity and indexed aortic valve area). Results: Patient mean age was $75 \pm 9$ years. Average peak aortic jet velocity was $4.1 \pm 0.8 \mathrm{~m} / \mathrm{s}$ and mean gradients were $41 \pm 18 \mathrm{~mm} \mathrm{Hg}$, both being similar between men and women $(\mathrm{p}=0.26$ and $\mathrm{p}=0.49$, respectively). AVC indexed to aortic annulus area was significantly lower in women compared to men ( $\mathrm{p}<0.0001)$, but valve weight indexed to aortic annulus area was not $(\mathrm{p}=0.17)$ (figure $1 \mathrm{a}$ and $1 \mathrm{~b})$. AVC correlated strongly with valve weight in men, but poorly in women $\left(\mathrm{r}^{2}=0.57 ; \mathrm{p} \leq 0.0001\right.$ vs. $\left.r^{2}=0.23 ; p=0.003\right)$ and the slope of the regression was lower in the female cohort as well $(\mathrm{p}=0.006)$. Moreover the ratio of aortic valve weight by AVC, representing the relative quantity of non-calcified tissue, was higher in women (figure 1c). After adjustment for age, calcification, BMI and left ventricular outflow tract diameter, female gender turned out to be and independent predictor for higher fibrosis scores as measured on anatomic

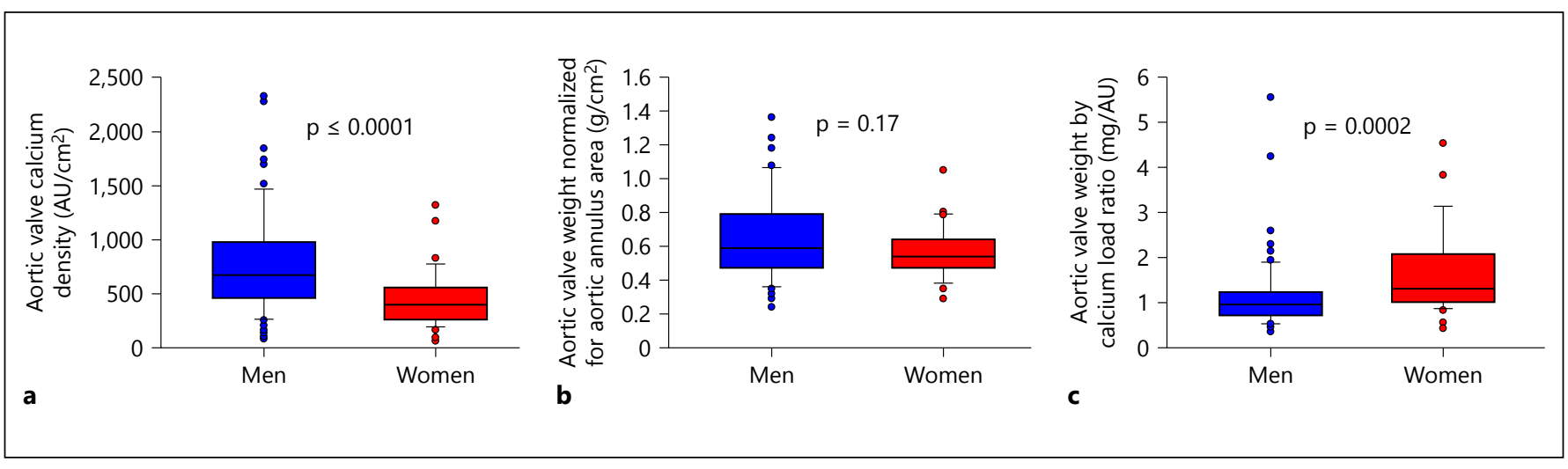

Fig. 1. (for Abstract of Louis Simard). 
specimen $(\mathrm{p}=0.015)$. Conclusions: In this subgroup of AS patients, for a given level of valvular weight, women have relatively more valvular fibrosis compared to men. This may explain why women reach hemodynamically severe AS at lower levels of AVC. These findings suggest that the pathophysiology of the disease and thus the therapeutic targeting for AS should individualized according to patient sex.

\section{Mechanisms of Fibrotic Aortic Stenosis}

Hardik Doshi, Yi Chu, Donald Lund, Henry Keen, Kevin Knudtson, Robert Brooks, Kathrine ZImmerman, Curt Sigmund, Robert M. Weiss, Donald Heistad

University of lowa, lowa City, IA, USA

Objective: We have previously described an experimental model of calcific aortic valve stenosis (CAVS). Children and some adults with AVS have only small amounts of valve calcification. We now describe a model of fibrotic aortic valve stenosis (FAVS), with minimal calcification, and examine molecular mechanisms preceding development of FAVS. Methods: Serial echocardiograms were performed in normotensive, normocholesterolemic mice (Control), hypertensive (HT, over-expression of human renin and angiotensinogen, $\mathrm{REN}+\mathrm{AGT}+)$, hypercholesterolemic (HC, Apoe-/-) and HC/HT mice (Apoe-/-REN+AGT+), ( N = $8-10$, in each group). Mice were euthanized at 12 months of age, earlier if they developed severe aortic stenosis. Collagen content, type and collagen fiber orientation were studied using pricrosirius red staining, polarized light microscopy and immunofluorescence staining for Collagen-1. Results: Only HC/HT mice developed hemodynamically significant aortic stenosis. Calcification of the valve (Alizarin Red) was minimal in Control and minimally increased in HT, HC, and HC/HT groups ( $\mathrm{p}=\mathrm{NS}$ ). Collagen-1 content was increased in $\mathrm{HC} / \mathrm{HT}$ valves $\left(0.10 \pm 0.004 \mathrm{~mm}^{2}\right.$, mean \pm $\mathrm{SE})$ compared to Control $\left(0.07 \pm 0.01 \mathrm{~mm}^{2},{ }^{*} \mathrm{p}<0.05\right)$. In HC/HT mice only, collagen fibers formed a mesh, which joined adjacent cusps at their bases. In groups that did not develop stenosis (Control, HC and HT) collagen fibers were oriented predominantly

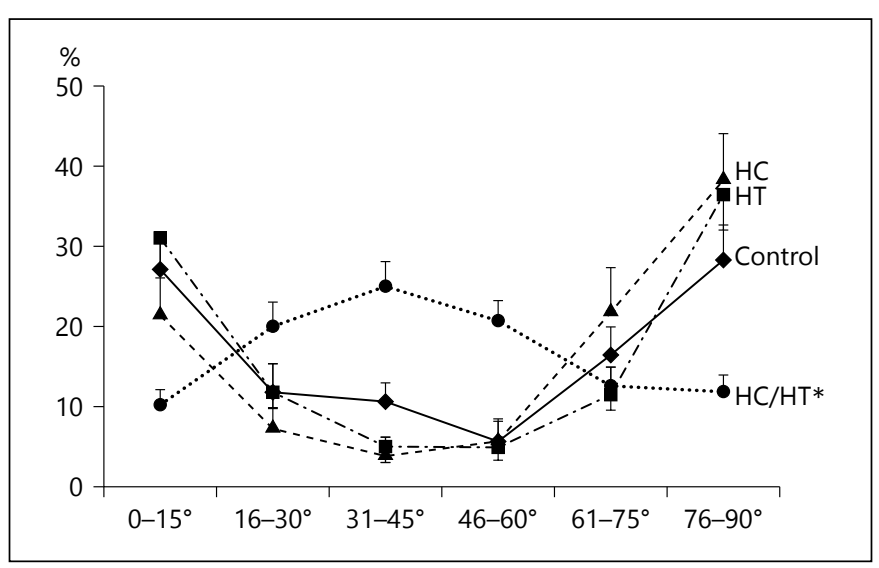

Fig. 1. (for Abstract of Hardik Doshi). parallel or normal to long axis of cusp attachment sites. In HC/HT mice, collagen fibers were oriented predominantly at $30-60^{\circ}(\mathrm{p}<$ 0.05 ) from reference axis (figure 1). Quantitative whole-genome mRNA sequencing performed during developmental phase of stenosis revealed differential expression of molecular targets with increased expression of both pro-calcific and anti-calcific genes and also increased expression of pro-fibrotic genes in HC/HT. Conclusions: Collagen-1 content in aortic valves increases in $\mathrm{HC} / \mathrm{HT}$ mice with FAVS, which develop aortic stenosis with minimal valve calcification. There is altered organization of collagen fibers in $\mathrm{HC} / \mathrm{HT}$ valves, which binds adjacent cusps at their bases, associated with severely reduced cusp mobility. No other aortic valve study, in humans or mice, has profiled genome-wide expression of stenosis-prone aortic valve prior to onset of overt valve dysfunction.

\section{Circulating Regulatory T Cells in Patients with Degenerative Aortic Valve Stenosis}

\section{Sara Shimoni, Iris Bar, Valeri Meledin, Gera Gandelman, Jacob George}

Kaplan Hospital, Rehovot, Israel

Objective: Severe aortic valve stenosis (AS) accounts for considerable morbidity and death, especially in older patients. There is increasing evidence to suggest a role for immune mod-

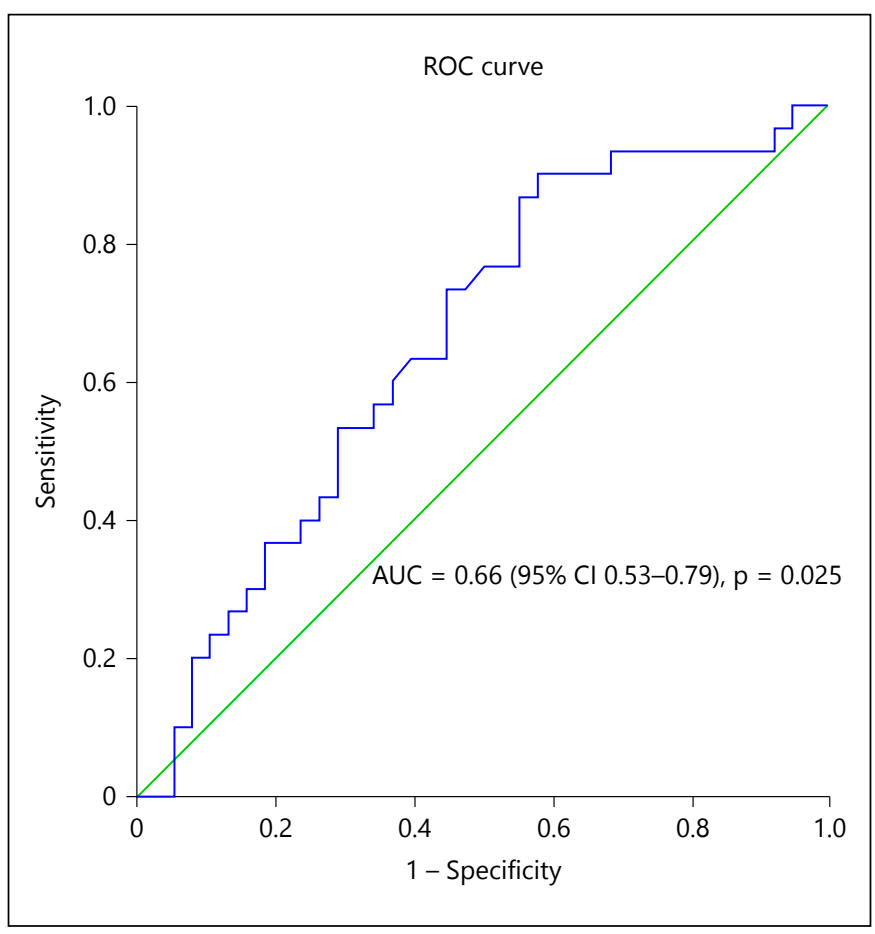

Fig. 1. AS progression (delta AVA) and baseline Tregs (for Abstract of Sara Shimoni). 
ulatory cells in AV thickening and calcifications. Regulatory $\mathrm{T}$ cells (Tregs) influence the development of autoimmunity and their use is increasingly proposed for clinical applications. The aim of the present study is to explore the level of circulating Tregs in patients with various stages of AS and to assess correlation with disease progression. Methods: The number of Tregs (CD4+CD25+Foxp3+) was determined by flow cytometry in 229 patients with AS and a control group of 69 patients. Thirtyeight, 51 and 140 patients had mild, moderate and AS, respectively. We evaluated the association between baseline level of Tregs and AS progression during follow up. Results: The level of Tregs was significantly higher in patients with AS as compared to the control group $(1.64 \pm 1.61$ vs. $1.13 \pm 0.97, \mathrm{p}=0.04)$. There was no difference in Tregs level between patients with mild, moderate and severe AS $(\mathrm{p}=0.18)$. Hundred patients underwent a second echocardiography study during follow up (median 13 (10-21) months). The median decrease in AVA was $0.1 \mathrm{~cm}^{2} /$ year. There was a borderline correlation between baseline Tregs and AVA progression $(\mathrm{r}=0.19, \mathrm{p}=0.056)$. In a subgroup of 68 patients with severe AS, the association between Tregs and AVA progression was significant ( $\mathrm{r}=0.374, \mathrm{p}-0.0017)$. The ROC curve showing the Tregs sensitivity for AS progression in severe AS patients is shown in the figure 1. One hundred and eleven patients underwent intervention during follow up. Forty five patients had Tregs assessments 3-6 month after intervention. The percent of Tregs significantly decreased after intervention $(1.86 \pm 1.6$ vs. $1.04 \pm 1.8, \mathrm{p}=0.0005)$. Conclusions: Circulating Tregs are elevated in patients with AS. The levels of Tregs are higher in patients with severe AS and accelerated progression of valve narrowing. These results may help to identify AS patients at increased risk for disease progression and possibly in need for earlier intervention.

\section{Left Ventricular Overload Is Required for Manifestation of Overt Cardiac Phenotypes in SIRT6-Deficient Mice}

\author{
Grace C. Verzosa, Michael A. Hagler, Carolyn M. Roos, \\ Bin Zhang, Jordan D. Miller
}

Mayo Clinic, Rochester, MN, USA

Objective: To determine whether the sex-dependent cardiac phenotypes in sirtuin 6-deficient mice are influenced by aging and/or calcific aortic stenosis. Methods: We studied two groups of mice. Group 1 was comprised of hyperlipidemic ( $\mathrm{Ldlr}^{-/}$ ApoB ${ }^{100 / 100}$ ) mice that were either wild type (WT) or heterozygous (HET) for sirtuin 6. Mice were fed with Western diet $(42 \%$ fat, $0.2 \%$ cholesterol) for 12 months. Group 2 was comprised of 18 month-old non-hyperlipidemic mice that were WT or HET for sirtuin 6 fed a regular chow diet. Two-dimensional and Doppler echocardiography were performed at 12-month time point. Left ventricular ejection fraction (EF), LV mass, and aortic valve cusp separation were measured. Results: Hypercholesterolemic mice developed aortic valve stenosis after 12 months of diet. Cusp separation distance was smaller in HET (WT $=0.85 \pm 0.02$ $\mathrm{mm}, \mathrm{HET}=0.75 \pm 0.02 \mathrm{~mm}, \mathrm{P}=0.002)$ and left ventricular mass was higher in female WT mice compared to HET littermates
$(\mathrm{WT}=118 \pm 6.3 \mathrm{~mm}, \mathrm{HET}=98 \pm 8.8 \mathrm{~mm}, \mathrm{P}=0.039)$. In contrast, aged mice did not develop aortic valve dysfunction, and left ventricular mass was comparable between genotypes $(\mathrm{WT}=$ $106 \pm 11 \mathrm{mg}, \mathrm{HET}=100 \pm 17.5 \mathrm{mg}, \mathrm{P} 0.78)$. Furthermore, there was no significant difference in ejection fraction between WT and HET in the aged, normocholesterolemic mice. Conclusions: Chronic increases in ventricular afterload are essential for development of overt ventricular hypertrophy in aging mice. Furthermore, our data suggest that SIRT6 plays an integral, context-dependent role not only in the pathogenesis of hypercholesterolemia-induced valvular dysfunction, but also in modulating the hypertrophic responses to progressive increases in left ventricular afterload.

\section{Multiscale Analysis of Blood Flow through Aortic Valve Stenosis: Implications on Red Blood Cell Membrane Damage}

Koohyar Vahidkhah ${ }^{1}$, Dan Cordasco ${ }^{2}$, Mostafa Abbasi $^{1}$, Liang Ge ${ }^{3}$, Elaine Tseng ${ }^{3}$, Prosenjit Bagchi ${ }^{2}$, All Azadani

${ }^{1}$ University of Denver, Denver, CO, ${ }^{2}$ Rutgers University, Piscataway, NJ, ${ }^{3}$ University of California San Francisco, San Francisco, CA, USA

Objective: Valvular hemolysis and thrombosis are common pathological conditions related to prosthetic heart valves. Exposure of blood cellular components, specifically red blood cells and platelets, to foreign surfaces as well as to considerably high shear rates have been recognized as major incentives to such complications. The present study focuses on the significance of shear-induced red blood cell damage via evaluation of viscous shear stress downstream of a severely calcified bioprosthesis along with simulations of a red blood cell membrane response to the measured shearing force environment. Methods: A degenerated $21 \mathrm{~mm}$ Carpentier-Edwards PERIMOUNT bioprosthesis explanted from a patient was tested in a custom-built pulse duplicator system (mean pressure: $61.2 \pm 0.4 \mathrm{~mm}-\mathrm{Hg}$ ). A multiresolution 2D PIV measurement was employed to capture instantaneous velocity and viscous shear stress fields downstream of the bioprosthesis. Microhydrodynamics of a red blood cell under the measured shear rates was then simulated using a $3 \mathrm{D}$ Immersed-Boundary/Front-Tracking method that coupled an FEM model of the cell membrane large deformation to a finitedifference CFD flow solver. Results: The experimental measurements provided the shear stress level at the boundary layer of the jet flow formed downstream the valve orifice. The measured value was observed to reach a maximum of $1700 \mathrm{dyn} / \mathrm{cm}^{2}$ which was within the range of thresholds for red blood cell damage that have been reported in the literature as $1000-2000 \mathrm{dyn} / \mathrm{cm}^{2}$. The results of the simulations demonstrated that the resultant maximum shear tension in the membrane of a red blood cell residing in the jet shear layer reached values that were larger than the yield shear for the cell membrane plastic failure and fragmentation $(0.02 \mathrm{dyn} / \mathrm{cm})$. The experimental measurements further showed that the shear stress level downstream the bioprosthesis was beyond the threshold values for platelet activation (100$1000 \mathrm{dyn} / \mathrm{cm}^{2}$ ). Conclusions: Formation of a shear layer in the 
Fig. 1. (for Abstract of Ali Azadani).

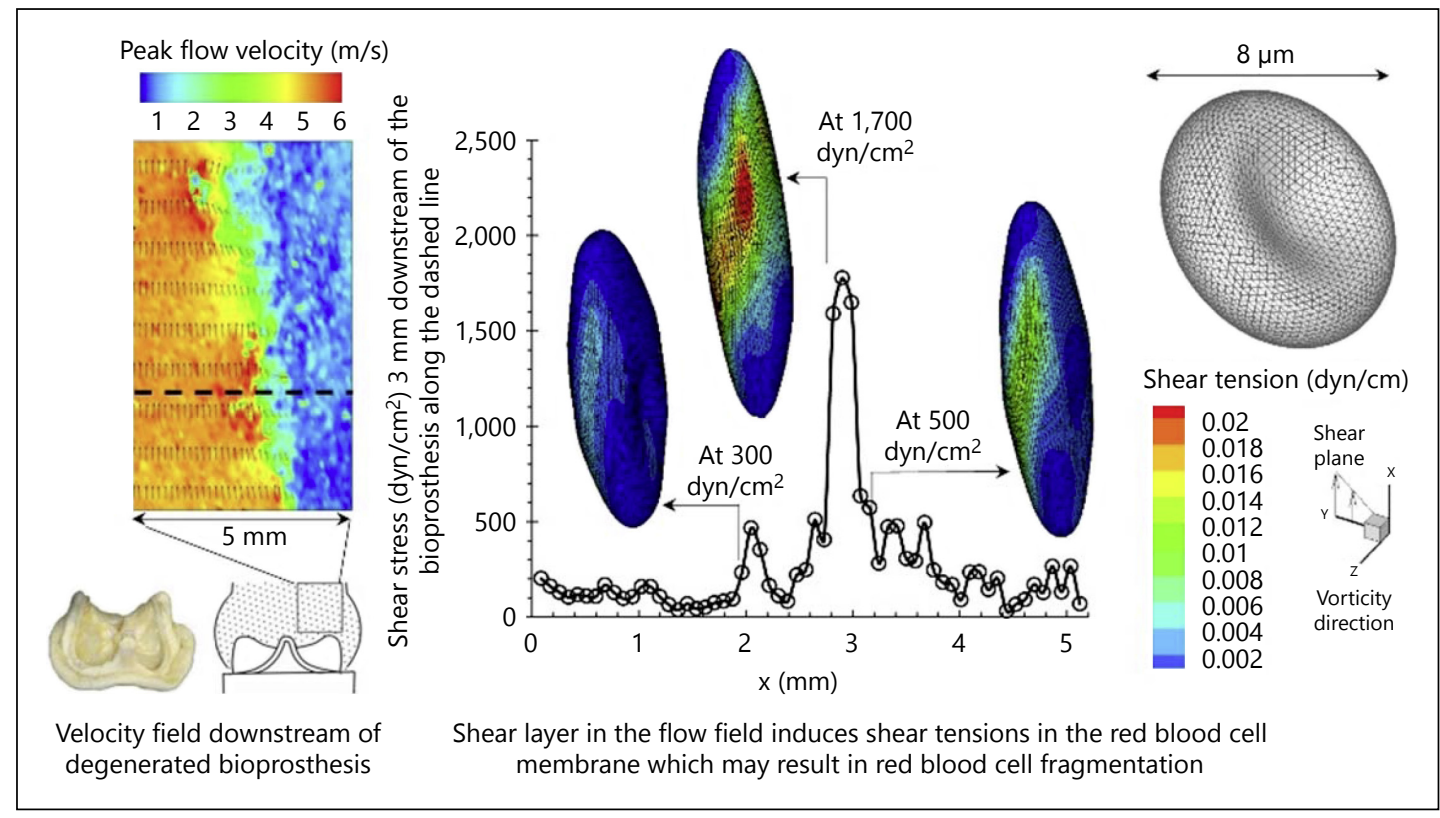

flow field of a severely calcified valve induces shear tensions in the red blood cell membrane beyond the shear yield and could result in red blood cell fragmentation.

\section{Fibroblast Activation Protein is Decreased in Fibro Elastic Deficiency Compared to Barlow's Disease: Implications in Valvular Lesion Progression}

K.-Raman Purushothaman ${ }^{1}$, Meerarani Purushothaman ${ }^{1}$, Prakash Krishnan ${ }^{1}$, David Adams ${ }^{1}$, William N. O'connor ${ }^{2}$, Annapoorna Kini ${ }^{1}$, Samin K. Sharma ${ }^{1}$, Carlos Cordon-Cardo ${ }^{1}$, Pedro R. Moreno ${ }^{1}$

${ }^{1}$ Icahn School of Medicine at Mount Sinai, New York, NY, ${ }^{2}$ University of Kentucky Medical Center, Lexington, KY, USA

Background: Fibroblast Activation Protein (FAP), a serine protease expressed by fibroblasts is involved in cell activation and extracellular matrix (ECM) remodeling. Surgically defined sub types of degenerative mitral valve disease, Fibroelastic deficiency (FED) and Barlow's disease (BD) differ in elastic fiber structure/ content and in extracellular matrix composition. In FED, lack of connective tissue triggers chordal rupture. Our study of degenerative mitral valve disease tested the hypothesis that decreased FAP protein expression in FED may be associated with reduced fibroblast content and alteration in elastin fibers in FED compared to BD. Methods: Twenty-four human posterior mitral valve leaflets (12 BD and 12 FED) were studied. Protein expression profiles of FAP and Fibroblast specific protein-1 (FSP-1) for fibroblasts were evaluated by immunohistochemistry. Elastin protein expression was identified and scored by immunohistochemistry. Elastic fibril fragmentation and disorganization were evaluated and graded using elastic trichrome stain. Elastic fibril fragmentation was scored by identifying discontinuity of elastic fibrils. Disorganization was identified by measuring a disordered and haphazard orientation of elastic fibrils. Results: FED was associated with decreased FAP and fibroblast protein expression with less elastic fiber fragmentation and disorganization. Furthermore, FED showed decrease in elastin protein expression grade compared to $\mathrm{BD}$. (See figure 1 and table 1). Conclusions: In FED, reduction in FAP expression and fibro-

Table 1. Histopathological profile of fibroelastic deficiency vs. Barlow's disease (for Abstract of K.-Raman Purushothaman)

\begin{tabular}{lrrr}
\hline Histological profile & \multicolumn{1}{l}{$\begin{array}{l}\text { FED } \\
(\mathrm{n}=12)\end{array}$} & \multicolumn{1}{c}{$\begin{array}{l}\mathrm{BD} \\
(\mathrm{n}=12)\end{array}$} & $\mathrm{p}$ value \\
\hline Mean fibroblast activation protein expression grade & $2.01 \pm 0.22$ & $3.54 \pm 0.05$ & 0.0001 \\
Mean fibroblast (FSP-1) protein expression density & $150.1 \pm 6.71$ & $171.9 \pm 4.74$ & 0.0001 \\
Mean elastin protein expression grade & $1.3 \pm 0.19$ & $3.6 \pm 0.10$ & 0.0001 \\
Elastin fiber degeneration grade & $1.08 \pm 0.13$ & $1.51 \pm 0.12$ & 0.03 \\
Elastin fiber fragmentation grade & $1.01 \pm 0.11$ & $1.39 \pm 0.11$ & 0.02 \\
\hline
\end{tabular}




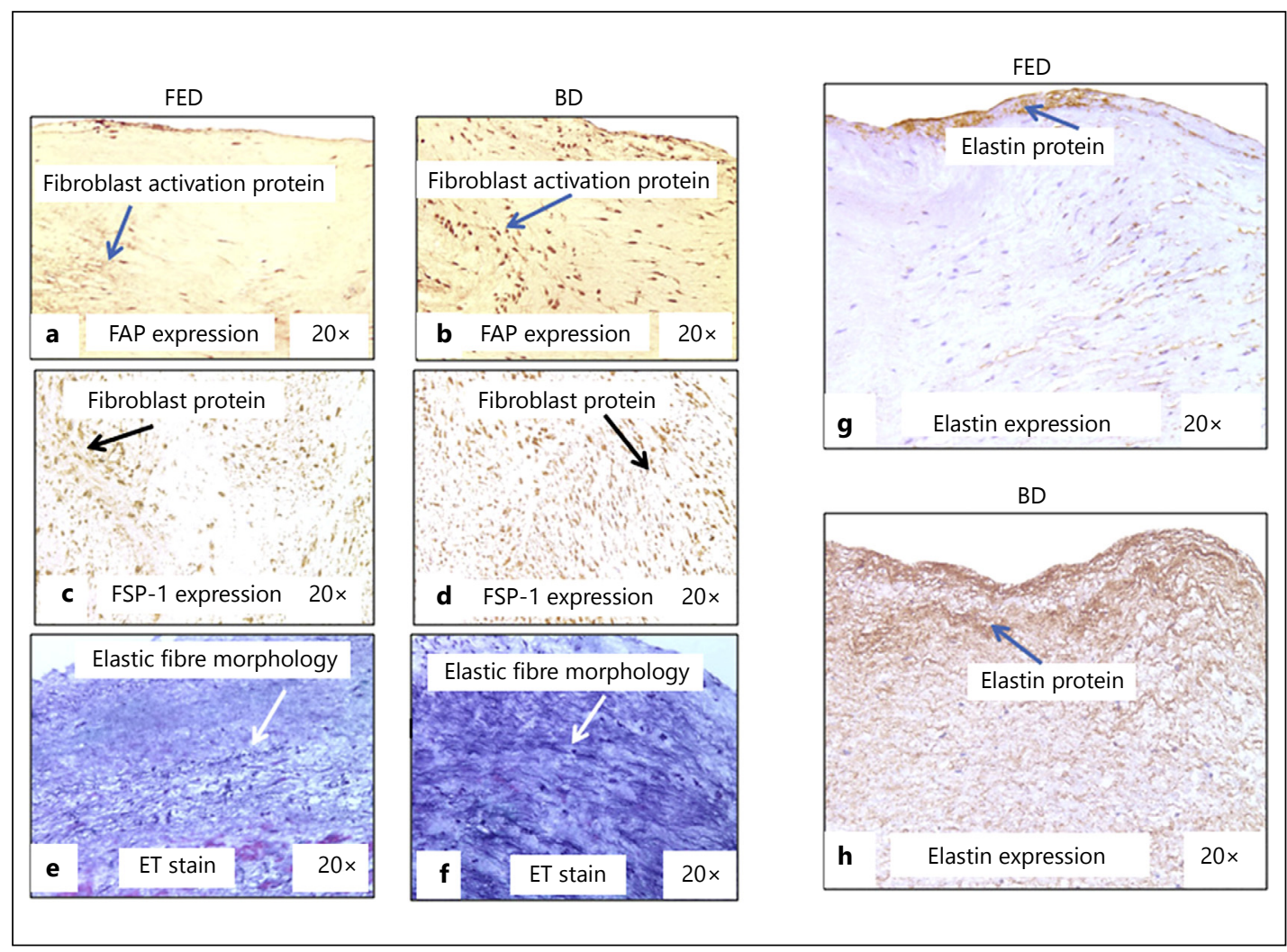

Fig. 1. FED vs. BD histopathological profiles (for Abstract of $\boldsymbol{K} .-\boldsymbol{R} \boldsymbol{a}$ man Purushothaman). blast content in valvular interstitial cells may be responsible for structural instability of the valves in addition to reduced elastic fibrils with alterations in ECM content. Further molecular studies will address the underlying mechanism involved in elastic fibril and ECM remodeling of FED valves and may help further understanding of the propensity for chordal rupture.

\section{A Study on the Role of Sex on Cellular Calcification within the Aortic Valve}

\section{Shirin Masjedi, Zannatul Ferdous}

University of Tennessee, Knoxville, TN, USA

Background: Aortic valve stenosis is a prevalent disease among the elderly in developed countries. Male sex is a risk factor for aortic stenosis based on clinical reports. In this study, we aim to determine whether valvular interstitial cells (VICs) isolated from male and female rats develop different amounts of early calcific markers and matrix remodeling proteins when cultured in osteogenic media. The results from this study would provide crucial information at the cellular level to understand whether VICs isolated from different sexes demonstrate different amounts of calcification in pathological culture environment. Methods: VICs were isolated from aortic valves of euthanized 9 week old male and female Sprague-dawley rats. The cells were cultured in growth media until passage 7 . The growth rates of the male and female VICs in

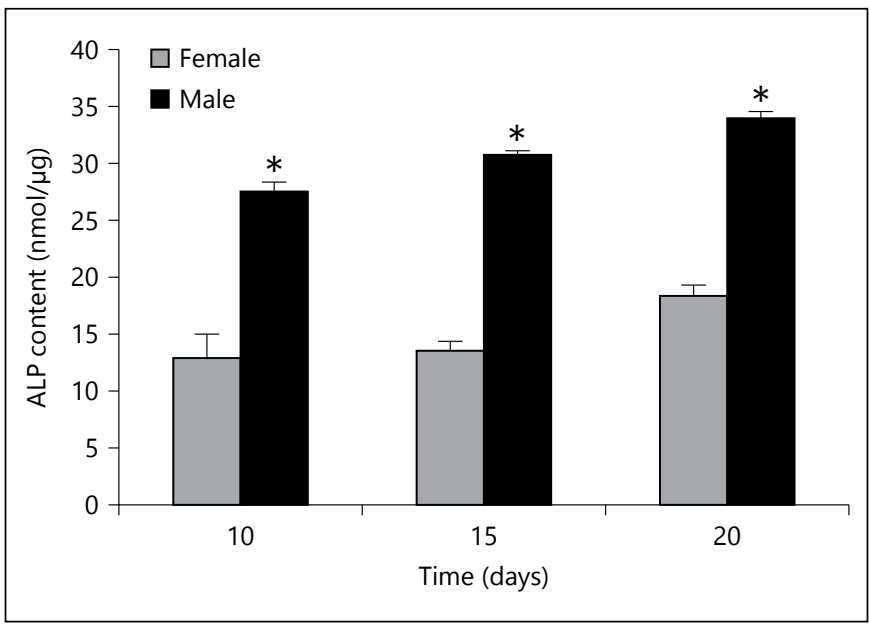

Fig. 1. ALP content of female and male VICs after 10, 15 and 20 days of culture in osteogenic media; ${ }^{*}$ p-value $<0.05$ (for Abstract of Shirin Masjedi).

osteogenic and regular culture media were compared by counting the cell number every 2 days for 12 days. Then, VICs were cultured in osteogenic media for a period of 10, 15, and 20 days. Calcification potential of the VICs was assessed using alizarin red and von kossa staining and alkaline phosphatase (ALP) enzyme activity measurement. The extracellular matrix (ECM) production and 
degradation was measured using gelatin zymography. Results: We observed that the growth rate for the VICs isolated from female rats were significantly greater than male. Furthermore, calcific nodule formation and mineral deposition in VICs isolated from male rats were greater than female at late-stages of calcification (20 days). The ALP enzyme content was significantly elevated in male compared to female VICs after 10, 15 and 20 days of culture in osteogenic media (figure 1). Additionally, the difference in expressed MMP-2 enzyme was significant in male VICs than female after 20 days of culture in osteogenic media. Conclusions: Our results suggest that there is a difference in calcification potential and ECM remodeling in male versus female rat aortic VICs.

\section{Effect of Cell Migration and Endothelial Cell Contact Upon Phenotype Activation of Valvular Interstitial Cells}

Mir Ali ${ }^{1}$, Xinmei Wang ${ }^{1}$, Mai Dinh ${ }^{1}$, Allison Curtis ${ }^{2}$, Monica Kim ${ }^{2}$, Elliot Hui ${ }^{2}$, Carla Lacerda ${ }^{1}$

${ }^{1}$ Texas Tech University, Lubbock, TX, ${ }^{2}$ University of California, Irvine, CA, USA

Objective: Increasing substrate stiffness has notable effects on collective cell migration speed, persistence, and directionality. Moreover, it is known that substrate stiffness has a determining effect on valvular cell phenotypes. Specifically, quiescent valvular interstitial cells become activated, or myofibroblastic, in monolayer culture on substrates of $33 \mathrm{kPa}$ or higher elastic moduli. Under similar conditions, valvular endothelial cells in co-culture are expected to provide reversal of phenotype activation. This study investigates the influence of cell migration and endothelial cell contact upon phenotype activation of valvular interstitial cells. Methods: Valvular interstitial and endothelial cells were seeded at $10^{4}$ cells $/ \mathrm{cm}^{2}$ on two different substrates, namely, polyacrylamide or polydimethylsiloxane. Each system provided different barriers for isolation of interstitial and endothelial cells. After 1 day in isolated culture, such barriers were removed and cells were allowed to migrate towards each other and share culture medium. Phenotype changes were visualized by immunofluorescence and quantified by immunoblotting of markers of quiescent and activated phenotypes. Applicable single culture controls were also evaluated. Results: Interstitial cells presented distinctive migrating patterns towards endothelial cells. Migrating interstitial cells presented higher expression of $\alpha$-SMA and TGF- $\beta$, characteristic of myofibroblastic morphology, and settled around endothelial cell clusters. At high cell densities, close contact with endothelial cells in co-culture resulted in increased interstitial cell activation, regardless of substrate stiffness. Conclusions: Cells in co-culture tended to migrate towards each other and interstitial cells were largely activated when in close contact with endothelial cells regardless of substrate stiffness. We conclude that endothelial cells are only capable of reversing phenotype activation at lower cell densities and at higher cell confluence the endothelial impact is reduced.

\section{Epidemiological Characteristics of Valvular Heart Disease in the 21st Century: A Nationwide Study of the Swedish Population}

\author{
Pontus Andell ${ }^{1}$, Xinjun $\mathrm{Li}^{2}$, Andreas Martinsson ${ }^{1}$, \\ Charlotte Andersson ${ }^{1}$, Bengt Zöller ${ }^{2}$, Kristina Sundquist ${ }^{2}$, \\ J. Gustav Smith ${ }^{1}$ \\ ${ }^{1}$ Department of Cardiology, Clinical Sciences, Lund University, \\ Lund, ${ }^{2}$ Center for Primary Health Care Research, Lund \\ University, Malmö, Sweden
}

Objective: Transitions in the spectrum of valvular heart diseases (VHDs) in developed countries over the 20th century have been reported from clinical case series. Well-powered and contemporary population-based studies of VHDs are scarce. Methods: We used nation-wide Swedish registers to identify all patients with a first diagnosis of VHD as inpatients or outpatients at all Swedish hospitals between 2001-2010. Incidence of each VHD from international classification of disease (ICD) codes was assessed across age, sex and comorbidity groups. Results: The overall incidence of VHD was 105.5 per 100,000 person years, with aortic stenosis (AS) (48.1\%), mitral regurgitation (MR) $(23.2 \%)$ and aortic regurgitation (AR) (17.8\%) being the most frequent. The majority of VHDs were diagnosed in the elderly (67.9\% in subjects aged $\geq 65$ years). Pulmonary valve disease and tricuspid stenosis (TS) had peak incidence in newborns. AR, AS and MR incidences were higher in men, while mitral stenosis (MS) incidence was higher in women (figure 1). A history of rheumatic fever was rare, and principally seen only in a few patients with mitral and aortic disease as well as tricuspid regurgitation (TR). More than half of AS cases had concomitant atherosclerotic vascular disease (51.4\%), whereas concomitant heart failure and atrial fibrillation was particularly common with tricuspid regurgitation and mitral valve disease. Pulmonary valve disease and TS frequently occurred in combination with non-valvular forms of congenital heart disease. Other common comorbidities were thoracic aortic aneurysm in AR (10.5\%), autoimmune disease in MS (18.8\%) and TR (16.7\%), and connective tissue disorders in MR (17.1\%) and TR (16.5\%). Conclusions: Clinically diagnosed VHD in the 21st century is primarily a disease of the elderly, although pulmonary valve disease and tricuspid stenosis are typically diagnosed during the first decade of life. Rheumatic heart disease is rare in Sweden; instead specific VHDs display different comorbidity profiles, indicating a wide range of potential etiologies in the current era. AR and MS display striking sex-specific patterns for which the mechanisms remain incompletely understood. 


\section{Comparison of Leaflet Kinematics in State-of-the-Art Bioprosthetic and Mechanical Valves with a Tri-Leaflet Valve Design}

\author{
Bernhard M. Vennemann 1, Silje E. Jahren², \\ Paul Philipp Heinisch ${ }^{3}$, Thomas Roesgen ${ }^{1}$, Dominik Obrist ${ }^{2}$ \\ ${ }^{1}$ Institute of Fluid Dynamics, ETH Zurich, Zurich, ${ }^{2}$ ARTORG \\ Center, University of Bern, Bern, ${ }^{3}$ Department of \\ Cardiovascular Surgery, Bern University Hospital, Bern, \\ Switzerland
}

Objective: The opening and closing of artificial heart valves is strongly related to their design. While bioprosthetic valves close relatively slowy, typical mechanical heart valves (MHVs) close abruptly causing high shear stresses which can lead to hemodynamic complications such as hemolysis or platelet activation. We aim at a comprehensive comparison of the kinematics of the individual leaflets of various valve designs. To this end, we compare results for a conventional mechanical bi-leaflet valve, a novel mechanical tri-leaflet valve and a bioprosthetic valve in an identical in vitro setting. The study of individual leaflets captures also asynchronous dynamic effects such that it provides a more detailed view of the valve's hemodynamic performance and thrombogenicity than studies using the projected dynamic valve area. Methods: A mock loop replicates physiological flow in a transparent, compliant model of the aortic root using a pulsatile pump (ViVitro Labs, Victoria, Canada). An Edwards Intuity, a St. Jude Regent bi-leaflet valve and a Lapeyre-Triflo FURTIVA valve were tested in this setup. The measurements were performed using high-speed imaging and motion tracking of the individual leaflets. In addition, opening area, transvalvular pressure drop and flow rate were measured. Results: The results show the time-resolved motion of the different heart valves during the full cardiac cycle including, for instance, fluttering and asynchronous closing of the valve. The results for the studied valve designs are compared with respect to their hemo-

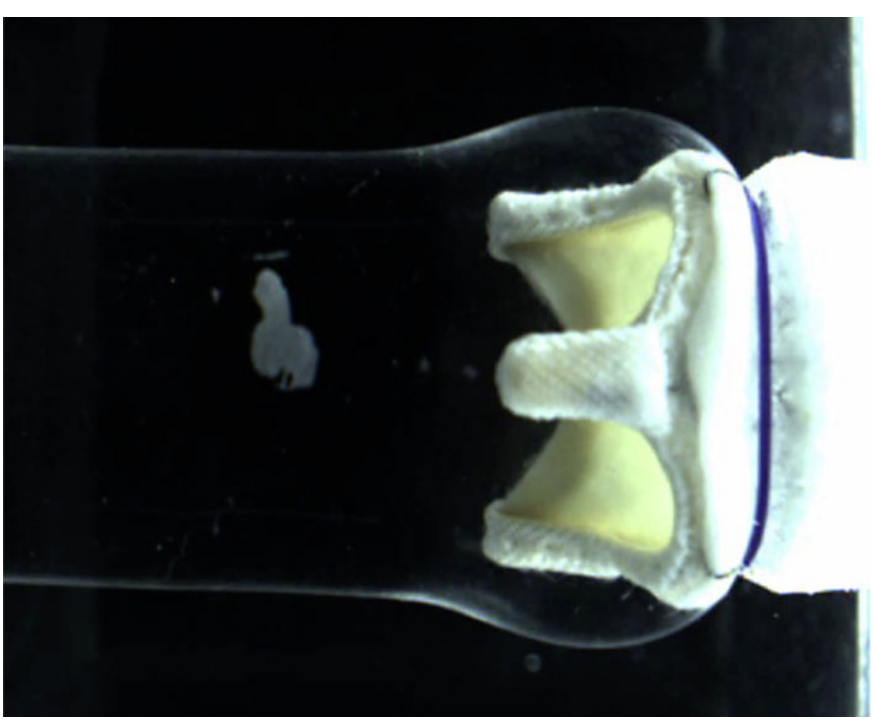

Fig. 1. (for Abstract of Bernhard M. Vennemann). dynamic parameters and leaflet kinematics. It will be discussed how the closing kinematics can affect the clinical performance of these valves. Conclusions: For the first time the novel tri-leaflet Lapeyre-Triflo FURTIVA valve is directly compared against stateof-the-art mechanical and bioprosthesic valves. A comprehensive analysis of the individual leaflet kinematics for all tested valves gives new insight into their hemodynamic performance studied under physiological conditions.

\section{Thirteen Years Durability of the Medtronic Mosaic Porcine Bioprosthesis in the Mitral Position at Single Institution}

Koki Ikemoto, Kiyoshi Doi, Satoshi Numata, Sachiko Yamazaki, Kiichi Itatani, Hidetake Kawajiri, Kazuki Morimoto, Kaichiro Manabe, Hitoshi Yaku

Kyoto Prefectural University of Medicine, Kyoto, Japan

Objective: Long-term durability of bioprosthesis impacts the clinical result of valve surgery. Objective of this study was to evaluate performance of the Mosaic bioprosthesis after implant in the mitral position. Method: From January 2000 to December 2014, 137 patients (mean age: $70.6 \pm 9.2$ years, range: $27-86$ years) underwent mitral valve replacement (MVR) with the Medtronic Mosaic bioprosthesis. The data was retrospectively analyzed and stratified according to patient age at surgery (group $1,<60$ years; group $2,61-70$ years; and group $3,>70$ years), using actuarial method and multivariable analysis. The mean follow-up period was 5.06 years (maximum 14.53 years). Results: The hospital mortality rate was $10.9 \%$. Of the 15 early deaths, 8 were cardiac and 7 were noncardiac. During follow-up period, survival rate was lower in group 3 $(p=0.004,0.025$; versus group 1,2$)$. Seventeen patients required reoperation. The etiology of reoperation was structural valve deterioration (SVD) (10 patients), paravalvular leak (4 patients), primary valve thrombosis (2 patients), and endocarditis (1 patient). The hospital mortality rate of reoperation was $5.9 \%$ (1 patient). Twelve SVD events occurred at $11.05 \pm 2.29$ (7.08-14.03) years on average, ten of them underwent reoperation. The others were one with end-stage cancer and another in observation. The echocardiographic findings of the explanted SVD prostheses were stenosis in 1 patient, regurgitation in 7 patients, and mixed in 2 patients. In two nonexplanted patients, the findings were stenosis. Leaflet tear was identified in 7 patients, leaflet structural dysfunction in 2 patients, and others in 3 cases. There was no significant difference in freedom from reoperation due to SVD among all groups. No independent predictor of the development of SVD was identified during follow-up period by Cox proportional hazard regression analysis. Conclusions: Up to 10 years, durability of the Mosaic bioprosthesis in mitral position was satisfactory. However, 13 year-follow-up results showed risk of SVD over 10 years after implant in all generation. Therefore, close observation is mandatory over 10 years after MVR with Mosaic bioprosthesis. 


\section{Time-Resolved Measurements of in-vitro Leaflet Motion for a Bioprosthetic Aortic Valve}

Oliver Häuselmann ${ }^{1}$, Andrin Landolt ${ }^{1}$, David Hasler ${ }^{2}$, Bernhard Winkler ${ }^{3}$, Dominik Obrist ${ }^{2}$, Thomas Rösgen ${ }^{1}$

${ }^{1}$ Institute of Fluid Dynamics, ETH Zurich, Zurich, ${ }^{2}$ ARTORG Center, University of Bern, Bern, ${ }^{3}$ Department of

Cardiovascular Surgery, Bern University Hospital, Bern, Switzerland

Objective: For some time now, calcification and structural failure of bioprosthetic valves has been suggested to be related to the local flow field. However, fully time- and space-resolved flow data is still not available. The objective of the current study is to determine the smallest necessary temporal and spatial scales to resolve the leaflet motion under various physiological flow conditions, especially regarding leaflet fluttering which is most pronounced during peak systole. Methods: An in-house experimental setup is used to investigate a Carpentier-Edwards Bioprosthesis Aortic Model 2625. A simplified, rigid aorta geometry is employed. A waterglycerol mixture is used as working fluid, allowing to adjust the viscosity within the full range of reported viscosities of blood in the literature. The flow is induced with a computer-controlled piston pump. The pressure is measured up- and downstream of the valve using fast pressure sensors with a response time of less than $5 \mathrm{~ms}$. The volume flow rate is measured using an electromagnetic flowmeter featuring a sampling rate of $150 \mathrm{~Hz}$. The high temporal resolution of the employed sensors allows to match in-vivo data from the literature by adjusting the piston movement accordingly, assuring physiological flow conditions. The leaflet motion is recorded using a high-speed camera. The camera is optically aligned with the principal flow direction, recording the heart valve as would be seen from the left ventricle. Various image processing techniques are employed to analyze the leaflet movement and determine the orifice area. Results: Time resolved pressure drop, volume flow rate, and valve orifice area are presented, characterizing the induced flow. Further, frequency analysis is performed on the valve edges to determine the relevant time-scales and amplitudes, especially regarding leaflet fluttering. Results are presented for two different heartbeats (70 bpm and $150 \mathrm{bpm})$ and two different dynamic viscosities in the range of $3.9-4.9 \mathrm{cP}$. Conclusions: The results address the necessary requirements for fully time-resolved measurements of the fluid dynamical phenomena related to bioprosthetic heart valves. The presented data (pressure differentials, flow rates, instantaneous leaflet shapes) are essential in the understanding of transient phenomena such as leaflet flutter which may be determinants for calcification and structural deterioration.

\section{Experimental and Computational Valve Tissue Studies}

\section{Cusp Interventions in Aortic Valve Repair: Are All Pericardial Patches Created Equal?}

\author{
Janet M. Ngu' ${ }^{1}$, Hadi D. Toeg ${ }^{1}$, Reza Jafar ${ }^{2}$, Benjamin Sohmer ${ }^{3}$, \\ Vincent Chan ${ }^{1}$, Michel Labrosse ${ }^{2}$, Munir Boodhwani ${ }^{1}$ \\ ${ }^{1}$ University of Ottawa Heart Institute, Division of Cardiac \\ Surgery, Ottawa, ON, ${ }^{2}$ University of Ottawa, Department of \\ Mechanical Engineering, Ottawa, ON, ${ }^{3}$ University of Ottawa \\ Heart Institute, Division of Cardiac Anesthesia, Ottawa, ON, \\ Canada
}

Background: The use of a pericardial patch in aortic valve (AV) repair $(\mathrm{AVr})$ is associated with increased repair failure and may be related to properties of the patch material. To determine the ideal pericardial patch material, we compared three commercially available bovine pericardial patches, namely CardioCel ${ }^{\circledR}$, St-Jude Medical $^{\mathrm{TM}}$, and Periguard ${ }^{\circledR}$, by using an ex-vivo porcine AV cusp replacement model, combined with biaxial biomechanical testing and finite element (FE) modeling. Methods: Porcine aortic roots with intact AVs (control, $n=9$ ) underwent baseline assessment in a ViVitro left heart simulator mounted with a high-speed camera and hemodynamic monitoring. $80 \%$ of the noncoronary cusp was replaced with CardioCel (CC, $n=8)$, St-Jude Medical (SJM, $n=9$ ), or Periguard $(\mathrm{PG}, \mathrm{n}=9$ ) patches. The biomechanical properties were measured using a CellScale biaxial testing system. FE models of the AVs were constructed to evaluate cusp geometry and leaflet

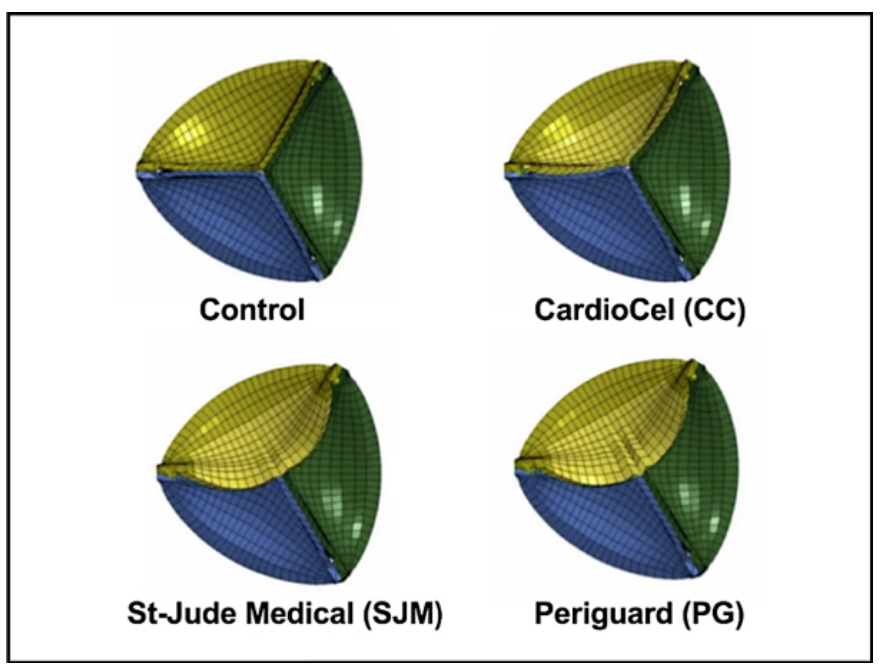

Fig. 1. Finite element (FE) modeling of porcine aortic valves at the end of diastole during a cardiac cycle. Green: left coronary cusp; Blue: right coronary cusp; Yellow (dark): native noncoronary cusp; Yellow (light): the replaced portion of noncoronary cusp (for Abstract of Janet $\mathbf{M}$. $\mathbf{N g u}$ ). 
stresses during the cardiac cycle. Results: The cardiac output and left ventricular work were preserved in all intervention groups. The geometric orifice area after repair was preserved in CC (1.62 \pm $\left.0.15 \mathrm{~cm}^{2}, \mathrm{p}=0.33\right)$ and SJM $\left(1.84 \pm 0.15 \mathrm{~cm}^{2}, \mathrm{p}=0.81\right)$, and was increased in PG $\left(2.36 \pm 0.23 \mathrm{~cm}^{2}, \mathrm{p}=0.004\right)$, as compared to control $\left(1.91 \pm 0.21 \mathrm{~cm}^{2}\right)$. CC demonstrated biomechanical properties resembling the native AVs, whereas both SJM and PG demonstrated lower strains, indicating higher stiffness (typical max radial Green strains of 0.15 vs. 0.13 vs. 0.06 vs. 0.05 for native porcine leaflets, CC, SJM and PG, respectively, under typical equibiaxial stress of $60 \mathrm{~N} / \mathrm{m}$ ). FE modeling showed preserved diastolic Von Mises stress in the replaced cusp (-2.6\%) and nonreplaced cusps $(+0.5 \%)$ in the CC group. The stresses in the replaced and nonreplaced cusps were increased in both SJM $(+31 \% ;+3.7 \%)$ and PG $(+70.3 \% ;+10.1 \%)$, respectively. When compared to control $\left(2.45 \mathrm{~cm}^{2} ; 10.4 \mathrm{~mm}\right)$, the leaflet coaptation area and effective height were preserved in the CC $\left(2.45 \mathrm{~cm}^{2} ; 10.2 \mathrm{~mm}\right)$; but were reduced in both SJM $\left(2.28 \mathrm{~cm}^{2} ; 9.4 \mathrm{~mm}\right)$ and PG $\left(2.15 \mathrm{~cm}^{2} ; 8.8 \mathrm{~mm}\right)$. Conclusions: Among all three pericardial patches, CardioCel demonstrates the closest profile to native AVs, rendering it best suited for AVr.

\section{Adherence of Staphylococcus Aureus to Ultra High Molecular Weight Polyethylene Fibers Heart Valve Leaflet and Clinically Used Cardiovascular Prosthesis}

\section{Paul F. Grundeman}

UMCU, Utrecht, Netherlands

Objective: Prosthetic valve endocarditis (PVE) is a medical entity with potentially fatal outcome. In early PVE bacterium Staphylococcus aureus predominates with $36 \%$ prevalence. Ultra High Molecular Weight Polyethylene fibers (UHMWPE) is a new prosthesis material which could circumvent some of the problems with the (biological) materials used currently. UHMWPE fibers are very thin, flexible, resistant to fatigue and abrasion, and have high strength. Therefore, prostheses made from UHMWPE fibers might be attractive for use in the (minimally invasive) treatment of vascular and valvular disease. To evaluate if the beneficial properties of these fibers are not annulled by a poor performance regarding bacterial adhesion and infection we tested both patches of UHMWPE fibers, and also UHMWPE membrane patches. The aim of this study was to assess Staphylococcus aureus adherence on

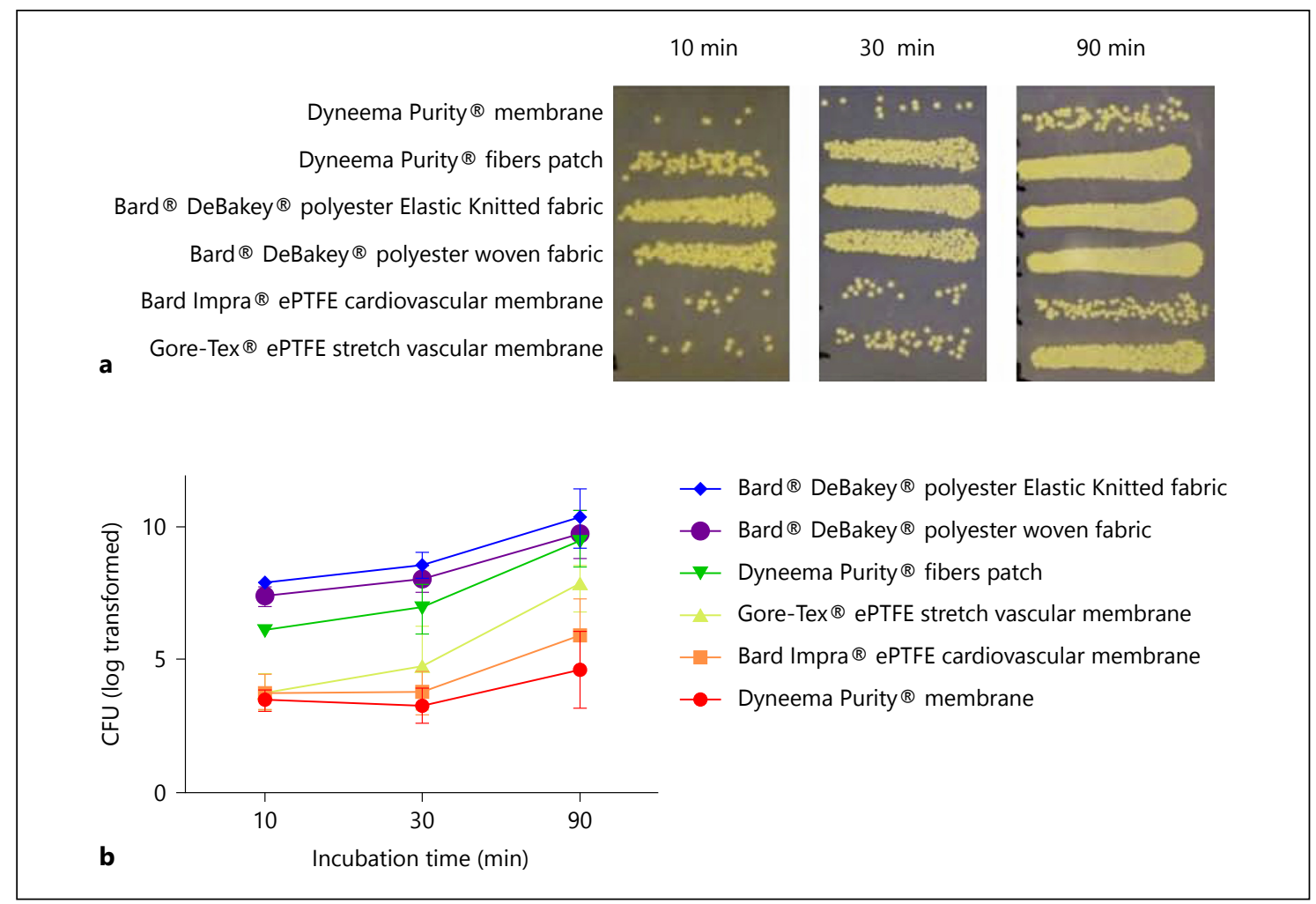

Fig. 1. Bacterial adhesion to the Dyneema Purity ${ }^{\circledR}$ and the control patches as measured by CFU counts. a Representative example of CFUs on agar plates after 10, 30, and 90 minutes of incubation of the 6 different yarncomposed and membrane based patches: Dyneema Purity ${ }^{\circledR}$ membrane; Bard Impra ${ }^{\circledR}$ ePTFE Cardiovascular Patch; Gore-Tex ${ }^{\circledR}$ Stretch Vascular Graft Standard Wall; Dyneema Purity ${ }^{\circledR}$ fibers patch; Bard ${ }^{\circledR}$ DeBakey ${ }^{\circledR}$ Elastic Knitted and woven polyester Fabric. b Log transformed average CFU of three experiment after 10, 30, 90 minutes incubation. Error bars represent STdev (for Abstract of Paul F. Grundeman). 
patches made of UHMWPE fibers and to compare these adherence with currently used cardiovascular prostheses. Methods: 3 different methods were used to compare the S. aureus adhesion to 2 different ( 1 membrane-based and 1 yarn-composed patch) with 5 clinically used yarn-composed and membrane-based expanded polytetrafluoroethylene and polyester patches. First patches were contaminated with S. aureus bacteria in the presence of $20 \% \mathrm{hu}-$ man serum, and incubated for 10, 30 and 90 minutes. After incubation, the bacteria on the patches were removed and quantified by 2 different methods; first by plating a serial dilution and counting the colony forming units (CFU) on plate and second by monitoring the outgrowth time as a value for initial inoculum by measuring the optical density (OD) in time. Also the bacterial adherence on patches was visualized in flow conditions. Results: $S$. aureus adherence to yarn-composed UHMWPE fibers patches after 10 and 30 minutes was lower compared to yarn-composed Bard ${ }^{\circledR}$ DeBakey ${ }^{\circledR}$ Elastic Knitted Polyester Fabric control patch. On membrane-based patches, less adherence was observed to Gore-Tex ${ }^{\circledR}$ Stretch vascular graft patch after 10 minutes. At 30 and 90 minutes there was less adherence to UHMWPEpatches compared to test patches. Conclusions: Patches of UHMWPE fibers might be attractive for use in cardiovascular applications such as catheter-based heart valves and endovascular prostheses by their noninferiority regarding bacterial adhesion.

\section{TAVR Valves: The Effect of Post Implantation Leaflet Distortion on Durability}

Yaghoub Dabiri, Janet Ronsky, Imtiaz Ali, Kishan Narine

University of Calgary, Calgary, $A B, C$ anada

Objectives: Bioprosthetic valve performance is determined by valve design and geometry. Variations in annular anatomy, calcification and in situ failed bioprosthetic valves result in recipient annular asymmetry and consequent stent distortion in percutaneous aortic valves. Leaflet malcoaptation post-implantation can adversely affect valve durability. Using a computational modeling approach, we evaluated and simulated the effect of distortion on leaflet stress as a predictor of valve durability. Methods: The finite element method was used to model a bioprosthetic valve $(19 \mathrm{~mm})$ and its leaflet. A neo-Hookean material was assumed for the leaflets. Four configurations were considered. In the normal case, the base of the valve was fixed. An array of variations of the fixed area was enforced such that the distortion approached an oval, rectangular and irregular annular shape. The fixed regions were enforced to move in the plane of valve cross section and diastolic backward pressure was applied at the aortic side of leaflets $(8 \mathrm{kPa})$. In all cases, coaptation was considered as contacts between leaflets in the normal and distorted configurations. Results: Leaflet stress was evaluated. The pattern of stress distribution as well as the values of leaflet stress was significantly altered by distortion. In the normal case, the maximum von Mises stress occurred at the commissures. The maximum von Mises stress in the distorted valves were at the areas of distortion and commissures as well as at newly created angular regions. The maximum stress observed ranged from approximately 4 to 10 fold depending on the distortion (normal 1.2 $\mathrm{MPa}$, oval 5.1 $\mathrm{MPa}$, rectangular $12.5 \mathrm{MPa}$ and irregular
8.2 MPa). Conclusions: Geometrical alterations consequent to annular and stent distortion of percutaneous valves result in a redistribution and increase in leaflet stress when compared that in the undistorted original design. Depending on the degree of distortion, percutaneous valves are predisposed to earlier structural deterioration.

\section{In vitro Calcification Testing of Biological Heart Valve Prostheses: A Proof of Principle Study}

\author{
Ulrich Steinseifer ${ }^{1}$, Svenja van Geul', Anne Babler ${ }^{2}$, \\ Christoph Schmitz ${ }^{1}$, Marc Utzenrath ${ }^{1}$, Thomas Schmitz-Rode ${ }^{1}$, \\ Wilhelm Jahnen-Dechent ${ }^{2}$ \\ ${ }^{1}$ Department of Cardiovascular Engineering, Helmholtz \\ Institute, RWTH Aachen University, Aachen, ${ }^{2}$ Biointerface \\ Laboratory, Helmholtz Institute, RWTH Aachen University, \\ Aachen, Germany
}

Objective: Calcification is a main problem of biological heart valves and responsible for most of their failures. Due to the lack of standardized in-vitro test methods, the calcification-affinity of newly developed or modified heart valves typically requires in vivo animal testing. However, studies on large animals are time and cost consuming, whereas studies on small animals are generally limited to subcutaneous implantation outside the main circulatory system. Thus, the development of appropriate in vitro calcification methods would be beneficial. This study introduces a new in vitro bench test to compare the calcification behavior of biological tissue as used in heart valve prostheses. Methods: Pericardium patches were calcified in vitro under constant conditions at accelerated frequency, pressure and phosphate concentration as well as physiological temperature, calcium concentration and $\mathrm{pH}$-value. The calcification affinity was then analyzed macroscopically and histologically (von Kossa, Alizarin Red, H\&E, Elastica von Giesson). In addition, the calcified pericardium patches were incubated with fluorescent-labeled Fetuin A (FetA), a prototypic systemic inhibitor protein of in vivo mineralization with a high affinity to Hydroxylapatite. This allows quantification of the amount of calcification via fluoroscopy. Results: The macroscopic observation of accelerated in vitro calcified pericardium patches showed calcification deposits. Histological observation revealed intrinsic and extrinsic calcifications. The fluoroscopy exhibited binding of fluorescentlylabeled FetA to the calcified areas. In comparison to the histological results, the same amount of calcification deposits could be detected by means of FetA. Analyses with computed tomography (CT) are ongoing. Conclusions: Pericardium patches were successfully calcified in vitro. The use of FetA as a proof-of-principle detection method is an appropriate tool for examination the calcification affinity of pericardium patches. The deposits generated in vitro were not only comparable to in vivo generated deposits by macroscopical and histological analysis, but also by high and comparable FetA binding to calcium-phosphate deposits in vitro and in vivo. 


\section{Heart Valve Outcomes}

\section{Single Center Experience with All Aspects of Tricuspid Valve Surgery, Including Long-Term Outcomes}

Mark A. Groh ${ }^{1}$, Alan M. Johnson ${ }^{1}$, Oliver A. Binns ${ }^{1}$, Steve W. Ely ${ }^{1}$, Gerard L. Champsaur ${ }^{2}$

${ }^{1}$ Asheville Heart, Asheville, NC, ${ }^{2}$ Mission Heart, Asheville, NC, USA

Objective: Evaluation of tricuspid valve surgery (TVS) has been rarely published outside of the association with mitral valve surgery (MVS), where recent data shows no impact of TVS on early and late mortality. We hypothesized that compared to mitrotricuspid surgery (MTV), other TVS have more severe early and long-term outcomes. Methods: From 2008 to 2014, we performed a series of 315 consecutive TVS procedures, 23 as an isolated procedure (ITV), 36 combined aorto-tricuspid surgeries (ATV), 60 triple valve operations (TVO) and 196 mitro-tricuspid (MTV) procedures with tricuspid regurgitation (TR) graded by clinical, echographic and hemodynamic assessment. Prospectively collected data from our STS database and hemodynamic files were analyzed retrospectively. Main baseline variables are depicted in Table 1 by interventions, pooling together 'other than MTV' procedures (OMT) for presentation purposes. Social Security database query provided long-term follow-up information. Results: Concomitant coronary bypass was performed in $47.90 \%$ of patients with OMT vs. $32.14 \%$ after MVT ( $\mathrm{p}=0.005)$. Early morbidity was similar in the two populations, with identical rates of major complications. Early (30-day) mortality was significantly higher after OMT (10.92\% vs. $3.06 \%$ after MTV, $\mathrm{p}=0.004)$. By sub-group, early mortality was $4.53 \%$ after ITV, $5.56 \%$ after ATV and $16.67 \%$ after TVO. Post-operative mean follow-up was 27.35 months after OMT and 33 months after MTV ( $\mathrm{p}=0.023)$. Cumulative probability of survival at 5 years (fig. 1) was $77.51 \%$ after MTV and $48.35 \%$ after OMT ( $\mathrm{p}=0.009$ ). Comparatively, individual 5 -year survival was $47.86 \%$ after ITV, $45.98 \%$ after ATV, and $52.40 \%$ after TVO procedures. Conclusions: TVS when associated with MVS has low

Table 1. Baseline variables in mitro-tricuspid (MTV) and other than MTV procedures (OMT) (for Abstract of Mark A. Groh)

\begin{tabular}{lccc}
\hline Variable & MTV & OMT & p value \\
\hline Age, years & $69.78 \pm 10.28$ & $69.81 \pm 12.33$ & 0.983 \\
Length of stay, days & $26.2 \pm 5.9$ & $27.7 \pm 6.0$ & 0.031 \\
Infect endocarditis & 9.3 & 3.0 & 0.018 \\
Tric. Annulus $\varnothing, \mathrm{mm}$ & $32.2 \pm 7.7$ & $41.1 \pm 7.2$ & $<0.01$ \\
LVEF, \% & $48.5 \pm 11.7$ & $47.2 \pm 12.5$ & 0.382 \\
Mean CVP, mm Hg & $11.2 \pm 5.5$ & $14.5 \pm 6.7$ & $<0.01$ \\
LV-Ao grad., mm Hg & $11.5 \pm 3.6$ & $36.4 \pm 13.9$ & 0.014 \\
Perfusion time, min & $147.4 \pm 50$ & $173.6 \pm 76.7$ & $<0.01$ \\
Ao. clamp time, min & $108.1 \pm 40.9$ & $173.6 \pm 76.7$ & $<0.01$ \\
\hline
\end{tabular}

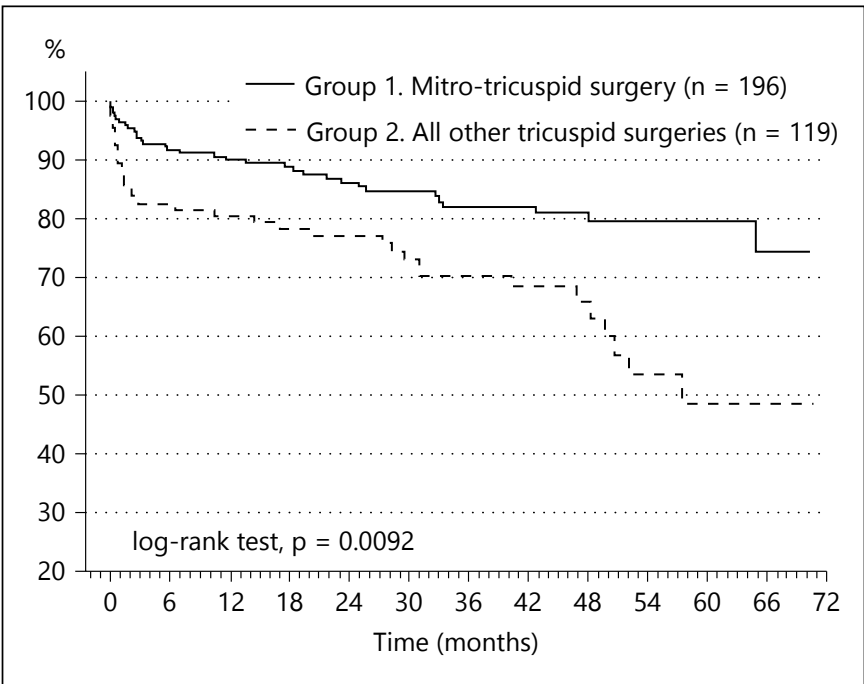

Fig. 1. Comparative cumulative proportion surviving (KaplanMeier) (for Abstract of Mark A. Groh).

early and late mortality, not different from isolated MVS as previously reported. However, when performed as an isolated procedure or associated with aortic valve or part of a triple valve procedure, early and late mortality rates increase significantly.

\section{Normal-Flow, Low-Gradient Discordance in Aortic Stenosis Severity Assessment: The Impact of Vascular Burden}

\section{Nancy Côté, Louis Simard, Anne-Sophie Zenses, Lionel Tastet, Mylène Shen, Marie-Annick Clavel \\ Centre de Recherche de l'institut Universitaire de Cardiologie et de Pneumologie de Québec, Quebec, QC, Canada}

Background: Fifty percent of Normal-Flow, Low-Gradient (NFLG) patients (i.e. low mean gradient [MG] and small aortic valve area) have severe aortic valve calcification (AVC) as measured by computed tomography. However NFLG patients are considered as moderate aortic stenosis (AS) in current ACC/AHA guidelines. Objective: The objective of this study was to evaluate the effect of hypertension and systemic arterial compliance on MG measurements. Methods: First, in 4 sheep with experimentally induced severe and critic AS at: (1) normal aortic pressure, (2) during hypertension (HTN) induced by banding of the aorta, and (3) with reduced aortic compliance (RC) induced by a dacron prosthesis around the aorta, Doppler echocardiography was performed. Second, a comprehensive echocardiography and computed tomography were obtained in 221 patients with AS and normal flow. MG and AVC divided by the cross-sectional area of the aortic annulus (AVCd) were studied according to hypertensive $(>140 \mathrm{~mm} \mathrm{Hg}$ ) and compliance [reduced indexed stroke volume/pulse pressure (SVi/ PP) $\left.<0.8 \mathrm{ml} / \mathrm{mm} \mathrm{Hg} / \mathrm{m}^{2}\right]$ status. Results: In sheep with severe and critic AS, HTN and RC induced a substantial decrease in MG com- 


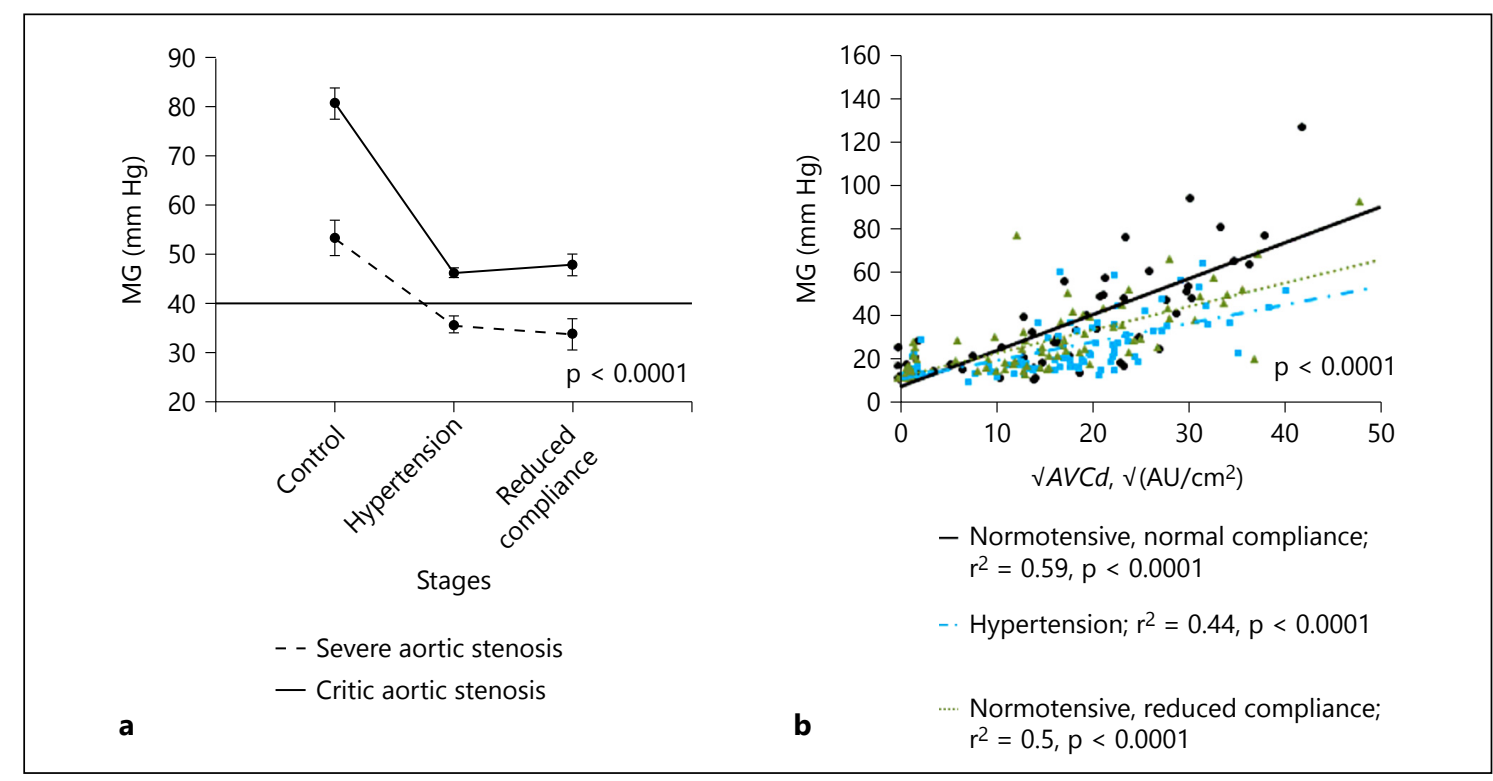

Fig. 1. (for Abstract of Nancy Côté).

pared to normal stage $(\mathrm{p}<0.0001)$ while the transvalvular flow remained stable ( $p=0.2$ severe AS and $p=0.7$ critic AS, figure $1 a)$. HTN and RC resulted in a greater reduction of MG in critic compared to severe AS $(\mathrm{p}=0.01)$. In patients, mean age was $69 \pm 13 \mathrm{yr}$, MG $29 \pm 18 \mathrm{~mm} \mathrm{Hg}$, and mean Stroke Volume index $44 \pm 7 \mathrm{ml} / \mathrm{m} 2$. The slope of the correlation between MG and AVCd was significantly reduced in patients with HTN and RC compared to normotensive patients ( $\mathrm{p}<0.001$; figure $1 \mathrm{~b})$. Accordingly, the number of patients with severe AS as documented by AVCd and NFLG were more frequent in HTN and RC groups ( $17 \%$ and $16 \%$, respectively) compared to the normotensive group $(\mathrm{p}=0.03)$. Conclusion: HTN and $\mathrm{RC}$ are associated with a substantial reduction in MG for similar AVC-load/AS severity, which may lead to a discordant NFLG pattern and underestimation of stenosis severity.

\section{Prognostic Value of Hemodynamic Parameters in Asymptomatic Aortic Valve Stenosis}

Tiffany Mathieu, Virginia Nguyen, Claire Cimadevilla, Maria Melissopoulou, Dimitri Arangalage, Isabelle Codogno, Xavier Duval, Virginie Huart, Alec Vahanian, David Messika-Zeitoun

Hopital Bichat, Paris, France

Background: Conflicting results regarding the most important hemodynamic prognostic parameter in patients with aortic valve stenosis (AS) have been published. The presence discordant grading in a substantial number of AS patients makes this issue even further complex. We aimed to evaluate the respective prognostic value of mean pressure gradient (MPG), aortic valve area (AVA) and Dimensionless Index (DI) in patients with AS. Methods: We prospectively enrolled 319 asymptomatic patients with AS (90 mild, 173 moderate, and 56 severe AS). All patients were followed on a yearly basis (clinical, biological and echocardiographic evaluation) and AS-related events (heart failure, sudden death or new onset of ASrelated symptoms) were collected. Results: After a mean follow-up of $3.1 \pm 1.7$ years, an AS-related event occurred in 84 patients $(26 \%)$. In univariate analysis, baseline MPG $(\mathrm{p}<0.0001)$, AVA $(\mathrm{p}<0.0001)$ and DI ( $\mathrm{p}<0.0001)$ predicted the occurrence of AS-related events. After adjustment for age, gender, history of coronary artery disease, aortic valve anatomy and left ventricular ejection fraction, MPG and AVA were both independent predictor of outcome $(\mathrm{p}<0.0007$ and $\mathrm{p}<0.0001$ respectively) but DI was not $(\mathrm{p}=0.61)$. The independent prognostic value of both parameters (MPG and AVA) was sustained after exclusion of the 31 patients $(10 \%)$ with discordant grading $(\mathrm{p}=$ 0.005 and $\mathrm{p}<0.0001$ respectively) or the 69 patients $(22 \%)$ with bicuspid valve. Conclusion: This study demonstrates that both MPG and AVA are independent and powerful predictors of outcome in patients with asymptomatic AS and highlights that both hemodynamics echocardiographic parameters should be used in association in the clinical management of these patients.

\section{Treatment of Mitral Bio-Prosthetic Valve Deterioration Using Trans Apical Valve in Valve Technique: Intermediate-Term Outcomes}

Ram Sharony, Abid Assali, Dan Aravot, Hana Vaknin-Assa, Yaron Shapira, Alex Sagie, Yaron Barac, Israel Kuznitz, Ran Kornowski

Rabin Medical Center, Petach Tiqva, Israel

Objective: The transcatheter approach for a failed bio-prosthetic valve is an emerging alternative to redo-valve surgery in patients at high surgical risk. We aim to analyze the mid-term 
outcomes of patients undergoing valve-in-valve implantation in the mitral position. Methods: We report our clinical experience in treating consecutive patients with symptomatic structural bioprosthetic mitral valve deterioration using the valve-in-valve technique via the trans-apical approach. Outcomes were rigorously assessed and reported based on VARC 2 criteria definitions. Results: The valve-in-valve procedure in the mitral position was performed in 15 patients, mean age $75 \pm 12.7$ years, mean STS score $10.0 \pm 7.3$. Balloon-expandable Sapien XT devices were used in all patients. The composite endpoint of device success was achieved in $15 / 15$ (100\%) of patients. Post procedural mean mitral transvalvular gradient was $5.5 \pm 3.3 \mathrm{~mm} \mathrm{Hg}$. No patients $(0 \%)$ had $\geq$ mild para-valvular leak. Follow up (up to 5 years, mean 29 months) demonstrated survival rates of $86 \%$. One patient sustained a large intractable pleural effusion that needed repeated drainage and died of sepsis 34 days following the procedure. The second patient died 18 months after surgery from a non cardiac cause. All surviving patients (100\%) were examined and are in NYHA-FC I/II. No valve migration was observed. The mean trans-mitral gradient was $7.2 \pm 2.3 \mathrm{~mm} \mathrm{Hg}$, and the preoperative pulmonary artery pressure decreased from $50.2 \pm 12.6$ to $35.5 \pm 12.2(\mathrm{p}=0.01)$. Conclusions: Intermediate-term clinical outcomes of transcatheter valve-in-valve implantation for the treatment of mitral prosthetic valve deterioration in high risk patient are encouraging. A residual elevated gradient across the valve were observed. This novel approach allows good survival rate and satisfactory quality of life during mid-term follow-up period.

\section{Low Intensive Anticoagulation Ought to Be Regarded as a Trusted Concept for Treatment of Selected Medtronic Open Pivot Mechanical Aortic Valve Patients}

Guido Van Nooten, Yves Van Belleghem, Katrien François, Frank Caes, Thierry Bové, Yves Taeymans

University Hospital Ghent, Ghent, Belgium

Objective: Since January 1993 the Medtronic Open Pivot Mechanical Heart Valve has been implanted routinely in the aortic position at the authors'institution. The study aim was to analyze, retrospectively, the 20-yearclinical results of the prosthesis under a lowered anticoagulation regime. Methods: Between January 1993 and December 2012 a total of 1,056 valves was inserted in the aortic position into consecutive patients. Patients in sinus rhythm, with good left ventricular function and without major vascular pathology $(n=552)$ were prospectively kept in a lower INR regime of 1.5-2.5. Mean age of the patients was $58 \pm 11.7$ years. Preoperatively, $38 \%$ of the patients were in NYHA class $\geq$ III. Frequent comorbidities included: hypertension $(n=228)$, vascular arteriosclerosis $(n=157)$ and coronary disease $(n=114)$. The $99 \%$ complete follow-up totaled 5,521 patient-years (range 24-267 months). Results: 90 -day mortality was $0.7 \%(\mathrm{n}=4$, none valve-related). Survival at 10 -years was $86 \%$ and $73 \%$ at 20 -years. Of the 153 total deaths, 46 were cardiac and 16 valve-related. Multivariable Coxregression analysis selected age as a continuous variable $(\mathrm{p}<$ 0.0001, HR 0.064/year), emergency operations $(\mathrm{p}=0.001, \mathrm{HR}$
$0.93)$, hypertension $(\mathrm{p}=0.003, \mathrm{HR} 1.8)$, renal failure $(\mathrm{p}=0.004 \mathrm{HR}$ $0.43)$, respiratory insufficiency $(\mathrm{p}=0.005, \mathrm{HR} 0.55)$ and poor ventricular function $(\mathrm{p}=0.019$, HR 0.02) as risk factors for death. Renal failure $(\mathrm{p}<0.0001)$ and hypertension $(\mathrm{p}=0.003)$ were considered risk factors for bleeding. Erratic INR $(\mathrm{p}=0.001)$ and vascular arteriosclerosis $(p=0.019)$ were considered as a risk factor for thromboembolism. Conclusions: This 22-year experience demonstrated excellent clinical outcomes with no structural valve failure. Odds ratio defined normotensive young patients as the lowest risk for adverse events. By contrast elderly age in combination with comorbidities and instable anticoagulation yielded the worst longterm results.

\section{Fusion of 3D Ultrasound and Cardiac-MRI Shows Motional Intra-Cardiac Blood Flow In the Whole Cardiac Cycle}

\author{
Koichi Sughimoto' ${ }^{1}$ Yoshiaki Shimamura², Hao Liü, \\ Ken'ichi Tsubota ${ }^{4}$, Kagami Miyaji', Takeshi Yoshii', \\ Shinzo Torii ${ }^{1}$, Tadashi Kitamura ${ }^{1}$, Mitsuhiro Hirata ${ }^{1}$, \\ Toshiaki Mishima' ${ }^{1}$, Tetsuya Horai ${ }^{1}$, Hideaki Haneishi ${ }^{2}$ \\ ${ }^{1}$ Kitasato University Hospital, Sagamihara, ${ }^{2}$ Center for Frontier \\ Medical Engineering, Chiba University, Chiba, ${ }^{3}$ Department \\ of Mechanical Engineering, Graduate School of Engineering, \\ Chiba University, Chiba, ${ }^{4}$ Department of Mechanical \\ Engineering, Graduate School of Engineering, Chiba, Japan
}

Objective: Introduction of three-dimensional ultrasound contributed to improve the quality of mitral valve (MV) repair, whereas development of cardiac-MRI enables to draw flow data in the heart and vessels. However, each modality has its limitations. This study aims to fuse the two modalities to illustrate the intra-ventricular streamline coordinating with valvular and ventricular wall motion. Methods: A 24-year old volunteer was enrolled in this study. Full-volume data sets of the left ventricle (LV) were acquired by real-time epicardial 3D echocardiography using a Philips iE33 handheld probe (Philips, The Netherland). MRI was performed using a Discovery MR750 3.0-T MR system (GE Healthcare, Milwaukee, Wisconsin), and image acquisition was done via ECG triggering with respiratory synchronization method. To illustrate the behavior of blood flow in the left ventricle during a single cardiac cycle, 3D flow visualization using ParaView software 3.12.0 (Kitware Inc., Los Alamos, NM) included time-resolved 3D streamline flow. Offline analysis was done manually to draw the streamline in the LV and wall motion, and by adjusting both images according to the time-frame of ECG. Results: The intra-ventricular blood flow and valvular motion were visualized to illustrate the smooth streamline from the left atrium (LA), MV, LV cavity and LV outflow tract (LVOT). A stroke of blood is conveyed to the next chamber in an efficient way. The anterior leaflet of the MV played an important role in carrying blood from the LA to LV in diastole. In systole, the anterior leaflet guided the flow of blood toward the aortic valve by bulging its sail of the leaflet. Conclusions: Combination of the two modalities of the $3 \mathrm{D}$ echocardiography and cardiac-MRI revealed the mechanism of efficient blood flow conveyance where the anterior mitral leaflet played an important role by pivoting itself and guiding 


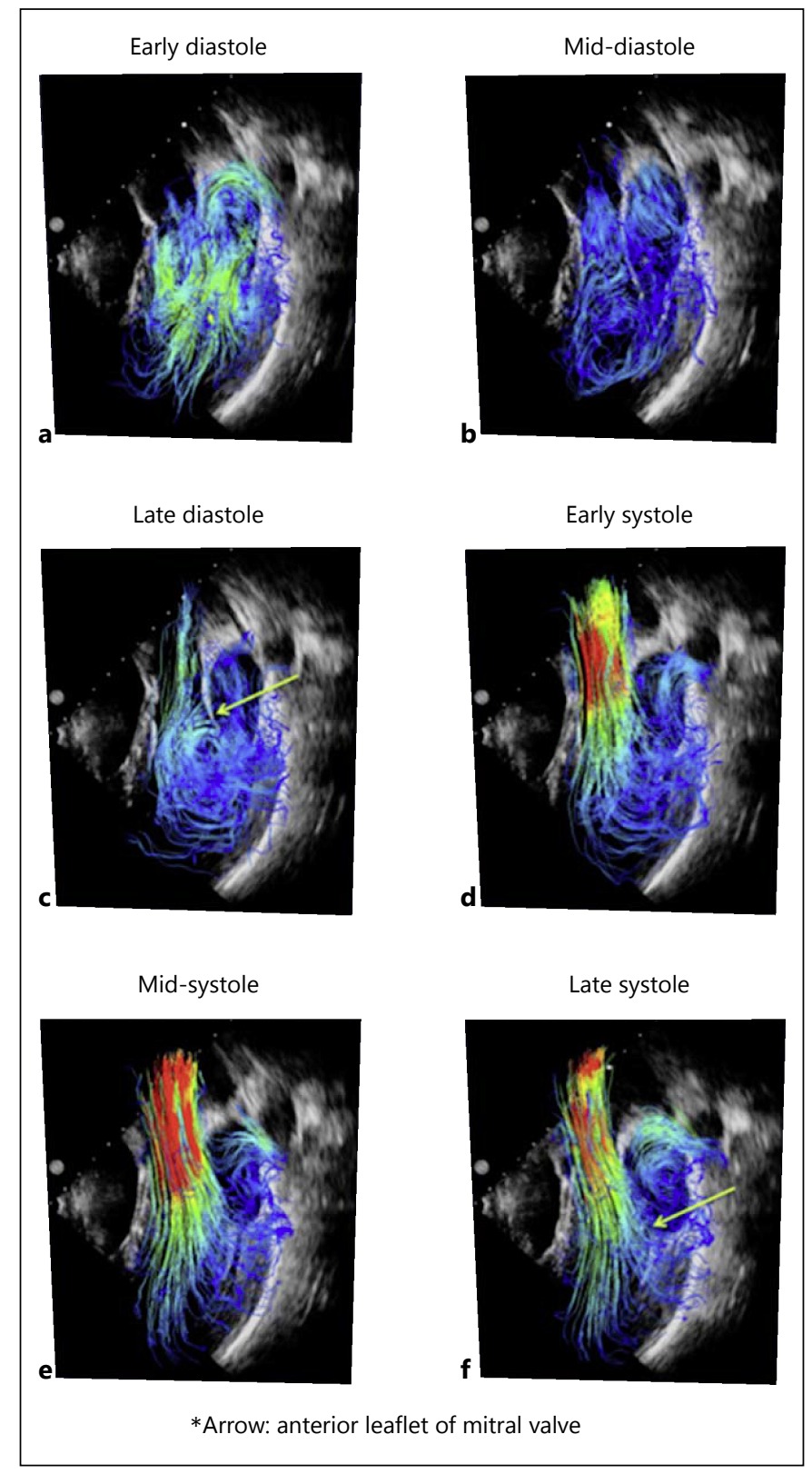

Fig. 1. a Early diastole; b mid-diastole; c late diastole; $\mathbf{d}$ early systole; e mid-systole; f late systole. * Arrow: anterior leaflet of mitral valve (for Abstract of Koichi Sughimoto).

blood flow from the LA, through LV and to LVOT. This fusion method can be applied to understand the complex mechanism of blood flow such as stagnation in dilated cardiomyopathy or congenital heart disease.

\section{Tricuspid Valve Replacement Using a Bileaflet Mechanical Valve: 35 Years of Experience}

Takahiro Shojima, Koichi Arinaga, Koji Akasu, Tohru Takaseya, Kazuyoshi Takagi, Satoshi Kikusaki, Hiroyuki Tanaka

Kurume University School of Medicine, Kurume, Japan

Objective: Compared to procedures involving left-heart valves, the application of tricuspid valve replacement (TVR) is rare. Hence, our experience with TVR procedures is currently limited and the optimal option for valve replacement remains controversial. We review our experience with TVR using a mechanical valve and assess the selection of an optimal valve. Methods: In this study, we reviewed the data of 43 patients who underwent TVR using bileaflet mechanical valves from 1980 to 2014. Among these cases, 25 SJM valves and 18 ATS valves were implanted in the tricuspid position. The median patient age was 51 years, and 30 patients were female. Twelve patients had undergone 1, 14 patients had undergone 2 , and 1 patient had undergone 3 previous cardiac surgeries. The mean follow-up duration was $12.0 \pm 9.4$ years. Results: There were 4 early deaths $(9.3 \%)$ and 13 late deaths. The overall survival rate, including early mortality, was $74 \%, 70 \%$, and $48 \%$ at 10,20 , and 30 years after surgery, respectively. Prosthetic valve thrombosis (PVT) developed a total of 12 times in 9 patients. The linearized rate of the PVT was $1.74 \% /$ patient-years. The rate of freedom from PVT was $84 \%, 64 \%$, and $64 \%$ at 10,20 , and 30 years after surgery, respectively. Thrombolysis with urokinase or tissue plasmin activator restored valve function a total of 6 times in 3 patients. Seven patients required reoperation due to PVT $(n=$ $6)$ and pannus ingrowth $(n=1)$. The linearized rate of the reoperation was $1.36 \% /$ patient-years. The rate of freedom from reoperation was $92 \%, 68 \%$, and $68 \%$ at 10,20 , and 30 years after surgery, respectively. Gastrointestinal hemorrhage and minor operation necessitating transfusion developed in 2 patients, whereas cerebral hemorrhage developed in 1 patient. The rate of freedom from a bleeding event was $92 \%, 80 \%$, and $80 \%$ at 10,20 , and 30 years after surgery, respectively. Valve thrombosis and reoperation were not observed in the ATS valve group. Conclusions: Unless patients have significant contraindications to oral anticoagulation or severe comorbidities that limit a long life expectancy, a bileaflet mechanical prosthesis can be considered as the valve of choice for TVR.

\section{Long Term Survival after Aortic Valve-Sparing (David and Yacoub) Operation with or without Type A Dissection}

Doreen Richardt, Stefan Klotz, Michael Petersen, Bence Bucsky, Thorsten Hanke, Ulrich Stierle, Hans H. Sievers

University Hospital of Schleswig-Holstein, Lübeck, Germany

Objective: Aortic valve-sparing reimplantion operation (David and Yacoub) is more and more used in patients with aortic root aneurysm and unimpaired cusps. But there are little long term data so far. Methods: We analyzed our long term survival and clinical data in patients with David's and Yacoub's procedure at long term follow up examination (FU). Results: In the years 1994 to 2004, 147 pa- 
Table 1. (for Abstract of Doreen Richardt)

\begin{tabular}{lcccc}
\hline Parameter & $\begin{array}{l}\text { Total } \\
(\mathrm{n}=147)\end{array}$ & $\begin{array}{l}\text { David } \\
(\mathrm{n}=58)\end{array}$ & $\begin{array}{l}\text { Yacoub } \\
(\mathrm{n}=89)\end{array}$ & p value \\
\hline Follow up, years & $10.9 \pm 4.7$ & $10.7 \pm 4.8$ & $11.1 \pm 4.7$ & 0.285 \\
Reoperation & $22(15.0)$ & $9(15.5)$ & $13(14.6)$ & 0.880 \\
Time to reoperation, years & $8.6 \pm 4.4$ & $11.1 \pm 5.2$ & $7.0 \pm 3.0$ & 0.030 \\
Deceased & $50(34.0)$ & $20(34.5)$ & $30(32.6)$ & 0.923 \\
Cause of death & $25(17.0)$ & $11(19.0)$ & $14(15.7)$ & 0.747 \\
$\quad$ Non-cardiac & $15(10.2)$ & $6(10.3)$ & $9(10.1)$ & 0.157 \\
$\quad$ Cardiac & $8.2 \pm 5.2$ & $10.5 \pm 5.8$ & $6.7 \pm 4.5$ & $7(7.9)$ \\
$\quad$ Time to cardiac death, years & $10(6.8)$ & $3(5.2)$ & & \\
$\quad$ Unknown & & & & \\
\hline
\end{tabular}

tients (mean age $53.3 \pm 15.6$ years) underwent David's or Yacoub's procedure including acute Type A dissections $(n=47)$. There have been 50 deaths ( 25 non cardiac, 15 cardiac and 10 unknown deaths, 6 in hospital deaths). 27 patients of the 50 deceased patients underwent surgery for Type A dissection $(\mathrm{p}<0.001)$, Time to cardiac death was significantly shorter for Type A dissection $(5.8 \pm 5.5$ years vs. $11.1 \pm 3.4$ years, $\mathrm{p}=0.008) .22$ patients had to be reoperated, time to reoperation was significantly shorter for Yacoub`s procedure. Compared to survival of the German population in both, David's and Yacoub`s procedure, was lower $(\mathrm{p}<0.001) .15 .3 \%$ of the patients had a neurological event and most patients (41.8\%) NYHA I in long term FU. Conclusions: There are nearly no statistical differences between David's and Yacoub's procedure. Both procedures seem to be feasible possibilities of aortic valve-sparing operations, but causes of late death need further exploration.

\section{Impact of Prosthesis-Patient Mismatch after Mitral Valve Replacement in Rheumatic Population}

\author{
Seung Hyun Lee, Young-Nam Youn, Kyung-Jong Yoo, \\ Byung Chul Chang, Sak Lee
}

Severance Cardiovascular Hospital, Seoul, Republic of Korea

Title: Impact of Prosthesis-Patient Mismatch After Mitral Valve Replacement In Rheumatic Population. Background: The purpose of this study was to determine the impact of prosthesis-patient mismatch (PPM) on late tricuspid valve regurgitation (TR) progression and clinical outcomes after mitral valve replacement (MVR) in rheumatic population. Methods: From 2000 to 2013, a total of 447 patients (mean age 54.2 \pm 11.7 years) underwent MVR for rheumatic disease were investigated. Mitral valve effective orifice area was determined by the continuity equation and index for body surface area (EOAi) and PPM was defined as EOAi $\leq 1.2 \mathrm{~cm}^{2} / \mathrm{m}^{2}$. Clinical and echocardiographic follow-up (mean follow up $8.7 \pm 4.0$ years) was performed. Results: $35 \%$ of patients $(n=157)$ had PPM. There were no significant differences in baseline and operative characteristics between patients with and without PPM except EOAi. A significant decreasing in mean trans-valvular pressure gradient (TVPG) over time followed MVR, however the change of
TVPG showed no differences between groups (No PPM vs. PPM: $8.9 \pm 4.7 \mathrm{~mm} \mathrm{Hg}$ à $3.6 \pm 1.2 \mathrm{~mm} \mathrm{Hg}$ vs. $8.7 \pm 4.5 \mathrm{~mm}$ Hgà $3.8 \pm$ $1.4 \mathrm{~mm} \mathrm{Hg}, p$-value $=0.28)$. In all patients, there was a decreasing of left atrium dimension $(58.6 \pm 12.0 \mathrm{~mm}$ à $53.2 \pm 12.0 \mathrm{~mm}$ vs. $57.9 \pm$ $8.9 \mathrm{~mm}$ à $52.2 \pm 8.9 \mathrm{~mm}, p$-value $=0.68)$ and left ventricular end diastolic diameter $(49.9 \pm 5.7 \mathrm{~mm}$ à $48.9 \pm 5.7 \mathrm{~mm}$ vs. $49.7 \pm 6.0 \mathrm{~mm}$ à $48.3 \pm 5.0 \mathrm{~mm}, p=0.24)$, however statistical significance was not found. Freedom from TR progression rates at 3 and 5 years $(99 \%$ vs. $98 \%, 99 \%$ vs. $98 \%, p$-value $=0.1)$, and overall survival rates at 3 and 5 years ( $97 \%$ vs. $96 \%, 94 \%$ vs. $94 \%, p$-value $=0.7$ ) were similar. Conclusion: PPM has not a significant effect on ventricular and atrial remodeling, tricuspid regurgitation, and might not influence late clinical outcome after MVR in rheumatic population.

\section{Comparison of Mild-Term Outcome between Surgical Aortic Valve Replacement with Stentless Bio-Prosthesis and Trans-Cutaneus Aortic Valve Replacement}

Amir Kramer, Ariel Finkelstein, Rephael Mohr, Yanai Ben Ben-Gal, Amit Gordon, Arie Steinvil, Dmitry Pevni

Sourasky Medical Center Tel Aviv, Tel Aviv, Israel

Objective: The decision to perform TAVR rather than SAVR is based mainly on assessment of operative risk using the current surgical risk calculators (STS score and EUROSCORE) The good results of TAVR in high risk patients have led a tendency to use TAVR in patients with lower risk. The purpose of this report is to compare intermediate term outcome of TAVR and SAVR and evaluate EUROSCORE as a criteria to differentiate patients with better outcome. Methods: Four hundred and ninty five consecutive TAVR patients were implanted from January 2009 to April 2014. They were compared to 279 SAVR patients, operated on between 19972014. Inclusion criteria was severe aortic stenosis (BSAx. EOA $<0.65 \mathrm{~cm}^{2} / \mathrm{m}^{2}$ ). Patients with bacterial endocarditis and patients with aortic root enlargement were excluded from the AVR group Additive Euro score I of 10 was used to differentiate between high risk and lower risk patients. Results: TAVR patients were older 
$(82+4$ vs. $71+9, \mathrm{P}<0.001)$. More patients in the TAVR group were females $(56.7 \%$ vs $47.3 \% \mathrm{p}=0.007)$ more had chronic obstructive lung disease (COPD) $(17.2 \%$ vs. $5.7 \%, \mathrm{P}<0.001)$, or prior coronary artery bypass surgery $(\mathrm{CABG})(18.4 \%$ vs. $7.2 \%, \mathrm{p}<0.001)$ and more had or chronic renal failure $(37.2 \&$ vs.9.7\% p < 0.001$)$ On the other hand. more patients in the SAVR group had congestive heart failure ( $29 \%$ vs. $17.6 \%, \mathrm{P}<0.001)$, Occurrence of diabetes and, peripheral vascular disease were similar. Thirty-day mortality of the two groups was similar $(2.4 \%$ vs. $4.3 \%$, in the TAVR and SAVR groups respectively, $\mathrm{p}=0.110$ ). Four years survival (Kaplan-Meier) of the SAVR patients with Additive Euro score I up to 10 was better ( $94.1 \pm 2 \%$ vs. $70.7 \pm 6 \%, p<0.001$ log-rank test). On the other hand patients with Euroscore above 10 had similar survival. Assignment to TAVR was also found to be a significant risk factor for decreased propensity adjusted survival (HR 3.052, 95\% CI 0.075-8.667) in patients with Euroscore,$<10$. Other predictors of decreased survival were: age, prior CABG and PVD. Conclusions: Midterm outcome of SAVR patients with Euroscore up to 10 is better. TAVI may be recommended for patients with euroscore $>10$.

\section{Hyperactive Delirium after Aortic Valve \\ Replacement: Preoperative and Operative Risk Factors}

Wilhelm P. Mistiaen ${ }^{1}$, Ivo Deblier ${ }^{2}$, Philip Van Cauwelaert ${ }^{2}$, Karl Dossche ${ }^{2}$, Philip Muylaert ${ }^{2}$, Anthony Vanermen ${ }^{2}$

${ }^{1}$ University of Antwerp, Faculty of Medicine and Health sciences, Department of Physiotherapy and Rehabilitation, Antwerp, ${ }^{2}$ General Hospital ZNA Middelheim, Department Cardiovasc Surgery, Antwerp, Belgium

Introduction: Delirium after aortic valve replacement (AVR) has been considered as a serious postoperative event. Identification of its predictors could be useful in its early detection and prevention. Methods: Between 2007 and 2014, 1000 consecutive patients underwent AVR with a biological prosthesis in a general teaching hospital. CABG was performed as concomitant procedure in 616 patients. The mean age was $76.2 \pm 6.5$ years and there were 582 males. In 93 patients, an episode of delirium was detected. Thirty preoperative (age, gender, diabetes, chronic kidney disease, chronic obstructive pulmonary disease, malignancy, hypertension, CVA or TIA, left ventricular function parameters, atrial fibrillation, ventricular arrhythmias, coronary, carotid and peripheral artery disease, prior cardiovascular procedures, severity indices of aortic valve stenosis) and operative factors (concomitant procedures, prolonged cross-clamp time) were evaluated for their effect on postoperative delirium by a Fisher-exact analysis. The significant variables were entered in a stepwise logistic regression analysis in order to detect the predictors. The results are given as OR (odds ratio) with 95\% CI (confidence interval) and p-value. Results: The results are listed within the table. Age and vascular (coronary, carotid and peripheral) factors have a significant effect. Delirium is associated with increased length of stay in an ICU $(55 / 91$ or $60.4 \%$ v. $233 / 888$ or $26.2 \%, \mathrm{p}<0.001)$ and with mortality $(13 / 91$ or $16.3 \%$ v. $33 / 890$ or $3.7 \%, \mathrm{p}<0.001)$. Complication rates were also significantly higher which indicates that delirium is a marker for potentially fatal complications. Conclusion: Old age and atheromatosis (coronary, carotid and peripheral) are important predictors for postoperative delirium. Some other cardiac factors, male gender and COPD have also an effect, but only in an univariate way. Remarkably, TIA and CVA did only show a trend.

\section{Chronic Use of Beta Blockade Is Associated with Increased Cardiac Event among Normotensive Patients with Severe Degenerative Mitral Regurgitation}

\author{
Phyllis G. Supino ${ }^{1}$, Ofek Y. Hai ${ }^{1}$, Clare Hochreiter ${ }^{2}$, \\ Edmund Herrold ${ }^{2}$, Jeffrey S. Borer ${ }^{1}$
}

${ }^{1}$ SUNY Downstate Medical Center, Beverly, MA, ${ }^{2}$ Weill Cornell Medical Center, Beverly, MA, USA

Objective: Impact of chronic beta blockade (BB) on cardiac events (CE) in patients (pts) with chronic severe mitral regurgitation (MR) has not been studied by randomized trials but is inferred as beneficial from observational data. However a recent random-

Table 1. Factors with an effect on postoperative delirium (for Abstract of Wilhelm P. Mistiaen)

\begin{tabular}{|c|c|c|c|c|c|c|}
\hline Preoperative factor & $\begin{array}{l}\text { Patients with } \\
\text { delirium: } \mathrm{n} / \mathrm{N}(\%)\end{array}$ & $\begin{array}{l}\text { Patients without } \\
\text { delirium: } \mathrm{n} / \mathrm{N}(\%)\end{array}$ & $\begin{array}{l}\mathrm{p} \\
\text { (univariate) }\end{array}$ & $\begin{array}{l}\text { Odds } \\
\text { ratio }\end{array}$ & $95 \% \mathrm{CI}$ & $\begin{array}{l}\mathrm{p} \\
\text { (multivariate) }\end{array}$ \\
\hline Age $>80$ years & $45 / 93(48.4)$ & $272 / 893(30.5)$ & 0.001 & 1.97 & $1.27-3.06$ & 0.003 \\
\hline Peripheral artery disease & $39 / 93(41.9)$ & $225 / 891(25.3)$ & 0.001 & 1.95 & $1.24-3.06$ & 0.004 \\
\hline Coronary artery disease & $70 / 93(75.3)$ & $569 / 893(63.7)$ & 0.040 & 1.47 & $1.06-2.83$ & 0.016 \\
\hline Prior carotid surgery & $11 / 92(12.0)$ & $41 / 890(4.6)$ & 0.006 & 2.31 & $1.12-4.74$ & 0.024 \\
\hline Concomitant CABG & $70 / 93(72.3)$ & $539 / 893(60.4)$ & 0.011 & - & - & not significant \\
\hline Carotid artery disease & $29 / 93(31.2)$ & $190 / 889(21.4)$ & 0.024 & - & - & not significant \\
\hline Atrial fibrillation & $32 / 93(34.4)$ & $215 / 886(24.4)$ & 0.024 & - & - & not significant \\
\hline Male gender & $62 / 93(66.7)$ & $512 / 893(57.3)$ & 0.051 & - & - & not significant \\
\hline Chronic obstructive pulmonary disease & $36 / 93(38.7)$ & $265 / 882(30.0)$ & 0.056 & - & - & not significant \\
\hline
\end{tabular}




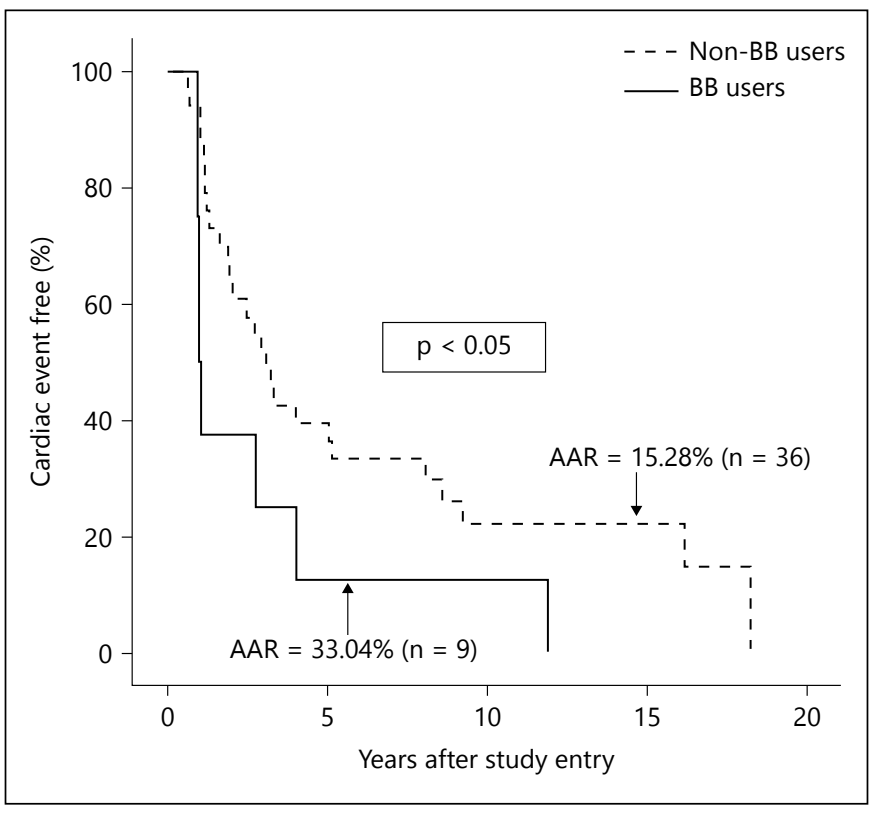

Fig. 1. Beta blockade vs. cardiac events among 45 patients with severe degenerative MR (for Abstract of Phyllis G. Supino).

ized preclinical study demonstrated increased mortality with BB in MR. Our objectives were to evaluate the relation of chronic use of $\mathrm{BB}$ to death or subsequent development of surgical indications in this patient population. Methods: We retrospectively analyzed 45 consecutive study-eligible pts (age $49 \pm 12 \mathrm{yrs}$ ) with isolated chronic severe non-ischemic MR without concomitant coronary disease, followed through $19 \mathrm{yrs}$, to assess impact of chronic BB use at entry on $\mathrm{CE}$ (death or indications for valve surgery). At entry, all pts were non-hypertensive (HTN) and free of surgical indications; 9 pts chronically received $\mathrm{BB}$ at study entry. $\mathrm{CE}$ rate differences were assessed by Kaplan-Meier log rank comparison. Cox model analysis was used to adjust for variations in other baseline covariates (age, gender, etiology, other cardiac drugs). Results: During follow-up, CE included: sudden death (1), heart failure (8), a-fib (6), left ventricular (LV) dimensions (IDs) $>4.5 \mathrm{~cm}$ (11), LV ejection fraction (EF) $<60 \%$ (6), right ventricular (RV) $\mathrm{EF}<35 \%$ (2), combination $\mathrm{CE}$ (7). CE risk was 2-fold higher among pts receiving $\mathrm{BB}$ at study entry (Avg annual risk $[\mathrm{AAR}]=15.28 \%$ ) vs. those not receiving $\mathrm{BB}$ $(\mathrm{AAR}=33.04 \%)(\mathrm{p}<0.05)$ (figure 1$) . \mathrm{A}>3$-fold relative $\mathrm{CE}$ risk was observed when pts on selective BB were excluded (AAR: BB vs. no
$\mathrm{BB}=56.18 \%$ vs. $15.28 \%, \mathrm{p}=0.01) . \mathrm{BB}$ effects remained statistically significant $(\mathrm{p}<0.05)$ when analyses were adjusted for other baseline covariates. Conclusions: Contrary to earlier observational data, in our series BB appears to increase CE risk in pts without HTN. Further research including larger numbers of MR pts, preferably via randomized design, is needed to clarify the discrepancy between the different findings among existing studies.

\section{Aortic Valve-Sparing Root Replacement in Patients Presenting with Congestive Heart Failure Results in Early Reverse Ventricular Remodeling}

\author{
Juan P. Umaña, Jaime Camacho, Nestor Sandoval, \\ Marisol Carreno Jaimes \\ Fundación Cardioinfantil - IC, Bogota, Colombia
}

Objetive: Aortic valve sparing (AVS) root replacement is an accepted procedure in patients with aortic root aneurysms and mild to moderate aortic regurgitation (AR). The purpose of the present study was to investigate the benefits of AVS in late stage $\mathrm{AR}$, and in patients presenting with congestive heart failure (CHF). Methods: All patients undergoing AVS for AR from January 1, 2002 to August30 2015 were identified from an institutional database that follows the guidelines of the Society of Thoracic Surgeons (STS). CHF was defined according to STS adult Cardiac Data Specifications 2.71-2.8. Data on demographics, preoperative, surgical, cardiopulmonary bypass $(\mathrm{CPB})$ and postoperative characteristics were analyzed. Follow-up was done at 30 days and yearly thereafter. Analysis included paired and Student's t-test, Mann-Whitney test, Chi square and Fisher's exact test. Survival analysis was done using the Kaplan-Meier method. Results: A cohort of 82 patients underwent AVS for AR. Fifteen patients (18.3\%) presented with CHF. Hemodynamic characteristics are depicted in Table 1 . Median intensive care unit stay for the CHF group was 2 days (IQR $1-3$ ), for a total median stay of 3 days (IQR 3-5). Follow-up at one year was $95.7 \%$ complete, with $1.6 \%$ mortality and $93 \%$ freedom from $2++\mathrm{AR}$. All CHF patients were in NYHA functional class I or II at last follow-up. Total follow-up was 534 patient/years. Conclusions: AVS represents an excellent operative choice for patients with annuloaortic ectasia presenting with late-stage AR and CHF. Preservation of the native aortic valve is associated with excellent hemodynamics, resulting in significant reverse ventricular remodeling and improvement in ejection fraction. Early functional recovery correlates well with short ICU and hospital stays.

Table 1. Hemodynamic characteristics (for Abstract of Juan P. Umaña)

\begin{tabular}{|c|c|c|c|c|}
\hline \multirow{2}{*}{$\begin{array}{l}\text { Variable } \\
\text { average } \pm \text { SD }\end{array}$} & \multicolumn{2}{|l|}{ Not CHF } & \multicolumn{2}{|l|}{$\mathrm{CHF}$} \\
\hline & preop & postop & preop & postop \\
\hline Ejection fraction $\%$ & $51.8 \pm 7.8$ & $53.6 \pm 4,6$ & $42.9 \pm 9.9$ & $49.7 \pm 12.1^{*}$ \\
\hline LVEDV, cc & $136 \pm 49.6$ & $118.1 \pm 78^{*}$ & $272 \pm 81.7$ & $127 \pm 99.3^{*}$ \\
\hline LVESV, cc & $63.4 \pm 35.3$ & $45.8 \pm 18.4$ & $164 \pm 99.4$ & $85.3 \pm 56.9^{*}$ \\
\hline Aortic root, $\mathrm{mm}$ & $56.1 \pm 8.6$ & $30.3 \pm 1.1^{*}$ & $64.1 \pm 12.2$ & $32.1 \pm 4.3^{*}$ \\
\hline
\end{tabular}




\section{Valve Replacement on a Perfused Beating Heart: Keeping Ischemic Injury at Bay}

\section{Amit Banerjee, Harpreet Singh, Aseem Srivastav, Nikhil Pendse}

Siksha'O'Anusandhan University, Bhubaneswar, India

Objective: Valve surgery on a perfused, beating heart can prevent deleterious ischemic damage caused by cardioplegic arrest during cardiopulmonary bypass (CPB). We present our experience of using different techniques to keep the heart perfused and beating during valve replacements at GB Pant Hospital, New Delhi. Methods: Between 1999 and 2014, 1510 patients (age 7 to 63 years; $57 \%$ males) received 1642 prosthetic heart valves. 581 patients were in NYHA class IV; 14 patients had an EF $<20 \%$. The procedures (all under $\mathrm{CPB}$ ), comprised isolated mitral valve replacement (MVR) with total excision (27) or posterior chordal preservation (98); mitral valve implantation (MVI) with total chordal preservation (809); isolated aortic valve replacement (AVR: 428); combined AVR and MVR (DVR: 31); 16 open mitral valvuloplasty with AVR; and 101 AVR with MVI. Techniques adopted for cardiac perfusion comprised: non-application of aortic cross-clamp (71 MVI); antegrade root perfusion (738 MVI and 125 MVR); of the 576 patients requiring AVR, we used retrograde coronary sinus perfusion (159); alternate antegrade and retrograde perfusion (273); and intermittent coronary ostial perfusion (144). Mechanical mitral valve prostheses (93 ball and cage; 206 tilting disc and 737 bileaflet) and aortic valve prostheses (320 tilting disc, 235 bileaflet and 5 ball and cage) were used. Bioprosthetic mitral and aortic valves were used in 31 and 16 cases respectively. Concomitant procedures included tricuspid annuloplasty (287); surgical ablation for AF (176); and CABG (4). 76 patients had a left atrial thrombus for which the heart was briefly arrested with normothermic potassium-rich antegrade infusion for a very brief period to facilitate safe and meticulous removal of thrombus. Results: Operative mortality occurred in 18 (1.2\%) patients. Reoperation for bleeding was needed in 3 patients $(0.2 \%)$. Postoperatively IABP was required in four patients and $44(3 \%)$ needed inotropic support. Conclusions: Sustained superior improvement, both objective and subjective, in up to 15-years followup distinctly endorse the surmise of negligible myocardial ischemia by avoiding cardioplegic arrest.

\section{Sudden Cardiac Death and Mitral Valve Prolapse: A Single Center Experience}

\author{
Allon Rafael ${ }^{1}$, Philipp Bartko' ${ }^{1}$, Ella I. Starobinska², \\ Anthony J. Choi ', Jodi L. Zilinski ${ }^{1}$, Keaney J. John ${ }^{1}$, Attila Roka', \\ Steven A. Lubitz' ${ }^{1}$ David J. Milan ${ }^{1}$ \\ ${ }^{1}$ Massachusetts General Hospital, Boston, MA, ${ }^{2}$ University of \\ Arizona, College of Medicine, Phoenix, AZ, USA
}

Introduction: MVP has been linked to ventricular arrhythmia and associated with sudden cardiac death. Delineating at risk patients has been the topic of ongoing controversy. High risk features in patients with MVP and cardiac arrest have been described: bileaflet MVP, female sex, young age, biphasic or inverted T waves (inferior leads), frequent complex ventricular ectopy. The purpose of this study is to analyze the clinical characteristics among the MVP and SCD population at our center in order to identify and compare high risk features and contribute to a developing risk stratification process. Methods: Patients with MVP were identified from a search of the ECHO lab database. This dataset was crossed with an electronic medical record search for Sudden Cardiac Death in problem lists and billing codes from years 20002014. Patient histories were reviewed to verify the diagnoses. Patients with primary causes for ventricular arrhythmias were excluded. Results: Our search yielded 32 subjects with MVP and cardiac arrest. 17 patients were excluded due to confounding etiologies including $\mathrm{CAD}$, systolic dysfunction, or ruptured subvalvular apparatus. Mean age was $54 \pm 12.5(46.7 \%$ male $)$. MVP affected both leaflets 11 patients (73\%) and 7 (46.7\%) underwent mitral valve surgery. Mitral regurgitation was severe in 7 subjects $(46.7 \%)$ and moderate in $6(40 \%)$. The first documented cardiac arrest rhythm was ventricular fibrillation (VF) in $85.7 \%$. All patients were treated with implantable cardiac defibrillators (ICDs) and 9 patients $(60.0 \%)$ received appropriate ICD therapies in follow-up. Three of 7 patients treated with surgical valve repair $(46.7 \%)$ received appropriate ICD therapy even after surgical repair. Frequent PVCs were noted in 7 of 15 subjects $(46.7 \%)$ for whom data were available (multifocal in at least one case). $T$ wave abnormalities were present in $46.7 \%$. Only one patient had magnetic resonance imaging which showed no evidence of cardiac fibrosis by late gadolinium enhancement. Conclusion: The majority of MVP and SCD subjects in this cohort had bileaflet MVP. Contrary to findings from previous reports, gender, ventricular ectopy, $\mathrm{T}$ wave inversions and severe MR were evenly distributed. Ventricular arrhythmia persisted despite surgical correction of the valve in a majority of subjects.

\section{Stentless Bioprostheses for Aortic Valve Replacement in Patients with Low Ejection Fraction}

\author{
Juergen Ennker ${ }^{1}$, Lena Hangleiter ${ }^{2}$, Bernd Niemann ${ }^{2}$, \\ Joern Pons-Kuehnemann ${ }^{3}$, Ina C. Ennker ${ }^{4}$, Andreas Boening ${ }^{2}$ \\ ${ }^{1}$ University Witten-Herdecke, Medical Faculty, Witten, \\ ${ }^{2}$ University Hospital Giessen, Giessen, ${ }^{3}$ Justus-Liebig University \\ Giessen, Giessen, ${ }^{4}$ Hannover Medical School, Hannover, \\ Germany
}

Objective: Valve replacement in patients with aortic valve stenosis or insufficiency has been shown to be beneficial especially if a valve prosthesis with a very low gradient is used. Because stentless valve prosthesis have the lowest gradients available, patients with heart failure $(\mathrm{EF}<30 \%)$ underwent aortic valve replacement with those valve substitutes. Methods: From 12/1996 to 12/2012, 4012 patients received stentless aortic valve prosthesis (Medtronic Freestyle) in a single centre. The 606 patients with an EF $<30 \%$ (62.5\% male; $37.5 \%$ female; $86 \%$ NYHA II+III; 1571 patient years) predominantly had aortic stenosis $95.2 \%$ and mixed aortic lesions (72.6\%). 53.6\% had coronary artery disease, 9\% peripheral artery disease. Concomitantly, CABG surgery was carried out in $41.6 \%$, and MVR in $1.2 \%$. Results: The 30 -day survival rate was $92 \%$. Perioperative complications (rethoracotomy 6.4\%, new-onset dialysis 
$10 \%$, myocardial infarction $2.7 \%$, stroke $2 \%$ ) occurred rarely. During the long-term follow-up of up to 17 years, long-term survival was $56 \pm 3.6 \%$ over 5 years, $17.8 \pm 5.3 \%$ after 10 and $11.8 \pm 4.9 \%$ after 15 years. Mean survival was 64.92 months. Reoperations of the aortic valve occurred in $1 \%(0.38 \% / 100$ patient years $)$ and endocarditis in $1.7 \%$ of the patients. Although $17.2 \%$ of the patients were on Warfarin, the bleeding rate $(2.8 \%)$ and the thromboembolic event rate $(0.7 \%)$ were low. Strokes occurred in $4.9 \%$ of the patients. 15 years after surgery, $23.1 \%$ of the patients with a low ejection fraction were in NYHA class 1 or 2 . Conclusions: Aortic valve replacement with stentless prostheses leads to comparably good results in patients with heart failure.

\section{Impact of the Learning Curve on Early Outcomes Following the Ross Procedure in the Current Era}

\author{
Ismail Bouhout, Aly Ghoneim, Raymond Cartier, Nancy Poirier, \\ Ismail El-Hamamsy
}

Montreal Heart Institute, Montreal, QC, Canada

Objective: The use of the Ross procedure has decreased in recent years, in part due to concerns over early operative risks. The aim of this study was to assess the impact of the learning curve on early outcomes following a Ross procedure in the current era in a dedicated starting program. Methods: From 2011 to 2015, 153 consecutive Ross procedures were performed in young adults $(<65$ years), representing our initial experience. One hundred and three (67\%) patients underwent isolated first-do Ross procedure \pm ascending aorta replacement. Twenty-four (16\%) patients underwent redo procedures. The cohort was divided into three consecutive tertiles of 51 patients according to period of surgery (T1, T2 and T3). The mean age was $45 \pm 12$ years and mean Euroscore II was $1.1 \pm 0.5 \%$. Perioperative outcomes were compared between groups and defined using STS guidelines. Safety (including mortality and major morbidity) and efficiency endpoints (cross-clamp and bypass times) were compared. Results: Overall, there were 2 operative mortalities (1.4\%). No early mortality occurred in isolated Ross procedure patients $(n=103)$. In terms of safety, there was a temporal trend towards reduction in the incidence of major complications after T1 (T1: 13 [26\%]; T2: 9 [18\%] and T3: 7 [14\%]; $\mathrm{p}=0.1)$. The most prevalent complication was acute kidney injury defined as $>2$-fold increase in serum creatinine (T1: 7 [14\%] vs. T2: 6 [12\%] vs. T3: 5 [10\%]; $\mathrm{p}=0.81)$. However, there was a trend towards lower rates of temporary dialysis between groups (T1: 4 [8\%] vs. T2: 1 [2\%] vs. T3: 1 [2\%]; $p=0.2$ ). In terms of efficiency, there was a statistically significant improvement across tertiles, after T1, in terms of cross-clamp times (T1: $201 \pm 33$ min vs. T2: $188 \pm 23$ min vs. T3: $184 \pm 31 \mathrm{~min} ; \mathrm{p}=0.008)$ and bypass times (T1: $237 \pm 44 \mathrm{~min}$ vs. T2: $212 \pm 36 \mathrm{~min}$ vs. T3: $210 \pm 41 \mathrm{~min} ; \mathrm{p}=0.001$ ). Conclusions: The Ross procedure is a reproducible operation in the current era. Nevertheless, safety and efficiency improve with experience in an unselected population of patients in a starting dedicated program. Improvements in these parameters are especially observed after the first 50 cases, representing the approximate learning curve.

\section{Perioperative Pain Management in the Elderly Following Transapical TAVR: Does IV Acetaminophen Improve Outcomes?}

\author{
Keith B. Allen, A. Michael Borkon, David J. Cohen, \\ Sanjeev Aggarwal, J. Russell Davis, Kevin Kennedy, Alex F. Pak
}

St. Luke's Mid America Heart Institute, Kansas City, MO, USA

Background: Guideline recommendations to incorporate nonopioid agents such as NSAIDS and Cox-2 inhibitors into multimodal pain management plans is challenging, particularly in elderly cardiac patients, who have contraindications to their use. Using intravenous acetaminophen may reduce overall opioid consumption, meet guideline requirements and improve outcomes in elderly patients undergoing cardiac procedures. Methods: A multimodal pain management strategy that incorporated IV acetaminophen was retrospectively evaluated in 43 patients undergoing transapical TAVR. Prior to formulary availability of IV acetaminophen, 23 transapical TAVR patients received standard post-operative pain management including IV narcotics followed by oral narcotic/acetaminophen. After IV acetaminophen became available, 20 subsequent transapical TAVR patients received IV acetaminophen (4 grams/day, minimum of four doses) in addition to supplemental intravenous and non-acetaminophen containing oral narcotics. Post-operative narcotic doses were standardized to morphine equivalents and clinical outcomes included length of stay and morphine equivalents used on post-operative day 0,1 and 2 were evaluated. Results: TAVR patients whom received IV acetaminophen versus non-IV acetaminophen pain management strategies were similar including STS mortality risk $(10.5 \%$ vs. $9.0 \%, \mathrm{p}=0.3$ ), age ( 86 vs. $85, \mathrm{p} 0.3)$, prior sternotomy $(48.7 \%$ vs. $47.4 \%, \mathrm{p}=0.9), \mathrm{EF}(60 \%$ vs. $60 \%, \mathrm{p}=0.9)$ and female gender $(50 \%$ vs. $61 \%, \mathrm{p}=0.5)$, respectively. Median doses of IV acetaminophen was 6.5 (interquartile range 4.0-18.5) with a median cost (hospital cost $\$ 34 /$ dose) per patient of $\$ 221$ (interquartile range $\$ 136-\$ 629$ ). After adjusting for STS risk score using a median regression model, multimodal pain management using IV acetaminophen was associated with a reduction in median post-operative length of stay from 7 to 5 days [ -1.9 days ( $95 \% \mathrm{CI},-0.9$ to -8.2 days), $\mathrm{p}=0.049$ ]. Patients that received IV acetaminophen used less morphine equivalents on POD zero (22.5 vs. $45.0, \mathrm{p}=0.03)$ with a strong trend towards reducing overall narcotic consumption through POD 2 (66.9 vs. 90.0, $\mathrm{p}=0.1$ ) compared with patients not receiving IV acetaminophen. Conclusion: A multimodal pain management strategy that incorporates IV acetaminophen resulted in a significant reduction in length of stay and narcotic use on the day of surgery with a trend towards reduced overall narcotic use through POD 2 in elderly patients undergoing transapical TAVR. 


\section{Predictors of Mortality in Prosthetic Valve Endocarditis}

\begin{abstract}
Antonio S. Santis, Guilherme Spina, Vitor Rosa, Tarso Accorsi, Joao Ricardo Fernandes, Roney Sampaio, Lucas Pires, Milena Paixão, Flavio Tarasoutchi
\end{abstract}

Instituto do Coração (Heart Institute) - Incor, São Paulo, Brazil

Objectives: Prosthetic valve endocarditis (PVE) is a feared complication in the postoperative period of cardiac valve surgery due to high mortality rates. The aim of this study is to assess the predictors of mortality related to PVE. Methods: The study comprised 100 patients with PVE admitted in a tertiary referral center from 2006 to 2012. Data were collected retrospectively and based on demographic, clinical and laboratory characteristics; echocardiographic findings; microbiological profile (etiology); treatment modality and in-hospital mortality. The diagnosis of PVE was made according to the modified Duke criteria. Statistical comparisons were performed using Student's t test or Fisher's exact test, as appropriate. Multivariate analyses were performed with the regression model. Results: The mean age at diagnosis was $56.9 \pm 16.7$ years, with male preponderance $(73 \%)$. The majority of patients had a biological prosthesis (87\%), with prevalence of late PVE (90\%). Blood cultures were positive in $77 \%$ of cases. Staphylococcus sp and Streptococcus sp infection had exactly the same incidence (29\%), followed by Enterococcus sp (8\%), HACEK group (8\%), fungus (2\%) and other agents (4\%). The overall mortality was $40 \%$. Surgical treatment was performed in $58 \%$ of the patients, mainly due to heart failure and mitroaortic junction abscess, with a mortality rate of $41 \%$. In the group that received medical therapy alone, mortality was $38 \%$. Factors associated with mortality were circulatory shock at presentation $(\mathrm{p}<0.001)$, female gender $(\mathrm{p}=0.02)$, high levels of $\mathrm{C}$ reactive protein $(\mathrm{CRP})$ at presentation $(88.9 \pm 55 \mathrm{mg} / \mathrm{dL}$ vs. $124.2 \pm$ $98.3 \mathrm{mg} / \mathrm{dL}, \mathrm{p}<0.001)$, lower hemoglobin level $(11.9 \pm 2 \mathrm{~g} / \mathrm{dL}$ vs. $10.5 \pm 2.1 \mathrm{~g} / \mathrm{dL}, \mathrm{p}=0.002)$, high systolic pulmonary artery pressure (SPAP) $(43.7 \pm 11.7 \mathrm{~mm} \mathrm{Hg}$ vs. $54.4 \pm 16.7 \mathrm{~mm} \mathrm{Hg}, \mathrm{p}=0.007)$. On multivariate analysis, circulatory shock was the only independent predictor of mortality $(\mathrm{p}=0.05)$. Conclusions: Overall mortality after PVE remains high. Female gender, circulatory shock at presentation, lower hemoglobin level, high CRP levels and high SPAP were predictors of mortality on the univariate analysis. Circulatory shock at presentation was an independent predictor of mortality in PVE.

\section{0-Year Experience with the Carpentier-Edwards PERIMOUNT Standard Aortic Valve at a Single Japanese Center}

Koichi Arinaga, Koji Akasu, Kazuyoshi Takagi, Takahiro Syojima, Tohru Takaseya, Hiroyuki Tanaka

Kurume University School of Medicine, Kurume, Japan

Background: The aim of this study was to evaluate the longterm results of the Carpentier-Edwards Pericardial (CEP) Standard valve at the aortic position. Methods: From January 1996 to December 2010, 275 patients who underwent aortic valve replacement with the CEP valve were enrolled. A $19-\mathrm{mm}$ valve was used in
42 patients, $21-\mathrm{mm}$ valve in 103 patients, $23-\mathrm{mm}$ valve in 97 patients, and $25-\mathrm{mm}$ valve in 33 patients. There were 27 patients 64 years or younger, 50 patients aged 65 to 70 years, 159 patients aged 71 to 79 years and 39 patients 80 years or elder. The early and late results were evaluated. Results: There were 5 early deaths (early mortality rate, $1.82 \%)$. Among the 270 survivors, 255 (94.4\%) were followed-up for a mean duration of 8.4 years (range, 2.3 months to 18.5 years) for a total of 2143.3 patient-years. There were 110 (40.7\%) late deaths during the follow-up period. Cardiac death and valve-related death occurred in 60 patients. Over all actuarial survival rate at 10, and 15 years was $59.0 \%$ and $43.6 \%$, respectively. Actuarial survival rate at 10 , and 15 years was $91.8 \%$ and $91.8 \%$ (64 years or younger), $75.9 \%$ and $37.8 \%$ (65 to 70 years), $55.5 \%$ and $35.4 \%$ ( 71 to 79 years), $22.3 \%$ and $0 \%$ (80 years or elder), respectively. Thromboembolism was observed in 18 patients $(0.84 \%$ /patient-year $)$, structural valve deterioration (SVD) in 8 patients $(0.37 \% / \mathrm{p}-\mathrm{y})$, and reoperation in $13 \mathrm{pa}-$ tients $(0.61 \% / \mathrm{p}-\mathrm{y})$. Actuarial freedom from thromboembolism, SVD and reoperation at 15 years was $87.6 \%, 85.0$ and $82.4 \%$, respectively. Freedom from SVD at 15 years $39.6 \%$ (64 years or younger), $77.1 \%$ ( 65 to 70 years), $100 \%$ (71 to 79 ), $100 \%$ (80 years or elder), respectively. Conclusion: The long-term results of CEP bioprosthesis in the aortic position were satisfactory. Especially, freedom from SVD at 15 year in Pts over 70 years was 100\% in our series.

\section{Maximising Homograft Yield from Cardiac Transplant Recipients}

Nicholas Kang, Jill Faulkner, Helen Gibbs

Green Lane Cardiothoracic Unit, Auckland, New Zealand

Objective: Live donation from transplant recipients is an important source of cardiac valve homografts. The purpose of this study was to investigate whether improvements in surgical technique at the

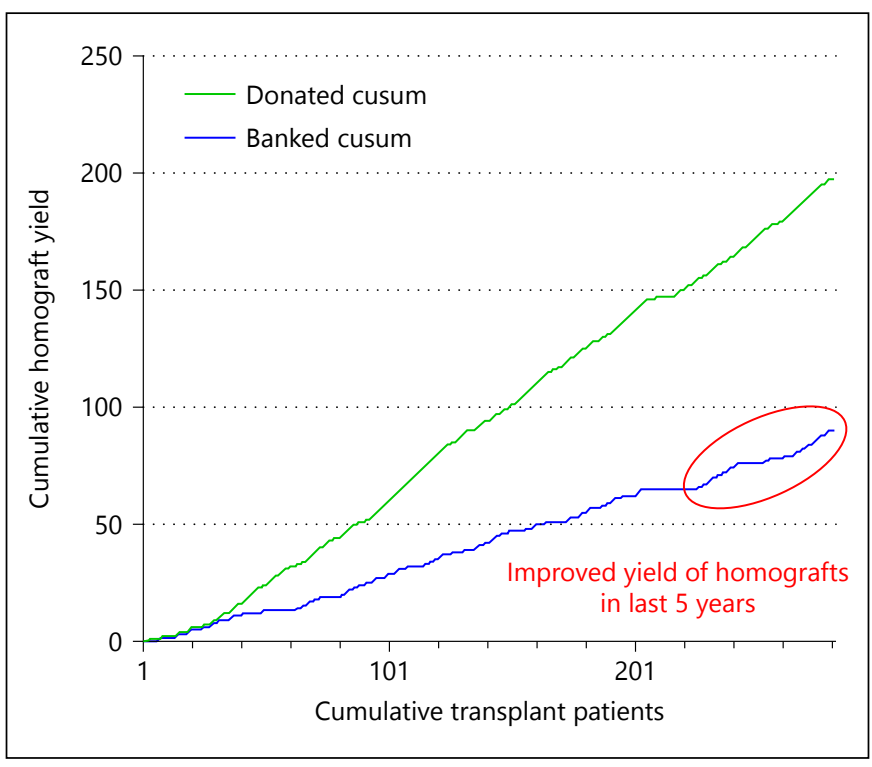

Fig. 1. (for Abstract of Nicholas Kang). 
time of transplantation improved our yield of homografts. Methods: Retrospective review of cardiac transplant and homograft bank registries was undertaken from the inception of the transplant programme at our institution. Analysis of homograft yield and reasons for non-donation were documented. Cumulative sum analysis was used to track the improvement in yield following modification in surgical technique. Results: 281 cardiac transplants were performed, of which 190 recipient hearts were donated (68\%). Reasons for non-donation included older age, valvular disease and congenital anomaly. Of donated hearts, 90 were banked (32\% of all transplants). Reasons for non-banking included inadequate length of the excised great arteries, poor quality valve tissue, and infection hazard. The banked hearts yielded 130 homografts. In the last 5 years, we were able to improve our yield of homografts from $29 \%$ to $47 \%$ of the total transplant cohort by attention to transplant cardiectomy technique. Conclusions: Only one-third of transplant recipients yielded bankable homografts in our overall experience. With better attention to surgical technique at the time of transplant cardiectomy, we were able to improve the overall yield by $60 \%$ in the last 5 years.

\section{The Influence of Frailty Markers on Post-Operative Outcomes among Patients Undergoing Surgical Aortic Valve Replacement with a Low STS Predicted Risk of Mortality}

\section{Alexander Iribarne, Joseph P. DeSimone, Sara B. Bucklin, Roseanne Palmer, Anthony W. DiScipio, Jock N. McCullough}

Dartmouth-Hitchcock Medical Center, Lebanon, NH, USA

Objective: To assess the influence of frailty markers on in-hospital outcomes among patients undergoing surgical aortic valve replacement (SAVR) with a low Society of Thoracic Surgeons predicted risk of mortality (STS-PROM). Methods: Data on 6 frailty makers (Katz score, IADL, grip strength, KCCQ, 5-minute walk test, and albumin) were prospectively collected as a pilot study on $15 \mathrm{pa}-$ tients with severe aortic stenosis considered low risk for SAVR based on their calculated STS-PROM. One point was assigned to each frailty marker if considered positive. Patients were then followed prospectively for the primary endpoints of: ventilator hours, length of ICU stay, length of hospital stay, discharge disposition, and mortality. Results: All patients in the analysis underwent successful
SAVR with a bioprosthetic valve. The mean age of the cohort was $78.3 \pm 6.6$ years, mean aortic valve gradient $48.3 \pm 19.2 \mathrm{~mm} \mathrm{Hg}$, and mean STS-PROM $3.9 \pm 1.3$. The distribution of frailty points was: 1 point $=33.3 \%(n=5), 2$ points $=33.3 \%(n=5), 3$ points or greater $=$ $33.3 \%(\mathrm{n}=5)$. The median length of ICU stay was 70.8 [27.3-98.9] hours, median length of hospitalization was 7 [5-9] days, there were no post-operative or 30-day mortalities, and no 30-day readmissions. When controlling for STS-PROM, the number of frailty score points per patient was not associated with ventilator hours $(\mathrm{p}=$ 0.295 ), length of ICU stay ( $\mathrm{p}=0.181$ ), or length of hospitalization $(\mathrm{p}=0.131)$, however, there was a slight trend towards significance (figure 1). There was also no association between the number of frailty score points and discharge disposition to home versus rehabilitation $(\mathrm{p}=0.741)$. Conclusions: Among patients with a low STSPROM who undergo SAVR, frailty markers alone do not appear to influence post-operative outcomes. As indications for TAVR expand to lower risk populations, additional markers beyond STSPROM will be needed to optimally risk stratify such patients.

\section{Re Entry Complications in Adults Undergoing Redo Cardiac Surgery: Role of Pre Op Imaging}

\section{Gwyn Beattie, Jennifer Whiteley, Geoffrey Berg}

Golden Jubilee National Hospital, Glasgow, United Kingdom

Objective: The percentage of patients requiring valve surgery has now reached $26 \%$ in our unit and the number of patients requiring redo cardiac surgery has subsequently increased. Surgical 'work-up' is usually initiated by the cardiology team to streamline patient flow however there is no consensus for preoperative imaging. This study investigates the requirement of preoperative imaging before redo median sternotomy. Methods: The prospective cardiac database was interrogated for all redo operations from Apr2011 to September2014. The demographics and operation notes identified complications occurring during the time of sternotomy and if femoral bypass was started prior to sternotomy. The national radiology system identified if cross sectional imaging (CT or MRI) was available before the operation. Results: 217 patients had a redo sternotomy during the study period with a median age of 44 . The median number of sternotomies was 2 with a maximium of 5.180 (83\%) cases were elective and 96 cases $(44 \%)$ were adult congenital cases. 20 patients had a complication during re-entry, these includ-
Fig. 1. ICU and hospital length of stay as a function of number of frailty markers (for Abstract of Alexander Iribarne,).

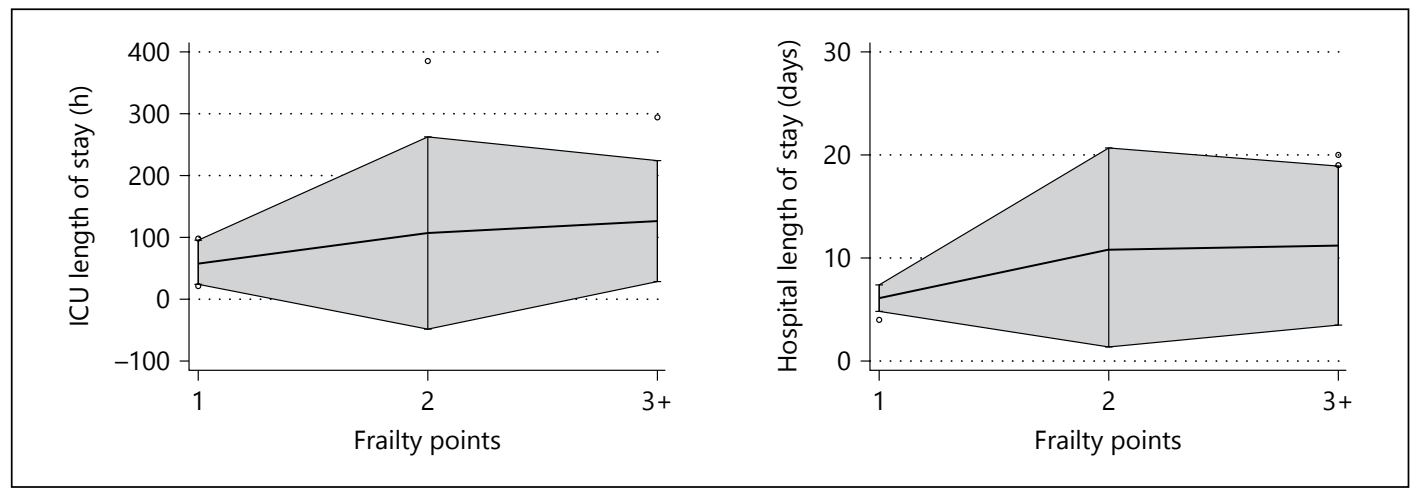


ed: VF [3], Damage to: Subclavian vein [1], LIMA [2], Previous bypass grafts [2], Innominate vein [1], Left ventricle [2], Right atrium [6], Aorta [1], Coronary artery [2]. The predicted mortality for patients with a re-entry complication was 15.58 but the observed mortality was $30 \%$. The observed mortality in patients without a complication was $4 \%$. Patients with imaging and a re-entry complication had an observed mortality of $22 \%$ however without imaging the mortality was $50 \%$. The rate of complications was consistent between the groups at $15.5 \%$ and $14.9 \%$. Conclusions: Complications from re entry injury double mortality from predicted. Cross sectional imaging reduces the observed mortality by $50 \%$.

\section{Aortic Valve Replacement with the First-Generation Mitroflow Bioprosthesis: A 10-Years Single Center Experience}

\author{
Thierry Bove, Jonas Verbeke, Liesbeth Lootens, \\ Yves Van Belleghem, Thomas Martens, Frank Caes, \\ Katrien François
}

University Hospital of Ghent, Ghent, Belgium

Objective: Recent reports raised concerns on the durability of the Mitroflow aortic bioprosthesis, especially for the 12A- and LXA-model, by lack of anticalcification treatment. This study reflects a single-center experience with the Mitroflow for aortic valve replacement (AVR). Methods: From June 2003 to December 2014, 634 patients underwent AVR with the Mitroflow prosthesis. The study focused on 510 consecutive patients that received the firstgeneration prosthesis (2003-2012), by addressing the end-points survival and prosthesis durability. Structural valve degeneration (SVD) was defined by a mean transprosthetic gradient $>30 \mathrm{~mm} \mathrm{Hg}$ at echocardiography and/or need for reoperation. Results: Mean patient age was $76 \pm 6$ years, with $14 \% 80$ y. Valve sizes 23 and 25 were used in $70 \%$, and sizes 19 and 21 in only $18 \%$, avoiding patient-prosthesis mismatch (PPM) in $91 \%$ of the patients. The mean follow-up time was $5.0 \pm 3.2$ years, cumulating a total of 2152 patient-years ( $\max 11.6 \mathrm{y}$ ). The 1-, 5-, and 8-year survival was $86 \pm$ $2 \%, 67 \pm 3 \%$, and $47 \pm 3 \%$ respectively. Freedom from SVD was $99 \pm 1 \%$ and $88 \pm 3 \%$ at 5 and 8 years. Reoperation for SVD was performed in 3.3\%, including redo-AVR (9) or TAVI (6) for cusp rupture (6) and stenotic calcified degeneration (9). Prosthetic explantation for endocarditis was done in 3 patients. No specific patient- nor prosthesis-related factors significantly affected valve durability. SVD was not observed with the more recent Mitroflow model-DLA with phospholipid reduction therapy (used since May 2012), within a maximal follow-up time of 2.8 y. Conclusions: Despite lacking anticalcification treatment, the first-generation $\mathrm{Mi}$ troflow bioprosthesis offered a reliable aortic valve substitute in patients older than 70 years. The low occurrence of PPM, enhanced by its specific design and a consistent supra-annular implantation technique, might have improved the valve durability. Further results with the Mitroflow model-DLA have to be awaited.

\section{Differential Effects of Blood Transfusion after Surgical and Transcatheter Aortic Valve Replacement: A Propensity Matched Analysis}

\author{
John N. Melvan, Vinod Thourani, Jose Binongo, \\ Hanna Jensen, Jose Condado, Jessica Forcillo, \\ Vasilis Babaliaros, Eric Sarin \\ Emory University School of Medicine, Atlanta, GA, USA
}

Background: Blood (PRBC) transfusion is a known risk factor for postoperative complications following cardiac surgery. However, the differential effects of blood transfusion on clinical outcomes after surgical (SAVR) and transcatheter aortic valve replace-

Table 1. Hemodynamic characteristics (for Abstract of John N. Melvan)

\begin{tabular}{|c|c|c|c|c|c|c|}
\hline Matched outcomes: OR (95\% CI) & $1-2$ vs. 0 pRBC & $3-4$ vs. 0 pRBC & $5-6$ vs. 0 pRBC & $>6$ vs. 0 pRBC & $\mathrm{p}$ value & $\mathrm{p}$ value \\
\hline \multicolumn{7}{|l|}{$\operatorname{SAVR}(n=476)$} \\
\hline Postoperative CVA & $1.70(0.35-8.16)$ & $11.60(2.92-46.06)$ & $3.25(0.14-73.55)$ & $4.56(0.66-31.34)$ & 0.27 & \\
\hline Postoperative pneumonia & $3.06(0.31-29.96)$ & $26.35(3.69-187.91)$ & $32.68(2.91-366.56)$ & $61.51(9.40-402.32)$ & 0.01 & \\
\hline Postoperative atrial fibrillation & $2.54(1.20-5.38)$ & $5.37(2.05-14.10)$ & $2.66(0.41-17.23)$ & $7.65(2.80-20.90)$ & 0.41 & \\
\hline Postoperative dialysis & $0.60(0.03-12.85)$ & $6.30(0.78-50.49)$ & $19.55(2.18-175.45)$ & $28.57(5.61-145.52)$ & 0.06 & \\
\hline $\operatorname{TAVR}(n=476)$ & & & & & & $\begin{array}{l}\text { SAVR vs } \\
\text { TAVR }\end{array}$ \\
\hline Postoperative CVA & $2.73(0.42-17.96)$ & $0.30(0.01-7.70)$ & $1.69(0.22-13.24)$ & $3.77(0.64-22.10)$ & 0.01 & 0.20 \\
\hline MACE & $4.04(0.66-24.70)$ & $0.92(0.09-9.15)$ & $6.20(1.05-36.57)$ & $9.50(1.74-51.76)$ & 0.00 & 0.05 \\
\hline Postoperative pneumonia & $0.48(0.10-2.32)$ & $0.10(0.01-1.87)$ & $1.48(0.42-5.17)$ & $2.69(0.91-7.93)$ & 0.00 & 0.01 \\
\hline
\end{tabular}


ment (TAVR) are not well described. This study compared the incidence and consequence of blood transfusion in SAVR and TAVR. Methods: We performed a retrospective review of 3,090 isolated aortic valve replacements at a single U.S. academic institution between 2007-2013. Demographics, timing and the number of units of blood transfused, and postoperative outcomes were compared for SAVR and TAVR. A propensity match of preoperative variables was created for SAVR and TAVR patients in order to assess the impact of blood transfusion volume on clinical outcomes. Results: Of the 3,090 patients undergoing AVR, 1,010 underwent TAVR and 2,080 SAVR. As expected, SAVR patients experienced a greater incidence of blood transfusion during hospitalization $(68.8 \%$ versus $37.4 \%)$. Moreover, transfusion-associated complications including MACE $(\mathrm{p}=0.0169)$, pneumonia $(\mathrm{p}=0.0271)$, and atrial fibrillation ( $\mathrm{p}=0.0164$ ) occurred proportionately more often for SAVR compared to TAVR patients for similar units of blood transfused. After propensity matching of 476 pairs, higher rates of pneumonia ( $p=$ 0.0119 ) and atrial fibrillation $(\mathrm{p}=0.0029)$ were still seen for SAVR compared to TAVR patients after comparable numbers of units of blood were transfused (Table). Conclusions: These results demonstrate higher rates of blood transfusion for SAVR compared to TAVR patients. Moreover, for similar units of blood transfused, transfusion-associated complications including pneumonia and atrial fibrillation were more likely after SAVR compared to TAVR. While TAVR patients are by definition high risk, the difference in surgical approach alone may predispose SAVR patients more to transfusion-associated complications than TAVR patients.

\section{Quality Control of Isolated Bioprosthetic Aortic Valve Replacement in the Post Tavi Period: Impact of Innovative Operative Techniques}

\begin{abstract}
Joseph Nader ${ }^{1}$, Charles-Henri Gautier ${ }^{1}$, Gilles Touati ${ }^{1}$, Christophe Tribouilloy ${ }^{1}$, Hélène Eltchaninoff ${ }^{2}$, Thierry Caus ${ }^{1}$

${ }^{1}$ Amiens-Picardy University Hospital, Amiens, ${ }^{2}$ Charles Nicole University Hospital, Rouen, France
\end{abstract}

Objective: To assess quality control of actual single center surgical outcomes for bioprosthetic isolated aortic valve replacement (AVR) by using sequential analysis of VARC-2 early outcomes. Methods: We retrospectively reviewed a consecutive series of 463 patients operated on for isolated AVR with conventional (369) or sutureless bioprostheses (94). Surgical approach was a complete sternotomy in 412 patients and an upper hemi-sternotomy in 51 patients. We considered overall mortality as well as the composite endpoints 'device success' and 'early safety'. Results of patient groups were screened through funnel plots lowering the confidence interval to $1.5 \mathrm{SD}$ before being scrutinized with CUSUM or SPRT methods whenever required. Results: Overall and 30-days mortality were 3.9 and $2.6 \%$ respectively and was not influenced by the type of bioprosthesis or of surgical approach (funnel plot). Early safety was $88 \%$ for the overall patients and was not significantly influenced by the type of bioprosthesis or of surgical approach (funnel plot). Device success was degraded to $87 \%$ for the sutureless valves $(p=0.057)$ through complete sternotomy (but not through upper sternotomy) and therefore plotted outside of the funnel of confidence interval. Subsequent SPRT accepted H1 for a doubling of the odds of device failure for sutureless valves. CUSUM analysis revealed a prolonged team learning curve after the 30th implant with bovine pericardial nitinol-stented bioprostheses as opposed to balloon-expanded bioprostheses, which rapidly competed with sutured valves. Conclusion: Introducing innovative operative techniques in a standard practice of surgical bioprosthetic AVR with can be performed safely especially concerning mini-invasive approach. However technical challenge with nitinol-stented bioprostheses must not be under-estimated and prolonged ad-hoc support may be required in the present product conformation.

\section{Performance of Edwards Magna in Aortic Position for Small Size Patient Population}

\section{Yuta Kume, Tomoyuki Fujita, Hiroki Hata, Yusuke Shimahara, Junjiro Kobayashi}

National Cerebral and Cardiovascular Center, Osaka

Prefecture, Japan

Objective: Few reports presented the efficacy of Edwards Magna valve in small size patient population. Thus, we analyzed the efficacy of Magna valve in aortic position for small size patient population. Methods: Between June 2008 and April 2015, 282 consecutive patients (113 female, mean age, $69.9 \pm 9.9$ years, mean body surface area (BSA), $1.59 \pm 0.19 \mathrm{~m}^{2}$ ) underwent AVR with a Magna $(n=139)$ and Magna Ease $(n=143)$. We divided the patients into 3 groups (Very Small group $(\mathrm{n}=49, \mathrm{VS})$ : BSA $\leqq 1.40$, Relatively Small group $(\mathrm{n}=103$, RS): BSA 1.40-1.60, Standard group $(n=130, S T)$ : BSA $>1.60)$. The average valve size were $20.0 \pm$ $1.5 \mathrm{~mm}$ in VS group, $20.5 \pm 1.6 \mathrm{~mm}$ in RS group, and $23.1 \pm 2.1$ $\mathrm{mm}$ in ST group ( $\mathrm{p}<0.05$ between VS and ST, RS and ST). The average EuroScore II was $3.4 \pm 4.6 \%$ (VS: $4.0 \pm 5.5 \%$, RS: $3.3 \pm 3.9 \%$, ST: $3.1 \pm 4.8 \%)$. The hemodynamic performance was evaluated by echocardiography pre- and post-operatively and during the follow-up. Results: The mean follow-up time was $3.1 \pm 2.1$ years with a $95.4 \%$ completion rate. Overall hospital mortality was $0.4 \%$. There were 5 explant due to infectious endocarditis and no explant due to structural valve deterioration. The measured effective orifice area (EOA) for each sizes were $1.25 \pm 0.27 \mathrm{~cm}^{2}$ for $19 \mathrm{~mm}$, $1.38 \pm 0.33 \mathrm{~cm}^{2}$ for $21 \mathrm{~mm}, 1.65 \pm 0.29 \mathrm{~cm}^{2}$ for $23 \mathrm{~mm}$ and $1.75 \pm$ $0.33 \mathrm{~cm}^{2}$ for $25 \mathrm{~mm}$. The trans-valvular mean pressure gradients (mPG) were $13.9 \pm 4.6 \mathrm{~mm} \mathrm{Hg}$ for $19 \mathrm{~mm}, 13.0 \pm 5.4 \mathrm{~mm} \mathrm{Hg}$ for $21 \mathrm{~mm}, 11.9 \pm 4.5 \mathrm{~mm} \mathrm{Hg}$ for $23 \mathrm{~mm}$ and $10.7 \pm 4.3 \mathrm{~mm} \mathrm{Hg}$ for $25 \mathrm{~mm}$. The follow up indexed EOA were $0.92 \pm 0.23 \mathrm{~cm}^{2} / \mathrm{m}^{2}$ in VS group, $0.90 \pm 0.21 \mathrm{~cm}^{2} / \mathrm{m}^{2}$ in RS group and $0.92 \pm 0.21 \mathrm{~cm}^{2} / \mathrm{m}^{2}$ in ST group, and the follow up trans-valvular mPG were $12.4 \pm 5.1$ $\mathrm{mm} \mathrm{Hg}$ in VS group, $13.6 \pm 5.1 \mathrm{~mm} \mathrm{Hg}$ in RS group and $11.9 \pm 4.7$ $\mathrm{mm} \mathrm{Hg}$ in ST group, and there were no significant difference between the groups. Follow-up echocardiography $(3.0 \pm 2.3$ years after surgery) revealed Left ventricular (LV) mass was significantly $(\mathrm{p}<0.05)$ reduced postoperatively with regression rate of $27.7 \%$ in VS group, $26.9 \%$ in RS group, and $30.9 \%$ in ST group. Conclusions: Implantation of Magna valve in aortic position was safe and effective procedure for small size patients, with low operative risks and similar hemodynamics and left ventricular mass regression with standard size patients. 


\section{The First United Kingdom (UK) Institutional Experience of the Sutureless (Perceval) Aortic Valve Replacement}

\section{Lay Ping Ong, Faruk Oezalp, Stephen Clark, Stephan Schueler, Thassee Pillay}

Freeman Hospital, Newcastle Upon Tyne, United Kingdom

Objective: Due to the ageing and higher risk population of patients requiring aortic valve replacement (AVR), sutureless valve implantation has been proposed as a safe, reproducible and comparable alternative to conventional techniques. We describe our institution's early and mid-term outcomes with the sutureless Perceval S bioprosthesis (Sorin Biomedica) compared with conventional AVR. Methods: A retrospective review was performed of all patients who underwent AVR with sutureless Perceval or conventional prostheses between January 2014 and June 2015. Patients were divided into Group A (Perceval) and Group B (conventional) for comparison of pre-op characteristics, in-hospital mortality and peri-operative outcomes. Results: Group A consisted of 83 patients (36 M:47 F, mean age $75.71 \pm 5.9$ years) whilst Group B consisted of 208 patients (122 M:86 F, mean age $67.78 \pm 11.1$ years). Patients in Group A were significantly older $(p<0.0001)$ with more females $(p-0.018)$ and higher Logistic Euroscore; (Group A = $9.65 \pm 6.9$ vs. Group B $=6.95 \pm 5.9, p-0.0014$ ), figure $1 a$. Mean CCS and NYHA for both groups were not significantly different; CCS Group $A=1.6 \pm 0.8$ vs. CCS Group B $=1.3 \pm 0.7$ and NYHA Group $\mathrm{A}=2.5 \pm 0.7$ vs. NYHA Group $\mathrm{B}=2.3 \pm 0.8$. Group A had significantly shorter cardiopulmonary bypass times (Group A $=76.68 \pm$ 49.1 mins vs. Group $B=94.52 \pm 37.1$ mins, $p$-0.0009) and crossclamp times (Group A $=46.44 \pm 23.4$ mins vs. Group B $=66.25 \pm$ 26.6 mins, $p<0.0001$ ), figure $1 b$. Post-operative neurological event were similar in both groups (Group A $=1.2 \%$ vs. Group B $=2.4 \%$, $\mathrm{p}$-0.847). Length of ICU stay were significantly longer in Group A (Group $\mathrm{A}=5.62 \pm 11.6$ nights vs. Group $\mathrm{B}=2.33 \pm 3$, p-0.0003). However, in-hospital mortality were similar for both groups (Group A $=2.4 \%$ vs. Group B $=2.4 \%$, p-1.0). Conclusions: Despite an older group with a greater female preponderance and longer
ICU stay, patients with the sutureless Perceval valve were observed to have comparable post-operative outcomes to conventional AVR. Of note, patients with Perceval valves had significantly shorter durations of cross-clamp and cardiopulmonary bypass, respectively. The advantages of the sutureless Perceval implantation technique may mitigate against early complications in elderly, higher risk patients compared to conventional prostheses.

\section{Patient Survey on Patient Information and Decision-Making in Congenital Aortic and Pulmonary Valve Surgery}

Jonathan R.G. Etnel, Willem A. Helbing, Jolien W. Roos-Hesselink, Ad J.J.C. Bogers, Johanna J.J.M. Takkenberg

Erasmus University Medical Center, Rotterdam, Netherlands

Objective: To optimize treatment of patients with congenital aortic (AV) and pulmonary valve (PV) disease, informing patients in an objective and understandable manner and involving them in decision-making is increasingly considered to be important. A cross-sectional survey was conducted to assess the current state of patient information and shared decision-making (SDM) in congenital aortic and pulmonary valve surgery. Methods: A questionnaire was sent to 189 patients who underwent surgery at $0-40$ years of age for congenital AV or PV disease between January 2005 and February 2014. For patients currently $<18$ years of age $(n=32)$, the questionnaire was sent to their parents/caregivers. Survey measures included patient knowledge, views on patient information, involvement in decision-making, decisional conflict, valve-specific quality of life (QoL), numeracy and preference for risk presentation. Results: Seventy-three subjects (38.6\%; 43 AV, 30 PV; 63 patients, 10 parents) were included. Mean time from surgery to survey was $6.2 \pm 2.9$ years. Mean age of the subjects was $36.3 \pm 8.7$ years. Of the included subjects (both patients and parents), only $61 \%$ could recall who their surgeon was and only $51 \%$ were able to answer basic valve-specific knowledge questions correctly. Cardi-

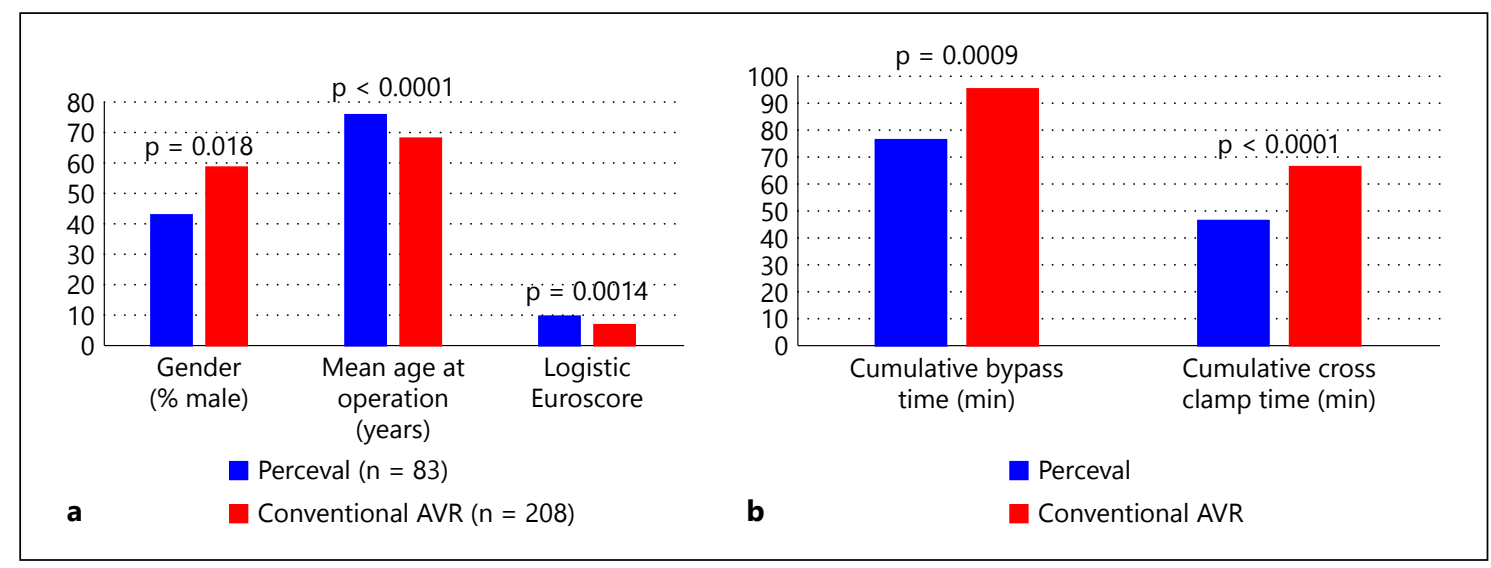

Fig. 1. a Comparison of pre-operative characteristics. b Comparison of intra-operative characteristics (for Abstract of Lay Ping Ong). 
ologists (88\%) and heart surgeons (26\%) were most frequently reported as primary sources of information, while the most important secondary source of information was the internet (30\%). Difficulty in finding reliable information was experienced by $39 \%$ of subjects and $11 \%$ encountered contradictions in the available information. Although $95 \%$ of subjects prefer being involved in decision-making, only $74 \%$ agreed that they were actually involved. Decisional conflict was experienced by $30 \%$ of subjects. In AV patients, QoL was most frequently impaired due to fear of reintervention (38\%), anticoagulation use (34\%) and fear of thromboembolism (30\%). Only $46 \%$ of subjects were numerate and the majority (61\%) preferred pie charts over bar graphs and icon arrays for risk presentation. Conclusions: After 6 years of follow-up, present survey found substantial shortcomings in the current practice of patient information and SDM, which underlines the need for innovative solutions, such as evidence-based online patient information portals. In all communication with patients, limited numeracy and patient preferences for information presentation and SDM should be taken into account.

\section{In vitro Analysis of the Thrombogenic Potential of Heart Valve Prostheses in Blood Testing}

\author{
Torsten Linde, llona Mager, Thomas Schmitz-Rode, \\ Ulrich Steinseifer \\ Department of Cardiovascular Engineering, Helmholtz \\ Institute, RWTH Aachen University, Aachen, Germany
}

Objective: Thrombotic complications are the major failure mode of cardiovascular prostheses, particularly heart valve prostheses. Due to the lack of standardized in-vitro test methods, the thrombogenic potential of newly developed or modified heart valves is typically assessed in lengthy and costly in vivo tests with large animals such as sheep. In vitro testing methods are usually limited to the assessment of the evaluation of the hydrodynamic potential of the prostheses. This study introduces a new method to analyze the thrombogenic potential of heart valve heart valve prostheses in a custom designed blood tester. Methods: A pulse duplicator was custom designed for the use with human or porcine blood. It consists of a linear drive system to generate physiological pressure and flow curves and biocompatible test chambers. The blood flow patterns in the tester were optimized via computational fluid dynamics and validated via experimental methods such as particle image velocimetry and leaflet kinematic studies. General tester function was validated via reference experiments with whole blood and subsequent analysis of thrombus depositions with and without heart valve prostheses. Finally, initial tests were performed on two different types of heart valve prostheses (St. Jude Medical, Triflo Medical) as proof of principle. Results: The blood tester was capable of generating a wide range of physiological and pathological conditions. The flow fields up and downstream of the heart valve prostheses were comparable to those in standardized pulse duplicators. This was confirmed by comparable leaflet kinematics in both test systems. However, the kinematics showed clear dependency on the compliance of the model aortic root. Macroscopic and lightmicroscopic inspection of the tester surfaces did not show any thrombus depositions on the tester surfaces despite substantial valve thromboses, proving the biocompatibility of the tester surfaces. Initial measurements showed higher levels for the coagulation activity, in particular CT and alpha of the HepNaTem, for the tri-leaflet Triflo prostheses in comparison to the bi-leaflet St. Jude prostheses. Conclusions: The custom designed blood tester for the in vitro analysis of the thrombogenic potential of heart valve prostheses may offer an alternative to costly and lengthy animal studies.

\section{Mid-Term Outcomes of Distal Aortic Arch Replacement via Left Thoracotomy Using Antegrade Cerebral Perfusion: A Retrospective Analysis}

\author{
Abdelsalam Elhenawy, Colleen Norris, David zicho, \\ Michael Moon, Roderick MacArthur \\ Mazankowski Alberta Heart Institute, University of Alberta, \\ Edmonton, AB, Canada
}

Objective: Patients with distal aortic arch aneurysms remain a challenging surgical cohort. Expanding treatment options include both open and endovascular approaches to repair. Endovascular strategies remain unattractive in some cases due to arch hypoplasia, angulation, pseudoaneurysm formation, chronic dissection and suboptimal proximal landing zones. Cerebral and spinal cord injury remain a significant morbidity risk to both repair strategies. We present mid-term results of open repair via left thoracotomy using an adjunctive antegrade cerebral perfusion (ACP) protective strategy. Methods: Over a ten-year interval ending in April 2015, a total of thirty five consecutive patients with distal aortic arch pathology were repaired via left thoracotomy using cardiopulmonary bypass and deep hypothermia. Pathology includes congenital, connective tissue and degenerative disorders of the distal aortic arch. Patients with Extent II thoracoabdominal aneurysms were excluded. Thirteen $(37 \%)$ patients presenting for reoperative surgery, fourteen $(40 \%)$ patients with coarctation of the aorta, and eleven (31\%) patients presenting with an acute aortic syndrome. Results: Twenty eight of thirty-five (80\%) patients received adjunctive ACP for cerebral protection during distal aortic arch reconstruction. Mean age was 48 years (range 16-71 years) with an average hospital length of stay 9.5 days. Their intraoperative perfusion data are shown in Table1. There were no in hospital deaths or patient requirement for permanent dialysis. Ten patients (29\%) received no blood products throughout the entire hospital stay. One patient developed permanent paraplegia peri-operatively and there were no new neurologic event. The patient developing paraplegia presented with frank rupture and hemorrhagic shock at the time of

Table 1. Intraoperative perfusion data (for Abstract of Abdelsalam Elhenawy)

Mean time ( $\mathrm{min}$ )

Cardiopulmonary bypass (CPB) 205

Hypothermic circulatory arrest (HCA) 23

Aortic arch reconstruction $\quad 56$

Antegrade cerebral perfusion (ACP) 33

Lowest bladder temperature (Celsius) 20 
surgery. One-year and 5-year survival are $100 \%$ with median follow up of 3.8 years, range 0.10 to 10.3 years. There has been no evidence for neurological events, recurrent coarctation or aneurysm formation and no reoperations on the aorta have been required. Conclusions: We present the largest case series (left thoracotomy) of open repair of distal aortic arch pathology as a safe and reproducible treatment option. Antegrade cerebral perfusion might be considered as an adjunctive measure in the overall cerebral protection strategy.

\section{The ATS Medtronic 3F Stentless Aortic Valve: An Excellent Prosthesis Unappreciated and Misunderstood. A Single Surgeon Experience with 220 Cases}

\section{Guglielmo Stefanelli, Fabrizio Pirro, Davide Trevisan, Massimo Longo, Alina Olaru, Vincenzo Smorto, Marco Meli}

Hesperia Hospital, Modena, IT, Italy

Background: Aim of this study was to evaluate the clinical and hemodynamic results after 220 consecutive implants of Medtronic $3 \mathrm{~F}^{\circledR}$ stentless equine pericardial aortic valve, and to confirm its ease and reproducibility of implantation. Methods: Between March 2007 and August, 2015220 consecutive patients affected by aortic valve disease received a $3 \mathrm{~F}$ valve at our unit by a single surgeon. The size ranged between 21 and 29, with prevalence of size 23 and 25 . Mean age at operation was $73.8 \pm 8.5$ years. $58.7 \%$ of patients were males, the mean logistic EuroScore was $9.76 \pm 6.3$ and $51.8 \%$ of patients received concomitant procedures. For isolated valve replacement the mean ECC time was 90' \pm 10 ', Cross Clamp time $72^{\prime} \pm 8^{\prime}$. In 31 pts the aortic prosthesis was included in a Dacron tube straight graft for a Bentall operation. Results: Early mortality in isolated AVR was $1.3 \%$ and $1.8 \%$ in the entire group. There have been 12 late all-causes deaths (9-non cardiac), with a survival of $93 \%$ at 8 yrs. $91 \%$ of patients were in NYHA class I or II at last control. Actuarial freedom from reoperation due to structural deterioration was $100 \%$ at 5 years, $96 \%$ at 8 years. Freedom from endocarditis and thromboembolic events at 8 years were $97 \%$ and $100 \%$ respectively. Mean aortic pressure gradient measured by echo was $11.5 \mathrm{~mm} \mathrm{Hg}$ at hospital discharge and $10.4 \mathrm{~mm} \mathrm{Hg}$ at the time of follow-up. The mean aortic valve area was $1.74 \mathrm{~cm}^{2}$ at last follow-up. Conclusions: The $3 \mathrm{~F}$ valve is an interesting new generation, user friendly stentless aortic valve substitute with excellent hemodynamics, resistance to infections, durability and freedom from structural deterioration at 8 yrs follow-up. Unfortunately, for many reasons mostly related to business strategies it has not obtained a great diffusion among cardiac surgeons.

\section{A Prospective Randomized Comparison of 3 Pericardial Aortic Stented Valves, Interimediate Results}

Nicolas d'Ostrevy, Lucie Ulmann, Bruno Pereira, Christian Duale, Nicolas Dauphin, Etienne Geoffroy, Benjamin Plateau, Lucie Cassagnes, Kasra Azarnoush, Lionel Camilleri

CHU G Montpied, Clermont-Ferrand, France

Objective: We wished to assess the hemodynamic performance of Sorin Mitroflow ${ }^{\circledR}$ (SM), Edwards-Lifesciences MagnaEase $^{\circledR}$ (ME) and St. Jude Trifecta ${ }^{\circledR}$ (ST) bioprosthesis (Trials, $2013 ; 14: 413$ ) by carrying out a prospective randomized single center study. Here we report intermediate results. Methods: Bioprosthesis performances were assessed by echocardiography on day 7 and at 6 postoperative months. During the intervention, native aortic ring size was measured with a universal sizer specifically designed. The primary endpoint was mean gradient at the sixth month. The secondary endpoints were the mean gradient at discharge, the maximum gradient, the surface and the indexed orificial surface at discharge and 6 months, without and after stratification on native annulus size. We also compared the efficiency ratio between the inner diameter of the implanted valve (from manufacturer's data) and the diameter of the native annulus (measured by the universal sizer). Results: 66 patients were randomized during the first part of the protocol (SM:21, ME:23 and ST:22). At 6 month, the mean gradient $(\mathrm{mm} \mathrm{Hg})$ was not statistically different between the groups (SM:12, ME:10 and ST:13; $\mathrm{p}=0.25)$. At discharge, the mean gradients ( $\mathrm{mm} \mathrm{Hg}$ ) (SM:14, ME:12 and ST:9; $\mathrm{p}=$ 0.006), maximum ( $\mathrm{mm} \mathrm{Hg})(\mathrm{SM}: 24, \mathrm{ME}: 22, \mathrm{ST}: 18 ; \mathrm{p}=0.06)$ and the orificial surface $\left(\mathrm{cm}^{2}\right)$ (SM:1.5, ME:1.7 ST:1.9, $\left.\mathrm{p}=0.01\right)$ were all statistically different in favor of the ST bioprosthesis. The efficiency ratio was also statistically higher in the ME and ST groups (SM 0.8, ME:0.93 and ST:0.93; p < 0.001). In the native ring <24 $\mathrm{mm}$ subgroup, at discharge, we found a difference in favor of the ST group on average gradients $(\mathrm{mm} \mathrm{Hg})(\mathrm{SM}: 15, \mathrm{ME}: 12 \mathrm{ST}: 9 ; \mathrm{p}=$ $0.004)$ and maximum (mm Hg) (SM:26, ME:25 and ST:18, p = $0.01)$. This difference was also significant on orificial surface $\left(\mathrm{cm}^{2}\right)$ (SM:1.4, ME:1.7 and ST:2; p = 0.001) and the indexed surface $\left(\mathrm{cm}^{2} / \mathrm{m}^{2}\right)(\mathrm{SM}: 0.8, \mathrm{ME}: 0.86$ and ST:1.1; $\mathrm{p}=0.02)$. These last two persist at the 6th postoperative month. Conclusions: Half of the inclusions performed, some measurements suggest higher performance of the prosthesis St. Jude Trifecta ${ }^{\circledR}$. In our opinion, its more efficient design allows replacement of the same native ring with larger inner diameter bioprosthesis. This element must be confirmed on all planned inclusions. 


\section{Myocardial Protection during Mitral Valve Surgery and Artery Bypass Grafting in High Risk Patient: Strategies and Cardioplegic Solutions}

\author{
Jens Garbade, Sven Lehmann, Denis Merk, Piroze Davierwala, \\ Martin Misfeld, Michael Borger, Friedrich-Wilhelm Mohr \\ Heart Center Leipzig, Leipzig, Germany
}

Objective: Optimal myocardial protection during complex cardiac procedures are challenging and demanding. Therefore, we hypothesized that a single administration of Bretschneider histidintryptophan-ketoglutarate (HTK) crystalloid solution (Custodiol) offers myocardial protection comparable to repeat blood cardioplegia in patients with ischemic cardiomyopathy receiving mitral valve surgery (MVS) and coronary artery bypass grafting (CABG). Methods: We reviewed a prospectively compiled single-center database containing all patients with ischemic cardiomyopathy (EF <30\%) received a MVS combined with CABG from 2000 to 2013. Preoperative demographic and investigative data, operative characteristics, early and longterm mortality and morbidity were compared between Custodiol solution and blood cardioplegia groups. Propensity score matching was performed to correct for any bias that may be associated with the use of HTK Bretschneider solution. The follow-up was made with a mean time span of 3.6 years (range $0-12.5$ years). Results: A total of 410 complex cardiac procedures were identified of which 159 (40.8\%) received Custodiol solution (group 1) and 231 (59.2\%) used blood cardioplegia (group 2) as the primary myocardial protection agent. Pre-operative characteristics were not significant different with a mean ejection fraction of $24.3 \pm 6.6 \%$ (group 1) vs. $23.8 \pm 6.8 \%$ (group 2). However, the mean logistic EuroSCORE was lower in patients received Custodiol (15.6 vs. 19.6; $\mathrm{p}=0.02$ ). Operation-, bypass- and cross clamp time was significant shorter in group 1: $204.9 \pm 56.0,117.8 \pm 39.0$ and $75.1 \pm 25.1$ minutes vs. group 2: $255.7 \pm 80.7,142.5 \pm 48.2$ and $82.0 \pm 29.7$ minutes. The incidence of postoperative mechanical support was similar in both groups. However, in patients who received Custodiol we found a statistical significant lower incidence of postoperative gastrointestinal complication, new pacemaker implantation, and dialysis. The 30-day, 1-year, 5 -year and 10 -year mortality was $12.1 \%, 23.8 \%, 36.7 \%$ and $55.6 \%$ for group 1 and $12.5 \%, 29.6 \%, 40.1 \%$, and $56.6 \%$ for group 2 respectively. Conclusions: Considering the impaired LV function and the complexity of procedure, we could show that the use of Custodiol is convenient, and at least as safe as blood cardioplegia with similar longterm survival in our study of those who received crystalloid cardioplegia. However, for further validation a randomized study is warranted.

\section{Current Outcomes after Isolated Aortic Valve Replacement in the Octogenarians}

Thomas Senage, Basile Marie, Caroline Cueff, Bertrand Rozec, Sabine Pattier, Jean-Christian Roussel, Le Tourneau Thierry

Nantes University Hospital, St-Herblain, France

Objective: To assess the actual hospital morbi-mortality of isolated aortic valve replacement (AVR) in a monocentric cohort of octogenarians between 2002 and 2014. Methods: Between 2002 and 2014,769 octogenarians patients, men in $46.4 \%(n=357)$ with a mean age of $82.7 \pm 2.4$ [80-94.4] underwent isolated AVR with implantation of a biological valvular prosthesis at our center. Surgical indication was aortic stenosis in $79.3 \%$ of patients. Redo surgeries were excluded. Results: Hospital mortality was $1.95 \%$ ( $\mathrm{n}=$ 15) with an Euroscore 2 average of $2.7 \pm 3$ [0.9-41.2]. Mean Intensive Care Unit stay was $3.1 \pm 4.5$ days for a total hospital stay of $13.5 \pm 7.9$ days. Main postoperative complications included renal failure $(15.6 \%)$ requiring dialysis in $2.7 \%$ of the cases and stroke (1.2\%). Multivariate predictors for hospital mortality were cardiogenic shock $(\mathrm{OR}=34.8$ [4.2-215.9]) and $\mathrm{BMI}$ greater than $30(\mathrm{OR}$ 4.5 [1.3-16.2]). Conclusions: Nowadays, aortic valve replacement in octogenarians is a reliable surgery, with a low hospital morbimortality rate and a improved identification of patients at risk.

\section{Echocardiographic Evaluation at Rest and Under Exercise of Sorin Freedom Stentless Aortic Valve in 184 Patients at 8 Years}

\author{
Guglielmo Stefanelli, Fabrizio Pirro, Clorinda Labia, \\ Giuseppe Danniballe, Massimo Longo, Marco Meli \\ Hesperia Hospital, Modena, IT, Italy
}

Objective: To emphasize the importance of evaluating the biological aortic prostheses under stress conditions. To encourage the choice of an aortic valve prosthesis in relation to a better quality of life of the patient, particularly in the younger population. Methods: Between March 2003 and April 2010184 patients operated in our unit for aortic valve replacement with a Sorin Pericarbon Freedom bovine pericardial stentless valve by a single surgeon underwent an Echocardiographic evaluation at rest and under stress at 8 years follow-up. The mean age at surgery was $69.7 \pm 10.9$ (range $17-89$ yrs). Mean Log Euroscore was $7.73 \pm 7.09$. Concomitant procedures were carried out in $41.3 \%$ of patients. Mean follow-up time was 6.8 years. The stress protocol consisted of a maximum exertion of $100 \mathrm{w}$ for 6 minutes at cycle-ergometer. Assessment of Peak/Mean aortic gradient, effective orifice area, effective orifice area index were obtained along with evaluation of Patient/prosthesis mismatch, NYHA functional class, and percentage of ventricular mass reduction. Results: A low increase of mean gradient (from $7.8 \mathrm{~mm} \mathrm{Hg}$ to $11.6 \mathrm{~mm} \mathrm{Hg}$ ) and peak gradient (from $14.9 \mathrm{~mm} \mathrm{Hg}$ to $21.6 \mathrm{~mm} \mathrm{Hg}$ ) even under maximum exercise has occurred The observed improvement in effective orifice area (from $1.86 \mathrm{~cm}^{2}$ to $1.96 \mathrm{~cm}^{2}$ and effective orifice area indexes (from $1.05 \mathrm{~cm}^{2} / \mathrm{m}^{2}$ to $1.10 \mathrm{~cm}^{2} / \mathrm{m}^{2}$ ) might be related to increased cardiac output during exercise, along with a possible stentless valve adaption to changes in patient's hemodynamics. Severe patient/prosthesis mismatch was present only in a few cases. Clinical conditions at follow-up were excellent ( $92 \%$ of patients were in NYHA class I). Conclusions: The Sorin Pericarbon Freedom prosthesis is a bovine pericardial stentless valve ideal for aortic valve replacement. The hemodynamic evaluation at rest and under stress demonstrate excellent performances, with a possible capability of valve adaption to increase in cardiac output. The SPF may be considered as a good alternative as aortic valve substitute in younger, active population and in cases of acute bacterial endocarditis. A longer follow-up time is mandatory in order to drop reliable conclusions. 


\section{Mitral Regurgitation and Increased Risk of All-Cause and Cardiovascular Mortality in Patients with Type 2 Diabetes}

Andrea Rossi ${ }^{1}$, Giacomo Zoppini ${ }^{1}$, Giovanni Benfari ${ }^{1}$, Giulia Geremia ${ }^{1}$, Stefano Bonapace ${ }^{1}$, Enzo Bonora ${ }^{1}$, Corrado Vassanelli ${ }^{1}$, Maurice Enriquez Sarano ${ }^{2}$, Giovanni Targher ${ }^{1}$

${ }^{1}$ University of Verona, Verona, Italy; ${ }^{2}$ Mayo Clinic, Rochester, MN, USA

Background: Mitral regurgitation (MR) is the most common heart valve disease in the general adult population and it is associated with cardiac remodeling and excess mortality. Objectives: We aimed to analyzed the prevalence, determinants and prognostic implications of MR in patients with type 2 diabetes (T2DM). Methods: We retrospectively analyzed the data from 814 type 2 diabetic outpatients, who had undergone a conventional echocardiography for clinical reasons during the years 1992-2007. Presence and severity of MR was evaluated by using an integrated multi-parametric echocardiographic approach. The study outcomes were all-cause and cardiovascular mortality. Results: At baseline, MR was present in $32 \%$ of these patients. The severity of MR increased progressively with advancing age and ventricular function impairment. Mitral valve abnormalities were also significantly associated with MR (occurring in $33 \%$ of patients with mild MR and $67 \%$ of those with moderate-to-severe MR; $\mathrm{P}<0.001$ ), but they were also present in $19 \%$ of patients without MR. Over a mean follow-up period of 9 years, 120 (14\%) patients died, 50 of them from cardiovascular causes. Compared with those without MR, patients with mild MR had a 3.3-fold increased risk of all-cause mortality, whereas those with moderate-to-severe MR had a 5.1-fold increased risk of allcause mortality. Results remained statistically significant after adjustment for multiple potential confounders. Similar results were also found for CVD mortality. Conclusions: Our results show that MR is very common in patients with T2DM and is independently associated with an increased risk of both all-cause and CVD mortality, even if the degree of MR severity is mild.

\section{Balloon-Expandable Device for Non-Aortic Valve in Valve Transcatheter Heart Valve Implantation}

Judson Williams, Matthew M. Sherwood, Andrew Wang, Jeffrey G. Gaca

Duke University Medical Center, Durham, NC, USA

Objective: To examine results with valve-in-valve (ViV) procedures for degenerated non-aortic bioprostheses as an alternative for the standard of care approach in high risk patients. Methods: Five patients have undergone non-aortic ViV at our institution, including 4 mitral and 1 tricuspid. Bioprosthetic failure was due to stenosis in 3 and regurgitation in 2. A balloon-expandable device was used for all patients (Edwards Lifescience, Irvine, CA). Results: All patients were determined to be at high-risk for conventional redo surgery. Successful transcatheter ViV replacement was accomplished by a transapical (mitral) or transfemoral vein (tricuspid) approach. One transapical patient required cardiopulmonary bypass due to ventricular tachycardia. Median length of stay postoperatively was 5 days. No deaths occurred through a median follow-up of 21 months. NYHA at follow-up was decreased to I-II from IV for all patients. No elevated transvalvular gradients or paravalvular leakage greater than trivial was encountered. Postoperative complications encountered were pleural effusion requiring drainage, hematuria, epistaxis, acute kidney injury, and atrial fibrillation. Conclusions: Transcatheter non-aortic ViV implantation for dysfunctional bioprostheses can be performed safely with favorable clinical outcomes using a balloon expandable device.

Table 1. Patient and prosthesis characteristics (for Abstract of Judson Williams)

\begin{tabular}{|c|c|c|c|c|c|c|c|c|c|}
\hline Age & $\begin{array}{l}\text { Initial prosthesis } \\
\text { type }\end{array}$ & $\begin{array}{l}\text { Mechanism } \\
\text { of failure }\end{array}$ & $\begin{array}{l}\text { Label size } \\
\mathrm{ED}(\mathrm{mm})\end{array}$ & $\begin{array}{l}\text { Label size } \\
\text { ID }(\mathrm{mm})\end{array}$ & $\begin{array}{l}\text { Type of } \\
\text { THV }\end{array}$ & $\begin{array}{l}\text { THV size } \\
(\mathrm{mm})\end{array}$ & $\begin{array}{l}\text { Follow-up time } \\
\text { (months) }\end{array}$ & Complications & $\begin{array}{l}\text { NYHA at } \\
\text { follow-up }\end{array}$ \\
\hline 71 & CE perimount & $\begin{array}{l}\text { Severe mitral } \\
\text { stenosis }\end{array}$ & 27 & 25 & $\begin{array}{l}\text { Edwards } \\
\text { SAPIEN }\end{array}$ & 26 & 30 & $\begin{array}{l}\text { Right pleural effusion } \\
\text { requiring drainage; } \\
\text { postoperative atrial } \\
\text { fibrillation; AKI without } \\
\text { need for RRT }\end{array}$ & I \\
\hline 65 & CE perimount & $\begin{array}{l}\text { Severe tricuspid } \\
\text { stenosis }\end{array}$ & 29 & 27 & $\begin{array}{l}\text { Edwards } \\
\text { SAPIEN XT }\end{array}$ & 29 & 9 & None & I \\
\hline 79 & Baxter porcine & $\begin{array}{l}\text { Severe mitral } \\
\text { regurgitation }\end{array}$ & 25 & 23 & $\begin{array}{l}\text { Edwards } \\
\text { SAPIEN XT }\end{array}$ & 23 & 9 & $\begin{array}{l}\text { Postoperative nausea } \\
\text { resolved with decreased } \\
\text { narcotics }\end{array}$ & $\mathrm{I}$ \\
\hline 85 & CE perimount & $\begin{array}{l}\text { Severe mitral } \\
\text { stenosis }\end{array}$ & 25 & 23 & $\begin{array}{l}\text { Edwards } \\
\text { SAPIEN }\end{array}$ & 26 & 25 & $\begin{array}{l}\text { Hematuria with urology } \\
\text { consultation for bladder } \\
\text { irrigation }\end{array}$ & II \\
\hline 79 & Medtronic mosaic & $\begin{array}{l}\text { Severe mitral } \\
\text { regurgitation }\end{array}$ & 33 & 28 & $\begin{array}{l}\text { Edwards } \\
\text { SAPIEN XT }\end{array}$ & 29 & 21 & $\begin{array}{l}\text { Epistaxis with ENT } \\
\text { consultation }\end{array}$ & II \\
\hline
\end{tabular}




\section{The Ross Operation in Neonates: The Best Choice?}

Stefano Congiu', Sergio De salvatore ${ }^{2}$, Antonio Segreto ${ }^{2}$, Subbarayalu Balaji', Chiusaroli Alessandro' ${ }^{2}$, Federico Bizzarri'

${ }^{1}$ Leeds Children Hospital, Leeds, United Kingdom; ${ }^{2}$ Università di Roma la Sapienza, Latina, Italy

Objective: The Ross procedure was first described in 1967 for the treatment of aortic valve disease in adults. This procedure has been lastly applied to neonates and infants expecially for severe left ventricular outflow tract obstruction (LVOTO) and severe aortic valve disease. The potential for growth and the proven durability make the autograft an ideal aortic valve substitute. Methods: 5 neonates less than 30 days of age underwent a Ross procedure. The
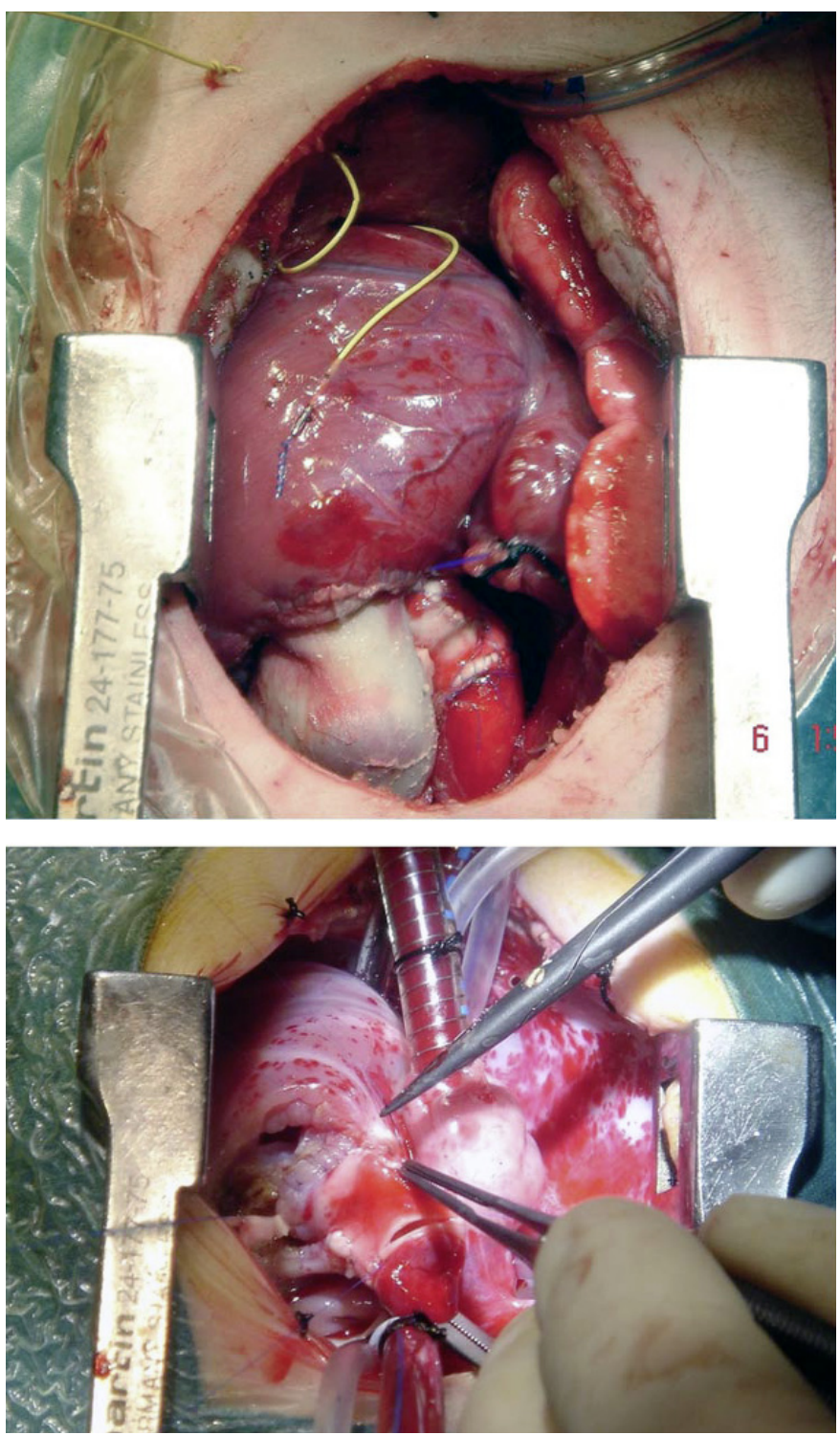

Fig. 1. (for Abstract of Antonio Segreto). median weight was $3.2 \mathrm{~kg}$. Three patients had a LVOTO with different degrees of endomiocardial fibroelastosis. Two of them had a severe aortic regurgitation with dysplastic aortic valve after aortic valvuloplasty. Three neonates required with a bovine yugular valve conduit and two of them a aortic homograft for the reconstruction of the RVOT. Results: There were no death with complete recovery of the left ventricular function in all cases. The autograft only showed trivial aortic insufficiency with no residual LVOTO on Doppler echocardiography. The telediastolic volume increased to more than $20 \mathrm{ml} / \mathrm{m}^{2}$ and telediastolic pressure decreased to normal values before discharged from the hospital. The median hospital stay was 16 days without complications. All the patients are free of operation or complications with a follow-up of 5 years. Conclusions: There were no death with complete recovery of the left ventricular function in all cases. The autograft only showed trivial aortic insufficiency with no residual LVOTO on Doppler echocardiography. The telediastolic volume increased to more than 20 $\mathrm{ml} / \mathrm{m}^{2}$ and telediastolic pressure decreased to normal values before discharged from the hospital. The median hospital stay was 16 days without complications. All the patients are free of operation or complications with a follow-up of 5 years.

\section{The Future of the Heart Valve Allografts: 25 Years of Banking in Brussels}

\section{Ramadan Jashari, Béatrice Van Hoeck, Richard Ngakam Noumanje, Rosario Diaz Rodriguez}

Saint Jean Clinic, Bruxelles, Belgium

Objective: Thousands of patients have benefitted of heart valve allografts since the initial orthotopic implantation of cadaveric valves by Ross and Barratt-Boyes in 1962. In Europe, about 1500 allograft valves have been implanted each year in children with congenital malformation and/or young patients. Methods: Traditionally, the allograft valves have been cryopreserved and stored in vapors of liquid nitrogen in the extreme low temperatures (below $-135^{\circ} \mathrm{C}$ ) using dimethylsulphoxide as cryoprotectant solution. We are presenting our results of the clinical application of the conventional allograft valves distributed in Europe over 25 years. Currently, divers decellularization techniques are used for cell removal of the allografts. EHB is a partner of the EU multicentric clinical trial ESPOIR with decellularized pulmonary allografts. Results: 6155 valves (3561-pulmonary, 2540-aortic and 54-mitral) have been successfully implanted for RVOTR, LVOTR and Mitral/Tricuspid replacement/repair. Treatment of endocarditis with abscesses and natvie/prosthetic valve destruction, are of the most important indications, as allografts almost neve show early recurrent endocarditis. The long term results are better than for tissue valves of animal origin. However, there is evidence of early allograft deterioration in the newborns and children of up to 5 years of age (60\% failure rate at 5 years after implantation). Conclusions: The results of clinical application of allograft valves are excellent in adult patients. However, due to accelerated deterioration in the newborns and children, decellularized allografts might be the real option for this patient subgroup. ESPOIR clinical trial will probably give this answer within few years. 


\section{Effect of Body Mass Index (BMI) on Outcomes in Cardiac Valve Surgery}

Siavash Saadat, Karan Chhabra, Viktor Dombrovskiy, Anthony Lemaire, Aziz Ghaly, George Batsides, Leonard Y. Lee

Rutgers - Robert Wood Johnson Medical Sc, New Brunswick, NJ, USA

Objectives: Previous research on the effect of body mass index (BMI) on cardiac valve surgery has shown mixed outcomes attributed to various mechanisms. Here we analyzed our experience with cardiac valve surgery and the potential risk factors that contribute to increased postoperative morbidity and mortality among patients across the BMI spectrum. Methods: A total of 716 patients who underwent cardiac valve surgery, including aortic valve replacement (AVR) \pm coronary artery bypass grafting $(\mathrm{CABG})$, mitral valve repair $(\mathrm{MVR}) \pm \mathrm{CABG}$, mitral valve replacement $\pm \mathrm{CABG}, \mathrm{AVR}+\mathrm{MVR}$, or any other combination of valve surgery from January 2012-June 2013. Patient demographics, comorbidities, postoperative complications and mortality were evaluated in BMI classes including underweight $(<18.5)$, normal (18.5-24.9), overweight (25-29.9), and obese $(>30)$. Chi-square test and logistic regression analysis were performed using SAS 9.4 software (SAS Institute, NC). Results: Of the 716 patients, $17 / 716(2.4 \%)$ were classified as underweight with BMI <18.5, 193/716 (29.9\%) were classified as normal weight BMI 18.5-24.9, 227/716 (31.7\%) were classified as overweight BMI 25-29.9, and 279/716 (38.9\%) were classified as obese with BMI $>30$. Rates of prolonged ventilator time ( $>4$ hours postoperatively) were higher in the underweight group (35.3\%) compared to normal weight $(10.9 \%)$, overweight $(13.2 \%)$, and obese $(15.1 \%)$ patients $(\mathrm{p}=0.05)$. Cases of postoperative acute renal failure tended to be higher in underweight patients $(17.7 \%)$ compared to normal weight $(5.18 \%)$, overweight $(4.41 \%)$, and obese $(8.24 \%)$ patients $(\mathrm{p}=0.065)$. The percentage rates of atrial fibrillation, bleeding, and stroke was higher in underweight patients compared to the other groups, however the differences were not statistically significant. Mortality in the underweight patients was $2 / 17(11.76 \%)$, normal weight $10 / 193$ (5.18\%), overweight $8 / 227$ (3.52\%), and obese $15 / 279$ (5.38\%); however this difference was not statistically significant. Conclusions: Underweight patients with $\mathrm{BMI}<18.5$ are at risk for poor outcomes after cardiac valve surgery, with a statistically significant higher rate of prolonged ventilator time and a trend towards higher rates of postoperative acute renal failure. Further research on the effects of BMI on outcomes in cardiac valve surgery is necessary.

\section{What Is the Predictor of Persistent High Trans-Prosthetic Velocity Early after Aortic Valve Replacement?}

Yukio Umedq ${ }^{1}$, Yasunari Yokota'2, Yukihiro Matsuno', Yoshio Mori', Yoko Kawamura ${ }^{2}$, Hiroshi Takuya ${ }^{1}$

${ }^{1} \mathrm{Gifu}$ Prefectural General Medical Center, Gifu, ${ }^{2} \mathrm{Gifu}$ University, Gifu, Japan

Objective: We sometimes experience the persistent high transprosthetic velocity (TPV) early after AVR in spite of appropriate EOAI. Little was addressed to this issue. In the present study, we focused on the patients with persistent high TPV after AVR and clarified its profile. And we explored the predictors for TPV. Methods: Thirty-six patients underwent isolated aortic valve replacement were recruited in this study. Patients with low EF $(<50 \%)$, aortic regurgitation ( $\mathrm{AR}>2$ ), and $\mathrm{PPM}\left(\mathrm{EOAI}<0.85 \mathrm{~cm}^{2} / \mathrm{m}^{2}\right)$ were excluded. Persistent high TPV was defined as $3.0 \mathrm{~m} / \mathrm{s}$ or greater. Clinical characteristics, cardiac parameters, and peripheral artery distensibility parameters were assessed. Cardiac parameters were obtained by echocardiography at pre-AVR and 1 week after AVR. At the same time, static and dynamic distensibility parameters of the carotid artery were also obtained by a real time echo-tracking system (e.g. $\beta, \mathrm{Ep}, \mathrm{AC}, \mathrm{dD} / \mathrm{dt}$, and tDPc). Un-paired t-test was used for comparing the results of the two groups. Stepwise multiple regression analysis was used to identify the generalized linear model to predict the post-AVR trans-prosthetic velocity. Results: Eight patients underwent AVR revealed persistent high TPV. Compared with low TPV group, high TPV group had greater pre-AVR peak AoV (pre-AoV; $5.5 \pm 0.9 \mathrm{~m} / \mathrm{s}$ vs. $4.8 \pm 0.7 \mathrm{~m} / \mathrm{s})$, less dD/dt $(2.89 \pm$ $1.30 \mathrm{~mm} / \mathrm{s}$ vs. $4.03 \pm 1.27 \mathrm{~mm} / \mathrm{s})$, and less BMI $\left(19.9 \pm 2.2 \mathrm{~kg} / \mathrm{m}^{2}\right.$ vs. $\left.22.6 \pm 2.8 \mathrm{~kg} / \mathrm{m}^{2}\right)$. There were no significant differences in comorbidities, medications, LVEF, FS, SVi, or static peripheral artery distensibility parameters. A stepwise multiple regression analysis identified two predominant predictors of the TPV; pre-AoV and the ratio of EOAI to pre-AVR AVAI (EOAI/AVAI). Correlation between the predicted value calculated using formula $(=2.31-$ $0.44^{*} \mathrm{EOAI} / \mathrm{AVAI}+0.345^{*}$ pre-AoV) and the TPV was significant with $\mathrm{R}$-value of $0.603, \mathrm{p}<0.001$. Conclusion: Two predictors (i.e. pre-AoV and EOAI/AVAI) found in this study would be reasonable when we regard heart and peripheral vascular system as an apparatus creating TPV. The formula found in the present study to predict the TPV could be significance to avoid the persistent high TPV early after AVR. 


\section{EuroSCORE II, BNP, C-Reactive Protein, Creatinine and Delayed Myocardial Enhancement Imaging are Predictors of 1-Year Mortality in Patients with Low-Flow and Low-Gradient True Severe Aortic Stenosis with Low Ejection Fraction}

Vitor E. Rosa, Roney O. Sampaio, Milena R. Paixão, Joao Ricardo C. Fernandes, Tarso A. Accorsi, Antonio S. de Santis, Guilherme S. Spina, Marcelo L. Vieira, Thamara C. Morais, Juliana H. Bello, Wilson Mathias, Jr., Carlos E. Rochitte, Carlos Manuel A. Brandão, Flavio Tarasoutchi

HC FMUSP InCor, Sao Paulo, Brazil

Objective: Low-Flow and Low-Gradient True Severe Aortic Stenosis with Low Ejection Fraction (LFLGAS) is a patology with higher mortality when compared to Normal Gradient Aortic Stenosis, but still benefits from aortic valve replacement. Risk stratification is complex and new parameters of mortality prediction are needed. Our objective is to asses mortality predictors in LFLGAS. Methods: This is a prospective study with 14 patients with LFLGAS. All patients underwent Dobutamine Stress Echocardiogram (DSE), 3D echocardiogram, Cardiac Magnetic Ressonance (CMR) and laboratory tests, including C-reactive protein, BNP and troponin. The variables were analyzed using the Student's t-test and Fisher's exact test. Results: The mean age was $68.5 \pm 10.3$ years, $7.14 \%$ female, $100 \%$ with degenerative aortic stenosis. All patients had heart failure symptoms, $7.14 \%$ syncope and $7.14 \%$ angina. Arterial sys- temic hypertension was found in $71.4 \%$, diabetes in $35.7 \%$ and coronary artery disease in $42.8 \%$. Creatinine was $1.41 \pm 0.39 \mathrm{mg} / \mathrm{dl}$, BNP $704 \pm 795 \mathrm{pg} / \mathrm{mL}, \mathrm{C}$-reactive protein $11.92 \pm 17.64 \mathrm{mgqdl}$ and troponin $0.04 \pm 0.02 \mathrm{ng} / \mathrm{ml}$. The mean EuroSCORE II was $3.74 \pm$ $3.52 \%$ and STS $3.07 \pm 4.42 \%$. All patients were submitted to surgery. The postoperative 30 -day mortality was $21.4 \%$ and 1 -year mortality of $35.7 \%$, all of cardiovascular cause. All patients had left ventricule flow reserve and the diagnosis of true severe aortic stenosis DSE. One-year mortality predictors are shown in table 1 . Conclusion: In this study, only EuroSCORE II, BNP, C-reactive protein, creatinine and Delayed Myocardial Enhancement in CMR were predictors of 1-year mortality in patients with LFLGAS.

\section{Are Patients with Prior Malignancy and Symptomatic Degenerative Aortic Valve Disease too Frail to Undergo Aortic Valve Replacement}

\author{
Wilhelm P. Mistiaen ${ }^{1}$, Ivo Deblier ${ }^{2}$, Philip Van Cauwelaert ${ }^{2}$, \\ Karl Dossche ${ }^{2}$, Philip Muylaert ${ }^{2}$, Anthony Vanermen ${ }^{2}$ \\ ${ }^{1}$ University of Antwerp; Faculty of Medicine, Division of \\ Physiotherapy and Rehabilitation, Antwerp, ${ }^{2}$ General Hospital \\ ZNA Middelheim, Antwerp, Belgium
}

Objective: Patients referred for aortic valve replacement (AVR) are usually elderly. A prior malignancy might be more common in this group. The 30 -day postoperative outcome is studied.

Table 1. (for Abstract of Vitor E. Rosa)

\begin{tabular}{lccc}
\hline & Live $(\mathrm{n}=9)$ & Dead $(\mathrm{n}=5)$ & p value \\
\hline EuroSCORE II & $2.27 \pm 1.63$ & $7.03 \pm 4.32$ & 0.0107 \\
BNP, pg/ml & $385 \pm 185$ & $1,280 \pm 1,092$ & 0.0292 \\
Creatinine, $\mathrm{mg} / \mathrm{dl}$ & $1.23 \pm 0.12$ & $1.74 \pm 0.49$ & 0.0100 \\
C-reactive protein, $\mathrm{mg} / \mathrm{dl}$ & $3.05 \pm 2.04$ & $26.11 \pm 21.8$ & 0.0069 \\
CMR aortic valve area, cm ${ }^{2}$ & $0.71 \pm 0.19$ & $0.79 \pm 0.13$ & 0.4215 \\
CMR ejection fraction, \% & $29.3 \pm 7.0$ & $24.0 \pm 8.4$ & 0.2290 \\
CMR delayed myocardial enhancement & $1 / 9$ & $4 / 5$ & 0.023 \\
3D ejection fraction, \% & $29.7 \pm 8.06$ & $26.4 \pm 8.3$ & 0.4738 \\
3D aortic valve area, $\mathrm{cm}^{2}$ & $0.65 \pm 0.13$ & $0.72 \pm 0.12$ & 0.3417 \\
\hline
\end{tabular}

Table 1. Relation between cancer and preoperative factor and 30-day outcome (for Abstract of Wilhelm P. Mistiaen)

\begin{tabular}{lccc}
\hline Factor & With cancer, n/N (\%) & Without cancer, n/N (\%) & p value \\
\hline Preoperative ventricular premature beat & $15 / 239(6.3)$ & $194 / 1,719(11.3)$ & 0.019 \\
Male gender & $148 / 241(61.4)$ & $944 / 1,729(54.6)$ & 0.027 \\
Obesity & $46 / 137(33.6)$ & $355 / 840(42.3)$ & 0.033 \\
FEV1 <70\% of the expected value & $95 / 814(11.7)$ & $23 / 131(17.6)$ & 0.044 \\
Need for urgent AVR & $16 / 239(6.7)$ & $68 / 1,710(4.0)$ & 0.044 \\
Coronary artery disease & $143 / 240(59.6)$ & $1,111 / 1,717(64.7)$ & 0.070 \\
Postop disorientation or delirium & $18 / 135(13.3)$ & $74 / 837(8.8)$ & 0.071 \\
Postop ventricular arrhythmias & $13 / 238(5.5)$ & $58 / 1,716(4.4)$ & 0.082 \\
Increased length of hospital stay (>8 days) & $34 / 131(26.0)$ & $260 / 804(32.3)$ & 0.086 \\
\hline
\end{tabular}


Methods: Setting: General hospital Patients: 2000 patients, operated between 1986 and 2014 (obesity, hyperlipidemia HLP, disorientation, forced expiratory volume FEV $1<70 \%$ of the expected theoretical value and length of stay have been recorded only for the last 1000 patients). Device: biological heart valve. Age: Median \pm IQR 76 (72-81) years. Prior malignancy: present in 241 patients. Statistics: Fisher exact test to calculate the relation between the presence of malignancy and the other factors. Results: The significant factors or those with a trend are given in the table, in order of increasing p-value. Factors without significant association for malignancy were prior CVA, hypertension, peripheral artery disease, left ventricular function parameters, prior coronary artery procedures, conduction defects, prior atrial fibrillation, history of smoking, pulmonary arterial hypertension, need for concomitant CABG, procedure on the ascending aorta or repair of the mitral valve, increased need red blood cells or other blood products, increased length of stay on ICU, valve related complications, conduction defects, congestive heart failure, renal or pulmonary complications or 30-day mortality. Conclusions: Patients with a history of malignancy do not have considerably more comorbid conditions. These patients are usually less obese and have less hyperlipidemia. They can withstand surgical AVR in an era where transcatheter aortic valve implantation becomes increasingly important. There is a trend for more postoperative disorientation and a weaker one for increase in length of stay.

\section{Stentless Bioprostheses in Octogenarians: Sensible or Too Much Surgery?}

\author{
Andreas Boening ${ }^{1}$, Bernd Niemann ${ }^{1}$, Peter Roth ${ }^{1}$, \\ Behnam Zadeh ${ }^{1}$, Joern Pons-Kuehnemann ${ }^{2}$, Ina C. Ennker ${ }^{3}$, \\ Juergen Ennker
}

${ }^{1}$ University Hospital Giessen, Giessen, ${ }^{2}$ Justus-Liebig University Giessen, Giessen, ${ }^{3}$ Hannover Medical School, Hannover,

${ }^{4}$ University Witten-Herdecke, Medical Faculty, Witten, Germany

Objective: In octogenarians, biological bioprostheses are the conduits of choice for aortic valve replacement. Because of a higher surgical time and effort compared to stented prostheses, stentless bioprostheses are rarely used in this age group. We sought to determine the results of stentless biological heart valve replacement in octogenarians. Methods: From 12/1996 to 12/2012, 4012 patients received stentless aortic valve prostheses (Medtronic Freestyle) in a single centre. The 635 patients older than 80 years of age at the time of implant had a mean age of $83 \pm 2$ (2320 patient years), the male/ female ratio was 40:60, NYHA class I and II in $22.5 \%$, preoperative atrial fibrillation in $20.6 \%$, coronary artery disease in $55.7 \%$, mitral valve disease in $12.5 \%$ and aortic disease in $3.6 \%$. The patients were followed in written form and by telephone interview, follow-up completeness was about $96.3 \%$. Results: Early after surgery, mortality $(10.2 \%)$ and new-onset dialysis (10.6\%), myocardial infarction (1.4\%) and stroke (2.6\%) occurred rarely. During the long-term follow-up of up to 17 years, the bleeding rate in the ctogenarians was $4 \%$ despite of many patients being on oral anticoagulants $(23 \%)$. While the pacemaker implantation rate $(10.7 \%)$ during long-term FU was high, astonishingly, the rate of neurological disorders was quite low (1.8\%). Long-term survival after 5 years $(55.1 \% \pm 2.1)$, after 10 years $(21.1 \% \pm 2.5)$, after 15 years $(3.9 \% \pm 3.1)$ was low due to the patients' age at the time of surgery. Mean survival was 70.7 (66.3) months. Reoperation rate was $0.8 \%$ (0.003 events per patient year). Conclusions: Despite more adverse events early after surgery than known for younger patients, octogenarians fare well in the long term with a stentless biological aortic valve prosthesis.

\section{Associations between Mitral Valve Prolapse, Psycho-Emotional Variables and HR-QoL}

\author{
Tali Bayer-Topilsky ${ }^{1}$, Maurice Enriquez-Sarano ${ }^{2}$
}

${ }^{1}$ Myers-JDC-Brookdale Institute, Jerusalem, Israel;

${ }^{2}$ Mayo Clinic, Rochester, MN, USA

Background: Associations between mitral valve prolapse (MVP) and the patient's psycho-emotional status (PES) has been disputed, but have not been rigorously addressed. Methods: MVP and MR were prospectively and comprehensively assessed in 281 patients (age $61 \pm 13$ years, $63 \%$ men); 216 MVP were compared to 65 without MVP (of similar age and sex). Simultaneously, we assessed the patient's PES (anxiety, depression, posttraumatic stress symptoms (PTS)) and health-related quality of life (HRQoL) using validated questionnaires. Results: $29 \%$ of the patients had either no or mild MR (effective regurgitant orifice [ERO] $\left.\leq 0.2 \mathrm{~cm}^{2}\right)$ and $71 \%$ had clinically significant $\mathrm{MR}\left(\mathrm{ERO}>0.2 \mathrm{~cm}^{2}\right)$. After stratifying the 2 study groups into patients with $\mathrm{MR}>$ or $\leq$ ERO $0.2 \mathrm{~cm}^{2}$, we found no differences in PES and mental HRQoL between the MVP vs. no-MVP groups, both in patients with significant (all $p \geq 0.5$ ) and without significant $M R$ (all $p \geq 0.5)$. In multivariate analysis, MVP was not independently associated with any of PES or HR-QoL outcomes (all $\mathrm{p} \geq 0.4$ ). In addition, while the objective severity of MR was not independently related to PES or HR-QoL (all $p \geq 0.2$ ), perceived severity of the illness predicted all PES (all $\mathrm{p}<0.03$ ) and HR-QoL outcomes (all $\mathrm{p}<0.003$ ). Conclusions: MVP is not a determinant of the patient's psycho-emotional status or quality of life. PES and HR-QoL are determined by the patient's perception of the severity of disease. Thus, a reassuring stance of the physician towards the patient is essential, particularly regarding an accurate presentation of the prognosis and the possibilities for an effective treatment of the mitral condition.

\section{Hemodynamic Results of Rapid Deployment Aortic Valves and Perimount Valves in a Matched Group of Patients}

Christelle Roduit ${ }^{1}$, Denis Berdajs ${ }^{1}$, Piergiorgio Tozzi ${ }^{1}$, Enrico Ferrari ${ }^{2}$

${ }^{1} \mathrm{CHUV}$, Lausanne, ${ }^{2}$ Cardiocentro Ticino Foundation, Lugano, Switzerland

Objective: Rapid deployment aortic valve (Intuity) represents a valid alternative to standard bioprosthesis. In particular, it helps in shortening minimal invasive aortic procedures and combined valve 
procedures in patients with higher surgical risk profiles. Postoperative hemodynamic results should be comparable to those of well established standard bioprosthesis. Methods: Sixty-four matched patients were operated for aortic valve replacement with Rapid Deployment Intuity valves (32 patients, Intuity group) and standard Perimount valves ( 32 patients Perimount group). Postoperative clinical and echocardiographic parameters were compared. Results: Mean age of Intuity and Perimount groups was $78 \pm 6$ years respectively. Hypertension, chronic renal failure and coronary disease were equally distributed. Concomitant coronary surgery was performed in 11 Intuity patients and 6 Perimount patients $(\mathrm{p}=$ $0.445)$. Mean cardiopulmonary bypass time was $68 \pm 27 \mathrm{~min}$ for Perimount and $72 \pm 76$ min for Intuity $(p=0.007)$; and mean clamp time was $50 \pm 25 \mathrm{~min}$ and $53 \pm 22 \mathrm{~min}(\mathrm{p}=0.0045)$. Mean valve size was $23.7 \mathrm{~mm}$ for Intuity and $24 \mathrm{~mm}$ for Perimount ( $\mathrm{p}=0.416)$. Postoperatively, no differences in hospital mortality $(0 \%)$, new pacemaker rate and stroke rate. Acute renal failure appeared more often in the Perimount group $(\mathrm{p}=0.004)$. Postoperative echocardiogram showed peak gradients of 18 vs. $21 \mathrm{~mm} \mathrm{Hg}(\mathrm{p}=0.033)$ and mean gradients of 9 vs. $12 \mathrm{~mm} \mathrm{Hg}(\mathrm{p}=0.035)$ in favour of Intuity valves. Conclusions: The rapid deployment aortic valves showed very good hemodynamic results and helps in shortening the procedural length in particular when combined procedures are concerned.

\section{Five Year Follow-Up of Haemodynamic Performance of the St. Jude Medical Trifecta Aortic Bioprosthesis}

\section{Jacob Chacko, Joy Edlin, Shirish Ambekar, Kulvinder Lall}

Bart's Heart Centre, London, United Kingdom

Objective: The St. Jude Medical Trifecta aortic supra-annular bioprosthesis is regarded as the next generation in pericardial stented tissue valves. The unique design of tissue leaflets attached to the exterior of the valve stent provides unrivalled in-vivo mean gradients and haemodynamics. The aim of this prospective study was to evaluate midterm haemodynamic performance. Methods: Two hundred and sixty four consecutive patients undergoing aortic valve replacement using the St. Jude Medical Trifecta valve at a single UK centre over a five year period were included in this study. Patients undergoing concomitant cardiac procedures were included. All implanted valves were 19, 21, 23, 25, 27 \& $29 \mathrm{~mm}$ in size. Assessment of haemodynamic function was carried out using transthoracic echocardiography pre-operatively and at follow-up, as well as transoesophageal echocardiography intra-operatively. Results: The study population consisted of 264 patients (162 male, 102 female). Mean age was $72.1 \pm 10.4$ years. Implanted valve sizes were $19 \mathrm{~mm}(\mathrm{n}=20), 21 \mathrm{~mm}(\mathrm{n}=77), 23 \mathrm{~mm}(\mathrm{n}=101), 25 \mathrm{~mm}$ $(\mathrm{n}=54), 27 \mathrm{~mm}(\mathrm{n}=11)$ and $29 \mathrm{~mm}(\mathrm{n}=1)$. Overall mean postoperative pressure gradients were $9.4 \pm 4.1 \mathrm{~mm} \mathrm{Hg}$ (mean) and $16.9 \pm 7.6 \mathrm{~mm} \mathrm{Hg}$ (peak). Subgroups mean post-operative pressure gradients were $11.6 \pm 4.3 \mathrm{~mm} \mathrm{Hg}, 10.4 \pm 5.8 \mathrm{~mm} \mathrm{Hg}, 9.8 \pm 2.7 \mathrm{~mm}$ $\mathrm{Hg}, 8.4 \pm 4.1 \mathrm{~mm} \mathrm{Hg}, 6.1 \pm 2.4 \mathrm{~mm} \mathrm{Hg}, 6.86 \pm 0 \mathrm{~mm} \mathrm{Hg}$, for the $19,21,23,25,27$ and $29 \mathrm{~mm}$ cohort respectively. Overall mean post-operative left ventricular ejection fraction was $57.2 \pm 10.1 \%$. Overall mean effective orifice area was $1.71 \pm 0.4 \mathrm{~cm}^{2}$. Conclusions: These results of our experience demonstrate excellent haemodynamic performance of the Trifecta bioprosthetic valve.

\section{Structural Valve Deterioration of Mitroflow Pericardial Aortic Bioprostheses Implanted in Children and Young Adults}

\author{
Mila Della Barbera ${ }^{1}$, Marialuisa Valente ${ }^{1}$, Aurelio Giaretta ${ }^{2}$, \\ Cristina Basso ${ }^{1}$, Gaetano Thiene, Sr. ${ }^{1}$ \\ ${ }^{1}$ Department of Cardiac, Thoracic and Vascular Sciences, \\ University of Padova, Padova, ${ }^{2}$ CNR-The Institute of \\ Geosciences and Earth Resources, Padova, Italy
}

Objective: Valve diseases affect also children and may require surgical valve replacement. Bioprosthetic valves (BPs) are employed, because they do not need anticoagulation, but the risks of early structural valve deterioration is high. The ideal valve substitution is not yet available in children. Methods: We report the pathological findings in 5 aortic Mitroflow LXA pericardial bovine bioprosthesis valves, size $19-23 \mathrm{~mm}$, implanted in children ( $3 \mathrm{fe}$ male and 2 male, mean age $14.19 \pm 4.77$, range $11.75-21.16$ years) and explanted at $26 \pm 8.24$ mos after aortic valve replacement because of severe stenosis or stenoincompetence. Clinical and surgical data have been previously reported elsewhere (Senage et al., Circulation 2014; 130:2012-2020). Investigation was accomplished through gross, $\mathrm{x}$-ray, histology, transmission electron microscopy (TEM) and spectroscopy for calcium $(\mathrm{Ca}++)$ and phosphorus (P) content. Results: Massive intrinsic cusp calcification was present, with mean $\mathrm{Ca}++$ and $\mathrm{P}$ content of $183.27 \pm 62.48$ and $94.35 \pm 33.76 \mathrm{mg} / \mathrm{g}$ dry weight, respectively, in the absence of significant inflammatory infiltrates. Exuberant fibrous tissue overgrowth was detected in 3 cases. At both histology and TEM, a significant collagen denaturation, pools of plasma insudation and massive calcific deposits on collagen fibers and cell debris were observed. Conclusions: Mineralization, fibrous pannus and collagen denaturation accounted for early structural valve deterioration of these BPs implanted in aortic position of children and young adults. Anti-mineralization treatment in new generation Mitroflow BPs, with phospholipids removal using long-chain alcohol solution, as well as improved collagen preservation might reduce this undesired complications and enhance long-term durability even in children.

\section{Effect of Ultrafilteration during on Pump Cardiac Surgery in High Risk Patients (EUROSCORE >6) Undergoing Complex Cardiac Surgery Procedures}

\section{Sumit Narang, Sr.}

Savodaya Hospital and Research Centre, Faridabad, India

Aim: The aim of this study was to see the effect of ultrafilteration during on pump cardiac surgery in high risk patients (EUROSCORE >6) undergoing complex cardiac procedures. Method: 58 patients with moderate renal dysfunction and various other risk factors with complex cardiac problems (EUROSCORE $>6$ ) were randomized to group a (n30) which underwent ultrafilteration during on pump surgery and group b (n28) in which ultrafilteration was not used. Patients were randomized depending on date of presentation, with those presenting on odd dates were 
assigned to group a and those presenting on even dates were assigned to group b. Results: 15 patients in group a and 12 patients in group b were acute MI with acute severe Mitral valve regurgitation with cardiogenic shock. 5 patient in group a and 7 pts in group b were post MI VSD with cardiogenic shock. 24 pts in group a and 23 pts in group b were on moderate to high dose ionotropic support. One month mortality in group a was $5(16.6 \%)$ and of group b was 7 (25\%). 7 patients in group a and 11 pts in group b required post operative hemodialysis/CRRT therapy. average ICU stay and hospital stay were 6 days and 13 days in group a and 8 and 16 days in group b. Conclusion: use of ultrafilteration during on pump cardiac surgery in high risk patients undergoing complex cardiac procedures significantly improves outcome and decreases mobidity and requirement of dialysis support. However large multi institutional study involving large number of patients is required to confirm these findings.

\section{Early Predictors of Permanent Pacemaker Implantation in Sutureless Aortic Valve Replacement with Perceval Bioprosthesis: A Conduction Disorders Analysis}

\section{Igor Belluschi, Stefano Moriggia, Mariachiara Calabrese Davide Schiavi, Ottavio Alfieri}

San Raffaele University Hospital, Milan, Italy

Objective: Sutureless aortic valve replacement (SU-AVR) with the Perceval bioprosthesis (Sorin S.p.A., Saluggia, Italy) has been developed as an alternative to conventional surgery. During the deployment of this sutureless prosthesis, the inflation at $4 \mathrm{~atm}$ of a ballon catheter-mounted is recommended. As for the transcatheter heart valves (THV), the implantation procedure could lead to postoperative conduction disorders. The aim of our study is to analyze the postoperative conduction disorders and identify the associated risk factors in patients undergoing SU-AVR with the Perceval bioprosthesis. Methods: In this observational study, patients who underwent the implantation of the Perceval prosthesis at the San Raffaele University Hospital (Milan) from January 2014 to September 2015 were analyzed. The conduction disorders were identified by reviewing the patients' electrocardiograms at baseline, postoperatively, before hospital discharge and at follow-up. All tracings were analyzed by an independent investigator and an expert cardiac surgeon to record the presence of first-, second- or third-degree AV block, right bundle branch block (RBBB), left bundle branch block (LBBB), left anterior hemiblock (LAHB), left posterior hemiblock (LPHB) and arrhythmias. Results: All patients $(\mathrm{n}=35)$ who underwent a SU-AVR with the Perceval bioprosthesis were included. At baseline, four patients (11.4\%) presented with pre-operative conduction disorders: two patients (5.7\%) had LBBB, one had RBBB (2.9\%) and one RBBB+LAHB. All the patients showed a sinus rhythm, except 3 cases of chronic atrial fibrillation. No one had a pre-existing permanent pacemaker (PPM). One patient with permanent AF received a concomitant radiofrequency ablation. Five patients (14.3\%) needed a PPM implantation: one for second-degree and four for third-degree AV block respectively. Among the 5 patients who implanted a PPM, three patients $(60 \%)$ had pre-operative conduction disorders
( $\mathrm{LBBB}, \mathrm{RBBB}$ and $\mathrm{RBBB}+\mathrm{LAHB}$ respectively; $\mathrm{p}=0.006$ ), one patient had anatomical risk factors (small aortic annulus and an offlabel concomitant mitral valve replacement), while only one patient had no preoperative conduction disorders. Conclusions: The presence of preoperative conduction disorders is a statistically significant risk factor for PPM implantation following a SU-AVR with the Perceval bioprosthesis. Therefore, an accurate analysis of the preoperative rhythm is to be considered.

\section{A Single Institution 16 Years' Experience of Aortic Valve Replacement Using the Mosaic Porcine Bioprosthesis}

\section{Alan Soo, Sofoklis Mitsos, Maria Carnoletta, John Pepper, Anthony de Souza}

Royal Brompton Hospital, London, United Kingdom

Background: Stented bioprosthesis remains the mainstay choice in aortic valve replacement. The Mosaic bioprosthesis (Medtronic Inc.) is a stented tissue valve composed of a porcine valve secured to a flexible frame. Studies have shown that using the Mosaic bioprosthesis to replace the aortic valve have comparable survival to the use of pericardial prosthesis despite some issues with elevated measured gradients. In this study, we report our experience of using the Mosaic porcine valve in the aortic position over a 16 year period. Methods: All patients who received a Mosaic bioprosthesis in the aortic position at our institution between 1998 to 2014 were included in the study. Pre-operative clinical characteristics were obtained from the local hospital PATS (Patient Analysis \& Tracking System) database. Echocardiographic findings were collected also from local hospital database retrospectively. Survival was assessed using the Kaplan Meier method. Results: Between April 1998 to February 2014, 1059 patients underwent aortic valve replacements and had the Mosaic tissue valve implanted. These patients had an average age of $71 \pm 11$ years. 299 patients $(28 \%)$ had unstable symptoms and were in heart failure pre-operatively. Ventricular function was measured as good in 693 (65\%) patients, moderate in $313(29.5 \%)$ patients, and poor in 53 $(0.05 \%)$ patients. Aortic valve replacement was a first time surgery in the majority of patients $(933,88.2 \%)$ whereas $126(11.8 \%)$ patients had previous surgery and underwent redo surgery. There were 39 in-hospital mortalities (3.7\%). Causes of death included cardiac failure (56.7\%), pulmonary complications (5.4\%), infective complications (5.4\%), neurological complications (2.7\%) and other unspecified causes (29.7\%). The 5 and 10 year survival were $88 \%$ and $74 \%$ respectively. Freedom from structural valve deterioration was also analysed. Conclusion: Our experience with the Mosaic bioprosthesis over a 16 years period demonstrated it to be a safe and durable bioprosthesis in the aortic position with good long term survival. 


\section{Twenty-Year Patient Survival and 17 Year Complications of Isolated Mitral Biocor Standard Porcine Valve}

Jonida Bejko ${ }^{1}$, Giulio Rizzoli ${ }^{1}$, Marina Comisso', Giuseppe Faggian' ${ }^{2}$, Aldo Milano², Mikhail Dodonov², Gino Gerosa ${ }^{1}$, Tomaso Bottio ${ }^{1}$, Tomaso Bottio ${ }^{1}$

${ }^{1}$ Padua University, Padova, ${ }^{2}$ Verona University, Verona, Italy

Objective: Performance of prostheses must be verified in the time domain. We report the 20 years survival of 230 patients operated on with a mitral Biocor Standard prosthesis at the cardiac surgery of Padova and Verona and the 17-year complication rates of 168 prosthesis applied in Padova. Methods: Two-hundred-thirty patients ( 150 females and 80 males, aged $71 \pm 6.3$ ) received 235 single mitral prostheses, in Verona (67) and Padova (168), between June 1989 and August 2004. Twenty-year survival follow-up, including 1698 patient-year, was complete. Prosthesis survival and events were evaluated only on Padova prostheses recipients: follow-up included 905 patients-year with $90 \%$ follow-up completeness. Results: Overall survival was 6\% (2-13\%). Thirty days mortality was $8.9 \%$ (5.6-13.3\%). Seventeen-year Kaplan-Meier and Actual freedom were as follows, respectively: from reoperation 80.5\% (55.3-92.3\%) and 92\% (86.4-94.5\%), from SVD $85.2 \%$ (52.6-96.1\%) and 94\% (88.2-96.7\%). Actuarial freedom from perivalvular-leak was $92.6 \%$ (86.5-96\%), from embolism $83.1 \%$ (73$89.7 \%)$, from hemorrhage $83.8 \%(71.5-91.1 \%)$, and from endocarditis $90.9 \%$ (82.4-95.4\%). Conclusions: The Biocor Standard prosthesis in mitral position shows an optimal durability (actuarial freedom 85.2\%, actual 94\%), low reoperation (actuarial freedom $80.5 \%$ and actual $92 \%$ ) and complication rates at 17 -years.

\section{Percutaneous Heart Valve Therapy}

\section{Does Undersizing of Transcatheter Aortic Valve Bioprostheses during Valve-in-Valve Implantation Avoid Coronary Obstruction? An in vitro Study}

\author{
Sina Stock ${ }^{1}$, Michael Scharfschwerdt ${ }^{1}$, Roza Meyer-Saraei ${ }^{2}$, \\ Doreen Richardt ${ }^{1}$, Efstratios I. Charitos ${ }^{3}$, Hans-Hinrich Sievers ${ }^{1}$, \\ Thorsten Hanke ${ }^{1}$ \\ ${ }^{1}$ Department of Cardiac and Thoracic Vascular Surgery, \\ University of Luebeck, Luebeck, ${ }^{2}$ Department of Cardiology, \\ Angiology and Intensive Care Medicine, University of Luebeck, \\ Luebeck, ${ }^{3}$ Department of Cardiac and Thoracic Surgery, \\ University of Halle, Halle, Germany
}

Background: The Transcatheter Aortic Valve-in-Valve Implantation (TAViVI) is an evolving treatment strategy for degenerated surgical aortic valve bioprostheses (SAVB) in patients with unacceptable high operative risk. For TAViVI, presently an oversizing of transcatheter aortic valve bioprostheses (TAVB) is recommended. Although hemodynamics are excellent, there is some concern regarding coronary obstruction, especially when TAViVI with oversized TAVB is performed in SAVB with externally mounted leaflet tissue. We investigated the hemodynamic performance and coronary flow after TAViVI with an undersized TAVB in an in vitro study. Methods: An aortic root model was constructed incorporating geometric dimensions known as risk factors for coronary obstruction. To prove the validity of this model, we performed oversized TAViVI with a TAVB Sapien XT'TM (size 26) in SAVB with externally mounted leaflet tissue (Trifecta ${ }^{\mathrm{TM}}$, size 25) in a mock circulation. This model led to coronary obstruction. Afterwards, hemodynamic performance and coronary flow (left and right coronary diastolic flow: LCF, RCF) after TAViVI with an undersized TAVB Sapien XT ${ }^{\mathrm{TM}}$ (size 23) in a $\operatorname{Trifecta}^{\mathrm{TM}}$ (size 25) were investigated at two different coronary ostia heights $(\mathrm{COH}$, $8 \mathrm{~mm}$ and $10 \mathrm{~mm}$ ). Results: Undersized TAViVI caused no significant reduction in coronary flow (lCF: $\mathrm{COH} 8 \mathrm{~mm} 0.90 \mathrm{ml} /$ stroke to $0.87 \mathrm{ml} /$ stroke, COH $10 \mathrm{~mm} 0.89 \mathrm{ml} /$ stroke to $0.82 \mathrm{ml} /$ stroke; rCF: COH $8 \mathrm{~mm} 0.64 \mathrm{ml} /$ stroke to $0.60 \mathrm{ml} /$ stroke, $\mathrm{COH}$ $10 \mathrm{~mm} 0.62 \mathrm{ml} /$ stroke to $0.58 \mathrm{ml} /$ stroke; all p-values non-significant). Mean transvalvular gradients ( $4 \mathrm{~mm} \mathrm{Hg}$ to $5 \mathrm{~mm} \mathrm{Hg}, \mathrm{p}<$ $0.001)$ and leakage volume ( $1 \%$ to $11 \%$ of stroke volume, $\mathrm{p}<0.001)$ increased significantly after TAViVI. Conclusions: In our in vitro model, undersized TAViVI in modern generation SAVB avoided coronary flow reduction, but mean transvalvular gradient and aortic regurgitation increased significantly albeit of questionable clinical relevance.

\section{Mechanical Load Analysis of Transcatheter Heart Valve Prostheses in Accelerated Wear Testers}

\author{
Jan Roggenkamp, Alexander Struth, Christoph Schmitz, \\ Thomas Schmitz-Rode, Ulrich Steinseifer \\ Department of Cardiovascular Engineering, Helmholtz \\ Institute, RWTH Aachen University, Aachen, Germany
}

Objective: Accelerated wear tests are established methods for the investigation of device durability. However, the requirements for appropriate accelerated wear tests are contradictory. On the one hand the test method shall represent the physiological loading conditions of the human cardiovascular system precisely. On the other hand the tests are performed at higher test frequencies, due to the required cycle numbers. The aim of this work is to investigate the impact of test frequency on the deforming of flexible heart valves prostheses under defined operating conditions. Methods: The tests were performed in the CVE-FT2 durability testers, developed at the Department of Cardiovascular Engineering, with a commercially available Medtronic CoreValve prosthesis. For the evaluation based on high-speed video analyses, the heart valve prosthesis was deployed in a tubular silicone aorta mounted into a standard transparent tester compartment. This compartment was modified with an optical access and with additional adapters for the CVE pulse duplicator. First, the radial deforming of the prosthesis was detected in the pulse duplicator with physiological pressure profiles at a differential pressure of $100 \mathrm{~mm} \mathrm{Hg}$ and at three working points with cardiac outputs of 2,5 and $71 / \mathrm{min}$. The results were then compared with the prosthesis deforming in the durability tester at $100 \mathrm{~mm} \mathrm{Hg}$ differential pressure and test frequencies 


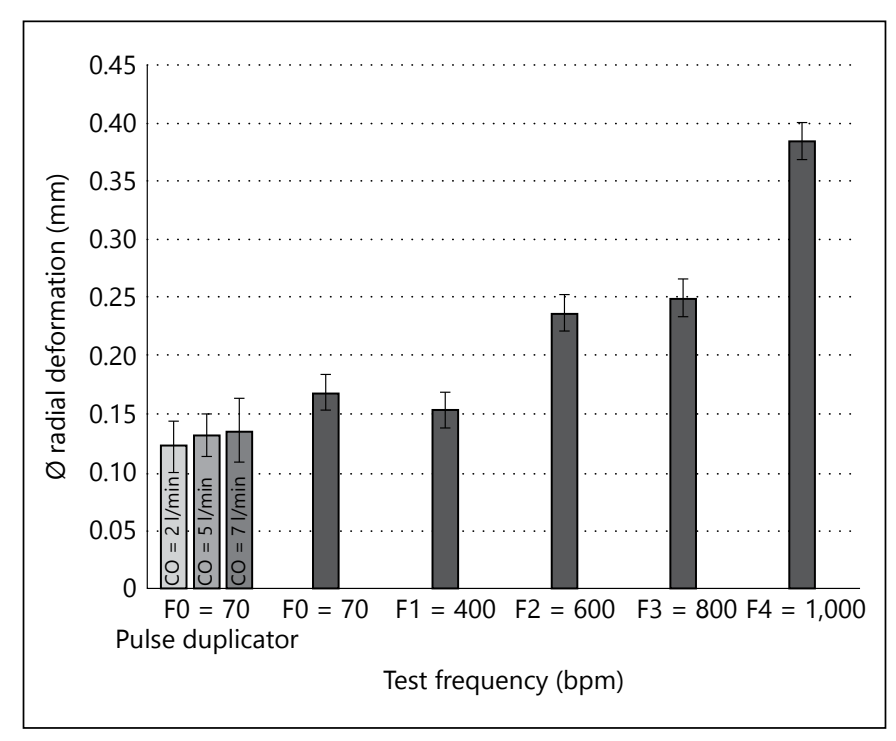

Fig. 1. Mean radial deformation (for Abstract of Jan Roggenkamp).

of 70, 400, 600, 800 and $1000 \mathrm{bpm}$. Results: The results show, that the prosthesis' radial deforming is comparable with physiological values for frequencies of 70 and $400 \mathrm{bpm}$. The deforming of the prosthesis rises with higher test frequencies. At a frequency of $1000 \mathrm{bpm}$ the detected deforming values are 2.5 times higher than measured under physiological conditions. Conclusions: Accelerated wear tests for transcatheter heart valve prostheses are highly frequency dependent. As structural component fractures are associated with strain values mainly, the dependencies between the test system configuration and the appropriate loading of the prosthesis have to be further investigated. The variation of operating parameters and the investigation of flexible vascular models will lead to more accurate accelerated wear tests.

\section{Predictors of Frailty at 30-Days in High-Risk Patients Who Undergo a Transcatheter Aortic Valve Replacement}

\section{Jessica Forcillo, Jose F. Condado, Yi-An Ko, Michael Yuan, Vasilis Babaliaros, Vinod H. Thourani}

Emory University, Atlanta, GA, USA

Objectives: Patients who undergo transcatheter aortic valve replacement (TAVR) are frequently elderly, frail, and carry multiple co-morbidities which can lead to increased early mortality and morbidity. Despite the increasing use of TAVR predictors of frailty on outcomes remains incompletely understood. The objective of this study is to evaluate the weight of four frailty parameters on 30-day outcomes in patients undergoing TAVR. Methods: A retrospective review of prospectively collected data from 2011 to 2015 on 361 patients undergoing TAVR at an academic US institution was performed. All patients had a 4 parameters frailty assess-
Table 1. Cut-off points for the frailty parameters assessed (for Abstract of Jessica Forcillo)

\begin{tabular}{ll}
\hline Frailty parameters & Cut-off points \\
\hline Albumin, g/dl & 3.4 \\
Grip strength, kg & \\
$\quad$ Male & $43-44$ \\
$\quad$ Female & $35-39$ \\
KATZ index ADLs & $\leq 4 / 6$ ADLs for frailty \\
5-meter walk test, s & $7.6-7.9$ \\
$\quad$ Male & $7.7-7.9$ \\
$\quad$ Female & \\
\hline
\end{tabular}

ment prior to TAVR: albumin, 5-meter walk, grip strength and KATZ questionnaire. Logistic regression was used to examine the association between the selected frailty markers and 30-day composite events. In the process of delineating a cutoff point for each frailty marker for adverse outcomes, an area under the receiver operating characteristic curve was used. The weight of each parameters was found by doing correlation with mortality and with a composite of: mortality, stroke, new heart block requiring permanent pacemaker, major or life threatening bleeding, acute renal failure, major vascular complication and $30-\mathrm{d}$ readmission rate. Results: Median age was 82 years [IQ: 76-86] and 46.3\% (167/361) were female. The majority of TAVR were performed using the transfemoral route $57.6 \%$ (208/361), The 30 -day mortality for the entire cohort was $5.8 \%$, stroke rate was $1.4 \%$ and re-admission rate was $3.9 \%$. The composite event for mortality and morbidity occured in $28 \%$ of patients. The odds ratio estimates for a 30 -day composite event outcome was only significant for albumin 0.38 $(p=0.02)$. The cut-off points for frailty for increased mortality and morbidity are enumerated in the table below. Conclusions: Approximately one-third of patients have a composite of mortality or morbidity within 30 days of TAVR. In these high-risk patients, a low albumin was the only frailty marker associated with increased risk for the 30-day composite outcome. Careful preoperative screening is required to minimize complications associated with this innovative procedure.

\section{Visual Assessment of Mitraclip Implantation and Functionality in the Physioheart Isolated Working Heart Model}

\author{
Arend de Weger', Frank van der Kley', Victoria Delgado', \\ Sjoerd van Tuiji', Nina Ajmone ${ }^{1}$, Martin Jan Schalij' \\ ${ }^{1}$ Leids Universitair Medisch Centrum, Leiden, ${ }^{2}$ LifeTec Group \\ Eindhoven, Eindhoven, Netherlands
}

Objective: Altough echocardiography and fluoroscopy are valubale tools for implantation direct visualisation can play an important part in training the physician to enter the era of new and increasingly complex technology and treatment of valvular disease. The Physioheart platform an ex-vivo beating heart platform enables technical implantation as well as direct visualisation in a 


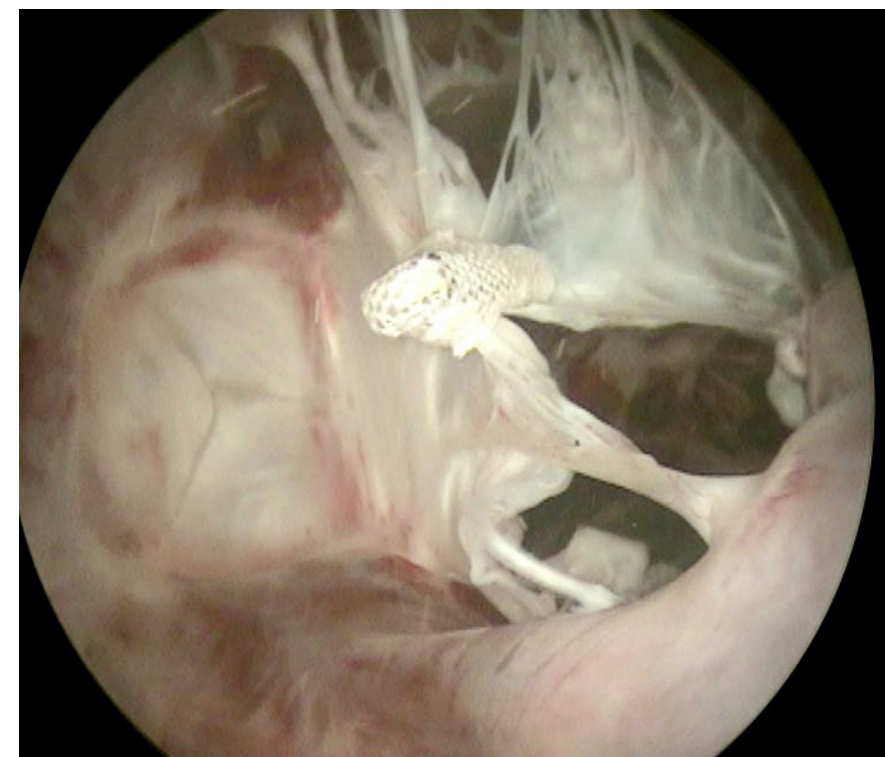

Fig. 1. (for Abstract of Arend de Weger).

beating working heart of intracardiac devices. In this movie we would like to demonstrate a MitraClip implantation. Methods: A MitraClip ${ }^{\circledR}$ (Abbot Vascular, IL, USA) was implanted into a physiological working beating pig heart. The procedure was performed resembling a clinical implantation under echocardiographic guidence and direct endoscopic vision. The PhysioHeart model is an ex-vivo working slaughterhouse pig heart model based on adapted human heart transplantation protocols and sophisticated engineering technology to create physiological performance. After reinstating coronary flow and installing preload and afterload normal physiological pressures and flow patterns are realized which enable us to study hemodynamic parameters under different physiological conditions. When replacing the blood with a buffered transparent solution we can film inside the heart and directly visualize the anatomical structures and functionality under normal working conditions. Results: The implantation was done under stable hemodynamic conditions with arterial blood pressure of 120/83 $\mathrm{mm} \mathrm{Hg}$ and cardiac output varying between 4 to 6 litres/ $\mathrm{min}$. All stages from introduction into the left atrium, crabbing of the mitral leaflets, closure of the clip and finally releasing of the clip were filmed (movie in supplement). The fully functional MitraClip ${ }^{\circledR}$ is assessed from the left atrial side as well as the left ventricular side. Conclusions: The Physioheart platform as shown in this movie is a unique platform that enables implantation or surgery on the intracardiac structures and facilitates the evaluation of the procedural results by means of direct visualisation. This direct visual feedback is a valuable tool for training the clinician to overcome a learning curve outside the patient and may lead to better procedural results, develop expertise and evaluate the success of a treatment.

\section{Transcatheter Aortic Valve Replacement Planning Imaging in Patients with Chronic Kidney Disease}

\author{
Lucy Safi, Nandini Meyersohn, Brian Ghoshhajra, \\ Jacob Dal-Bianco, Sammy Elmariah, Ignacio Inglessis, \\ Igor Palacios, Serguei Melnitchouk, Gus Vlahakes, \\ Jonathan Passeri
}

Massachusetts General Hosptial, Boston, MA, USA

Background: Chronic kidney disease (CKD) prevalent in the aging population. Low-dose contrast computed tomography (LDC-CT) protocols have been established to reduce the dose of contrast given to patients with CKD. When evaluating for transcatheter aortic valve replacement (TAVR), accurate aortic annulus measurement is important for transcatheter heart valve (THV) sizing. The accuracy of measurements using LDC-CT has not been well established. This study aims to compare the accuracy of LDCCT measurements in comparison to 3D-TEE measurements. Methods: Single-center, retrospective analysis of 57 patients who underwent LDC-CT protocol (mean contrast dose $24 \pm 6 \mathrm{cc}$ ) between November 2012 to October 2015 in evaluation for TAVR were analyzed. Aortic valve annulus diameter, circumference, and area obtained by LDC-CT were compared to measurements obtained by intra-procedural 3D-TEE, our protocol's valve sizing standard. Ideal TAVR valve size based on measured areas was determined for each modality and results were compared. Results: A self-expanding THV was used in 18 patients and 39 received a balloon expandable THV. Access included: 38 transfemoral, 16 transapical and 3 subclavian, with no major vascular complications. The mean area by 3D-TEE and LDC-CT were $451 \pm 93$ and $424 \pm 80$ $\mathrm{mm}^{2}$ respectively $(\mathrm{p}=0.09)$. The mean absolute difference in area between CT and 3D-TEE was $49 \pm 46 \mathrm{~mm}^{2}$. Compared to low-dose CT, a different THV size was selected in 17 (30\%) patients based on 3D-TEE recommendations. Of those patients, a larger THV was used in 13 (76\%). At the conclusion of the TAVR, paravalvular leak was moderate in 2 patients, mild-to-moderate in 8 patients and mild or less in 47 patients. Conclusions: Compared to $3 \mathrm{D}$ TEE, the LDC-CT may both overestimate and underestimate the annulus size. In patients with chronic kidney disease, the use of $3 \mathrm{D}$-TEE is complementary in determining an accurate annulus size for TAVR planning.

\section{Acquired Ventricular Septal Defect Following Transcatheter Aortic Valve Replacement: A Systematic Review}

\author{
Tomo Ando ${ }^{1}$, David Slovut ${ }^{2}$, Joseph DeRose ${ }^{2}$ \\ ${ }^{1}$ Mount Sinai Beth Israel, New York, NY, ${ }^{2}$ Montefiore Medical \\ Center, Bronx, NY, USA
}

Background: Ventricular septal defect (VSD) has been rarely reported as a complication following transcatheter aortic valve replacement (TAVR). We sought to evaluate the baseline characteristics, management and outcomes of these patients. Methods: Relevant studies were searched through PUBMED and EMBASE between 2002 to September 2015. The references were 
also manually reviewed for articles those included in the full article review. Congress abstracts were excluded. Articles that included baseline characteristics or hospital outcomes were included in the analysis. Results: A total of 16 studies including 18 patients were identified. The mean age was $83 \pm 8,38 \%$ were male and median Logistic EuroSCORE was 19.8 (range 11.7-33.8). There were $71 \%$ transfemoral approach. Pre-dilation was performed in 10 patients and post-dilation in 3. Self-expandable balloon was used in majority (89\%) of the cases. The clinical presentation varied from asymptomatic to progressive heart failure. The timing of the diagnosis also varied significantly from immediately post valve implantation up to 1 year. There were 2 cases of Gerbode type defect while the rest were inter-ventricular defect. The location was mostly membranous or perimenbranous (94\%), adjacent to the valve landing zone. Total of 7 interventions ( 1 open surgery and 6 percutaneous closure) were performed. The mortality rate was $(17.6 \%)$. Those who survived had overall clinically stable course (range 12 days to 2 year). Conclusions: The clinical course of reported cases of VSD following TAVR varied significantly from no symptoms to death. Self-expansion valve was implanted in majority of the cases and predilation was performed in more than half of the cases. High clinical suspicion is needed when patient is deteriorating and no other obvious etiology is identified as the condition is often treatable with percutaneous approach.

\section{Percutaneous Closure of the Insufficient Aortic Valve in a Heart Failure Patient on a Left Ventricular Assist Device Patient}

\author{
Hartmuth Bittner, Robert Hooker, George Makdisi, \\ Joseph H. Boyer
}

Gulf Coast Cardiothoracic Surgery Institute, Tampa, FL, USA

Objective: Aortic insufficiency (AI) is increasingly recognized as a complication of continuous flow left ventricular assist device (CF-LVAD) support and may lead to clinical decompensation requiring correction. The incidence of 'de novo' AI in patients supported with the CF-LVAD Heartmate II (Thoratec Corp, Pleasanton, CA) is greater than $25 \%$. Methods and Results: In this case report, we present a patient status post Heartmate II implantation as a third time redo sternotomy for ischemic cardiomyopathy complicated by de novo aortic insufficiency. He underwent coronary bypass surgery in 2002 with redo surgical revascularization in 2010. At 11 months postimplant, this 74 year old hard working architect presented with severe right and left heart failure, multi-organ dysfunction, and hepato-renal failure. Multiple echocardiograms revealed an opening aortic valve and near normal right ventricular function in the past. Six weeks ago, when the worsening AI was diagnosed, the decrease of Heartmate II CF-LVAD flow through decreasing the rounds per minute (RPM) from 8800 RPM to 8200 RPM attenuated the AI markedly. Ultimately, the patient underwent percutaneous, transcatheter closure of the aortic valve (see echo and heart catheter images and video) with an multi-fenestrated $30 \mathrm{~mm}$ Amplatzer Cribiform closure device (AGA Medical Corp, Plymouth, MN). Under echocardiography the flow of the CF-LVAD was increased to 9400 RPM. Two weeks post procedure, the patient remains stable with improved symptoms and functional status, and without evidence of further aortic insufficiency or device migration. Conclusion: Percutaneous transcatheter closure of the aortic valve effectively treats LVAD-associated AI and reduces pulmonary capillary wedge pressure. This procedure should be considered to treat LVAD-associated AI in patients who are poor candidates for repeat operation and catheter-based aortic valve procedures.

\section{Development and Analysis of a Purification System for Accelerated Heart Valve Wear Testers}

\section{Benjamin Grossmann, Jan Roggenkamp, Peter Molatta, Christoph Schmitz, Thomas Schmitz-Rode, Ulrich Steinseifer}

Department of Cardiovascular Engineering, Helmholtz Institute, RWTH Aachen University, Aachen, Germany

Objective: A well-established method to investigate long-term durability of heart valve prostheses are accelerated wear tests. Those tests are typically performed using a test fluid of isotonic saline, blood or a blood-equivalent fluid. These fluids all provide an excellent breeding ground for bacteria, which can settle on the valve tissue and can affect the performance. The aim of this work is to investigate the efficiency of a developed purification system for accelerated wear testers under operating conditions according to ISO 5840-3:2013. Methods: The purification system was integrated into the custom developed CVE-FT2 durability tester with three commercially available CoreV alve protheses. Therefore each test compartment was modified with an in- and outlet and connected to one central reservoir. A pump provided a constant circulation of the testing fluid trough the reservoir, a heating system, an ultraviolet lamp and back to the test compartments. The differential pressure across the closed valve was adjusted to $100 \mathrm{~mm} \mathrm{Hg}$. First, the system was contaminated with bacteria. Then, test samples were extracted in weekly intervals. Afterwards the samples were incubated at $30^{\circ} \mathrm{C}$ for two days on two different nutrient

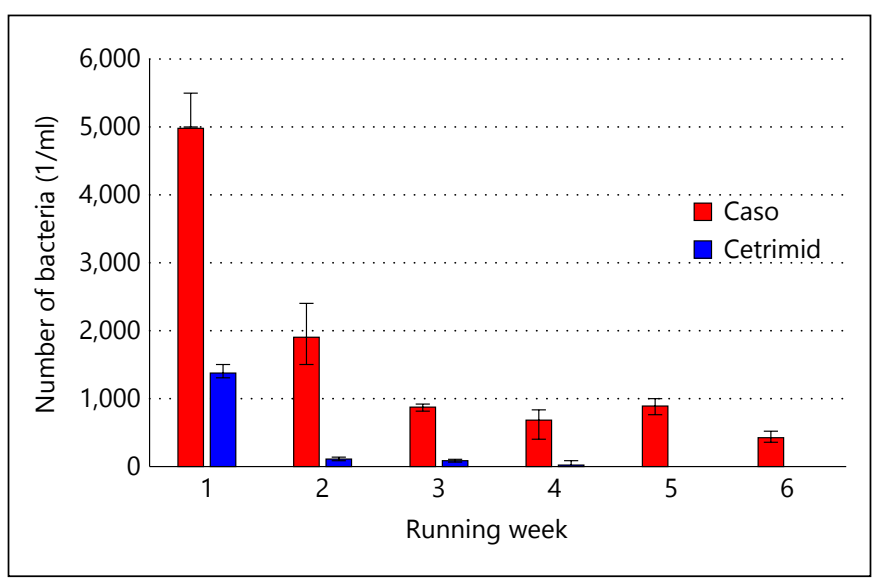

Fig. 1. Quantitative determination of bacteria (for Abstract of Benjamin Grossmann). 
agars. A Caso agar was used to determine general bacteria growth. For the evaluation of Pseudomonas Aeruginosa the samples were incubated on a Cetrimid agar. The number of bacteria per milliliter fluid was determined using Matrix-assisted Laser Desorption/Ionization (MALDI). The test period ended after six weeks. Results: The results show a reduction over time on both used nutrient agars. On Caso the number of bacteria was reduced by $91 \%$. On Cetrimd the reduction rate was $99 \%$. Conclusions: The efficiency of the developed purification system was identified with at least $90 \%$ of bacteria reduction. To get a completely bacteria-free test environment, some pre-test cleaning procedures and the ability of the purification system to keep a pure system clean have to be further investigated.

\section{Patients at Low Risk for Atrial Thrombosis Can Safely Perform Percutaneus Balloon Mitral Valvuloplasty without Transesophageal Echocardiography}

\author{
Vitor E. Rosa, Joao Ricardo C. Fernandes, Tarso A. Accorsi, \\ Antonio S. de Santis, Milena R. Paixão, Guilherme S. Spina, \\ Roney O. Sampaio, Flavio Tarasoutchi \\ HC FMUSP InCor, Sao Paulo, Brazil
}

Objective: Percutaneus Balloon Mitral Valvuloplasty (PBMV) remains the treatment of choice for patients with mitral stenosis in the absence of severe mitral regurgitation, atrial thrombosis and with a favorable valve anatomy. International guidelines indicate that transesophageal echocardiography (TEE) should be used to assess atrial thrombosis before PBMV. However, in patients with low-risk for thrombosis (LRT) (without atrial fibrillation, previous thromboembolism or left atrial diameter smaller than $55 \mathrm{~mm}$ ) the rate of embolic events after the procedure is low. Our aim is to evaluate the effectiveness and safety of performing solely Transthoracic Echocardiography (TTE) in the evaluation of patients with mitral stenosis and LRT undergoing PBMV. Methods: This retrospective study included 82 patients undergoing PBMV in the period between 2011 and 2014. All patients underwent TTE prior the procedure in order to assess contra-indications for the procedure. Those with atrial fibrillation and prior

Table 1. (for Abstract of Vitor E. Rosa)

\begin{tabular}{lll}
\hline & $\begin{array}{l}\text { High-risk for } \\
\text { thrombosis } \\
(\mathrm{n}=27)\end{array}$ & $\begin{array}{l}\text { Low-risk for } \\
\text { thrombosis } \\
(\mathrm{n}=55)\end{array}$ \\
\hline Mean Wilkins score & $7.73 \pm 0.5$ & $7.47 \pm 0.76$ \\
Wilkins scores $>8$ & $11.1 \%(\mathrm{n}=3)$ & $7.2 \%(\mathrm{n}=4)$ \\
Atrial fibrillation & $85.1 \%(\mathrm{n}=23)$ & 0 \\
Left atrial thrombosis & $3.7 \%(\mathrm{n}=1)$ & 0 \\
Left atrium $>55$ mm & $7.4 \%(\mathrm{n}=2)$ & $5.4 \%(\mathrm{n}=3)$ \\
Spontaneous echo contrast & $3.7 \%(\mathrm{n}=1)$ & 0 \\
Previous embolism & $25.9 \%(\mathrm{n}=7)$ & 0 \\
Use of anticoagulants & $100 \%(\mathrm{n}=27)$ & 0 \\
\hline
\end{tabular}

history of embolism were considered at high-risk for thrombosis (HRT), undergoing TEE before the procedure. Fifty-five (67\%) patients were considered of LRT in which TEE was not performed before PBMV and 27 (32.9\%) patients were considered HRT. Mortality and embolic events were assessed in the procedure and at 30 -days. Results: Mean age was $46.97 \pm 12.23$ years, $89 \%$ women and $100 \%$ of rheumatic etiology. Clinical characteristics are shown in Table 1 . No patient in either group had thromboembolic events after the procedure. Mortality in the procedure was $2.4 \%$ ( 2 patients, both due to pericardical tamponade during emergency procedures) and 30-day mortality was $4.8 \%$, all in the LRT group. Even so there was no significant mortality difference according to risk status $(\mathrm{p}=0.29)$. Conclusion: In this cohort, the exclusive use of TTE before PBMV in patients with LRT was sufficient and safe.

\section{Heart Valve Repair}

\section{In-vivo Evaluation of Flexible Mitral Annuloplasty Rings - Are They All the Same?}

\author{
Jonas Rasmussen, Soren Nielsen Skov, Marcell Juan Tjornild, \\ Christine Ilkjaer, Diana M. Ropcke, Sten Lyager Nielsen
}

Aarhus University Hospital, Aarhus, Denmark

Objective: Several different flexible mitral annuloplasty rings (FMARs) are available. Their efficacy for surgical repair of the mitral valve is in general considered equal. However, clinical experience indicates remodelling differences that have not been quantitatively assessed yet. The objective of this study was to compare biomechanical properties of two commonly used FMARs. Methods: Two FMARs were evaluated in an acute $80 \mathrm{~kg}$ pig model; the Medtronic Simulus Flexible $(n=5)$ and the Medtronic Duran AnCore $(n=7)$. Rings were normo-sized to the mitral annulus (Simulus size $29(110 \mathrm{~mm})$; Duran ring size 29 $(110 \mathrm{~mm}))$. The native mitral valve was used as reference $(\mathrm{n}=7)$. Mitral annular dynamics were assessed using 11 sonomicrometry crystals placed in the mitral apparatus and left ventricle. Mitral annular circumference (MAC), intercommissural (CC) and septal-lateral (SL) distances were measured. A dedicated force transducer was used to detect deformational force transmission between the annuloplasty ring and the mitral annulus. Results: The cyclic changes of the MAC and CC distance were significantly reduced in the Simulus group compared to the Duran group (MAC: $\mathrm{p}=0.025$ and CC: $\mathrm{p}<0.01$ ). There were no significant differences between the Duran group and the native group ( $p \geq$ 0.19 ). In the Simulus group, significantly smaller deformational forces were recorded compared to the Duran group $(0.53 \mathrm{~N} \pm$ $0.58 \mathrm{~N}$ vs. $2.14 \mathrm{~N} \pm 1.30 \mathrm{~N}, \mathrm{p}=0.028$ and $0.90 \mathrm{~N} \pm 0.53 \mathrm{~N}$ vs. $3.07 \mathrm{~N} \pm$ $1.29 \mathrm{~N}, \mathrm{p}=0.006$ for the SL and the CC force measurements, respectively). Conclusion: The Simulus ring showed significantly more remodelling capacity than the Duran ring. Differences in material properties of the rings may explain the different remodelling and downsizing effects on the mitral annulus, and suggest a more differentiated and patient-tailored use of FMARs. In- 
Table 1. (for Abstract of Jonas Rasmussen)

\begin{tabular}{|c|c|c|c|c|c|c|}
\hline & \multicolumn{2}{|l|}{ MAC } & \multicolumn{2}{|c|}{ SL distance } & \multicolumn{2}{|c|}{ CC distance } \\
\hline & simulus & duran & simulus & duran & simulus & duran \\
\hline Max distance, $\mathrm{mm}$ & $114 \pm 8$ & $107 \pm 10$ & $33 \pm 6$ & $33 \pm 4$ & $36 \pm 4$ & $35 \pm 4$ \\
\hline Min distance, $\mathrm{mm}$ & $111 \pm 7$ & $99 \pm 10$ & $30 \pm 7$ & $29 \pm 4$ & $35 \pm 4$ & $30 \pm 4$ \\
\hline Difference, $\mathrm{mm}$ & $3 \pm 2$ & $7 \pm 3^{\S}$ & $3 \pm 1$ & $4 \pm 2$ & $1 \pm 0$ & $4 \pm 1^{\S}$ \\
\hline
\end{tabular}

$\S \mathrm{p}<0.05$.

sights into these biomechanical differences may facilitate strategies for surgical reconstruction in degenerative mitral valve disease, especially in patients with excess amount of tissue and risk of systolic anterior motion.

\section{Surgical Strategy for Ischemic Mitral Regurgitation Adopting Subvalvular and Ventricular Procedures}

\section{Pierpaolo Maietta, Giustino Rispo}

Aorn Moscati, Mercogliano, Italy

Objective: The progression of left ventricular (LV) remodeling and subsequent mitral valve tethering impair the results of reduction annuloplasty for ischemic mitral regurgitation (MR). Methods: We studied 90 patients who underwent surgical repair of ischemic MR between 1999 and 2013 according to our surgical strategy adding submitral and ventricular procedures to annuloplasty as follows: annuloplasty alone (stage $1, \mathrm{n}=30$ ), additional papillary muscle approximation (PMA) for progression of tethering (stage $2, \mathrm{n}=26$ ), and additional left ventriculoplasty with PMA for progression of LV remodeling and tethering (stage $3, \mathrm{n}=34$ ). Results: The preoperative New York Heart Association (NYHA) functional classes $(2.5 \pm 0.7,3.1 \pm 0.7$ and $3.3 \pm 0.7$ for stages 1,2 and 3 , respectively, $\mathrm{P}<0.001)$, $\mathrm{LV}$ end-diastolic diameters $(56 \pm 7 \mathrm{~mm}$, $66 \pm 5 \mathrm{~mm}$ and $70 \pm 7 \mathrm{~mm}, \mathrm{P}<0.001$ ), and LV ejection fractions $(45 \pm 12 \%, 32 \pm 9 \%$ and $27 \pm 9 \%, P<0.001)$ significantly differed among the stages. In contrast, the MR grades did not significantly differ $(2.9 \pm 0.8,3.0 \pm 1.0$, and $2.9 \pm 1.1$, respectively; $\mathrm{P}=0.93)$. Both the rates of cardiac-related survival and freedom from reoperation were comparable among the 3 groups $(\log$-rank $\mathrm{P}=0.92$ and 0.58 , respectively). Conclusion: Additional submitral and ventricular procedures can compensate for the possible impairment of the outcomes after annuloplasty alone for ischemic MR in patients with severe LV remodeling and tethering.

\section{Aortic Valve Preserving Procedures: Our Management Approach and Current Outcomes}

Pallav Shah, Nasir Shaikh, Kaz Czaplinski, Carly Lodewyks, Kanwal Kumar, Karln Love, Edward Pascoe, Alan Menkis, James Tam

Cardiac Sciences Program, Winnipeg, MB, Canada

Background: Valve sparing root replacement (VSRR) and primary valve repair (PVR) are well established procedures used to preserve the native aortic valve. This study was undertaken to examine the clinical and echocardiographic outcomes following aortic valve preserving procedures (AVPP). Methods: This is a single surgeon experience with 90 patients who underwent AVPP from

Table 1. Aortic value etiology and morphology based on type of surgical repair performed (for Abstract of Pallav Shah)

\begin{tabular}{|c|c|c|c|c|}
\hline & \multicolumn{2}{|c|}{$\operatorname{VSRR}(\mathrm{n}=50)$} & \multicolumn{2}{|c|}{$\operatorname{PVR}(\mathrm{n}=40)$} \\
\hline & $\mathrm{n}$ & $\%$ & $\mathrm{n}$ & $\%$ \\
\hline \multicolumn{5}{|l|}{ Etiology } \\
\hline Normal cusp motion (type 1a) & 0 & 0 & 0 & 0 \\
\hline Root dilatation (type $1 \mathrm{~b}$ ) & 46 & 92 & 0 & 0 \\
\hline Annular dilatation (type 1c) & 0 & 0 & 5 & 12 \\
\hline Type 1d & 0 & 0 & 0 & 0 \\
\hline Cusp prolapse (type II) & 0 & 0 & 23 & 58 \\
\hline Cusp restriction (type III) & 0 & 0 & 1 & 2 \\
\hline Type A dissection & 4 & 8 & 11 & 28 \\
\hline \multicolumn{5}{|l|}{ Aortic valve morphology } \\
\hline Bicuspid & 9 & 18 & 12 & 30 \\
\hline Tricuspid & 41 & 82 & 27 & 68 \\
\hline Quadricuspid & 0 & 0 & 1 & 2 \\
\hline \multicolumn{5}{|l|}{ Severity of aortic insufficiency } \\
\hline Moderate-severe & 29 & 58 & 38 & 95 \\
\hline \multicolumn{5}{|l|}{ Type of repair } \\
\hline Reiplantation & 46 & 92 & 0 & 0 \\
\hline Remodeling & 4 & 8 & 0 & 0 \\
\hline Cusp prolapse repair & 32 & 64 & 33 & 83 \\
\hline
\end{tabular}

VSRR = Valve sparing root replacement $\mathrm{PVR}=$ primary valve repair. 
September 2008 to September 2015. Preoperative investigations included 2D and 3D TTE, TEE, and CT scan. 56\% (50/90) underwent VSRR and 44\% (40/90) of patients had a PVR procedure. The repair oriented functional classification of aortic insufficiency (AI) was used to classify disease and guide treatment. (Table 1). Associated elective procedures were CABG in 14 and Hemiarch in 9 patients. Clinical and echocardiographic review was done at 6 weeks, then every 6 months for the 1st year, then yearly thereafter. $100 \%$ of patients were followed for a mean of 40 months. Results: Thirty day-mortality was $1.1 \%$ (1/90, redo aortic dissection); Thirty day morbidity included reexploration for bleeding in $2.2 \%(2 / 80)$ and pacemaker implantation in $4.45 \%$ (4/90). There was one late death due to pulmonary embolism. 79\% (70/89) were in NYHA class 1 , $92 \%(82 / 89)$ patients were on aspirin, $2.2 \%(2 / 89)$ had infective endocarditis and $4.5 \%(4 / 89)$ had stroke. Reoperation rate was $3.4 \%(3 / 89)$. There were no cases of anticoagulant or antiplatelet related hemorrhage. In the VSRR group, 85\% (42/49) had none to trivial central non progressive AI and mean gradients were 2-6 mm $\mathrm{Hg}$. One patient with a dysmorphic right coronary cusp had intraoperative conversion to a mechanical valve and one patient with bicuspid aortopathy developed severe AI 3 months postoperatively requiring reoperation. $68 \%(27 / 40)$ of PVR patients had trivial to mild central non progressive AI with mean gradients of 8-14 mm $\mathrm{Hg}$. One patient required reoperation secondary to healed endocarditis. In those undergoing type A dissection repair, 73\% (11/15) had trace to mild central non progressive AI. Conclusion: AVPP can be performed safely with acceptable outcomes. Long-term clinical and echocardiographic follow-up is essential.

\section{Functional Tricuspid Regurgitation in Rheumatic Heart Disease: Results of De Vega Annuloplasty}

\section{Zahira Zouizra, Yassin Boukaidi, Rachid El Houati, Drissi Boumzebra}

Ibn Tofail Hospital, Marrakech, Morocco

Objectives: Tricuspid regurgitation (TR) in patients with rheumatic heart disease is associated with poor outcome and predicts poor survival, heart failure, and reduced functional capacity. We review our experience in DeVega annuloplasty for functional TR. Methods: Between 2007 and 2015: 520 patients (mean age, 37 years) underwent a De Vega tricuspide annuloplasty with concomitant rheumatic mitral and or aortic valve disease operated. All patients were investigated perioperatively by Doppler echocardiography. Echocardiographic assessment of tricuspid incompetence was on the basis of gradation of tricuspid regurgitation severity according to color Doppler flow criteria, the flow convergence region proximal to the regurgitant orifice (PISA), the size of the tricuspid annulus, and estimation of the pulmonary artery systolic pressure by continuous Doppler. Median tricuspid insufficiency was $3+$. All patients underwent De Vega tricuspid annuloplasty at the time of mitral and or aortic surgery. Results: There were 23 postoperative deaths. Four patients (who had a severe pulmonary hypertension and or right ventricular dysfunction) had developed a right cardiac failure 1 to 2 weeks after surgery. The postoperative course was uneventful in all other patients. The mean follow was 5.5 years. Post-operative echo study: There was $1+$ or less TR in
453 patients (87\%), moderate TR $2+$ in 37 patients (7\%) and severe TR $3+$ in 7 (1.34\%) patients who had a severe pulmonary hypertension. Conclusions: DeVega tricuspid annuloplasty is a simple, short, and inexpensive procedure with few complications. In a developing country like Morocco, De Vega annuloplasty remains the procedure of choice in the management of functional tricuspid valve incompetence.

\section{Mid-Term Outcomes after Tricuspid Annuloplasty Repair Using Medtronic Contour 3D Annuloplasty Ring}

Umair Aslam, Yasir Ahmed, Joseph George, Prakash Nanjaiah, Jennifer Williams, Pankaj Kumar

ABM NHS Trust, Swansea, United Kingdom

Objective: Contour 3D annuloplasty ring allows tricuspid valve repair with a prosthesis that mirrors the 3 -dimensionsal shape of the anatomical annulus. We report our 5 year outcomes of tricuspid annuloplasty using the 3D Medtronic Contour prosthesis. Methods: We retrospectively analysed prospectively collected data (Dendrite Systems, UK) of all patients having undergone tricuspid annuloplasty (TVA) with 3D Contour tricuspid annuloplasty rings (Medtronic, USA) between January 2011 and October 2015 at our institution. SPSS v22 (IBM, USA) was used to analyse results. Results: 56 patients with a mean age of 69.4 years (49-83) with a mean logistic euroSCORE of 14.33 (2.08-72.35) underwent tricuspid annuloplasty (TVA) with 3D Contour rings. Pre-operatively, there was moderate-severe tricuspid regurgitation (TR) in $81.1 \%$, moderate-severe RV dysfunction in $14.5 \%$ and mean LV ejection fraction was $48.0 \%$. Post-operatively, TV annulus size reduced by $1.62 \mathrm{~cm}$ from $4.960 .48 \mathrm{~cm}$ to 3.340 .50 ( $\mathrm{p}<$ $0.001)$, and RA size reduced from $5.180 .83 \mathrm{~cm}$ to $4.630 .73 \mathrm{~cm} \mathrm{(p<}$ 0.001 ). Median post-operative stay was 7 days. There were no inhospital deaths; 30 -day mortality was $0 \%$, with 4 late deaths $(12.9 \%$ ) at the most recent follow-up (2-24 months). TR remained mild in $89.1 \%$ at follow-up, and freedom from TV-related re-interventions was $0 \%$. Conclusion: From our experience over 5 years, we conclude that tricuspid annuloplasty with Medtronic Contour 3D ring gives excellent outcomes at an average of 9 months follow-up. Specifically, there was no 30-day mortality and there was significant reduction in the mean tricuspid annulus $(7.25 \mathrm{~mm})$, and achieved control of TR in $89.1 \%$ of patients at follow-up.

\section{An Easy and Fast Technique to Repair Mitral Valve: U Technique}

\section{Giuseppe Chiarella, Valerio Mazzei, Domenico Benvenuto}

Mater Dei Hospital, Bari, Italy

Objective: We applied in our institution the $U$ technique for correction of mitral valve regurgitation in 54 pts. We report the early and mid-term results. Methods: 54 pts affected by mitral regurgitation were operated on with this new technique from April 


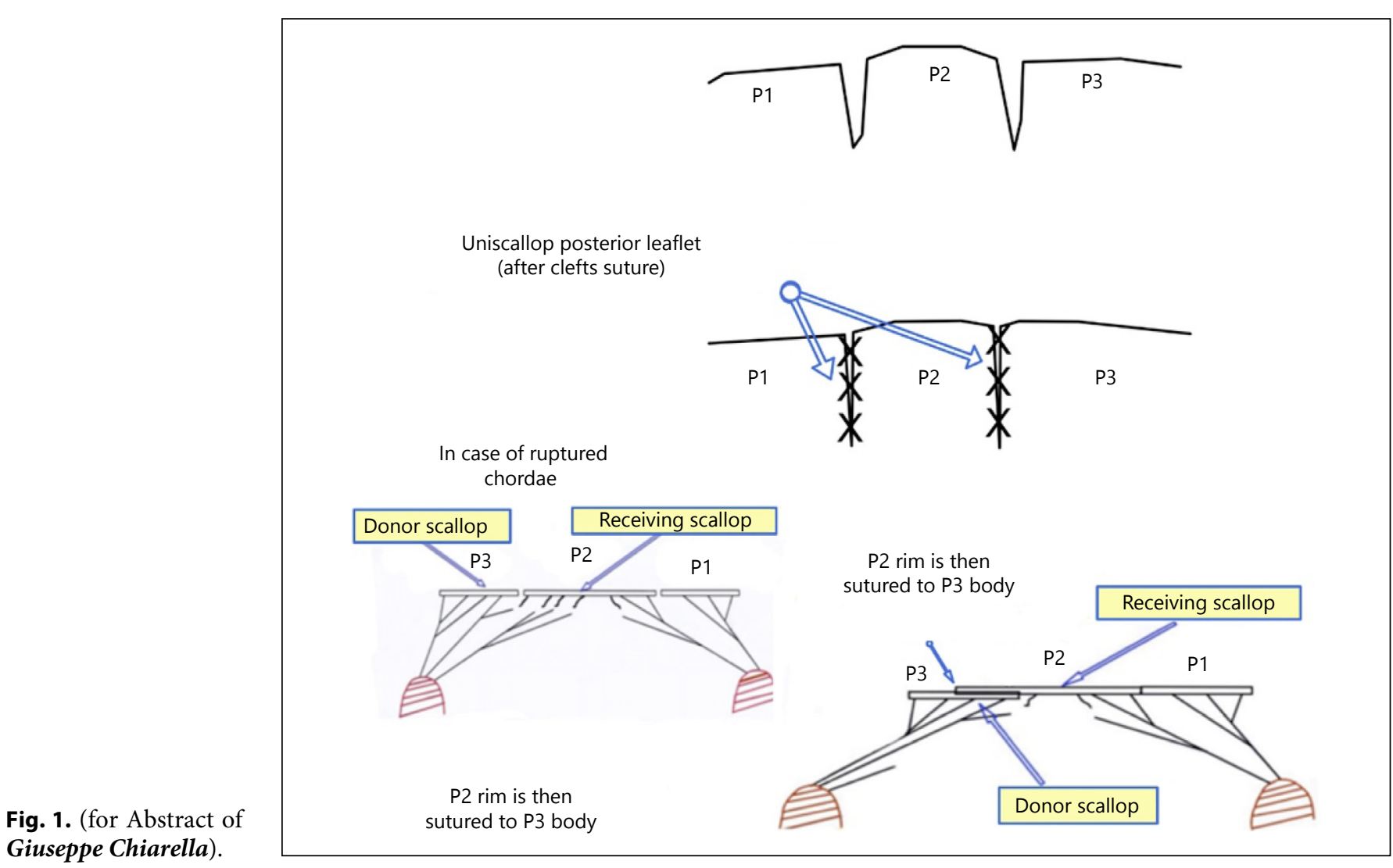

2014 to September 2015. The U technique is based only on suture of scallops of posterior leaflet ( $\mathrm{U}=$ Uniscallop posterior leaflet). In this simple way we eliminate the prolapse of posterior leaflet without any resection. In case of ruptured chords the rim of donor scallop (that has valid chords) is sutured over the body of receiving scallop (that has ruptured cords). We conclude mitral valve plasty suturing a ring on the annulus. Any kind of rings and bands may be applied. 29 patients were male and 25 female. Median age was $68 \pm 12.5$. Isolated posterior leaflet prolapse was present in $42 \mathrm{pts}$ and bileaflet prolapse in 12 (in these cases we implanted one or two chords on the anterior leaflet). Median sternotomy was used in 40 cases and small right thoracotomy in 14. Preoperative Ejection Fraction (EF) was $51 \pm 16 \%$, Mitral Regurgitation (MR, 1 to 4 ) was $3.5 \pm 0.5$. Results: Cardiopulmonary bypass time was $50 \pm 20 \mathrm{~min}-$ utes, Aortic Clamping time was $35 \pm 18$ minutes. There was one in-hospital death due to intestinal infarct. No patients showed Systolic Anterior Motion. At discharge mean EF was $50 \pm 13 \%$, mean MR was $0.3 \pm 0.2$, with 1 patient showing MR of grade 1.5 . At follow-up evaluation (50 pts 1-18 months) EF was $53 \pm 7 \%$, MR was $0.4 \pm 0.2$. All patients who underwent small thoracotomy approach had no residual MR. Conclusions: The $\mathrm{U}$ technique has shorter $\mathrm{CPB}$ and Clamping time. It is simply, fast and reproducible also for surgeons that have not much expertize in mitral valve repair and minimally invasive cardiac surgery. Mid term results are excellent and comparable with those ones from conventional tecniques.

Abstracts

\section{Early Results of Aortic Valve Reconstruction with Autologous Pericardium for Elderly Aortic Stenosis Patients}

Kentaro Amano, Koji Hattori, Mika Noda, Yusuke Sakurai, Yoshiro Higuchi, Masato Tochii, Michiko Ishida, Hiroshi Ishikawa, Yasushi Takagi

Fujita Health University, Tyoake, Aichi, Japan

Objective: In Japan, many elderly patients with aortic stenosis (AS) have a small aortic annulus, carrying a potential risk of patient-prosthesis mismatch (PPM). We examined the hypothesis that aortic valve reconstruction provides advantages to valve functioning in comparison with conventional aortic valve replacement in elderly AS patients. Methods: 153 patients with AS underwent aortic surgery between February 2014 and August 2015. We performed aortic valve reconstruction in 11 elderly AS patients (AVrC group: $79.4 \pm 3.1$ years old, $45.5 \%$ males, body surface area [BSA] $1.5 \pm 0.1 \mathrm{~m}^{2}$ ). In the same period, conventional aortic valve replacement without concomitant surgery was performed in 13 AS patients (AVR group: $73.3 \pm 6.5$ years old, $61.5 \%$ males, BSA $1.6 \pm 0.9 \mathrm{~m}^{2}$ ). The AVrC procedure involved independent tricuspid replacement using autologous pericardium based on the sizing apparatus and template designed by Ozaki. We retrospectively analyzed the surgical outcomes and echocardiographic data of these patients. Results: Concomitant procedures 
were performed in four patients of the $\mathrm{AVrC}$ group as follows: coronary artery bypass grafting in one, maze procedure in one, and Morrow's resection in two. In the AVR group, a mechanical valve was implanted in seven patients and a bioprosthetic valve in six. The operation time and aortic cross-clamp time were significantly longer in the AVrC group ( $380 \pm 43$ and $177 \pm 38 \mathrm{~min})$ than in the AVR group $(317 \pm 64$ and $118 \pm 31 \mathrm{~min})(\mathrm{p}=0.0095$ and $\mathrm{p}=0.0004$, respectively). There was no mortality in either group. The preoperative peak velocity through the stenotic aortic valve measured by echocardiography was similar in both groups $(4.7 \pm$ 1.8 and $5.0 \pm 0.6 \mathrm{~m} / \mathrm{s}, \mathrm{p}=0.2781$ ). Postoperative measurement of peak velocity through the aortic valve 1 month after surgery was lower in the AVrC group $(1.9 \pm 0.3$ versus $2.9 \pm 0.4 \mathrm{~m} / \mathrm{s}, \mathrm{p}<$ $0.0001)$. Conclusions: Aortic valve reconstruction was considered to be a feasible procedure for elderly AS patients. Although the procedure times were longer in the $\mathrm{AVrC}$ group, aortic valve reconstruction delivers minimal peak velocity through the aortic valve and can reduce the risk of PPM.

\section{Short and Midterm Experience with Valve Sparing Aortic Root Reconstruction Using the Caviaar-Technique: A Single Center Experience}

\author{
Christian Dinges, Johannes Steindl, Roman Gottardi, \\ Michael Kirnbauer, Rainald Seitelberger
}

Paracelsus Medical University Salzburg, Salzburg, Austria

Objective: Aortic root pathology is the most common cause of aortic valve insufficiency (AI). Valve sparing aortic root reconstruction using the CAVIAAR-technique (Conservative Aortic Valve Surgery for Aortic Insufficiency and Aneurysms of the Aortic Root) is an alternative for prosthetic valve replacement in selected patients. Methods: Aortic root reconstruction using CAVIAAR was performed in 25 patients with aortic root aneurysm and in 3 patients with isolated AI between September 2012 and September 2015. Twenty five patients underwent elective procedures, 3 patients had an acute aortic dissection. In one case CAVIAAR was done as a reoperation after a previous Ross-procedure. Mean patient age was $56.3( \pm 12.3)$ years. Echocardiography was performed intraoperatively, at hospital discharge and once per year as a follow up. Results: Mortality was $0 \%$ both perioperatively and during follow up. Successful valve sparing operation was performed in 25 patients (89.3\%). In three patients aortic valve replacement had to be done due to failed valve repair. In all of these patients preoperative Echo showed a tricuspid valve with a highly excentric jet. Mean aortic crossclamp and cardiopulmonary bypass time were $145.1 \mathrm{~min}( \pm 43.7)$ and $214.6 \mathrm{~min}( \pm 79.5)$, respectively. No pacemakers had to be implanted perioperatively. In one patient mitral annuloplasty had to be done due to moderate mitral insufficiency after CAVIAAR. Accumulated postoperative follow up accounted for 234 (3-36) months. In 24 out of 25 patients (96\%) aortic insufficiency was rated grade 0 or I during follow up. One patient underwent aortic valve replacement due to recurrent severe aortic insufficiency after 1 year. Conclusions: Our series showed safety and feasibility of the CAVIAAR technique. In our experience, the clearly defined step by step approach facilitates a safe start for an aortic valve repair program. Repair failures only occurred in patients with tricuspid valves and highly excentric jets. Consequently, we consider those patients at a higher risk for intraoperative valve repair failure.

\section{Surgical Treatment of Ischemic Mitral Regurgitation Triggers Reverse Remodeling of Left Heart Chambers}

\author{
Plamen Panayotov' ${ }^{1}$, Milen Slavov' ${ }^{1}$ Daniela Panayotova ${ }^{1}$, \\ Natalia Nikolova ${ }^{2}$ \\ ${ }^{1}$ Department of Cardiac Surgery, Varna, ${ }^{2}$ N.Y. Vaptsarov Navil \\ Academy, Varna, Bulgaria
}

Objective: Mild-to-moderate ischemic mitral regurgitation still poses debates on the necessity of adding mitral repair to the surgical revascularization. CABG with or without concomitant valve surgery trigger left atrial and ventricular reverse remodeling and improve the prognosis. Methods: 69 patients with ischemic heart disease and mild-to-moderate ischemic mitral regurgitation were subjected to isolated revascularization (Group A). In another 71 patients mitral repair was additionally performed (Group B). Mean follow-up was 28 (6 to 52) months. Left atrial and ventricular dimensions and volumes were evaluated preoperatively and at the follow-up. Results: The average improvement in effective left ventricular ejection fraction (LVEF) for all survivors was $6.2 \pm 14.1 \%$ in Group A and $21.2 \pm 12.3 \%$ in Group B $(p<0.05)$. Significant left ventricular reverse remodeling was observed in $45 \%$ of Group A and in $55 \%$ of Group B survivors. The average reduction in left ventricular end-systolic volume index was $10 \pm 23 \mathrm{ml} / \mathrm{m}^{2}$ vs. $13 \pm 26$ $\mathrm{ml} / \mathrm{m}^{2}(\mathrm{p}=\mathrm{NS}$ ). Left atrial reverse remodeling was significant in $21 \%$ of Group A and $57 \%$ of Group B. The average left atrial volume index slightly increased in Group A with $4 \pm 26 \mathrm{ml} / \mathrm{m} 2$, but reduction with $15 \pm 23 \mathrm{ml} / \mathrm{m}^{2}$ was observed in Group B ( $\left.\mathrm{p}<0.05\right)$. Conclusions: Surgical revascularization alone and in combination with mitral repair trigger reverse left ventricular remodeling which is significant for about half of the patients. Left atrial reverse remodeling strongly depends on the restoration of the valvular function. Still in about $21 \%$ of patients with isolated revascularization significant left atrial reverse remodeling is also observed.

\section{The Transaortic 'Edge-to-Edge' Mitral Valve Plasty in Combined Treatment of Obstructive Cardiomyopathy}

\author{
Alexei V. Evtushenko, Vladimir V. Evtushenko, \\ Konstantin A. Smyshlyaev, Elena N. Pavlyukova, Valery Kh Vaizov, \\ Vadim A. Katkov, Alexandr l. Maksimov, Yana Anfinogenova
}

Research Institute of Cardiology, Tomsk, Russian Federation

Objective: The aim of the study was to compare hemodynamic efficacy of the combined and isolated myectomy in patients with hypertrophic cardiomyopathy (HCMP). Methods: The study included 22 patients ( 9 males and 13 females) with obstruction of the left ventricular outflow tract (LVOT). Mean age was $52.81 \pm 15.57$ years. All patients presented with transthoracic echocardiography 
signs of HCMP: LVOT gradient was $85.5 \pm 25.7 \mathrm{~mm} \mathrm{Hg}$, thickness of the interventricular septum (IVS) was $23.9 \pm 6.8 \mathrm{~mm}$, end-diastolic volume (EDV) was $75.35 \pm 18.72 \mathrm{~mL}$, end-systolic volume (ESV) was $20.31 \pm 7.72 \mathrm{~mL}$, end diastolic dimension was $39.95 \pm$ $5.24 \mathrm{~mm}$, end systolic dimension was $23.85 \pm 5.57 \mathrm{~mm}$, left atrium dimension was $45.43 \pm 4.61 \mathrm{~mm}$, and ejection fraction was $73.91 \pm$ $6.99 \%$. All patients were administered with surgical treatment due to inefficacious drug therapy. Patients were randomized into three groups: group 1 included 7 patients with isolated ESM; group 2 comprised 9 patients with ESM in combination with transaortic 'edge-to-edge' MVP according to O. Alfieri; and group 3 comprised 6 patients with ESM in combination with 'sliding leaflet' MVP by A. Carpentier. Results: All patients were followed up for up to 72 months. In early postoperative period, 1 patient of group 1 required implantation of two-chamber pacemaker due to AV block; 4 patients of group 2 required implantation of two-chamber pacemaker; patients of group 3 did not require pacemaker implantation. Degree of residual mitral regurgitation after operation was $1 \pm 0.81$ (1.83 \pm 0.75 before surgery) (group 1), $0.66 \pm 0.5$ (1.55 \pm 0.52 before surgery) (group 2), and $0.82 \pm 0.32$ (1.76 \pm 0.62 before surgery) $(\mathrm{p}<0.05)$. The thickness of IVS was $16.33 \pm 3.21 \mathrm{~mm}$ (group 1), $15 \pm 2.23 \mathrm{~mm}$ (group 2), and $13 \pm 2.14 \mathrm{~mm}$ (group 3) (non-significant). EDV was $60.33 \pm 1.52 \mathrm{~mL}$ (group 1), $75.78 \pm 7.78$ $\mathrm{mL}$ (group 2), and $64.38 \pm 5.78 \mathrm{~mL}$ (group 3) (non-significant). ESV was $14.33 \pm 4.04 \mathrm{~mL}$ (group 1), $24.18 \pm 7.66 \mathrm{~mL}$ (group 2), and $26.02 \pm 4.46 \mathrm{~mL}$ (group 3) (non-significant). Conclusions: There is no difference in combined treatment of obstructive cardiomyopathy between the 'edge-to-edge' MVP by O. Alfieri versus 'sliding leaflet' by A. Carpentier in long-term period. Possible advantages of 'edge-to-edge' MVP are the ability to perform it by the mini-sternotomy access and shorter aortic cross-clamping.

\section{Left Atrial Reverse Remodeling and Atrial Fibrillation after Combined Surgical Revascularization and Mitral Repair}

\section{Plamen Panayotov', Milen Slavov' ${ }^{1}$ Daniela Panayotova', Natalia Nikolova ${ }^{2}$ \\ ${ }^{1}$ Department of Cardiac Surgery, Varna, ${ }^{2}$ N.Y. Vaptsarov Navil Academy, Varna, Bulgaria}

Objective: Chronic volume overload triggers left atrial (LA) structural and functional remodeling in the settings of ischemic mitral regurgitation and often complicates with atrial fibrillation (AF). Contemporary cardiac surgery allows safe and effective revascularization and mitral repair when needed. Thus the left atrium is unloaded and reverse changes potentially start. Methods: 63 patients with significant ischemic mitral regurgitation and in sinus rhythm were subjected to isolated surgical revascularization (Group A). In another 54 patients mitral repair was added (Group B). Left atrial dimensions and volumes as well as serial electrocardiograms were evaluated and follow-up was performed between 6 and 52 months after surgery. Results: In Group A LA size decrease early after surgery from от $47 \pm 5 \mathrm{~mm}$ to $45 \pm 5 \mathrm{~mm}, \mathrm{p}<0.05$, but later dilatation progresses and sizes at follow-up are larger than preoperative $(48 \pm$ $5 \mathrm{~mm}, \mathrm{p}<0.05)$. At least one episode of AF is recorded in $27 \%$ of patients. New onset AF is registered in $1.6 \%(1 / 63)$ of patients at discharge and $5.5 \%$ of all survivors at follow-up. In Group B we found more obvious reduction of LA size (from $50 \pm 6 \mathrm{~mm}$ to $47 \pm$ $5 \mathrm{~mm}, \mathrm{p}<0.05$ ), which appears stable over time (at follow-up $-48 \pm$ $5 \mathrm{~mm}, \mathrm{p}<0.05)$. One or more AF episodes complicated $43 \%$ of all patients correlating to the more advanced LA remodeling. In AF were $5.6 \%(3 / 54)$ of the patients at discharge and $7 \%$ of the survivors at follow-up. Conclusions: Despite the significant LA decrease in both groups, LA sizes do not return to normal even after correction of mitral regurgitation. When early postoperative LA sizes are comparable and the rhythm is sinus, the rate of $\mathrm{AF}$ at follow up is lower after combined surgery compared to isolated revascularization.

\section{Perioperative Outcome of Fundaro Annuloplasty in Patients with Carpentier Type-3 Mitral Regurgitation}

\author{
Serkan Mola ${ }^{1}$, Omer Faruk Cicek ${ }^{1}$, Ersin Kadirogullari ${ }^{1}$, \\ Ibrahim Erkengel ${ }^{1}$, Eren Gunertem ${ }^{1}$, Ali Baran Budak ${ }^{2}$, \\ Emre Yasar ${ }^{1}$, Adem Ilkay Diken ${ }^{3}$, Gokhan Lafci ${ }^{1}$, \\ Serdar Gunaydin ${ }^{2}$, Kerim Cagli ${ }^{1}$ \\ ${ }^{1} Y$ uksek Ihtisas Training and Research Hospital, Ankara, \\ ${ }^{2}$ Numune Training and Research Hospital, Ankara, ${ }^{3}$ Hitit \\ University, School of Medicine, Corum, Turkey
}

Objective: Chronic ischemic mitral regurgitation is traditionally a complex lesion to repair. Only restrictive annuloplasty has become an accepted strategy to avoid valve replacement, but results are unsatisfactory in some subgroups of patients. Fundaro described an original technique that addresses the pathophysiologic mechanisms responsible for one of the most common subtypes of ischemic mitral regurgitation (MR), asymmetric tethering of the mitral leaflets after inferior MI. The technique includes partial detachment of the posterior leaflet from the mitral annulus, annular plication, and posterior cusp plasty. In this report, we document the perioperative outcome of this technique applied on posterior leaflet for repair in patients with type $3 \mathrm{~b}$ MR. Methods: We retrospectively evaluated 30 patients undergoing mitral valve repair with 'Fundaro Annuloplasty' technique by same team during the period from August 2007 until August 2013. 16 patients were male (53.3\%) and mean age was $55.5 \pm 16.20$ patients had concomitant CABG, 5 aortic surgery, 6 tricuspid repair and $3 \mathrm{LV}$ aneurysm repair. Results: 17 patients $(56.7 \%)$ received flexible, $6(20 \%)$ rigid ring plasty and 7 teflon strip plasty. Mean follow up was $35.8+20.3$ months.

Table 1. Perioperative evaluation of patients (for Abstract of Serdar Gunaydin)

\begin{tabular}{lcll}
\hline & Preoperative & $\begin{array}{l}\text { Postoperative } \\
(24 \text { months })\end{array}$ & p value \\
\hline MR (grade) & $3.1 \pm 0.5$ & $1.07 \pm 0.8$ & 0.001 \\
NYHA class & $2.1 \pm 0.4$ & $1.1 \pm 0.2$ & 0.001 \\
Pulmonary artery & & & \\
$\quad$ pressure, mm Hg & $43 \pm 11.8$ & $34.5 \pm 9.72$ & 0.007 \\
LVEDD, mm & $57.7 \pm 6.3$ & $52.2 \pm 4.4$ & 0.001 \\
LA, mm & $49 \pm 9$ & $43.6 \pm 6$ & 0.001 \\
\hline
\end{tabular}


Echocardiography demonstrated no MR in 23 (76.7\%), mild in 1 (3.3\%) and severe in $2(6.7 \%)$ patients. One (3.3\%) patient underwent second repair and one (3.3\%) recieved valve replacement. Early and late mortality rates were $6.7 \%$ (3 patients each). Perioperative data is summarized in table 1. Conclusion: Fundaro technique is safe, durable and has excelent outcomes with fewer recurrence rates and allows comfortable concomitant surgery. Positive effects on ventricular remodeling are also other advantages of this technique.

\section{Mitral Annular Dynamics after Mitral Valve Repair - Cardiac Magnetic Resonance Analysis}

\author{
Carlos M. Brandao, Ahmad A. Abdouni, Carlos E. Rochitte, \\ Elinghton T. Veronese, Rodrigo J. Melo, Antonio Santis, \\ Flavio Tarasoutchi, Pablo M. Pomerantzeff, Fabio B. Jatene \\ Heart Institute, Sao Paulo, Brazil
}

Background: The mitral valve repair techniques alter the morphology and dynamics of the mitral annulus during the cardiac cycle. Our goal is to analyze the mitral ring behavior by Cardiac Magnetic Resonance (CMR) in patients undergoing mitral valve repair by quadrangular resection without ring annuloplasty ('Double Teflon Technique'). Methods: Sixteen patients with degenerative mitral insufficiency underwent mitral valve repair using the technique described between August 2014 and July 2015. The age ranged between 40 and 75 years and $75 \%$ were male. Patients underwent MRI in the preoperative and postoperative period (up to a month follow-up) using a specific protocol for the evaluation of the mitral valve. CMR studies were performed with a Philips Achieva 1.5 Tesla machine, using a 5-element phased array coil. Contiguous long-axis cine images were obtained across the mitral valve, perpendicular to the valve commissures. Measurements were carried out of the ring diameter, area, circumference, and circularity index of the mitral annulus in the different phases of the cardiac cycle (diastole, early systole, mid systole, late systole), as well as measures of the leaflets, regurgitant volume, ventricular and atrial volumes. Results: There was no operative mortality. We observed the decrease of the mitral annulus area, with a significant reduction in the antero-posterior diameter of the mitral annulus, from $39.06 \mathrm{~mm}$ in the preoperative period to $31.81 \mathrm{~mm}$ in the postoperative period $(\mathrm{p}<0.01)$, without significant reduction in the medio-lateral distance $(\mathrm{p}=0.055)$. The mitral annulus contractility was preserved in the postoperative period. Changes in the mitral valve area values are presented in the table 1 . Conclusion: CMR

Table 1. (for Abstract of Carlos M. Brandao)

\begin{tabular}{llll}
\hline $\begin{array}{l}\text { Mitral valve area, } \\
\mathrm{cm}^{2}\end{array}$ & $\begin{array}{l}\text { Preoperative, } \\
\%\end{array}$ & $\begin{array}{l}\text { Postoperative, } \\
\%\end{array}$ & $\begin{array}{l}\text { Reduction, } \\
\%\end{array}$ \\
\hline Diastole & 15.05 & 10.84 & 27.97 \\
Early systole & 15.03 & 11.09 & 26.21 \\
Mid systole & 16.51 & 11.94 & 27.68 \\
Late systole & 18.04 & 12.91 & 28.43 \\
Annulus shortening & 16.68 & 16.03 & \\
\hline
\end{tabular}

is able to provide quantitative assessment of mitral regurgitation severity, insights into the mechanism of regurgitation and assessment of mitral valve dynamics. Furthermore, the use of this technique of mitral valve repair preserved the contractility of the mitral annulus during the cardiac cycle.

\section{Three Dimensional Transesophageal Echocardiography as a Predictive Tool to Assess the Outcome of Mitral Valve Plasty}

\section{Robin Bruggink, Jan Grandjean}

Medisch Spectrum Twente, Enschede, Netherlands

Objective: Mitral valve plasty has shown its advantages in comparison with mitral valve replacement. However, a lot of patients get a replacement while repair is preferred, which can be explained by high complexity and large performance gap of the surgery. The aim of this study was to create a model to predict the annuloplasty ring size based on perioperative $3 \mathrm{D}$-TEE datasets. This gives the surgeon more scientific rationale for choices made during surgery which makes the procedure more evidenced based and as result hopefully increasing the number of mitral valve repairs. Methods: Retrospective perioperative 3D-TEE data was collected from patients who underwent mitral valve plasty. The annulus circumference, leaflet lengths, leaflet area and inter-commissural distance were determined with use of Mitral Valve Navigator (Philips Electronics, The Netherlands, Version 10.2) and in house made software created with Matlab. The parameters were compared with the implemented ring size for creating univariate prediction models. A model which takes the average of the outcome of each parameter was created to create a more robust prediction. Initial validation was performed to test the performance of the model. Results: 28 patients were included into this study. The individual inter-commissural distance, leaflet area and annulus circumference models are significant predictors for the implanted ring size $\left(\mathrm{p}<0.01, r^{2}\right.$ respectively $0.76,0.74$ and 0.74 ). The average model correlated best $\left(r^{2}=0.77\right)$. No multivariate model could be created due to multicollinearity. Initial validation was performed with 9 patients, in which the average model has a $r^{2}$ of 0.85 , which is promising. However, more patients have to be included into the validation for a more accurate assessment. The correlation between the implemented and predicted ring size. Conclusions: In this study, a new promising tool was created, to increase the standardization of the current annuloplasty sizing technique. However, adaptations in the measurement software have to be made to enhance the intra-/inter-observer variability. A model to predict the increase of the effective leaflet lengths during annuloplasty is introduced, but yet not validated. 


\section{Surgical Challenge for Active Infective Endocarditis with Multiple Valvular Involvement or Periannular Abscess}

\author{
Hiroshi Furukawa, Taishi Tamura, Hiroki Takiuchi, \\ Masahiko Kuinose, Kazuo Tanemoto
}

Kawasaki Medical School, Kurashiki, Japan

Background: Clinical and surgical management for active infective endocarditis (AIE) with multiple valvular involvement (MVI) or periannular abscess (PA) is still challenging. We retrospectively analyzed these high-risk complicated AIE surgical patients with MVI or PA. Methods: Of 55 consecutive AIE patients who underwent surgical intervention in our institute between April 2000 and September 2015, 23 patients (mean age: $66 \pm 14$, female:male $=10: 13$ ) preoperatively diagnosed with having MVI or PA due to AIE. MVI was recognized in 11 patients (47.8\%) and PA was shown in $14(60.9 \%) .12$ patients $(52.2 \%)$ had large $(>10 \mathrm{~mm})$ vegetation which was diagnosed by preoperative echocardiography. Emergent or urgent surgical intervention was performed in 12 patients $(52.2 \%)$. Double or triple valve surgery was performed in 15 patients $(65.2 \%)$, with AVR+MVR in 6, AVR+MVP in 6, AVR+TVR in 1, and AVR+MVR+TVR in 1. In cases of $\mathrm{PA}$, complete resection of infectious tissues and aggressive debridement was performed followed by local administration and dispersion of bacteriostatic antibiotics (powder minocycline) at not only valvular annulus and periannular cavity but also prosthetic cuff and ring was accomplished, thereafter surgical repair was achieved without extensive reconstruction. Because of the fragile aortic annulus due to $\mathrm{PA}$, an anterior leaflet of mitral valve had to be used to fix the new prosthesis; therefore, concomitant prophylactic mitral valve annuloplasty was performed in 4 patients to prevent unexpected residual severe mitral valve regurgitation. Results: Surgical mortality was $8.7 \%$ and hospital mortality was $17.4 \%$ which seems to be acceptable compared with previous clinical reports. Only one patient $(4.3 \%)$ who underwent the third operation for recurrent PVE died of septic problem 51 days after surgery. There was three patients $(13.0 \%)$ who had significant $(>\bmod -$ erate) PVL following surgery, however, only one patient $(4.3 \%)$ required reoperation for PVL with hemolysis about 10 years following primary surgery. Survival by Kaplan-Meier analysis was $66.2 \%$ at 1 -year and $58.9 \%$ at 5 -year, respectively. Conclusions: These results demonstrated that our surgical pitfall and technique may be effective for high-risk AIE patients with MVI or PA, however, some manipulation or early aggressive surgical intervention is mandatory to improve clinical outcomes and prognosis.

\section{Reoperative Aortic Valve Replacement by Deconstruction of the Prosthetic Valve in Patients with Previous Aortic Root Replacement Using a Biologic Composite Valve Graft: A Technique for Avoiding Redo Aortic Root Replacement}

\author{
Mario Gaudino, Christopher Lau, Monica Munjal, \\ Mohamed Elsayed, Erin Mills, Benjamin C. Degner, \\ Ivancarmine Gambardella, Leonard Girardi
}

Cornell University, New York, NY, USA

Objective: Structural valve deterioration in patients who have had previous biologic composite valve grafts has generally required reoperative aortic root replacement. We examine the results of a technique for avoiding repeat aortic root replacement by piecemeal deconstruction of the prosthetic valve within the aortic graft, leaving the aortic root undisturbed. Methods: From July 2009 to October 2014, 10 consecutive patients who had previous aortic root replacement with biologic composite valve grafts underwent reoperative aortic valve replacement. The indication for reoperation was prosthetic aortic stenosis in all patients and all patients requested another biologic valve. Exposure was via redo median sternotomy and transverse aortotomy through the Dacron graft. The valve was removed without dissection of the aortic root or coronary buttons. Results: Patients presented with New York Heart Association Class II $(8,80 \%)$ or Class III $(2,20 \%)$ symptoms. Mean age was $53.5 \pm$ 15.9 years, $7(70 \%)$ of patients were male, and mean time since the previous operation was $8.4 \pm 1.8$ years. There were no sternal reentry complications. Cardiopulmonary bypass time was $99.5 \pm 19.2$ minutes and crossclamp time was $70.7 \pm 15.3$ minutes. The majority of patients received an aortic valve that was the same size as the original valve; mean size of the original valve was $23.8 \pm 2.0 \mathrm{~mm}$ and new valve was $23.4 \pm 2.0 \mathrm{~mm}$. On postoperative transesophageal echocardiogram, no patient had a significant paravalvular leak with only one having trace paravalvular aortic insufficiency. There were no perioperative mortalities and no patients had myocardial infarction, stroke, respiratory failure, renal failure, or sternal wound infection. One (10\%) patient required a pacemaker. Conclusions: A degenerated bioprosthetic aortic valve in a composite valve graft can be safely replaced without a full redo aortic root replacement. Early results are promising but long-term follow-up is necessary.

\section{The Use of a Pre-Operative Mathematical Model to Guide Mitral Valve Repair}

Virimchi Pillutla ${ }^{1}$, Tom Goodwin ${ }^{1}$, Elli Tutungi ${ }^{2}$, Aubrey Almeida ${ }^{3}$

${ }^{1}$ Monash University, Melbourne, ${ }^{2}$ Epworth Hospital,

Melbourne, ${ }^{3}$ Epworth Hospital and Monash Medical Centre,

Melbourne, Australia

Objective: Trans-Esophageal Echocardiogram (TEE) is routinely performed to assess the mechanism of mitral regurgitation to guide repair. However, currently surgeons do not use TEE information regarding the approximate dimensions of the valve they should aim for in order to achieve optimal valve function and avoid adverse outcomes. Using pre-operative TEE measurements, we aimed to 


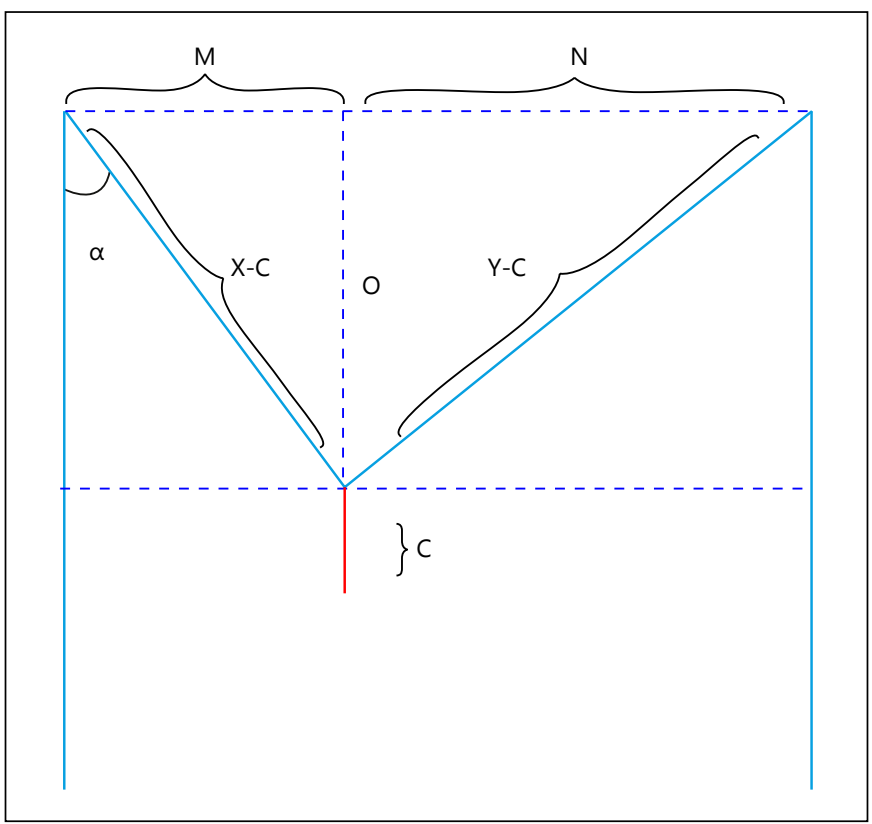

Fig. 1. (for Abstract of Virimchi Pillutla).

develop a mathematical model that can help predict optimal annuloplasty AP diameter. Methods: A mathematical model can be used to calculate the AP diameter of the mitral valve post repair to achieve an optimal coaptation using the geometrical relationship of various mitral valve components and certain assumptions.

Variables:

Let posterior leaflet length $=\mathrm{X}$

Let anterior leaflet length $=\mathrm{Y}$

Let $\mathrm{AP}$ diameter $=\mathrm{AP}$

Let $\alpha=$ angle in degrees between posterior leaflet and ventricular wall

Let coaptation $=\mathrm{C}$

Equations:

$\mathrm{M}=(\mathrm{X}-\mathrm{C}) \times \operatorname{Sin}(\alpha)$

$\mathrm{O}=(\mathrm{X}-\mathrm{C}) \times \operatorname{Cos}(\alpha)$

$\mathrm{N}=$ Square root of $\left[(\mathrm{Y}-\mathrm{C})^{2}-[(\mathrm{X}-\mathrm{C}) \times \operatorname{Cos}(\alpha)]^{2}\right]$

AP Diameter $=\mathrm{M}+\mathrm{N}$

To examine the accuracy of our model we compared actual post-operative TEE measurement of the AP diameter to a modelpredicted AP diameter. Results: In $87 \%$ of cases, the model predicted AP diameter using post-operative TEE measurements fell within $25 \%$ of actual measured AP diameter. Variables such as im- age quality and types of views available could have contributed to any significant discrepancies between model predicted and actual AP diameters. Conclusion: In mitral valve repair surgery, a mathematical model can be used to pre-operatively guide surgeons in order to achieve optimal valvular dimensions.

\section{External Basal Annuloplasty Device for Ischeamic Mitral Regurgitation (Basal Annuloplasty of the Cardiac Externally Device Implantation) - Single Center Early Experience}

Chandrasekar Padmanabhan ${ }^{1}$, Mahalakshmi Shanmugam ${ }^{1}$ Jai Shankar Raman²

${ }^{1}$ GKNM Hospital, Coimbatore, India; ${ }^{2}$ Rush University, Chicago, IL, USA

Background: Ischemic Mitral Regurgitation (IMR) is primarily a disease of the ventricle. Present surgical treatment options mostly address the mitral annulus \& leaflets. The BACE device is an external annuloplasty system which corrects the MR by improving the annular geometry,. It also addresses the ventricular component by providing a support for the postero basal segment from progressive dilatation. The advantage is that it can be done off pump, no anticoagulation and can be remotely adjusted. Methods: Three patients with coronary artery disease and ischeamic mitral regurgitation satisfied inclusion criteria. All patients had moderate-Severe MR (Grade $>2$ ) with Impaired LV function. They underwent implantation of BACE device along with CABG. After off pump revascularisation, The device was deployed by inflating the ports with saline under TEE control to achieve optimal reduction in MR. The device was then fixed in position with tacking sutures, with the ports are placed subcutaneously in the sub costal area for future adjustment. Results: There were three patients, 2 male and 1 female. The mean age was 60 years. TWo patients had Diabetes Mellitus and all three were Hypertensive. There were no perioperative deaths. The degree of MR improved significantly (Ref Table). The improvement in MR was sustained over the six-month follow-up period. Conclusion: This initial experience with the BACE device has shown its efficacy in correcting IMR. The procedure can be performed Off pump, and MR also be dynamically corrected on a beating heart under TEE control. The external nature of the device precludes the need for anticoagulation. The subcutaneous ports allow late adjustment guided by echocardiography as an outpatient procedure.

Table 1. (for Abstract of Chandrasekar Padmanabhan)

\begin{tabular}{|c|c|c|c|c|c|c|c|c|}
\hline \multicolumn{5}{|l|}{ Pre-op } & \multicolumn{4}{|l|}{ Post-op } \\
\hline patient & $\begin{array}{l}\text { NYHA } \\
\text { class }\end{array}$ & $\begin{array}{l}\mathrm{EF}, \\
\%\end{array}$ & $\begin{array}{l}\text { grade of } \\
\text { MR }\end{array}$ & $\begin{array}{l}\text { discharge } \\
\mathrm{EF}, \%\end{array}$ & $\begin{array}{l}\text { discharge } \\
\text { grade of MR }\end{array}$ & $\begin{array}{l}6 \text { months } \\
\text { NYHA }\end{array}$ & $\begin{array}{l}6 \text { months } \\
\text { EF, \% }\end{array}$ & $\begin{array}{l}6 \text { months } \\
\text { grade of MR }\end{array}$ \\
\hline 1 & II & 35 & Moderate & 35 & Mild & I & 40 & Mild \\
\hline 2 & III & 30 & Severe & 28 & Moderate & I & 35 & Mild \\
\hline 3 & $\mathrm{I}$ & 40 & Severe & 45 & Mild & I & 47 & Mild \\
\hline
\end{tabular}




\section{Simulation Technique for Extended Posterior Mitral Leaflet Prolapse}

Tatsuhiko Komiya, Hirohumi Tauneyoshi, Takeshi Shimamoto

Kurashiki Central Hospital, Kurashiki, Japan

Objective: Repair of the posterior leaflet of the mitral valve has been traditionally performed by resection. In case of extensive prolapse, large resection would result in restricted leaflet motion (curtain effect). The exact determination of resection area is not always feasible. Methods: Using several stitches to plicate excess leaflet toward left ventricle, we can simulate postrepair mitral valve shape and estimate the resection area properly. After marking the fold with a pen, resection lines become apparent. Approximation of resected leaflets is a simple running suture for a triangular resection. For extended prolapse, in order to reduce the height of the leaflet, $\mathrm{L}$ or inverted $\mathrm{T}$ configuration is intended by simulation stitches. Just to image pushing down the protruded portion into the left ventricle to obtain flat surface. Cutting the stitches unravel the part to be resected and its shapes are various forms of quadrangles. The reconstruction of the leaflets is accomplished by simple reproduction of the simulation. No focal annuloplasty is required. Results: Since 2009, we introduced this technique for posterior prolapse of the mitral valve. A triangular resection in 87 cases and a quadrangle resection without local annuloplasty in 24 cases were performed with good results. Conclusions: Simulation technique is beneficial for determination of resected area especially for extended posterior prolapse.

\section{Minimally Invasive Valve Surgery}

\section{Aortic Valve Replacement Through Right Anterior Minithoracotomy: Early Outcomes and One-Year Survival} Rainer Leyh, Constanze Bening, Christoph Schimmer,

University of Wuerzburg, Wuerzburg, Germany

Objective: Right anterior minithoracotomy (RAT) for aortic alve replacement (AVR) is regarded as a difficult innovative surgical technique for minimal invasive AVR. The aim of this study was to evaluate the early outcome and 1-year survival of patients undergoing RAT-AVR for severe aortic stenosis in a center starting with a RAT-AVR program. Methods: From 09/2013 to 07/2015, 70 patients (mean age $76 \pm 6$ years; mean EuroScore II $6.7 \pm 4.3$ ) underwent RAT-AVR. In 45 patients the Elite Intuity rapid deployment valve and in 25 patients a conventional stented biological aortic valve was implanted. Results: The mean aortic cross-clamp time and cardiopulmonary bypass time were $51 \pm 20 \mathrm{~min}$ and $89 \pm$ $36 \mathrm{~min}$, respectively. Conversion rate and hospital mortality was $2.86 \%(2 / 70)$ each. Median stay in the ICU was 1 day, with assisted ventilation necessary for a median of 11 hours. There was no paravalvular leakage and no postoperative stroke. Incidence of atriventricular block requiring pacemaker and new onset atrial fibrillation was $1.4 \%(1 / 70)$ and $8.6 \%(6 / 70)$, respectively. At a mean follow-up

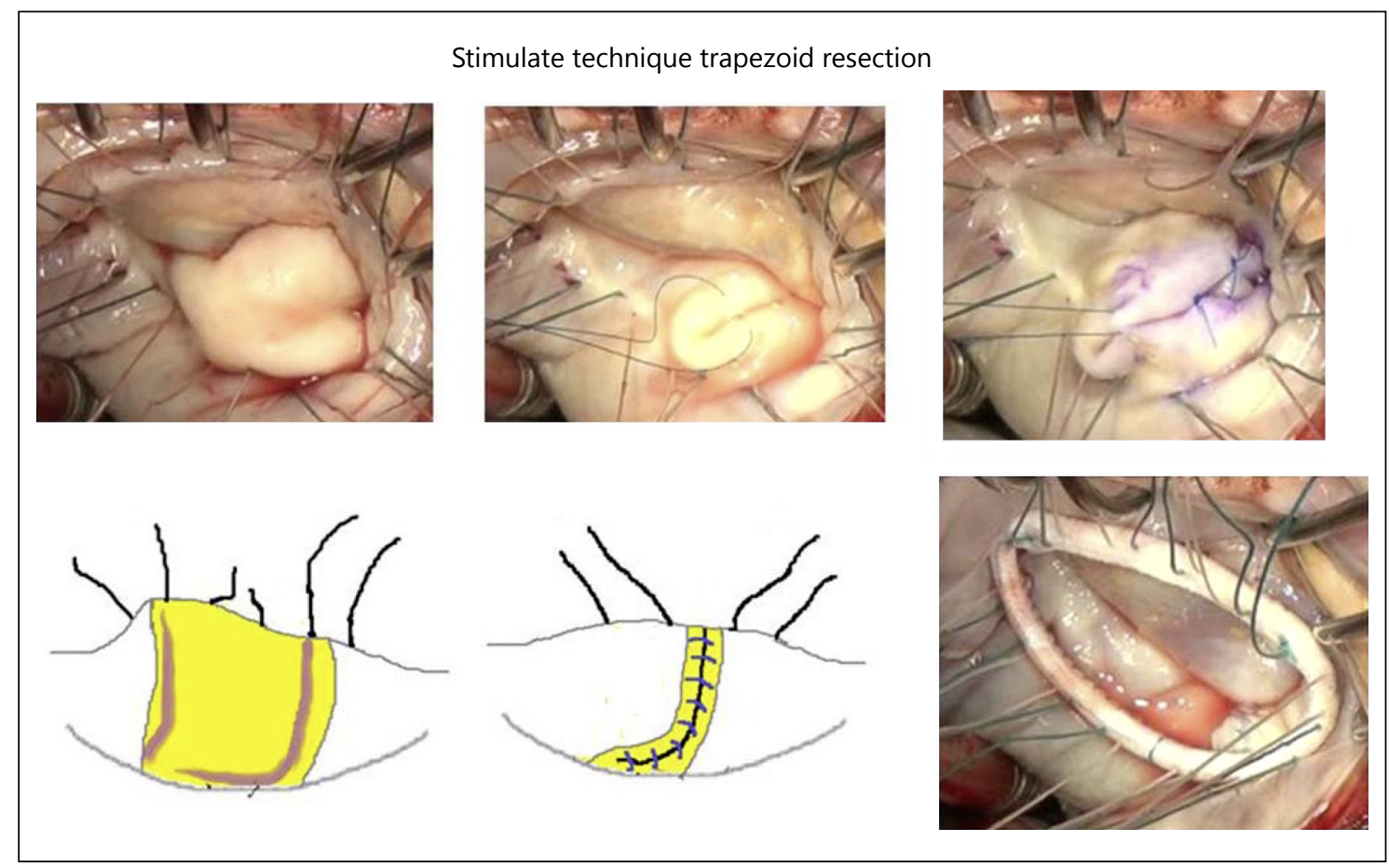

Fig. 1. Simulation technique trapezoid resection (for Abstract of Tatsuhiko Komiya). 
of $11.4 \pm 6$ months, the overall survival was $95.7 \%$. Conclusions: These preliminary data shows that RAT-AVR is reproducible and safe with a low perioperative complication rate and satisfactory survival rate. This technique could amplify indications for surgical minimal invasive aortic valve replacement in high risk patients and might be an alternative for transcatheter aortic valve replacement.

\section{Advantages of Minimal Access versus Conventional Aortic Valve Replacement in Elderly or Severely Obese Patients}

\author{
Calogera Pisano, Pasquale Totaro, Sabrina Ferrante, \\ Alberto Allegra, Oreste Fabio Triolo, Eduardo Tulumello, \\ Cesira Palmeri, Vincenzo Argano \\ University of Palermo, Palermo, Italy
}

Objective: Potential clinical advantage of minimal access versus conventional surgery in the subgroup of elderly and obese patients undergoing aortic valve replacement (AVR). Materials and Methods: We retrospectively collected data on 153 consecutive patients undergoing isolated primary AVR with standard prosthesis in our Cardiac Surgical Unit over two years period. 93 AVR were performed with full sternotomy (FS) and 42 with an upper J ministernotomy (MS). Propensity score matching was used to compare 42 selected FS patients with the $42 \mathrm{MS}$ patients in terms of postoperative outcomes. Older and severely obese patients were further analysed as a separate subgroup. Results: After propensity matching score the two groups were comparable in terms of preoperative characteristics. No differences were observed in the incidence of major and minor postoperative complications or related morbidity. Cardiopulmonary bypass time was significantly longer in MS group compared to the FS group (107 \pm 22 vs. $92 \pm$ $18 \mathrm{~min}, \mathrm{p}=0.001$ ), on the other hand no significant difference was observed in aortic cross clamp time $(76.9 \pm 19.4$ vs. $72 \pm 16.5 \mathrm{~min}$, $\mathrm{p}=0.2)$. Significant shorter ventilation time ( $13 \pm 0.7$ vs. $24 \pm$ 3 hours, $\mathrm{p}=0.001$ ), intensive care unit (ICU) stay ( $1 \pm 0.1$ vs. $2 \pm$ 0.2 days, $\mathrm{p}=0.001)$ and hospital stay $(8.5 \pm 0.9$ vs. $13 \pm 1.2$ days, $\mathrm{p}=0.003$ ) were shown in MS group. The age subgroup analysis showed that statistical significance for mechanical ventilation and
ICU stay was specific for patients over 75 years (fig. 1a and fig. 1b). The analysis of BMI quartile showed that statistical significance for mechanical ventilation was specific for patients in the fourth quartile (fig. 1c). Conclusions: Mini-access AVR is a reproducible, safe and effective surgical option in patients candidate for isolated AVR. Clinical advantages in terms of faster recovery using such technique seems to be more specific for severely obese or older patients. Therefore we support this approach as the technique of choice for isolated AVR is this particular subgroups of patients.

\section{Our Experiences with Adopting Partial J-Sternotomy as the Standard Incision for Aortic Valve Replacement Surgery}

\section{Petr Budera, David Talavera, Richard Fojt, Miroslav Kolesar, Zbynek Straka \\ University Hospital Kralovske Vinohrady, Prague 10, Czech Republic}

Background: Minimally invasive procedures are becoming integral component of modern cardiac surgery. In aortic valve replacement (AVR), this trend is forced by a strong competition of TAVI with expected expansion of its indications in the future. We present our experiences with the J-sternotomy as the standard incision for AVR. Methods: Since 2014, the partial J-sternotomy, incised to the right fourth intercostal space, has been adopted as an incision of choice for isolated AVR in our department, instead of previously used full sternotomy. Central cannulation is done through the ascending aorta and right atrium, vent is placed through the pulmonary artery or right superior pulmonary vein. Perioperative and 30-days clinical and echocardiography data were analyzed for purpose of this study. Results: Out of 116 isolated AVRs performed between January 2014 and October 2015, 100 (86\%) was operated via the partial J-sternotomy. Mean age was 70 years (3488 ), mean left ventricule ejection fraction $58 \%$ (20-75), mean body mass index $30 \mathrm{~kg} / \mathrm{m}^{2}(20-47)$. Mean operation time was $143 \mathrm{~min}$ (100-270), mean cardiopulmonary bypass time $62 \mathrm{~min}$ (37-131), mean cross clamp time $49 \mathrm{~min}$ (31-110), right mammary artery has not been damaged in any patient. Mechanical valve was implanted

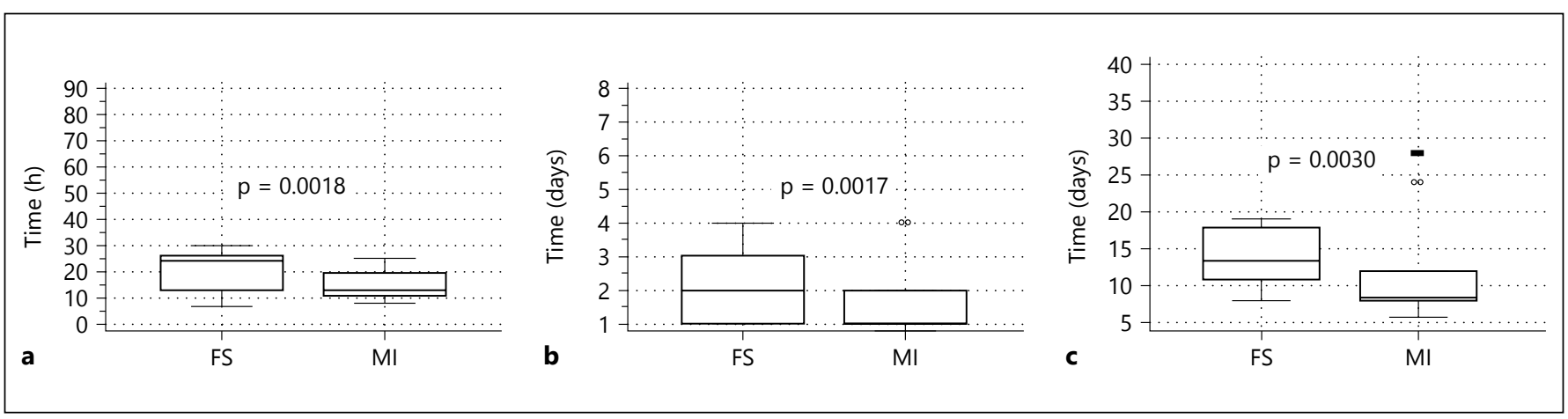

Fig. 1. a Ventilation time by surgical approach. b ICU stay by surgical approach. c Hospital stay by surgical approach (for Abstract of Calogera Pisano). 
in $20(20 \%)$, stented biological in $61(61 \%)$ and sutureless in 19 (19\%) of patients. Mean postoperative blood loss was $359 \mathrm{ml}(70-$ $1400)$, we had $3(3 \%)$ conversions on sternotomy and 1 (1\%) reexploration for bleeding. Thirty-days mortality was 3\%, 4 (4\%) patients had a pericardial effusion with a need of thoracoscopic pericardial fenestration, 1 (1\%) had a transient ischaemic attack, 1 (1\%) had a pneumonia, $11(26 \%)$ patients had a postoperative atrial fibrillation, $1(1 \%)$ had a prosthetic valve endocarditis. No deep wound infections occured. Three patients had a mild paravalvular leak on 30-days echocardiography. Conclusions: Partial J-sternotomy has been quickly accepted as an incision of choice for isolated AVR by all surgeons in our center. It presents a safe and feasible minimally invasive approach with very-low conversions and postoperative complications rates. Our experiences support its wide adoption as a standard method of choice for AVR, especially in current TAVI era.

\section{Staged Percutaneous Coronary Intervention Followed by Minimally Invasive Mitral Valve Surgery versus Combined Coronary Artery Bypass Graft and Mitral Valve Surgery for Multi-Vessel Coronary Artery Disease and Severe Ischemic Mitral Regurgitation}

\author{
Christos G. Mihos ${ }^{1}$, Andres M. Pineda ${ }^{2}$, \\ Maiteder C. Larrauri-Reyes ${ }^{2}$, Nirat Beohar ${ }^{2}$, Orlando Santana ${ }^{2}$ \\ ${ }^{1}$ Massachusetts General Hospital, Boston, MA, ${ }^{2}$ Coulmbia \\ University, Division of Cardiology, Mount Sinai Heart Institute, \\ Miami Beach, FL, USA
}

Background: The optimal treatment for multi-vessel coronary artery disease (CAD) and ischemic mitral regurgitation (IMR) has not been established. A staged approach of percutaneous coronary intervention followed by minimally invasive valve surgery (PCI+MIVS), rather than combined median sternotomy coronary artery bypass and valve surgery (CABG+MVS), may be a viable option. Methods: We retrospectively evaluated patients with multivessel CAD and IMR who underwent PCI+MIVS $(\mathrm{N}=9)$ or CABG+MVS $(\mathrm{N}=15)$ at our institution between February 2009 and April 2014. All patients had severe IMR, which was characterized by systolic restricted leaflet motion and preserved leaflet pliability (Carpentier type IIIb dysfunction). Results: Baseline characteristics between the groups were similar, except for a higher clopidogrel use in the PCI+MIVS group (78\% versus $27 \%, \mathrm{p}=0.02)$. Patients undergoing $\mathrm{CABG}+\mathrm{MVS}$ had a higher incidence of left main and right coronary artery disease ( $33 \%$ versus $0 \%, p=0.05)$, while left anterior descending and left circumflex coronary artery disease was more prevalent in PCI+MVS group (78\% versus $27 \%$, $\mathrm{p}=0.02$ ). The median time from PCI to MIVS was 40 days [IQR $8-71$ ], with a mean of $2.3 \pm 1$ stents placed per patient. All patients undergoing $\mathrm{CABG}+\mathrm{MVS}$ received a left internal thoracic artery to left anterior descending coronary artery graft, with a mean of $2.5 \pm$ 0.5 total grafts per patient. PCI+MIVS was associated with decreased cardiopulmonary bypass ( $111 \pm 41$ versus $167 \pm 49$ minutes, $\mathrm{p}=0.01)$ and aortic cross-clamp $(79 \pm 32$ versus $129 \pm 35$ minutes, $\mathrm{p}=0.003)$ times, and fewer intraoperative packed red blood transfusions (2 [IQR 0-2] versus 3 [IQR 1-4] units, $\mathrm{p}=0.05$ ), when com- pared with $\mathrm{CABG}+\mathrm{MVS}$. The rate of mitral valve repair was $80 \%$ for $\mathrm{CABG}+\mathrm{MVS}$ versus $67 \%$ for PCI+MVS $(\mathrm{p}=0.47)$. There was no difference between the surgical approaches in regards to postoperative complications, or 30-day mortality. At 1-year follow-up, survival between the CABG+MVS (100\%) and PCI+MVS (89\%) groups was similar $(\mathrm{p}=0.19)$, and no patient required reoperation for recurrent mitral regurgitation. Conclusions: A staged approach of PCI+MIVS for concomitant multi-vessel CAD and IMR is associated with decreased operative times and intraoperative blood transfusions, and may be a safe alternative to CABG+MVS.

\section{Minimally Invasive Aortic Valve Replacement in Patients with Coronary Artery Disease}

\section{Matthew Thomas, Alon Aharon, Oleg Orlov, loannis Paralikas, Scott M. Goldman, Konstadinos Plestis}

Lankenau Medical Center, Wynnewood, PA, USA

Objective: Minimally invasive aortic valve replacement (mAVR) is becoming the standard of care for patients with isolated aortic valve disease. Decreased pain, shorter lengths of stay, and reduced morbidity are well documented. In patients with asymptomatic CAD not involving the left main, isolated mAVR may be a viable option. We examined our population of $\mathrm{mAVR}$ patients with concomitant distal coronary artery disease. Methods: From January 2006 to May 2015, 436 patients underwent mAVR at our institution. 69 patients had concurrent CAD. Their mean age was 74.1 years. $84 \%$ were men. Comorbidites included hypertension (82.6\%), hypercholesterolemia (88.4\%), diabetes (27.5\%), COPD (2.9\%), and CHF (29\%). Hypertension and hypercholesterolemia were significantly more prevalent these patients than those without CAD ( $\mathrm{p}<0.05)$. Preoperative EF was $58.4+11.2$. Surgical indications included aortic stenosis in $94.2 \%$ and aortic insufficiency in $28.8 \%$. Results: The mean cardiopulmonary bypass and crossclamp times were 114.9 and 86.9 minutes. Operative blood requirements included of 2.3 units of PRBC, 0.73 units of FFP, 1.06 units of PLTS, and 1.2 units of cryoprecipitate. Postoperative morbidities occurred in 39 patients $(56.5 \%)$, including stroke in 2 (2.9\%), atrial fibrillation in 29 (33.3\%), new onset renal insufficiency in $5(7.2 \%)$, and gastrointestinal bleeding in $1(1.4 \%)$. In hospital mortality occurred in 4 patients $(5.8 \%)$. 1 patient died of complete heart block, 1 died of sudden cardiac arrest after a protracted hospital course including pulmonary insufficiency and GI bleeding, 1 patient died secondary to profound sepsis, MSOF, and circulatory collapse, and 1 patient died secondary to postcardiotomy shock requiring ECMO and subsequent massive stroke and brain death. Kaplan-Meier survival data was determined with 1-, 3 -, and 5-year survival rates of $0.91,0.87$, and 0.74 respectively. Conclusion: mAVR offers proven benefits over traditional fullsternotomy. The presence of concomitant CAD complicates clinical decision-making. The presence of CAD in patients undergoing mAVR resulted in increased length of stay, more frequent early complications, and worsened KM survival data in matched $\mathrm{mAVR}$ without coronary disease. However, the observed morbidity and mortality rates are congruent with STS risk assessment scores for AVR-CABG patients with similar comorbidities. In addition, no late ischemic related complications were noted. 


\section{Tissue Engineered Heart Valves}

\section{Novel Pericardial Tissue Demonstrates Significant Improvement in Resistance to Calcification Over Commercial Tissues Using Rabbit Intramuscular Model}

Hao Shang, Steven M. Claessens, Bin Tian, Gregory A. Wright

Edwards Lifesciences LLC, Irvine, CA, USA

Objective: The objective of the study is to investigate the calcification resistance of a novel pericardial tissue in comparison with commercially available tissues. The novel tissue aims at reducing calcification and increasing the durability of the bioprosthesis. Methods: The newly developed bovine pericardial tissue (test) was processed through a novel integrity preservation technology with permanent free aldehyde capping and glycerolization. The tissue discs from the test sample, a commercial bovine pericardial bioprosthesis, and a commercial porcine bioprosthesis were implanted intramuscularly in 30 rabbits for $60 \pm 5$ days. Glutaraldehyde fixed bovine pericardium was used as a positive control. The explanted samples were digested in acid and the calcium content was quantified by ICP-OES. Histology was performed to observe the collagen structure and integrity. In addition, the infiltration of host cells were examined in related to inflammatory responses. The free aldehyde groups in the pre-implanted tissue were also measured. Results: The results show that the test samples have significantly lower calcium content than tissues from commercial bioprosthesis. The test sample showed median calcium of $5.08 \mathrm{mg} / \mathrm{g}$ as opposed to $141 \mathrm{mg} / \mathrm{g}(\mathrm{p}<0.05)$ for commercial bovine pericardial tissue and $96.6 \mathrm{mg} / \mathrm{g}(\mathrm{p}<0.05)$ for commercial porcine leaflet tissue. In addition, histology analysis showed less inflammatory response for test samples than commercial tissues. Aldehyde content in the tissue was found to correlate with calcium content. Conclusions: The study demonstrates that the novel tissue can reduce calcification by $95 \%$ in comparison with commercially available tissues in the intramuscular rabbit model. The new tissue can potentially improve the durability of the bioprosthesis. Further studies need to be conducted to establish the relationship between preclinical effectiveness and clinical outcomes.

\section{Heart Valve Imaging}

\section{Flow Acceleration Time for the Diagnosis of Severe Aortic Stenosis}

Israel Mazin, Moshe Katz, Ori Vaturi, Rafael Kuperstein, Roy Beigel, Elad Asher, Micha S. Feinberg, Sagit Ben Zekry

Sheba Medical Center, Tel Hashomer, Israel

Background: Current echocardiographic diagnosis of the severe aortic stenosis (AS) is based on valve gradients (peak velocity $\geq 4 \mathrm{~m} / \mathrm{sec}$ or mean gradient $\geq 40 \mathrm{~mm} \mathrm{Hg}$ ) and area calculation $\left(\leq 1 \mathrm{~cm}^{2}\right.$ or index valve area $\left.\leq 0.6 \mathrm{~cm}^{2} / \mathrm{m}^{2}\right)$. Stenotic aortic valve has a characteristic systolic flow pattern with late peaking velocity which can be a helpful parameter in assessing severity of challenging cases. Our aim was to describe systolic time parameters and their diagnostic potential in severe AS. Methods: Seventyfive patients (Mean age 80.7 years, 53\% men) were studied. Of whom, 54 patients were with severe, 11 with moderate and 10 with mild AS. All had normal ejection fraction $(\geq 50 \%)$ with no significant other valvular conditions. Systolic time intervals were studied; acceleration time (AT) defined as the time from the beginning of systolic flow to maximal velocity, ejection time (ET) was defined as the time from onset to end of systolic flow. The relation of AT/ET was calculated as well. Results: Acceleration time was significantly longer for patients with severe AS (86.611.8 msec, $92.815 .4 \mathrm{msec}$ and $132.920 .5 \mathrm{msec}$ for mild, moderate and

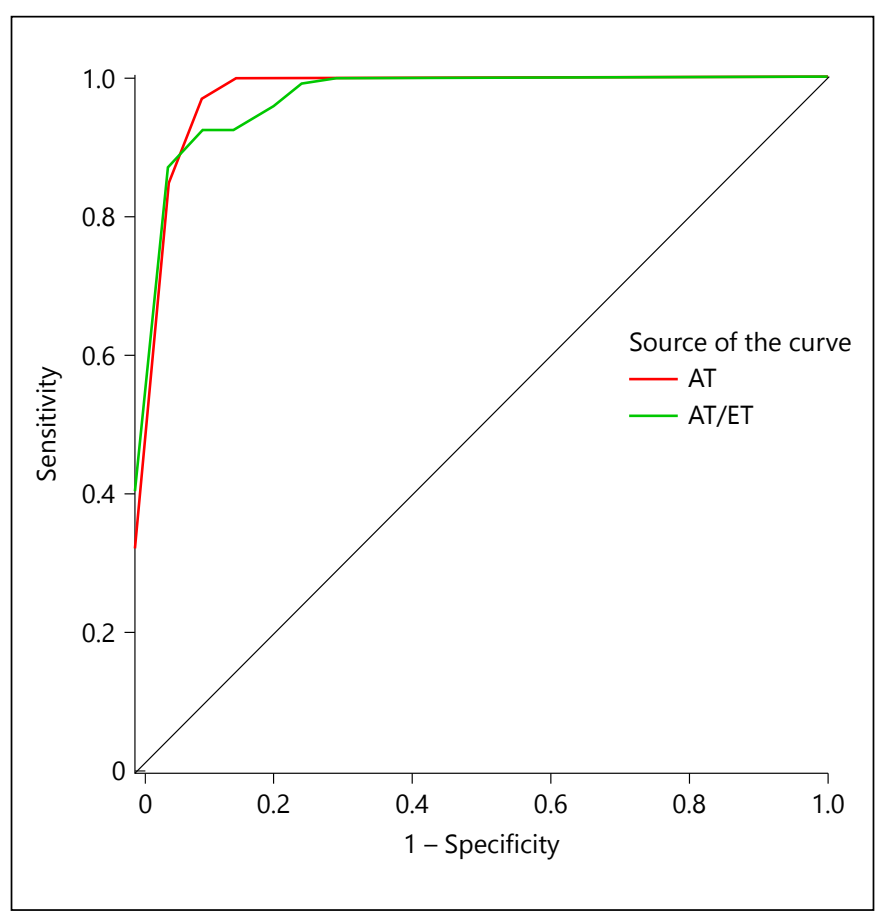

Fig. 1. Receiver operator curve (for Abstract of Israel Mazin). 
severe AS respectively, $\mathrm{p}<0.001)$. There was no significant difference in ET among groups (312.830.3 $\mathrm{msec}, 292.443 .2 \mathrm{msec}$ and $32631.1 \mathrm{msec}$ for mild, moderate and severe AS respectively, $\mathrm{p}=$ 0.1 ). Patients with severe AS presented with a longer AT/ET ration $(0.280 .03,0.310 .03$, and 0.410 .05 for mild, moderate and severe AS respectively, $\mathrm{p}<0.001)$. Receiver-operator characteristic curve (ROC) analysis has shown that AT and AT/ET can identify patients with severe AS (area ROC 0.98 for both, figure 1). Acceleration time $>112 \mathrm{msec}$ and AT/ET ratio $>0.35$ separate severe AS to non-severe AS (sensitivity 93\% and specificity 95\%). Conclusion: Acceleration time and the ratio of AT/ET are reliable measurements for identifying patients with severe AS. Those measurements are not angle dependent and can have additive value in challenging cases.

\section{Association between Bicuspid Aortic Valve Raphe Morphology and Aortopathy Phenotype}

\author{
Maude Page ${ }^{1}$, Maxime Laflamme ${ }^{2}$, Omar Nawaytou ${ }^{3}$, \\ Laurent De Kerchove ${ }^{4}$, Gébrine El-Khoury ${ }^{4}$, \\ Jean-Louis Vanoverschelde ${ }^{4}$ \\ ${ }^{1}$ Hôpital du Sacré-coeur de Montréal, Outremont, QC, ${ }^{2}$ Institut \\ de Cardiologie et Pneumologie de Québec, Outremont, QC, \\ Canada; ${ }^{3}$ University Hospitals Birmingham, Birmingham, \\ United Kingdom; ${ }^{4}$ Cliniques Saint-Luc, Brussels, Belgium
}

Background: Heterogeneity of bicuspid aortic valve (BAV) disease is increasingly recognized and pathophysiology of aortopathy remains debated. Recent data suggest that valve-related hemodynamics mediate BAV aortopathy via increased shear stress and altered aortic wall matrix architecture. On the other hand, BAV classifications are currently reconsidered to account for the wide spectrum of phenotypes and to tailor surgical approaches. How these newly described phenotypes relate to BAV aortopathy is poorly documented. Objectives: To assess whether BAV phenotype, according to a new classification based on raphe presence and morphology, was associated with specific dilatation patterns. Methods: 86 consecutive BAV patients undergoing valve-sparing surgery for aortic regurgitation and/or aneurysm at our institution were included (76 males; mean age 4312 years). BAV were classified according to the absence (type 0 ) or presence of a complete (type 1A) or incomplete/restrictive (type 1B) raphe on pre-operative transoesophageal echocardiography. Results: Eleven patients had a type $0 \mathrm{BAV} ; 7$ had antero-posterior orientation of the free edges and 4 had a right-left orientation. A complete raphe (type 1A) was identified in 57 patients; an incomplete, restrictive raphe (1B) was identified in 18. The indexed diameters of the ventriculoaortic junction (VAJ), sinuses of Valsalva (SOV) and sino-tubular junction (STJ) were significantly larger among type 0 compared to types $1 \mathrm{~A}$ and $1 \mathrm{~B}$ (VAJ $16.1 \pm 1.5$ vs. $14.4 \pm 1.9 \mathrm{~mm} / \mathrm{m}^{2}, \mathrm{p}=0.009$; SOV $22.3 \pm 2.4$ vs. $19.3 \pm 2.3 \mathrm{~mm} / \mathrm{m}^{2}, \mathrm{p}<0.001 ;$ STJ $19.4 \pm 4.3$ vs. $16.9 \pm 2.8 \mathrm{~mm} / \mathrm{m}^{2}, \mathrm{p}=0.013$; proximal ascending aorta $19.3 \pm 4.3$ vs. $18.8 \pm 3.7 \mathrm{~mm} / \mathrm{m}^{2}, \mathrm{p}=0.72$ ). Aortic phenotypes were divided into three clusters: A: Diameter of the ascending aorta $\left(\mathrm{D}_{\mathrm{ASC}}\right)>\mathrm{D}_{\mathrm{SOV}}>\mathrm{D}_{\mathrm{ST}}$; B: $\mathrm{D}_{\mathrm{SOV}} \mathrm{D}_{\mathrm{ASC}}$ and $\mathrm{D}_{\mathrm{SOV}}>\mathrm{D}_{\mathrm{ST}}$; C: $\mathrm{D}_{\text {SOV }} \mathrm{D}_{\mathrm{STJ}}$. Distribution of the aortic patterns is shown in figure 1 ( $p<0.001$ for comparison across groups). Conclusions: When classified ac-

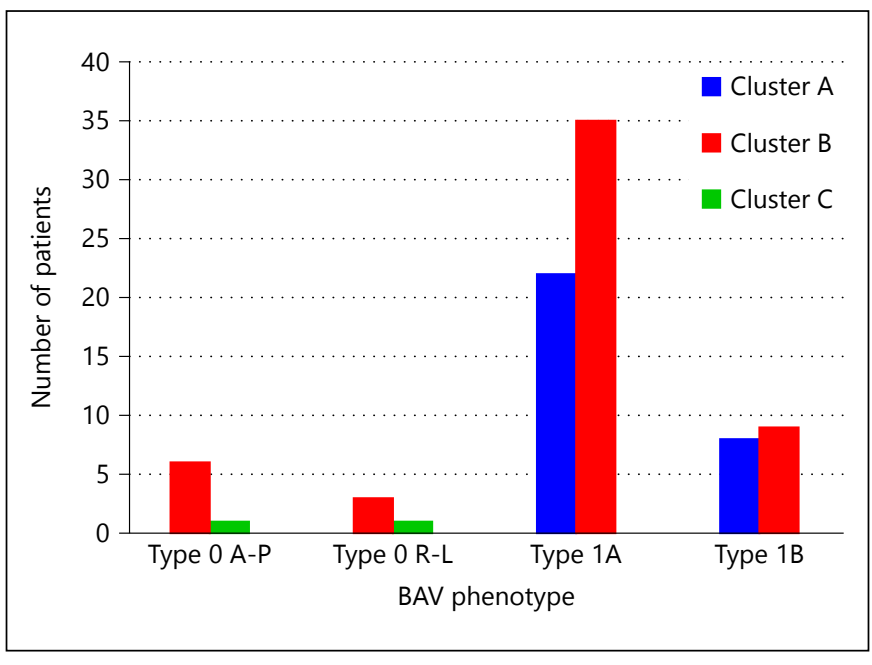

Fig. 1. (for Abstract of Maude Page).

cording to raphe presence and morphology, BAV phenotypes seem to be associated with distinct aortic dilatation patterns. Type 0 are more frequently associated with large VAJ and SOV. Investigation into dynamic flow patterns according to this repair-oriented BAV classification would allow further understanding of $\mathrm{BAV}$ aortopathy and perhaps better risk-stratification to guide aortic intervention.

\section{Impact of Right Ventricular Function on Outcomes of Patients with Organic Mitral Regurgitation}

\section{Oumhani Toubal \\ Institut Universitaire de Cardiologie et Pneumologie de Québec, Quebec, QC, Canada}

Objective: In patients with asymptomatic severe organic mitral regurgitation (MR), there is a dilemma with regard to the type of treatment that should be used: i.e. watchful waiting versus early prophylactic surgery. It is thus important to develop novel biomarkers to assist the clinician in this difficult therapeutic decision making. The triggers for performing mitral valve surgery are essentially based on MR severity, symptoms, and LV function. In this study, we examined the impact of right ventricular function on prognosis in patients with asymptomatic mitral regurgitation. Methods: 120 asymptomatic patients with at least moderate MR were recruited in the prospective study PROGRAM. The RV function was assessed in a CoreLab and included the following measurements: tricuspid annular plane systolic excursion (TAPSE), tricuspid annular plane velocity (TAPSV), right ventricular fractional shortening (RVFS) and right ventricular free wall longitudinal strain (RVLS). The primary study end-point was the composite of occurrence of atrial fibrillation, symptoms, hospitalization for heart failure, or death. Results: During a mean follow-up of $5.6 \pm 3.6$ years, there were 33 events. The 3 years 
event-free survival was lower in patients with RV dysfunction defined as RVLS $<20 \%$ compared to patients with no evidence of RV dysfunction ( $33 \pm 19 \%$ versus $74 \pm 6 \%$ ). RVLS correlated with the MR effective regurgitant orifice area (EROA; $\mathrm{r}=0.45, \mathrm{p}<0.0001$ ), the RVFS $(\mathrm{r}=0.6, \mathrm{p}<0.001)$, and the TAPSE $(\mathrm{r}=0.46, \mathrm{p}<0.001)$. Independent predictors of events were the EROA $(\mathrm{P}=0.006)$ and RV strain ( $\mathrm{p}=0.05)$. Patients with RVLS $<20 \%$ had a $1.42(95 \%$ confidence interval: 1.08-1.95) fold increase in cardiac events after adjustment for other risk factors. Conclusions: The results of this study suggest that the assessment of RV function with the use of RV free wall longitudinal strain provides incremental prognostic value beyond the other echocardiographic parameters of MR severity and LV function. This information could be helpful to identify the patients with asymptomatic severe MR who may benefit from early surgery.

\section{Functional Mitral Regurgitation as a Determinant of Symptomatic Status in Patients with Aortic Valve Stenosis}

\author{
Giovanni Benfari ${ }^{1}$, Andrea Rossi ${ }^{2}$, Caterina Maffeis ${ }^{2}$, \\ Giulia Geremia ${ }^{2}$, Stefano Nistri ${ }^{3}$, Corrado Vassanelli', \\ Maurice E. Sarano ${ }^{1}$
}

${ }^{1}$ Department of Cardiovascular Diseases, Mayo Clinic, Rochester, MN, USA; ${ }^{2}$ Section of Cardiology, Department of Medicine, University of Verona, Verona, ${ }^{3}$ CMSR-Veneto Medica, Vicenza, ${ }^{4}$ Department of medicine, Section of Cardiology, University of Verona, Verona, Italy

Background: Symptom onset in the presence of severe aortic valve stenosis (AS) has long been considered the main indication for aortic valve replacement. However symptoms can be challenging to detect or unassociated with the AS severity; further understanding of predictors and pathophysiological mechanism leading to symptoms is needed. Aims of the present study were: (i) to identify the determinants of heart failure symptoms in patients with AS and (ii) to analyse whether mitral regurgitation (MR) has an independent role in defining symptoms severity. Methods: Consecutive patients with AS, defined as a thickened aortic valve with a transaortic flow velocity $>2.5 \mathrm{~m} / \mathrm{s}$, were prospectively enrolled between October 2008 and February 2014. End-diastolic and endsystolic left ventricular volumes and left atrial volume were measured. Longitudinal shortening velocity (S-DTI), early and late lengthening velocities were assessed. The maximal transaortic velocity was evaluated from any window and the highest velocity identified; the aortic valve area (AVA) was calculated by the continuity equation. The effective regurgitant orifice area (ERO) and regurgitant volume (RV) were obtained with proximal isovelocity surface area method PISA in the presence of MR. Results: 218 patients (mean age $78 \pm 9$ years; $53 \%$ male) formed the study population. They were subdivided in 2 groups: 84 clearly symptomatic patients (NYHA III and IV) and 134 pauci-symptomatic patients (NYHA I e II). Differences in systolic and diastolic function were: EF $49 \pm 16$ vs. $61 \pm 12 \%$, p < 0.0001; stroke volume $62 \pm 17$ vs. $81 \pm$ $20 \mathrm{~mL}, \mathrm{p}<0.0001$; S-DTI $0.05 \pm 0.01$ vs. $0.07 \pm 0.01 \mathrm{~m} / \mathrm{s}, \mathrm{p}<0.0001$; E/e' $15 \pm 7$ vs. $11 \pm 5 \mathrm{p}<0.0001$; mean AVA resulted smaller $0.8 \pm$ 0.3 vs. $\left.1.07 \pm 0.3 \mathrm{~cm}^{2}, \mathrm{p}<0.0001\right)$. What is more, symptomatic pa- tients showed higher prevalence of MR, larger ERO $(0.10 \pm 0.07$ vs. $\left.0.05 \pm 0.06 \mathrm{~mm}^{2} \mathrm{p}<0.0001\right)$ and $\mathrm{RV}(19 \pm 13 \mathrm{vs} .12 \pm 13 \mathrm{~mL}, \mathrm{p}=$ $0.001)$ on average. ERO resulted a predictor of the symptomatic status $(\mathrm{OR}=1.08$ [1.003-1.15], $\mathrm{p}=0.04)$ in a multivariate model including EF, stroke volume, S-DTI, E/e' and AVA. In addition ERO remained the only independent predictor of NYHA class when the subgroup of patients with preserved $\mathrm{EF}(>50 \%)$ was analyzed. Conclusion: MR can independently affect heart failure symptoms, even when its degree is mild, independently from common systolic and diastolic function parameters.

\section{Cardiac and Respiratory-Gated Aortic Valve Ultrasound Imaging in Mice}

\section{Arvin H. Soepriatna, Pavlos P. Vlachos, Craig J. Goergen}

Purdue University, West Lafayette, IN, USA

Objective: A small animal cardiac imaging is challenging due to their small size and fast heart rates. As such, echocardiographic assessment of cardiac valves in mice relies on one-dimensional Mmode measurements and simple flow quantification from Doppler imaging. The aim of this study is to use a gated imaging technique capable of capturing aortic valve leaflet motion with high spatiotemporal resolution. Methods: We used a high-frequency small animal ultrasound system (Vevo2100, VisualSonics) and a $50 \mathrm{MHz}$ transducer (MS700) to image the aortic orifice of adult C57BL/6 mice ( $\mathrm{n}=4$; male $38 \pm 14$ weeks old). The animals were anesthetized with $2-3 \%$ isoflurane, positioned supine on a heated stage, and monitored for both cardiac and respiratory rates. We placed the transducer on the left thorax to acquire short-axis views of the heart and aorta. ECG-gated kilohertz visualization (EKV) imaging mode was used with respiratory gating to visualize the motion of aortic valve leaflets during the cardiac cycle with a sampling rate of 1000 frames-per-second. Color Doppler images were then acquired to capture the directionality of blood flow through the aortic valve. Results: The EKV high-resolution cine loops provided both structural and dynamic information of the aortic valve leaflets and annulus throughout the cardiac cycle. Using manual segmentation, cross-sectional areas of aortic valve annuli significantly increased from $2.20 \pm 0.16 \mathrm{~mm}^{2}$ during diastole to $2.64 \pm 0.16 \mathrm{~mm}^{2}$ during systole $(\mathrm{p}<0.05)$. Diastolic area measurements of the three valve leaflets - the right coronary cusp (RCC), the left coronary cusp (LCC), and the non-coronary cusp (NCC) - showed that NCC is significantly larger than RCC $(\mathrm{p}<0.05)(\mathrm{RCC}=0.59 \pm$ $\left.0.09 \mathrm{~mm}^{2}, \mathrm{LCC}=0.75 \pm 0.08 \mathrm{~mm}^{2}, \mathrm{NCC}=0.85 \pm 0.11 \mathrm{~mm}^{2}\right)$. This is consistent with previous work that shows the inter-commissural distance and leaflet height are largest in the NCC. Color Doppler cine loops confirmed the presence of blood flow only during systole, when the aortic valve leaflets are opened. Conclusions: The proposed cardiac and respiratory-gated technique allows for both structural and dynamic characterization of aortic valve leaflets and annulus, a potentially powerful tool in the study of aortic valve disease. 


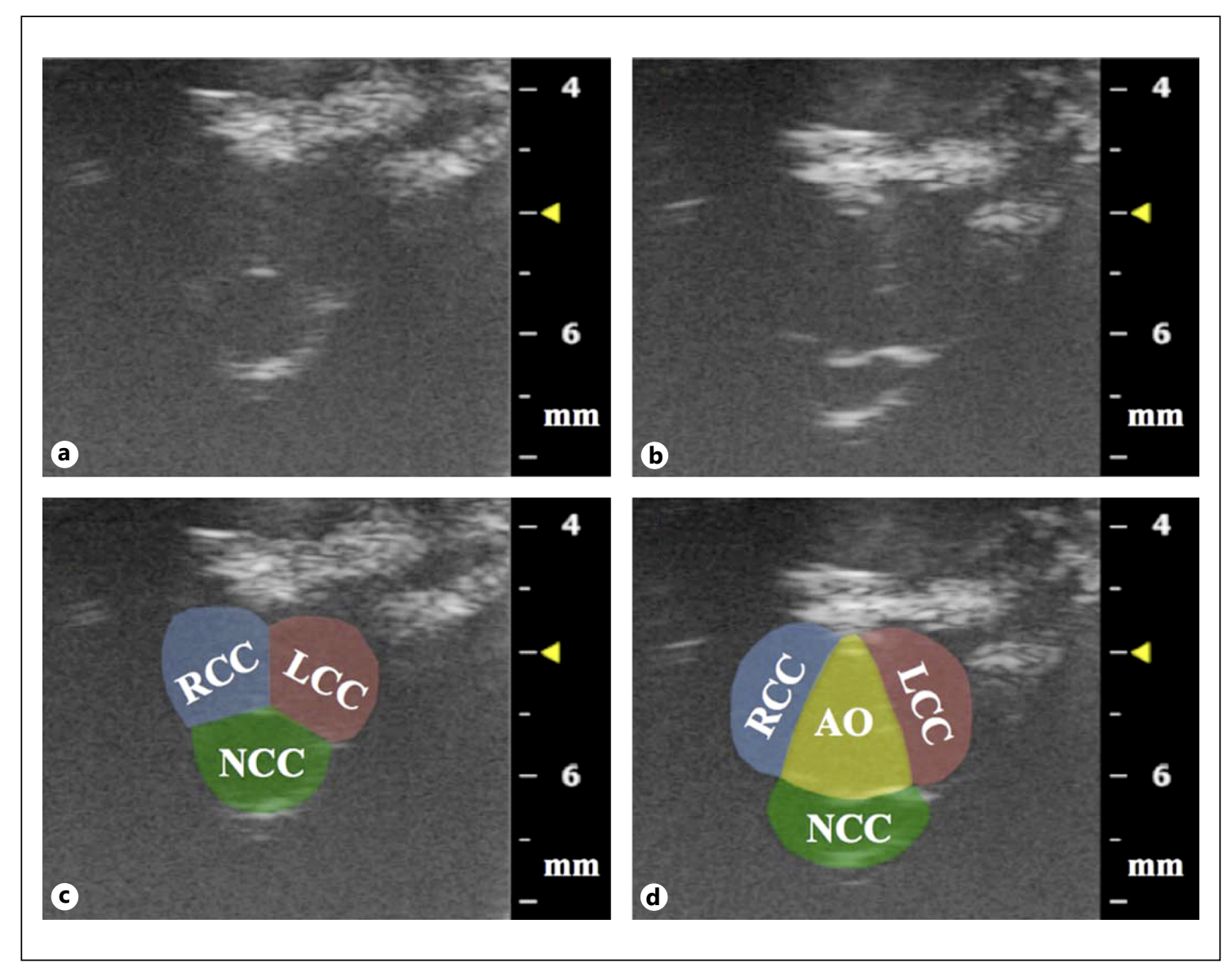

Fig. 1. Representative ultrasound images of a mouse aortic valve during (a) diastole and (b) systole with the three aortic leaflets and the aortic orifice highlighted $(\mathbf{c}, \mathbf{d})$. RCC = Right coronary cusp; LCC = left coronary cusp; $\mathrm{NCC}=$ non-coronary cusp; $\mathrm{AO}=$ aortic orifice (for Abstract of Craig J. Goergen).

\section{The Reproducibility and Optimisation of 18F-Fluoride Positron Emission Tomography and Computed Tomography in Patients with Aortic Stenosis}

Tania A. Pawade, Timothy R. Cartlidge, David E. Newby, Marc R. Dweck

University of Edinburgh, Edinburgh, United Kingdom

Background: 18F-Fluoride positron emission tomography (PET) and computed tomography (CT) can measure disease activity and progression in aortic stenosis. We aimed to optimize imaging methodology, analysis and scan-rescan reproducibility. Methods: Fifteen patients with aortic stenosis underwent $18 \mathrm{~F}$-fluoride PET-CT twice within 1 month. We compared non-gated PET and non-contrast CT, with a modified approach that incorporated contrast CT and ECG-gated PET. We explored a range of image analysis techniques including estimation of blood pool activity at differing vascular sites and a most-diseased segment (MDS) ap- proach. Results: Contrast-enhanced ECG-gated PET-CT provided superior spatial localization of $18 \mathrm{~F}$-fluoride uptake that permitted localization to individual valve leaflets (figure 1). Scan-rescan reproducibility was markedly improved using enhanced analysis techniques leading to a reduction in variability from $25 \%$ to $<10 \%$ (tissue-to-background MDS: mean value 1.55, difference 0.05, limits of agreement $-0 \cdot 10$ to $0 \cdot 20$ ). Conclusion: Optimized 18F-fluoride $\mathrm{PET}$-CT provides excellent spatial resolution and scan-rescan reproducibility. It holds major promise as a marker of disease activity in aortic stenosis and has major potential as a biomarker endpoint in trials of novel therapies in aortic stenosis. 

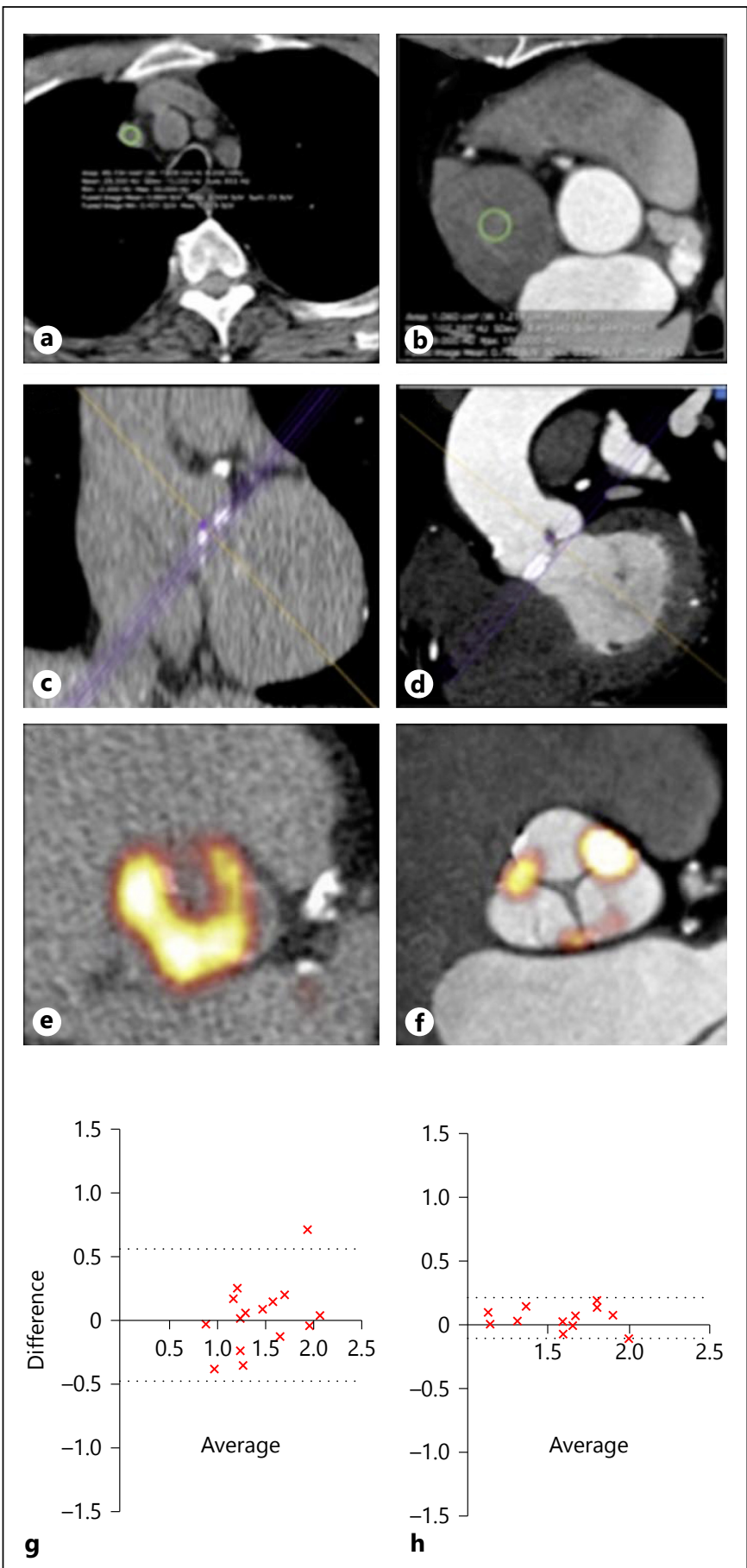

Fig. 1. a, b Blood pool correction was performed by sampling from the right atrium instead of the brachio-cephalic vein. $\mathbf{c}, \mathbf{d}$ The most diseased segment method was used to quantify the PET signal within 2 hottest slices rather than the whole valve. e, $\mathbf{f}$ Contrast CT fused with ECG-gated PET facilitated better localization to leaflets compared to the original approach. $\mathbf{g}$, $\mathbf{h}$ Bland-Altman plots demonstrated superior scan-rescan reproducibility for the modified technique (d) compared to the previous approach (c) (for Abstract of Tania A. Pawade).

\section{Pre-Procedural Imaging to Determine Sizing for Transcatheter Mitral Valve Intervention with a Tendyne Device}

\author{
Alison Duncan ${ }^{1}$, Cesare Quarto', Simon Davies', \\ Michael Rubens', Philipp Blanke', Jonathan Leipsic ${ }^{2}$, Neil Moat ${ }^{1}$ \\ ${ }^{1}$ The Royal Brompton Hospital, London, United Kingdom; \\ ${ }^{2}$ Center for Heart Valve Innovations, Vancouver, BC, Canada
}

Background: Transcatheter mitral valve implantation (TMVI) is an alternative to conventional surgery for high risk patients with severe native mitral regurgitation (MR). The Tendyne TMVI system consists of a porcine pericardial valve in a nitinol frame tethered to the left ventricular (LV) apex using an epicardial pad. A variety of device sizes are available depending on mitral annular size. Complications of inaccurate sizing include device migration, paravalvular leak, and left ventricular outflow tract obstruction (LVOTO). Patients with a narrow aorto-mitral angle, small LVOT, and long anterior mitral valve leaflet (AMVL) may develop LVOTO after TMVI. Mitral annulus anatomy is complex (asymmetrical, non-tubular, and changes shape during the cardiac cycle). Thus, multi-modality imaging is imperative for pre-procedural device sizing. Methods: All patients being considered for TMVI in our institution underwent pre-procedural cardiac multi-sliced computerized tomography (MSCT) scan and three-dimensional transoesophageal echocardiography (3DTOE). MSCT were performed on a Siemens (Surrey, United Kingdom) Flash scanner, with images analyzed retrospectively at 35\% (systole) and 75\% (diastole) of the cardiac cycle. Real-time 3DTOE was performed with a Philips ie33 echocardiogram (Andover, Massachusetts) with built-in Mitral Valve Quantification (MVQ) using QLab 9. Results: End-systolic MSCT frames were used to measure mitral annular dimensions in intra-commissural (fig. 1a) and anterio-posterior (fig. 1b) planes, and the aorto-mitral angle (fig. 1c). As TMVI devices are large and may sit below the mitral annulus, an estimation of potential sub-mitral annular dimension was simulated at approximate LVOT depth (fig. 1d). On 3DTOE imaging, LV length (apical epicardium to the mitral annulus) was measured in 4 conventional LV mid-oesophageal views (fig. 1e, f). After confirming MR aetiology (fig. 1i), LVOT dimensions in 2D and 3D (fig. $1 \mathrm{j}, \mathrm{k}$ ) and the length of the anterior mitral valve leaflet (AMVL) were measured (fig. 1l). Intra-commissural and anterio-posterior annular measurements were confirmed with X-plane (fig. $1 \mathrm{~m})$ and 3DTOE (fig. $1 \mathrm{n}-\mathrm{p}$ ) imaging. Conclusion: To facilitate TMVI and reduce potential complications (device migration, paravalvular leak, LVOTO), pre-procedural diagnostic MSCT and 3DTOE are mandatory to provide detailed evaluation of mitral annular, mitral leaflet, and LVOT anatomy. 


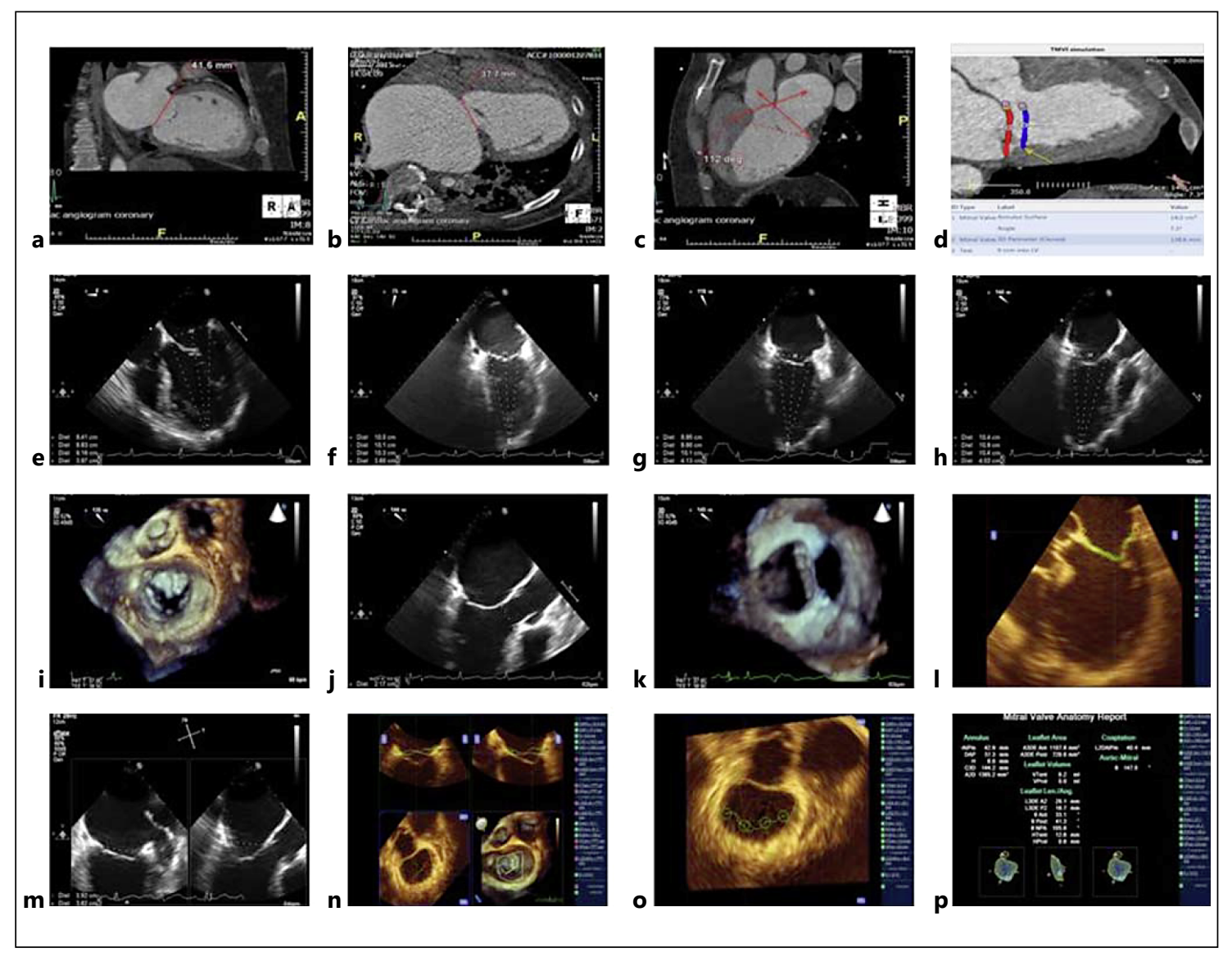

Fig. 1. (for Abstract of Alison Duncan).

\section{Hemodynamic Behavior of Two 4th Generation Aortic Valve Bioprosthesis during Exercise}

Christian Auer, Michael Scharfschwerdt, Junfeng Yan, Hans-Hinrich Sievers, Thorsten Hanke

University Medical Center Schleswig-Holstein, Luebeck, Germany

Objective: Hemodynamic behavior of biological aortic valve prosthesis during exercise is essential for final valve judgement but is seldomly performed. Opening characteristics of the prosthetic valve leaflets as well as the valve stent design itself contribute to maintain optimal pressure gradients during exercise, resulting in better hemodynamics. It was the aim of the present study to examine the in vitro opening behavior of two different modern 4th generation aortic valve bioprosthesis (SJM Trifecta, Edwards Magna Ease) in a mock circulation at different flow levels. Methods: In order to evaluate the different performances of the SJM Trifecta size $23 \mathrm{~mm}(\mathrm{n}=4)$ and the Edwards MagnaEase size $23 \mathrm{~mm}(\mathrm{n}=$ 4) we used an aortic model in which all valves have been embedded for measurement. In the mock circulation the hemodynamic parameters (dp mean $[\mathrm{mm} \mathrm{Hg}], \mathrm{EOA}\left[\mathrm{cm}^{2}\right]$, GOA $\left.\left[\mathrm{cm}^{2}\right]\right)$ were assessed stepwise at different flow levels (from $3 \mathrm{l} / \mathrm{min}$ up to $8 \mathrm{l} / \mathrm{min}$ ).

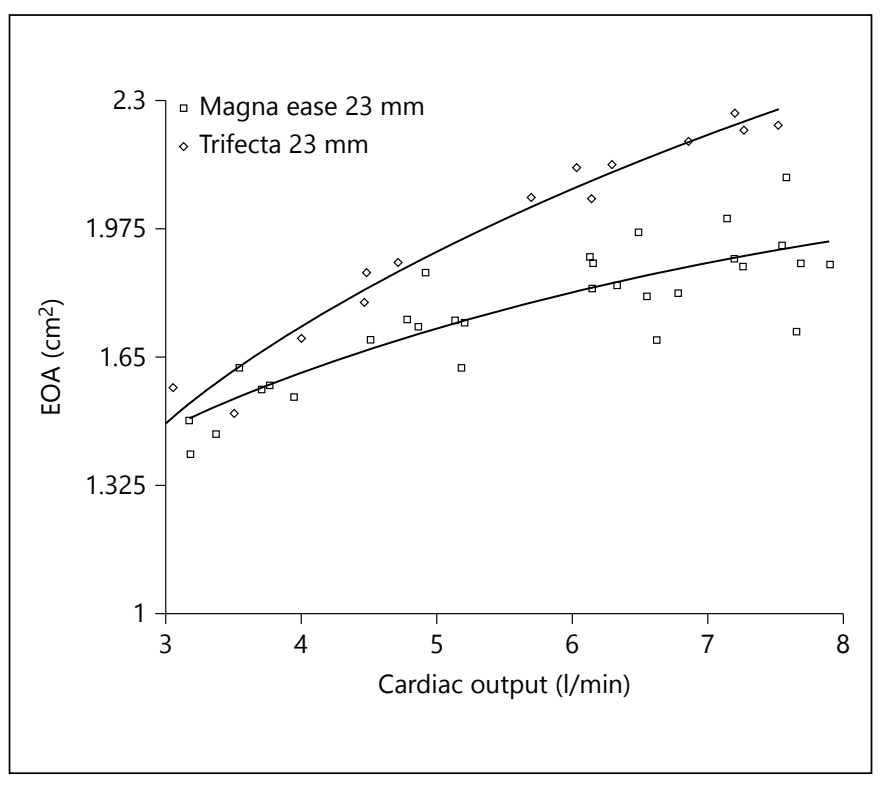

Fig. 1. (for Abstract of Christian Auer). 
Results: Hemodynamic behavior of the Edwards MagnaEase was significantly inferior to the SJM Trifecta at higher flow levels $(>4.5$ $1 / \mathrm{min})$ with respect to all parameters being measured $(\mathrm{p}<0.05)$ Conclusions: In our in vitro exercise model, the SJM Trifecta aortic valve bioprosthesis revealed a lower pressure gradient when compared to another 4th generation aortic valve bioprosthesis (Edwards MagnaEase). This is presumably caused by the capability of an additional dynamic stent opening at high exercise levels.

\section{New Insights into Unicuspid Aortic Valve Disease in Adults: Not Just a Subtype of Bicuspid Aortic Valves}

Pierre-Emmanuel Noly, Lauren Basmadjian, Ismail Bouhout, Viet Le, Nancy Poirier, Ismail El-Hamamsy

Montreal Heart Institute, Montreal, QC, Canada

Objective: The clinical presentation, echocardiographic features and patterns of aortic dilatation in unicuspid aortic valves (UAV) are not well defined. Methods: From 2011 to 2015, all data from adult patients with confirmed UAV $(\mathrm{n}=42)$ undergoing aortic valve surgery were reviewed. Results: Mean age was 33.9 \pm 1.7 years, 33 were male (78\%). Mixed aortic valve disease (stenosis + regurgitation) was the most common mode of presentation $(n=25 ; 59 \%)$. Preoperative diagnosis of UAV was made in only 6 patients $(14 \%)$. Preoperative TEE in the operating room increased the diagnosis to $69 \%$ of cases. The peak and mean aortic gradients were $80 \pm 29 \mathrm{~mm}$ $\mathrm{Hg}$ and $50 \pm 20 \mathrm{~mm} \mathrm{Hg}$, respectively. Aortic valve area was $1.1 \pm 0.4$ $\mathrm{cm}^{2}$. Overall, $21 \%$ of patients $(\mathrm{n}=9)$ had aortic dilatation $(>45 \mathrm{~mm})$ : 6 patients (14\%) had isolated ascending aortic dilatation, 1 patient
(2\%) had isolated aortic root dilatation, and 2 patients (5\%) had combined aortic root and ascending aortic dilatation. In contrast, $71 \%$ of patients $(\mathrm{n}=30)$ had a dilated aortic annulus $(>25 \mathrm{~mm})$. Mean aortic diameters measured on TEE were: aortic annulus: $25.8 \pm 3.4 \mathrm{~mm}$; sinus of Valsalva: $32.1 \pm 5 \mathrm{~mm}$, sino-tubular junction: $28.1 \pm 5 \mathrm{~mm}$ and ascending aorta: $36.2 \pm 5 \mathrm{~mm}$. The valve was repaired using a bicuspidization technique in 8 patients (19\%) and replaced using a Ross procedure in 34 patients (81\%). Conclusions: UAV remains significantly underdiagnosed in young adults with aortic valve disease. UAVs are associated with a distinct pattern of aortic dilatation, which is mainly present at the level of the aortic annulus. In a small proportion of patients, the valve can be surgically repaired.

\section{Association between Left Ventricular Ejection Fraction, Ventricle Structural Changes and Clinical Status in Patients with Severe Aortic Stenosis Referred for Surgery}

Ricardo A. Spampinato ${ }^{1}$, María Agustina Spampinato ${ }^{2}$, Jaqueline G. da Rocha e Silva', Valerie Schloma', Yaroslava Dmitrieva ${ }^{1}$, Elfriede Strotdrees ${ }^{1}$, Friedrich-Wilhelm Mohr ${ }^{1}$

${ }^{1}$ Heart center Leipzig, Leipzig, Germany; ${ }^{2}$ National University of Cuyo Faculty of Medicine, Mendoza, Argentina

Background: Guidelines describe a left ventricular ejection fraction (LVEF) $<50 \%$ as a marker of LV decompensation, being a threshold for referral to aortic valve replacement (AVR) in severe

Table 1. Clinical, echocardiographic, and laboratory indices (for Abstract of Ricardo A. Spampinato)

\begin{tabular}{|c|c|c|c|c|c|}
\hline & LVEF <50\% $(\mathrm{n}=20)$ & LVEF $51-59 \%(n=42)$ & LVEF $60-65 \%(n=41)$ & LVEF > 66\% $(n=34)$ & $\mathrm{p}$ value \\
\hline Age & $73 \pm 9$ & $68 \pm 11$ & $71 \pm 8$ & $69 \pm 10$ & 0.282 \\
\hline Male & $16(80)$ & $28(67)$ & $22(54)$ & $13(38)$ & 0.012 \\
\hline Renal Injury & $8(40)$ & $5(12)$ & $6(15)$ & $3(9)$ & 0.029 \\
\hline COPD & $5(25)$ & $3(7)$ & $6(15)$ & 0 & 0.013 \\
\hline CAD & $9(45)$ & $15(36)$ & $6(15)$ & $11(32)$ & 0.048 \\
\hline A Fib & $5(25)$ & $4(9.5)$ & 0 & $3(9)$ & 0.008 \\
\hline NYHA III-IV & $13(65)$ & $12(29)$ & $8(19.5)$ & $6(18)$ & 0.002 \\
\hline AVAi, $\mathrm{cm}^{2} / \mathrm{m}^{2}$ & $0.31 \pm 0.08$ & $0.35 \pm 0.08$ & $0.37 \pm 0.09$ & $0.40 \pm 0.09$ & 0.005 \\
\hline Mean gradient, $\mathrm{mm} \mathrm{Hg}$ & $42 \pm 15$ & $51 \pm 16$ & $56 \pm 16$ & $51 \pm 14$ & 0.013 \\
\hline $\mathrm{SVi}, \mathrm{ml} / \mathrm{m}^{2}$ & $28 \pm 8$ & $35 \pm 7^{*}$ & $40 \pm 11^{*}$ & $41 \pm 9 *, \dagger$ & $<0.0001$ \\
\hline Mass- $\mathrm{i}, \mathrm{gr} / \mathrm{m}^{2}$ & $154 \pm 43$ & $147 \pm 44$ & $145 \pm 45$ & $139 \pm 38$ & 0.621 \\
\hline Mass/EDV & $2.8 \pm 1.2$ & $3.6 \pm 1.2$ & $4.1 \pm 1.5^{*}$ & $4.0 \pm 1.6^{*}$ & 0.005 \\
\hline RWT & $0.43 \pm 0.1$ & $0.52 \pm 0.1$ & $0.57 \pm 0.13^{*}$ & $0.56 \pm 0.14^{*}$ & 0.001 \\
\hline $\mathrm{EDVi}, \mathrm{ml} / \mathrm{m}^{2}$ & $61.5 \pm 22$ & $43.4 \pm 14^{*}$ & $38 \pm 13^{*}$ & $38 \pm 14.5^{*}$ & $<0.001$ \\
\hline $\mathrm{ESVi}, \mathrm{ml} / \mathrm{m}^{2}$ & $38 \pm 15.5$ & $19 \pm 6^{*}$ & $14 \pm 5^{*, \dagger}$ & $12 \pm 5^{*, \dagger}$ & $<0.001$ \\
\hline $\mathrm{EF}, \%$ & $39 \pm 7$ & $55 \pm 3$ & $62 \pm 2$ & $68 \pm 2$ & - \\
\hline GLPS, \% & $-10.3 \pm 3.5$ & $-14.6 \pm 2.4^{*}$ & $-17.3 \pm 2.7^{*, \dagger}$ & $-17.2 \pm 3.0^{*, \dagger}$ & $<0.001$ \\
\hline BNP, ng/l, median (IQR) & $5,535(1,544-8,613)$ & $698(285-1,696)^{*}$ & $506(274-967)^{*}$ & $368(217-1,020)^{*}$ & $<0.001$ \\
\hline Activated BNP, n (\%) & $19(95)$ & $31(74)$ & $25(61)$ & $22(65)$ & 0.016 \\
\hline hs-TNT, ng/l, median (IQR) & $20(13-57)$ & $13(9.5-16)$ & $13.5(10-21)$ & $9(6-13)^{*}$ & $<0.001$ \\
\hline
\end{tabular}

Values expressed as mean \pm SD $\mathrm{n}(\%)$, or median (IQR).

* $\mathrm{p}<0.05$ vs. LVEF $<50 \% ;{ }^{\dagger} \mathrm{p}<0.05$ vs. LVEF $51-59 \%$. 
asymptomatic aortic stenosis (AS). Actual evidence suggests that this threshold does not detect early LV impairment. We investigated the association of different LVEF strata with clinical and echocardiographic parameters in patients with severe AS referred to AVR. Method: Echocardiograms were performed on 137 patients with severe AS (valve area index [AVAi] $0.36 \pm 0.09 \mathrm{~cm}^{2} / \mathrm{m}^{2}$ ) referred for AVR. Clinical characteristics, LVEF (biplane method), global longitudinal peak systolic strain (GLPS), high sensitive troponin T (hs-TNT), and pro-B-type natriuretic peptide (BNP) were all determined at the time of admission. Patients were classified according to LVEF in 4 groups: $\leq 50 \%$ in 20 (15\%) patients, $51 \%-$ $59 \%$ in $42(31 \%), 60 \%-65 \%$ in $41(30 \%)$, and $\geq 66 \%$ in $34(25 \%)$. Results: Patients with lower LVEF, particularly those with LVEF $<50 \%$, were more likely to be men, to have renal injury, coronary artery disease, and atrial fibrillation; to be treated with aspirin and diuretics, and to have a NYHA class III-IV. BNP values were higher with LVEF $<50 \%$. There was a slightly different in AS severity, with lower stroke volume index across the 4 groups, and a higher rate of 'low-flow' state in groups LVEF <50\% and 51-59\% (18 [90\%] vs. $21[50 \%]$ vs. $12[29 \%]$ vs. $10[29 \%] ; \mathrm{p}<0.0001)$. The relative wall thickness (RWT) was lower in groups with LVEF $<59 \%$, with an increase in LV end-diastolic volume index (EDVi). The reduction in SVi despite increased EDVi was accompanied with an increase in end-systolic volumes, even with LVEF 51-59\%. Finally, although group LVEF $51-59 \%$ had better GLPS values compared with LVEF $<50 \%$, they were worse than in LVEF $\geq 60 \%$, with higher hs-TnT values. Conclusions: In a cohort of patients with severe AS undergoing AVR, those with LVEF $\leq 59 \%$ have a tendency to eccentric LV remodeling and higher ESVi, with worse GLPS and higher rate of 'low flow' state, associated with advanced symptoms and higher values of BNP and hs-TnT.

\section{Tricuspid Regurgitation as a Result of Chronic Atrial Fibrillation; An Under Appreciated Clinical Syndrome}

\section{Geraldine Ong, Kim Connelly, Robert Chisholm}

\section{St. Michael's Hospital, Toronto, ON, Canada}

Objective: Tricuspid annular dilatation is a well-recognised sequelae following right ventricular enlargement, most frequently occurring as a result of pulmonary hypertension. It is less well recognized that dilatation of the right atrium may also lead to tricuspid annular dilatation and secondary functional tricuspid regurgitation (TR). We therefore hypothesized that different disease processes would manifest with different disease remodelling patterns, as assessed by the ratio of RA area to RV area. Method: We retrospectively analyzed right atrial (RA) and right ventricle (RV) areas in 3 separate groups (patients with longstanding atrial fibrillation (AF), patients with $\mathrm{PH}$ and in healthy controls). Thirteen patients with severe TR and longstanding AF were identified (group 1) and compared to 10 patients with severe TR and severe $\mathrm{PH}$ (group 2). Twenty six young healthy adults were used as controls (group 3). Only patients with functional TR were included. All patients had undergone a complete transthoracic echocardiogram as per American Society of Echocardiography recommendations. Clinical and echocardiographic findings were recorded. RA areas, RV
Table 1. Echocardiographic data (for Abstract of Geraldine Ong)

\begin{tabular}{lcccc}
\hline & $\begin{array}{l}\text { Group 1: } \\
\text { AF } \\
(\mathrm{n}=9)\end{array}$ & $\begin{array}{l}\text { Group 2: } \\
\text { PHT } \\
(\mathrm{n}=11)\end{array}$ & $\begin{array}{l}\text { Group 3: } \\
\text { controls } \\
(\mathrm{n}=26)\end{array}$ & $\begin{array}{l}\text { p value } \\
\text { group 1 c/w } \\
\text { group 2) }\end{array}$ \\
\hline Age & $79.7 \pm 4.6$ & $78.3 \pm 10.9$ & $19 \pm 0$ & n.s. \\
BSA & $1.6 \pm 0.1$ & $1.8 \pm 0.3$ & $1.9 \pm 0.2$ & n.s. \\
RA area, cm ${ }^{2}$ & $39.3 \pm 13.7$ & $29.1 \pm 9.8$ & $14.5 \pm 2.3$ & $<0.0001$ \\
RV area, cm & $26.4 \pm 8.0$ & $27.8 \pm 6.5$ & $21.9 \pm 3.1$ & n.s. \\
RA:RV ratio & $1.5 \pm 0.4$ & $1.1 \pm 0.3$ & $0.7 \pm 0.1$ & $<0.0001$ \\
RA annulus, cm & $4.8 \pm 0.6$ & $4.6 \pm 0.5$ & $3.8 \pm 0.4$ & n.s. \\
RVSP, mm Hg & $45.1 \pm 13.4$ & $83.5 \pm 7.7$ & $16.5 \pm 4.9$ & $<0.0001$ \\
RAP, mm Hg & $12.7 \pm 3.5$ & $15 \pm 0$ & $3 \pm 0$ & $<0.0001$ \\
Degree TR & 4 & 4 & 1 & n.s. \\
\hline
\end{tabular}

areas and RA:RV ratio were obtained and compared between the groups. Results: The mean age, BSA and degree of TR are presented in table 1 . Both group 1 and group 2 demonstrated higher $\mathrm{RV}$ systolic pressure, RA pressure, and RA/RV areas as compared to controls $(\mathrm{p}<0.01)$. Patients in group 2 had significantly higher RVSP as compared to group 1 patients. RV areas were similar across groups 1 and 2 . Patients in group 1 demonstrated a larger RA area as compared to the patients in group 2, along with a higher RA:RV ratio $(\mathrm{p}<0.01)$. Conclusion: Different aetiologies predispose to different patterns of atrial remodelling. Massive atrial dilatation and severe function TR secondary to long-standing AF may represent a distinct syndrome. Further research with this group of patients and mechanisms underlying atrial remodelling should be undertaken.

\section{Impact of the Ross Procedure on Left Ventricular Mechanics by Speckle-Tracking Echocardiography}

\author{
Stéphanie Deneault-Bissonnette ${ }^{1}$, Donato Barile ${ }^{2}$, \\ François-Pierre Mongeon ${ }^{2}$, Patrick Garceau ${ }^{2}$, \\ Ismail El-Hamamsy ${ }^{2}$, Frédéric Poulin ${ }^{1}$
}

${ }^{1}$ Hôpital du Sacré-Coeur de Montréal, Montreal, QC, ${ }^{2}$ Institut de Cardiologie de Montréal, Montreal, QC, Canada

Background: The Ross procedure (pulmonary autograft) results in excellent valve hemodynamics. However, prolonged ischemic time, coronary reimplantation, and interventricular septal scarring can result in subclinical LV dysfunction, especially in patient with aortic stenosis (AS) and LV hypertrophy. We aimed to assess the impact of the Ross procedure on LV myocardial mechanics in patients with AS and to determine predictors of relative improvement in global longitudinal strain (GLS). Methods: From 2011 to 2014,32 patients ( $45 \pm 11$ yrs; $75 \%$ male) with severe AS (aortic valve area (AVA) $0.5 \pm 0.1 \mathrm{~cm}^{2} / \mathrm{m}^{2}$, mean gradient $64 \pm$ $18 \mathrm{~mm} \mathrm{Hg}$ ) underwent a Ross procedure. Speckle-tracking echocardiography was used to assess longitudinal myocardial deformation prior to and at midterm follow-up ( $9 \pm 3$ months) after the procedure. Results: Despite overall preserved LVEF (63 $\pm 7 \%)$, LV 
longitudinal deformation was impaired at baseline. At $9 \pm 3$ months after surgery, there was a significant improvement in systolic and diastolic longitudinal deformation: GLS from $-17.3 \pm 2.9$ to $-18.8 \pm 2.0 \%(\mathrm{p}=0.002)$; and early diastolic strain rate from $0.82 \pm$ 0.26 to $0.97 \pm 0.21 \mathrm{sec}^{-1}(\mathrm{p}=0.002)$. Additionally, there was a significant decrease in LV diastolic volume (from $127 \pm 45$ to $102 \pm$ $27 \mathrm{ml} / \mathrm{m}^{2} ; \mathrm{p}<0.001$ ), systolic volume (from $47 \pm 22$ to $38 \pm 10 \mathrm{ml} /$ $\mathrm{m}^{2} ; \mathrm{p}=0.004$ ) and LV mass (from $123 \pm 42$ to $86 \pm 23 \mathrm{~g} / \mathrm{m}^{2} ; \mathrm{p}<$ $0.001)$, with no change in LVEF (64 \pm 7 versus $63 \pm 4 \% ; \mathrm{p}=0.50)$. By univariate analysis, determinants of relative improvement in GLS were: baseline AVA $\left(\beta_{1}\right.$ coefficient $=-8.8$ per $0.1 \mathrm{~cm}^{2} / \mathrm{m}^{2} ; \mathrm{p}=$ $0.001)$, LV mass $\left(\beta_{1}\right.$ coefficient $=2.3$ per $\left.10 \mathrm{~g} / \mathrm{m}^{2} ; \mathrm{p}=0.004\right)$, LVEF $\left(\beta_{1}\right.$ coefficient $\left.=-1.3 ; \mathrm{p}=0.003\right)$, absolute GLS $\left(\beta_{1}\right.$ coefficient $=$ $-5.4 ; \mathrm{p}<0.001)$ and post-operative change in indexed AVA $\left(\beta_{1}\right.$ coefficient $=0.8$ per $10 \%$ increase; $p=0.02$ ). Neither pre- or postoperative aortic regurgitation nor cross-clamp time, were associated with LV functional deterioration. Conclusions: The Ross procedure in AS restores LV structure and function towards physiological myocardial mechanics. Patients with lower preoperative systolic function, more severe AS and LV hypertrophy derive the most benefit in terms of longitudinal reverse remodeling.

\section{Dynamic Assessment of Mitral Apparatus in Acute Myocardial Infarction by 3D Quantitative Echocardiography}

\author{
Toshiyuki Kimura ${ }^{1}$, Nozomi Watanabe ${ }^{2}$, William Jenkins ${ }^{1}$, \\ Veronique Roger ${ }^{1}$, Maurice Sarano ${ }^{1}$ \\ ${ }^{1}$ Mayo Clinic, Rochester, MN, USA; ${ }^{2}$ Miyazaki Medical \\ Association Hospital, Miyazaki, Japan
}

Background: The mitral apparatus combines the left ventricle (LV), mitral leaflet, annulus and papillary muscles and changes dynamically throughout the cardiac cycle. It is unknown how myocardial infarction (MI) impact on 3-dimensional (3D) dynamics of this mitral apparatus. The aim of our study is to determine the relationship between LV remodeling and mitral apparatus remodeling using real-time 3D echocardiography in incident MI. Methods: We prospectively performed 3D echocardiography in patients with first anterior ST-elevated MI (STEMI) $(\mathrm{n}=10)$ and inferior STEMI at 3.1 \pm 2.4 days after incident of MI. LV remodeling was measured using $3 \mathrm{D} \mathrm{LV}$ volume quantification and ejection fraction (EF), and mitral apparatus remodeling was measured with 3D-echo dynamic imaging and quantitative analysis in diastole and early-, mid- and late-systole. Results: Both groups were similarly distributed by age and sex. Assessing LV remodeling, end systolic volume (ESV) was larger in anterior-STEMI compared with inferior-STEMI $(66.3 \pm 19.4$ vs. $48.0 \pm 17.4 \mathrm{~mL}, \mathrm{p}=$ $0.04)$. Similarly, end diastolic volume (EDV) tended to be larger and EF lower in anterior-STEMI (EDV; $128.6 \pm 18.6$ vs. $106.6 \pm$ $30.5 \mathrm{~mL}, \mathrm{p}=0.07, \mathrm{EF} ; 49.0 \pm 9.9$ vs. $55.6 \pm 5.2 \%, \mathrm{p}=0.08)$. Assessing mitral apparatus remodeling, annular height in early-systole was lower in anterior-STEMI $(3.9 \pm 1.8$ vs. $6.0 \pm 2.3 \mathrm{~mm}, \mathrm{p}=0.04)$ and visually, the saddle shape of mitral annulus was flattened throughout the cardiac cycle in anterior-STEMI. Although annular area was similar, there was a smaller change through systole in anterior-STEMI compared to persistent dynamic changes in infe-
rior-STEMI $\left(-0.7 \pm 10.9\right.$ vs. $\left.-10.1 \pm 6.8 \mathrm{~cm}^{2}, \mathrm{p}=0.03\right)$. Papillary muscle position in diastole varied between groups, with wider papillary muscle separation in anterior-STEMI $(22.5 \pm 5.3$ vs. $17.5 \pm 3.0 \mathrm{~mm}, \mathrm{p}=0.019)$ and a wider angle from fibrosa $(34.4 \pm$ 10.2 vs. $\left.26.3 \pm 5.7^{\circ}, \mathrm{p}=0.039\right)$. The combination of these dynamic alterations led to a similar tenting height and volume between groups throughout the cardiac cycle. Conclusions: Three-dimensional quantitative analysis of remodeling affecting LV and mitral valve demonstrates complex interactions following MI. In anterior-STEMI, greater LV remodeling results in a wider displacement of papillary muscle and less saddle shape accentuation of mitral annulus in anterior-MI. Through these different pathways however, mitral tenting is similarly affected in anterior- and inferiorSTEMI. Hence, 3D quantitative mitral and LV assessment provides new insights into cardiac remodeling post-MI.

\section{Is the Continuity Equation the Proper Way to Measure the Aortic Annulus?: The Surgeon Point of View}

\author{
Andrea Mangini ${ }^{1}$, Monica Contino ${ }^{1}$, Caludia Romagnoni ${ }^{1}$, \\ Rubina Rosa ${ }^{1}$, Guido Gelpi ${ }^{1}$, Carlo Antona ${ }^{2}$ \\ ${ }^{1}$ L. Sacco Hospital, Milano, ${ }^{2}$ Università di Milano, Milano, Italy
}

Objective: Being the aortic annulus elliptic, the continuity equation provides an underestimated value since it is obtained by the measurement of the distance between the nadir of the Non Coronary and the Right Coronary Valsalva sinuses (NC-RC) that usually corresponds to the ellipse short axis. Aim of the study was to validate a simple correction to the continuity equation in order to improve estimation accuracy. Methods: We considered 13 patients, 10 male and 3 female with a mean age of $59.73 \pm 12.18$ years. They have normal aortic root dimensions and a echocardiogram negative for valve diseases. All the patients underwent an ECG-triggered thorax CT-scan that were then analysed and measured with the software Osirix. Of all these patients we tabulated all the aortic measurements. In the CT-scan images we measured the distance between the nadir of the $\mathrm{NC}$ and the RC Valsalva sinuses that is the diameter considered in the echo formula. We considered the echo diameter as the side of an equilateral triangle and through the Heron's formula we calculated the area of the circumference circumscribed to this triangle. We compared the area obtained with the continuity equation and the one estimated with Heron's formula to the one directly measured at the CT-scan by paired t-test. Results: The aortic area measured at the CT-scan resulted $4.53 \pm 1.01$, the area estimated with the continuity equation was $3.43 \pm 0.85$ and the area calculated with our corrected equation was $4.57 \pm 1.13$. The difference between the continuity equation estimation of the aortic valve area and the measured one turned out to be statistically significant $(>0.01)$ while no statistically significant difference was found between the estimation with our corrected equation and the CT-scan measurements $(\mathrm{p}=0.93)$. Conclusions: The functional aortic area calculated with the continuity equation is underestimated by $33 \%$. Our correction is easy to apply and seems to perfectly fit the elliptical area of the LVOT leading to a more correct aortic valve stented prosthesis estimation. 
Fig. 1. (for Abstract of Andrea Mangini).

The distance between the nadir of the NC and RC Valsalva Sinuses measured at CT-Scan was $2,07 \pm 0,26 \mathrm{~cm}$

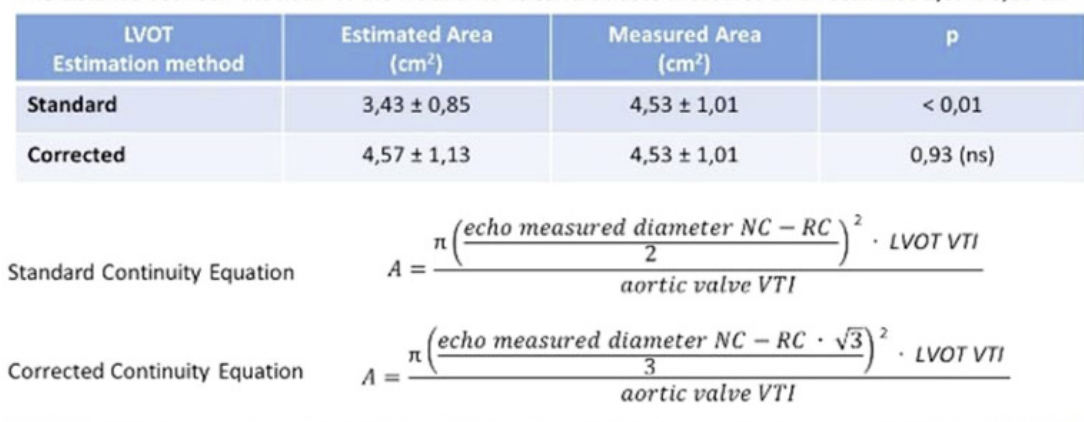

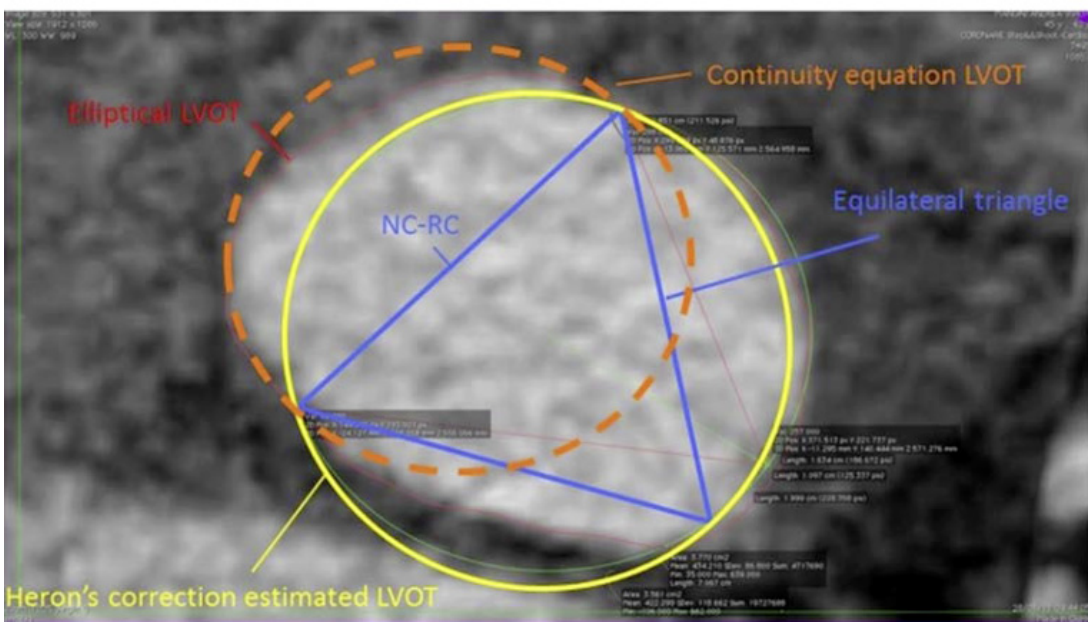

\section{Left Ventricular Remodelling after Mitral Valve Reconstruction Using cMRI: 1-Year Follow-Up}

\section{Andrea Mangini, Christian Frank, Falk-Udo Sack}

Department of Cardiac Surgery Academic City Hospital Ludwigshafen, Ludwigshafen, Germany

Objective: The aim of this prospective study is the evaluation of the postoperative remodelling of the left ventricle $(\mathrm{LV})$ after mitral valve reconstruction (MVR) in patients with isolated mitral valve disease using cardio magnetic resonance imaging (cMRI) within 12 months. Methods: Since 201173 patients were enrolled. In a prospective trial, LV geometry was assessed by cMRI pre-, before discharge and 12 months postoperatively. Of these, 57 (78\%) were men, the mean age was $59+14$ years. Minimal invasive operation technique (MIC) using of Goretex-Neo-Chordae to repair mitral valve regurgitation was done in more than $90 \%$ cases. The pre-surgical estimate of regurgitation was correlated with the postoperative decrease in LV volume parameters. Results: One patient died 4 months after MVR due to an aortic dissection Stanford A. The 1 year freedom from reoperation or severe recurrent insufficiency was $89 \%(n=65)$. The mean ischemia time observed $70+19$ min, the mean hospital duration was $10+3$ days. The posterior leaflet correction (PML) was done using $34+2 \mathrm{~mm}$ ring size (mean). The remodelling measurement of cMRI showed significant reduction of nearly all parame-ters from preoperative to 12 months: Enddiastolic volume 56 vs. $47 \mathrm{ml}$, Endsystolic vol-ume 63 vs. $44 \mathrm{ml}$, Enddiastolic diameter 56 vs. 47, cor weight 156 vs. 148 gr ( $\mathrm{p}<0.001)$ with decrease of Ejection Fraction $63 \%$ vs. 54\% ( $p<0.001$ ) (Table I). Conclusion: Remodelling measurements using cMRI proved to be important imaging tools in assess-ing reverse LV remodelling although the technical measurement was always not possible. There is a clear tendency for postoperative remodelling.

\section{Late Posttraumatic Tricuspid Regurgitation with Right Ventricular and Right Atrial Dilatation Successfully Repaired by Artificial Chordae Replacement}

Kemal Korkmaz', Serhat Bahadir Genc ${ }^{2}$, Ali Baran Budak', Hikmet Selcuk Gedik', Serdar Gunaydin ${ }^{1}$, Kerim Cagli ${ }^{2}$

${ }^{1}$ Numune Training and Research Hospital, Ankara, ${ }^{2}$ Yuksek Ihtisas Training and Research Hospital, Ankara, Turkey

Patient Demographics: A 45 year-old lady was admitted with progressive shortness of breath and palpitation. Echocardiography demonstrated severe tricuspid regurgitation (TR) and moderate mitral valve regurgitation (MR). MRI confirmed right ventricular 
dilatation $(8.9$ X5.5 cm). Right ventricular ejection fraction was $56.6 \%$ and right atrium was 8.9 X $9.0 \mathrm{~cm}$. Relevant History: Patient had a history of blunt chest trauma due to a car accident 15 years ago. After 3 years, she became symptomatic and referred to cardiology. Due to the progression of symptoms, deterioration of heart function and enlargement of cardiac chambers, patient was consulted to cardiac surgery. Pre-Operative Plan: TR occurred following chordae rupture due to trauma but late diagnosis and concomitant mitral valve disease accelerated right atrial and ventricular dilatation within a 10 -year period. We preferred valve repair with neo-chordae insertion despite it is more challenging with these chamber dimensions. Discussion of what was actually done and the challenges, deaths and complications encountered. (Unless case is still pending): Mitral valve was exposed via trans-atrial approach. Neo-chordae length was measured as $23 \mathrm{~mm}$ concordant to15-Hegar dilator. 4-0 PTFE was looped three times, two neochordae were placed on anterior mitral leaflet $5 \mathrm{~mm}$ apart and were reinforced by a 27 -mitral ring. Flail anterior tricuspid valve leaflet was plicated in a triangular fashion. Secondary chordae that were tethering and restraining anterior leaflet motion were cut. Edge of the neo-chordae was implanted to the moderator band. Free sides of loops were attached to valve by 5-0 PTFE with $5 \mathrm{~mm}$ gaps and were reinforced by a 31-flexible ring. Saline test and echocardiography confirmed optimal coaptation for both valves. Horizontal incision of right atrium was sutured vertically for plication. Former right atrium became two-chambered atrium, caval part and functional right atrium. Postoperative period was uneventful and patient was discharged on postoperative 7th day. Follow up echocardiography demonstrated satisfactory integrity of both valves at first and sixth months. Exact measurement of artificial chordae length, adequate plication without reducing valve area and increasing coaptation zone are the most important points in this successful repair.

\section{Redo Suturless Aortic Valve Replacment and Mitral Valve Repair in Patient with Infected Calcific Aortic Homograft}

\section{Ahmed Attia, Farouk Oueida, Mostafa Alrefaei, Khalid Alkhamees, Khalid Eskander}

Saud Albabtain Cardiac Center, Dammam, Saudi Arabia

Introduction: Aortic valve replacement in patients who have undergone previous aortic root replacement with an aortic homograft remains a technical challenge because of homograft degeneration and the need for a redo bentall operation. Methods: We report a case of aortic valve replacement (valve in valve) with a sutureless valve in an infected degenerated aortic homograft and mitral valve repair by miniband annuloplasty. Discussion: This technique allows rapid aortic valve replacement in a heavily calcified aortic root. It also avoids aortic valve size affection after mitral valve repair by ordinary methods especially in patients with small aortic annulus. Conclusion: This technique is particularly suitable in redo procedures for homograft degeneration, It avoids performing a redo Bentall operation with its known proplems as well as to avoid patient prosthesis mismatch.
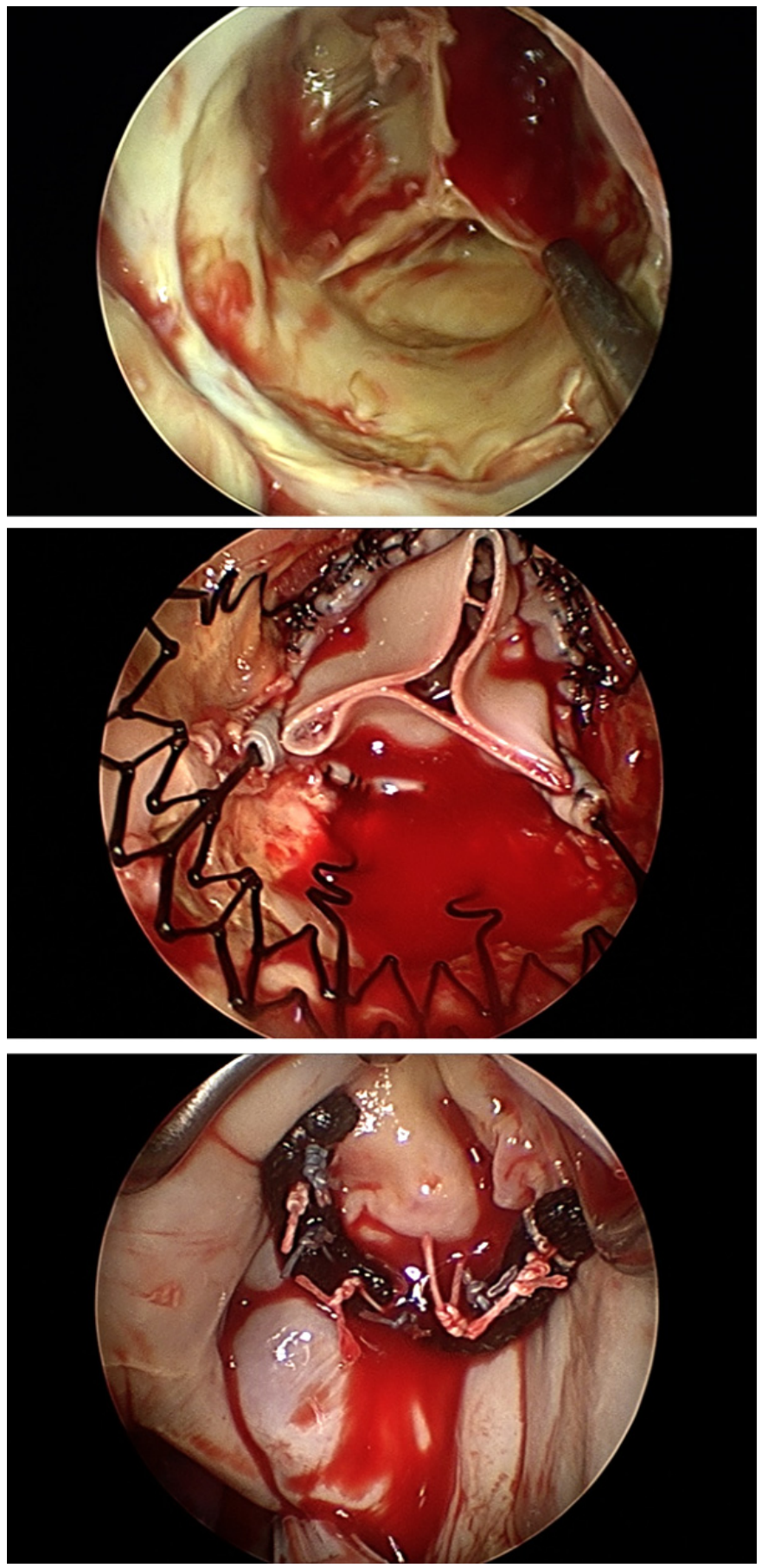

Fig. 1. (for Abstract of Farouk Oueida). 


\section{Uncle Sam}

Paolo Denti, Giovanni La Canna, Nicola Buzzatti, Ottavio Alfieri

San Raffaele Hospital, Milan, Milan, Italy

Patient Demographic: 59 years male, $113 \mathrm{~kg}, 190 \mathrm{~cm}$.

Relevant history: Advanced epatopathy $(\mathrm{HCV}+$, Child Pugh class C), thrombocytopenia and portal hypertension causing esophageal erosive congestive gastropathy. Mild Renal Insufficiency.

2013: Diagnosis of hypertrophic septal cardiomyopathy, with no significant mitral regurgitation (MR) and persistent atrial fibrillation.

NYHA Class III despite medical therapy.

After several hospital admissions for HF, we evaluated in Our Institution with a basal and stress transoesophageal echocardiography (TOE).

Basal TOE: Hypertrophic asymmetric cardiomyopathy and malposition of the anterior papillary muscle, not determining significant basal obstruction in the outflow tract, absence of MR. EDD $40 \mathrm{~mm}$, LVEF 60\%. Stress TOE: severe dynamic obstruction in the outflow tract ( $\mathrm{G} \max 74 \mathrm{~mm} \mathrm{Hg}$ - IVS $16 \mathrm{~mm}$ ) due to systolic anterior motion (SAM), concomitant significant MR (++++) obstruction-dependent.

STS mortality $0.8 \%$, morbidity $13 \%$.

Log EuScore 1.5\%.

Pre-Operative Plan: Since the presence of severe liver dysfunction and the interventricular septum was not amenable for Morrow intervention or alcoholic ablation, Heart Team decided was too high risk for surgical; mitral repair with edge to edge or replacement. We planned percutaneous procedure. Discussion: We use isoprenalin (IS) infusion in operatory room to evoke SAM, since it was present only under stress condition. The overall effect of IS is to decrease mean arterial pressure and induce tachycardia, plasma half-life is two minutes. We describe all the phases (trajectory, grasping, grasping assessment under basal and stress condi- tion and final result) of a straightforward one central clip procedure with complete abolition of SAM, MR and obstruction. Patient was quickly estubated and discharged home on 3rd post-operative day without nursing facilities. At 6 months follow up, patient is asympthomatic and echo shows good result of Mitraclip implantation (no residual MR - no obstruction - No SAM) and good LV function (60\%). We conclude Mitraclip procedure is effective in SAM abolition in patient affected by MR obstruction-dependent with same immediate and short term result as surgery but different impact on day-to-day life.

\section{Mitral Valve Prolapse and Sudden Cardiac Death Risk}

Allon Rafael, Theofanie Mela, David J. Milan

Massachusetts General Hospital, Boston, MA, USA

Patient Demographics: A 46-year-old woman with mitral valve prolapse and history of premature ventricular contractions associated with palpitations was seen in the Arrhythmia Clinic after an episode of syncope. Relevant History: She had lost consciousness and collapsed while talking with colleagues five minutes after standing up from her desk. A similar episode occurred nine years prior. Her PVCs significantly improved on low-dose betablockers, but had recently recurred. Holter monitoring on atenolol revealed $7.9 \%$ ventricular ectopy with 2 PVC foci in frequent bigeminy, trigeminy, couplets and triplets. Echocardiography showed bileaflet MVP with mild mitral regurgitation and normal ventricular size and function, unchanged from a prior study. Her mother was recently deceased at age 66 of sudden cardiac death; she had also been diagnosed with mild MR and PVCs before her ventricular fibrillation arrest. The patient had no personal history of drug, alcohol or stimulant abuse. Her resting blood pressure and pulse were 100/70 mm $\mathrm{Hg}$ and $58 \mathrm{bpm}$. Her BMI was 22. Cardiac auscultation revealed a regular rate and rhythm, normal S1 and S2,

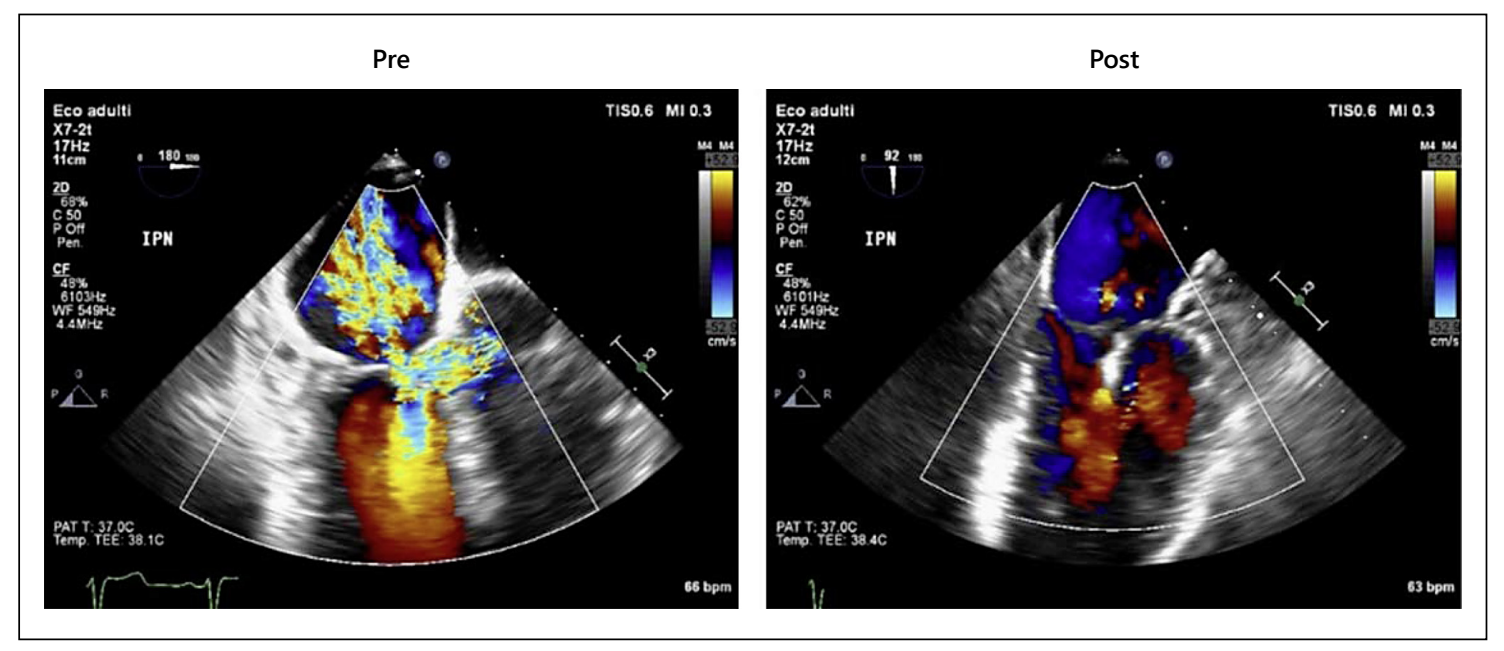

Fig. 1. (for Abstract of Paolo Denti). 
a mid-systolic click and a soft blowing systolic murmur. ECG showed normal sinus rhythm with inferior T wave inversions. She achieved 13 METS during stress testing without arrhythmias or perfusion abnormalities. Pre-Operative Plan: News of her mother's recent SCD prompted an extensive discussion about her own risk amidst a paucity of guideline recommendations and limited risk stratification algorithms. Her high risk features including bileaflet MVP, complex ventricular ectopy, resting T wave abnormalities, female gender and recurrent syncope were underlined and we agreed to implant a single chamber ICD for primary SCD prevention. Discussion of what was actually done and the challenges, deaths and complications encountered. (Unless case is still pending): Her St. Jude Medical Durata lead dislodged the following day during non-invasive program stimulation and required replacement. Five weeks later her new lead dislodged and was again replaced. This lead subsequently developed a precipitous rise in capture threshold, however the patient ultimately preferred not to undergo a third revision. She has had two detections for nonsustained ventricular tachycardia episodes, however no sustained VT/VF detections or therapies.

\section{New Onset of Cardiac Failure after Treatment with Trastuzumab for Breast Cancer: Case Report in an Adult with Previous Aortic Valve Replacement}

\section{Vincenzo Caruso, Haytham Sabry Elsayed, Inderpaul Birdi}

Basildon and Thurrock University Hospital, Basildon, United Kingdom

Patient Demographics: A 69 year old female patient with diagnosis of breast cancer, was referred for treatment of her severe aortic stenosis, prior to initiation of chemotherapy with Trastuzumab, a recombinant humanized monoclonal antibody directed against the human epidermal growth factor receptor 2. Relevant History: She underwent tissue aortic valve replacement, with good hemodynamic result, via a mini-sternotomy. After three weeks, she was started on Trastuzumab. She developed symptoms and signs of heart failure after her first and second doses, those were medically treated. Her transesophageal echocardiogram showed a new para-valvular leak related to the non-coronary sinus with moderate aortic regurgitation, severe mitral regurgitation, severe tricuspid regurgitation and an impairment of the ejection fraction. Pre-Operative Plan: Trastuzumab was abandoned and patient was medically stabilized. It was decided to take the patient to theatre for a redo-aortic valve replacement; atrioventricular valves appeared without any morphologic alterations. Discussion: An opaque white thick fluid was encountered in the anterior mediastinum. There were signs of tissue inflammation with whitish discoloration of the aortic wall. The valve leaflets were intact. A small area of valve sutures at the non-coronary sinus was disrupted. Aortic valve replacement was carried out with same size tissue valve. Post bypass transesophageal echocardiogram showed a well-seated prosthesis, mild mitral and tricuspid regurgitations. The sampled fluid showed no growth on direct culture or on enrichment. This report describes a case of sudden heart failure due to druginduced myocarditis and uncontrolled hypotension after Trastuzumab therapy. The pathological implications of monoclonal

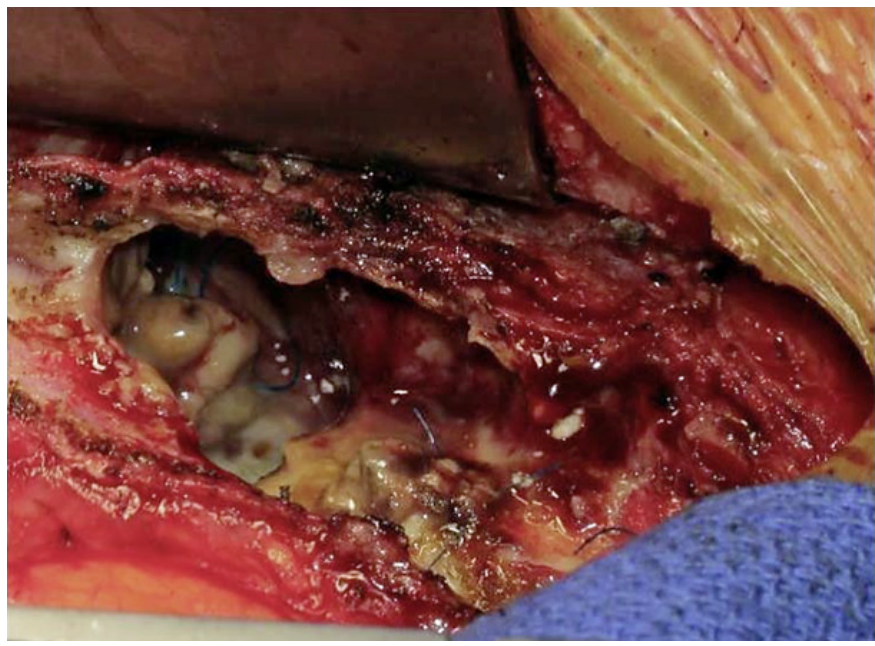

Fig. 1. (for Abstract of Vincenzo Caruso).

agents on myocytes, especially in the patients with previous heart diseases, are poor understood. Hypotensive crisis, following administration of Trastuzumab, after recent valve surgery, should be investigated.

\section{Aortic Valve Reconstuction with Autologous Pericardium in Young Patient with Parathyroid Adenoma and Severe Regurgitation of Bicuspid Aortic Valve}

Tomasz Niklewski, Roman Przybylski, Michael Zembala, Tomasz Kukulski, Marian Zembala

Silesian Center for Heart Diseases, Zabrze, Poland

Patient Demographics: 23 years old man in NYHA class III with severe IV degree regurgitation of bicuspid aortic valve (BAV). Relevant History: In medical history parathyroid adenoma (hyperparathyroidism) was diagnosed, complicated with renal failure and mental disorders. In transoesophageal echo examination (TEE) significant prolapse of both cusps, calcification of pseudoraphe and enlargement of aortic annulus to $3.0 \mathrm{~cm}$. Patient's parents (caregivers) not agree for valve replacement due to hypercalcemia and mental status not suitable for future anticoagulation treatment control. Pre-Operative Plan: Because of suspected uncertain effect of patient's valve plasty we decided about aortic valve reconstruction with Ozaki method with autologous pericardium. Preparation was started by cleaning fat, calcium and other redundant tissue on the outer surface of pericardium with Harmonic scalpel. Pericardium was excised at last $8 \times 12 \mathrm{~cm}$ and treated with $0.6 \%$ glutaraldehyde solution with a buffer for 10 minutes and then rinsed 3 times using $0.9 \% \mathrm{NaCl}$ solution for 6 minutes. Then, the distance between each commissure was measured with an originally invented sizing apparatus by giving the appropriate tension, similar to reproducing the annulus during diastole. The new 3 
cusps with the size corresponding to the measured value $31 \mathrm{~mm}$ was trimmed with an original template. Last, the annular margin of the pericardial cusp was sutured with running 4-0 monofilament stitches. New commissures were reinforced Prolene 5-0 with pericardial patchs. The pericardial cusps were sewn thoroughly up to the top of commissure. To have a deep coaptation secured with additional 4-0 monofilament sutures. In postoperative TEE we confirmed a very good coaptation without any prolapse or residual regurgitation. After 5 days patient was discharged home in very good condition. Discussion of what was actually done and the challenges, deaths and complications encountered. (Unless case is still pending): Aortic valve reconstruction became feasible with low mortality in many patients with aortic regurgitation. It is showing better results in terms of freedom from valve-related complications compared with conventional aortic valve replacement especially in specific cases. This procedure is effective for a wide variety of aortic valve diseases. Assessment of long-term data, especially concerning valve durability, will be disclosed in the future.

\section{Mitral Valve Endocarditis in Severe Pectus Excavatum: Planning Access and Timing for Surgery}

\section{Joseph George, Umair Aslam, Pankaj Kumar \\ Morriston Hospital, Swansea, United Kingdom}

Patient Demographics: 55-year-old handyman with no significant past medical history. Relevant History: The patient presented to the emergency department with headaches, malaise, weight loss and anorexia. On examination, marked pectus excavatum was noted. No other significant abnormalities were evident. His blood tests showed anaemia and mildly-elevated white cell count. Endoscopic and radiographic investigations were negative for malignancy and the septic screen was negative. A change in behaviour with new onset depression and emotional lability was noted. He developed facial droop and slurred speech. CT head revealed multiple embolic ischaemic infarcts. He developed fevers, and blood cultures grew Streptococcus viridans. A diagnosis of native mitral valve infective endocarditis was confirmed on echocardiogram showing multiple vegetations on both of the mitral valve leaflets. Antibiotics were commenced. Operative intervention was planned after further assessment. Pre-Operative Plan: Due to his acute inflammatory phase, the recent multiple cerebrovascular events, extremely poor nutritional state, emotional instability and unwillingness to undergo an operation, surgery was delayed for pre-operative optimisation. CT chest demonstrated severe pectus with a Haller index of 5.2. Coronary angiogram revealed a leftdominant system with no significant disease. 3D CT reconstructions were used to plan access to the mitral valve. Discussion of what was actually done and the challenges, deaths and complications encountered. (Unless case is still pending): Access for cardiac procedures, especially when mitral surgery is planned, is a challenge in the setting of severe pectus deformity. An inverted-T upper partial sternotomy was used in this case, the patient cannulated centrally and placed on cardiopulmonary bypass. The native valve with multiple perforations and vegetations was not suitable for repair and therefore mitral valve replacement was undertaken using a $33 \mathrm{~mm}$ CardiaMed mechanical prosthesis through left atriotomy. This case highlights the role of $3 \mathrm{D}$ reconstruction in facilitating the choice of incision in cases with severe skeletal abnormalities especially when minimal access approach is planned. An alternative approach avoiding the high-risk sternotomy would be a right thoracotomy approach. Factors influencing the timing of surgical intervention in this complex setting will also be discussed.

\section{Mitral Valve Repair and 3-Year Follow-Up in a Patient with Isolated Double-Orifice Mitral Valve}

\author{
Alexandr Bogachev-Prokophiev, Sergey Zheleznev, \\ Alexander Afanasyev, Rustem Tuleutaev, Alexey Pivkin, \\ Darya Prokhorova, Alexander Karaskov
}

State Research Institute Blood Circulation Pathology, Novosibirsk, Russian Federation

Patient Demographics: A 41-year-old man was referred to our hospital because of severe mitral regurgitation. Although, a murmur typical of mitral regurgitation was revealed in 2009, the patient remained asymptomatic until 2011, when he developed weakness and dyspnea. Relevant History: Severe mitral regurgitation and double-orifice mitral valve (DOMV) was confirmed by a TTE, which revealed a left ventricular end-diastolic dimension of $6.0 \mathrm{~cm}$, a large left atrium $(5.5 \times 7.9 \mathrm{~cm})$, and a left ventricular ejection fraction of $68 \%$. An electrocardiogram showed a sinus rhythm and a coronary angiography was normal. Pre-Operative Plan: The left atrium was significantly enlarged, and a DOMV was found with a central fibrous bridge dividing the two unequal orifices, a lateral larger and a medial smaller orifice. The anterior leaflet A2 segment had an $8-\mathrm{mm}$ cleft, the P1 segment was prolapsed due to chordae elongation, and the mitral annulus was dilated. The central fibrous bridge was connected with anterior leaflet using a continuous running 6-0 prolene suture, but the smaller orifice was excluded from the suturing, and the cleft of the A2 segment was closed with four interrupted sutures. Following a P1 triangular resection and confirmation of a central jet by a saline test, a mitral annuloplasty was performed using a $28-\mathrm{mm}$ annuloplasty ring (CardiaMed, Penza, Russia). At discharge TTE showed trivial mitral regurgitation with a mean transmittal gradient of $5 \mathrm{~mm} \mathrm{Hg}$ and an orifice area of $3.1 \mathrm{~cm}^{2}$. Discussion of what was actually done and the challenges, deaths and complications encountered. (Unless case is still pending): At the 3-year follow-up the patient's dyspnea was classified as NYHA Class I, and the TTE showed a single mitral orifice with an area of $3.4 \mathrm{~cm}^{2}$, a mean transmitral gradient of $3.2 \mathrm{~mm} \mathrm{Hg}$, and the absence of mitral regurgitation. Results reported by other surgeons and our mid-term followup dates have shown that mitral valve repair is the operation of choice for DOMV. 


\section{Concomitant Valve Sparing Root Remodeling with Extra Aortic Ring Annuloplasty and E-vita Stented Elephant Trunk Implantation}

\author{
Igor Rudez, Marko Kusurin, Mislav Planinc, Josip Varvodic, \\ Davor Baric, Daniel Unic, Ante Bosnjak, Robert Blazekovic, \\ Zeljko Sutlic \\ University Hospital Dubrava, Zagreb, Croatia
}

Patient Demographics: From November 2014 to October 2015 valve sparing root remodeling technique with external ring annuloplasty was used in twenty patients. From November 2011 to August 2015 E-vita stented elephant trunk procedure was used in eighteen patients, of which fourteen in acute setting. Relevant History: A 49-year-old male, with chronic Stanford B dissection presented to hospital due to paroxysmal atrial fibrillation and chest pain. Transthoracic echocardiography revealed bicuspid aortic valve with $\mathrm{AR} 2+$, dilatation of aortic root up to $40 \mathrm{~mm}$ and $42 \mathrm{~mm}$ at the level of ST junction. MSCT showed chronic Stanford type B aortic dissection. Dissection spread through the descending aorta down to the level of Th 8 , with true lumen diameter $10 \times 28 \mathrm{~mm}$, and false lumen diameter $29 \times 36 \mathrm{~mm}$ at the level of pulmonary bifurcation. Pre-Operative Plan: Valve sparing root remodeling with external subvalvular ring annuloplasty technique is a newly implemented procedure at our department. It represents excellent alternative to patients with $\mathrm{AR}$ and concomitant root and ascending aortic dilatation who were previously treated with modified Bentall procedure. E-vita stented elephant trunk is a good modality for treatment of complex cases of aortic pathology which were previously treated with frozen elephant trunk in two stages. Plan was to preserve native aortic valve and stabilize dissected aorta without need for secondary intervention. Discussion of what was actually done and the challenges, deaths and complications encountered. (Unless case is still pending): Peripheral cannulation using axillary artery was performed, aortic root was dissected, cusp free edges were aligned and root remodeling with Gelweave Valsava graft was preformed. Cusps resuspension followed by subvalvular extra-aortic Coroneo ring positioning with coronary ostia reimplantation was done. Circulatory arrest with bilateral antegrade cerebral perfusion was established for 43 minutes during which the stent was deployed and the graft sutured to the distal aorta with separate reimplantation of the supraaoritc branches. Patient recovered uneventfully and early TTE findings show no AR. MSCT showed exclusion of the false lumen. The patient was discharged from hospital nine days after surgery and 3 months following surgery is doing well.

\section{Successful Use of a Balloon-Expandable and Recollapsible Sheath in Transcatheter Aortic Valve Replacement with Prior Endovascular Aortic Repair with Endologix Stent Graft}

\author{
Anoshia Raza, Syed Zaid, Gilbert Tang, Hasan Ahmad, \\ Cenap Undemir, Martin Cohen
}

Westchester Medical Center, Valhalla, NY, USA

Patient Demographics: 75 year old frail female with hypertension, NYHA-III diastolic heart failure, porcelain aorta from prior chest irradiation from malignancy complicated by left arm lymphedema, moderate coronary artery disease and stage-IV chronic kidney disease. Relevant History: Prior EVAR for AAA with an Endologix endograft, complicated by endoleak requiring Palmaz stent. Was diagnosed with symptomatic severe aortic stenosis and deemed inoperable. Given comorbidities and frailty, transfemoral TAVR was her only option. Pre-Operative Plan: CT showed aortic annular dimensions $26.6 \times 22.0 \mathrm{~mm}$, area $457 \mathrm{~mm}^{2}$ and perimeter $76.5 \mathrm{~mm}$, with severe annular and LVOT calcifications. $29 \mathrm{~mm}$ CoreValve was selected. Transfemoral CoreValve implantation combined with EVAR had been reported once but TAVR preceded EVAR. Our case proves challenging for two reasons. First, the endoskeleton design of the Endologix overlapped by a Palmaz stent could risk catheter trapping between two prostheses. Advancing large-bore introducer sheath in such rigid aortoiliac anatomy could risk contact with the metal frame of the Endologix graft and the Palmaz stent, risking migration and dislodgement of the vascular prostheses and catastrophic complication. Second, the introducer sheath must be long enough for its tip to be positioned proximal to the Palmaz stent in the native aorta to avoid advancing the valve delivery catheter through the stented portion of the aorta. Discussion of what was actually done and the challenges, deaths and complications encountered. (Unless case is still pending): 11/19-French balloon-expandable, recollapsible Terumo Solopath sheath has been used in TAVR with CoreValve in small and tortuous iliofemoral access but not through an Endologix endograft. In our case, $29 \mathrm{~mm}$-CoreValve was successfully implanted with only $40 \mathrm{~mL}$ of IV contrast, under conscious sedation and TTE monitoring. Completion abdominal aortic angiogram showed no migration of Endologix and Palmaz prostheses. She required a permanent pacemaker but otherwise was discharged home uneventfully in 2 days, with mild paravalvular aortic regurgitation at 30 days due to her severe LVOT calcification, but excellent hemodynamics with mean gradient $2 \mathrm{~mm} \mathrm{Hg}$ and prosthetic valve area $2.53 \mathrm{~cm}^{2}$. 


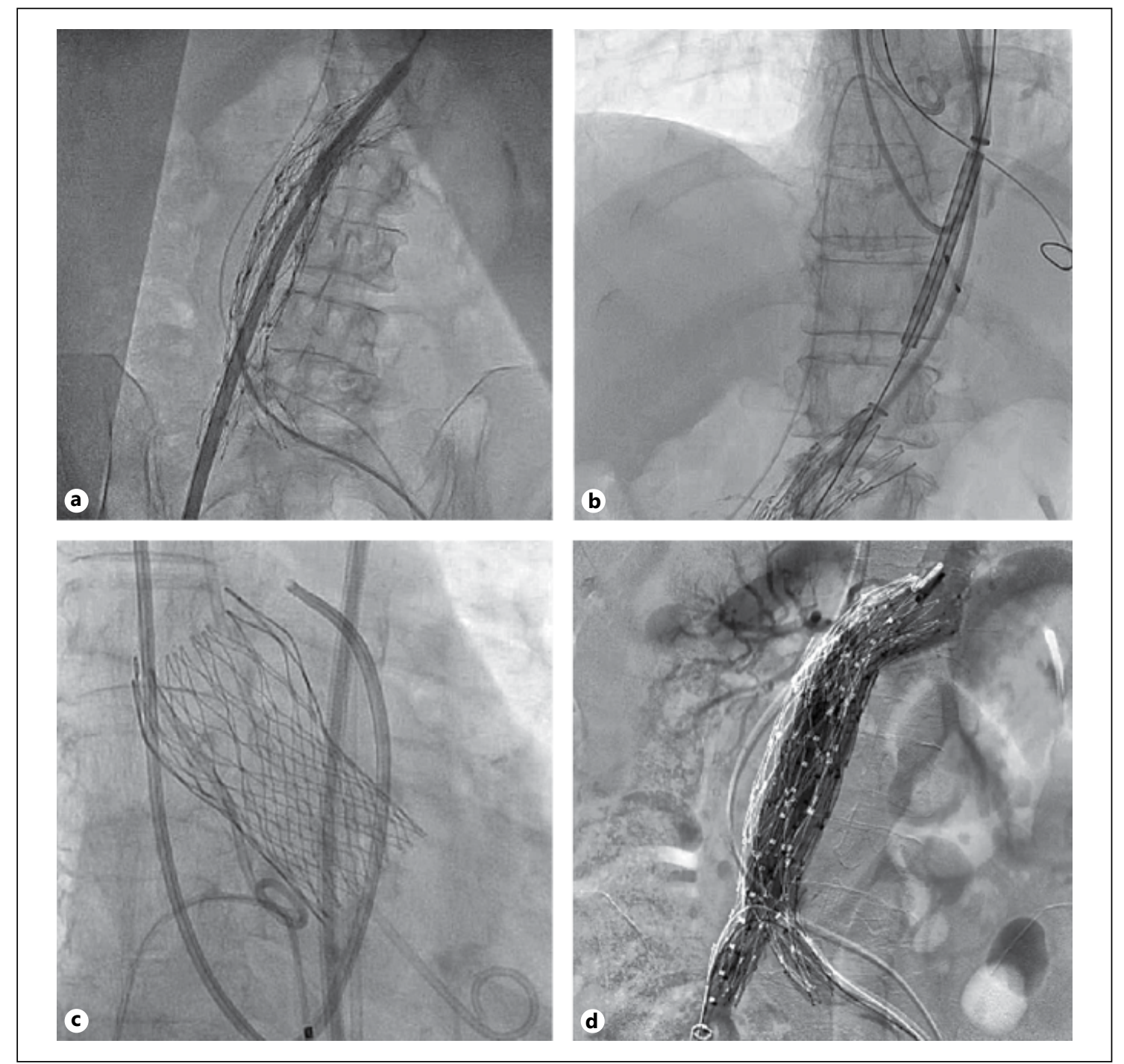

Fig. 1. (for Abstract of Anoshia Raza).

\section{Two Consecutive Cases of Fulminant S. aureus Endocarditis Complicated by Hepatitis-C/HIV Presenting during the Second Trimester of Pregnancy: Consolidation of Evolving Gestation-Based Surgical and Cardiopulmonary-Bypass (CPB) Principles in the Collaborative Management of High Risk Surgery during Pregnancy Promises Improved Fetal Salvage without Compromising Maternal Survival}

\section{Taras R. Mycyk, Mark Rosin, Jo-anne Marcoux}

Royal University Hospital, Saskatoon, SK, Canada

Objective: Cardiopulmonary-bypass (CPB)-supported cardiac surgery during pregnancy is generally performed under urgent/ emergent conditions in the face of failure of medical/intervention- al management. Management of fulminant endocarditis during the second trimester has not been subject to rigorous prospective randomized trials. $\mathrm{CPB}$-supported cardiac procedures during the second trimester face unique challenges presented by the competing demands of two inseparable but physiologically diverse patients at risk. Whereas maternal surgical mortality approaches that of age/disease-matched non-pregnant females, reported fetal mortality (up to $30 \%$ and more) remains disproportionately high (often attributed to CPB and maternal pathophysiology). We present two consecutive cases of complicated second trimester S. aureus endocarditis. Our objective was to define, refine and apply gestation-based surgical/CPB principles to reduce the pronounced historic gap between maternal and fetal morbidity/mortality without compromising maternal surgical risks. Methods: Two 27 year-old Hep-C/HIV-positive (IVDU) females in their 19th and 22nd weeks of gestation complicated by intractable $\mathrm{CHF}$, sepsis and recurrent emboli due to $S$. aureus endocarditis underwent emergent: 
i) subaortic abscess debridement+AVR

and

ii) preoperative IABP insertion + MVR respectively.

Gestation-based management adjustments included:

- Collaborative patient-individualized multi-disciplinary team care

- Pre/post-op fetal scans

- Operative positioning

- Perioperative fetal monitoring

- MgSO4-enriched blood pump-prime

- Maintain HCT $\geq 0.30$

- Maximal-sized aortic/bicaval cannulas

- Minimize impairment of uterine venous drainage

- Minimize arterial tubing length

- Normothermic, $\mathrm{FiO}_{2}$ 100\%, high flow (3.4-4.6 1/min/m²) pulsatile perfusion at a MAP of 70-80 $\mathrm{mm} \mathrm{Hg}$

- Tepid crystalloid maintenance cardioplegia after warm induction protocol

- Active multi-site potassium scavenging

- Ultrafiltration (CHF)

- Pre/post-op thromboelastograph (TEG) monitoring to address maternal hypercoagulable status

- Frequent intra-op ACT + [Heparin] determinations

Results:

- No perioperative maternal-fetal morbidity/mortality

- Both pregnancies carried successfully to term

- No significant perioperative fetal distress by FHR parameters

- Normal post-op fetal scans

- Uteroplacental hemodynamics substantially improved by IABP in setting of severe MR and intractable/hypotensive $\mathrm{CHF}$

- Both healthy (HIV and Hep-C free) children adopted by respective grandmothers

- To date, one mother survives. One reused/expired.

Conclusions: Gestation-based adjustments to CPB-supported procedures during pregnancy serve to normalize maternal hemodynamics and the fetal-placental milieu with the promise of optimized fetal salvage and uncompromised maternal survival. The described evolving principles/adjustments are applicable to all $\mathrm{CPB}$-supported procedures during pregnancy.

\section{Mycotic Aneurysm of the Mitral Valve: Extension of Bicuspid Aortic Valve Endocarditis}

\section{Ana Kadkhodayan, Nandan Anavekar Chandrasekaran Krishnaswamy}

Mayo Clinic, Rochester, MN, USA

Patient Demographics: 20 year old Caucasian male. Relevant History: 20 year old male with a history of bicuspid aortic valve, told it was 'leaky' in the past, presented to the CCU after 4 days of new onset dyspnea, orthopnea, cough, and intermittent hemoptysis. Two weeks prior he had an upper respiratory infection. He recovered without any antibiotic use. He denied any fever, chills, night sweats, or weight loss. He had no history of IV drug use or recent dental work or infections. TTE showed complex endocarditis affecting the bicuspid aortic valve with mobile vegetations on the ventricular surface with posterior cusp perforation and aortic

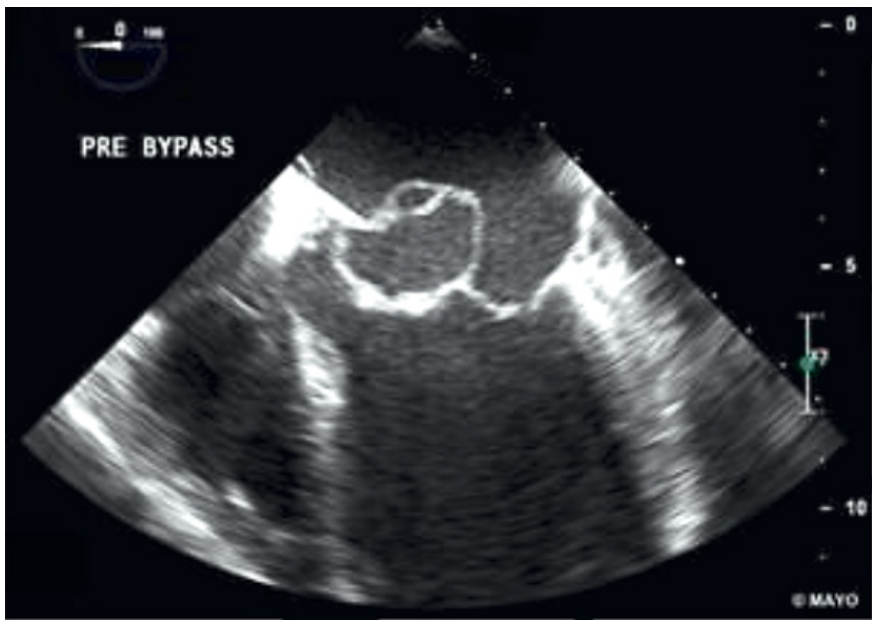

Fig. 1. (for Abstract of Ana Kadkhodayan).

root abscess with severe aortic regurgitation. There was extension into the intervalvular fibrosa and the anterior mitral leaflet with mycotic aneurysm formation ( $21 \times 22 \times 25 \mathrm{~mm}$ echo-lucent space) and eccentric, severe mitral regurgitation. He had severe left ventricular enlargement with ejection fraction $59 \%$ and a normal right ventricle. Pre-Operative Plan: Plan was to medically stabilize the patient prior to surgery. Antibiotics with Ceftriaxone, based on positive blood cultures showing Strep Mitis, were given. He was medically optimized from a heart failure standpoint prior to undergoing surgery. Discussion of what was actually done and the challenges, deaths and complications encountered. (Unless case is still pending): Five days after he was admitted to the CCU and antibiotics were started, he underwent surgery with aortic root and mitral valve debridement with excision of the infected aortomitral continuity and aneurysm. He underwent reconstruction of the roof of the left atrium and aortomitral continuity using a bovine pericardial patch. He then had mechanical mitral valve replacement with a 31-mm St. Jude and had aortic root replacement with a 27-mm CarboMedics valved conduit. Fortunately, the patient survived the operation without any major complications. Challenges during surgery included ensuring that all infected tissue was removed, and that the tissue that the prosthetic material was sewed onto was durable enough to prevent future dehiscence. 


\section{Severe Septicaemic Outcome of Infection Endocarditis of Mitral Valve with Erisypelothrix Rusiopathiae in Slaughterhouse Worker}

Tomasz Niklewski, Michael Zembala, Krzysztof Filipiak, Tomasz Kukulski, Marian Zembala

Silesian Center for Heart Diseases, Zabrze, Poland

Patient Demographics: 53 years old man without any previous medical history. Relevant History: Patient was admitted to General Hospital with circulatory insufficiency, oedema and sepsis, with only non specific blood culture bacteria (Streptococcus mittis). The empiric antibacterial treatment with 3 antibiotics was started, but patient's status still worsened - complicated with renal failure, diabetes, thrombocytopaenia and microcellular anaemia. Control physical examination present a new, loud murmur above the mitral valve (MV) confirmed in transoesophageal echo (TEE) as severe, IV degree MV regurgitation caused by 2 big $(>4.5 \mathrm{~cm})$ longitudinal, flagellet vegetations on anterior mitral leaflet. Intubated, deteriorated patient was urgently transmitted to Cardiac Surgery Department. Pre-Operative Plan: Mitral valve was replaced with OnX 29 mechanical valve. The fast bacterial inoculation of MV material shown active cultures of animal bacteria Erysipelothrix rusiopathiae and targeted treatment with Ceftriaxone was deploy. During next few days the significant improvement of patient status with retreat of all complications was observed. Reevaluation of social history revealed that patient is a slaughterhouse worker with every day contact with pigs. After 7 days patient was discharged home in good condition. Discussion of what was actually done and the challenges, deaths and complications encountered. (Unless case is still pending): In human most often manifestation of Erysipelothrix rusopathiae infection is single cutaneous erysipeloid or generalised cutaneous form. Septicaemic form is very rare (50 cases/15 years 1995-2010), but with more serious manifestation and almost always linked to endocarditis ( $90 \%$ cases) with mortality over $40 \%$ - double rate more than endocarditis of other bacteria, because of difficult bacteriological diagnostics.

\section{Posterior Mitral Leaflet Augmentation and Artificial Chordae Reconstruction for a Hemodialysis Patient with Mitral Regurgitation and Mitral Annulus Calcification}

Yusuke Sakurai, Koji Hattori, Mika Noda, Kentaro Amano, Yoshiro Higuchi, Masato Tochii, Michiko Ishida, Hiroshi Ishikawa, Yasushi Takagi

Fujita Health University, Toyoake, Aichi, Japan

Patient Demographics: A 77-year-old woman with mitral regurgitation (MR) and calcified aortic stenosis was referred to our institution for mild effort dyspnea. She had been repeatedly hospitalized for congestive heart failure. Echocardiography demonstrated severe MR with segmental shortness of a posterior mitral leaflet (P2), elevation of the systolic aortic transvalvular gradient (peak gradient $129 \mathrm{~mm} \mathrm{Hg}$ ), and a left ventricular ejection fraction of 59\%. Computed tomography showed extensive posterior mitral annular calcification (MAC) extending into the left ventricular muscle. Relevant History: Her medical history included hemodialysis over an 18-year period. Pre-Operative Plan: Although aortic valve replacement (AVR) was necessary in this patient, concomitant mitral valve replacement requiring debridement of the MAC would be a high-risk procedure. As motion of the short segment of $\mathrm{P} 2$ was restricted, we considered that posterior leaflet augmentation with autologous pericardial patch while avoiding contact with the calcified mitral annulus would effectively control the MR. Therefore, we decided to perform mitral valve plasty and AVR. Discussion of what was actually done and the challenges, deaths and complications encountered. (Unless case is still pending): The posterior side of the P2 leaflet was thick and had shrunk to about $5 \mathrm{~mm}$ in height. Thick and short marginal chordae were attached to the P2 leaflet. A $20-\mathrm{mm}$ incision was made on the posterior mitral leaflet along the posterior mitral annulus from P2 to P3. An ellipse-shaped piece of autologous pericardium $\left(25^{*} 10\right.$ $\mathrm{mm}$ ) was sewn on to augment the posterior mitral leaflet. A water test revealed that the anterior mitral leaflet (A2) was slightly prolapsed from P2 and that the posterior side of P2 was not sufficiently elevated toward the coaptation line. Therefore, we cut the thick marginal chordae and performed reconstruction with artificial chordae for A2 and P2. After confirmation of good control of MR with a water test, AVR was performed with a bioprosthetic aortic valve (Magna Mitral EASE $19 \mathrm{~mm}$; Edwards). Post-operatively, echocardiography demonstrated no MR and a normal diastolic mitral transvalvular gradient (mean gradient $4 \mathrm{~mm} \mathrm{Hg}$ ). Despite suffering post-operative vocal cord paralysis due to the intra-tracheal intubation, the patient recovered well.

\section{A Rare Case of Triple Valve Repair for Stenotic Lesions of Mitral, Tricuspid and Pulmonary Valves in Rheumatic Heart Disease}

\author{
Rajeev Gehlot, Ashish Saraogi, Deepak Tiwari, \\ Ramesh K. Madhok, Munish Batheja, Anu Yadav, Anuj Verma
}

NIMS University Jaipur, Jaipur, India

Patient Demographics: Rheumatic Heart Disease (RHD) is significant cause of Valvular Heart Disease (VHD) in developing countries. We report a case of 30 year old married female patient hailing from rural low socio-economic population. Relevant History: Patient presented with breathlessness on exertion (NYHA Class III) which gradually progressed over 3 years to present state with limitation of physical activities (Specific Activity Scale - SAS Class III). She had history of recurrent abortions and found to be hypothyroid during assessment. Pre-Operative Plan: 2D Transthoracic Echocardiography (TTE) revealed-moderate to severe Mitral Valve stenosis (MS) and trace regurgitation; severe Tricuspid Valve stenosis (TS) and regurgitation; severe Pulmonary Valve stenosis (PS); mild Aortic Valve regurgitation with normal left atrial size, dilated right atrium (RA), dilated and hypertrophied right ventricle, moderate pericardial effusion. Decision to repair or replace mitral valve was based on intra-operative findings of mitral valve apparatus. Repair of tricuspid valve (Commissurotomy sup- 
ported by suture/ring annuloplasty) and open pulmonary valvotomy were planned. Discussion of what was actually done and the challenges, deaths and complications encountered. (Unless case is still pending): On cardiopulmonary bypass, by standard technique mitral valve accessed by trans-septal approach through RA. Anterior mitral leaflet was thickened hence thickened covering was peeled off. Condition of subvalvular apparatus was satisfactory hence only open mitral commissurotomy of both mitral commissures was done. Commissurotomy of fused antero-septal, septo-posterior and commissure between posterior and anterior leaflets of tricuspid valve were done and supported with De-Vega's annuloplasty. Significant leak from site of septo-posterior commissure was found during saline insufflation test which was repaired. All three cusps of pulmonary valve were thickened and fused. Commissurotomy of all commissures of tight stenotic pulmonary valve was done. Patient is in NYHA Class I and SAS Class I after two follow ups within six months of surgery. 2D TTE in follow-up showed mild MS, trace mitral regurgitation, mild to moderate tricuspid regurgitation with no stenosis, no pulmonary stenosis or regurgitation. This extremely rare $(<0.013 \%)$ case of PS with MS and TS in RHD was repaired successfully keeping in view of young female patient planning to conceive and avoiding anticoagulation.

\section{ECMO as a Bridge to Mitral Valve Repair in the Setting of Acute Non-Ischemic Mitral Regurgitation}

\section{Federico Milla, Randolph Martin}

Piedmont Heart Institute, Atlanta, GA, USA

Objective: Patient Demographics: 66 year old male with a remote history of mitral prolapse and no active medical conditions. Relevant History: Sudden onset shortness of breath without prodrome that progressed to pulmonary edema and cardiogenic shock within 48 hours. Echocardiography demonstrated Flail posterior leaflet and severe mitral regurgitation (MR), normal ventricular function, and normal coronary anatomy was seen on catheterization. Urgent transferred to our institution with IABP, high dose vasopressors, and full vent support with acidosis, anuria, hypoxia on arrival. Methods: Preoperative Plan: Patient underwent femoral veno-arterial Extra-Corporeal Membrane Oxygenator (ECMO) placement for 24 hours for resuscitation. His acidosis was corrected and renal function improved. Successful mitral valve repair was then performed. Results: The patient weaned from cardiopulmonary bypass successfully and had a prolonged recovery requiring tracheostomy. He was discharged to a rehab facility and made full recovery after a month and is currently off the ventilator and fully ambulatory. Conclusions: Acute MR is often the result of coronary ischemia or endocarditis. Spontaneous chordal rupture can also lead to acute MR. Acute MR leading to cardiogenic shock requires emergent intervention and patients usually undergo mitral valve replacement. ECMO can be used for resuscitation in the setting of acute MR, which may allow for the performance of mitral repair.

\section{Unusual Rare Indication for Extracorporeal Membrane Oxygenation Immediately after Successful Sequential Bilateral Lung Transplantation for the Treatment of Severe Systolic Anterior Motion and Mitral Valve Regurgitation Induced Cardiogenic Shock}

Hartmuth B. Bittner, George Makdisi, Robert Hooker, Joseph H. Boyer, Andres Pelaez

Gulf Coast Cardiothoracic Surgery Institute, Tampa, FL, USA

Patient Demographics: We report an unusual case of primary graft dysfunction grade III following uneventful off-pump bilateral sequential lung transplantation (LTX) caused by paradoxical left ventricular failure due to systolic anterior motion (SAM) of the mitral valve-induced left ventricular outflow tract obstruction. Relevant History: A 53-year-old man with idiopathic pulmonary fibrosis and moderate secondary pulmonary arterial hypertension underwent uncomplicated LTx. The patient was transferred to intensive care on norepinephrine $(0.08 \mu \mathrm{g} / \mathrm{kg} / \mathrm{min})$ and vasopressin ( 0.8 units $/ \mathrm{min}$ ) with very restrictive iv fluid maintenance. Pre-Operative Plan: Ischemia-reperfusion injury-induced primary graft dysfunction after LTx is a major cause of early morbidity and mortality; it is characterized by pulmonary edema with diffuse alveolar damage manifesting clinically as progressive hypoxemia with radiographic pulmonary infiltrates. Discussion of what was actually done and the challenges, deaths and complications encountered. (Unless case is still pending): Despite aggressive volume resuscitation and additional inotropic support (epinephrine $0.15 \mu \mathrm{g} / \mathrm{kg} / \mathrm{min}$ ), cardiac index remained below $1.5 \mathrm{~L} /[\mathrm{min} \cdot \mathrm{m} 2]$. TTE demonstrated severe mitral valve regurgitation (MR). Persistent hemodynamic and respiratory instability required further resuscitation and emergency VA-ECMO. Intra-op TEE confirmed severe MR but also documented near total LVOT obstruction by the very elongated anterior mitral valve leaflet $(3.4 \mathrm{~cm})$. SAM disappeared and MR immediately improved. Our case of transient heart failure was paradoxically induced by extreme dehydration and catecholamine therapy precipitating intraventricular obstruction and significant MR, and thereby inducing an acute drop in cardiac output. Classically, cardiogenic shock is characterized by increased pulmonary capillary wedge pressure (PCWP) and decreased cardiac output, whereas hypovolemic shock includes low PCWP and reduced cardiac output. Due to different management strategies, distinguishing between cardiogenic and hypovolemic shock is critical. In the present case, pulmonary infiltrates were notable and were considered a consequence of elevated PCWP due to increased left arterial pressure which resulted from intracardiac dynamicobstructionand significantMR.Conclusion:Hypovolemia together with a hyperdynamic state resulting from catecholamine administration may result in the development of dynamic LVOT obstruction secondary to SAM. Early detection and intensive efforts to reverse the underlying conditions including cessation of catecholamine therapy and correction of hypovolemia are essential. 


\section{Late Complication of a Previous Bentall's Procedure - A Large Pseudo-Aneurysm of the Aortic Composite Graft}

\section{Anthony J. Barron, Shelley L. Rahman Haley, Jullien Gaer}

Royal Brompton and Harefield NHS Foundation Trust, Harefield, United Kingdom

Patient Demographics: A 73 year-old, hypertensive female presented to the cardiothoracic clinic for elective follow up. 18 months' earlier she had undergone a Bentall's procedure with a replacement of the aortic root and ascending aorta using a $21 \mathrm{~mm}$ BioValsalva composite graft for a $7 \mathrm{~cm}$ ascending aortic aneurysm with moderate aortic regurgitation. Relevant History: The patient had recovered fully after surgery and LV function on echocardiography improved to normal. However, she presented for clinic follow up with a 6-8 week history of being rather drowsy and tired - her husband and family suspected depression. The routine transthoracic echocardiogram was reported as showing moderate-severe paraprosthetic regurgitation - but this was clearly impossible, due to the composite graft. A chest X-ray showed a bulging opacity which had not been present 3 months' earlier. Further review of the transthoracic images revealed a large cavity posterior to the aortic root. The cavity filled with blood during systole and emptied during diastole, giving the impression on colour Doppler of paravalvar AR. Aortic arch doppler showed greatly reduced blood flow in the aortic arch. CT scanning and transoesophageal echocardiography revealed an extremely large pseudo-aneurysm due to a partial dehiscence of the proximal aortic suture line. There was no evidence of infection. Pre-Operative Plan: Case discussed by the

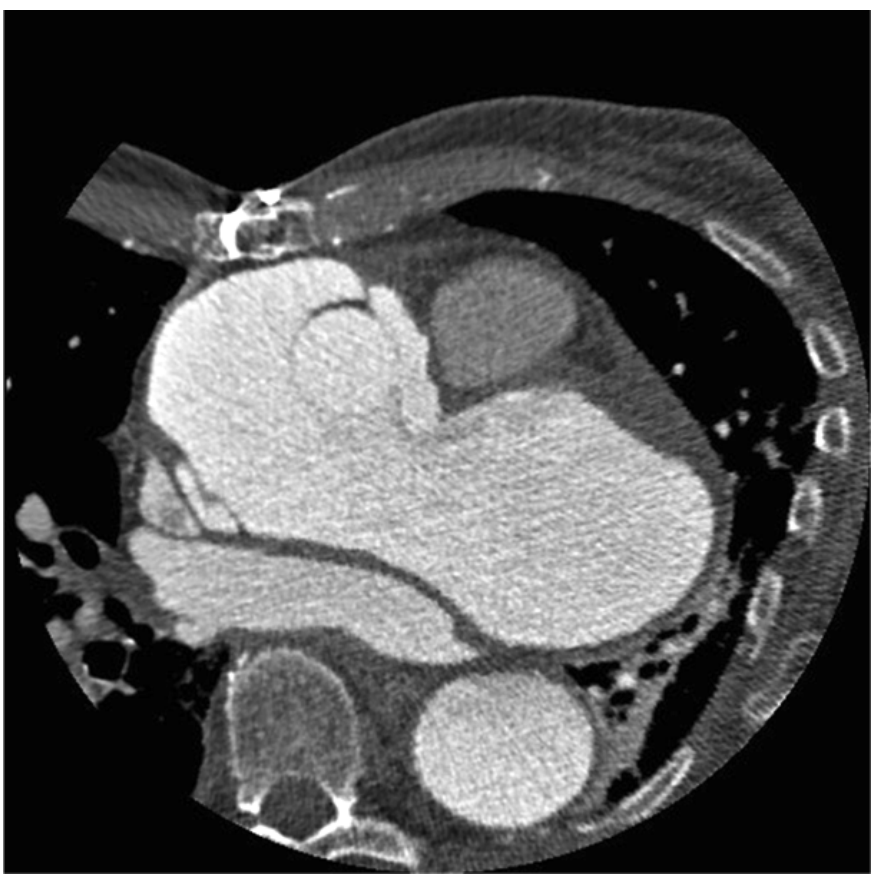

Fig. 1. (for Abstract of Anthony J. Barron). heart team in the joint cardiology/cardiothoracic meeting and agreed for redo surgery. Discussion of what was actually done and the challenges, deaths and complications encountered. (Unless case is still pending): With femoro-femoral bypass, the patient was cooled to $18^{\circ} \mathrm{C}$. The sternum was divided without incident. The aneurysm was opened and the defect repaired with interrupted sutures. Post-operatively recovery was slow, with a long period of inotropes and Dressler's syndrome. She was discharged over a month later to another hospital for on-going rehabilitation, and subsequently made a full recovery.

\section{Rare Cause of Heart Failure: Aorta to Right Atrium Fistula}

Steven A. Plato, II', Samhati Mondal', Yakov Elgudin ${ }^{1}$

${ }^{1}$ Univeristy Hospitals/Case Western Reserve University, Cleveland, $\mathrm{OH},{ }^{2}$ Metro Health Medical Center, Cleveland, $\mathrm{OH}, \mathrm{USA}$

Relevant History: Recurrent heart failure admissions, heart murmur since childhood, imaging with aorta to right atrium fistula, preoperative diagnosis was ruptured sinus of Valsalva aneurysm. Preoperative Plan: Biatrial cannulation, atriotromy and aortotomy to visualize the defect from both sides, patch angioplasty. Discussion: Exploration revealed normal valvular morphology with large defect at the valve annulus along the non-coronary sinus. This occupied one half of the sinus, opening into the right atrium adjacent to the septal leaflet of the tricuspid valve. Repair was completed using CorMatrix patch of the non-coronary sinus and primary repaired on the atrial side. Post -repair transesophageal echocardiogram showed no flow from the aorta to the right atrium. He was weaned from cardiopulmonary bypass and was able to be extubated and weaned from inotropic agents later that day. The most likely etiology is a ruptured sinus of Valsalva aneurysm from the non-coronary cusp of aorta into the right atrium; this accounts for 20 to $30 \%$ of ruptured sinus of Valsalva aneurysms $s^{1,2}$. This defect was located adjacent to the annulus of the aortic valve near the left coronary sinus, so the only place for the aneurysm to expand was intracardiac, preventing free rupture into the pericardium, cardiac tamponade, and death. Sinus of Valsalva Aneurysm is a congenital defect due to lack of continuity of the elastic lamina of the aortic media and the heart ${ }^{3}$. This defect can present in any of the sinuses of Valsalva, but is most common within the right coronary sinus ${ }^{4}$. Surgical repair is well tolerated with in hospital mortality less than $2 \%$ and significant improvement in symptoms after repair ${ }^{5,6}$, This operations has $95 \% 20$ year surviv$\mathrm{al}^{1}$, and $88 \%$ of those presenting without aortic regurgitation have NHYA class I symptoms at 10 years post op, a benefit preserved whether repaired primarily or with a patch ${ }^{1}$. These symptoms, imaging, and intraoperative findings are consistent with type IV ruptured sinus of Valsalva aneurysm in a 53 year old patient with new onset of heart failure. 


\section{Forgetting the Aorta after Bicuspid Aortic Valve Replacement: A Potential Catastrophe}

Jae Yoon Park, Naser M. Ammash, Maurice E. Sarano, Hector I. Michelena

Mayo Clinic, Rochester, MN, USA

Patient Demographics: A 39-year-old Caucasian man presented for evaluation of cough. Relevant History: History was notable for bicuspid aortic valve (BAV) stenosis status post $19-\mathrm{mm}$ mechanical aortic valve replacement at age 13 . On his last evaluation 4 years prior, his ascending aorta measured $53-\mathrm{mm}$. He had been lost to follow-up. Examination was remarkable for a grade III/VI mid-to-late peaking systolic murmur at the base and a dull mechanical S2. Chest X-ray demonstrated a widened mediastinum (Panels A-B), prompting computed tomography (CT) scan. PreOperative Plan: CT chest demonstrated a massive ascending aortic (Ao) aneurysm with effacement of sinotubular junction and dissection flap (arrow; Panel C) extending from mid-ascending aorta to left subclavian artery. The maximal dimension was $96-\mathrm{mm}$ at mid-ascending aorta and $50-\mathrm{mm}$ at sinus of Valsalva, with a markedly compressed right pulmonary artery (PA) from the true lumen (TL) of the aneurysm (Panel D). Transthoracic echocardiogram demonstrated evidence of severe aortic valve prosthetic stenosis with a mean systolic Doppler gradient of $40 \mathrm{~mm} \mathrm{Hg}$, confirming physical exam findings. Discussion of what was actually done and the challenges, deaths and complications encountered. (Unless case is still pending): Given the findings, he was referred for urgent surgery. Intra-operative transesophageal echocardiogram confirmed evidence of massive ascending aortic aneurysm and significant prosthetic aortic valve stenosis. He underwent explantation of the prosthetic aortic valve, aortic root and ascending aorta replacement with a composite mechanical valve conduit, as well as total aortic arch replacement. The prosthesis exhibited pannus formation on the ventricular aspect. The patient's recovery was uneventful. BAV is the most common congenital heart defect, occurring in approximately $1 \%$ of the popula-
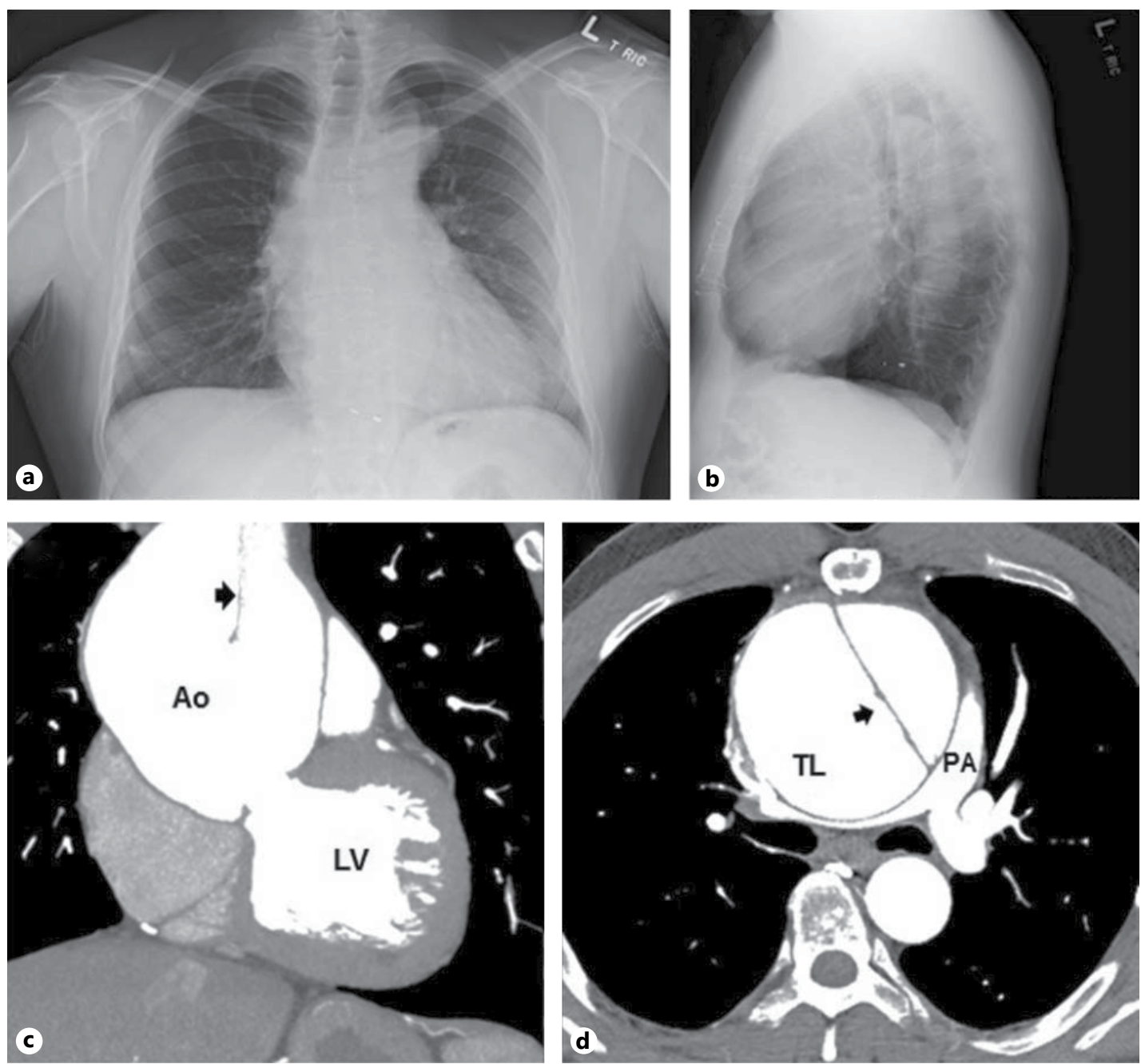

Fig. 1. (for Abstract of Jae Yoon Park). 
tion. It is characterized by valvulo-aortopathy. Despite early correction of our patient's aortic valve dysfunction, silent progression of his aortopathy resulted in a massive dissecting aneurysm that was fortuitously identified. Compulsory periodic screening of the thoracic aorta in BAV patients with echocardiography and/or CT/ $\mathrm{MR}$ is critical even after aortic valve replacement.

\section{Systemic Hyperkalemia and Mild Hypothermia in Redo Aortic and Mitral Valves Replacement for the Patient with Fungal Endocarditis and Patent LIMA}

\section{Mohammad H. Naseri, Alireza Jalali, Mohsen Sadeghi,} Arezoo Khosravi, Hamid Shafiee

Baqiyatallah University of Medical Sciences, Tehran, Iran

Patient Demographics: A 70 year old Diabetic man with fever, asthenia and narcosis 2 months ago with Hx. of CABGs (4 grafts) 7 years ago. In TTE and TEE, there was a small pediculated mass on ventricular site of AMVL, severe valvular MR and A I, PAP \# $65 \mathrm{~mm} \mathrm{Hg}$. The underwent of antibiotic for 3 weeks. Relevant History: With worsening of his general condition, TTE and TEE was repeated, the mass was larger, mobile and extended to both atrial and ventricular site of Mitral Valve, severe MR and AI. Pre-Operative Plan: Patient was candidate for surgery with untreated Endocarditis for Mitral and Aortic valves replacement. In coronary angiography, LIMA, OM and PDA grafts were patent. Discussion of what was actually done and the challenges, deaths and complications encountered. (Unless case is still pending): After AXC, infusion of $2000 \mathrm{ml}$ of low pressure tepid cardioplegia from aortic root and vein grafts, successful cardiac arrest was not obtained and decided for systematic Hyperkalemic arrest. The patient was mildly cold ( $29 \mathrm{C})$. Now $\mathrm{K}$ was about 5 , after infusion of $40 \mathrm{ml}$ of $\mathrm{K}$ solution, $\mathrm{K}$ was 8.8 and $\mathrm{HR}$ was $25 / \mathrm{min}$. In 3 steps $\mathrm{K}$ solution was delivered $20 \mathrm{ml}$ and finally $\mathrm{K}$ was 9.8 and complete cardiac arrest obtained. In continuance, low pressure tepid car- dioplegia delivery from coronary ostia and vein grafts was sustained and $\mathrm{K}$ was stayed on 9.5-9.8. After successfully removal of all vegetations and replacement of Mitral and Aortic valves with 29 and $21 \mathrm{~mm}$ of Edwards, Bioprosthetic, rewarming performed with Z-BUF method and hemofiltration with $4000 \mathrm{ml}$. For preventing of acidosis, each $1000 \mathrm{ml}$ hemofiltration, $50 \mathrm{ml}$ bicarbonate was infused. In 2 steps, 25 IU of Insulin infused. The patient stood at metabolic alkalosis and during rewarming, about $600 \mathrm{ml}$ of warm blood in $25 \mathrm{~min}$ infused from aortic root and with systematic $\mathrm{K}$ of about 7, the patient was weaned of $\mathrm{CPB}$ without Pacing and with low doses of inotrop. TEE was done with excellent results and the patient was transferred to CVS-ICU with stable hemodynamic state.

\section{Complex Mitral Valve Reconstruction in a Patient with Endocarditis and Associated Annular Abscess}

Alexander Iribarne, Joseph P. DeSimone, Jock N. McCullough, Anthony W. DiScipio

Dartmouth-Hitchcock Medical Center, Lebanon, NH, USA

Patient Demographics: 21 year-old woman with no past cardiac history who presents with two days of chest pain and fever. Relevant History: History is notable for IV drug use and hepatitis C. Blood cultures were positive for gram positive cocci in clusters. TTE showed a moderate effusion, $1.1 \mathrm{~cm}$ mobile vegetation on the posterior leaflet of the mitral valve, and echolucency between the mitral annulus and appendage concerning for possible abscess. A pericardiocentesis was purulent and the patient required increasing vasopressor support prompting surgery. Pre-Operative Plan: Annular debridement and patch closure, mitral valve replacement, possible repair. Discussion of what was actually done and the challenges, deaths and complications encountered. (Unless case is still pending): There was dense pericarditis with purulent adhesions. The mitral valve contained a complex vegetation on the

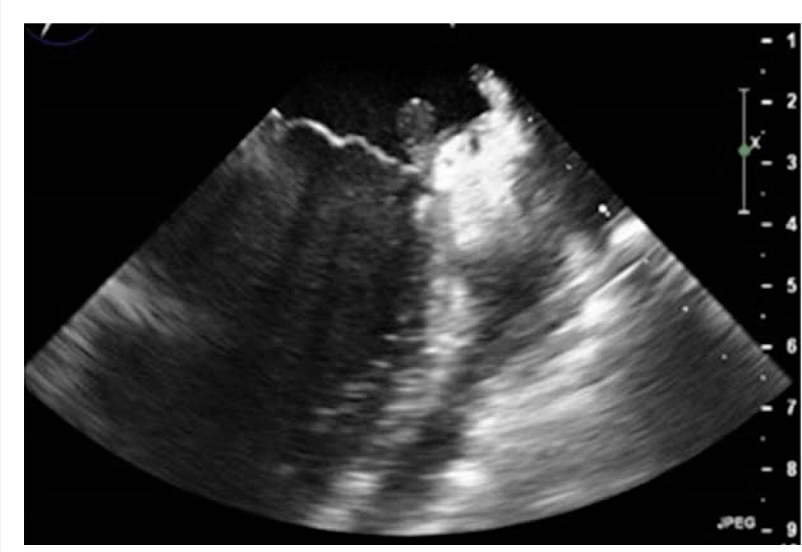

Pre-op TEE

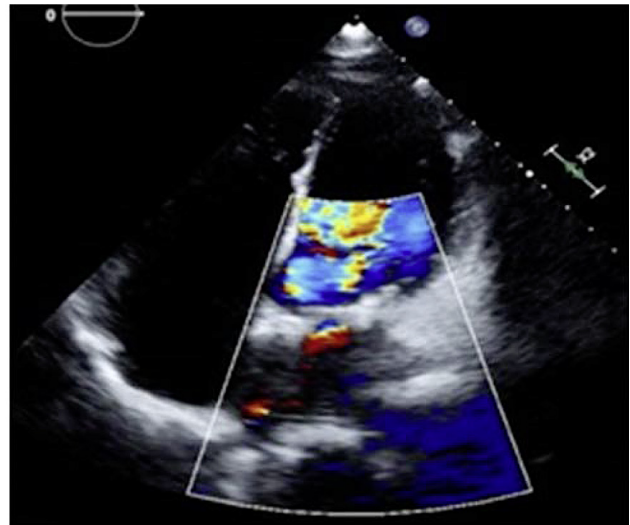

Post-op TEE

Fig. 1. (for Abstract of Alexander Iribarne). 
atrial and ventricular side of the annulus near the base of P1 and P2 consistent with early abscess. The posterior leaflet edges were intact and the anterior leaflet was not affected. P1 and P2 were detached, and the annulus and myocardium debrided below the valve. The resultant annular defect was covered with a $2.5 \times 1.5 \mathrm{~cm}$ bovine pericardial patch extending from the ventricular muscle and then along the atrial wall. Additional sutures were used along the ventricular edge of the patch to buttress the muscular suture line. Small vegetations on P3 were locally debrided. P1 and P2 were then sutured back to the neoannulus after augmentation of the base of P1 with additional pericardial patch. A $28 \mathrm{~mm}$ annuloplasty ring was placed. There were no complications. Post-op TEE demonstrated trace MR and 30 day follow-up TTE showed trace MR. This case demonstrates the technical challenges of mitral valve endocarditis with annular abscess. Even if a mitral replacement was performed, annular involvement would have likely resulted in an inferior outcome without aggressive debridement and patch closure. Chordal detachment and debridement with neo annular re-attachment offered this young patient a likely superior alternative to mitral replacement.

\section{Streptococcus Agalactiae Infective Endocarditis in a Post-Partum Female}

Vinod Chainani ${ }^{1}$, Yashwant Agarwal ${ }^{2}$, Vishal Gupta ${ }^{3}$

${ }^{1}$ Aventura Hospital and Medical Center, Miami, FL, ${ }^{2}$ Western Michigan University Homer Stryker School of Medicine, Kalamazoo, MI, ${ }^{3}$ Borgess Medical Center, Kalamazoo, MI, USA

Patient Demographics: 34-year old caucasian female. Relevant History: Postpartum day 13 with fever. Two sets of blood cultures, vaginal cultures, and cervical cultures grew streptococcus agalactiae. Examination revealed purulence at the vaginal suture site. Ceftriaxone therapy was started. Subsequent cultures didn't show any growth. During the hospital course, patient developed a petechial rash, right heart failure features, and acute kidney injury with a nephritic syndrome picture. Echocardiogram (ECHO) was performed on day seven of admission, which revealed tricuspid valve endocarditis and moderate tricuspid regurgitation. Conservative management continued in hope for the vegetation to regress to make valve repair more feasible. Patient improved clinically and was discharged with close outpatient follow up. However, her heart failure symptoms gradually worsened and she had to be started on digoxin. Pre-Operative Plan: Valvular repair/replacement was planned. In the interim, patient had to be admitted with possible septic embolism to the lungs. Tricuspid valve replacement was performed approximately two months after the initial presentation. The post-operative course was complicated by development of cardiac tamponade and intra-operative cardiac arrest during mediastinal exploration. Patient had a prolonged course in the Intensive Care Unit and eventually recovered. Discussion of what was actually done and the challenges, deaths and complications encountered. (Unless case is still pending): Streptococcus agalactiae infective endocarditis (IE) is rare, and is known to have an aggressive clinical course. It is more common in non-pregnant patients with underlying chronic immunosuppressive conditions. The incidence has been shown to be higher in older patients, and it has no gender preponderance. Incidence in the setting of pregnancy and parturition is very low. The possibility of streptococcus agalactiae IE should be given adequate consideration in postpartum females with positive blood cultures, especially in the presence of other clinical features suggestive of endocarditis. Review of literature suggest that streptococcus agalactiae IE is an aggressive disease; early detection with ECHO and early valvular surgery can reduce morbidity and mortality.

\section{Right-Sided Endocarditis in an Adult without Any History of Intravenous Drugs Abuse: A Case Report}

\author{
Vincenzo Caruso, Antonio Bivona, Haytham S. Elsayed, \\ Arvind V. Singh \\ Basildon and Thurrock University Hospital, Basildon, United \\ Kingdom
}

Patient Demographics: A 68 years old man was referred for urgent surgical treatment of a severe tricuspid valve regurgitation due to a right-side endocarditis. Relevant History: Patient presented with shortness of breath and fever without any significant past medical history except surgical correction of inguinal herniation of the urinary bladder eight months ago. He was not an intravenous drugs abuser. He was treated with oral antibiotic therapy for chest infection in community. He continued to deteriorate despite 15 days of treatment leading to admission to local hospital where intravenous antibiotics were started. Blood cultures identified Pseudomonas aeruginosa. Urine culture and flexible cystoscopy did not show any abnormality. A total body tomography scan was normal. A petechial rash was observed on his lower limb. Patient continued to worsen clinically spiking temperature, developed atrial fibrillation, oliguria and signs of right side heart failure. Transthoracic and trans oesophageal echocardiogram revealed $18 \times 16 \mathrm{~mm}$ vegetation on tricuspid valve causing a severe tricuspid regurgitation. Pre-Operative Plan: He was transferred to our tertiary care centre for close monitoring with a plan to continue antifailure treatment and antibiotics. Discussion of what was actually done and the challenges, deaths and complications encountered. (Unless case is still pending): Patient did not respond to conservative treatment with increasing shortness of breath at rest and escalating need of oxygen. Urgent surgery had to be performed for tricuspid valve endocarditic. During the operation a white opalescent friable vegetation was found attached to the anterior leaflet of the tricuspid valve. Posterior leaflet appeared to be completely destroyed. Small vegetations identified adherent to the anterior and inferior papillary muscle as well as adjacent right ventricular wall. This tricuspid valve could not be saved, all leaflets were excised, ventricular cavity was washed and tricuspid valve was replaced with a tissue valve. The postoperative course was uneventful without any complications. Patient was transferred back to referring hospital with a plan for 6 weeks of intravenous antibiotics. Right-side endocarditis in the absence of risk factors leads to diagnostic delays. This case report describes a tricuspid valve in an adult man without any risk factors necessitating tricuspid valve replacement. 


\section{Infective Endocarditis Requiring Complex Mitral Valve Repair Surgery}

\section{Jennifer Williams, Umair Aslam, Pankaj Kumar}

ABM NHS Trust, Swansea, United Kingdom

Patient Demographics: 23-year old man with past medical history of eczema. Relevant History: The patient presented with a 3 -week history of fevers, weight loss, abdominal pain with diarrhoea and vomiting, and confusion, seeminly preciptated after a take-away meal. Examination revealed a systolic murmur in the apex with signs of septic shock. Echocardiography revealed a 2x3 columnar vegetation on the posterior mitral leaflet. Subsequent to increasing confusion and right-sided hemiparesis, MR and CT revealed multiple cerebral septic emboli. Pre-Operative Plan: Multidisciplinary team input with microbiology and stroke teams were sought and the patient commenced on antimicrobial treatment to Staphylococcus aureus grown from blood cultures, and anti-platelet agent. Surgery was scheduled urgently and intra-operative examination from TEE and direct visualisation steered definitive management. Discussion of what was actually done and the challenges, deaths and complications encountered. (Unless case is still pending): $3 \times 3.5 \mathrm{~cm}$ PMVL vegetation was visualised with complete destruction of the P1 leaflet. The infected segment was excised and the leaflet reconstructed with a bovine pericardial patch, supported by PTFE neo-primary chords and a $30 \mathrm{~mm}$ annuloplasty ring. The patient had an unremarkable recovery and echocardiogram revealed no MR. This case juxtaposes the strive for repairing and retaining the native valve in a young patient with MR, with the principle of complete debridement of any infected-looking material in endocarditis surgery. In addition, the unusual nature of the vegetation with respect to suitability for repair is also discussed.

\section{Bailout Balloon Mitral Valvuloplasty for Severe Degenerative Mitral Stenosis in a Patient with Refractory Cardiogenic Shock and Severe Pulmonary Arterial Hypertension}

\author{
Vinod Chainani ${ }^{1}$, Dhruti Mankodi ${ }^{1}$, Enrique Gorin ${ }^{1}$, \\ Edward Savage ${ }^{2}$, Roberto J. Cubeddu ${ }^{2}$ \\ ${ }^{1}$ Aventura Hospital and Medical Center, Miami, FL, \\ ${ }^{2}$ Cleveland Clinic Florida, Weston, FL, USA
}

Patient Demographics: A 77 year-old obese Caucasian woman. Relevant History: Patient presents with acute decompensated heart failure resulting in respiratory failure requiring mechanical respiratory support. Echocardiographic examination showed densely calcified and degenerative mitral valve with mean gradient $19 \mathrm{~mm} \mathrm{Hg}$, with trace regurgitation and preserved left ventricular systolic function. Catheterization exhibited patent coronary arteries with severe pulmonary arterial hypertension (peak 92; mean $62 \mathrm{~mm} \mathrm{Hg}$ ), calculated Fick cardiac index of 1.9 and pulmonary vascular resistance of 8.2 Wood units. Pre-Operative Plan: Despite intensive medical therapy for 8 consecutive days with intravenous inotropic support, the patient remained in refractory shock, respiratory failure and was unable to wean off the ventilator. Secondary pre-renal azotemia ensued. Cardiothoracic surgical team evaluation deemed patient inoperable due to prohibitive risks. End of life support was reviewed with family. Bailout palliative PBMV was discussed and ultimately considered. Discussion of what was actually done and the challenges, deaths and complications encountered. (Unless case is still pending): PMBV was performed using standard transseptal approach with $26 \mathrm{~mm}$ Inoue balloon and a single balloon inflation at $24 \mathrm{~mm}$, that resulted in significant improvement in MVA from 1.1 to $1.7 \mathrm{~cm}^{2}$, with corresponding decrease mean gradient from 13 to $5 \mathrm{~mm} \mathrm{Hg}$ and drop in mean left atrial pressure from 25 to $14 \mathrm{~mm} \mathrm{Hg}$. The baseline mean pulmonary artery pressure of $70 \mathrm{~mm} \mathrm{Hg}$ markedly decreased to $54 \mathrm{~mm} \mathrm{Hg}$ immediately following PMBV. The degree of baseline mitral regurgitation of $1+$ remained unchanged. Over the followings 48 hours, the patient was successfully extubated, weaned off of all support, and subsequently discharged 5 days later in stable conditions. Three months later the patient underwent successful mitral valve replacement using a $27 \mathrm{~mm}$ Hancock porcine mitral valve. At three years of follow-up she remains asymptomatic, with estimated peak RVSP of $45 \mathrm{~mm} \mathrm{Hg}$, and properly functioning mitral bioprosthesis. Contrarily to calcific degenerative mitral stenosis (MS), percutaneous balloon mitral valvuloplasty (PBMV) is widely approved with acceptable results in the selected patient population with rheumatic heart disease only. As displayed by this case, palliative PBMV should be considered as a bridge to definitive MVR in selected inoperable patients.

\section{A Case of Mitral Valve Replacement in a Patient with Dextrocardia - A Review of the Literature}

\author{
Shawn Robinson, Berhane Worku, losif Gulkarov, \\ Anthony Acinapura, Anthony J. Tortolani
}

New York Methodist Hospital, Brooklyn, NY, USA

Patient Demographics: 84-year-old Hispanic male with diabetes and hypertension presented with syncope secondary to hypoglycemia. Relevant History: He was incidentally found to have elevated troponins with unremarkable electrocardiogram. Transthoracic echocardiogram and cardiac MRI demonstrated dextrocardia, situs inversus, and severe mitral regurgitation associated with severely reduced left ventricular function (ejection fraction 14\%) and left ventricular dilation. Cardiac catheterization was unremarkable. Pre-Operative Plan: Given patient's severe mitral regurgitation associated with severely reduced left ventricular function and left ventricular dilation, surgical intervention was planned. Discussion of what was actually done and the challenges, deaths and complications encountered. (Unless case is still pending): Via median sternotomy, cardiopulmonary bypass was established with ascending aortic and bicaval cannulation with the surgeon on the left side. Anterograde cardioplegia was utilized and the mitral valve was replaced via a transseptal approach with a $29 \mathrm{~mm}$ pericardial tissue valve. The patient required pacemaker placement for heartblock and was discharged on postoperative day seven. A literature search was performed with regards to mitral valve surgery for noncongenital etiologies in adults with dextrocardia, yielding a total of eight reports. Situs solitus was present in 


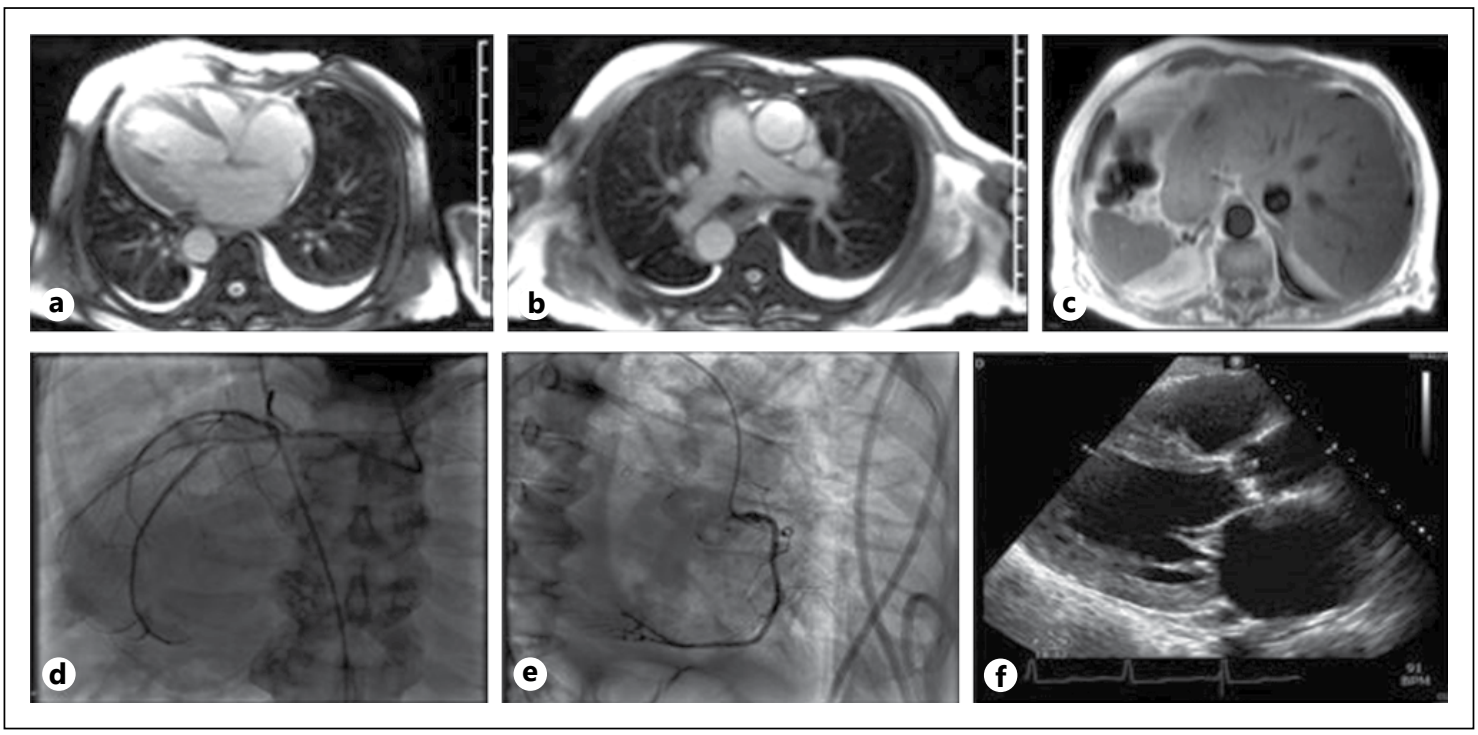

Fig. 1. a Cardiac MRI view of our patient's heart (note mirror position). b Cardiac MRI view of our patient's great vessels (note mirror position). c MRI view of patients intraabdominal organs (note mirror position). d, e Cardiac cath images of our patient. $\mathbf{f}$ Echocardiographic view of our patient's mitral and aortic valves (for Abstract of Shawn Robinson).

$63 \%$. Median sternotomy was utilized in $100 \%$ of cases with the surgeon standing on the left. Central cannulation was utilized exclusively in six with a combination of peripheral and central cannulation techniques in the remaining two due to difficult anatomy. The mitral valve was accessed via the left atrium in six and via a transeptal approach in the remaining two and replaced in all cases. All cases had uncomplicated postoperative courses. Available evidence suggests that mitral valve replacement may be performed safely in patients with dextrocardia, but careful planning with chest and abdominal imaging is mandatory to assess for the presence of situs inversus or other abnormalities which may alter cannulation strategy. The left side appears to be the preferred side for the surgeon to stand, and left atrial or transeptal approaches may both be utilized. Larger studies are required to make further conclusions.

\section{Thoracic Aortic Dissection Combined with Dilated Cardiomyopathy in a Patient with Autosomal Dominant Polycystic Kidney Disease}

\section{Harveen K. Lamba, Steven Plato, Yakov Elgudin}

University Hospitals Case Medical Center, Cleveland, $\mathrm{OH}$, USA

Patient Demographics: 34 year old white male, end stage renal disease (ESRD), autosomal dominant polycystic kidney disease (ADPKD). Relevant History: 34 year old man presented with dyspnea on exertion and cough productive of grey sputum. Transesophageal echocardiography showed ejection fraction (EF) of $20 \%$ and possible vegetation. He was treated medically for heart failure and culture negative endocarditis. Upon readmission for

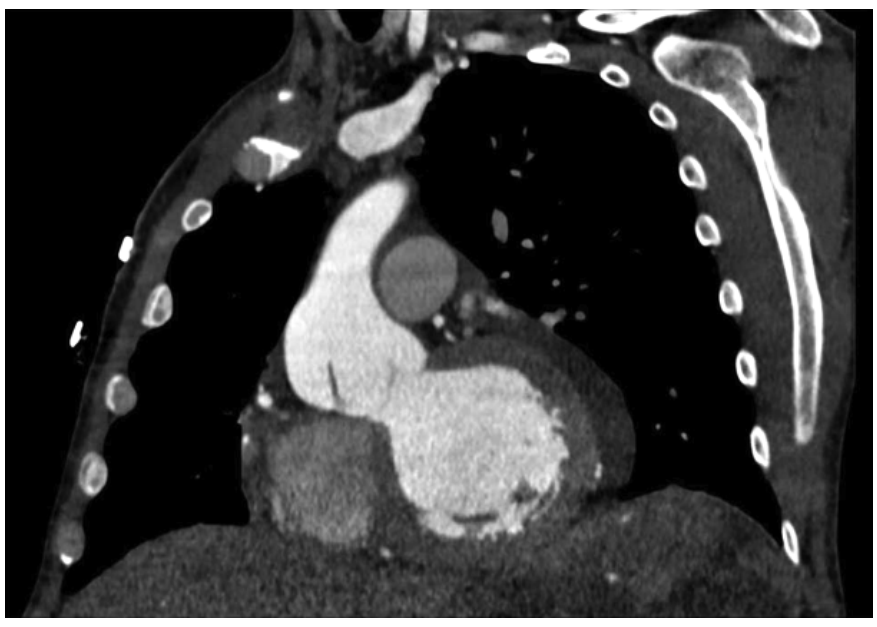

Fig. 1. CT angiography showing large aortic root aneurysm with dissection into right coronary (for Abstract of Harveen K. Lamba).

exacerbation, echocardiography showed linear opacity in the proximal aorta suggestive of focal dissection and EF of $25 \%$. CT angiography demonstrated aortic root dilation to $5.6 \mathrm{~cm}$ with sinotubular junction effacement and a linear structure in the proximal ascending aorta extending into the right coronary sinus (See figure 1). Pre-Operative Plan: Echocardiography and CT findings consistent with type A dissection. Plan to replace ascending aorta with tube graft. Discussion of what was actually done and the challenges, deaths and complications encountered. (Unless case is still pending): Dissection included the right coronary sinus 
of valsalva, but spared valve cusps and coronary arteries. Valve sparing aortic root replacement was completed without any complications. He tolerated surgery well and was discharged home. ADPKD is a common inherited disorder, responsible for $6-10 \%$ of cases of ESRD. However, the leading cause of morbidity and mortality in ADPKD is cardiovascular; hypertension, heart valve lesions, cerebral aneurysms, aortic root dilation, and abdominal aortic aneurysm. Thoracic aortic dissection has been rarely reported. Also rare, ADPKD can be associated with heart failure due to idopathic dilated cardiomyopathy (IDCM). Mutations in PKD1 and PKD2 genes cause most cases of ADPKD and are thought to cause loss of vascular integrity and heart failure. Often, patients with ADPKD and aortic aneurysms also have underlying Marfan syndrome, a genetic disorder of connective tissue caused by fibrillin-1 deficiency. Interestingly, our patient does not have a diagnosis of Marfan. Our patient presents a unique and rare constellation of symptoms associated with ADPKD; polycystic kidney disease, IDCM and thoracic aortic dissection without underlying Marfan. No reports of these co-existing conditions exist to our knowledge.

\section{Valve-in-Valve TAVI for Regurgitant Sutureless Aortic Prosthesis}

Shuli Silberman, Yaron Almagor, Jonathan Balkin, Arik Wollak, Daniel Fink, Ofer Merin, David Rosenmann, Daniel Bitran

Shaare Zedek Medical Center, Jerusalem, Israel

Patient Demographics: 48 year old woman, history of chest irradiation for Hodgkin lymphoma in the past. Relevant History: Four months previously was admitted for mitral and aortic valve replacement. The mitral valve was replaced with a mechanical valve. The aortic annulus was calcified, and found to be too small to accomodate a mechanical valve, therefore a sutureless bioprosthesis was implanted. The patient was presently admitted due to severe CHF with evidence of severe AI (central and paravalvular). CT scan showed kinking of the stent of the implanted aortic valve resulting in both central and paravalvular leakage. Pre-Operative Plan: Options for reoperation included replacement of the failing aortic prothesis. Implantation of a second valve was not considered because of the anatomy of the annulus. Replacement of the aortic root was an option, but was considered high risk beacause of heavy calcification of the root and coronary ostia. Discussion of what was actually done and the challenges, deaths and complications encountered. (Unless case is still pending): Trans-cutaneous aortic valve implantation (valve-in-valve TAVI) using a balloon-expandable valve was undertaken. The procedure was done under general anesthesia. Access was via the femoral artery and TEE (2-D and 3-D) was employed to guide positioning and to avoid entering the para-valvular space. The patient was prepared for femoro-femoral bypass in the event of hemodynamic collapse. The procedure was uneventful and the patient was extubated several hours later.

\section{How Low Can You Go? < 4 mm Transfemoral Transcatheter Aortic Valve Replacement with Balloon-Expandable, Recollapsible Sheath}

\author{
Syed Zaid, Anoshia Raza, Gilbert Tang, Hasan Ahmad, \\ Cenap Undemir, Martin Cohen
}

Westchester Medical Center, Valhalla, NY, USA

Patient Demographics: 88 year old male with hypertension; stage-III chronic kidney disease; bilateral severe ilio-femoral stenosis; moderate COPD; pulmonary hypertension; atrial fibrillation and prior CABG. Relevant History: Patient was experiencing claudication and NYHA-III heart failure with low-flow, low-gradient severe aortic stenosis, aortic valve area $0.63 \mathrm{~cm}^{2}$ and mean gradient $20 \mathrm{~mm} \mathrm{Hg}$. Cardiac catheterization demonstrated severe triple coronary disease with occluded LAD, significant proximal RCA stenosis and LCx stenosis with patent LIMA to LAD. Pre-Operative Plan: Severe aortic stenosis was recommended for treatment prior to iliac artery stenting. STS score was $9.47 \%$ for reoperative AVR and was deemed prohibitive risk given patient's comorbidities and frailty. Combined TAVR and iliac stenting procedure was therefore considered. Patient was deemed unsuitable for alternative access given comorbidities and hostile mediastinum with only one patient LIMA supplying coronaries. Transfemoral approach with iliac balloon angioplasty was considered to enable insertion of $11 / 19$-French balloon-expandable, recollapsible Terumo Solopath sheath, which would facilitate advancement of the Medtronic-CoreValve delivery system for implantation. Bilateral iliac stenting was planned after the valve delivery system was removed, given risk of stent migration if iliac stenting were to precede TAVR. Discussion of what was actually done and the challenges, deaths and complications encountered. (Unless case is still pending): Bilateral balloon angioplasty was performed at the common iliac arteries, but the Solopath sheath was unable to advance past the right common iliac artery. Instead, the Solopath sheath tip was successfully positioned at the aorto-iliac bifurcation via left ilio-femoral artery. During initial deployment of $29 \mathrm{~mm}$-Evolut-R-CoreValve across the aortic valve, patient developed cardiac arrest with successful resuscitation. The Evolut-R-CoreValve was recaptured and withdrawn. After hemodynamics improved, second attempt to deploy CoreValve again resulted in severe hypotension. CoreValve was withdrawn and procedure was aborted. Completion aortoiliac angiography after Solopath sheath removal demonstrated no vascular complication, following successful deployment of both iliac stents. The patient required 7 days of ICU stay with inotropic support and was discharged uneventfully to rehabilitation facility after 10 days. 


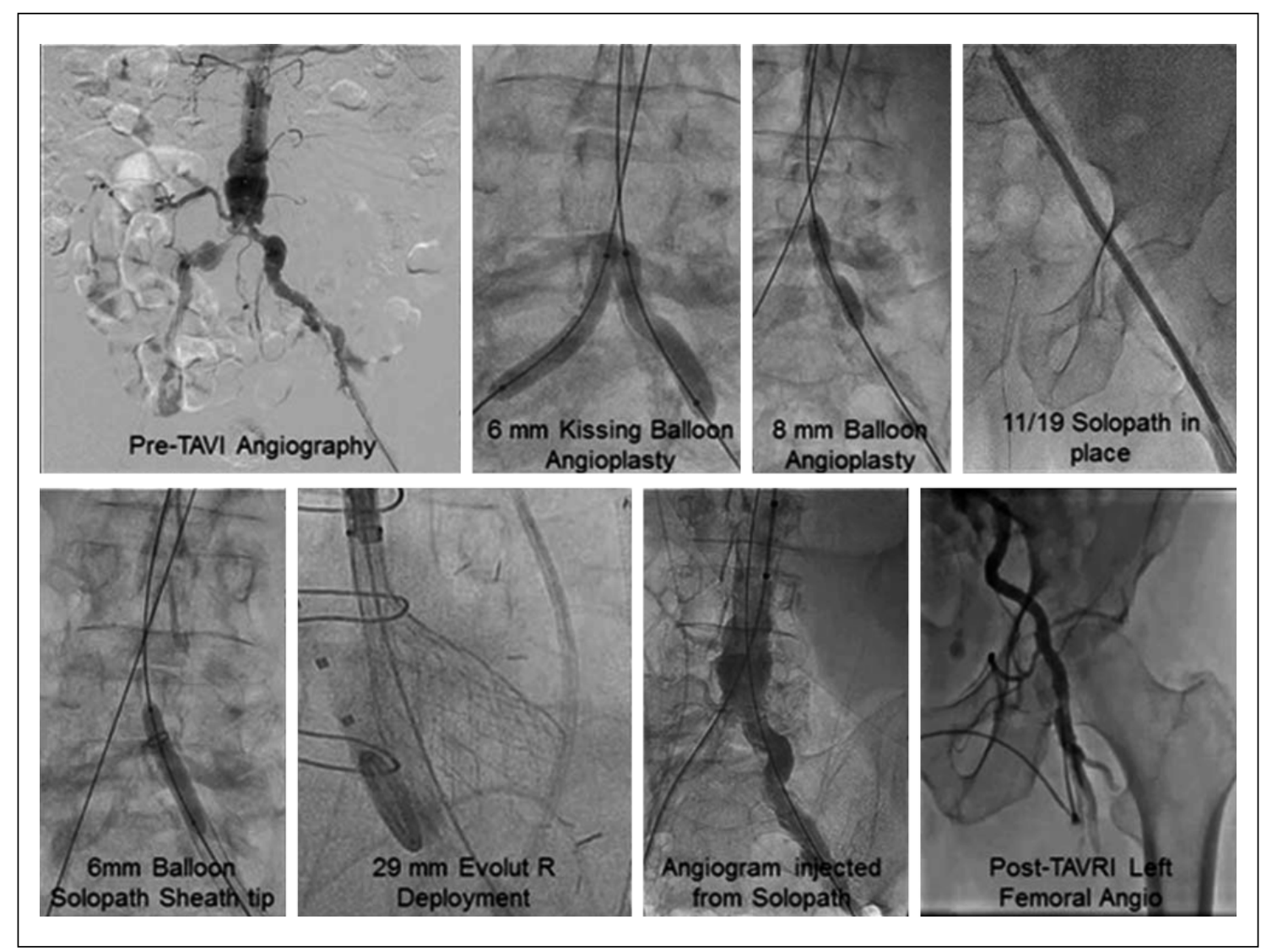

Fig. 1. (for Abstract of Syed Zaid).

\section{How to Do It: Bail-Out Transapical/Transaortic Valve Implantation in No-Option Patients on the ECMO}

Nikolaos Bonaros, Markus Kofler, Thomas Schachner, Daniel Höfer, Johannes Holfeld, Wolfgang Franz, Michael Grimm, Guy Friedrich

Medical University of Innsbruck, Innsbruck, Austria

Patient Demographics: Two male patients 60 and 74 years old respectively with severe aortic stenosis (mean gradients 40 and 55 respectively) and severly impaired left ventricular function $(\mathrm{EF}=24$ and $18 \%$ respectively) were presented to our department after several episodes of congestive heart failure. Relevant History: The first patient had a history of previous CABG with open grafts was oxygen dependent, with a moderate COPD and a severe pulmonary hypertension. The second patient had a systolic pulmonary pressure of $85 \mathrm{~mm} \mathrm{Hg}$ and a coronary artery disease with a proximal LAD stenosis. Pre-Operative Plan: Both patients were turned down for conventional surgery by major departments in the country and were scheduled for transcatheter valve implantation. The first patient was scheduled for a transapical implantation under femoro-axilar venoarterial ECMO because of limitations for vascular access. The second patient was planned for coronary bypass to the LAD and a transaortic implantation under femoro-femoral
ECMO. Discussion of what was actually done and the challenges, deaths and complications encountered. (Unless case is still pending): Both procedures were performed without intraoperative complications as scheduled. The video focuses on patients' selection and the pitfalls and tricks of the implantation technique as well as weaning from the ECMO. Both patients left the hospital after 14 and 18 days respectively. The postoperative echo showed an excellent implantation result and an improved left ventricular function.

\section{Pulmonic Valve Papillary Fibroelastoma as Seen on Echocardiography, Cardiac Magnetic Resonance Imaging and at Surgery}

Prasanthi Sunkesula, Berhane Worku, Shawn Robinson, losif Gulkarov, Anthony Acinapura, Anthony Tortolani

New York Methodist Hospital, Brooklyn, NY, USA

Patient Demographics: A 62 year old male with hypertension, diabetes mellitus, obesity, and coronary stenting presented with chronic dyspnea and pedal edema. Relevant History: Workup included echocardiography and cardiac magnetic resonance imaging which revealed normal ventricular function and a $16 \times 14 \mathrm{~mm}$, 


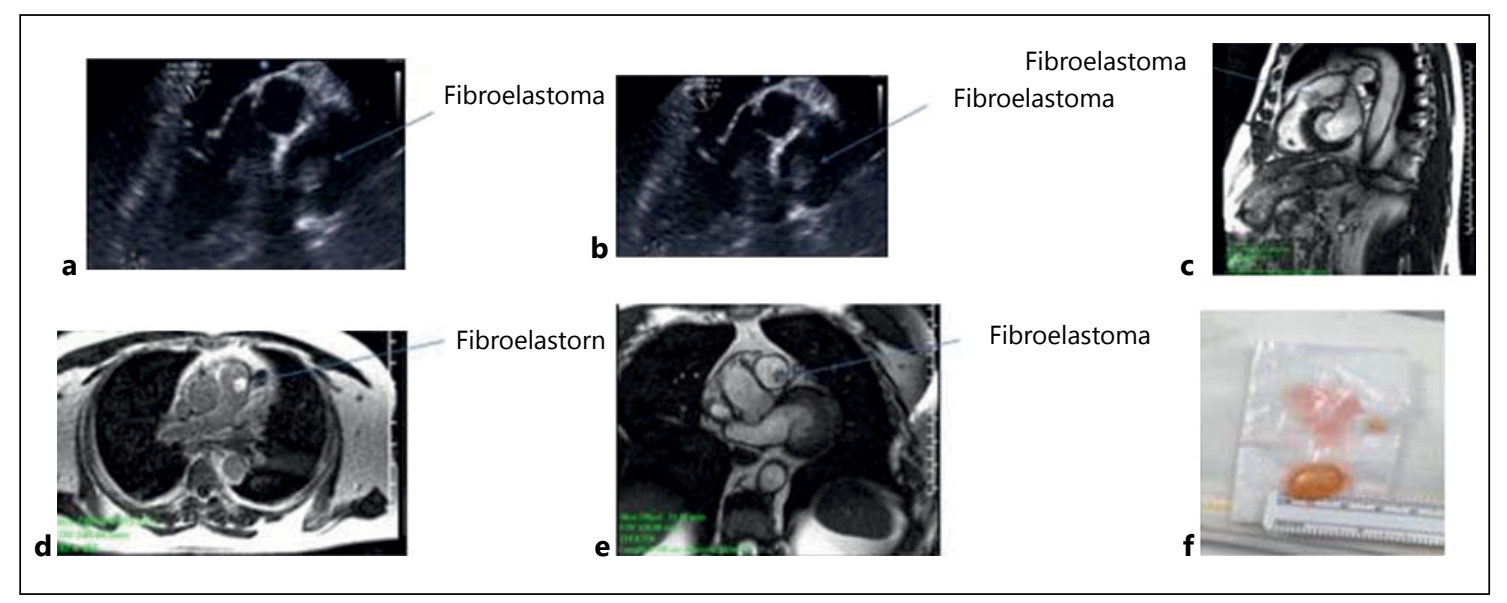

Fig. 1. a, b Transesophageal echocardiogram demonstrating a $16 \times 14 \mathrm{~mm}$, pedunculated, mobile hyperechogenic mass attached to the pulmonic aspect of the pulmonic valve with no regurgitation. c-e Cardiac magnetic resonance imaging demonstrating a $12 \times 11 \times 9 \mathrm{~mm}$ mobile mass on the left cusp of the pulmonary valve. f Surgical specimen (for Abstract of Shawn Robinson).

pedunculated, mobile mass attached to the pulmonic aspect of the pulmonic valve with no regurgitation, likely a papillary fibroelastoma (PFE). Cardiac catheterization demonstrated non obstructive coronary artery disease. Pre-Operative Plan: Surgical excision was recommended to prevent embolic complications. Discussion of what was actually done and the challenges, deaths and complications encountered. (Unless case is still pending): The patient underwent surgical excision via sternotomy with cardiopulmonary bypass and aortic crossclamping. The mass was excised via a pulmonary arteriotomy with sparing of the native valve. The patient recovered uneventfully and was discharged home on postoperative day seven. Final pathologic analysis revealed a PFE. PFE is the second most common primary cardiac tumor after atrial myxoma, accounting for approximately $7 \%$ of cases and representing $70 \%$ to $80 \%$ of heart valve tumors. The majority are found on the aortic (44.5\%) and mitral valves (36.4\%). When found in the right heart, the tricuspid valve is affected more commonly than the pulmonic valve. With regards to pulmonic valve PFE, the mean age at detection is 60 years, with an equal distribution amongst both sexes. A combination of imaging modalities including cardiac magnetic resonance imaging was useful to make a preoperative diagnosis with reasonable certainty. Given his low surgical risk, excision was performed to prevent potential embolic complications. Although the literature regarding pulmonic valve PFE consists mainly of case reports, the available evidence suggests that low risk patients with large $(>1 \mathrm{~cm})$, mobile lesions undergo surgical excision for diagnosis and cure. There is no definitive data on the efficacy of anticoagulation in this scenario.

\section{Minimal Invasive Bicuspid Aortic Valve Repair with External Anuloplasty}

Thomas Schachner, Julia Dumfarth, Ludwig Mueller, Michael Grimm, Nikolaos Bonaros

Innsbruck Medical University, Innsbruck, Austria

Patient Demographics: 45 year old male patient. Relevant History: Dyspnea on exertion, Severe regurgitation of a bicuspid aortic valve type $1 \mathrm{LR}$, aortic anulusdilatation $30 \mathrm{~mm}$. Pre-Operative Plan: Minimal invasive aortic valve repair or mechanical aortic valve replacement. Discussion of what was actually done and the challenges, deaths and complications encountered. (Unless case is still pending): Partial upper sternotomy and cannulation of aorta and right atrium. Aortic valve repair with cusp plications (fig. 1), superficial resection of localised raphe - cusp thickening. External aortic anuloplasty with tailored ring of a $32 \mathrm{~mm}$ Dacron prosthesis which is fixed by subanular sutures (fig. 2). Postoperative TEE showed completely competent valve. Uneventful postoperative course. HD video of all important steps of the operation is shown.

\section{Aortic Valve Stenosis in Patient with Hypereosinophilic Syndrome}

\author{
Harveen K. Lamba' ${ }^{1}$, Salil V. Deo', Salah E. Altarabsheh", \\ Yakov Elgudin', Soon J. Park ${ }^{1}$ \\ ${ }^{1}$ University Hospitals Case Medical Center, Cleveland, OH, USA; \\ ${ }^{2}$ Queen Alia Heart Institute, Amman, Jordan
}

Patient Demographics: 61 year old Indian male, Multiple Myeloma (MM), Atrial Fibrillation (AF), Hypereosinophilic syndrome (HES), Non-bacterial Endocarditis. Relevant History: 61 year old male with known MM presented with new onset of dys- 

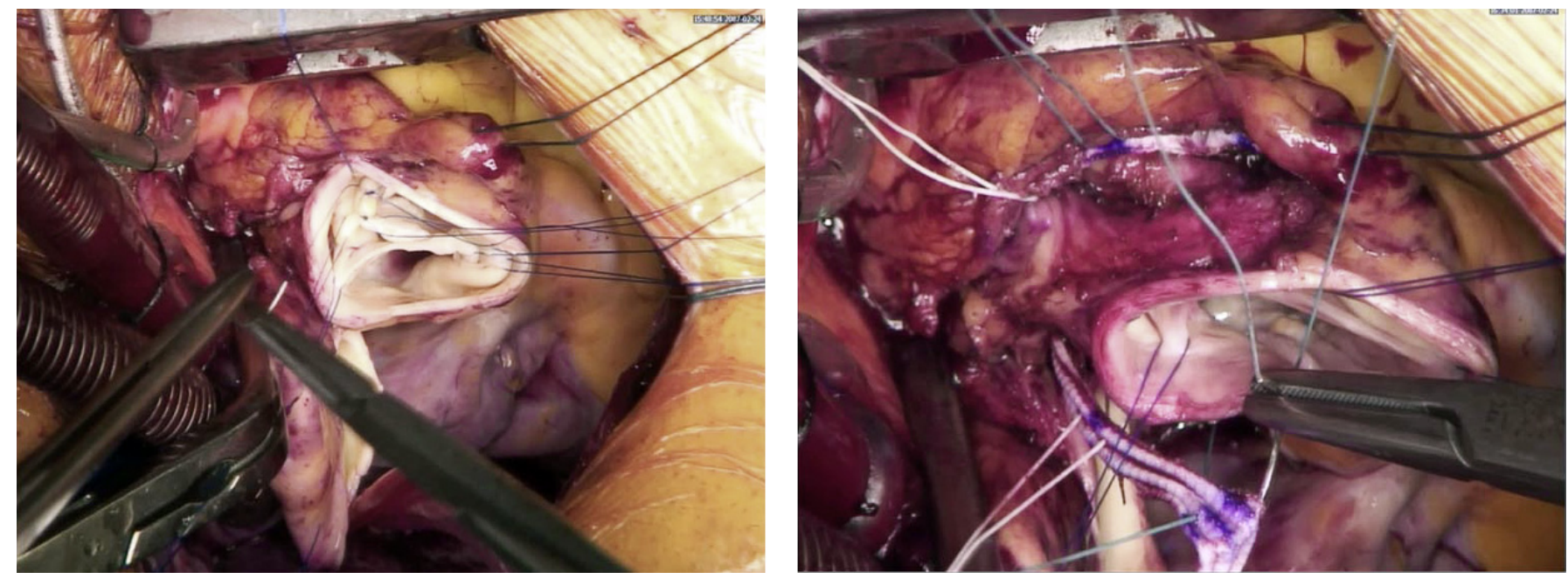

Fig. 1. (for Abstract of Thomas Schachner).
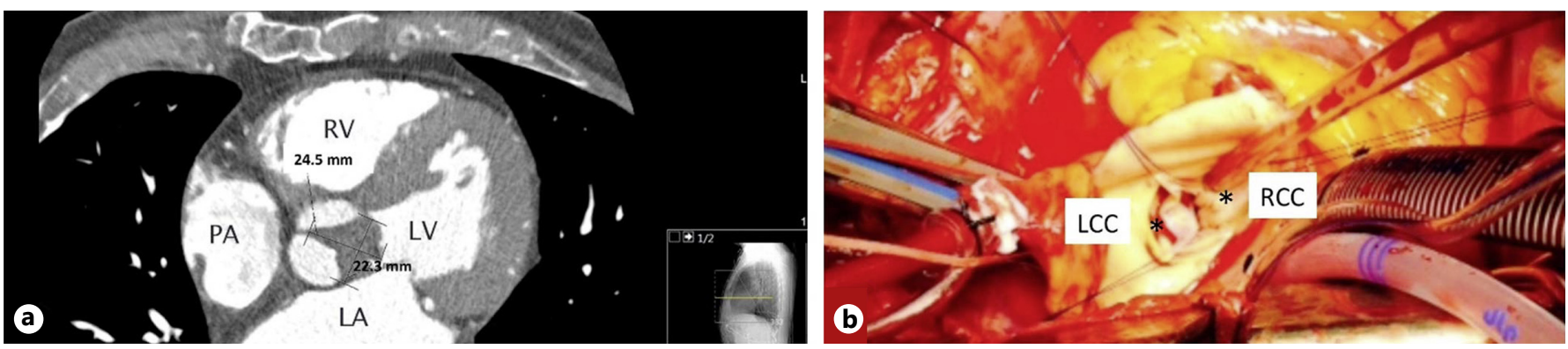

Fig. 1. CT angiography $(\mathbf{a})$ and intraoperative picture $(\mathbf{b})$ demonstrating large mass $\left(^{*}\right)$ on the right coronary and non-coronary cusp of the aortic valve. (for Abstract of Harveen K. Lamba).

pnea, chest pain upon exertion, and syncope. Workup demonstrated HES and a $24.5 \times 22.3 \mathrm{~mm}$ mass on the right coronary and non-coronary cusps of the aortic valve on CT angiogram (CTA) (figure 1a). Transthoracic echocardiography demonstrated severe aortic stenosis with peak gradient of $85 \mathrm{~mm} \mathrm{Hg}$ and aortic insufficiency grade of 2 . Left ventricular function was preserved with ejection fraction of $60 \%$ and no regional wall motion abnormality. Pre-Operative Plan: Laboratory, echocardiography and CTA findings consistent with atrial myxoma secondary to HES with otherwise normal coronary anatomy. Plan for aortic valve replacement (AVR) with bio-prosthetic valve. Discussion of what was actually done and the challenges, deaths and complications encountered. (Unless case is still pending): Inspection of the aortic valve revealed diffusely thickened valve cusps with spongynodular nodular masses almost obliterating the orifice (figure 1b). AVR performed with $19 \mathrm{~mm}$ Perimount Magna Ease valve without any complications. Patient tolerated surgery well and was discharged to home on post operative day 12 with dual anticoagulation therapy. Pathology report confirmed inflammatory process consistent with HES. Follow up echocardiography at one year demonstrated no recurrence of myxoma. HES is a rare hematological entity characterized by elevated number of eosinophils in the bone marrow and peripheral circulation with varying involvement of multiple organ systems. Cardiac involvement in HES involves formation of granulation tissue resulting in restrictive cardiomyopathy and valvular dysfunction of mainly mitral and tricuspid valves with concurrent aortic valve involvement. To our knowledge, this case is unusual in that the atrio-ventricular valves were spared and only the aortic valve was affected. We show that a bio-prosthetic valve is safe choice in HES patients requiring valve replacement.

\section{Triple Valve Endocarditis in a IV Drug User with Hepatic and Renal Insufficiency - Appropriate Resource Allocation or Exercise in Futility?}

\section{Joshua S. Hall, Sreekumar Subramanian}

TriStar Centennial Medical Center, Nashville, TN, USA

Patient Demographics: The patient is a 32 year old white man with a history of hepatitis $\mathrm{C}$, ascites and polysubstance abuse. Relevant History: He had a prior history of a right empyema requiring decortication. He presented with persistent MSSA and fungal endocarditis of his aortic, mitral and tricuspid valves with septic em- 
bolization to his brain, and had significant liver and renal dysfunction. He self-referred to our institution from a hospice facility. PreOperative Plan: Given the extensive endocarditis, polysubstance abuse history, and combined hepatic and renal dysfunction, the patient was considered to have a $90 \%$ mortality with operative intervention. Cardiology evaluation felt operation could be refused, based on overall poor prognosis. After a frank discussion of the risks and benefits, a salvage triple valve operation was offered, as operative extirpation was felt to be the only chance at survival. Discussion of what was actually done and the challenges, deaths and complications encountered. (Unless case is still pending): A median sternotomy was performed, and a triple valve replacement using conventional bioprostheses for the aortic and mitral valve replacement. The tricuspid valve was replaced using an extracellular matrix cylinder. Due to the severely dilated tricuspid valve annulus, the patient developed dehiscence of the ECM cylinder with recurrent severe TR and profound RV dysfunction on postoperative day 1 . This necessitated return to the OR for explantation of the cylinder and tricuspid valve replacement with a conventional bioprosthesis and intra-aortic balloon pump placement. The patient was discharged home at 2 weeks after surgery. At 3 months postoperatively, he has been hospitalized for recurrent ascites requiring paracentesis, and a subarachnoid bleed with recurrent bacteremia, but has spent the majority of time at home with family.

\section{Pulmonary Artery Aneurysm Evolving in Fatal Consequences}

\section{Giulio Tessitore, Giuseppe Petrone, Mario Fabbrocini}

Policlinico di Monza - Heart Centre - Città di Alessandria, Alessandria, Italy

Patient Demographics: We describe the case of a 66-years-old caucasian male presented with sudden onset of chest pain described as severe and associated with a month-long history of progressive shortness of breath. Relevant History: He had a history of pulmonary thromboembolism and deep vein thrombosis known since 1999 and successfully treated by the combination of Inferior Vena Cava Filter installation and anticoagulant therapy. Pre-Operative Plan: General examination was unremarkable. There were no signs of cardiac failure. Chest radiography revealed enlargement in the right heart silhouette. The Transthoracic echocardiography showed an aneurysm of the main pulmonary artery (PA) measuring approximately $5.9 \mathrm{~cm}$ and dilatation of the left and right pulmonary arteries. Enlargement of the left atrium and the right heart chambers as well as moderate tricuspid regurgitation were detected. The PA aneurysm was not associated with pulmonary regurgitation. Post-contrast chest computed tomography (CT) was done and showed a dilated main PA with a maximum diameter of $6 \mathrm{~cm}$ and its 2 branches, with a huge (partially calcified) aneurysm of the right branch (about $24 \times 14 \mathrm{~cm}$ ) partially occupied by a mural thrombus compressing the left atrium, together with marked pericardial effusion. Surgical correction of the aneurysm with placement of a pulmonary allograft was recommended, but was refused by the patient. Discussion of what was actually done and the challenges, deaths and complications encountered. (Unless case is still pending): Pericardiocentesis was undertaken on day 2 , immediately followed by subxiphoid drainage, wherein 2500 cc of fluid was aspirated. On day 3 , the patient suddenly developed marked dyspnea and tachycardia, and passed into shock with marked hypoxemia and hypocapnia, suggesting a massive pulmonary embolism. The echocardiography revealed an intimal flap noted in the lateral wall of the pulmonary trunk, with suggestion of flow within the flap. Then, he went into cardiac arrest and died. Pathologic examination showed mild myxoid degeneration of the endothelial surface and confirmed the presence of dissection within the aneurysmal wall. Clinical presentation of pulmonary aneurysms remains nonspecific and dissection must be suspected in the face of sudden hemodynamic decompensation in a patient with documented pulmonary artery aneurysm.

\section{Partial Dehiscence of a Mitral Valve Annuloplasty Ring}

Sara M. Negrotto, Ana Kadkhodayan, Jeffrey B. Geske

Mayo Clinic Rochester, Rochester, MN, USA

Patient Demographics: 79 year old Caucasian male.

Relevant History: 79 year old male with history of previous tricuspid bioprosthesis for severe tricuspid regurgitation presented to clinic for evaluation of worsening dyspnea and lower extremity edema, found to have hypotensive response with exercise. Echocardiogram showed a normally-functioning tricuspid prosthesis, but severe mitral regurgitation that was difficult to quantify. He had severe left atrial enlargement with pulmonary vein systolic reversals and LVEF 59\%. Therefore, he underwent mitral valve repair using a 34-mm Carbomedics annuloplasty band. Three months postoperatively, he developed worsening dyspnea and lower extremity

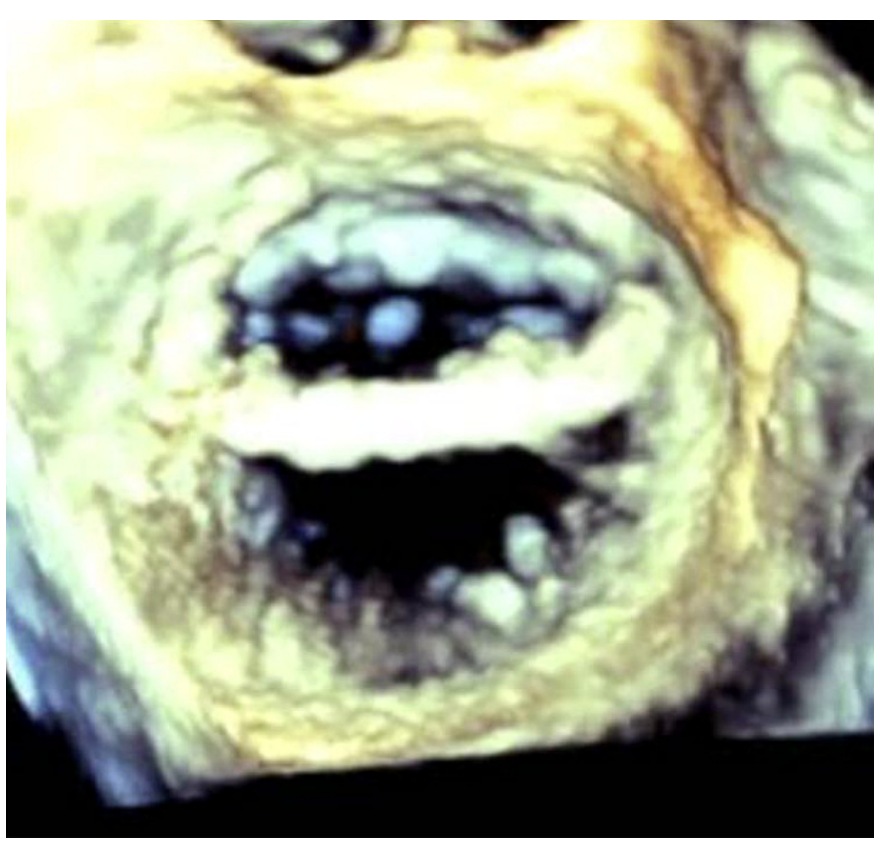

Fig. 1. 3D reconstruction of mitral valve on TEE. (for Abstract of Sara M. Negrotto). 
edema with repeat echocardiogram showing partial dehiscence of the mitral annuloplasty band anteriorly with recurrent moderate mitral regurgitation (PISA 37cc); no endocarditis. There was no history to suggest recent infection; blood cultures were negative. Pre-Operative Plan: Evaluation for MitraClip versus third sternotomy and mitral valve replacement. Discussion of what was actually done and the challenges, deaths and complications encountered. (Unless case is still pending): He was evaluated for MitraClip with transesophageal echocardiogram, but the dehisced portion of the annuloplasty ring was directly over the mitral valve on the atrial side with only 3 to $4 \mathrm{~mm}$ between the ring and the valve plane. Given the close proximity making placement of the clip challenging, he underwent a third sternotomy and mitral valve replacement with a 33-mm Hancock mitral bioprosthesis 6 months after his last operation. On postoperative day 4 , he developed hypotension and significant increase in bloody chest tube output. Echocardiogram showed small-moderate pericardial effusion but no classic signs for tamponade. He was taken back to the OR for exploration, evacuation of blood clots in mediastinum and pleural spaces, and ligation of bleeding sites. He was dismissed home on postoperative day 9 with symptomatic improvement. Echocardiogram at time of dismissal showed a normal functioning mitral prosthesis with a mildly elevated gradient in the setting of postoperative anemia with only trivial mitral regurgitation; EF 57\%.

\section{Perceval Bioprosthesis Intraoperative Echocardiographic Assessment: Impact of Hemodynamics to Evaluate the Residual Intra-Valvular Regurgitation}

\section{Stefano Moriggia, Igor Belluschi, Fabrizio Monaco,} Ottavio Alfieri

San Raffaele University Hospital, Milano, Italy

Patient Demographic: A 74 years old woman was admitted at our Centre with severe aortic stenosis. Cardiovascular risk factors were: obesity (BMI 31.6), arterial hypertension and dyslipidemia. The patient suffered from COPD with a history of reccurent bronchitis. EuroSCORE II was 1.48\%, STS predicted mortality was $2.044 \%$ and STS mortality and morbidity $15.35 \%$. Relevant History: Pre- operative transoesophageal echocardiography (TOE) showed EF $61 \%$, transaortic gradient $77 / 51 \mathrm{~mm} \mathrm{Hg}$, planimetric aortic valve area $0.7 \mathrm{~cm}^{2}$ (indexed area $0.38 \mathrm{~cm}^{2} / \mathrm{m}^{2}$ ). The aortic annulus was $20 \mathrm{~mm}$. Mild mitral regurgitation (MR) and mild-to-moderate tricuspidal regurgitation were observed. Severe left ventricular hypertrophy was present. The coronary angiogram did not revealed coronary artery stenosis. Preoperative Plan: To minimize cardiopulmonary bypass and cross-clamp time, a minimally invasive approach via ministernotomy with a sutureless aortic valve replacement (SU-AVR) with a Perceval valve was planned. Discussion: An M-size Perceval prosthesis was implanted and after aortic cross-clamp removal, the intraoperative TOE showed appropriate aortic prosthesis position and no paravalvular leakage. Since the initial weaning from CPB, a continuous TOE was performed. With hypovolemia and low blood pressure (BP: $80 / 40 \mathrm{~mm} \mathrm{Hg}$ ), echocolor-doppler images revealed an intraprosthetic central residual mild-to-moderate AR (fig. 1a) with left ventricular kissing. After hemodynamic optimization with vasoconstricor agents and fluid challenge achieving normal BP (130/80 mm $\mathrm{Hg}$ ), we observed a gradual but complete resolution of the central AR (fig. 1b, c). The PVLs with the Perceval prosthesis are no longer an issue after the recommendation of complete annular decalcification. Nevertheless, a certain quota (trivial-to-moderate) of central AR can be observed with pericardial bioprosthesis. Moreover, with the Perceval valve, the sizing and the correct expansion of the device may play a role in the intraprosthetic AR. In conclusion, in case of central AR during a SU-AVR with the Perceval bioprosthesis, hemodynamic tests which could increase the blood pressure are important for the correct assessment of the residual regurgitation.

\section{The Challenges of Assessing Dysfunction of a Mechanical Prosthesis in the Context of Heart Failure and Atrial Fibrillation}

\section{Anthony Barron, Shelley L. Rahman Haley, Lydia Sturridge Toufan Bahrami \\ Royal Brompton and Harefield NHS Foundation Trust, Harefield, United Kingdom}

Patient Demographics: A 59 year old lady with a background history of mechanical mitral valve replacement with a MedtronicHall tilting disc and tricuspid annuloplasty for rheumatic mitral

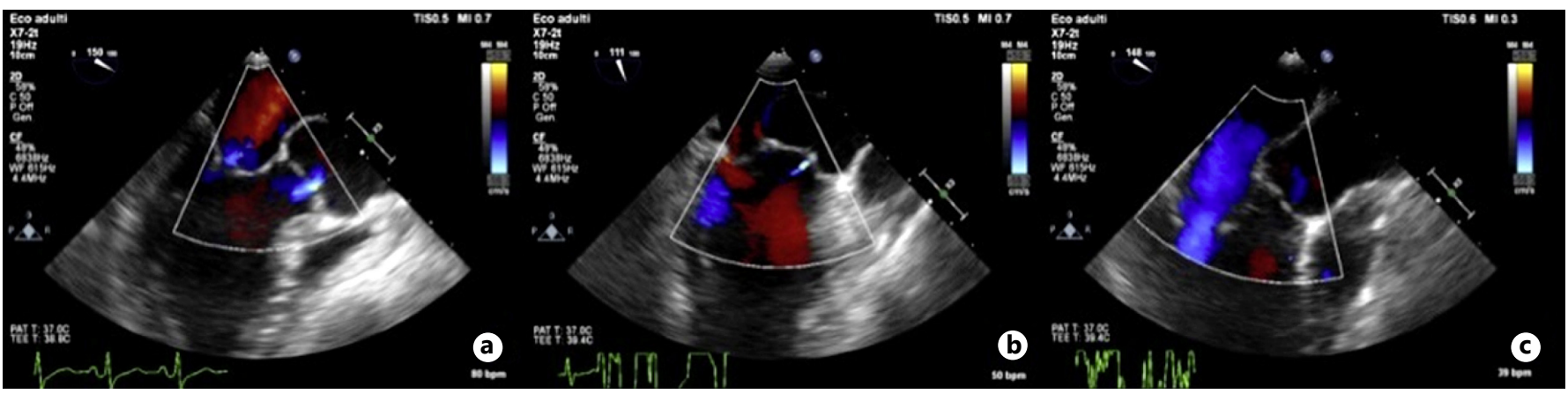

Fig. 1. (for Abstract of Stefano Moriggia). 
stenosis and tricuspid regurgitation 30 years previously in Hong Kong. Relevant History: She had developed increasing shortness of breath over a number of months. She had been in atrial fibrillation for 30 years, with a history of previous pulmonary embolism, treated right breast cancer and a transient ischaemic attack. Her clinical examination revealed signs of pulmonary hypertension and right heart strain, with reduced volume of her mechanical 1st heart sound. She had a microcytic anaemia in keeping with haemolysis. Transthoracic echocardiogram (TTE) suggested intermittent failure of transmitral flow, with intermittent faint transmitral Doppler VTIs. Transoesophageal echocardiogram (TOE) confirmed intermittent obstruction of the mitral valve single tilting disc, which failed to open on every beat with no relation to the varying RR interval: obstruction-alternans. RT3DTEE suggested pannus, predominantly anteriorly on the atrial side of the valve. There was moderate tricuspid regurgitation. Pre-Operative Plan: The patient was discussed in a tertiary centre multidisciplinary team joint cardiology/cardiothoracic meeting and re-do mitral valve replacement and tricuspid valve repair was agreed. Discussion of what was actually done and the challenges, deaths and complications encountered. (Unless case is still pending): Following sternotomy and cardioplegia, the left atrium was opened and the mitral valve was found to be covered in pannus and was therefore unable to fully close. This was completely removed with insertion of a $33 \mathrm{~mm}$ mechanical Carbomedics valve with interrupted sutures. The tricuspid valve was repaired with removal of the previous De Vega sutures and Teflon, and the insertion of a $30 \mathrm{~mm}$ Physio III D Edwards annuloplasty ring. The left atrial ap- pendage was occluded with a $50 \mathrm{~mm}$ AtriClip after confirmation of a thrombus. The patient made a successful recovery. Postoperative transthoracic echocardiogram showed a well-seated mitral valve replacement without regurgitation, and only mild residual tricuspid regurgitation. The patient's symptoms steadily improved and she was discharged home on standard heart failure therapy.

\section{Percutaneous Transcatheter Repair of Bioprosthetic Mitral Valve Dehiscence and Associated Severe Mitral Regurgitation}

\author{
Manavjot Sidhu' ${ }^{\text {, Anmol Dhillon }}{ }^{2}$, Leon Varjabedian ${ }^{1}$, \\ Marcel Zughaib ${ }^{1}$, Martin Alpert ${ }^{3}$
}

${ }^{1}$ Providence Heart Institute, Providence Hospital, Southfield, MI, ${ }^{2}$ University of Michigan, Ann Arbor, MI, ${ }^{3}$ University of Missouri, Columbia, MO, USA

Patient Demographics: A 76 year old female with past medical history of mitral valve replacement with $25 \mathrm{~mm}$ bioprosthetic valve for severe mitral regurgitation and mild mitral stenosis. Relevant History: A year after surgery, patient presents with progressive dyspnea. Physical examination was positive for a grade $2 / 6$ ejection systolic murmur heard best at left upper sternal border. TTE showed a left ventricular ejection fraction of $60 \%$ and a normal functioning mitral valve prosthesis with mean \& peak gradients of

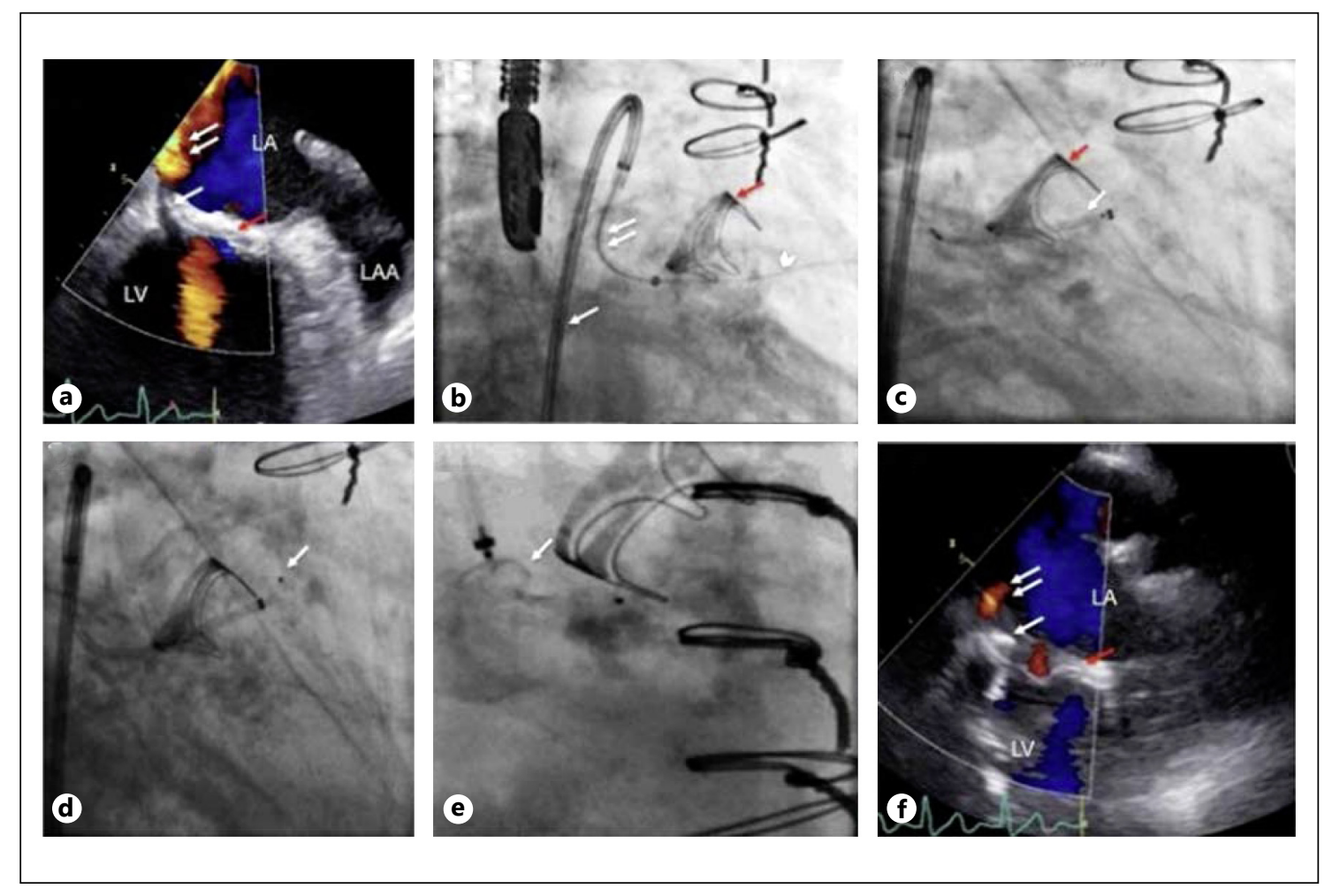

Fig. 1. (for Abstract of Manavjot Sidhu). 
5 and $15 \mathrm{~mm} \mathrm{Hg}$ respectively. No mitral valve regurgitation was noticed. Due to her continued complaints of shortness of breath, a TEE was performed. On careful examination of the color flow Doppler imaging, a prominent jet of mitral insufficiency measuring 6 meters/second directed towards the posterior left atrial wall was detected. This jet was coming from a defect located posterior to the sewing ring of the bioprosthetic valve confirming a severe paravalvular mitral regurgitation. Additionally, the valve seemed to have a back and fourth motion consistent with prosthetic mitral valve dehiscence. Pre-Operative Plan: She was referred for right heart catheterization, valve fluoroscopy and repair of this paravalvular defect under TEE and fluoroscopic guidance. On valve fluoroscopy, a pericardial bioprosthesis was identified in the mitral position. A back and forth (rocking) motion of the mitral valve prosthesis confirmed mitral prosthesis dehiscence. Right heart catheterization showed mean pulmonary artery pressure of $45 \mathrm{~mm}$ $\mathrm{Hg}$. Discussion of what was actually done and the challenges, deaths and complications encountered. (Unless case is still pending): The mitral valve dehiscence and an associated severe peravalvular regurgitation was treated with a 6 X6 ADO II Amplatz vascular plug.

\section{Acute Myocardial Infarction Secondary to Giant Lambl's Excrescence of the Aortic Valve}

\section{Pallavi Khattar ${ }^{1}$, Puneet Bedi ${ }^{2}$, John T. Fallon ${ }^{1}$}

${ }^{1}$ Westchester Medical Center, Valhalla, NY, ${ }^{2}$ Brookdale University and Medical Center, Brooklyn, NY, USA

Cardiac papillary fibroelastomas (CPFEs) are rare benign tumours of the endocardium and represent the most common primary valvular tumours of the heart. CPFE is sporadically reported with an incidence between $0.002 \%$ and $0.33 \%$ at autopsy. Although many papillary fibroelastomas do not cause symptoms, early diagnosis of CPFE is of prior importance to prevent patients from fatal complications. Nevertheless, this tumour can present with a variety of clinical manifestations, making diagnosis challenging. We report an unusual case of aortic valve papillary fibroelastoma, which was found on autopsy in a $64 \mathrm{y} / \mathrm{o}$ male patient with evidence of myocardial ischemic damage. Histopathological examination of heart revealed a $1.5 \times 1.0 \mathrm{~cm}$, bulky tan-white non-encapsulated, rubbery lesion comprised of multiple papillary fronds on the aortic valve. The tumour mass completely occluded the right coronary ostium. An acute inferior wall myocardial infarction was also present. Histology showed a benign papillary lesion comprised of a single layer of endocardial cells overlying a thin layer of mucopolysaccharide matrix and underlying, almost acellular, avascular stroma composed predominantly of elastic fibres and a small amount of collagen. Transesophageal echocardiography is known to have high sensitivity to detect excrescences and should always be included in the diagnostic assessment. Asymptomatic patients who are found to have evidence of CPFE should be monitored closely. Here, we describe a rare case of acute myocardial infarction secondary to cardiac papillary fibroelastomas of the aortic valve completely occluding the right coronary ostium leading to sudden cardiac death.
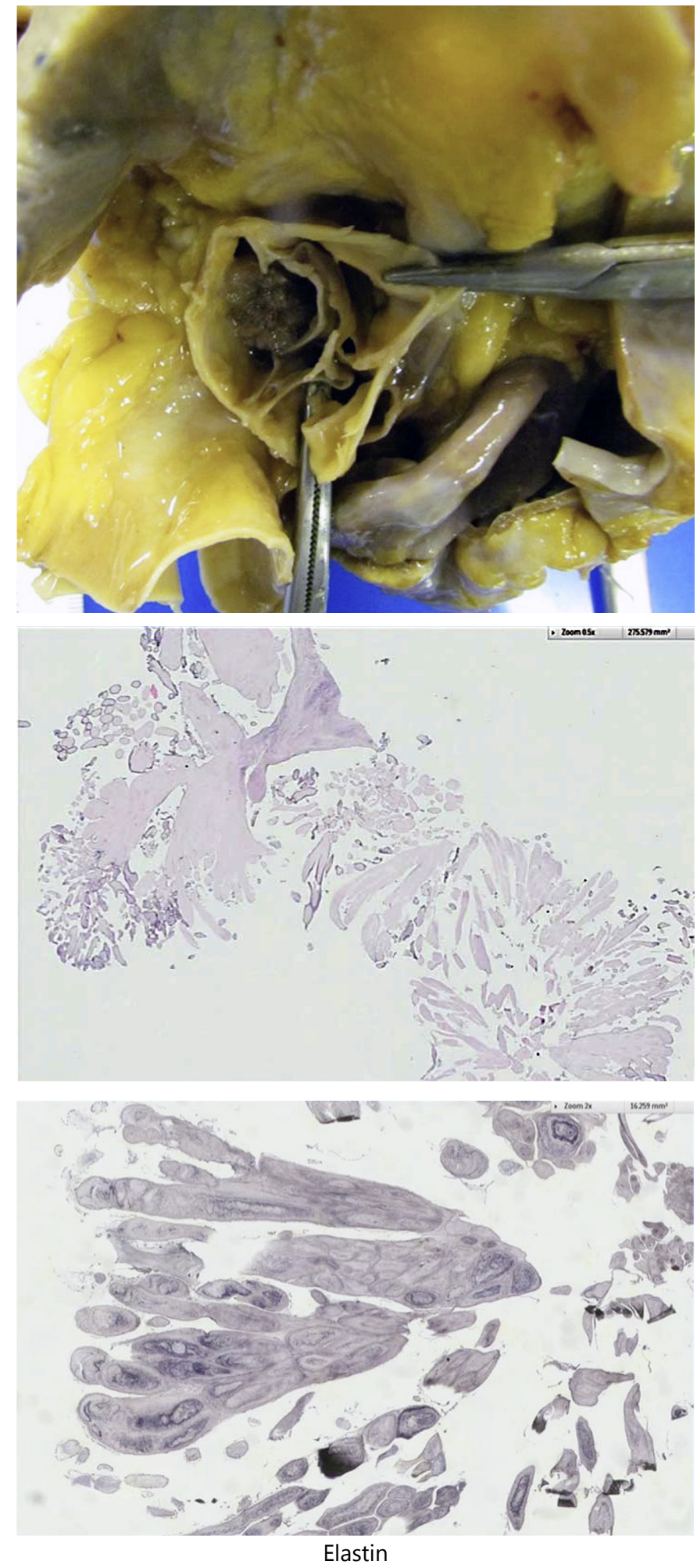

Fig. 1. (for Abstract of Pallavi Khattar). 


\section{Fibroelastoma Recurrence in Left Ventricle: Rarity of Primary Cardiac Tumor}

\section{Inna Kammerer, Reinhard Besser, Falk-Udo Sack}

Department of Cardiac Surgery Academic City Hospital

Ludwigshafen, Ludwigshafen, Germany

Objective: Cardiac papillary fibroelastoma (CPF) is a primary cardiac neoplasm usually detected by echocardiography. Left ventricular fibroelastomas are extremely rare. The incidence of $\mathrm{CPF}$ is to be found between $0.0017-0.33 \%$ during autopsy studies. Methods: We report a case of a 70 years old male patient who was re-operated with a bioprosthesis mi-tral valve replacement in 2014. The reason for the re operation was the recurrence of papillary fibroelastoma which was primarily discovered in 2005. The tumor was rapidly growing with increase in size over the follow-up period year 2013-2014. Due to the location and nature of the recurrent tumor, mitral valve replacement was treatment of choice in order to prevent a third recurrence of the fibroelastoma. Results: Due to the location and nature of the recurrent tumor, mitral valve replacement was treatment of choice in order to prevent a third recurrence of the fibroelastoma. Conclusions: The patient was discharged from the hospital on 9 th post operative day.

\section{An Odd Case of Hypertrophic Cardiomyopathy}

Colleen E. Lane, Hector I. Michelena, Maurice Enriquez-Sarano

Mayo Clinic, Rochester, Rochester, MN, USA

Patient Demographics: A 46 year-old male with hypertension, diabetes and recently diagnosed hypertrophic obstructive cardiomyopathy (HOCM) was referred from an outside hospital with diffuse alveolar hemorrhage and transfusion requiring anemia. Relevant History: Two weeks earlier, he presented to an outside hospital with persistent cough, progressive dyspnea, and hemoptysis. He required intubation for hypoxia. Bronchoscopy demonstrated diffuse alveolar hemorrhage with lavage negative for infection/malignancy. Due to continued hypoxia, a lung biopsy was performed which showed hemosiderosis without capillaritis. He was extubated and transferred to our care. On arrival, he was asymptomatic and hemodynamically stable. Exam showed no neck vein distention, systolic murmur and lower extremity edema. Chest x-ray demonstrated diffuse bilateral infiltrates. Laboratory demonstrated hemolytic anemia. Comprehensive autoimmune panel was negative. The night of admission, he became acutely hypoxic responding to IV diuresis. A transthoracic echocardiogram demonstrated hyperdynamic LV function, HOCM with SAM-induced severe mitral regurgitation and resting peak outflow gradient of $88 \mathrm{~mm} \mathrm{Hg}$. Right-left heart catheterization after diuresis revealed LVEDP of $22 \mathrm{~mm} \mathrm{Hg}$ at rest and wedge of $25 \mathrm{~mm} \mathrm{Hg}$, consistent with left-sided diastolic failure. Pre-Operative Plan: Despite diuresis and beta-blockade, the nightly paroxysmal dyspnea, hemolytic anemia and hemoptysis persisted. Discussion of what was actually done and the challenges, deaths and complications encoun- tered. (Unless case is still pending): The patient underwent surgical septal myectomy with resolution of outflow obstruction and mitral regurgitation. Two months after surgery, he was asymptomatic with complete resolution of hemoptysis, hemolysis, and dyspnea. This case demonstrates an atypical presentation of HOCM, in which alveolar hemorrhage, mechanical hemolysis and resulting anemia were the debuting features. HOCM with severe obstruction and severe mitral regurgitation may present with episodic flash pulmonary edema leading to respiratory insufficiency. In our patient, these episodes of acute pulmonary hypertension resulted in bland alveolar hemorrhage. Additionally, his resting outflow obstruction $(>50 \mathrm{~mm} \mathrm{Hg}$ ) caused significant RBC shear stress resulting in hemolysis. Hemoptysis secondary to bland alveolar hemorrhage is a rare sign of LV heart failure and should be considered if there is evidence of elevated left sided filling pressures or valve disease. The presence of mechanical hemolytic anemia in patients with HOCM is rare but must be recognized.

\section{Simple and Reliable Technique in Barlow Disease, Bileaflet Foldoplasty; Report of a case}

Toshinobu Kazui, Badi Rawashdeh, Phat L. Tran, Zain I. Khalpey

The Univeristy of Arizona/Banner Univeristy Medical Center Tucson, Tucson, AZ, USA

Patient Demographics: A 37-year-old male with no previous significant history. Relevant History: The patient had become short of breath over the few months with exertion. His transthoracic echocardiography (TTE) demonstrated severe mitral valve regurigitation (MR) with anterior and posterior leaflet prolapse and billowing with redundant but no torn chordae. Aortic valve was normal. There was trace tricuspid valve regurgitation. Left ventricular ejection fraction was $58 \%$, Left ventricular end-diastolic dimension $5.5 \mathrm{~cm}$, Left ventricular end-systolic dimension $4.0 \mathrm{~cm}$. Based on the TTE, he underwent mitral valve repair. The patient discharged post-operative day 6 . The TTE at 6 months of follow up demonstrated no MR. Pre-Operative Plan: Application of foldoplasty to anterior and posterior leaflet to reduce the leaflet height, and to repair the prolapsed segments, and ring annuloplasty was planed. Discussion of what was actually done and the challenges, deaths and complications encountered. (Unless case is still pending): The saline test demonstrated no torn chords, a leak from anterolateral commissure, and prolapse in P1, P2, and A2. The thick leaflets with multiple segments of prolapse billowing suggested a Barlow disease. The annuloplasty ring stitches were placed at the beginning. The posterior leaflet height was confirmed to be longer than $15 \mathrm{~mm}$. A double-armed 4-0 polypropylene sutures were inserted from the leaflet edge to the annulus on the A2. The foldoplasty was also applied to P1 and P2 to reduce the posterior leaflet height by at least $50 \%$. A annuloplasty ring ( $34 \mathrm{~mm}$ Physio II Ring) was implanted. The saline test demonstrated leak from the anterolateral commissure and A2-P2 coaptation. This was repaired with a figure-of-eight commissuroplasty stitch using a 5-0 polypropylene suture and edge-to-edge repair on A2-P2 with a 4-0 polypropylene suture. The MR diminished after the repair. Foldoplasty is a simple and reproducible technique. It effectively lowers the height 
of the redundant leaflet and creates an optimal coaptation zone without leaflet resection. This case demonstrated feasibility of this technique to Barlow disease. Furthermore, the pre-operative plan was easy and reproducible.

\section{Severe Aortic Paravalvular Regurgitation Treated with Valve-in-Valve Implantation through Brachiocephalic Trunk}

\author{
Mauro Del Giglio, Fausto Castriota, Diego Magnano, \\ Marco Panzavolta, Marco Paris, Elisa Mikus
}

Maria Cecilia Hospital, GVM for Care \& Research, Cotignola (Ra), Italy

Patient Demographics: In this case report, we detailed a case of successful transcatheter aortic valve implantation (TAVI) in a 71-year old male affected by diabetes mellitus and hypertension with an history of coronary artery disease (occlusion of right coronary artery), carotid artery disease (Previous Ischemic Stroke, and left carotid artery endarterectomy), Leriche Syndrome with occlusion of both iliac-femoral axes and renal insufficiency (glomerular filtrate $30 \mathrm{ml} / \mathrm{min} / 1.73 \mathrm{~m}^{2}$ ). The challenge in this case was the patient's surgical approach through a brachiocephalic trunk. Relevant History: Since March 2014 a diagnosis of severe aortic stenosis complicated by pulmonary edema and heart failure was done. Due to estimated high operative risk a TAVI approach was chosen and a $29 \mathrm{~mm}$ CoreValve implanted. This procedure was performed through a trans-aortic access because of subclavian axes were not suitable due to a small diameter and iliac-femoral vessels diseased. A mild-moderate residual aortic regurgitation after valve implantation was present before discharge. In January 2015 evidence of severe paraprosthetic aortic regurgitation complicated again by acute pulmonary edema and heart failure (EF 30\%). Pre-Operative Plan: The Heart Team decided for a valve in valve procedure using a surgical approach through a brachiocephalic trunk avoiding the resternotomy thanks to a sovraclavear skin incision. A 29 mm CoreValve again was implanted uneventfully under fluoroscopic and transoesophageal echocardiography guidance. Patient was discharged 8 days after the operation without aortic regurgitation. Discussion of what was actually done and the challenges, deaths and complications encountered. (Unless case is still pending): A possible late complication of TAVI procedure is the prosthesis migration towards the left ventricle, causing worsening of post-procedural aortic regurgitation. A valve-in-valve implantation is a good therapeutic option for this paraprosthetic aortic leaks. Our experience shows that surgical brachiocephalic access is feasible in case of previous transaortic access or in absence of femoral and subclavian accesses.

\section{Leaflet Perforation Cause of Endocarditis}

Marcelo J. Figueroa a , Jose L. Serra ${ }^{\text {b }}$, Jorge O. Bronzia

${ }^{a}$ Cardiac Surgery, ${ }^{a}$ Electrophysiology Department, Sanatorio Allende, Cordoba, Argentina

Patient Demographics: The use of electronic devices including permanent pacemakers (PPM), implantable cardioverter-defibrillators (ICD) and cardiac resynchronizations therapy (CRT) has become an important tool for the treatment of various cardiac pathologies. The indications are growing. The infection of these devices is a serious and potentially fatal, especially in elderly patients with comorbid conditions. Relevant History: The case is a patient of 62 years, Caucasian, who was implanted a dual chamber PPM five years ago, in a health institution in another province. She was referred to our institution by the presence of fever of more than 2 months. Blood cultures were negative. A transthoracic echocardiogram showed the presence of vegetation of more than $4 \mathrm{~cm}$ at the level of the tricuspid valve, with moderate tricuspid regurgitation. Pre-Operative Plan: The patient was prepared for surgery, which would be consisting in a infective lead extraction and tricuspid valve repair, and implant of definitive epicardial leads. Median sternotomy, bicaval cannulation and antegrade cardioplegia was used. The most important finding was the presence

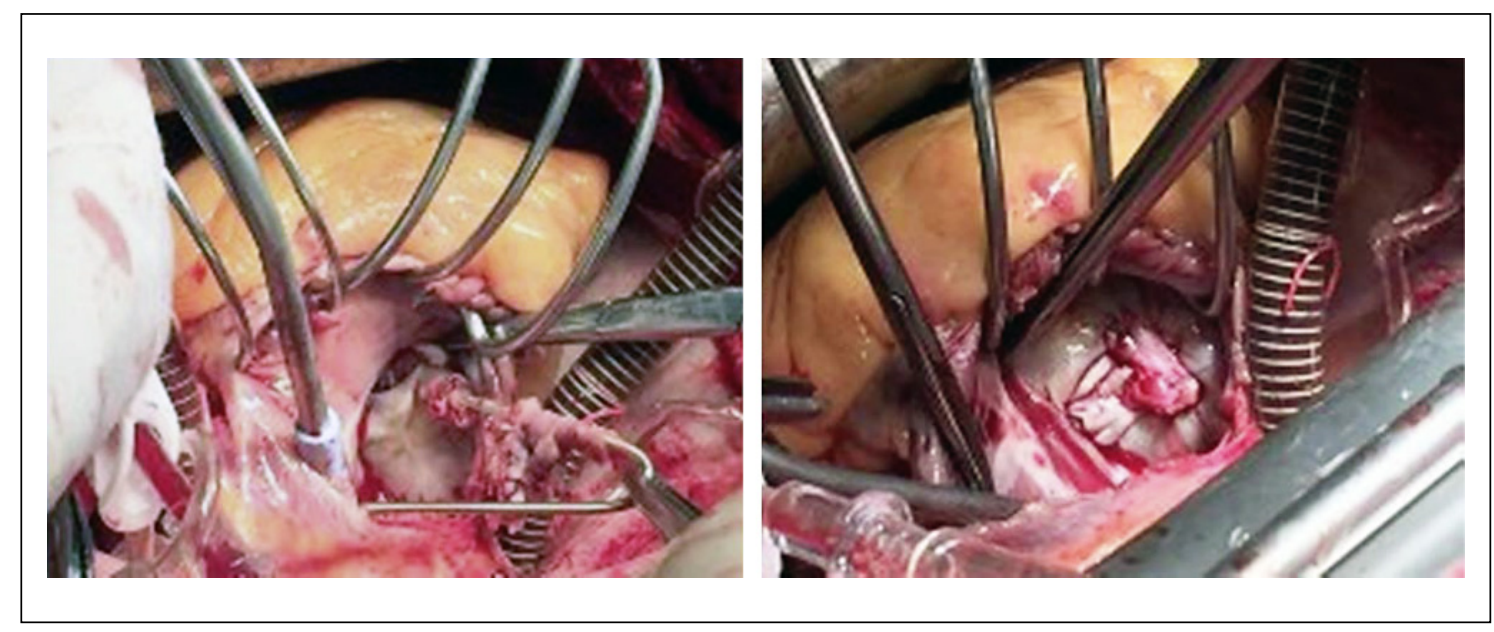

Fig. 1. (for Abstract of Marcelo J. Figueroa). 
of a perforation in the posterior leaflet, produced by the ventricular lead with large vegetations on the ventricular and atrial side, as shown in the photo attached. We performed a lead extraction and a tricuspid valve repair, which consisted of resection of the edges of the perforation and placement of untreated autologous pericardial patch. The patient had an uneventfullness recovery and was discharged at POD 5. Discussion of what was actually done and the challenges, deaths and complications encountered. (Unless case is still pending): The cultures of the extracted material were negative, due to prolonged antibiotic therapy. In this case, the important thing is that the perforation of the leaflet is a starting place of infection.

\section{Combined Upper Extremity Complex Percutaneous Coronary Intervention and Transcatheter Aortic Valve Replacement with No Alternative Access}

\section{Syed Zaid, Anoshia Raza, Gilbert Tang, Hasan Ahmad, Cenap Undemir, Martin Cohen}

Westchester Medical Center, Valhalla, NY, USA

Patient Demographics: 68 year old male with symptomatic severe aortic stenosis, NYHA class-II heart failure symptoms and Canadian cardiovascular society grade-III angina was referred for transcatheter aortic valve replacement. Relevant History: Past medical history included hypertension, stage-III COPD by GOLD criteria, HIV, severe peripheral vascular disease with prior right

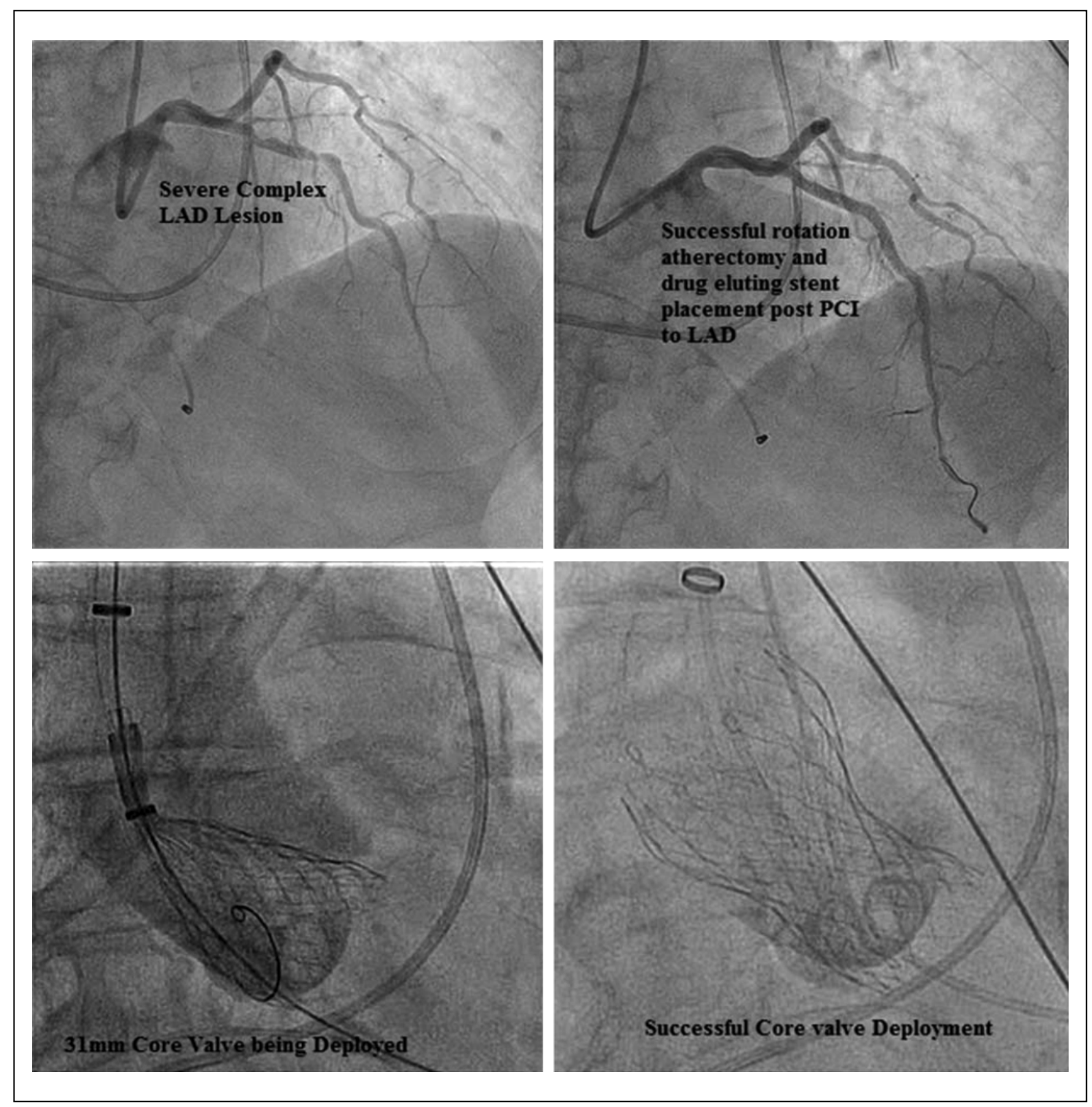

Fig. 1. (for Abstract of Syed Zaid). 
axillary-femoral and femoral-femoral artery bypass with occluded abdominal aorta, cerebrovascular disease with carotid stenosis and pulmonary hypertension. The predicted operative mortality for surgical aortic valve replacement based on the Society for Thoracic Surgeons score and EuroScore II was $4.55 \%$ and $2.92 \%$ respectively, but he was deemed inoperable given his history and predicted risks. Pre-Operative Plan: Cardiac catheterization demonstrated $90 \%$ mid left anterior descending artery stenosis. An attempt at percutaneous coronary intervention was unsuccessful during diagnostic catheterization due to patient agitation. Computed tomography showed the patient was a candidate for transaxillary TAVR. The patient's habitus and comorbidities made him unsuitable for transaortic or transapical approach while his peripheral anatomy eliminated the possibility of a transfemoral approach due to the occlusion of abdominal aorta. A combined PCI + TAVR was decided to minimize the risk of myocardial ischemia during the procedure. Discussion of what was actually done and the challenges, deaths and complications encountered. (Unless case is still pending): Using a right brachial surgical access, the left anterior descending artery underwent rotation atherectomy and drug-eluting stent placement with excellent angiographic results. A $31 \mathrm{~mm}$ Medtronic CoreValve was successfully implanted via the left axillary artery using the Gore Dryseal introducer sheath placed into the ascending aorta. The patient was discharged home uneventfully in 3 days. Echo at one month showed trivial paravalvular aortic regurgitation and valve area $1.72 \mathrm{~cm}^{2}$. $\mathrm{He}$ is currently NYHA-class I, physically active, with no further cardiac symptoms.

\section{Quadricuspid Aortic Valve - Case Report and Literature Review}

\section{Ana Paula K. Nobrega, Amarildo B. Almeida \\ Hospital Beneficencia Portuguesa De Sao Paulo, Sao Paulo, Brazil}

Objective: Quadricuspid aortic valve is a rare congenital anomaly often requiring aortic valve replacement. Prompt symptom recognition is pivotal. Surgeons should be aware of the possibility of anatomical variations. The objective is to make cardiologists and surgeons aware of the malformation and anomalys that come within. Methods: Performed a retrospective review of patients diagnosed with quadricuspid aortic valve. Results: There have been, approximately, 200 cases described in the literature. This case report is a patient, 39 years old, female, that went to ambulatory care with shortness of breath. The echocardiogram showed quadricuspid aortic valve, with moderate/severe insufficiency. Performed aortic valve replacement, with no complications. Conclusions: Quadricuspid aortic valse is a rare malformation, with few cases described in the literature. The echocardiogram is the most applied method to diagnose. Aortic valve insufficiency was the prevalent dysfunction. Aortic valve replacement was the most acceptable procedure. Patients with quadricuspid aortic valve should be carefully checked, because there is a possibility of another cardiac malformation.

\section{My Most Interesting Case - A Cause of Fever, New Murmur and Elevated Mood}

\author{
Gareth Barnes ${ }^{1}$, Chun So ${ }^{2}$, Soroosh Firoozan², \\ Shelley Rahman Haley', Toufan Bahrami ${ }^{1}$ \\ ${ }^{1}$ Royal Brompton and Harefield NHS Foundation Trust, \\ Harefield, ${ }^{2}$ Wycombe General Hospital, High Wycombe, \\ United Kingdom
}

Patient Demographics: 55 year old Caucasian male, previously well, was sent to the cardiac emergency admissions unit of a local hospital by his family doctor after presenting with a fever and new systolic murmur. Relevant History: He gave a one week history of rapidly progressing shortness of breath on exertion and peripheral oedema on a three month background of diarrhoea, $8 \mathrm{~kg}$ weight loss, hot flushes and night sweats. During history-taking and examination it was noted that the patient appeared to have a remarkably (inappropriately) cheerful affect.

Pre-Operative Plan: Echocardiogram: 24/06

Thickening and restriction of the tricuspid valve leaflets with coaptation failure, free tricuspid regurgitation and flow reversal in the hepatic veins. The pulmonary valve also thickened and there is severe/free PR. Severe right ventricular dilatation with preserved long axis function and moderate right atrial dilatation. Two masses also identified within the liver. CT chest abdomen and pelvis $25 / 06$. There is ascites. The liver is irregular in and there are multiple lesions within the liver ranging in size up to just over $10 \mathrm{~cm}$, likely metastases to be metastases. A $3.2 \mathrm{~cm}$ mass within the mesentery likely to be the primary tumour. Carcinoid would be a strong possibility. Conclusions: Ascites, liver metastases and mesenteric mass.? Carcinoid:

Blood tests 25/06.

Chromogranin A $303 \mathrm{pmol} / \mathrm{L}$ (0-60).

Chromogranin B 205 (0-150).

Serotonin $42.3 \mathrm{nmol}$.

Discussion of what was actually done and the challenges, deaths and complications encountered. (Unless case is still pending): Diagnosis of carcinoid syndrome and patient referred to oncologist for assessment. Tumour assessed as slow growing and octreotide therapy initiated. In view of right sided heart failure, tissue tricuspid and homograft pulmonary valve replacement scheduled - surgery pending.

\section{A Sutureless Valve Used in a High Risk Reoperation Procedure Suffering from Active Infective Bioprosthetic Valve Endocarditis}

\section{Johanna Konertz, Marc Kastrup, Sascha Treskatsch, Pascal M. Dohmen}

Charite Hospital, Berlin, Germany

Objective: We present a 72 -years-old male patient suffering from active infective aortic prosthetic endocarditis two months after emergency surgery with aortic valve implantation and aortic arch reconstruction using a Dacron patch, due to spontaneous 
post-procedural migration of a transcatheter valve (TAVI). Methods: The heart team considered a TAVI procedure due to increased operative risk demonstrated by a $10.9 \%$ euroSCORE II at this time, including chronic obstructive pulmonary disease GOLD 3 , cirrhosis (Child-B), esophageal varicose stage 2 , chronic renal failure stage 3 and reduced left ventricular ejection fraction. Results: Today, however the euroSCORE II was extremely high (55\%) due to active infective prosthetic endocarditis and aortic arch reoperation. A sutureless aortic valve was implanted and pericardial patch repair of the aortic ascending and arch. Post-operative sternal re-stabilization was needed. Conclusions: The patient was discharge home after 42 days. No recurrent endocarditis was diagnosed during follow-up.

\section{Heart Valve Outcomes}

\section{Early Experience of TAVR Arising from a Long Standing Valve Clinic in a Veteran Population}

\author{
Kendal Endicott ${ }^{1}$, Dominic Emerson ${ }^{1}$, Conor Hynes ${ }^{1}$, \\ Jason Morrisette' , Amy Kirkpatrick', Christian Nagy', Jon Reiner', \\ Ramesh Mazhari ${ }^{1}$, Farzad Najam ${ }^{1}$, Frederick Mordini ${ }^{1}$, \\ Shawn Robinson ${ }^{2}$, Michael Greenberg ${ }^{1}$, Gregory Trachiotis ${ }^{1}$ \\ ${ }^{1}$ Division of Cardiology and Cardiothoracic Surgery, The \\ George Washington University and Veterans Affairs Medical \\ Center, and The Heart Center, Washington DC Veterans Affairs \\ Medical Center, Washington, DC, ${ }^{2}$ Baltimore Veterans Affairs \\ Medical Center, Baltimore, MD, USA
}

Objective: Transcatheter aortic valve replacement (TAVR) has emerged as an increasingly utilized strategy for treatment of severe aortic stenosis in high risk surgical candidates. We report our early experience in a Veteran population utilizing a long-standing multidisciplinary valve clinic as a means for patient selection. Methods: From August 2014 to September 2015, 12 patients were deemed appropriate candidates for TAVR after presentation in our institution's multidisciplinary valve clinic. The mean age of the patients was $75 \pm 9.7$ years. The majority of patients $(83 \%)$ were NYHA class III with the remainder class II. Of the 12 attempted cases, 11 patients underwent transcatheter valve replacement (8 TF-TAVR, 1 TA-TAVR, 2 TA-VIV-TAVR) with the SAPIEN transcutaneous valve. Results: Of the 11 procedures performed, there were no reports of 30-day mortality, stroke, MI, prosthetic valve endocarditis, or conversion to surgical aortic valve replacement. Pre-implantation mean and maximal aortic valve gradients were measured as $43.7 \pm 12.4$ and $71.6 \pm 19.1$ respectively. Postimplantation mean and maximal aortic valve gradients were measured as $12.2 \pm 9.7(\mathrm{p}<0.001)$ and $23.6 \pm 17.3$ respectively $(\mathrm{p}<$ $0.001)$. Mean preoperative EF was $50.4 \pm 8.5$ with postoperative EF $54.7 \pm 12.5(\mathrm{p}=0.12)$. Post-procedure, two patients had trace paravalvular leak (18\%), and one patient had mild paravalvular leak (9\%). Complications include the need for transfusion in 4 patients (36\%) and post-operative arrhythmias in 2 patients (18\%). Hours spent in the ICU averaged $28 \pm 10.1$ with average hospital stay of
$5 \pm 2.25$ days. Two patients were discharged to short term rehab. On follow up analysis which was $100 \%$ complete, there were no deaths (mean duration $7.1 \pm 4.4$ months; range $1-15$ months). Heart failure class improved at 30 day follow up in all but one patient $(\mathrm{V}=55, \mathrm{p}=0.003)$. Conclusions: Early experience with transcatheter aortic valve replacement within a high risk Veteran population demonstrates that the procedure is safe and effective. Our $100 \%$ survival rate highlights the benefits of a multidisciplinary valve conference to optimize patient selection for TAVR.

\section{Percutaneous Heart Valve Therapy}

\section{Transcatheter Heart Valve Implantation (THVI) as an Alternative to Redo Surgery}

Gry Dahle, Kjell Arne Rein, Arnt Fiane

Rikshospitalet, Oslo University Hospital, Oslo, Norway

Objective: Redo surgery after failed valve repair and degenerated bioprosthesis is high risk procedures, even more in patients with comorbidities. THVI may be beneficial, also in extraanatomical positions. We present the experience from our center. Methods: The patients were evaluated by echo, CT scan reconstruction as well as Bapats application was consulted. The procedure was performed in a hybrid operation room in general anesthesia with fluoroscopy and echo guidance. Results: Twentyfive patients, ten female, mean age 72 years (27-87) were treated with implantation in all valve positions: aorta 16 , mitral 5 , tricuspid 1 , pulmonal/tricuspid 1, extra-anatomic 1 (GUCH patient with Rastelli tunnel) and native aortic valve with supracoronary graft (A-dissection) 1 .

Access routes: transfemoral 4, transapical 17, transaortic 2, transatrial 2. Failure of the prostheses and repairs was: regurgitation 14, stenosis 7, combined 5. The previous implant was: stented bioprostheses 12 , stentless prostheses 5 , annuloplastyrings 4 , homografts 2, THV 1, extra-anatomic 1 and native aorta with supracoronary graft 1 . The implanted catheter valve was Edwards Sapien XT/S3:16/3, CoreValve 2, Engager 4. Five patients had their 3 rd or 4 th redo procedure and three were in severe heart failure. Time interval from last operation to implantation was mean 6.4 years $(0.1-14)$. The procedural success was $100 \%$. Five patients had reoperation femoral reconstruction 2 , hemothorax 1 , open redo valve surgery 1 , VSR closure (after MI). The transvalvular pressure gradients were acceptable, though upper limit for the smallest prostheses. One patient died at day 28 , the overall survival was $92 \%$. Conclusions: There is an increasing number of bioprosthesis implanted as well as valve repairs perfomed. Our results demonstrate the feasibility of transcatheter treatment for failed repair and degenerated bioprostheses in all valve positions. The most convenient access route is choosen, and the most suitable catheter valve should carefully be selected to prevent high gradients, paravalvular leak, migration or embolization. This may in the future play a significant role to reduce mortality in complex redo valve surgery. Surgeons in the transcatheter team make this possible. 


\section{Case Reports}

\section{Redo Transapical Procedure in a Calcified Transapical Edwards Sapien XT Prosthesis}

Doreen Richardt, Thorsten Hanke, Holger Thiele, Hans H. Sievers, Thomas Kurz

University Hospital of Schleswig-Holstein, Lübeck, Germany

Objective: Patient Demographics: The 77-year-old female underwent a transapical $26 \mathrm{~mm}$ Sapien XT (Edwards) procedure due to severe aortic stenosis in November 2010. Methods: Relevant History: Her medical history mainly included a heavily peripheral artery disease, porcelain aorta and chronic obstructive lung disease with lung emphysema, diabetes and renal insufficiency. In the last two years she had progressive symptoms of a progressive symptomatic severe aortic stenosis with combined aortic insufficiency with recurrent decompensations, hospitalization and pneumonia. In September 2015 we performed a high risk transapical redo of aortic valve implantation with a $23 \mathrm{~mm}$ Sapien 3 (Edwards) as a Valve in Valve procedure. Results: Pre-Oerative Plan: In preparation of the procedure we did not found a case like that described before. After analyzing CT and Echo several times we decided against a $26 \mathrm{~mm}$ Sapien 3 and for a $23 \mathrm{~mm}$ Sapien 3 with predilation due to impressive calcification of the Sapien XT. Conclusions: Discussion: During implantation procedure we oriented to the upper margin of the frame of the Sapien XT and implanted the Sapien S3 very centrally with an excellent result (maximum gradient $3 \mathrm{~mm} \mathrm{Hg}$ ). This case emphasizes that we will have to deal with reoperations also in the transapical procedure in patients, whose diseases are even worse than in the first procedure. In this case the main difficulties were the adhesiolysis of the pericardium and to identify a possible puncture site at the apex. Another important point is, to calculate the exact valve size respecting the anatomy of the valve prosthesis implanted before.

\section{Three-Dimensional Transoesophageal-Guided Transmitral Valve Implantation with a TENDYNE Device}

\section{Alison Duncan, Cesare Quarto, Simon Davies, Neil Moat}

The Royal Brompton Hospital, London, United Kingdom

Patient Demographics: Transcatheter mitral valve implantation (TMVI) is an alternative to conventional surgery for high risk patients with severe mitral regurgitation (MR). The TendyneTM TMVI system consists of a porcine pericardial valve in a nitinol frame tethered to the left ventricular (LV) apex using an epicardial pad. The device can be used to treat primary and secondary $\mathrm{MR}$, and is fully repositionable and retrievable even after complete deployment. Implantation is guided by three-dimensional transoesophageal echocardiography (3DTOE). Relevant History: A frail 87 year-old man with previous myocardial infarction and angioectasia of stomach presented with severe mitral regurgitation due to P2 chordal rupture. He had reduced LV ejection fraction $43 \%$, with right ventricular impairment and severe pulmonary hypertension (75 mm Hg). He had significant renal dysfunction (creatinine clearance $34 \mathrm{ml} / \mathrm{min}$ ) and raised brain naturetic peptide $470 \mathrm{ng} / \mathrm{L}$ (normal $20 \mathrm{ng} / \mathrm{L}$ ). His logistic EuroSCORE was 20.2 and his STS PROM score was $12.6 \%$. Pre-Operative Plan: He was accepted for TMVI by the Heart Team. Discussion of what was actually done and the challenges, deaths and complications encountered. (Unless case is still pending): TMVI was performed via a transapi-

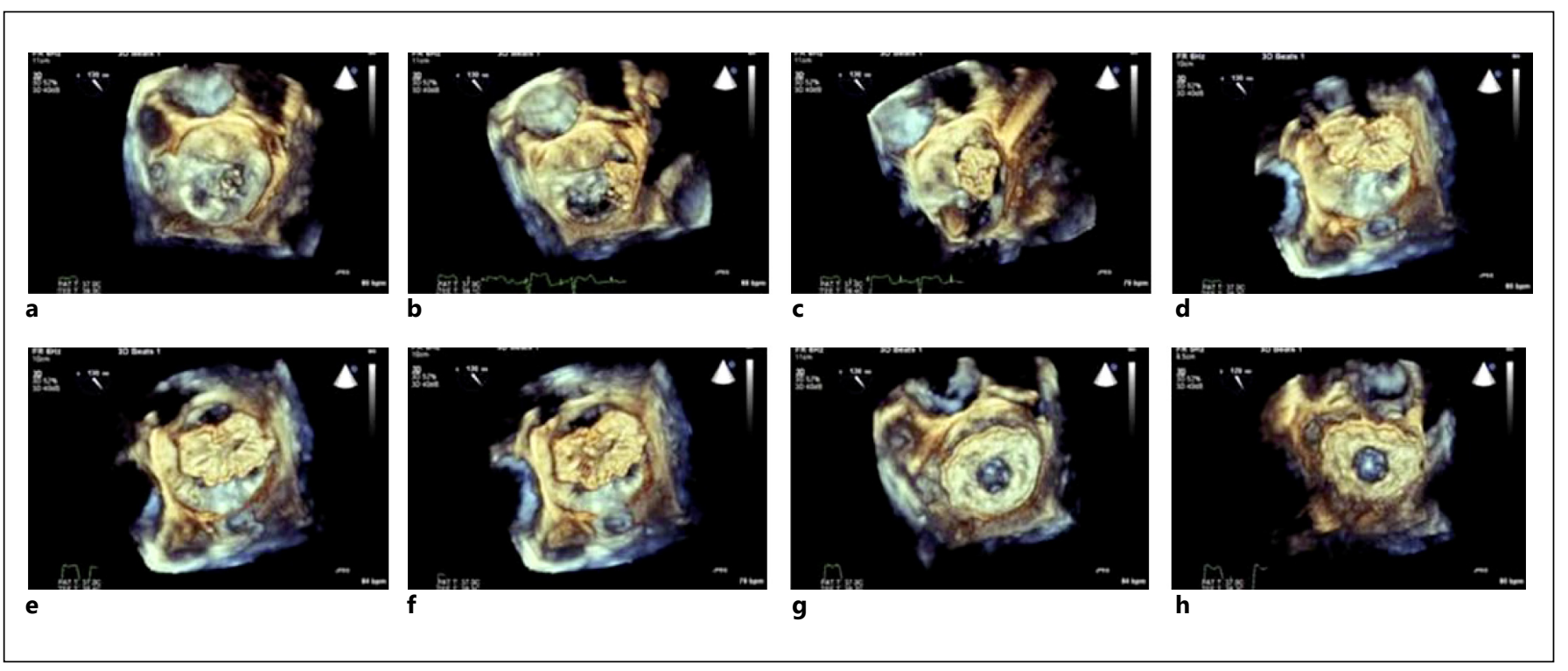

Fig. 1. (for Abstract of Alison Duncan). 
cal approach using a left mini-thoracotomy. The procedure was guided by 3DTOE. Apical tether tension was adjusted to optimize device position (figure 1): a: guide sheath placed across mitral valve; b-d: device progressively opened in left atrium; e, f: anterior flange of device lined up with aorta; g: device fully opened by still high in left atrium resulting in anterior paravalvular leak; $\mathrm{H}$ : fully deployed and correctly positioned device with no MR or paravalvular leak. TOE also established no LV outflow tract obstruction and peak mitral pressure gradient was $5 \mathrm{~mm} \mathrm{Hg}$. There were no VARC-2 procedural complications. The patient was discharged home on 5 th post-operative day.

\section{Reversible Thrombotic Aortic Restenosis after Valve-in-Valve Transcatheter Aortic Valve Replacement}

\section{Patrick J. Goleski, Creighton W. Don}

University of Washington, Seattle, WA, USA

Patient Demographics: Three patients with bioprosthetic aortic valve failure who underwent valve-in-valve (VIV) transcatheter aortic valve replacement (TAVR) presented with severe restenosis within one year of the procedure. Relevant History: All patients experienced significant heart failure due to stenosis of their bioprosthetic valves. Each was considered extreme risk for surgery, and underwent VIV TAVR procedures. Two patients had stented bioprosthetic valves and one had a homograft (Table). Pre-Operative Plan: The two patients with stented valves had pre-TAVR valve sizing based on the specified inner diameter of the valves, and the patient with a homograft was sized based on the CT evaluation. All patients had placement of Edwards SAPIEN XT balloon expandable valves. Discussion of what was actually done and the challenges, deaths and complications encountered. (Unless case is still pending): The TAVR procedures were uncomplicated and the patients were discharged uneventfully. The post-TAVR valve hemodynamics showed a significant reduction in gradients. Case 1 developed worsening heart failure symptoms at 6 months, associ- ated with worsening valve hemodynamics. He was anticoagulated with warfarin and his gradients decreased over the next 6 months with an improvement in symptoms. By 3 years his valve hemodynamics had normalized. Case 2 also did well until his clopidogrel was stopped at 6 months. Within a month the patient's pre-TAVR symptoms returned and his aortic valve velocity increased (figure 1). Transesophageal echocardiography showed thickening of the left anatomic bioprosthetic leaflet. The patient was anticoagulated and 2 months later his symptoms had resolved and his valve gradients had normalized. Case 3 did well until 10 months post-TAVR, when he developed worsening heart failure. His echocardiogram showed worsening valve gradients and he was anticoagulated. The gradients decreased gradually and returned to baseline after 7 more months. All patients have continued anticoagulation and their valve gradients continue to be normal at follow-up.

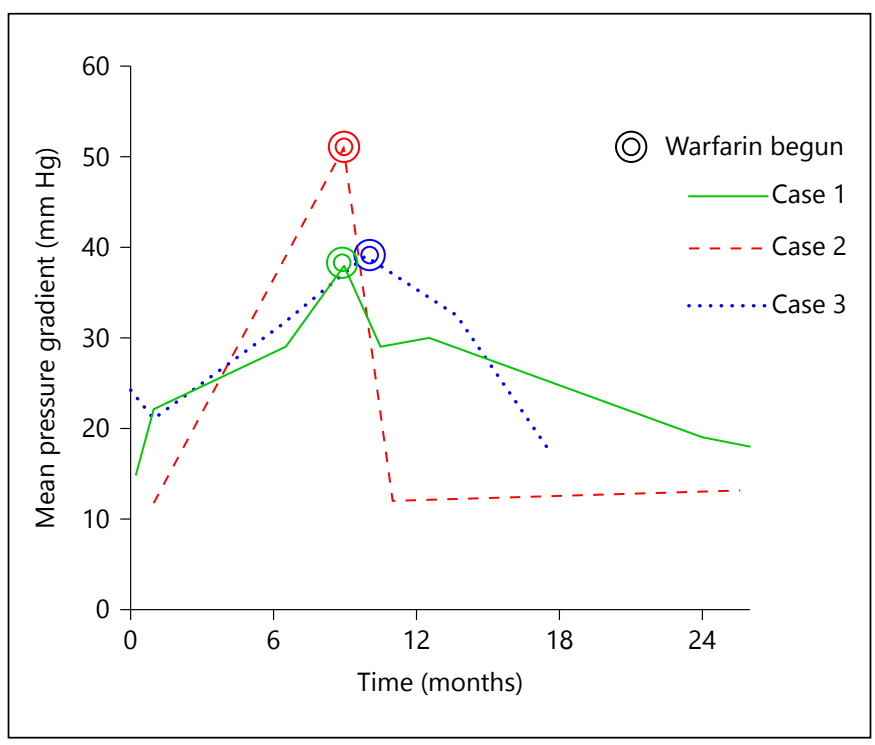

Fig. 1. (for Abstract of Patrick J. Goleski).

Table 1. Echocardiographic data (for Abstract of Patrick J. Goleski)

\begin{tabular}{llll}
\hline & Case 1 & Case 2 & Case 3 \\
\hline Age & 96 & 68 & 57 \\
\hline Sex & Male & Male & Male \\
\hline AVR & $23 \mathrm{~mm}$ Hancock II & $24 \mathrm{~mm}$ homograft & $23 \mathrm{~mm}$ CE \\
\hline Inner diameter, mm & 18.5 & 29.4 & 20 \\
\hline VIV-TAVR & $23 \mathrm{~mm}$ SAPIEN & $26 \mathrm{~mm}$ SAPIEN XT & 23 mm SAPIEN XT \\
\hline Diameter oversizing percentage, $\%$ & 24 & -12 & 15 \\
\hline Initial antithrombotic therapy & DAPT $\times 6$ months & DAPT $\times 6$ months & $\begin{array}{l}\text { DAPT } \times 2 \text { weeks } \\
\text { Warfarin } \times 2 \text { months } \\
\end{array}$ \\
& & & ASA chronically \\
\hline
\end{tabular}




\section{Hyper-Acute Early Transcatheter Prosthesis Thrombosys in a Patient with Polycythemia Vera}

Laura Besola, Augusto D'Onofrio, Eleonora Bizzotto, Erica Manzan, Chiara Tessari, Gianbattista Isabella, Gaetano Thiene, Gino Gerosa

University of Padua, Padua, Italy

Patient Demographics: 86 year-old lady with severe symptomatic aortic valve stenosis and frequent hospital admissions for pulmonary edema. EuroSCORE II and STS Score were $4.88 \%$ and $2.85 \%$, respectively. Relevant History: The patient presented history of chronic cerebrovascular disease, previous bilateral saphenectomy, right mastectomy and orthopedic intervention. The patient was affected by polycythemia vera with JAK-2 mutation, treated with cytoreductive therapy. Preoperatively she presented normal red blood cells count, lymphocytosis (white blood cells count of $21.12 \times 10^{\wedge} 9 / \mathrm{L}$ ) and a severe thrombocytosis (platelets count of $\left.1102 \times 10^{\wedge} 9 / \mathrm{L}\right)$. Pre-Operative Plan: The patient underwent standard preoperative screening (transthoracic echocardiography (TTE), coronary angiography, Angio-CT) and for the advanced age and comorbidities she was scheduled for TAVI. For severe tortuosity of the abdominal aorta and femoral arteries the transapical approach was preferred. Preoperative thromboelastogram and aggregometry were performed and demonstrated an slightly hypercoagulant state but an hematological evaluation did not contraindicate the procedure and did not suggest preoperative anticoagulation nor antiplatelet therapy. Discussion of what was actually done and the challenges, deaths and complications encountered. (Unless case is still pending): The patient successfully underwent TA-TAVI with a $26 \mathrm{~mm}$ Edwards Sapien-3 bioprosthesis. The angiographic and transesophageal echocardiographic (TEE) controls performed in the cath-lab at the end of the procedure showed good bioprosthesis position and function with no paravalvular leak. Hemodynamic conditions were good. After three hours she unexpectedly presented rapid deterioration of hemodynamic conditions followed by cardiac arrest. She underwent cardiopulmonary resuscitation and emergent femoro-femoral ECMO implantation after standard heparinization. A TEE performed during CPR showed massive bioprosthesis thrombosis. Furthermore, sudden coagulation of the ECMO oxygenator occurred and ECMO replacement as well as complete heparinization were required. A coronary angiography showed no coronary obstruction and confirmed the prosthesis dysfunction due to acute thrombosis. The patient died after few hours for multi-organ failure. Autopsy showed massive thrombosis of the bioprosthesis with involvement of all leaflets causing severe obstacle to valve opening. To the best of our knowledge this is the first case of transcatheter valve early acute thrombosis.

\section{Transcatheter Aortic Valve Replacement in Type B Aortic Dissection}

\author{
Iosif Gulkarov' , Akanibo Da-Wariboko' , Ashwad Afzal', \\ Arash Salemi ${ }^{2}$, Berhane Worku' \\ ${ }^{1}$ New York Methodist Hospital, Brooklyn, NY, ${ }^{2}$ Weill Cornell \\ Medical Center, New York, NY, USA
}

Patient Demographics: A 66-year old African American male with a history of hypertension, end-stage renal disease, hepatitis $\mathrm{C}$ with cirrhosis and multiple myeloma, presented with chest pain and shortness of breath occurring at rest. Relevant History: Transthoracic echocardiography revealed normal left ventricular function, and severe aortic stenosis with mean gradient of $66 \mathrm{~mm} \mathrm{Hg}$ and a valve area of $0.95 \mathrm{~cm}^{2}$. Cardiac catheterization revealed an $80 \%$ circumflex artery stenosis. Computerized tomography angiogram revealed a type $B$ aortic dissection from the left subclavian to both common iliac arteries. Patient's Society of Thoracic Surgeon risk score was $9.6 \%$. Preoperative Plan: In light of his multiple comorbidities, including cirrhosis with portal hypertension and thrombocytopenia and multiple myeloma, he was deemed high risk for standard surgical intervention and transcatheter aortic valve replacement (TAVR) was entertained. A transaortic (TAo) approach was planned in order to avoid instrumenting the dissected descending aorta. Preoperatively the patient underwent successful bare metal stenting of the left circumflex artery. Discussion: The procedure was performed under general anesthesia with transesophageal echocardiographic guidance. A pigtail catheter was advanced into the ascending aorta via the right femoral arterial artery and an aortogram was performed. The ascending aorta was exposed via partial upper sternotomy. Via a 24F Ascendra 3 Introducer Sheath placed in the ascending aorta, balloon valvuloplasty was performed and a $23 \mathrm{~mm}$ Edwards Aortic Valve (Edwards Lifesciences Inc., Irvine, California, USA) was deployed. The patient did well and was discharged home. We report the first case in the literature, to our knowledge, of TAVR in a patient with a type $\mathrm{B}$ dissection. As the transfemoral approach is contraindicated, transapical (TA) and TAo approaches were considered. The TAo approach avoids the need to traverse the dissected proximal thoracic aorta with a stiff wire as done with the TA approach. Furthermore, if emergent cardiopulmonary bypass becomes necessary, access to the ascending aorta avoids the need for femoral cannulation in the setting of a dissected thoracic aorta. The presence of a type B aortic dissection should not preclude TAVR. The TAo route offers access to aortic valve intervention with minimal to no manipulation of the descending aorta. 


\section{Multiple Valve Surgery for Advanced Rheumatic Heart Disease: Results and Risk Factors in $\mathbf{1 5 0}$ Patients}

\section{Zahira Zouizra, Yassin Boukaidi, Rachid El Houati, Drissi Boumzebra}

Ibn Tofail Hospital, Marrakech, Morocco

Objective: Multiple valve surgery (MVS) is still of choice for advanced rheumatic heart disease (RHD), which has been associated with reported poor early and late outcomes. We review our experience and results after MVS. Methods: between 2007 and 2015: 150 patients (54\% men, $46 \%$ women), with mean age of 37 years (16-64 Y), underwent MVS for isolated advanced RHD. Criteria for selecting patients: New York Heart Association (NYHA) class III or IV, Cardiomegaly, lower left ventricular ejection fraction (LVEF), severe pulmonary hypertension and or right ventricular dysfunction, Valvular disease with deterioration of more than one vital function. All patients received replacement procedures in mitral and aortic position (145 mechanical, 5 bioprosthetic), with concomitant De Vega tricuspide annuloplasty (140 patients), tricuspide annuloplasty ring (10 patients) and or other complex tricuspide plasty procedures. The mean cardiopulmonary bypass time: $130 \mathrm{~min}$ (75-240 min), Mean cross-clamping time: $65 \mathrm{~min}$ (45-120 min). Results: The 30-day hospital mortality was $17 \%$ $(\mathrm{n}=26), 30$ patients had postoperative complications: Low Cardiac output syndrome (12), 6 Infections (1 mediastinitis, 5 Pneumonia), 5 Acute kidney failure (Dialysis: 1 case), 7 Transient atrioventricular Block, 5 transient tachyarrythmias, 3 Neurologic events (2 Stroke, 1 Coma), 4 Right sided Heart failures. Follow-up 6-48 months after surgery: The freedom from thromboembolism and anticoagulation-related hemorrhage was $81 \%$. Of the 120 patients still alive, 96 were in NYHA class I and II. Conclusions: Surgery of advance multiple valves disease has a significant high morbidity and mortality rates. Results may be improved by early surgical treatment before NYHA class IV or deterioration of LVEF occurs.

\section{Progressive Aortic Valve Stenosis: Is it All About the Calcium Deposition?}

Geoffrey D. Huntley, Jeremy J. Thaden, Marie-Annick Clavel, Maurice E. Sarano, Vuyisile T. Nkomo

Mayo Clinic, Rochester, MN, USA

Patient Demographics: Case 1 is a 67 year-old male with a 19year diagnosis of bicuspid aortic valve (BAV) stenosis. Case 2 is a 40 year-old female with a 13-year diagnosis of BAV stenosis. Relevant History: Both Cases developed symptoms of severe aortic stenosis with NYHA Class III symptoms during active surveillance for their known aortic stenosis. On cardiac exam, Case1 had a normal S1 and an absent aortic component of S2 with a grade $3 / 6$ systolic murmur at left sternal border which radiated to the carotids. While Case 2 had an S1 with an opening click and soft S2 with a 4/6 late-peaking systolic murmur heard best in the aortic area with a thrill radiating all over the precordium to the ca-
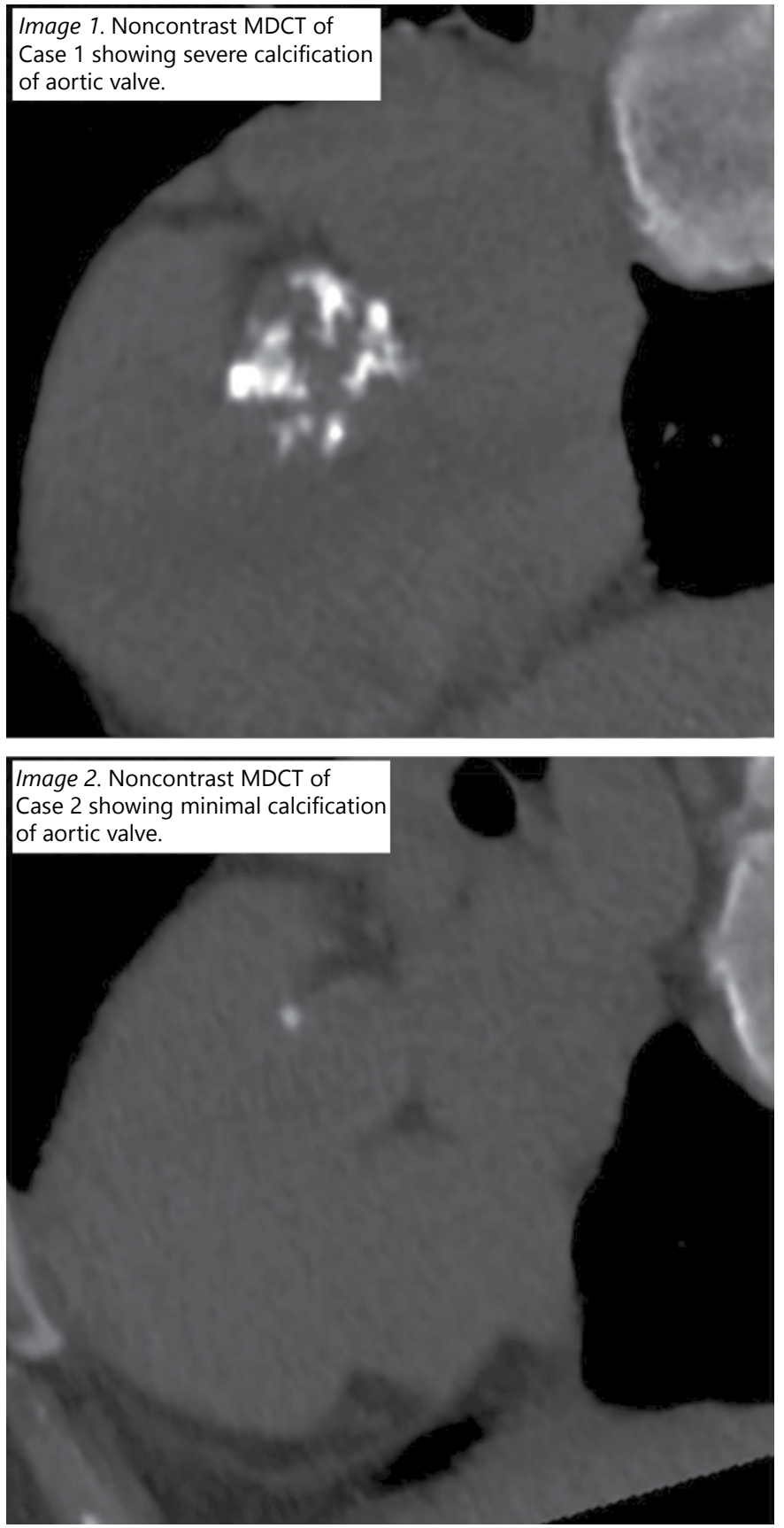

Fig. 1. (for Abstract of Geoffrey D. Huntley).

rotids. In both cases, severe BAV stenosis was suspected. Pre-Operative Plan: Both patients met criteria for severe BAV stenosis requiring replacement after undergoing transthoracic echocardiography and MDCT with aortic valve calcium quantification (Table 1). However, CT of Case 1 (Image 1) demonstrated a heavily calcified valve with a calcium score of 4.238 AU while CT of Case 2 (Image 2) revealed a calcium score of only 54 AU. Discussion: Case 1 represents routine BAV stenosis: severe calcification of cusps and consequential abnormal hemodynamics by the $7^{\text {th }}$ 
Table 1. Comparison of transthoracic echocardiographic and MDCT with calcium results (for Abstract of Geoffrey D. Huntley)

\begin{tabular}{|c|c|c|}
\hline & Case 1 & Case 2 \\
\hline Demographics & 67 year-old male & 40 year-old female \\
\hline Aortic valve area, $\mathrm{cm}^{2}$ & 0.93 & 0.98 \\
\hline $\begin{array}{l}\text { Transaortic maximum velocity, } \\
\mathrm{m} / \mathrm{s}\end{array}$ & 5.19 & 4.60 \\
\hline Mean pressure gradient, $\mathrm{mm} \mathrm{Hg}$ & 64 & 47 \\
\hline Ejection fraction, $\%$ & 66 & 72 \\
\hline CT calcium score, $\mathrm{AU}$ & 4,238 & 54 \\
\hline Valve weight, $g$ & 2.03 & 1.62 \\
\hline
\end{tabular}

decade requiring replacement. However, Case 2 exhibits severe stenosis with minimal calcification at 40 years of age requiring replacement, an interesting contradiction. Certainly, calcification is not the only mechanism for BAV stenosis, and Case 2 raises many questions about the mechanism behind stenosis in younger patients. This case comparison cautions that CT calcium quantification may not be as reliable in younger patients presenting with BAV stenosis.

\section{Apical Left Ventricular Mycotic Aneurysm with Prosthetic Aortic Valve Endocarditis}

Kazuyoshi Takagi, Hiroyuki Otsuka, Koichi Arinaga, Koji Akasu, Tohru Takaseya, Takahiro Shojima, Yuichiro Hirata, Kumiko Wada, Satoshi Kikusaki, Keishi Hashimoto, Hiroyuki Tanaka

Kurume University, Kurume, Japan

Patient Demographics: A 83-year-old male was hospitalized with general fatigue and fever 5 months after AVR and mitral valve repair. Transthoracic echocardiography (TTE) demonstrated multiple vegetations on the bioprosthesis, the aortic annular abscess and the left ventricular aneurysm (LVA). Computed Tomography $(\mathrm{CT})$ confirmed the presence of aneurysm at the apical aspect of left ventricle. Enterococcus was grown on blood culture. He was diagnosed with the prosthetic valve endocarditis (PVE). The patient was admitted to our hospital for surgical treatment. Relevant History: The postoperative period during 3 months after his previous surgery had been uneventful. Pre-Operative Plan: We planned to undergo urgent re-AVR with debridement of the annular abscess, patch closure of the defect, and resection of the LVA and closing with a patch. To avoid injuring of the LVA, we consid-

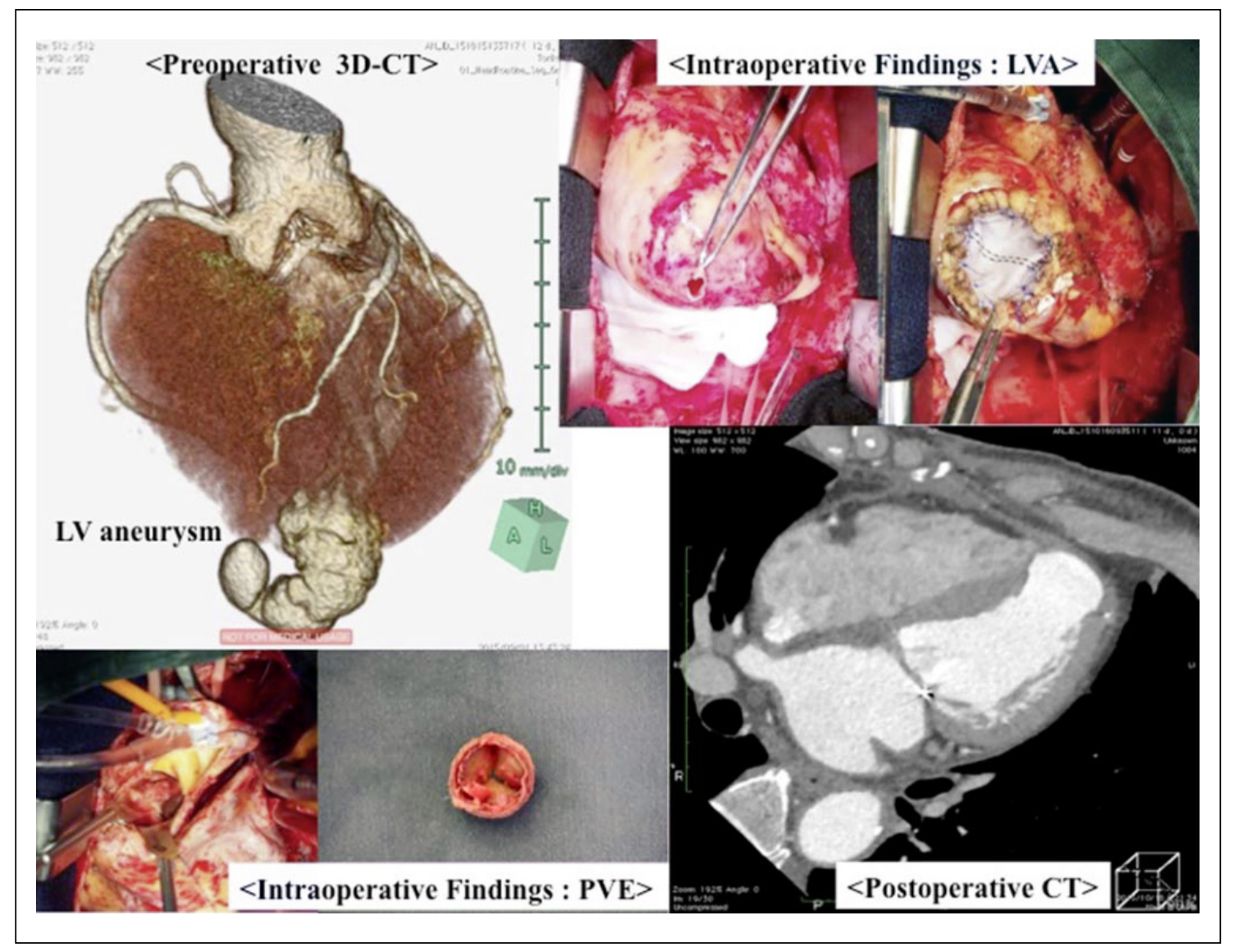

Fig. 1. (for Abstract of Kazuyoshi Takagi). 
ered the re-sternotomy under the cardiopulmonary bypass (CPB) through the femoral artery and vein. Discussion of what was actually done and the challenges, deaths and complications encountered. (Unless case is still pending): Careful re-sternotomy and dissection of the LVA were done under the CPB. After cardioplegic arrest, the LVA was opened in scarred myocardium. The LVA was shown multi-cystic cavities and the chamber's diameter was estimated to be $3 \mathrm{~cm}$. Necrotic tissue, consistent with abscess formation, was excised and the defect was closed with a Dacron patch sutured with continuous and interrupted pledgetted mattress sutures. The bioprosthesis with massive vegetations was removed and the annular abscess was evacuated and closed. A new bioprosthesis was implanted. The ventricular pacemaker was implanted 14 days after surgery because of atrioventricular conduction disturbance due to debridement of the annular abscess. Postoperative TTE and CT confirmed no recurrence of PVE and the LVA. The patient was discharged after six weeks of antibiotics therapy with ampicillin and gentamicin. According to pathological findings, a local septic embolism to the left anterior descending caused the mycotic LVA, that is a rare complication of the PVE. We report this interesting case including discussion from previous reports.

\section{Concomitant Tricuspid Valve Replacement Using an Extracellular Matrix Cylinder and Pulmonary Thromboembolectomy}

Sreekumar Subramanian, Joshua S. Hall, Bryan T. Fisher, Sr.

TriStar Centennial Medical Center, Nashville, TN, USA

Patient Demographics: The patient is a 30 year old white man. Relevant History: He has a history of polysubstance abuse and presented with MRSA bacteremia and epidural abscess. His hospital course was complicated by saddle pulmonary embolus treated with anticoagulation, and an IVC filter. Additionally, he had celiac artery and branch thromboemboli, which was treated with local thrombolysis. Pre-Operative Plan: TEE revealed severe tricuspid valve endocarditis and severe TR requiring tricuspid valve replacement. Given the drug use history, an extracellular matrix cylinder was chosen to reduce the risk of reinfection. The pulmonary embolism was refractory to anticoagulation and warranted concomitant treatment. Discussion of what was actually done and the challenges, deaths and complications encountered. (Unless case is still pending): Intraoperatively, a large portion of the anterior leaflet of the tricuspid valve was missing and the posterior leaflet was flail. Extracellular matrix was fashioned into a cylinder and used to replace the tricuspid valve by anchoring the cylinder to the RV papillary muscles and the tricuspid annulus. A pulmonary embolectomy was performed, and blood was perfused retrograde via the left atrium to backflush the pulmonary arteries to evacuate the residual emoblic material, which was embolized tricuspid leaflet tissue. Echocardiogram at 1 month revealed good function of the tricuspid cylinder and the patient remains clinically well at 6 months postoperatively.

\section{The Challenge of Sizing Valsalva Prostheses for Autograft Reinforcement in the Ross Procedure}

Alfred Kocher, Claudia Oeser, Martin Andreas, Guenther Laufer

Medical University of Vienna, Vienna, Austria

Patient Demographics: Patient 1: 32-year old man Patient 2: 42-year old man. Relevant History: Patient 1: bicuspid aortic valve with severe stenosis and moderate regurgitation. Patient 2: bicuspid aortic valve with severe regurgitation, dilated left ventricle with moderately impaired systolic function. Pre-Operative Plan: Both patients were scheduled for the Ross procedure, using the root replacement technique. Since progressive dilatation of the neo-aortic root can occur with subsequent valvular incompetence, we decided to reinforce the autograft with a Valsalva Dacron prosthesis in patients with aortic valve regurgitation and dilatation of the native aortic annulus. Discussion of what was actually done and the challenges, deaths and complications encountered. (Unless case is still pending): Different approaches are described for selecting the suitable diameter of a Valsalva prosthesis for pulmonary autograft reinforcement: a prosthesis with a diameter 2-5 $\mathrm{mm}$ larger than the autograft's inner diameter or a prosthesis whose diameter equates the autograft's commissural height. Patient 1: The autograft's diameter measured $26 \mathrm{~mm}$, and accordingly, a Valsalva prosthesis with a diameter of $30 \mathrm{~mm}$ was selected. The autograft was competent and the patient did well. 10 months later echocardiography showed severe autograft valve regurgitation. Computed tomography scanning showed a pseudoaneurysmatic dilatation of the neo-aortic root (maximum diameter 54 $\mathrm{mm}$ ). During reoperation a tear in the non-coronary sinus and the right commissure with a prolapse of the corresponding leaflet and of the autograft wall into the left ventricular outflow tract was observed. The pseudoaneurysm extended in the prosthesis into the area of the right atrium and the aorta. A mechanical Bentall procedure was performed. Patient 2: The autograft's inner diameter measured $35 \mathrm{~mm}$, the commissural height $24 \mathrm{~mm}$. According to one sizing recommendation a $40-\mathrm{mm}$, according to another a 24 $\mathrm{mm}$ Valsalva prosthesis was supposed to be used. A $28-\mathrm{mm}$ Valsalva prosthesis was chosen with a $4 \mathrm{~mm}$ autograft skirt left in place. Echocardiography showed good valve function. In conclusion, an unsuitable Valsalva prosthesis diameter may result in a catastrophic outcome. In both patients, the currently suggested measurement methods were not applicable. The careful and wellconsidered selection of the Valsalva prosthesis diameter is of utmost importance. 


\section{Right Atrial Myxoma Clinically Mimicking Recurrence of Mitral Valve Stenosis after Open Mitral Valve Repair for Rheumatic Severe Mitral Stenosis}

Gauranga Majumdar, Surendra Agarwal, Shantanu Pande, Bipin Chandra

Sanjay Gandhi Post Graduate Institute of Medical Sciences, Lucknow, India

Patient Demographics: Right atrial myxomas are rare. Its occurrence in a previously operated patient of rheumatic mitral stenosis posed clinical diagnostic challenge. We share our experience of successfully managing this patient who underwent urgent repeat cardiac surgery for right atrial myxoma removal along with mitral valve replacement. Relevant History: 37/f, school teacher underwent open mitral valve repair for rheumatic severe mitral stenosis at the age of 16 yrs in our hospital. As she was asymptomatic, stopped following up in hospital. Now she presented with 3 months history of dyspnoea, rapidly progressing to class 4 . She was emaciated, had hepatomegaly, raised JVP with bilateral pedal oedema. She was admitted for antifailure treatment with diagnosis of RHD, severe mitral restenosis with CHF. Surprisingly Echocardiography revealed MV area $>2 \mathrm{~cm}^{2}$ and regurgitant valve. There was a large right atrial mass measuring $76 \times 33 \mathrm{~mm}$, pedunculated and arising from coronary sinus ostium, occupying most of the RA and RV. Mass was heterogenic in echogenicity with hypo echoic region suggestive of either RA myxoma or large RA thrombus. PreOperative Plan: Patient was taken for urgent redo surgery, CPB established with asc aorta, SVC and femoral vein cannulation. Giant RA myxoma retrieved from RA and RV and it was arising from coronary sinus ostium. MV needed replacement as valve was regurgitant and diseased. Postoperative recovery was uneventful. She was followed up yearly for the last 5 yrs. There was no recurrence of tumor and normal prosthetic valve function with functional class I. Discussion of what was actually done and the challenges, deaths and complications encountered. (Unless case is still pending): We are presenting this patient with very unusual finding of a giant right atrial myxoma arising from coronary sinus ostium which reaches RV through tricuspid valve producing clinical features of severe TV stenosis. It occupies whole of right ventricle cavity. Another important salient feature of this patient was occurance of right atrial tumour in a previously operated case of rheumatic mitral stenosis thus offering clinical diagnostic challenges. This occurance is quite exceptional and was not mentioned in recent reviews of patients with intracardiac myxomas.

\section{Successful Bailout Transcatheter Valve Implantation for Prosthetic Aortic Valve Endocarditis during Open Re-Do Surgery}

\author{
Lorenz Hansen, Klaudija Bijuklic, Jan-Hendrik Stripling, \\ Joachim Schofer, Friedrich-Christian Rieß
}

Albertinen-Krankenhaus, Hamburg, Germany

Patient Demographics: 30 months after aortic valve replacement (Hancock $23 \mathrm{~mm}$ ) and aortic root enlargement, a 68-yearold woman was admitted in septic shock for infectious tissue-valve endocarditis. Relevant History: Upon Emergency redo-surgery and debridement, a wide circular aorto-ventricular discontinuity and a VSD became apparent. Annulus reconstruction and VSD defect-closure was carried out using three patches of equine pericardium. The newly formed annulus significantly narrowed the LVOT to $17 \mathrm{~mm}$. Even after patch-enlargement of the former noncoronary annulus, extraanatomic anchoring of even the smallest root prosthesis or stented valve was not considered possible. PreOperative Plan: A $23 \mathrm{~mm}$ Sapien-XT valve was crimped on an Ascendra-2 catheter and deployed in an 'anatomical' orientation. As partial obstruction of the LCA ostium by the sealing-skirt occurred, the valve was removed and crimped again. After a second deployment using a stay-suture to facilitate positioning, both ostia were unobstructed. Two additional sutures were used to further secure the stent to the enlargement-patch. Intraoperative TEE showed complete competence of the prosthesis with a mean systolic gradient of $12 \mathrm{~mm} \mathrm{Hg}$. Eleven months follow-up TEE showed stable systolic valve performance with mild to moderate paravalvular regurgitation and no sign of prosthesis migration or endocarditis with discontinued oral antibiotic therapy. Discussion of what was actually done and the challenges, deaths and complications encountered. (Unless case is still pending): THV in-native-ring is a trade-off between secure anchoring and the risk of rupture. Because a $23 \mathrm{~mm}$ stented valve had been in place, the narrowed aortic annulus was considered to accommodate for a $23 \mathrm{~mm}$ balloon-expandable valve with considerable oversizing and minimal risk of annulus disruption. Additionally, appropriate positioning of the valve in the annulus is crucial to prevent coronary ostia occlusion and to securely seaten the valve. Unfavorable anatomy and the balloon being inflated will obscure the surgeon's vision so that we found it helpful to add a stay suture to the stent frame. In a non-calcified annulus with leaflets removed, only a large frame/ tissue contact, considerable oversizing and use of additional sutures resulted in secure placement of the valve. However, upon follow-up, mild to moderate paravalvular leakage of the valve occurred. 


\section{Importance of the Prosthesis Choice in the Valve-in-Valve Era: Role of the Sutureless Perceval Valve}

Stefano Moriggia, Igor Belluschi, Andrea Blasio, Nicola Buzzatti, Fabrizio Monaco, Ottavio Alfieri

San Raffaele University Hospital, Milan, Italy

Objective: Biological prosthesis in aortic position are widely used even in younger patients and the possibility of transcatheter treatment of an eventual valve degeneration supports the shift from mechanical to biological prosthesis, minimizing the relevance of the patient's age at time of implant. The prosthesis selection used to be durability driven but nowadays it must be Valve-in-Valve feasability driven. So far, a very accurate analysis is mandatory. The aim of this study is to demonstrate how sutureless valves can play a pivotal role in this choice beyond the operative time reduction. Methods: In this perspective observational study, between September 2014 and September 2015, we examined 18 patients who underwent a sutureless-AVR with a Perceval bioprosthesis (Sorin Group S.p.A., Saluggia, Italy), in which an additional measurement with an Hancock II Ultra valve sizer has been performed during surgery. The rationale of double sizing is to compare the sutureless and sutured prosthesis that could be implanted in any single patient of the population examined. According to the ViV App, we analyzed the theoretical feasability of the Valve-in-Valve procedure in this serie, with particular attention to the models and sizes that can be used. Results: Our analysis showed that for any patient the feasability of ViV in case of sutureless valve is $100 \%$, while the feasability drops to $22.2 \%-50 \%$ using a standard stented valve, depending on the choice of the ViV prosthesis. Moreover, considering TAVI sizes in any given patient who implanted Perceval and who could receive a Medtronic Hancock II Ultra bioprosthesis, there is a clear statistical difference in favour of Perceval for the majority of TAVI devices $(p<0.001)$. Conclusions: In the transcatheter implantation era, the choice of a sutureless valve ensures $100 \%$ Valve-in-Valve feasability with most of the last generation transcatheter heart valves, providing an adequate EOA in all patients. This rate decreases to less than $50 \%$ in case of a standard sutured bioprosthesis. Sutureless valve can be a good choice even in the younger population who could benefit from a transcatheter ViV in their future.

\section{D TOE-Guided Mitral Valve-in-Valve Procedure}

\section{Alison Duncan, Cesare Quarto, Simon Davies, Neil Moat}

The Royal Brompton Hospital, London, United Kingdom

Objective: Transcatheter mitral valve-in-valve (ViV) implantation is an alternative to conventional surgery for high risk patients with severe mitral regurgitation $(\mathrm{MR})$ due to bioprosthestic

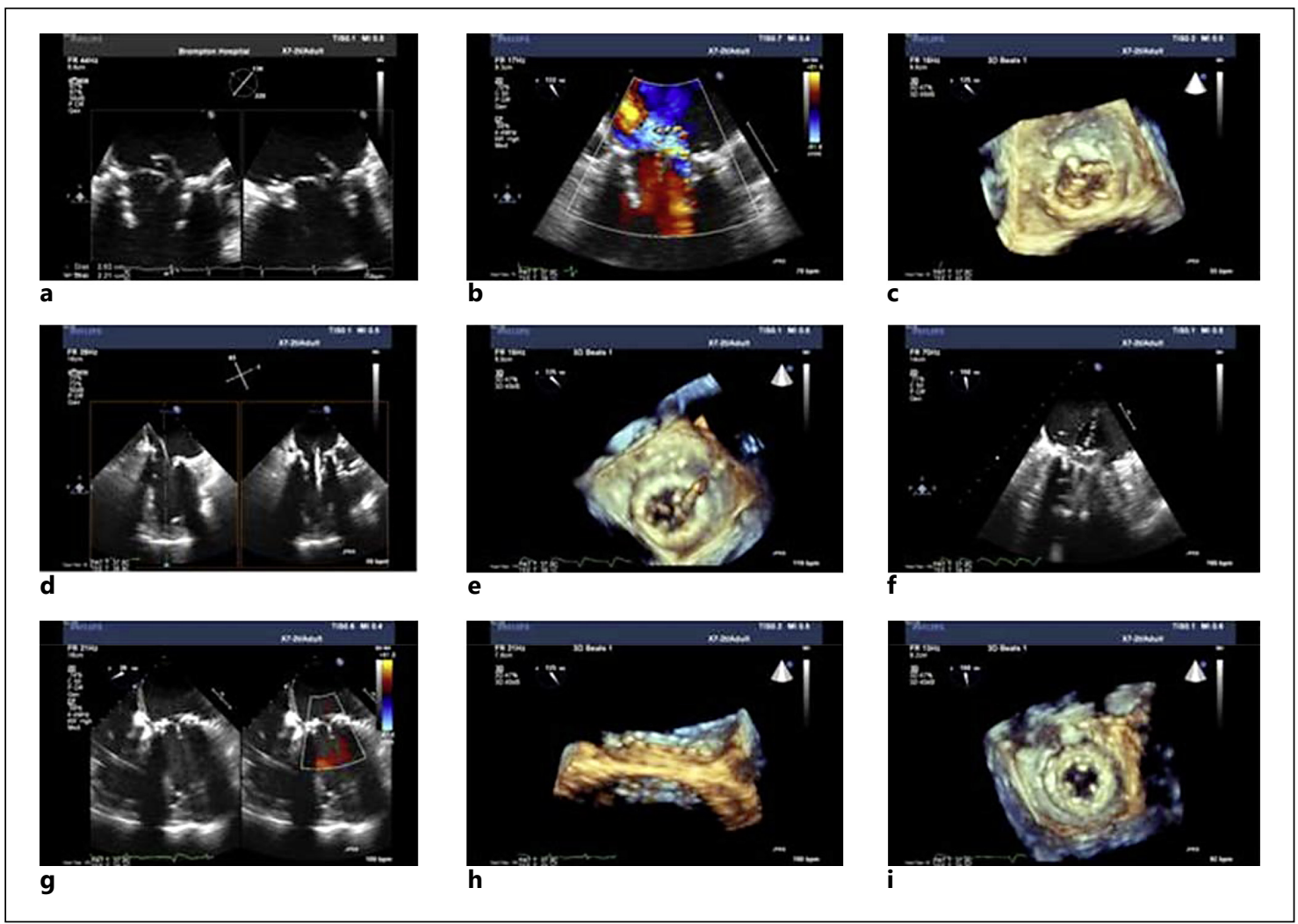

Fig. 1. a-i Intra-procedural TOE guidance for mitral valve-in-valve (for Abstract of Alison Duncan). 
device failure. Methods: A 57 year-old man with previous rheumatic fever and severe mitral stenosis underwent mitral and tricuspid repair in January 2015 (mitral position: $32 \mathrm{~mm}$ Physio II ring, tricuspid position: $34 \mathrm{~mm}$ incomplete annuloplasty ring). Sixmonths later, he was admitted to our intensive care unit with pulmonary and peripheral oedema and severe liver derangement. Transthoracic echocardiography confirmed severe mitral and tricuspid regurgitation, with right ventricular dysfunction and pulmonary hypertension (104 mm Hg). STS PROM score was 27.9 and logistic EuroSCORE was 37.8. Despite elective ventilation, he became progressively acidotic. The Heart Team proposed an emergency mitral ViV procedure. Results: Three-dimensional transoesophageal echocardiography (3DTOE) was used to confirm mitral biprosthetic valve failure (figure 1a-c). A small left anterolateral thoracotomy was made, and through a standard transapical approach, a size $29 \mathrm{~mm}$ SAPIEN device was implanted into the physio II ring under continuous 3DTOE guidance. Biplane images at $120-130^{\circ}$ (left) and $90^{\circ}$ (right) were used to guide the introducer sheath from the apex to the left atrium, across the degenerate mitral valve (figure 1d), and its position confirmed on zoomed 3D TOE (figure 1e). The balloon-expandable device was then positioned so that one-third of the final fully expanded device sat on the atrial aspect of the mitral annulus, and was deployed under rapid ventricular pacing (figure 1f). On balloon deflation, biplane (figure $1 \mathrm{~g}$ ) and 3D zoomed TOE (figure $1 \mathrm{~h}, \mathrm{i}$ ) views confirmed position and full expansion of the SAPIEN device. Post-operatively, the patient recovered quickly. On pre-discharge transthoracic echocardiography, there were 2 very small jets of paravalvular MR, peak trans-mitral gradient was $9 \mathrm{~mm} \mathrm{Hg}$, tricuspid regurgitation had become mild, and pulmonary pressures had reduced to $45 \mathrm{~mm}$ $\mathrm{Hg}$. He was discharged home on 16th post-operative day, independent of all activities of daily living. Conclusions: High-level periprocedural real-time $3 \mathrm{DTOE}$ is fundamental to guide effective transcatheter mitral valve-in-valve therapy.

\section{Transcatheter Mitral Valve-in-Ring Replacement: A Novel Therapy in a Challenging Case of Symptomatic Mitral Regurgitation}

\section{Risheen S. Reejhsinghani, Lucas S. Zier, Mark L. Villalon, Scot H. Merrick, Georg M. Wieselthaler, Atif N. Qasim, Vaikom S. Mahadevan}

University of California, San Francisco, San Francisco, CA, USA

Patient Demographics: Our patient is a 78 year-old female with a body-mass index of 15 and history of hypertension, restrictive lung disease, coronary artery disease (CAD) with right coronary artery (RCA) stent placement, and severe degenerative mitral regurgitation (MR). Three years prior, she underwent surgical repair of the posterior mitral leaflet and placement of a complete annuloplasty ring, with interval improvement in symptoms. Relevant History: The patient presented to our hospital with profound respiratory distress in the setting of pulmonary edema and hypertensive emergency. Echocardiography revealed severe MR with a flail posterior mitral leaflet. Despite medical therapy, she experienced eight episodes of flash pulmonary edema during the admission, with frequent ICU transfers. As the patient's co-morbidities

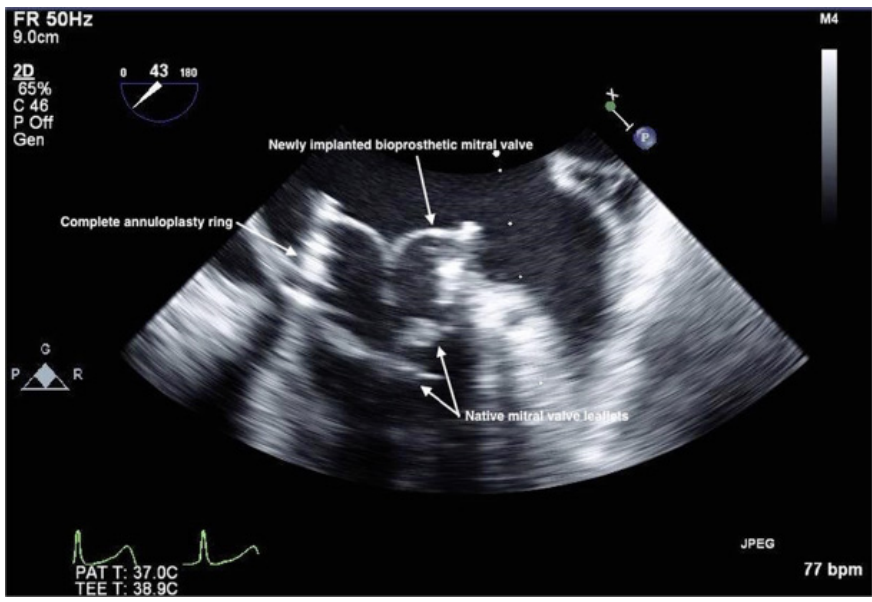

Fig. 1. (for Abstract of Risheen S. Reejhsinghani).

precluded further cardiac surgery, a decision was made to proceed with transcatheter mitral valve replacement within the existing annuloplasty ring. Pre-Operative Plan: Pre-operative cardiac catheterization revealed non-obstructive CAD with a patent RCA stent, $3+\mathrm{MR}$, mean pulmonary capillary wedge pressure of $31 \mathrm{~mm}$ $\mathrm{Hg}$ and mean pulmonary artery pressure of $36 \mathrm{~mm} \mathrm{Hg}$. Transesophageal echocardiography (TEE) showed a hyperdynamic left ventricle and partially flail posterior mitral leaflet with severe MR and systolic flow reversal in the pulmonary vein. Discussion of what was actually done and the challenges, deaths and complications encountered. (Unless case is still pending): The case was performed under TEE and fluoroscopic guidance. Following placement on venoarterial extracorporeal membrane oxygenation, transapical access was obtained and sizing-balloon valvuloplasty was performed. Under rapid pacing, a $29 \mathrm{~mm}$ Edwards Sapien XT valve was deployed across the native mitral valve, within the existing annular ring. Post-procedure TEE revealed two trace paravalvular leaks and mild-moderate central MR, significantly reduced from prior. Post-operatively, the patient had no further episodes of flash pulmonary edema. Repeat echocardiography showed significantly reduced $\mathrm{MR}$, and the patient reported marked symptomatic improvement at a post-discharge clinic visit. This case demonstrates the ability of novel valve replacement techniques to provide symptomatic benefit, even among complex patients with prior surgical interventions and limited therapeutic options. 


\section{Double-Sided Mitral Valve Repair: Transventricular Approach Augmented by Undersized Annuloplasty via Conventional Left Atriotomy}

\author{
Kerim Cagli ${ }^{1}$, Ali Baran Budak², Kemal Korkmaz², \\ Hikmet Selcuk Gedik², Eren Gunertem', Omer Cicek1, \\ Gokhan Lafci ${ }^{1}$, Serdar Gunaydin ${ }^{2}$ \\ ${ }^{1} Y u k s e k$ Ihtisas Training and Research Hospital, Ankara, \\ ${ }^{2}$ Numune Training and Research Hospital, Ankara, Turkey
}

Patient Demographics: A 59-year-old male patient was referred from emergency unit for urgent operation. Echocardiography showed severe ischemic mitral regurgitation (IMR), LV aneurysm with apical thrombi and minimally enlarged LA. LV ejection fraction was measured as 35\%, PAP as $50 \mathrm{~mm} \mathrm{Hg}$ and LVEDD as $70 \mathrm{~mm}$. Coronary angiography indicated occluded right coronary artery and significant lesions in D1 and OM3. Relevant History: Patient admitted to emergency unit with acute onset angina and dyspnea. Sudden cardiac arrest developed. After successful CPR, he underwent angiography and following insertion of IABP and medical stabilization, the patient was transferred to OR. Pre-Operative Plan: On evaluation of echocardiographic and angiographic data, we planned urgent $C A B G$ and $L V$ restoration as well as mitral valve repair from the ventricular side combined with undersized annuloplasty via conventional left atriotomy. Discussion of what was actually done and the challenges, deaths and complications encountered. (Unless case is still pending): LV aneurysm was incised and thrombi were extracted. One of the posterior papillary muscles was necrotic. Both papillary muscles were fixed using 4/0 pledgeted suture. Then anterior papillary muscle was approximated to those two using the same suture creating a singlestemmed bunch form. Restricted mitral leaflets were relieved by cutting tethered secondary chordae. LV aneurysmectomy was closed with internal double valor patch and plicated with external Teflon strips. Mitral ring annuloplasty was done by using \#28 Carpentier-Edwards rigid ring via conventional left atriotomy. Coronary revascularization was performed with saphenous vein grafts to PDA, OM3 and D1. Control echocardiography showed no significant MR in early postoperative period. Patient was discharged on the postoperative 7 th day. In patients with acute IMR and LV aneurysm, the absence of atrial dilation favors the transventricular approach by providing a better exposure for subvalvular apparatus, shortening CPB exposure and allowing more effective repair. The surgeon should pay attention not to interfere with anterior/ posterior leaflet chordae and papillary muscles with the annuloplasty sutures that may result in MR. Surgical restoration of LV causes changes of geometry and contraction resulting in surgical reverse modeling. Mitral valve repair should include annuloplasty to increase leaflet coaptation when feasible.

\section{Transapical Mitral Valve in Ring Procedure: Intraoperative Challenge and Late Complication}

\author{
Laura Besola, Augusto D'Onofrio, Erica Manzan, \\ Eleonora Bizzotto, Chiara Tessari, Tomaso Bottio, \\ Giuseppe Tarantini, Gino Gerosa \\ University of Padua, Padua, Italy
}

Patient Demographics: 77 year-old male with severe mitral regurgitation (MR) five years after mitral valve repair, with NYHA functional class III. History of atrial fibrillation, prostate carcinoma treated with hormone therapy, recurrent acute pancreatitis with following distal left spleen-pancreasectomy and diagnosis of intraductal mucinous papillary cancer. Euroscore II and STS score were $6 \%$ and $15 \%$, respectively. Relevant History: In 2010 the patient underwent mitral valve repair with annuloplasty with a 30 mm SJM Saddle Ring, 2 PTFE chordae implantation for P2 scallop prolapse, tricuspid annuloplasty with De Vega stitch and concomitant surgical treatment of atrial fibrillation with radiofrequency. Since July 2014 the patient presented severe mitral regurgitation together with symptoms of heart failure refractory to optimal medical therapy. Pre-Operative Plan: Preoperative Transesophageal echocardiography (TEE) was performed and showed severe MR due to P2-P3 prolapse. The patient was scheduled for mitral valvein-ring procedure through a transapical approach due to the high surgical risk. Discussion of what was actually done and the challenges, deaths and complications encountered. (Unless case is still pending): The patient underwent transapical mitral Valve-inRing implantation with a $26 \mathrm{~mm}$ Sapien-3 prosthesis. The angiographic control showed severe intra e para-prosthetic regurgitation consequent to an incomplete prosthesis deployment along the major axis of the mitral ring. The dysfunction was corrected with a 'double balloon' prosthesis postdilatation with a $19 \mathrm{~mm}$ and a 22 $\mathrm{mm}$ balloon. At final angiographic and TEE control in the cath-lab we observed mild PVL. The patient was discharged on 8th postoperative day in good clinical status; the transthoracic echocardiography showed only trivial residual MR and low transprosthesis gradients. After three months the patients was referred for pulmonary edema with diagnosis of severe intra and peri-prosthetic regurgitation. The TEE showed a big anterior leak and a smaller posterior leak together with incomplete coaptation of the leaflets. Therefore the patient underwent surgical mitral valve replacement. The intraoperative evaluation demonstrated a small anterior paravalvular leak and a large detachment of the Saddle ring along the anterior portion of the mitral annulus. The postoperative period was free from major adverse events and the patient was discharged in good clinical status. 


\section{Late presentation of Annular Rupture Initially Mimicking Prosthesis Dysfunction after Transcatheter Aortic Valve Implantation}

Sami M. Alnasser, Christopher Fiendle, Jennifer Day, Mark Osten, Robert J Cusimano, Ashish Shah, Eric Horlick

Peter Munk Cardiac Center, Toronto General Hospital, University of Toronto, Toronto, ON, Canada

Case Report: 75 year-old women with history of lymphoma treated with chest irradiation and chemotherapy 10 years ago. She was more recently diagnosed with oral cancer requiring mandibulofacial surgery. This however was hindered by the discovery of concomitant severe aortic valve stenosis on preoperative evaluation. Given the invasiveness of the planned oral surgery, aortic valve replacement was suggested. Transcatheter approach was favoured to minimize morbidity. Under conscious sedation, the patient underwent transfemoral TAVI using Sapien XT ${ }^{\circledR}$ size $26 \mathrm{~mm}$ valve, based on annular area of $410 \mathrm{~cm}^{2}$ by computed tomography (CT) with adequate deployment. Intraprocedural TransThoracic Echocardiography (TTE) was notable for a valvular, as well as paravalvular, colour-jet felt to be moderate in intensity. Post deployment aortogram showed no angiographic indication of com- plications. The patient was discharged home after three days of asymptomatic ambulation and stable hemodynamics. TTE on day three was comparable to prior study and unremarkable for major complications. Day five, the patient fainted while in washroom and presented to peripheral hospital where large pericardial thrombus is recognized. New cavity suggestive of pseuoaneurysm was also visualized between left and right coronary cusp with evidence of continuous flow. The patient was transferred urgently for further evaluation and management. Cardiac CT scan suggested subannular defect, contrast extravasation, and hemopericardium. Intraoperatively, mediastinal hematoma and subannular myocardial rupture were identified. After THV excision, a Bentall operation was performed using $25 \mathrm{~mm}$ Freestyle ${ }^{\circledR}$ porcine aortic root, and a bovine patch repair of the rupture site. Patient did well postoperatively with tracheostomy in place while awaiting oral surgery. Conclusion: Annular rupture can be subtle at initial stages and requires high index of suspicion. TransEsophageal Echo has been a workhorse tool for monitoring and diagnosis of procedural complications during TAVI. Given the practise shift toward 'Minimalist approach', forgoing TEE guidance, recognition of complications signs on surface echocardiography becomes vital. Therefore, subtle and explained TTE findings should prompt further evaluation TEE or CT imaging. 